Lehrstuhl für Massivbau

Institut für Baustoffe und Konstruktion

Technische Universität München

\title{
Verhalten von unbewehrtem Mauerwerk unter Erdbebenbeanspruchung
}

\author{
Detleff Clemens Schermer
}

Vollständiger Abdruck der von der Fakultät für Bauingenieur- und Vermessungswesen der Technischen Universität München zur Erlangung des akademischen Grades eines

Doktor-Ingenieurs

genehmigten Dissertation.

Vorsitzender:

Prüfer der Dissertation:
Univ.-Prof. Dr.-Ing. H. Kreuzinger

1. Univ.-Prof. Dr.-Ing. K. Zilch

2. Univ.-Prof. Dr.-Ing. E. Fehling, Universität Kassel

Die Dissertation wurde am 3.4.2003 bei der Technischen Universität München eingereicht und durch die Fakultät für Bauingenieur- und Vermessungswesen am 3.12.2004 angenommen. 
1. EINLEITUNG 1

$\begin{array}{lr}\text { 1.1. } & \text { Problemstellung }\end{array}$

$\begin{array}{ll}\text { 1.2. Zielsetzung und Lösungsweg } & 1\end{array}$

2. ERDBEBENEINWIRKUNG UND BAUWERKSVERHALTEN 3

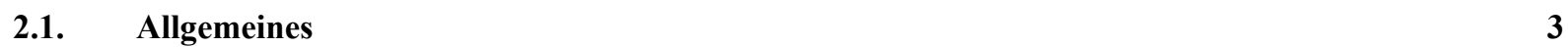

2.2. Erdbebenkenngrößen $\quad 3$

$\begin{array}{ll}\text { 2.2.1. Magnitude } & 3\end{array}$

$\begin{array}{ll}\text { 2.2.2. Intensität } & 4\end{array}$

2.2.3. Bodenbewegungskenngrößen $\quad 5$

2.2.4. Erdbebendauer $r$

2.2.5. Bodenbeschleunigungszeitverläufe $\quad 8$

$\begin{array}{lr}\text { 2.3. Baudynamik } & 9\end{array}$

2.3.1. Einmassenschwinger $r$

\begin{tabular}{lr} 
2.3.2. & 10 \\
\hline
\end{tabular}

$\begin{array}{lr}\text { 2.3.3. Dämpfung } & 10\end{array}$

$\begin{array}{lr}\text { 2.3.4. Modalanalyse } & 11\end{array}$

$\begin{array}{lr}\text { 2.3.5. Antwortspektrum } & 11\end{array}$

2.4. Tragfähigkeit und Duktilität $\quad 12$

$\begin{array}{lr}\text { 2.5. Normung } & 15\end{array}$

\begin{tabular}{lr} 
2.5.1. Allgemeines & 15 \\
\hline
\end{tabular}

\begin{tabular}{lr} 
2.5.2. & Erdbebengefährdung \\
\hline
\end{tabular}

$\begin{array}{lr}\text { 2.5.3. Antwortspektrum } & 17\end{array}$

\begin{tabular}{lr} 
2.5.4. & Untergrund \\
\hline
\end{tabular}

3. STAND DER FORSCHUNG 21

\begin{tabular}{lr} 
3.1. & Bruch- und Materialmodelle \\
\hline
\end{tabular}

$\begin{array}{lr}\text { 3.2. } & \text { Bauteilversuche }\end{array}$

$\begin{array}{ll}\text { 3.3. } & 36\end{array}$

$\begin{array}{llr}\text { 3.4. } & \text { Schadensbilder - allgemeine Untersuchungen } & 38\end{array}$

4. MATERIALEIGENSCHAFTEN 41

$\begin{array}{lr}\text { 4.1. Steine } & 43\end{array}$

$\begin{array}{lr}\text { 4.1.1. Druckversuche } & 43\end{array}$

4.1.2. Biegezugversuche $\quad 45$

$\begin{array}{ll}\text { 4.1.3. } & \text { Verformungskenngrößen }\end{array}$

\begin{tabular}{lr} 
4.2. & Mörtel \\
\hline
\end{tabular}

4.3. Mauerwerk $r$

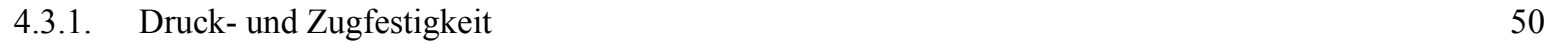

4.3.2. Kennwerte zur Schubfestigkeit $\quad 52$

$\begin{array}{lr}\text { 4.3.3. Verformungskenngrößen } & 54\end{array}$ 


\section{EXPERIMENTELLE UNTERSUCHUNGEN}

5.1. Übersicht 57

5.1.1. Allgemeines

5.1.2. Statisch-zyklische Versuche

5.1.3. Pseudodynamik

5.1.4. Zielsetzung und Vorgehensweise

5.2. Grundlagen der Pseudodynamik

5.2.1. Prinzip

5.2.2. Numerik

5.2.2.1. Explizites Zeitschrittverfahren $\quad 61$

$\begin{array}{lll}\text { 5.2.2.2. } & \text { Implizites Zeitschrittverfahren } & 62\end{array}$

$\begin{array}{lll}\text { 5.3. } & \text { Erdbebeneinwirkung } & 64\end{array}$

5.4. Erste Simulationsstufe - Einmassenschwingersystem $\quad 65$

5.4.1. Allgemeines 65

5.4.2. Gebäudemodell - Ersatzsystem $\quad 69$

5.4.3. Ablauf 70

$\begin{array}{lll}\text { 5.4.4. Ergebnisse } & 72\end{array}$

$\begin{array}{ll}\text { 5.4.5. Vergleichsrechnungen } & 76\end{array}$

$\begin{array}{lll}\text { 5.4.6. Zusammenfassung } & 80\end{array}$

5.5. Zweite Simulationsstufe - Mehrmassenschwingersystem $\quad 80$

5.5.1. Allgemeines $\quad 80$

5.5.2. Gebäudemodell - Ersatzsystem $\quad 82$

$\begin{array}{llr}\text { 5.5.3. Sub-Struktur } & 85\end{array}$

$\begin{array}{ll}\text { 5.5.4. Ablauf } & 91\end{array}$

5.5.5. Ergebnisse 94

5.5.5.1. Versagensbilder 95

$\begin{array}{llr}\text { 5.5.5.2. Steifigkeit } & 99\end{array}$

$\begin{array}{lll}\text { 5.5.6. } & \text { Vergleichsrechnung } & 102\end{array}$

$\begin{array}{lll}\text { 5.5.7. Tragfähigkeit } & 104\end{array}$

$\begin{array}{ll}\text { 5.5.8. Zusammenfassung } & 110\end{array}$

6. MATERIALMODELL 113

$\begin{array}{lll}\text { 6.1. } & \text { Einleitung } & 113\end{array}$

6.1.1. Anisotropie 113

6.1.2. Makro- und Mikromodellierung 113

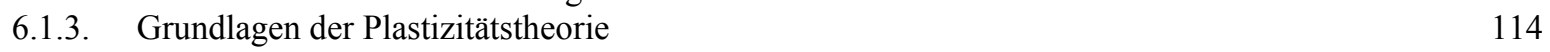

6.2. Materialmodell, Bruch- und Fließbedingungen 116

6.2.1. Schubtragmodell $\quad 116$

6.2.2. Fließregeln $\quad 119$

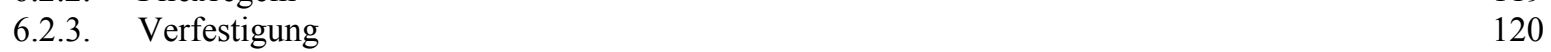

$\begin{array}{lll}\text { 6.2.3.1. Normalbeanspruchung } & 120\end{array}$

$\begin{array}{lll}\text { 6.2.3.2. Schubbeanspruchung } & 123\end{array}$

$\begin{array}{ll}\text { 6.3. Implementierung des Stoffgesetzes } & 127\end{array}$

$\begin{array}{ll}\text { 6.3.1. Allgemeines } & 127\end{array}$

$\begin{array}{ll}\text { 6.3.2. Ablauf } & 128\end{array}$

$\begin{array}{lll}\text { 6.4. } & \text { Verifizierung des Modells } & 130\end{array}$

6.4.1. Wand A 131

6.4.2. Wand E 133

$\begin{array}{ll}\text { 6.4.3. Wand KS4 } & 135\end{array}$ 
\begin{tabular}{ll} 
6.5. & Parameterstudie \\
\hline
\end{tabular}

$\begin{array}{lr}\text { 6.6. } & 140\end{array}$

7. ZUSAMMENFASSUNG UND AUSBLICK 143

LITERATURVERZEICHNIS 145

$\begin{array}{ll}\text { BEZEICHNUNGEN } & 157\end{array}$

$\begin{array}{ll}\text { ANHANG } & 161\end{array}$

$\begin{array}{lr}\text { A.1 Zeitverläufe und Antwortspektren } & 161\end{array}$

$\begin{array}{lr}\text { A.2 Rissbilder } & 165\end{array}$

$\begin{array}{lr}\text { A.2.1 Erste Versuchsstufe } & 165\end{array}$

$\begin{array}{lr}\text { A.2.2 Zweite Versuchsstufe } & 167\end{array}$

$\begin{array}{lr}\text { A.3 Kraft-Verformungs-Beziehungen } & 173\end{array}$

$\begin{array}{lr}\text { A.3.1 Erste Versuchsstufe } & 173\end{array}$

$\begin{array}{lr}\text { A.3.2 Zweite Versuchsstufe } & 176\end{array}$ 


\section{Einleitung}

\subsection{Problemstellung}

Im Zuge der Harmonisierung der europäischen Baunormen wird mit der ENV 1998 [27] ein im Vergleich zur momentan gültigen deutschen Erdbebennorm DIN 4149-1 (1981-04) [20] neues semi-probabilistisches Sicherheitskonzept angesetzt. Der Nachweis der Erdbebensicherheit hat dabei, wie international üblich, für ein Beben mit einer Referenzwiederkehrperiode von 475 Jahren zu erfolgen. Für deutsche Erdbebengebiete ergeben sich bei der darauf aufbauenden Überarbeitung der nationalen Norm E DIN 4149 (2002-10) [22] deutliche Lasterhöhungen.

In der neuen Normengeneration wird der günstige Einfluss des nichtlinearen Bauwerksverhaltens durch einen $u$. a. vom Baustoff und der Konstruktionsart abhängigen Verhaltensfaktor $q$ beschrieben. Für unbewehrte Mauerwerkskonstruktionen wird dieser mit 1,5 angesetzt, was deutlich unter den Werten für Stahlbeton-, Stahl- und Holzkonstruktionen liegt. Durch diese Festlegung wird der rechnerische Nachweis mit den üblichen Bemessungsansätzen für eine Vielzahl von Mauerwerksgebäuden nicht mehr eingehalten.

In teilweisem Widerspruch dazu stehen die Erfahrungen in deutschen Erdbebengebieten, denen zufolge bei Beben, die bereichsweise an die Bemessungsintensität heranreichten, an vorschriftsmäßig ausgeführten Mauerwerkskonstruktionen keine die Standsicherheit gefährdende Schäden auftraten.

Für die Mehrzahl üblicher Hochbauten in deutschen Erdbebengebieten ist beim Nachweis der Horizontaleinwirkungen momentan der Lastfall Wind maßgebend. Die Bemessung der aussteifenden Bauteile unter kombinierter Normalkraft- und Schubbeanspruchung erfolgt dabei nach DIN 1053-1 [17]. Der Schubnachweis basiert hier auf der Theorie von Mann / Müller $[73,74]$ aus den 70er Jahren, mit welcher die bei den Versuchen an damals gebräuchlichen Mauerwerksarten aufgetretenen Versagenslasten und -bilder gut beschrieben werden konnten. Die Entwicklung in den letzten 25 Jahren führte zu veränderten Steinformaten, z.B. großformatigen Planelementen mit reduziertem Überbindemaß, zu veränderten Lochbildern und zu neuen Mörtelarten, z.B. Dünnbett- oder Leichtmörtel. Zudem werden vermehrt Stoßfugen aus Wärmeschutz- und Rationalisierungsgründen planmäßig unvermörtelt ausgeführt.

In Anbetracht dieser Neu- und Weiterentwicklungen und den Besonderheiten der Beanspruchungen im Lastfall Erdbeben sind die vorhandenen Berechnungsmodelle kritisch zu hinterfragen.

\subsection{Zielsetzung und Lösungsweg}

Es stellt sich daher die Aufgabe, das Trag- und Verformungsverhalten unbewehrter Mauerwerkskonstruktionen des üblichen Hochbaus für den Lastfall Erdbeben realitätsnah zu untersuchen und zu beschreiben. Aufgrund der Tatsache, dass passende experimentelle Untersuchungen an in Deutschland heutzutage üblichen Stein-Mörtel-Kombinationen - bis auf wenige Ausnahmen - nicht vorhanden sind, wurden bereits im Vorfeld dieser Arbeit derartige Versuche als dringend erforderlich angesehen.

In einem ersten Schritt wird in Kapitel 2, aufbauend auf Literaturangaben, die Erdbebeneinwirkung mit den besonderen Bedingungen in Deutschland dargestellt.

Anschließend wird in Kapitel 3 im Zuge einer Literatursichtung der Stand der Forschung zum Erdbebentragverhalten von unbewehrten Mauerwerksbauten dargestellt und bewertet. Dabei 
werden neben vorhandenen Bruch- und Materialmodellen auch Bauteilversuche sowie allgemeine Untersuchungen zu aufgetretenen Schadensbildern erfasst.

Die Ergebnisse eigener experimenteller Untersuchungen werden in Kapitel 5 vorgestellt. An geschosshohen Wänden wurden pseudodynamische Erdbebenversuche durchgeführt. Die gewählte Versuchsmethode erlaubt eine realitätsnahe Betrachtung des dynamischen Verhaltens von Strukturen, ohne auf die äußerst aufwändigen Einrichtungen von Rütteltischversuchen zurückzugreifen. Durch die Berücksichtigung einer Sub-Struktur konnten die Randbedingungen der untersuchten Tragwand im Gesamtgebäude wirklichkeitsnah erfasst werden.

Als Materialien dienten einige der in Deutschland gebräuchlichsten Stein-MörtelKombinationen, deren Materialparameter in Kapitel 4 zusammengefasst sind. Insgesamt wurden zehn Wände mit den Abmessungen von 2,5 $\mathrm{m} \cdot 2,5 \mathrm{~m} \cdot 0,175 \mathrm{~m}$ in 40 Versuchen geprüft.

Für weitergehende Untersuchungen wird in Kapitel 6 ein Materialmodell auf Basis der Plastizitätstheorie entwickelt und an den eigenen Versuchsergebnissen überprüft. Das Modell hat den Vorteil, dass nur eine geringe Zahl von Eingangsparametern benötigt wird, die entweder in Standardversuchen bestimmt oder der Literatur entnommen werden können. Im Zuge einer Parameterstudie werden die verschiedenen Einflussfaktoren auf das Trag- und Verformungsverhalten untersucht.

Eine abschließende Zusammenfassung und Wertung der experimentellen und rechnerischen Ergebnisse erfolgt in Kapitel 7. 


\section{Erdbebeneinwirkung und Bauwerksverhalten}

\subsection{Allgemeines}

Der Großteil der weltweiten Erdbebengefährdung geht von tektonischen Beben aus. Hierbei wird die durch die Plattenbewegung aufgestaute Energie während eines Bruchvorganges schlagartig freigesetzt. Der Großteil dieser Energie wird dabei in Wärme umgewandelt und nur ein geringer Anteil als Wellenenergie abgestrahlt.

$\mathrm{Zu}$ weiteren Entstehungsursachen von Erdbeben zählen zudem:

- Vulkanaktivität

- Einsturz von unterirdischen Hohlräumen (Einsturzbeben)

- stauseeinduzierte Beben

- künstliche Ereignisse, z. B. Sprengstoffexplosionen

Da die freigesetzten Energiemengen im Vergleich zu tektonischen Beben relativ gering sind, und sie nur lokal begrenzte Wirkung aufweisen, werden sie bei der Beurteilung der Erdbebengefährdung üblicherweise vernachlässigt.

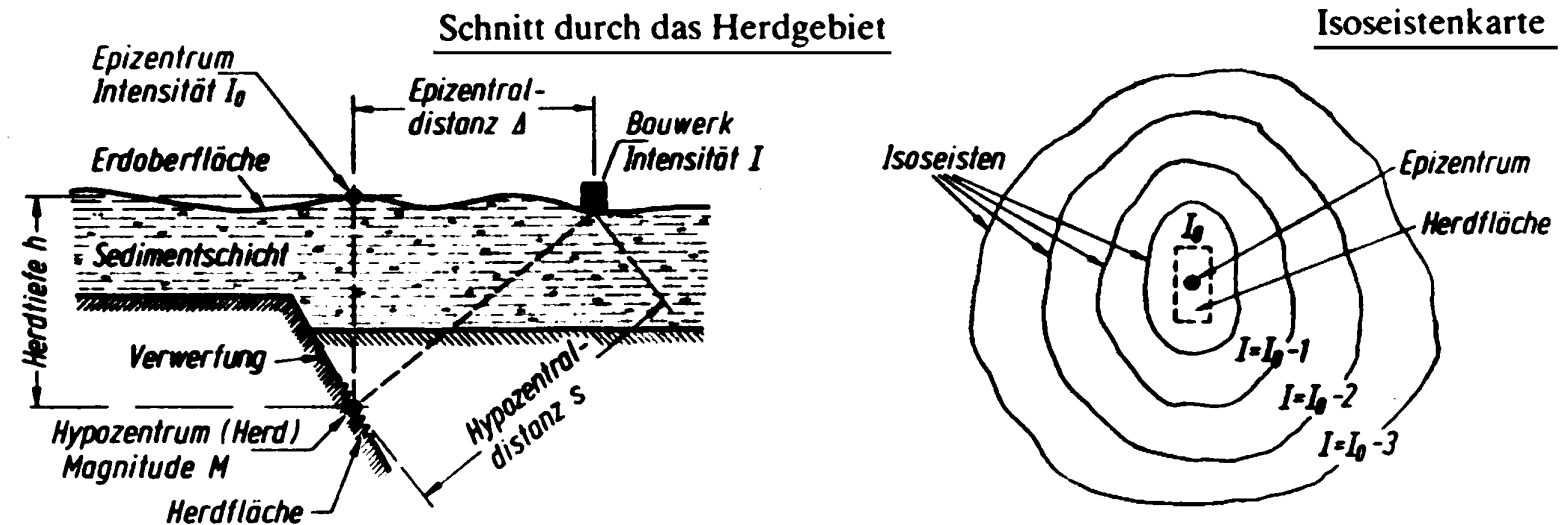

Abbildung 1: Bezeichnungen bei einem Dislokationsbeben - aus Müller / Keintzel [91]

Bei tektonischen Beben können als Herdmechanismen Horizontalverschiebungen, Abschiebungen sowie Über- und Unterschiebungen auftreten. Für die Lokalisierung des Bebenherdes wird die Bruchfläche vereinfacht auf ein punktuelles Hypozentrum reduziert.

Die Differenzierung der bei einem Beben ausgesandten Wellen erfolgt nach der Geschwindigkeit sowie der Ausbreitungs- und Bewegungsart. Es wird zwischen $P_{-}, S-$, Rayleigh- und Love-Wellen unterschieden. Die beiden ersteren werden als Raumwellen bezeichnet, die sich nur im Erdinneren ausbreiten und an der Oberfläche bzw. an Schichtgrenzen reflektiert bzw. gebrochen werden. Die Love- und Rayleigh-Wellen treten als Oberflächenwellen nur nahe der Geländeoberfläche auf und werden mit zunehmender Epizentralentfernung stark gedämpft.

\subsection{Erdbebenkenngrößen}

\subsubsection{Magnitude}

Mit der Magnitude $M$ wird die bei einem Erdbebenereignis freigesetzte Wellenenergie $E[\mathrm{~J}]$ beschrieben. Die Definition erfolgt mit: 


$$
M=\frac{2}{3}(\lg E-4,8)
$$

Die Bestimmung der Magnitude eines aufgetretenen Erdbebens erfolgt aus den gemessenen maximalen Geschwindigkeiten der Erdbebenwellen. Entsprechend ihrer Ermittlung wird dabei zwischen

- Lokalmagnitude $M_{l}$ (gilt für Nahbeben bis zu einer Epizentraldistanz von ca. $500 \mathrm{~km}$ ),

- Oberflächenmagnitude $M_{S}$ (aus dem Maximalwert der Oberflächenwellengeschwindigkeit) und

- Fernmagnitude $M_{b}$ (aus dem Maximalwert der Raumwellengeschwindigkeit) unterschieden.

Durch den logarithmischen Verlauf der Magnitudenskala (1) ergibt eine Differenz von einer Stufe eine um den Faktor $f=10^{1,5}=32$ größere Menge an freigesetzter Energie. Zwischen Magnitude und Größe der Herdfläche und -dislokation können empirische Zusammenhänge (s. Klein [57] und Müller / Keintzel [91]) dargestellt werden. Die maximal theoretisch auftretende Magnitude ist durch die Größe der Bruchfläche und der Gesteinsfestigkeit beschränkt und liegt in der Größenordnung von etwa 9,0. Nachfolgend ist die entsprechend der Referenzwiederkehrperiode bzw. Eintretenswahrscheinlichkeit zu erwartende Magnitude für das Gebiet des mittleren und unteren Rheins sowie für die gesamte Erde grafisch dargestellt.

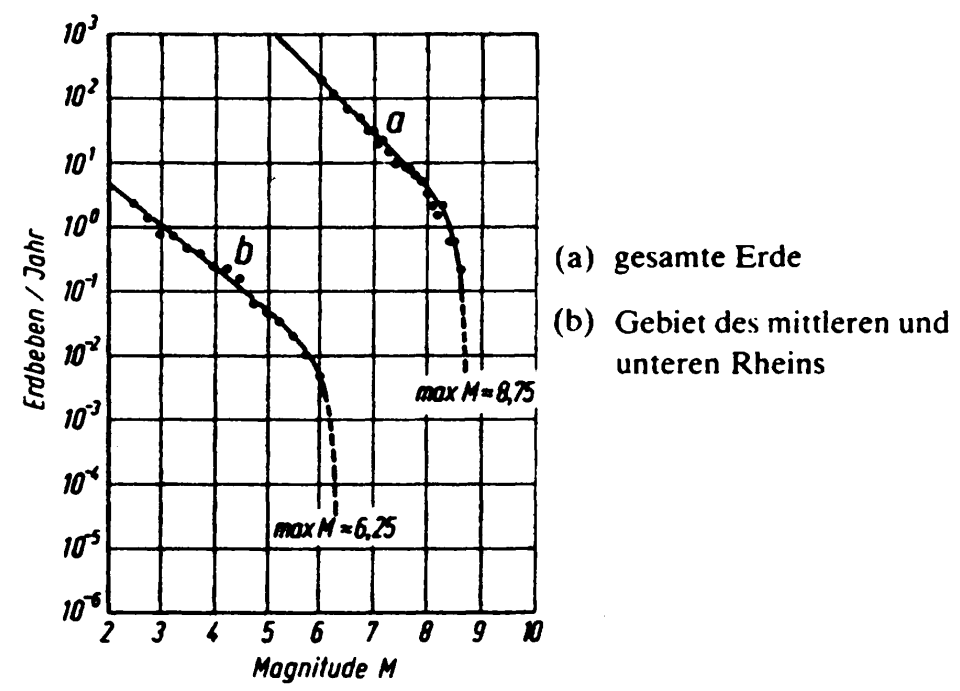

Abbildung 2: Abhängigkeit der zu erwartenden Magnitude von der Wiederkehrperiode für verschiedene Gebiete - aus Müller / Keintzel [91]

\subsubsection{Intensität}

Die Wirkung eines Bebens auf der Erdoberfläche, d.h. auf Menschen, Bauwerke und die Umwelt allgemein, wird durch die Intensität $I$ beschrieben. Da diese Wirkung von der Magnitude, der Herdentfernung und von den lokalen Verhältnissen, wie z. B. dem lokalen Baugrund bestimmt wird, ist die Intensität abhängig vom Ort der Betrachtung und bezogen auf das Ereignis eine lokale Größe. Für die Beschreibung existiert eine Vielzahl von Skalen, z. B. die Modifizierte-Mercalli-Skala (MM), die Medvedev-Sponheuer-Karnik-Skala (MSK 64) oder die aktuelle Europäische Intensitätsskala EMS 1992, die nachfolgend dargestellt ist. 


\begin{tabular}{|c|c|c|}
\hline $\begin{array}{c}\text { EMS } \\
\text { Intensität }\end{array}$ & Definition & Beschreibung der maximalen Wirkung \\
\hline $\bar{I}$ & nicht fühlbar & Nicht fühlbar. \\
\hline II & $\begin{array}{c}\text { kaum } \\
\text { bemerkbar }\end{array}$ & Nur sehr vereinzelt von ruhenden Personen wahrgenommen. \\
\hline III & schwach & $\begin{array}{l}\text { Von wenigen Personen in Gebäuden wahrgenommen. Ruhende Personen fühlen } \\
\text { ein leichtes Schwingen oder Erschüttern. Lampen schwingen leicht. }\end{array}$ \\
\hline IV & deutlich & $\begin{array}{l}\text { Im Freien vereinzelt, in Gebäuden von vielen Personen wahrgenommen. Einige } \\
\text { Schlafende erwachen. Geschirr und Fenster klirren, Türen klappern. }\end{array}$ \\
\hline $\mathbf{V}$ & stark & $\begin{array}{c}\text { Im Freien von wenigen, in Gebäuden von den meisten Personen wahrgenommen. } \\
\text { Viele Schlafende erwachen. Wenige werden verängstigt. Gebäude werden insge- } \\
\text { samt erschüttert. Hängende Gegenstände pendeln stark, kleine Gegenstände } \\
\text { werden verschoben. Gelegentlich treten Haarrisse im Verputz auf und in wenigen } \\
\text { Fällen Abfall kleiner Putzstücke. }\end{array}$ \\
\hline VI & $\begin{array}{l}\text { leichte } \\
\text { Gebäude- } \\
\text { schäden }\end{array}$ & $\begin{array}{l}\text { Viele Personen erschrecken und flüchten ins Freie. Einige Gegenstände fallen um. } \\
\text { An einigen Häusern entstehen leichte Schäden (Risse im Verputz), vornehmlich } \\
\text { an Häusern in schlechterem Zustand, feine Mauerrisse, Abfallen von Verputz- und } \\
\text { Schornsteinteilen. }\end{array}$ \\
\hline VII & $\begin{array}{l}\text { Gebäude- } \\
\text { schäden }\end{array}$ & $\begin{array}{l}\text { Die meisten Personen erschrecken und flüchten ins Freie. Möbel werden verscho- } \\
\text { ben. Gegenstände fallen in großen Mengen aus Regalen. An vielen Häusern } \\
\text { solider Bauart treten mäßige Schäden auf (Mauerrisse). Vornehmlich Gebäude in } \\
\text { schlechterem Zustand zeigen größere Mauerrisse, vereinzelt Einsturz von Zwi- } \\
\text { schenwänden. }\end{array}$ \\
\hline VIII & $\begin{array}{l}\text { schwere } \\
\text { Gebäude- } \\
\text { schäden }\end{array}$ & $\begin{array}{c}\text { Viele Personen verlieren das Gleichgewicht. Selbst schwere Möbel werden } \\
\text { verschoben und zum Teil umgeworfen. An vielen Gebäuden einfacher Bausub- } \\
\text { stanz treten schwere Schäden auf; d. h. Giebelteile und Dachgesimse stürzen ein. } \\
\text { Einige Gebäude sehr einfacher Bauart stürzen ein. }\end{array}$ \\
\hline IX & zerstörend & $\begin{array}{l}\text { Allgemeine Panik unter den Betroffenen. Sogar gut gebaute gewöhnliche Bauten } \\
\text { zeigen schwere Schäden und teilweisen Einsturz tragender Bauteile. Viele schwä- } \\
\text { chere Bauten stürzen ein }\end{array}$ \\
\hline $\mathbf{X}$ & $\begin{array}{l}\text { sehr zerstö- } \\
\text { rend }\end{array}$ & Viele gut gebaute Häuser werden zerstört oder erleiden schwere Beschädigungen. \\
\hline $\mathbf{X I}$ & verwüstend & $\begin{array}{c}\begin{array}{l}\text { Die meisten Bauwerke, selbst mit guter erdbebengerechter Konstruktion, werden } \\
\text { zerstört. }\end{array} \\
\end{array}$ \\
\hline XII & $\begin{array}{l}\text { vollständig } \\
\text { verwüstend }\end{array}$ & Nahezu alle Konstruktionen werden zerstört. \\
\hline
\end{tabular}

Tabelle 1: Verkürzte Europäische Intensitätsskala EMS 1992 - aus Grünthal, Mayer-Rosa, Lenhardt [38]

Es existieren mehrere empirische Verknüpfungen zwischen Epizentralintensität $I_{0}$ auf der einen Seite und der Magnitude $M$ und Herdtiefe $h$ auf der anderen Seite. Für europäische Verhältnisse mit Herdtiefen $h<50 \mathrm{~km}$ kann z. B. nach Müller / Keintzel [91] folgender Zusammenhang angegeben werden:

$$
M=0,5 \cdot I_{0}+\lg h+0,35
$$

Allgemein ist der Variationsbereich der Beziehungen als groß zu bezeichnen.

\subsubsection{Bodenbewegungskenngrößen}

Für die Wirkung eines Bebens auf Bauwerke sind von den auftretenden drei Bodenbewegungsgrößen Verschiebung, Geschwindigkeit und Beschleunigung die ersten beiden i. d. R. von untergeordneter Bedeutung. Bei angenommener steifer Gründung und fehlender Verbindung zu Nachbarbauwerken resultieren aus diesen beiden Größen keinerlei Beanspruchungen in der Konstruktion. Die Bodenbeschleunigung dagegen, die als Fußpunkterregung auf das 
System wirkt, erzeugt über die dynamische Wirkung in dem Tragwerk Beanspruchungen. Diese hängen von deren Maximalwert $a_{0}$ sowie von der Frequenzverteilung der Anregung und dem zeitlichen Verlauf ab. Die Beschreibung dieser Wirkung erfolgt üblicherweise mit Hilfe von Antwortspektren (s. Abschnitt 2.3.5). Bei dieser Vorgehensweise im Frequenzbereich geht die Information des zeitlichen Verlaufes der Größen verloren, da nur der zumeist einmalig aufgetretene Extremwert angegeben ist.

Entscheidend für das Bauwerksverhalten ist die horizontale Einwirkung, da die Bauwerke abgesehen von Windlasten - üblicherweise nicht für größere horizontale Lasten ausgelegt werden. Vertikale Bewegungsgrößen können für übliche Hochbauten vernachlässigt werden, da der Sicherheitsabstand der hauptsächlich auf Vertikallasten ausgelegten Konstruktion ausreichend groß ist. Eine Ausnahme hiervon stellt die Schubbemessung von unbewehrtem Mauerwerk dar, bei der durch eine Erhöhung oder Reduzierung der Normaldruckspannungen die aufnehmbaren Schubspannungen abgemindert werden können (s. Abschnitt 6.2.1).

Der Maximalwert der horizontalen Bodenbeschleunigung $a_{0}\left[\mathrm{~m} / \mathrm{s}^{2}\right]$ kann mit Hilfe empirischer Beziehungen aus der Intensität I ermittelt werden, z. B. nach Müller / Keintzel [91]

$$
I=b \cdot \lg a_{0}+c
$$

mit den Parametern $b=2,0 \div 3,0$ und $c=1,5 \div 2,7$. Weitere Angaben zur Beziehung von Intensität und Maximalwert der Bodenbeschleunigung sind in der Literatur, z. B. Hosser [51], Klein [58], Paulay / Bachmann / Moser [102] und Zilch [160] zu finden.

Eine weitere Möglichkeit der Ermittlung von $a_{0}$ besteht in Abhängigkeit von der Hypozentraldistanz $s$ und der Magnitude $M$ :

$$
a_{0}=\frac{1230 \cdot e^{0,8 \cdot M}}{\left(s+s_{0}\right)^{2}}
$$

Der Parameter $s_{0}[\mathrm{~km}]$ ist für mitteleuropäische Verhältnisse mit 5,0<M<6,0 nach Müller / Keintzel [91] zu $s_{0}=13 \mathrm{~km}$ anzusetzen.

\subsubsection{Erdbebendauer}

Die Gesamtdauer eines Erdbebenereignisses hängt von einer Vielzahl von Parametern ab, wie z. B. von

- der Art des Bruchvorganges, d. h. Hebe-, Tauch- oder Scherbruch,

- der Herdtiefe und Hypozentraldistanz,

- der Größe der Bruchfläche mit der resultierenden Herddauer (die wiederum direkt von der Magnitude abhängt),

- der Ausbreitungsvorgänge (Reflexionen, Absorption, Frequenzmodifikation) und

- lokalen topologischen Verhältnissen.

Entscheidend für die Beschreibung der Wirkung eines Erdbebens auf Bauwerke ist die sog. Starkbebendauer $T_{S B D}$. Neben mehreren anderen Definitionen (s. Hosser [51], Meskouris [79, 80, 81]) wird am gebräuchlichsten die Spanne bezeichnet, die zwischen den beiden Zeitpunkten liegt, in denen 5\% und 95\% der Bebenenergie freigesetzt sind. Der zeitliche Verlauf der 
Energiefreisetzung wird durch das Husid-Diagramm als Quadrat der Bodenbeschleunigung beschrieben:

$$
H(t)=\frac{\int_{t=0}^{t} a^{2} d t}{\int_{t=0}^{t=s} a^{2} d t}
$$

Die Abhängigkeit der Starkbebendauer von der Magnitude kann über empirische Zusammenhänge bestimmt werden, wie beispielhaft in Abbildung 3 dargestellt.

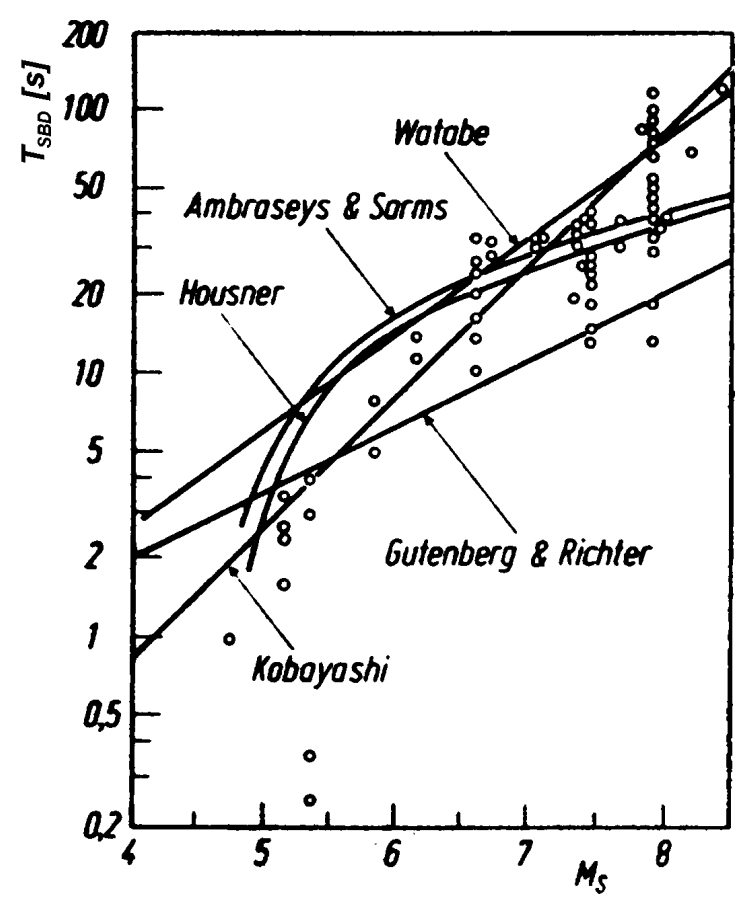

Abbildung 3: Zusammenhang zwischen Magnitude und Starkbebendauer - aus Müller / Keintzel [91]

In einem vorläufigen Entwurf des Nationalen Anwendungsdokumentes zur ENV 1998 [92] wurden Vorschläge zur Mindestzeitdauer des stationären Teils des Beschleunigungszeitverlaufes $T_{s}$ angegeben. Diese dienen der Generierung künstlicher Zeitverläufe oder der Beurteilung aufgezeichneter Zeitverläufe.

\begin{tabular}{|l|c|c|c|}
\hline & \multicolumn{3}{|c|}{ Erdbebenzone } \\
& $\mathbf{1}$ & $\mathbf{2}$ & $\mathbf{3}$ \\
\hline $\boldsymbol{T}_{s}[\mathbf{s}]$ & 1,5 & 2,0 & 3,0 \\
\hline
\end{tabular}

Tabelle 2: Mindestzeitdauer des stationären Teils $T_{s}$ des Beschleunigungszeitverlaufes - aus $V$-NAD zur ENV 1998 [92]

Für nichtlineare Berechnungen nach dem Zeitverlaufsverfahren sind dabei nach ENV 1998 [27] allgemein mindestens drei Bodenbeschleunigungszeitverläufe anzusetzen. Die maximale Abweichung zwischen elastischem Antwortspektrum und Zielantwortspektrum darf jedoch nicht mehr als 10\% betragen. Liegen keine genaueren Erkenntnisse (z.B. NAD zur ENV 1998 
[92] bzw. Tabelle 2 oder Abbildung 3) vor, ist der stationäre Teil des Zeitverlaufes zu $T_{S}=10 \mathrm{~s}$ anzunehmen. Zusätzlich ist der Zeitverlauf auf den Einhängewert von $\gamma_{l} \cdot S \cdot a_{g}$ bei $T=0 \mathrm{~s}$ zu skalieren.

Mit einer Intensitätsfunktion kann bei künstlich generierten oder aufgezeichneten Zeitverläufen der Verlauf der Größe der Erdbebeneinwirkung gesteuert werden. Eine einfache Möglichkeit besteht in Form einer tri-linearen Funktion, die sich aus einem linearen Anstieg, einem konstanten Plateau und einem linearen Abfall zusammensetzt (s. Kapitel 5).

Untersuchungen zur Beschreibung realistischer seismischer Lastannahmen für deutsche Erdbebengebiete wurden von Hosser [51] durchgeführt (s. a. Klein [59]). Als Ergebnisse liegen u. a. Vorschläge für Bemessungsspektren in Abhängigkeit der Intensitäts- und Untergrundklassen sowie statistische Parameter der Starkbebendauer vor.

\begin{tabular}{|c|c|c|c|c|}
\hline \multirow[t]{2}{*}{ Untergrundklasse } & \multicolumn{3}{|c|}{ Intensitätsklasse } & \multirow{2}{*}{$\begin{array}{c}\text { Hypozentral- } \\
\text { distanz } \\
s<60 \mathrm{~km}\end{array}$} \\
\hline & $\begin{array}{c}1 \\
\left(I_{M S K}=6 \div 7\right)\end{array}$ & $\begin{array}{c}2 \\
\left(I_{M S K}=7 \div 8\right)\end{array}$ & $\begin{array}{c}3 \\
\left(I_{M S K}=8 \div 9\right)\end{array}$ & \\
\hline $\begin{array}{l}\text { A: Holozän, Lockersedimente und } \\
\text { Böden geringer Impedanz, } d_{\min }=5 \mathrm{~m}\end{array}$ & $4,5 \pm 3,7$ & $5,9 \pm 1,3$ & $3,5 \pm 1,3$ & $5,0 \pm 3,3$ \\
\hline $\begin{array}{l}\text { M: mittelsteife, halbverfestigte } \\
\text { Sedimente }\end{array}$ & $2,6 \pm 1,0$ & $2,7 \pm 1,4$ & $2,5 \pm 1,3$ & $2,7 \pm 2,1$ \\
\hline $\begin{array}{l}\text { R: Fels, gut verfestigtes wenig porö- } \\
\text { ses Gestein }\end{array}$ & $1,1 \pm 0,8$ & $1,8 \pm 1,3$ & $3,1 \pm 1,8$ & $1,7 \pm 1,4$ \\
\hline
\end{tabular}

Tabelle 3: Statistische Parameter der Starkbebendauer $T_{S D B}$ in Abhängigkeit der Untergrund-IntensitätsKombination - aus Hosser [51]

Weitere Angaben zu der Starkbebendauer sind in der Literatur, z. B. Kaiser [53], Klein [58], Müller / Keintzel [91], Paulay / Bachmann / Moser [102] und Rackwitz [109] zu finden.

\subsubsection{Bodenbeschleunigungszeitverläufe}

Für die Untersuchung des Erdbebentragverhaltens von Bauwerken im Zeitbereich sind geeignete Zeitverläufe - entweder synthetische oder aufgezeichnete - erforderlich. Bei natürlichen Zeitverläufen ist zu beachten, dass sie die Charakteristik des aufgetretenen Bebens zusammen mit dem Ort der Messung und den Randbedingungen der Wellenweiterleitung vom Herd zum Beobachter widerspiegeln. Die aufgezeichneten Starkbebenzeitverläufe können bei Bedarf zur Korrektur lokaler Einflüsse noch modifiziert werden, z. B. um die Charakteristik der Laufwege zum Messpunkt herauszufiltern (s. Vanmarcke et. al. [152, 153]).

In Deutschland sind keine geeigneten Aufzeichnungen von Starkbebenzeitverläufen vorhanden, bzw. können nur durch Umrechnung von in großer Entfernung gemessenen Geschwindigkeitszeitverläufen erstellt werden. Für Berechnungen nach dem Zeitverlaufsverfahren ist man daher darauf angewiesen, synthetische Zeitverläufe anzusetzen oder Aufzeichnungen aus anderen Erdbebengebieten mit ähnlichen Bedingungen heranzuziehen.

Die Generierung künstlicher Zeitverläufe erfolgt i.d. R. auf Basis des elastischen NormZielantwortspektrums mit dem Maximalwert der Bodenbeschleunigung. Eine Möglichkeit der Generierung besteht mit Hilfe des weißen Rauschens, das mehrere Frequenzfilterungen durchläuft und damit an das Zielspektrum angepasst wird (Meskouris [79, 80, 81], Rackwitz [109]). Die Intensität über die gesamte Erdbebendauer kann über eine Funktion angepasst werden - z. B. zur Erfassung der Gesamtbeben- und Starkbebendauer. Diese Vorgehensweise wurde auch bei der Generierung der für die Versuche erforderlichen Zeitverläufe in Abschnitt 5.3 gewählt. 
Eine weitere Vorgehensweise zur Generierung von Freifeldzeitverläufen stellt die Abbildung des Bodens zwischen Fels und Oberfläche als Halbraum mit eigener Frequenzcharakteristik dar. Das dabei üblicherweise angewendete Kanai-Tajimi-Spektrum [54] ist als semiempirische Beziehung zu bezeichnen.

Da bei der Generierung künstlicher Zeitverläufe das elastische Ziel-Spektrum die Basis darstellt, welches die geglättete Einhüllende - üblicherweise der 84\%-Fraktilwert (= Mittelwert + Standardabweichung) oder 50\%-Fraktilwert (s. Hosser [51]) - aller der Sicherheitsphilosophie entsprechend zu erwartender Erdbeben darstellt, ist der Energieinhalt dieser Zeitverläufe größer als jener der zu erwartenden Einzelereignisse.

\subsection{Baudynamik}

Nachfolgend soll ein kurzer Einblick in die Grundlagen der Baudynamik und vorhandener Berechnungsverfahren gegeben werden. Eine ausführliche Darstellung dieses Themengebietes ist zudem in der Literatur zu finden, z. B. Meskouris [81], Petersen [103] und Zilch [160, $161]$.

\subsubsection{Einmassenschwinger}

Die dynamische Anregung eines Bauwerks im Lastfall Erdbeben kann als Fußpunkterregung eines Mehrmassenschwingers beschrieben werden. In einer Vielzahl von Fällen kann der Mehrmassenschwinger vereinfacht auf einen Einmassenschwinger reduziert werden.

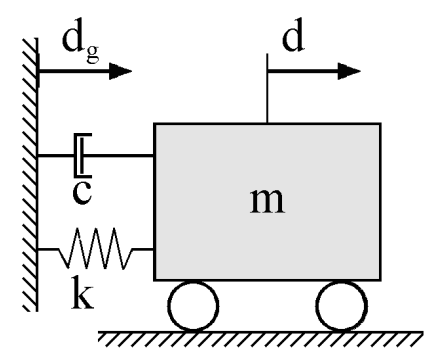

Abbildung 4: Fußpunkterregter, viskos gedämpfter Einmassenschwinger

Bei einem viskos gedämpften Einmassenschwinger ergibt sich bei einer Fußpunkterregung eine Differentialgleichung 2. Ordnung. Aus dem Kräftegleichgewicht mit den relativen GröBen $d_{\text {rel }}=d-d_{g}$ resultiert:

$$
m \cdot \ddot{d}_{r e l}+c \cdot \dot{d}_{r e l}+k \cdot d_{r e l}=-m \cdot \ddot{d}_{g}
$$

bzw. in der Standardform

$$
\ddot{d}_{r e l}+2 \cdot \zeta \cdot \omega \cdot \dot{d}_{r e l}+\omega^{2} \cdot d_{r e l}=-\ddot{d}_{g}
$$

$$
\text { mit: } \begin{array}{rlrl}
\omega & =\sqrt{\frac{k}{m}} & & : \text { Eigenkreisfrequenz des ungedämpften Einmassenschwingers } \\
\omega^{\prime} & \approx \omega & : \text { Eigenkreisfrequenz des gedämpften Einmassenschwingers }
\end{array}
$$




$$
\begin{array}{ll}
\zeta=\frac{c}{2 m \omega} & : \text { Dämpfungsma } \beta \\
T=\frac{2 \pi}{\omega}=\frac{1}{f} & : \text { Eigenperiode }
\end{array}
$$

Für kleine Dämpfungsmaße $\zeta<<1$ ist der Fehler der oben gemachten Vereinfachung $\left(\omega=\omega^{\prime}\right) \mathrm{zu}$ vernachlässigen.

\subsubsection{Mehrmassenschwinger}

Bei komplexen Strukturen erfolgt in der Praxis eine Reduktion des Systems auf die wesentlichen Freiheitsgrade, z. B. bei üblichen Hochbauten auf die Horizontalverschiebungen in Höhe der Geschossdecken. Diesen wird die Gebäudemasse punktuell zugeordnet, was durch eine diagonale Massenmatrix $\underline{m}$ beschrieben wird. Die Systemsteifigkeit wird ebenfalls auf die zu berücksichtigenden Freiheitsgrade in Form einer symmetrischen Matrix $\underline{k}$ angepasst. Entsprechend erweitert sich die Schwingungsdifferentialgleichung (6) in die Matrizenform (19).

\subsubsection{Dämpfung}

Wird einem frei schwingenden System von außen keine Energie zugeführt, so ist zu beobachten, dass die Schwingungsamplitude mit der Zeit abnimmt. Dieser bewegungshemmende Effekt resultiert aus der Energiedissipation des Systems durch verschiedene Dämpfungsmechanismen.

In der Baudynamik wird allgemein zwischen innerer und äußerer Dämpfung unterschieden. $\mathrm{Zu}$ ersterer gehören die materialabhängige Baustoffdämpfung sowie die Dämpfung in den Kontaktflächen der Konstruktion oder zwischen verschiedenen Materialien - die sog. Struktur- oder Bauteildämpfung. Die äußere Dämpfung stellt im Wesentlichen die Abstrahldämpfung in den Baugrund dar. Auf Gebrauchslastniveau kann die Summe aller Dämpfungseffekte durch einen viskosen Dämpfungsansatz i. d. R. ausreichend genau beschrieben werden. Der geschwindigkeitsproportionale Ansatz besitzt den Vorteil der einfachen rechnerischen Handhabbarkeit.

Zur Vereinfachung werden die in Wirklichkeit bei nichtlinearen Systemen auftretenden Dämpfungscharakteristiken nach Möglichkeit auf eine äquivalente viskose Dämpfung zurückgeführt. Diese kann bei harmonischer Anregung durch Gleichsetzen der in einem Zyklus vom nichtlinearen System dissipierten Energie mit der von einem äquivalenten viskosen Dämpfer mit der Dämpfungskonstanten c' in einem linearen System dissipierten Energie bestimmt werden. Bezogen wird die Dämpfung üblicherweise auf die sog. kritische Dämpfung, die den Übergang von einem schwingenden zu einem kriechenden System beschreibt. Anhaltswerte für den Dämpfungsgrad $\zeta$ (Lehr'sches Dämpfungsmaß) sind in der Literatur (z. B. Petersen [103]) zu finden.

Soll der Dämpfungsgrad durch Messung an einem Bauwerk bestimmt werden, so eignet sich dafür ein Ausschwingversuch mit Bestimmung des logarithmischen Dekrements $\Lambda$. Von dieser Methode wurde in Kapitel 5 Gebrauch gemacht, um die Dämpfung einer auskragenden Mauerwerkscheibe ohne äußere Belastung zu ermitteln.

Zur Lösung der Schwingungsdifferentialgleichung (19) ist es sinnvoll, die Dämpfungsmatrix $\underline{c}$ im Ansatz zu entkoppeln, was auf zwei Wegen erfolgen kann. Beim vollständigen modalen Dämpfungsansatz sind die Elemente außerhalb der Diagonalen zu Null zu setzten. Beim Ansatz der Rayleigh-Dämpfung wird als Dämpfungsmatrix $\underline{c}$ eine Linearkombination aus 
Steifigkeits- und Massenmatrix $\underline{c}=\alpha \cdot \underline{m}+\beta \cdot \underline{k}$ herangezogen. Die Bestimmung der Parameter $\alpha$ und $\beta$ erfolgt über den Zieldämpfungsgrad im abzudeckenden Periodenbereich.

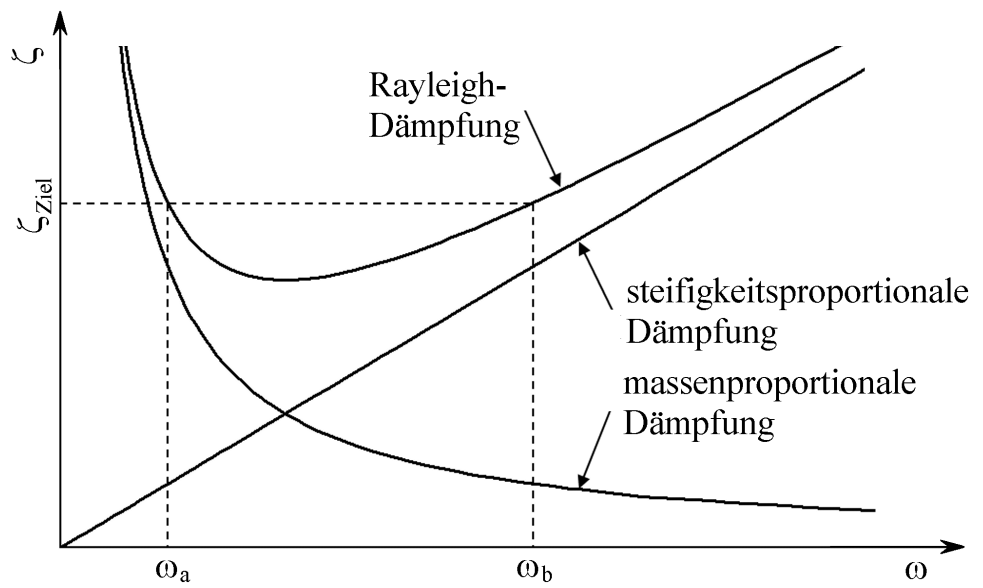

Abbildung 5: Rayleigh-Dämpfungsansatz

\subsubsection{Modalanalyse}

Zur Lösung des Eigenwertproblems erfolgt die Modalanalyse vereinfacht an einem ungedämpften System, wobei der Fehler für kleine Dämpfungsmaße $\zeta<<1$ vernachlässigbar ist. Die Eigenwerte $\omega_{i}^{2}$ bestimmen sich aus der Bedingung, dass die Determinante zu Null wird.

$$
\begin{gathered}
\underline{m} \cdot \underline{\ddot{d}}+\underline{k} \cdot \underline{d}=\underline{0} \\
! \\
\left|\underline{k}-\omega^{2} \cdot \underline{m}\right|=0
\end{gathered}
$$

Jeder Eigenform $i$ ist ein Eigenvektor $\varphi_{i}$ mit der Eigenperiode $T_{i}=2 \pi / \omega_{i}$ zugeordnet. Unter bestimmten Bedingungen, z. B. bei schlanken Bauwerken, ist zusätzlich auch eine nachgiebige Einspannung in den Baugrund mit zu berücksichtigen.

Die Eigenschwingzeiten von Bauwerken können daneben noch nach empirischen oder semiempirischen Verfahren abgeschätzt werden (in Bachmann [2] und Müller/Keintzel [20] dargestellt).

\subsubsection{Antwortspektrum}

Mit Hilfe von Antwortspektren wird in Abhängigkeit der Eigenschwingdauer eines Einmassenschwingers mit definiertem viskosen Dämpfungsmaß für einen Beschleunigungszeitverlauf die maximale Systemantwort dargestellt. Bei linearen Antwortspektren werden Einmassenschwinger mit verschiedenen Eigenschwingdauern $T_{i}$ dem Bodenbeschleunigungszeitverlauf unterworfen und die Systemantwort in Form der Bewegungsdifferentialgleichung ausgewertet. Die Berechnung kann zweckmäßigerweise mit dem Duhamel- oder Faltungsintegral erfolgen (Zilch [160]).

Die Darstellung erfolgt in Form von Verschiebungs-, Geschwindigkeits- und Beschleunigungsantwortspektren, d.h. $S_{d}, S_{v}$ und $S_{a}$. Für kleine Dämpfungsmaße $\zeta<<1$ kann mit $S_{p v}$ als 
Pseudorelativgeschwindigkeitsspektrum die nachfolgend dargestellte Vereinfachung durchgeführt werden, wodurch eine einfache Darstellung zusammen mit dem Verschiebungs- und Beschleunigungsantwortspektrum in einer doppelt-logarithmischen Zeichnung möglich wird (Beispiel: s. Anhang A.1).

$$
S_{p v} \approx \omega \cdot S_{d} \approx \frac{1}{\omega} \cdot S_{a}
$$

Für die Konstruktion von Bemessungsspektren gilt diese Vereinfachung nicht (s. Abschnitt 2.4).

Bei der Ermittlung der Systemantwort eines Mehrmassenschwingers mit Hilfe des elastischen Antwortspektrums sind die Antworten der Einzeleigenformen nach statistischen Gesichtspunkten zu überlagern, z. B. aus der Wurzel der Quadratsummen.

\subsection{Tragfähigkeit und Duktilität}

Mit der Duktilität wird das plastische Verformungsvermögen unter Beibehaltung der Tragfähigkeit bezeichnet. Entsprechend der betrachteten Verformungsgröße kann zwischen Krümmungs- bzw. Rotationsduktilität sowie Dehnungs- und Verschiebeduktilität unterschieden werden. Die lokale Duktilität bezieht sich hierbei auf einzelne Bauteile, wie z. B. die Ausbildung eines plastischen Gelenkes in einem Riegel, wohingegen die globale Duktilität eines Bauwerks durch die (Verschiebe-) Duktilität der Gesamtkonstruktion beschrieben wird.

Für das Trag- und Verformungsverhalten ist es entscheidend, in welche Form die durch eine Erdbebeneinwirkung in ein Bauwerk eingetragene Energie umgewandelt wird. Bleibt das Bauwerk unter Erdbebeneinwirkung linear-elastisch, so resultieren entsprechend höhere Beanspruchungen in der Konstruktion. Wird dagegen bei einem Bauwerk mit ausreichendem Verformungsvermögen die Fließlast überschritten, so stellen sich deutlich größere Verformungen ein. Die auftretende Beanspruchung und somit auch die erforderliche Tragfähigkeit reduzieren sich dementsprechend.

Das Verhältnis von der Beanspruchung eines elastischen Systems zu der eines elastoplastischen, duktilen Systems wird durch den Verhaltensfaktor $q$ beschrieben. Üblicherweise werden für die Bemessung in der Praxis anstelle der genauen Berücksichtigung des elastoplastischen Verhaltens eines Systems vereinfacht die bei einer linear-elastischen Berechnung resultierenden Schnittgrößen mit dem $q$-Faktor reduziert. In den normativen Angaben für $q$ eingeschlossen sind die Einflüsse des Materials, des Tragsystems, der konstruktiven Durchbildung sowie der Bemessungsmethode. Die infolge Duktilität gegenüber der elastischen Einwirkung $F_{e l}$ reduzierte Beanspruchung $F_{y}$ bestimmt sich zu:

$$
F_{y}=\frac{1}{q} F_{e l}
$$


Bei einem Einmassenschwinger kann die mathematische Bestimmung von $q$ nach folgenden beiden Ansätzen erfolgen (Bachmann [2], Paulay / Bachmann / Moser [102], Zilch [160]):

- Prinzip der gleichen Formänderungsarbeit:

$$
\begin{aligned}
& E_{\text {lin-el }}=\frac{1}{2} F_{e l}\left(\Delta y \frac{F_{e l}}{F_{y}}\right)^{!}=E_{e l-p l}=F_{y}\left(\Delta u-\frac{\Delta y}{2}\right) \\
& \Rightarrow q=\frac{F_{e l}}{F_{y}}=\sqrt{2 \mu_{\Delta}-1}
\end{aligned}
$$

mit der Verschiebeduktilität: $\mu_{\Delta}=\frac{\Delta u}{\Delta y}$

- Prinzip der gleichen Verschiebung:

$$
q=\frac{F_{e l}}{F_{y}}=\frac{\Delta u}{\Delta y}=\mu_{\Delta}
$$

Die Darstellung in einem Kraft-Verformungsdiagramm ergibt folgendes Bild:
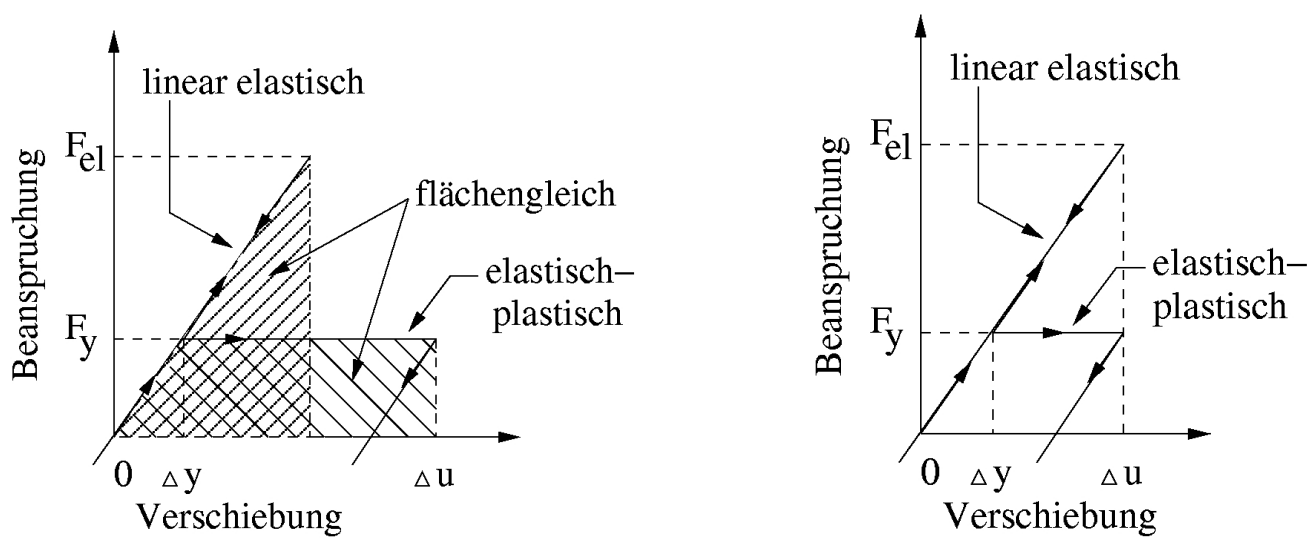

Abbildung 6: Prinzip der gleichen Formänderungsarbeit und Prinzip der gleichen Verschiebung

Beim Vergleich der beiden Ansätze zeigt sich, dass mit zunehmender Verschiebeduktilität der Abstand zunimmt. Im Bereich kleiner Duktilitätswerte, die für Mauerwerkskonstruktionen üblicherweise zu erwarten sind, ist der Unterschied dagegen vernachlässigbar. 


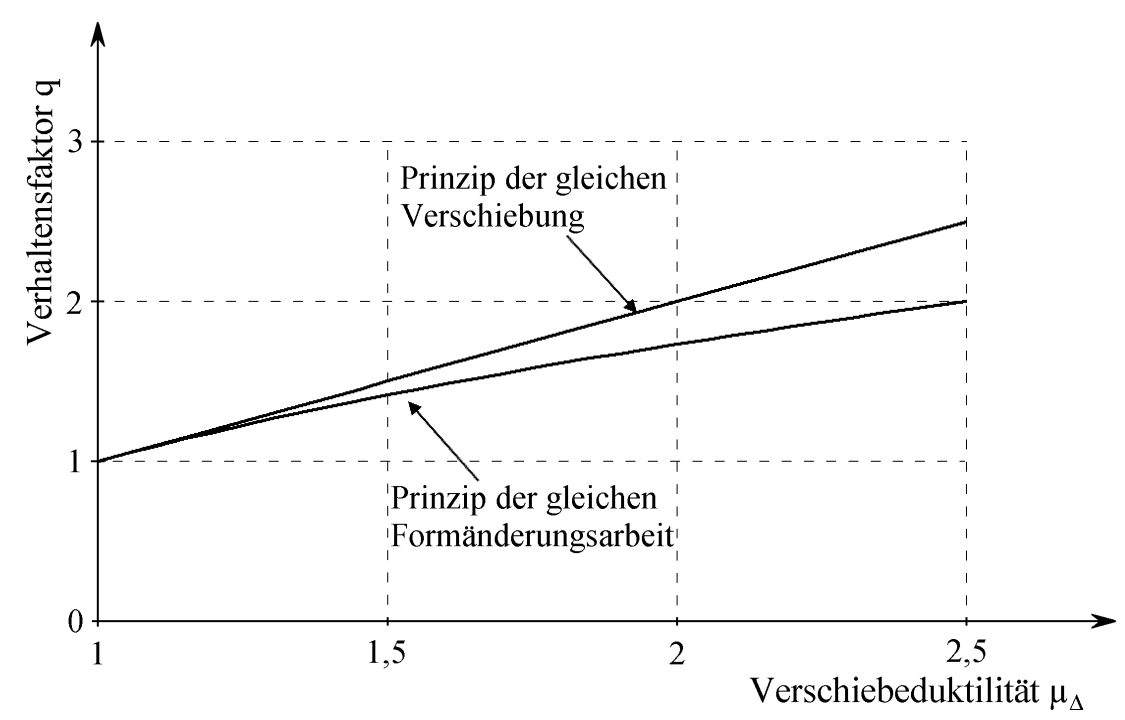

Abbildung 7: Verhaltensfaktor $q$ in Abhängigkeit der Verschiebeduktilität für verschiedene Ansatzprinzipien

Der erste Ansatz nach dem Prinzip der gleichen Formänderungsarbeit ist im Wesentlichen für mittlere Eigenschwingdauern 0,5 $\mathrm{s}>T>0,1 \mathrm{~s}$ geeignet, wohingegen der Ansatz über die gleiche Verschiebung das Verhalten bei hohen Eigenschwingdauern $T>1,4 \mathrm{~s}$ besser beschreibt. Im Bereich kleinerer Eigenschwingdauern $T<0,05 \mathrm{~s}$ ist der Effekt der plastischen Verformungen praktisch nicht ausgeprägt und kann daher vernachlässigt werden.

Eine weitere Möglichkeit, den Verhaltenswert $q$ in Abhängigkeit der Verschiebeduktilität zu bestimmen, besteht in Form eines empirischen Verfahrens (s. Bachmann [2], Paulay / Bachmann / Moser [102]). Dabei werden elastisch-plastische Einmassenschwinger mit unterschiedlicher Verschiebeduktilität unter verschiedenen Bodenbeschleunigungszeitverläufen untersucht. Über das Verhältnis von elastischer Beanspruchung zur Fließlast in Abhängigkeit der Eigenschwingzeit für verschiedene Erdbeben-Zeitverläufe kann $q$ empirisch bestimmt werden.

Dieses Verfahren ist nicht nur auf Einzelbauteile bzw. Einmassenschwinger beschränkt, sondern es kann auch auf komplexe Strukturen angewendet werden. In einem ersten Schritt erfolgt hierbei die linear-elastische Berechnung unter den vorgegebenen Zeitverläufen. In einem zweiten Schritt wird die nichtlineare Berechnung - bzw. die Versuchsdurchführung bei experimentellen Untersuchungen - der Struktur vorgenommen. Dabei sind mehrere Durchgänge mit verschieden hohen Laststufen notwendig. In Abhängigkeit des Lastfaktors kann anschließend die Reduktion von rechnerischer Beanspruchung bei linear-elastischer Berechnung und der tatsächlich aufgetretenen Beanspruchung bei nichtlinearem Verhalten (aus dem zweiten Schritt) bestimmt werden. Im Allgemeinen kann bei dieser Vorgehensweise nur ein punktueller $q$-Faktor ermittelt werden, der sämtliche nichtlineare Einflüsse der Gesamtkonstruktion erfasst. Eine Differenzierung nach der Eigenschwingzeit erfolgt dabei nicht.

Die direkte Konstruktion von Bemessungsantwortspektren kann nach einem Verfahren von Newmark (in Bachmann [2] dargestellt) erfolgen. Basis ist das Pseudogeschwindigkeitsspektrum $S_{p v}$ aus dem das inelastische Beschleunigungsspektrum $S_{a}$ und das inelastische Verschiebespektrums $S_{d}$ in Abhängigkeit des Duktilitätsfaktors $\mu_{\Delta}$ generiert werden.

Im Bereich kleiner Eigenfrequenzen bis etwa $2 \mathrm{~Hz}$ erfolgt die Abminderung vom $S_{a, e l}$. auf $S_{a}\left(\mu_{\Delta}\right)$ mit dem Faktor $1 / \mu_{\Delta}$ (Prinzip der gleichen Verschiebung) und im Plateaubereich bei konstanter Beschleunigung mit dem Faktor $\left(2 \mu_{\Delta}-1\right)^{-1 / 2}$ (Prinzip der gleichen Formänderungs- 
arbeit). Bereiche höherer Eigenfrequenzen ab ca. $33 \mathrm{~Hz}$ erhalten keinerlei Abminderung - der dazwischen liegende Bereich wird linear verbunden.

Die Generierung des inelastischen Verschiebungsspektrums erfolgt im Bereich ab einer Eckfrequenz von ca. $2 \mathrm{~Hz}$ aus dem inelastischen Beschleunigungsspektrum $S_{a}\left(\mu_{\Delta}\right)$ durch Multiplikation mit dem Faktor $\mu_{\Delta}$. Für den Bereich kleinerer Eigenfrequenzen ist das elastische Verschiebungsspektrum ohne Abminderung anzusetzen.

\subsection{Normung}

\subsubsection{Allgemeines}

Der Nachweis von Hochbauten in deutschen Erdbebengebieten erfolgt zurzeit nach der Norm DIN 4149 (1981-04) [20, 21] (s. a. [52]). Als Bezugsgröße des nachzuweisenden Referenzbebens wird die jeweils maximal historisch aufgetretene und damit maximal zu erwartende MSK-64-Intensität (s. Abschnitt 2.2.2) angesetzt. Diese Norm basiert auf einer Sicherheitsphilosophie, die im Katastrophenlastfall Erdbeben lediglich die Standsicherheit der Gebäude und damit die Vermeidung von Personenschäden fordert. Größere Schäden der Gebäude - bis hin zum erforderlichen Abriss nach einem Schadensbeben - werden für übliche Hochbauten toleriert. Der Nachweis der Erhaltung der Gebrauchstauglichkeit bei Erdbeben mit geringerer Intensität und damit höherer Eintretenswahrscheinlichkeit ist nach diesem deterministischen Sicherheitskonzept nicht vorgesehen.

Im Gegensatz dazu basiert die ENV 1998 [27 $\div$ 29] (s. a. [30]) und der aktuelle Normentwurf E DIN 4149 (2002-10) [22] auf einer Sicherheitsüberlegung, die den Lastfall Erdbeben als ein häufiger wiederkehrendes Ereignis definiert. Die anzusetzende Belastung für den Nachweis der Tragfähigkeit entspricht dabei einem Erdbeben mit einer Referenzwiederkehrperiode von 475 Jahren (= eine Nichtüberschreitenswahrscheinlichkeit von $10 \%$ in 50 Jahren), welchem ein Bedeutungsbeiwert $\gamma_{1}=1,0$ zugeordnet wird. Dabei ist zusätzlich zum Nachweis der Tragfähigkeit mit definiertem Sicherheitsabstand ( $\gamma_{1}$-fache Erdbebenlasten und einem z. B. bei Mauerwerk von $\gamma_{m}=1,5$ auf $\gamma_{m}=1,0$ reduzierten Materialsicherheitsfaktor) prinzipiell auch ein Nachweis der Gebrauchstauglichkeit - hier als Anforderung an die Schadensbegrenzung - zu führen. Das bedeutet, dass bei Erdbeben mit einer höheren Eintrittswahrscheinlichkeit und damit geringerer Intensität, keine oder nur geringe Schäden am Bauwerk zugelassen sind. Eine genaue Definition der Referenzwiederkehrperiode dieser Gebrauchstauglichkeitsbeben erfolgt in E DIN 4149 jedoch nicht. Es wird davon ausgegangen, dass außer dem explizit zu führenden Nachweis der Begrenzung der Geschossverschiebungen die Gebrauchstauglichkeit durch den Nachweis der Tragfähigkeit mit abgedeckt ist. Im Entwurf zur ENV 1998 [30] wird diese Referenzwiederkehrperiode als damage limitation requirement zu 95 Jahren vorgeschlagen, was einer Überschreitungswahrscheinlichkeit von $10 \%$ in 10 Jahren entspricht.

Bei Bauwerken mit besonderer Bedeutung für die Allgemeinheit oder für Gebäude, die empfindliche Anlagen enthalten, sind eigene Kriterien individuell festzulegen, beispielsweise durch eine Erhöhung des Bedeutungsbeiwertes $\gamma_{1}$. Für kerntechnische Anlagen, die deutlich höheren Sicherheitsanforderungen unterliegen, gelten abweichende Vorschriften (s. KTA 2201 $[63,64])$.

\subsubsection{Erdbebengefährdung}

Die normative Beschreibung der Erdbebengefährdung eines Gebietes erfolgt üblicherweise in Form einer Erdbebenzonenkarte, nach der die anzusetzende Intensität bzw. Bodenbeschleunigung bestimmt werden kann. Sowohl in dem aktuellen Normentwurf E DIN 4149 als auch in 
der ENV 1998 wurde die Referenzwiederkehrperiode - wie international gebräuchlich - zu 475 Jahren festgelegt. Die Zuordnung der Bezugsintensität (Tabelle 1) zum Bemessungswert der Bodenbeschleunigung $a_{g}$ erfolgt bei ersterer nach der Beziehung (13) (s. a. Gleichung (2)). Die Abstufung in den Intensitätsintervallen der drei Erdbebenzonen ist in Tabelle 4 zusammengefasst.

$$
\lg a_{g}=0,241 \cdot I_{E M S}-1,866
$$

\begin{tabular}{|l|c|c|c|}
\hline $\begin{array}{c}\text { Erdbeben- } \\
\text { zone }\end{array}$ & $\begin{array}{c}\text { Bemessungswert der } \\
\text { Bodenbeschleunigung } \\
\boldsymbol{a}_{\boldsymbol{g}}\left[\mathbf{m} / \mathbf{s}^{\mathbf{2}}\right]\end{array}$ & $\begin{array}{c}\text { Bezugsintensität } \\
\boldsymbol{I}_{\boldsymbol{r e f}}[-]\end{array}$ & $\begin{array}{c}\text { Intensitäts- } \\
\text { intervall }\end{array}$ \\
\hline $\mathbf{1}$ & 0,4 & 6,25 & $6,5 \leq \mathrm{I}<7,0$ \\
\hline $\mathbf{2}$ & 0,6 & 7,0 & $7,0 \leq \mathrm{I}<7,5$ \\
\hline $\mathbf{3}$ & 0,8 & 7,5 & $7,5 \leq \mathrm{I}<8,0$ \\
\hline
\end{tabular}

Tabelle 4: Bemessungswert der Bodenbeschleunigung und zugehörige Intensitäten - nach E DIN 4149 (102002) [22] und Schwarz / Grünthal [123]

Gebiete mit geringer oder sehr geringer seismischer Gefährdung, in denen rechnerisch die Intensität 6,5 bzw. 6,0 nicht überschritten wird, werden in dem Normentwurf $E$ DIN 4149 ohne Zonenangabe bzw. als Zone 0 klassifiziert. In diesen Gebieten ist der Lastfall Erdbeben für allgemeine Hochbauten nicht zu berücksichtigen. Intensitäten über 8 sind für Deutschland als äußerst unwahrscheinlich anzusehen und entfallen bei einer Referenzwiederkehrperiode von 475 Jahren.

Der angegebene Bemessungswert der Bodenbeschleunigung $a_{g}$ bezieht sich auf felsigen bzw. steifen Untergrund. Er stellt jedoch nicht die tatsächlich beobachtete und in den Zonen zu erwartende Spitzenbeschleunigung dar, sondern sein effektives Niveau berücksichtigt die Tatsache, dass die effektive Beschleunigung - d.h. der für die Bauwerksreaktion maßgebende Wert - bei den in mitteleuropäischen Erdbebengebieten relevanten nahen Ereignissen kleiner als die gemessene Spitzenbeschleunigung ist (s. a. Kaiser [53]).

Nachfolgend ist die Gefährdungskarte von Grünthal / Mayer-Rosa / Lenhardt [38] abgebildet, die die Basis für die Erdbebenzonenkarte nach E DIN 4149 darstellt (vgl. Tabelle 1). 


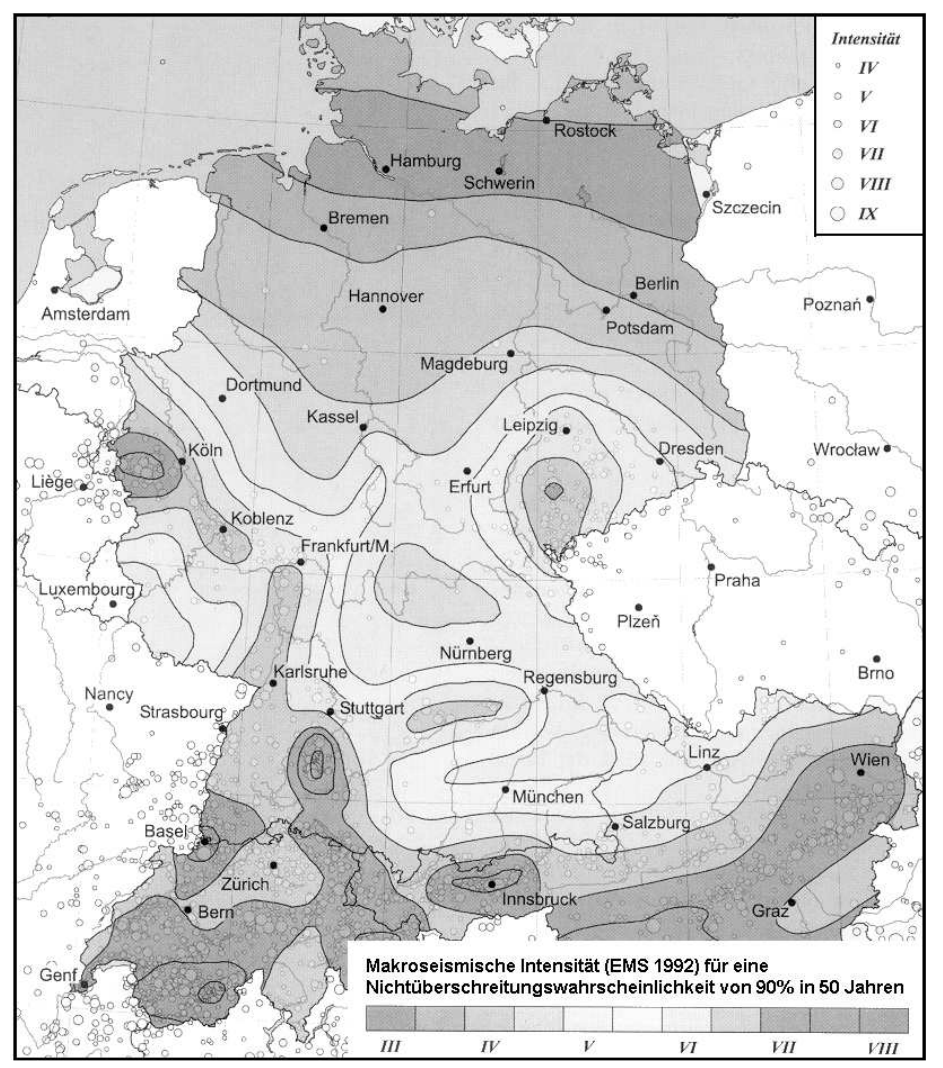

Abbildung 8: Erdbebengefährdung in Form berechneter EMS-1992-Intensitäten für eine Referenzwiederkehrperiode von 475 Jahren - aus Grünthal / Mayer-Rosa / Lenhardt [38]

Eine seismische Risikokartierung für deutsche Erdbebengebiete mit gefährdungskonsistenten Spektren im Vergleich zu aktuellen Normanforderungen wird von Schwarz, Grünthal und Golby [124] gegeben.

\subsubsection{Antwortspektrum}

Die Lasteinwirkung wird von der Normenseite im Wesentlichen durch das Antwortspektrum (s. Abschnitt 2.3.5) mit seiner Form und dem Einhängewert des Maximalwertes der Bodenbeschleunigung beschrieben. Die elastischen Antwortspektren stellen geglättete Einhüllende gemessener bzw. der Sicherheitstheorie entsprechend zu erwartender Erdbeben dar. Für die Bemessung wird neben dem elastischen Antwortspektrum i. d. R. ein abgemindertes Bemessungsspektrum angewendet. Diese Abminderung wird entweder explizit angegeben oder ist in der Form des Antwortspektrums bereits eingearbeitet (DIN 4149-1 (04-1981) [20]).

Die Ordinate $S_{e}(T)$ des elastischen, horizontalen Antwortspektrums von E DIN 4149 (200210) [22] wird abschnittsweise definiert zu:

$$
\begin{aligned}
0 \leq T \leq T_{B}: & S_{e}(T)=a_{g} \cdot S \cdot\left[1+\frac{T}{T_{B}} \cdot\left(\eta \cdot \beta_{0}-1\right)\right] \\
T_{B} \leq T \leq T_{C}: & S_{e}(T)=a_{g} \cdot S \cdot \eta \cdot \beta_{0} \\
T_{C} \leq T \leq T_{D}: & S_{e}(T)=a_{g} \cdot S \cdot \eta \cdot \beta_{0} \cdot \frac{T_{C}}{T} \\
T_{D} \leq T: & S_{e}(T)=a_{g} \cdot S \cdot \eta \cdot \beta_{0} \cdot \frac{T_{C} \cdot T_{D}}{T^{2}}
\end{aligned}
$$

mit: $\quad a_{g}: \quad$ Bemessungswert der Bodenbeschleunigung (s. Tabelle 4) 


$$
\begin{aligned}
\beta_{0}: & \text { Verstärkungsbeiwert (s. Tabelle 6) } \\
\eta: & \text { Dämpfungskorrekturbeiwert (s. Tabelle 6) } \\
\mathrm{S}: & \text { Bodenparameter (s. Tabelle 6) } \\
T_{B}, T_{C}, T_{D}: & \text { Kontrollperioden (s. Tabelle 6) } \\
T: & \text { Schwingungsdauer des linearen Einmassenschwingers }
\end{aligned}
$$

Die Berücksichtigung des nichtlinearen Bauwerksverhaltens in Form des Verhaltensfaktors $q$ erfolgt in Abhängigkeit der Eigenschwingdauer $T$. Im Bereich mittlerer und großer Eigenschwingzeiten $T \geq T_{B}$ erfolgt die Abminderung voll, und im Bereich kleiner Eigenschwingdauern $T<T_{B}$ wird die Abminderung linear von $q$ bei $T=T_{B}$ bis auf 1 bei $T=0 \mathrm{~s}$ abnehmend angesetzt.

Beispielhaft ist nachfolgend das elastische Antwortspektrum $S_{e}$ sowie das Bemessungsspektrum $S_{d}$ zusammen mit dem Verhaltensfaktor $q$ für unbewehrtes Mauerwerk in der Erdbebenzone 1 (Boden-Baugrund-Kombination A1) nach E DIN 4149 dargestellt.

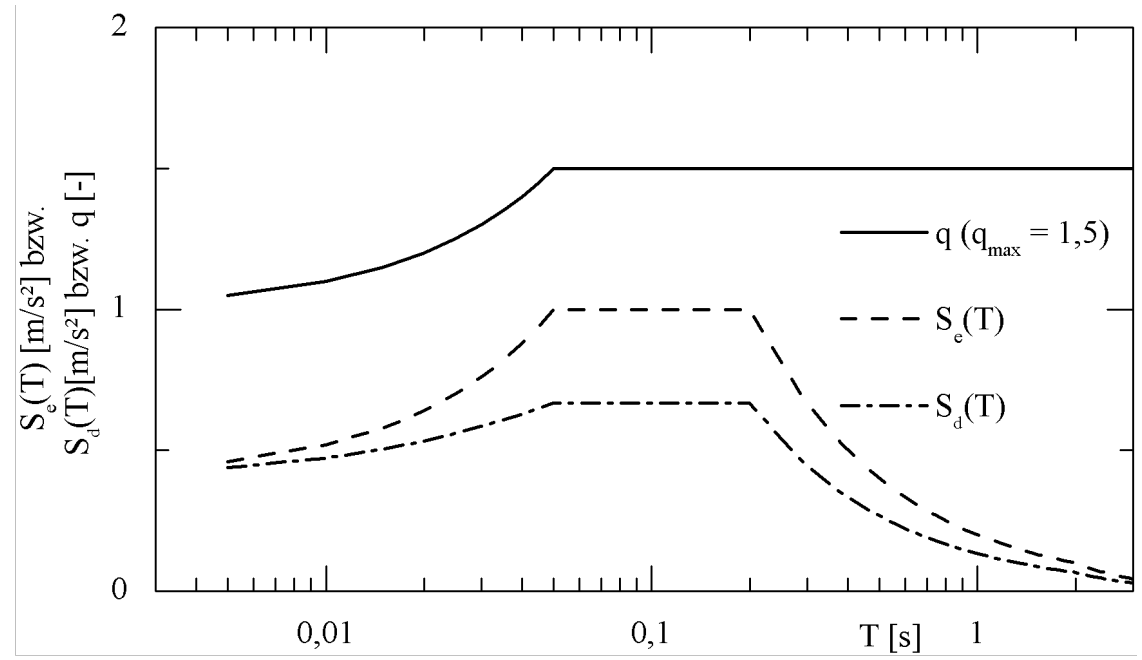

Abbildung 9: Elastisches- und Bemessungs-Antwortspektrum mit $q$-Faktor für unbewehrtes Mauerwerk (Boden-Baugrund-Kombination A1, $\left.a_{g}=0,4 \mathrm{~m} / \mathrm{s}^{2}\right)-\operatorname{nach} E$ DIN 4149 (2002-10) [22]

Die vertikale Erdbebeneinwirkung kann üblicherweise zwischen $50 \%$ und $75 \%$ der Horizontalen betragen. In E DIN 4149 wird neben einer Veränderung des Plateaubereiches des vertikalen, elastischen Antwortspektrums $\left(T_{C, v e r t i k a l}=0,2 \mathrm{~s}\right)$ die Vertikaleinwirkung zu dem 0,7fachen der horizontalen Einwirkung festgelegt. Deren Berücksichtigung wird jedoch nur für Träger mit direkten Stützenlasten gefordert. Der ungünstige Einfluss für die Schubbemessung von Mauerwerksscheiben durch eine Erhöhung oder Reduzierung der Normalkraft (vgl. Abschnitt 6.2.1) muss demnach nicht berücksichtigt werden.

Die Abschätzung des Einflusses einer Vertikaleinwirkung auf die Änderung der Normalkraft (Tabelle 5 mit $\left.\gamma_{1}=1,0\right)$ ergibt in Abhängigkeit der Erdbebenzone, der Boden-BaugrundKombination und der vertikalen Eigenperiode einen Wertebereich zwischen $\pm 2 \%$ und $\pm 21 \%$.

\begin{tabular}{|c|c|c|c|}
\hline Erdbebenzone & Boden-Baugrund-Kombination & $\boldsymbol{T}_{\boldsymbol{V}}[-]$ & Änderung [\%] \\
\hline $\mathbf{1}\left(\boldsymbol{a}_{\boldsymbol{g}}=\mathbf{0 , 4} \mathbf{~ m} / \mathbf{s}^{\mathbf{2}}\right)$ & $\mathrm{C} 3(S=0,75)$ & $T_{V}<<T_{B}=0,1 \mathrm{~s}$ & \pm 2 \\
\hline $\mathbf{3}\left(\boldsymbol{a}_{\boldsymbol{g}}=\mathbf{0 , 8} \mathbf{~ m} / \mathbf{s}^{\mathbf{2}}\right)$ & $\mathrm{A} 3(S=1,5)$ & $T_{B}=0,05 \mathrm{~s}<T_{V}<T_{C}=0,2 \mathrm{~s}$ & \pm 21 \\
\hline
\end{tabular}

Tabelle 5: Änderung der Vertikalkräfte bei Berücksichtigung der Vertikaleinwirkung nach E DIN 4149 


\subsubsection{Untergrund}

Die Berücksichtigung des Einflusses des Untergrundes auf die Höhe der Einwirkung erfolgt in den Normen über eine Klassifizierung des Baugrundes. Dabei wird sowohl die maximale Überhöhung als auch die Form des Antwortspektrums beeinflusst. International üblich wird vereinfachend dazu die mittlere Scherwellengeschwindigkeit der obersten $30 \mathrm{~m}$ als Kriterium herangezogen. Diese Beschränkung hat ihren Ursprung in den begrenzten messtechnischen Möglichkeiten zu Beginn systematischer Untersuchungen Anfang der 60er Jahre in Nordamerika. In Erdbebengebieten mit nahe an die Erdoberfläche reichendem festen Untergrund, z. B. an der Westküste Nordamerikas oder im Mittelmeerraum, hat sich diese Vereinfachung als ausreichend erwiesen. Im Gegensatz dazu treten in Gebieten mit tiefen tertiären Sedimentbecken besondere Effekte hervor, die zu einer Reduzierung der Erdbebenlasten führen können. Diese resultieren aus der Absorption und den Frequenzänderungen der vom Hypozentrum ausgestrahlten Wellen. Mit der Berücksichtigung sowohl des bis zu einer Tiefe von $20 \mathrm{~m}$ reichenden Baugrundes als auch des geologischen Untergrundes können die Besonderheiten der Erdbebengebiete Deutschlands deutlich besser erfasst werden. Diese Klassifizierung wurde bei der Neubearbeitung der E DIN 4149 (2002-10) [22] angewendet (s. Brüstle / Schneider [8]).

Für den geologischen Untergrund wird zwischen den Klassen A, B und C unterschieden:

- Untergrundklasse A: Gebiete mit felsartigem Gesteinsuntergrund mit für Festgestein typischen Scherwellengeschwindigkeiten.

- Untergrundklasse B: Übergangsbereiche zwischen den Gebieten der Untergrundklasse A und $\mathrm{C}$, sowie Gebiete relativ flachgründiger Sedimentbecken.

- Untergrundklasse C: Gebiete tiefer Beckenstrukturen mit mächtiger Sedimentfüllung (z. B. Niederrheinische Bucht und Oberrheingraben).

Bei dem lokalen Baugrund bis zu einer Tiefe von ca. $20 \mathrm{~m}$ erfolgt die Unterscheidung nach den Klassen 1, 2 und 3:

- Baugrundklasse 1: Feste bis mittelfeste Gesteine

- Baugrundklasse 2: Lockergesteine (Kies bis Grobsand, Mergel)

- Baugrundklasse 3: Feinkörnige Lockergesteine (Feinsand) bzw. Lößauflagen

Als Kombination können in Deutschland die Untergrundverhältnisse A1, A2, A3, B2, B3 sowie C3 vorkommen. Nachfolgend sind die Parameter des elastischen horizontalen Antwortspektrums dargestellt.

\begin{tabular}{|l|c|c|c|}
\hline $\begin{array}{c}\text { Baugrund- } \\
\text { verhältnisse }\end{array}$ & $\begin{array}{c}\boldsymbol{S} \\
{[-]}\end{array}$ & $\begin{array}{c}\boldsymbol{T}_{\boldsymbol{B}} \\
\text { [s] }\end{array}$ & $\begin{array}{c}\boldsymbol{T}_{\boldsymbol{C}} \\
{[\mathbf{s}]}\end{array}$ \\
\hline \hline $\mathbf{A 1}$ & 1,00 & 0,05 & 0,20 \\
\hline $\mathbf{A 2}$ & 1,25 & 0,05 & 0,25 \\
\hline $\mathbf{A 3}$ & 1,50 & 0,05 & 0,30 \\
\hline $\mathbf{B 2}$ & 1,00 & 0,10 & 0,30 \\
\hline $\mathbf{B 3}$ & 1,25 & 0,10 & 0,40 \\
\hline $\mathbf{C 3}$ & 0,75 & 0,10 & 0,50 \\
\hline$T_{D}=2,0$ s \\
$\boldsymbol{\beta}_{0}=2,5$ (bei 5\% viskoser Dämpfung) \\
$\eta=1$ (bei 5\% viskoser Dämpfung) \\
\hline
\end{tabular}

Tabelle 6: Parameter des elastischen, horizontalen Antwortspektrums von E DIN 4149 (2002-10) [22] 


\section{Stand der Forschung}

Nachfolgend wird ein Überblick über den Stand der Forschung zum Erdbebentragverhalten von unbewehrtem Mauerwerk gegeben. Arbeiten auf dem Gebiet der Pseudodynamik werden gesondert in Abschnitt 5.1.3 behandelt.

\subsection{Bruch- und Materialmodelle}

\section{$\underline{\text { Mann / Müller }}$}

Mann und Müller stellten in den 70er Jahren [73, 74] eine Bruchtheorie für infolge Schubund Normalkraft beanspruchtes unbewehrtes Mauerwerk auf, welche auch die Basis der Schubbemessung nach DIN 1053-1 [17] darstellt. Hierbei wird davon ausgegangen, dass in den Stoßfugen keinerlei Spannungen übertragen werden können. Aus der Gleichgewichtsbetrachtung am Einzelstein ergibt sich eine blockförmige Verteilung der Normalspannungen in der Lagerfuge (Abbildung 10 rechts). Daraus leiten sich folgende vier Versagenskriterien ab:

- Klaffen der Lagerfuge in der Steinhälfte mit der geringeren Normaldruckspannung

- Reibungsversagen in der Steinhälfte mit der geringeren Normaldruckspannung

- Steinzugversagen infolge Überschreitung der schrägen Steinzugfestigkeit in Steinmitte

- Druckversagen in der Steinhälfte mit der höheren Normaldruckspannung

Eine ausführliche Darstellung dazu ist in Abschnitt 6.2.1 im Rahmen der Entwicklung eines Materialmodells zu finden.

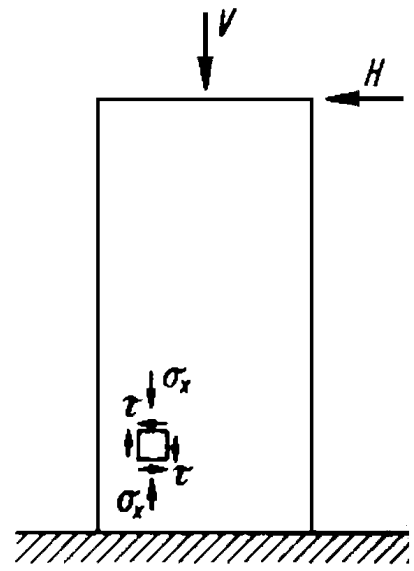

Windscheibe

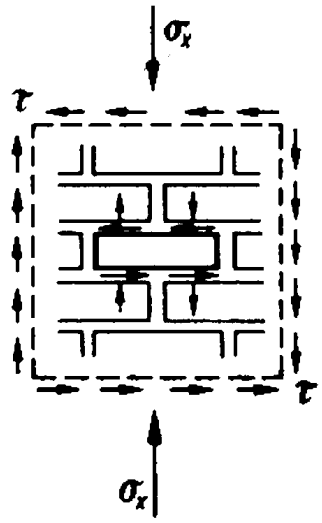

Element

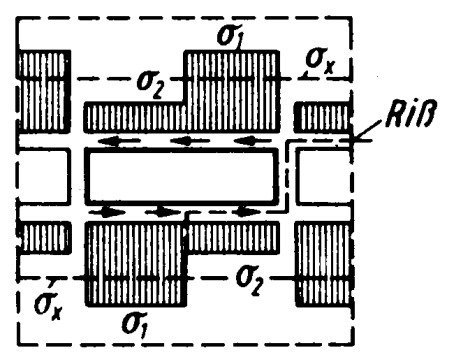

Spannungen am Stein

Abbildung 10: Spannungszustand für kombiniert beanspruchtes Mauerwerk - aus Mann / Müller [74]

Für den Sonderfall großformatiger Steine, bei denen das planmäßige bezogene Überbindemaß $\ddot{u} / \Delta y$ aus baupraktischen Gründen deutlich kleiner als bei kleinformatigen Steinen ist, modifizierte Simon [133, 134] aufbauend auf Untersuchungen von Schneider / Wiegand / Jucht [115] die Kriterien des Schubmodells von Mann / Müller. Mit Berücksichtigung eines Aufreißens der Lagerfuge wird als zusätzliches Kriterium ein Steinzugversagen im Randbereich eingeführt, das im Fall geringer bezogener Überbindemaße und geringer Normaldruckkraft maßgebend wird. 


\section{Hamid / Drysdale}

Hamid und Drysdale [42, 43] stellten 1981 für unbewehrtes Mauerwerk Bruchkriterien in Abhängigkeit der beiden Hauptspannungen und dem Winkel zu den Lagerfugen auf. Die Traglast wird zum einen durch ein Stein-Spaltzugkriterium und zum anderen durch Reibungsversagen entlang der Stoß- und Lagerfugen bestimmt.

Nach der Umformung erfolgt die Darstellung des Versagens über die Differenz der Hauptspannungen in Abhängigkeit des Reibungswinkels, der Haftscherfestigkeit sowie der Neigung der Hauptspannungen zu den Lagerfugen. Die Theorie wird von den Autoren mit eigenen Versuchsergebnissen verglichen und eine gute Übereinstimmung - insbesondere im Bereich geringer Winkel - festgestellt.

\section{$\underline{\text { Page }}$}

Aufbauend auf mehreren zweiaxialen Versuchen an Mauerwerkskörpern unter Variation der Lagerfugenneigung stellte Page [99, 100] die Versagenshüllflächen auf. Als Kriterien zur Beschreibung des Spannungszustandes in der Scheibe dienten die beiden Hauptspannungen und die Lagerfugenneigung.

Als Erweiterung werteten Dhanasekar, Page und Kleeman [12] insgesamt 180 biaxiale Versuche an Modellmauerwerk im Maßstab 1:2 aus und stellten ein modifiziertes dreidimensionales Bruchkriterium auf. Als Parameter zur Beschreibung des Spannungszustandes in der Scheibe fungieren dabei die Spannungen senkrecht und parallel zu den Lagerfugen sowie die Schubspannungen. Es ergibt sich im $\sigma_{x}-\sigma_{y^{-}} \tau$-Spannungsraum die Form von drei elliptischen Kegeln (s. Abbildung 11).

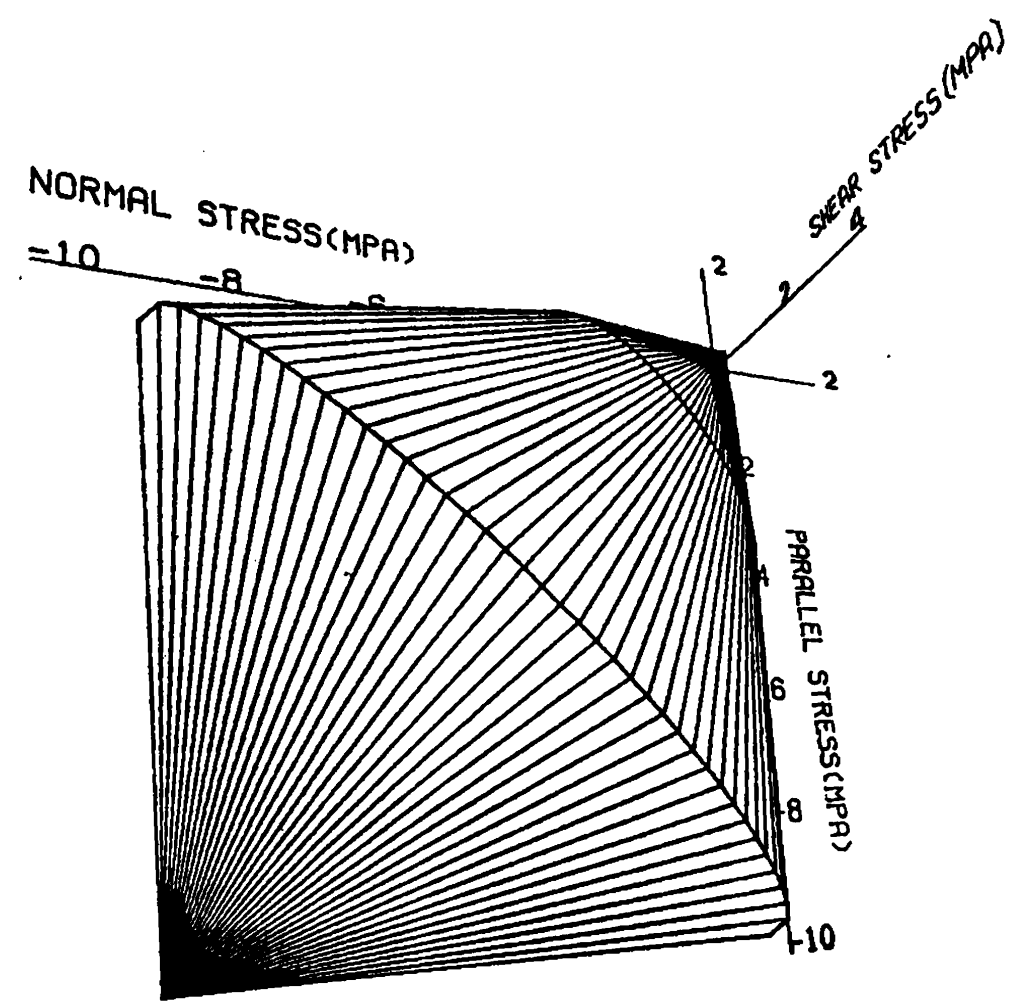

Abbildung 11: Versagenshüllflächen nach Dhanasekar / Page / Kleeman [12] (Mauerwerk aus Vollsteinen) 


\section{Ganz / Thürlimann}

Für kombiniert beanspruchte Mauerwerksscheiben stellten Ganz und Thürlimann [32, 140] 1984 ein zweiaxiales Bruchmodell auf. Die Unterscheidung der Versagensformen für unbewehrtes Mauerwerk ohne Zugfestigkeit erfolgt anhand von fünf bzw. für unbewehrtes Mauerwerk mit Zugfestigkeit anhand von elf Bruchkriterien. Nachfolgend sind für Mauerwerk mit Zugfestigkeit die Versagensflächen im dreidimensionalen Spannungsraum dargestellt.

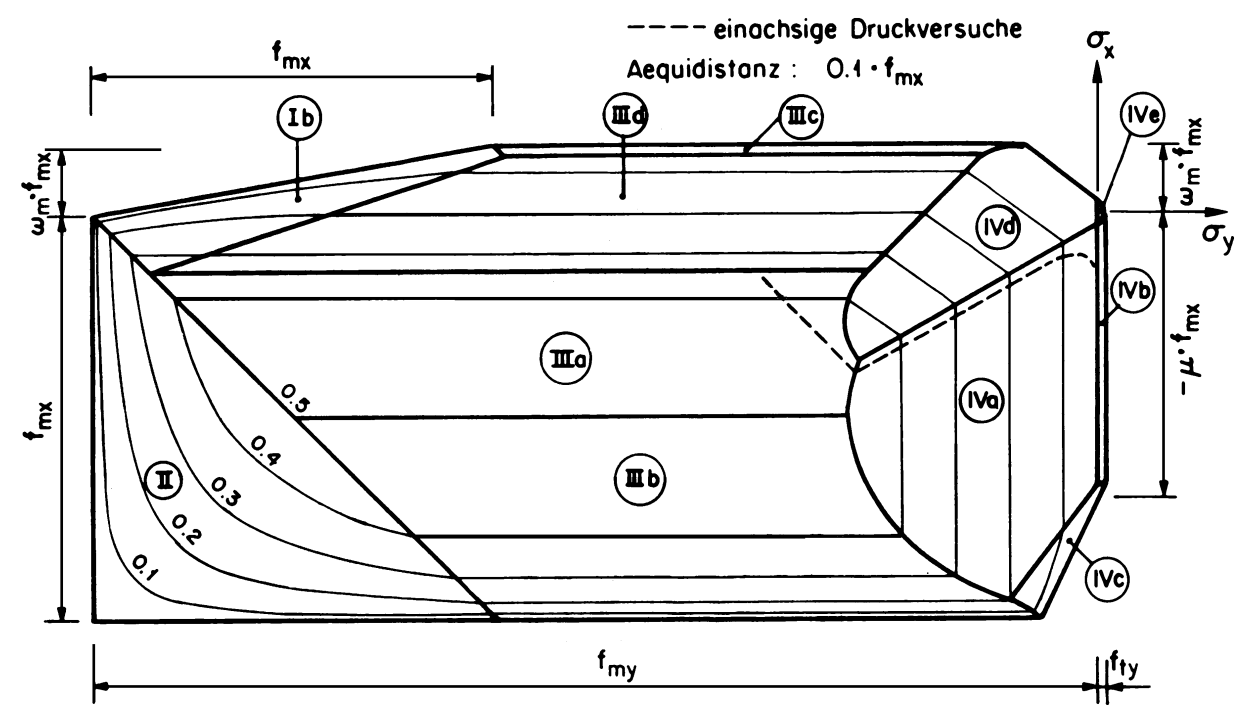

Abbildung 12: Bruchkriterien und Versagensflächen von Ganz / Thürlimann [32] für Mauerwerk mit Zugfestigkeit

Als Eingangsparameter dienen neben den Druckfestigkeiten senkrecht und parallel zu den Lagerfugen $f_{m y}$ bzw. $f_{m x}$ (Koordinatensystem analog zur allgemeinen Festlegung in dieser Arbeit) noch die Haftscherfestigkeit $k$ und der Reibungswinkel in der Lagerfuge $\varphi$. Bei der Erweiterung auf Mauerwerk mit Zugfestigkeiten ist des Weiteren die Kenntnis der zugehörigen Zugfestigkeiten, der geometrischen Parameter von Lagerfugen- und Stoßfugenabstand sowie der Abminderungsfaktoren unter Zug-Druck-Beanspruchung erforderlich.

Die Bestimmung der einzelnen Eingansparameter hat im Rahmen von Nebenversuchen zu erfolgen oder kann anhand der Angaben aus der Literatur (Ganz / Thürlimann [33, 34], Ganz et. al. [35], Guggisberg / Thürlimann [40], Lurati / Graf / Thürlimann [69], Schubert [120]) abgeschätzt werden.

\section{$\underline{\text { Dialer }}$}

Dialer [11] untersuchte an zweiachsig beanspruchten Modellmauerwerkselementen mit geneigten Fugen das Bruch- und Verformungsverhalten. Mit Hilfe der Ergebnisse konnte die Theorie von Mann / Müller und Ganz / Thürlimann bestätigt werden und einige Einflussparameter detailliert überprüft werden.

\section{Vratsanou}

In dem Beitrag von Vratsanou [155] wurde das ursprünglich auf Beton ausgerichtete Modell von Darwin / Pecknold auf unbewehrtes Mauerwerk angepasst (Motta / d'Amore [90]). Mit Hilfe einer äquivalenten einachsigen Dehnung wird dabei der Bezug des zweiachsigen Spannungszustandes auf den einachsigen Referenzspannungszustand hergestellt. Die Verifizierung 
des Modells unter zyklischer Schubbeanspruchung erfolgte anhand eines von Tomazevic [150] durchgeführten Versuches an einer Modellmauerwerksscheibe.

In einer Parameterstudie wurde von der Autorin anschließend ein Kragscheibensystem mit den Abmessungen 3,0 m $\cdot 3,0 \mathrm{~m} \cdot 0,3 \mathrm{~m}$ unter zwei Bodenbeschleunigungszeitverläufen untersucht. Die Kopfmasse von 3 bzw. 20 t wurde in Abhängigkeit der zwei untersuchten Mauerwerksfestigkeiten so bestimmt, dass die erste Eigenperiode jeweils $0,1 \mathrm{~s}$ betrug. Als Erregung wurde ein Sinusverlauf der Periode 0,2 s sowie die Aufzeichnung des Friaul-Bebens von 1976 gewählt. Bei den Berechnungen wurde jedoch keine Vertikallast angesetzt, was zu einer Verfälschung des Spannungszustandes in der Scheibe führte.

Die Auswertung der rechnerischen Untersuchungen erfolgte in Form der Darstellung von Bruchkurven in Abhängigkeit der Starkbebendauer und des Spitzenwertes der Bodenbeschleunigung. Als qualitatives Ergebnis wurde festgestellt, dass sich Mauerwerk unterhalb einer bestimmten Laststufe annähernd linear verhält und dass nach dem Überschreiten der Maximallast das Versagen generell schlagartig eintritt.

\section{$\underline{\text { Seim }}$}

Basierend auf die Theorie von Ganz / Thürlimann entwickelte Seim [127] ein Materialmodell nach der Plastizitätstheorie mit dem verschiedene historische Konstruktionen nachgerechnet und überprüft wurden. Für die untersuchten Systeme und Beanspruchungsfälle erwies sich die Vereinfachung zur idealen Plastizität nach dem Erreichen der Fließfläche als ausreichend.

\section{$\underline{\text { Mojsilovic }}$}

Mojsilovic [88] erweiterte das Modell von Ganz / Thürlimann [32, 140] auf den allgemeinen Fall exzentrisch einwirkender Scheibenkräfte. Dabei wird ein Sandwichmodell mit zwei Lamellen eingeführt, bei dem den Komponenten - d.h. Steinlamelle, Lager- und Stoßfuge ideal-plastisches Verhalten zugewiesen wird. Die Lagerfuge wird mit einer modifizierten Coulomb'schen Fließbedingung ohne Haftzugfestigkeit beschrieben. Stoßfugen können lediglich Druckspannungen übertragen, d.h. Schub- und Zugspannungen werden vernachlässigt. Das Steinmaterial besitzt eine Zugfestigkeit - eine Erhöhung der Festigkeit unter zweiaxialem Druck erfolgt nicht.

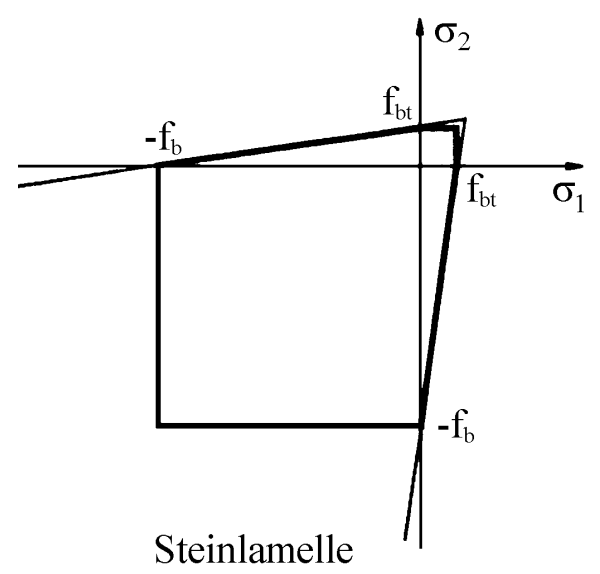

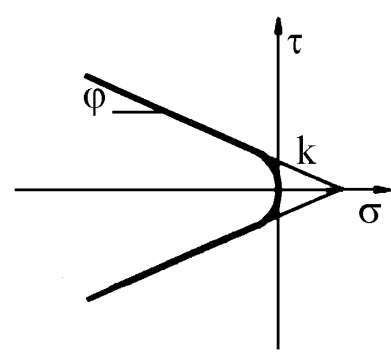

Lagerfuge

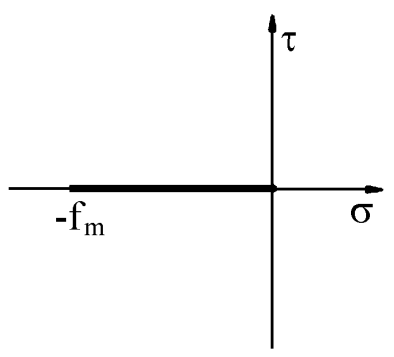

Stoßfuge

Abbildung 13: Fließbedingungen der Einzelkomponenten - nach Mojsilovic [88] 
Der Scheibenspannungszustand in den Steinlamellen wird mit Hilfe von Spannungsfeldern beschrieben. Anhand der Einwirkungen aus $n_{x}, n_{y}$ und $n_{x y}$ werden die einzelnen Beanspruchungszustände untersucht und die Versagenskriterien überprüft.
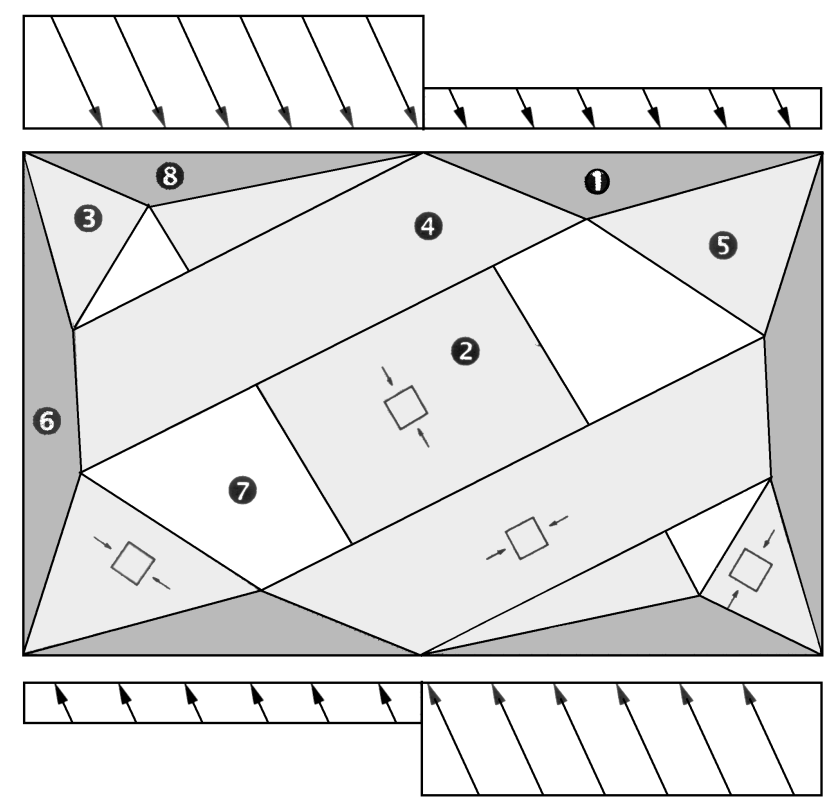

Abbildung 14: Spannungsfelder in der Steinlamelle unter Normal- und Schubbeanspruchung - nach Mojsilovic [88]

Das Modell wurde anhand eigener Versuche und Versuche aus der Literatur verifiziert.

\section{$\underline{\text { Lourenco }}$}

Eine umfassende Arbeit zur numerischen Beschreibung des Trag- und Verformungsverhaltens von Mauerwerk lieferte Lourenco [66, 67]. Zu Beginn werden mit Hilfe eines Mikromodells die verschiedenen Versagensarten von Mauerwerksscheiben untersucht und bewertet. Anschließend entwickelt der Autor ein eigenes Makromodell, bei dem die plastischen Verformungen und die Bruchenergie berücksichtigt werden. Die Beschreibung der Fließ- und Versagensflächen erfolgt im Druckbereich durch ein Hill-Kriterium und im Zugbereich durch ein Rankine-Kriterium.

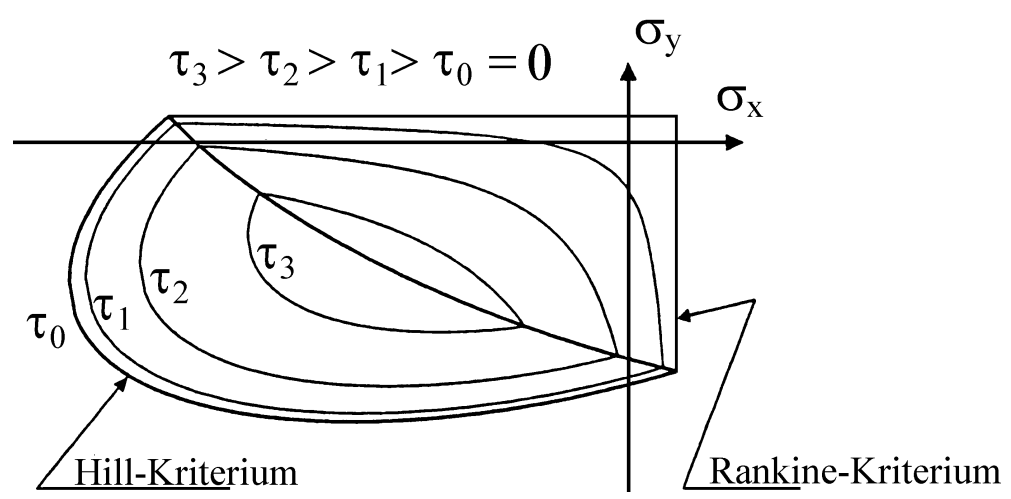

Abbildung 15: Fließ- und Versagensflächen von Lourenco [66]

Die Nachrechnung verschiedener Versuche ergab allgemein eine gute bis sehr gute Übereinstimmung, sowohl der erreichten Bruchwerte als auch des qualitativen Verlaufes. Des Weite- 
ren wurde das Modell für Untersuchungen zur Rissgefährdung zwangsbeanspruchter Wandscheiben verwendet.

\section{Fehling}

Anhand zweier Beispielkonstruktionen verglichen Fehling et. al. [31] verschiedene Rechenmodelle beim Nachweis im Lastfall Erdbeben. Zum einen wurde ein auf die Bruchkriterien von Mann / Müller aufbauendes anisotropes Makro-Modell mit verschmierten Materialeigenschaften verwendet und zum anderen wurde ein lokaler Riss am Fußpunkt der Konstruktion diskret abgebildet. Als Erweiterung wurden bei letzterem zusätzlich vertikale Massenträgheitskräfte sowie in der untersten Fuge Reibungsversagen über eine hysteretische Lagerfeder berücksichtigt. Des Weiteren wurde die Erdbebeneinwirkung ( $\rightarrow$ gemessener bzw. generierter Zeitverlauf/Lastfaktor) sowie die Wandgeometrie variiert.

Im Zuge der Berechnungen stellte sich die günstige Wirkung der erhöhten Verformbarkeit beim Klaffen in der untersten Lagerfuge als entscheidender Punkt dar. Durch die entsprechend reduzierte Sekantensteifigkeit war der nichtlineare Zusammenhang zwischen Bodenbeschleunigungsniveau und resultierender Querkraft in der Wand erkennbar.

Als Ergebnis konnte gezeigt werden, dass bereichsweise Verhaltensfaktoren deutlich über 1,5 möglich werden, wenn die verschiedenen Effekte berücksichtigt werden. Die Größe der Lastreduktion hing bei den Untersuchungen stark vom System, dem Beanspruchungsniveau, der Form des Antwortspektrums, der Versagensart sowie von der untersuchten Einwirkungsgröße (Querkraft / Moment) ab.

\section{Schlegel}

Schlegel [113] erweiterte aufbauend auf die Arbeit von Seim [127] das Bruchmodell von Ganz / Thürlimann auf die allgemeine räumliche Beanspruchungssituation. Das nichtlineare Spannungs-Dehnungsverhalten wird dabei durch angepasste Verfestigungs- und Entfestigungsfunktionen (s. a. van der Pluijm [104], Lourenco et. al. [66, 68]) und die zugehörigen Bruchenergien beschrieben. Die Zug- und Schubentfestigung wird mit Hilfe einer $e$-Funktion abgebildet. Für die Beanspruchung senkrecht zu den Lagerfugen wird die in Eurocode 2 bzw. DIN 1045-1 (2001-07) für die nichtlineare Schnittgrößenermittlung und Verformungsberechung von Beton vorgeschlagene Spannungs-Dehnungs-Funktion modifiziert und im Nachbruchbereich durch einen bi-linearen Abschnitt ergänzt.

\subsection{Bauteilversuche}

Die Mehrzahl der in der Vergangenheit weltweit durchgeführten Erdbebenversuche an Mauerwerksbauteilen erfolgte an bewehrtem Mauerwerk. Der Grund liegt zum einen an der deutlich höheren Tragfähigkeit mit der einhergehenden erheblich gesteigerten Duktilität und zum anderen an der Tatsache, dass in Starkbebengebieten, z. B. Südeuropa, tragende Bauteile aus Mauerwerk standardmäßig bewehrt ausgeführt werden.

Die Untersuchungen im Rahmen dieser Arbeit beschränken sich auf das Trag- und Verformungsverhalten unbewehrter Mauerwerksscheiben, wie sie in Deutschland gebräuchlich sind. Aus der Auswertung scheiden, neben Versuchen an bewehrtem Mauerwerk, somit auch die Untersuchungen an dem in den USA gebräuchlichen block-masonry, aus mit Beton verfüllten Steinen, aus (Mayes / Clough [77, 78], Shing / Lofti [131], Seible et. al. [125, 126]). Ebenfalls grundsätzlich außer Betracht fallen Untersuchungen an tragendem und nichttragendem Ausfachungsmauerwerk von Stahlbeton- oder Stahlrahmenkonstruktionen. 


\section{Clough, Gülkan und Mayes}

Clough, Gülkan und Mayes [10, 41] untersuchten Ende der 70er Jahre das Verhalten einfacher, einstöckiger Mauerwerksgebäude unter verschiedenen Bodenbeschleunigungen auf dem Rütteltisch. Als Material wurden ausbetonierte Mauersteine $(6 \cdot 4 \cdot 16$ inch concrete-blocks) verwendet. Es wurden Versuche mit bewehrtem, unbewehrtem sowie nachträglich verstärktem Mauerwerk durchgeführt. Die horizontale Aussteifung am Wandkopf der einstöckigen Gebäude erfolgte durch eine Holzdeckenkonstruktion. Es zeigte sich, dass die Gebäude aus unbewehrtem Mauerwerk erst bei Erdbebenlasten mit einer Bodenbeschleunigung von $a^{g}=0,2 \cdot \mathrm{g}$ die ersten Risse aufwiesen. Bei einer Erdbebenlast von $a^{g}=0,45 \cdot \mathrm{g}$ wuchsen die Verformungen - vor allem senkrecht zur Wandebene - stark an, und das Versagen stellte sich ein.

\section{Tomazevic und Weiss}

Anhand von Rütteltischversuchen an zwei Gebäudemodellen im Maßstab 1:5 untersuchten und verglichen Tomazevic und Weiss [148, 149] das Verhalten von bewehrtem und unbewehrtem Mauerwerk (s. a. $[142 \div 147,151,158]$ ).
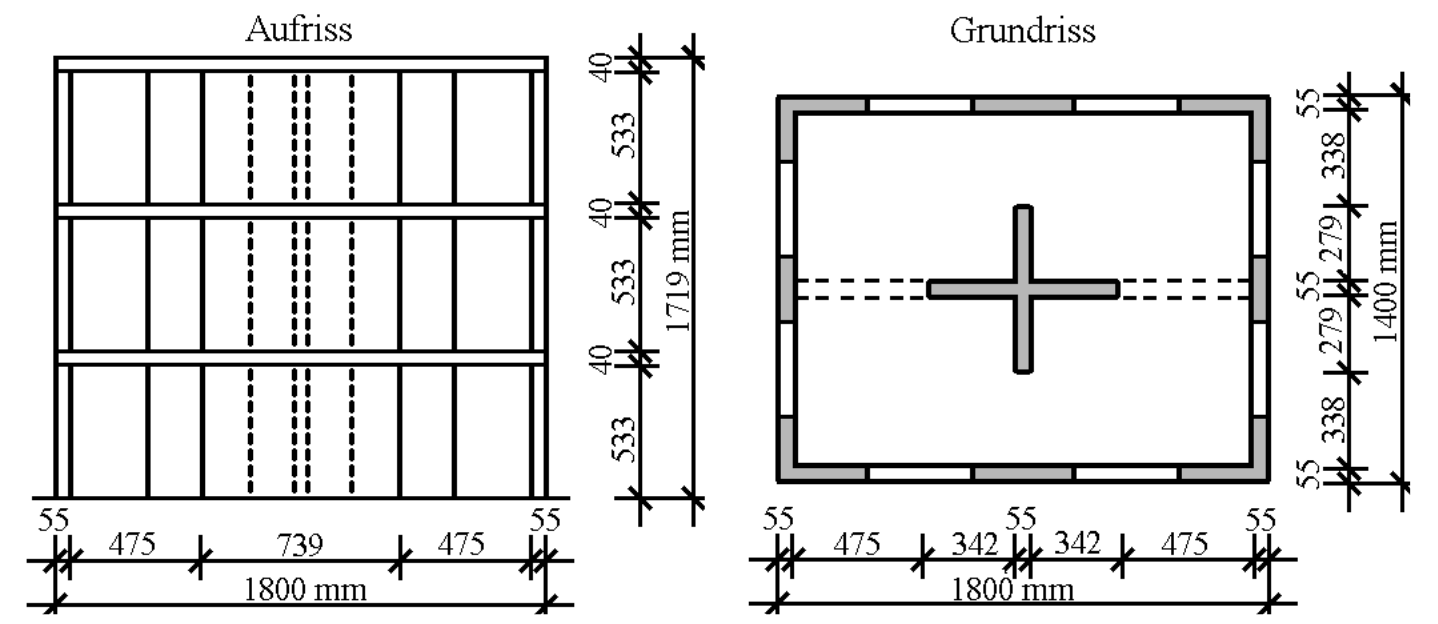

Abbildung 16: Gebäudemodell der Versuche von Tomazevic / Weiss [148]

Die Bestimmung der dynamischen Parameter für die verschiedenen Belastungsstufen ist in Tabelle 7 angegeben. Neben dem Abfall der Systemsteifigkeit mit der Belastungshöhe zeigt sich auch eine deutliche Zunahme des äquivalenten, viskosen Dämpfungsmaßes.

\begin{tabular}{|c|c|c|c|c|c|c|c|c|c|}
\hline \multirow[t]{2}{*}{ Wandbaustoff } & \multirow[t]{2}{*}{$\begin{array}{c}\text { Art der } \\
\text { Ermittlung }\end{array}$} & \multicolumn{2}{|c|}{$\begin{array}{c}\text { Ausgangs- } \\
\text { zustand }\end{array}$} & \multicolumn{2}{|c|}{$\begin{array}{c}\text { elastischer } \\
\text { Grenzzustand }\end{array}$} & \multicolumn{2}{|c|}{$\begin{array}{c}\text { maximaler } \\
\text { Widerstand }\end{array}$} & \multicolumn{2}{|c|}{ Bruchzustand } \\
\hline & & $f_{1}$ & $\zeta$ & $f_{1}$ & $\zeta$ & $f_{1}$ & $\zeta$ & $f_{1}$ & $\zeta$ \\
\hline \multirow{2}{*}{$\begin{array}{c}\text { bewehrtes } \\
\text { Mauerwerk }\end{array}$} & ASV & & 13,82 & 3,8 & 11,09 & 6,6 & 7,38 & 6,1 & 5,54 \\
\hline & VER & & & & 8,00 & & 4,74 & & 3,60 \\
\hline \multirow{2}{*}{$\begin{array}{l}\text { unbewehrtes } \\
\text { Mauerwerk }\end{array}$} & ASV & & 13,81 & 2,2 & 11,87 & 2,9 & 5,63 & 3,7 & - \\
\hline & VER & & & & 10,10 & & 3,60 & & - \\
\hline \multicolumn{10}{|c|}{$\begin{array}{l}f_{l} \text { erste Eigenfrequenz }[\mathrm{Hz}] \text { (Ermittlung durch FFT) } \\
\zeta \text { äquivalente viskose Dämpfung - in [\%] der kritischen Dämpfung } \\
\text { 1) ASV: Ausschwingversuch } \\
\text { VER: FFT der gemessenen Verschiebungs- und Beschleunigungsz }\end{array}$} \\
\hline
\end{tabular}

Tabelle 7: Dynamische Parameter der Modellgebäude von Tomazevic / Weiss [148]

Beim Vergleich der beiden Systeme über die Erdbebenzeit zeigen sich signifikante Unterschiede in der Verteilung der in das System eingetragenen Energie (Abbildung 17). Während 
das System aus bewehrtem Mauerwerk den Großteil der Energie über die hysteretische Dämpfung dissipiert, beträgt dieser Anteil bei dem System aus unbewehrtem Mauerwerk nur etwa $50 \%$. Die restliche Energie wird hier durch sonstige - als viskos bezeichnete - Dämpfungseffekte aufgefangen.
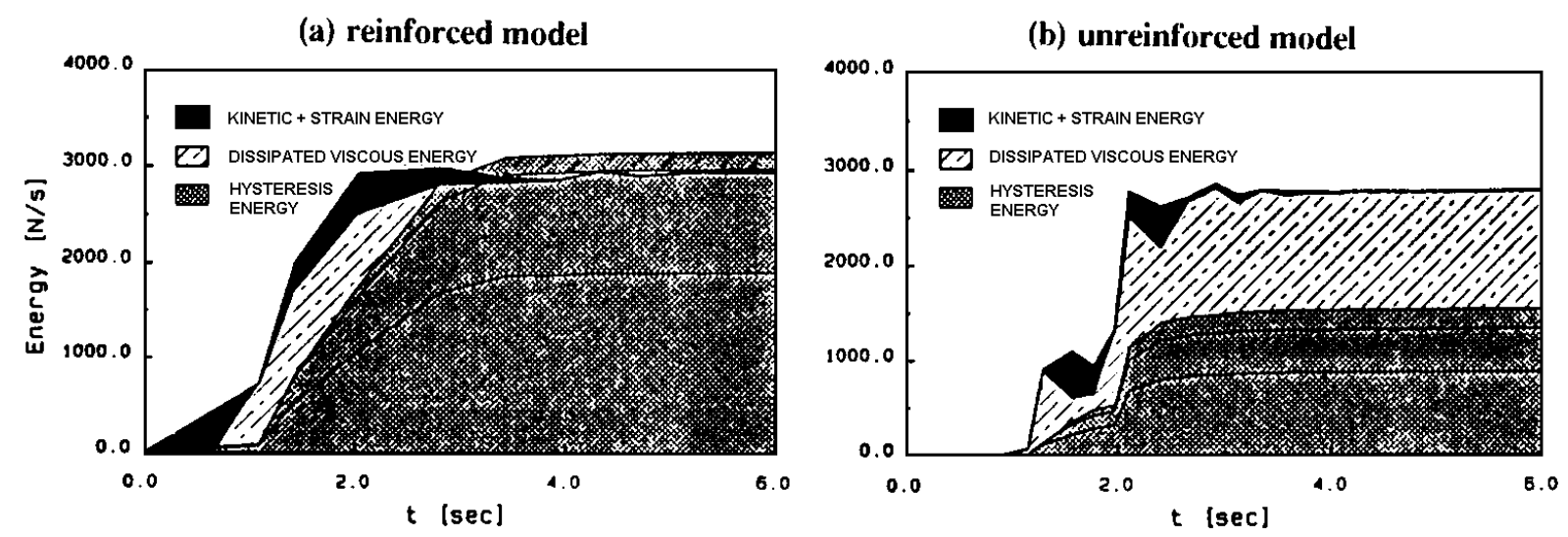

Abbildung 17: Aufteilung und Dissipation der Energie der beiden Gebäudemodelle über die Erdbebenzeit - aus Tomazevic / Weiss [148]

\section{König, Mann und Ötes}

In einer größeren Untersuchungsreihe wurden 1988 von König, Mann und Ötes auf dem Rütteltisch der TU Darmstadt an 10 unbewehrten Mauerwerkswänden insgesamt 62 Erdbebenversuche durchgeführt [60, 61, 72]. Als Materialien wurden Kalksandsteine mit und ohne Grifflöcher und Hochlochziegel jeweils mit der Mörtelgruppe IIa verwendet. Als Beschleunigungszeitverlauf wurde der Messschrieb in Tolmezzo während des Friaul-Erdbebens 1976 angesetzt. Bei der Versuchsdurchführung wurde die Kopfmasse von $7 \mathrm{t}$ über eine dazwischen angeordnete Stahlfeder auf dem Wandkopf fixiert. Dadurch wurde die Eigenschwingdauer des Systems auf einen für übliche Gebäude charakteristischen Wert von ca. $5 \mathrm{~Hz}$ eingerichtet. Die Wandabmessungen betrugen $1,24 \mathrm{~m} \cdot 1,14 \mathrm{~m} \cdot 0,115 \mathrm{~m}$.

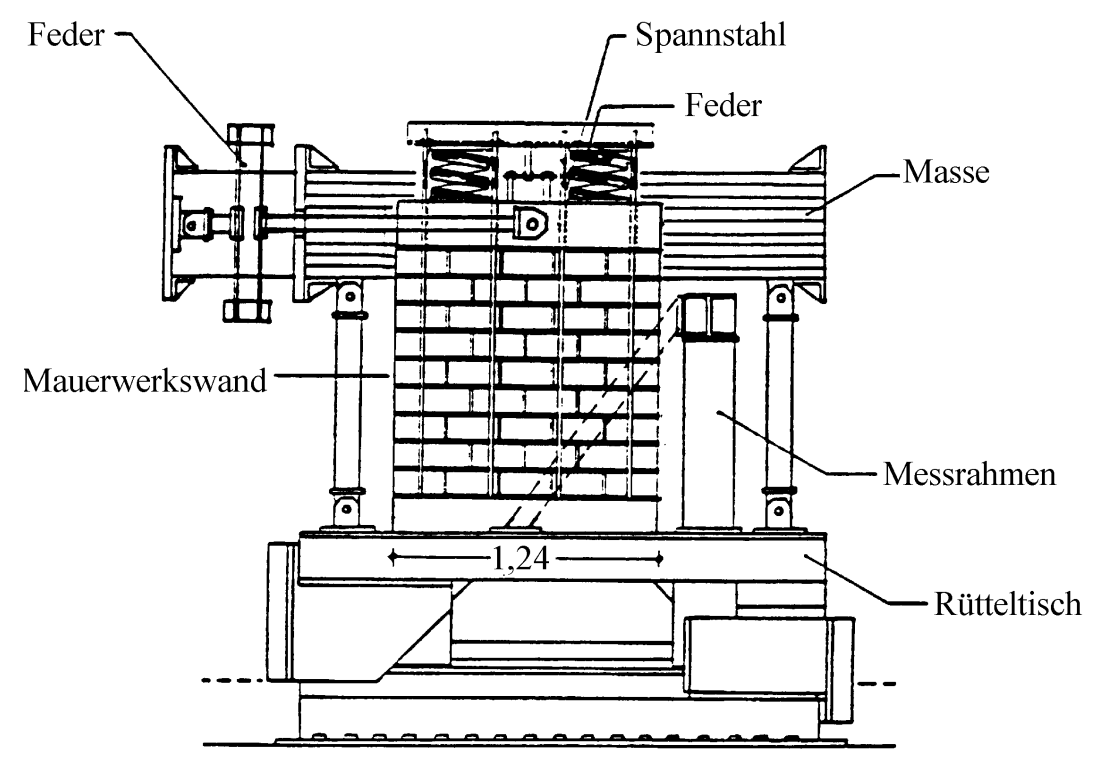

Abbildung 18: Versuchsaufbau der Rütteltischversuche von König / Mann / Ötes [60]

Nachfolgend sind in Tabelle 8 die wichtigsten Versuchsparameter zusammengestellt. 


\begin{tabular}{|c|c|c|c|c|c|}
\hline Versuch $^{1)}$ & Steinart & $\begin{array}{l}\text { Stein- / Mörtel- } \\
\text { druckfestigkeit } \\
f_{b} / f_{m o}\left[\mathrm{~N} / \mathrm{mm}^{2}\right]\end{array}$ & $\begin{array}{l}\text { Vertikal- } \\
\text { spannung } \\
\sigma\left[\mathrm{N} / \mathbf{m m}^{2}\right]\end{array}$ & $\begin{array}{l}\text { Versuchs- } \\
\text { anzahl } \\
n[-]\end{array}$ & $\begin{array}{c}\text { Horizontallast } \\
\text { - Faktor zum } \\
\text { Zeitverlauf } \\
\text { Tolmezzo } \\
\end{array}$ \\
\hline V01 D & \multirow{6}{*}{$\begin{array}{c}\text { KSV } 2 \text { DF, mit } 3 \text { Grifflöchern, } \\
\text { Rohdichte } 1,8 \mathrm{~kg} / \mathrm{dm}^{3} \text { (KSV 12) } \\
\text { SFK } 12, \text { NM IIa }\end{array}$} & Vorversuch & $-0,3$ & 1 & Vorversuch \\
\hline V02 D & & $14,7 / 4,1$ & $-0,6$ & 9 & $0,2 \div 1,0$ \\
\hline V03 D & & $14,7 / 7,7$ & $-1,0$ & 7 & $0,2 \div 1,0$ \\
\hline V04 D & & $14,7 / 7,7$ & $-1,5$ & 9 & $0,2 \div 1,4$ \\
\hline V02 S & & $14,7 / 9,2$ & $-0,6$ & 6 & statisch \\
\hline V04 S & & $12,8 / 6,9$ & $-1,5$ & 4 & statisch \\
\hline V11 D & \multirow{2}{*}{$\begin{array}{c}\text { KSV } 2 \text { DF, ohne Griffloch, } \\
\text { Rohdichte } 1,8 \mathrm{~kg} / \mathrm{dm}^{3} \text { (KSV 28) } \\
\text { SFK } 28, \text { NM IIa }\end{array}$} & $26,1 / 6,1$ & $-1,0$ & 12 & $\begin{array}{c}0,2 \div 1,2 \\
\text { (bzw. }-1,4)\end{array}$ \\
\hline V12 D & & $26,1 / 6,4$ & $-0,8$ & 6 & $\begin{array}{c}0,4 \div 1,2 \\
\text { (bzw. }-1,2)\end{array}$ \\
\hline V21 D & \multirow{2}{*}{$\begin{array}{c}\text { Hochlochziegel mit in Stein- } \\
\text { längsrichtung versetzten Stegen, } \\
\text { Rohdichte } 0,9 \mathrm{~kg} / \mathrm{dm}^{3} \text { (HLZ 12) } \\
\text { SFK } 12, \mathrm{NM} \mathrm{IIa}\end{array}$} & $10,9 / 7,0$ & $-1,0$ & 4 & $0,4 \div 1,2$ \\
\hline V22 D & & $10,9 / 7,9$ & $-0,6$ & 4 & $0,4 \div 1,0$ \\
\hline
\end{tabular}

Tabelle 8: Ausgangswerte der Versuche von König / Mann / Ötes [60]

Anhand der Ergebnisse der Versuche konnte gezeigt werden, dass das Versagensbild entscheidend für das Erdbebentragverhalten ist. Laufen die Risse abgetreppt durch die Fugen und tritt das Versagen nicht schlagartig auf, so kann von einem gutmütigen Verhalten gesprochen werden. Die bleibenden Dehnungen traten ohne wesentlichen Verlust der Tragfähigkeit auf (Versuche V02D, V11D und V12D). Die Auswertung führte in diesen Fällen zu einem Verhaltensfaktor der Einzelwand von $q_{\text {Wand }}=1,25 \div 1,30$.

Beim Auftreten von Rissen durch die Steine versagten die Wände schlagartig. Die Reaktionskraft entsprach dabei im Wesentlichen dem aus einer linear-elastischen Berechnung bestimmten Wert, was einem Verhaltensfaktor $q_{\text {Wand }}=1$ entspricht.

Untersuchungen zu der Wandsteifigkeit und deren Verlauf über die Belastungshöhe wurden nicht durchgeführt.

\section{Corderoy, Thambiratnam und Zhuge}

An acht unbewehrten Ziegelwänden mit den Abmessungen 1,4 $\mathrm{m} \cdot 0,96 \mathrm{~m} \cdot 0,11 \mathrm{~m}$ und $0,93 \mathrm{~m} \cdot 0,48 \mathrm{~m} \cdot 0,11 \mathrm{~m}(l \cdot h \cdot d)$ wurden von Corderoy, Thambiratnam und Zhuge [159] zyklische Schubversuche durchgeführt.

\begin{tabular}{|c|c|c|c|c|c|}
\hline Versuch & $\begin{array}{l}\text { Abmessung } \\
l \cdot h \cdot d[\mathrm{~m}]\end{array}$ & $\begin{array}{l}\text { Mörteldruckfestig- } \\
\text { keit } f_{m o}\left[\mathrm{~N} / \mathrm{mm}^{2}\right]\end{array}$ & $\begin{array}{c}\text { Haftscherfestigkeit } \\
k\left[\mathrm{~N} / \mathbf{m m}^{2}\right] \\
\end{array}$ & $\begin{array}{c}\text { Vertikallast } \\
N[\mathbf{k N}] \\
\end{array}$ & $\begin{array}{c}\text { Horizontallast- } \\
\text { aufbringung }\end{array}$ \\
\hline 1 & $1,4 \cdot 0,96 \cdot 0,11$ & 5,4 & 0,75 & 70 & zyklisch \\
\hline 2 & $1,4 \cdot 0,96 \cdot 0,11$ & 5,4 & 0,75 & 70 & zyklisch \\
\hline 3 & $0,93 \cdot 0,48 \cdot 0,11$ & 5,4 & 0,75 & 35 & zyklisch \\
\hline 4 & $0,93 \cdot 0,48 \cdot 0,11$ & 5,4 & 0,75 & 35 & zyklisch \\
\hline 5 & $0,93 \cdot 0,48 \cdot 0,11$ & 6,2 & 0,9 & 20 & monoton \\
\hline 6 & $0,93 \cdot 0,48 \cdot 0,11$ & 6,2 & 0,9 & \multicolumn{2}{|c|}{ Versagen vor Lastbeginn } \\
\hline 7 & $1,4 \cdot 0,96 \cdot 0,11$ & 6,2 & 0,9 & 70 & zyklisch \\
\hline 8 & $1,4 \cdot 0,96 \cdot 0,11$ & 6,2 & 0,9 & 70 & zyklisch \\
\hline
\end{tabular}

Tabelle 9: Ausgangswerte der Versuche von Zhuge, Thambiratnam und Corderoy [159]

Die verwendeten Vollziegel hatten die Abmessungen $0,225 \mathrm{~m} \cdot 0,07 \mathrm{~m} \cdot 0,11 \mathrm{~m}$ bei einer Fugenstärke von $10 \mathrm{~mm}$. Die Stoßfugen wurden voll vermörtelt. Der verwendete KalkZement-Mörtel hatte eine mittlere Druckfestigkeit von $5,8 \mathrm{~N} / \mathrm{mm}^{2}$ und eine mittlere Haft- 
scherfestigkeit von $0,825 \mathrm{~N} / \mathrm{mm}^{2}$. Die Mauerwerksdruckfestigkeit zum Zeitpunkt der Prüfung betrug $11,75 \mathrm{~N} / \mathrm{mm}^{2}$.

\begin{tabular}{|l|c|c|c|c|}
\hline Versuch & $\begin{array}{c}\text { Risslast } \\
{[\mathbf{k N}]}\end{array}$ & $\begin{array}{c}\text { Bruchlast } \\
{[\mathbf{k N}]}\end{array}$ & $\begin{array}{c}\text { Kersagensart }{ }^{\mathbf{1}} \\
\text { plebstoff zwischen Fundament- } \\
\text { platte und unterster Lagerfuge }\end{array}$ \\
\hline $\mathbf{1}$ & 35 & 90 & FV in der zweiten LaFu & ja \\
\hline $\mathbf{2}$ & 50 & 98,5 & FV in der untersten LaFu & nein \\
\hline $\mathbf{3}$ & 20 & 40 & FV in der untersten LaFu & nein \\
\hline $\mathbf{4}$ & 20 & 40 & FV in der untersten LaFu & nein \\
\hline $\mathbf{5}$ & 20 & 43,6 & FV in der untersten LaFu & nein \\
\hline $\mathbf{6}$ & 1,5 & 1,5 & Versagen bei erster Lastaufbringung & ja \\
\hline $\mathbf{7}$ & 40 & 80 & Stufenförmiges LaFu & ja \\
\hline $\mathbf{8}$ & 30 & 110 & FV in der obersten und untersten LaFu & \\
\hline
\end{tabular}

Tabelle 10: Ergebnisse der Versuche von Zhuge, Thambiratnam und Corderoy [159]

Eine Auswertung der Versuche bezüglich Steifigkeits-, Festigkeitsverlust und bleibenden Verformungen ist aufgrund fehlender Angaben nicht möglich. Der Vergleich kann lediglich mit den ersten Risslasten und den maximal erreichten Lasten durchgeführt werden.

\section{$\underline{\text { Modena }}$}

Modena $[5,86]$ stellte aufbauend auf einer umfangreichen Untersuchungsreihe an bewehrtem und unbewehrtem Mauerwerk ein einfaches Modell für die Beschreibung der KraftVerformungskurve auf. Als Eingangsgrößen für die Einhüllende (analog zu Abbildung 23) dienen die Parameter $\delta_{c r}, \lambda=H_{c r} / H_{\max }$ und $\mu=\lambda \cdot \delta_{H \max } / \delta_{c r}$. Für die Zyklische Beanspruchung gilt der in Abbildung 21 dargestellte Verlauf. Daneben ist der Vergleich von rechnerischer Kurve und dem Ergebnis eines statisch-zyklischen Versuches dargestellt.
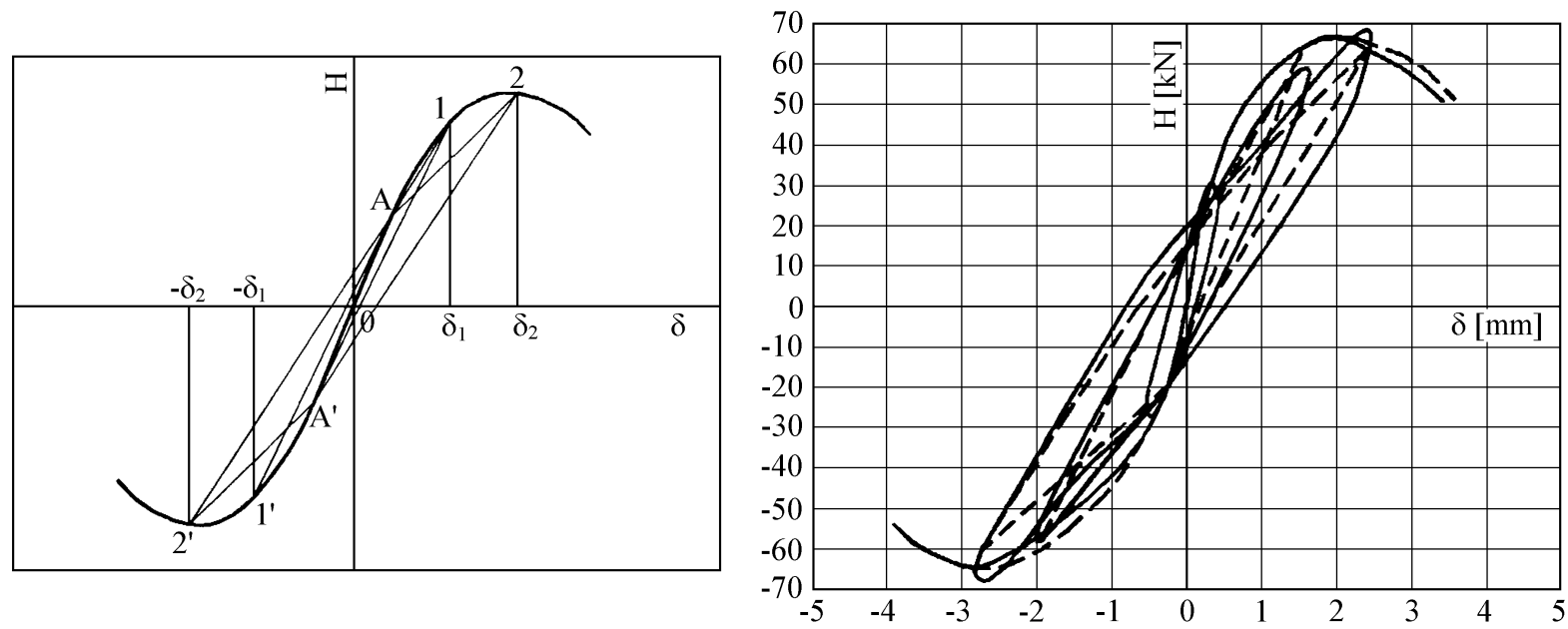

Abbildung 19: Zyklisches Modell von Modena [86] und Vergleich der rechnerischen Kraft-VerformungsLinie mit Versuchsergebnissen

Mit Hilfe des Modells und einem mittleren Duktilitätsfaktor $\mu=2,35$ wurde der Verhaltensfaktor $q$ rechnerisch bestimmt. Dazu wurde ein Einmassenschwinger unter zehn synthetisch generierten Zeitverläufen nichtlinear berechnet. Als Ergebnis stellte sich im Bereich der für Mauerwerksbauten charakteristischen Eigenperioden von $0,2 \div 0,4$ s ein Verhaltensfaktor von $1,2 \div 1,6$ ein. 


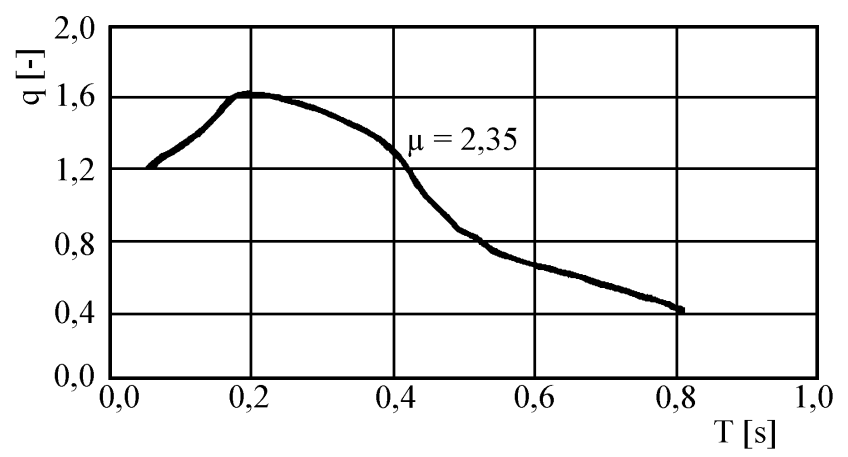

Abbildung 20: Rechnerisch ermittelter Verhaltensfaktor $q$ in Abhängigkeit der Eigenschwingdauer - aus Modena [86]

Der abfallende Verlauf des Graphen im Bereich ab $T>0,4 \mathrm{~s}$ mit Verhaltensfaktoren unter 1,0 ist auf die Form der Antwortspektren der angesetzten Zeitverläufe und die rechnerische Anfangssteifigkeit zurückzuführen.

\section{Naraine und Sinha}

Naraine und Sinha [93, 94, 95] führten an mehreren Mauerwerkskörpern im Maßstab 1:2 zyklische Druckversuche senkrecht und parallel zu der Lagerfuge durch. Nach erfolgter Entlastung schneidet die Spannungs-Dehnungslinie bei Wiederbelastung die Erstentlastungskurve in einem common point. Wird die Entlastung- und Wiederbelastung mehrmals wiederholt, so sinkt dieser Schnittpunkt weiter bis zu einem stabilen stability point ab. Dieser ist als Schnittpunkt von Wiederbelastungskurve zu Erstentlastungskurve für unbeschränkte Lastzykluszahlen definiert. Weiter zeigt sich, dass die Einhüllende der Spannungs-Dehnungslinie der zyklischen Versuche mit der Spannungs-Dehnungslinie bei monotoner Belastung zusammenfällt.
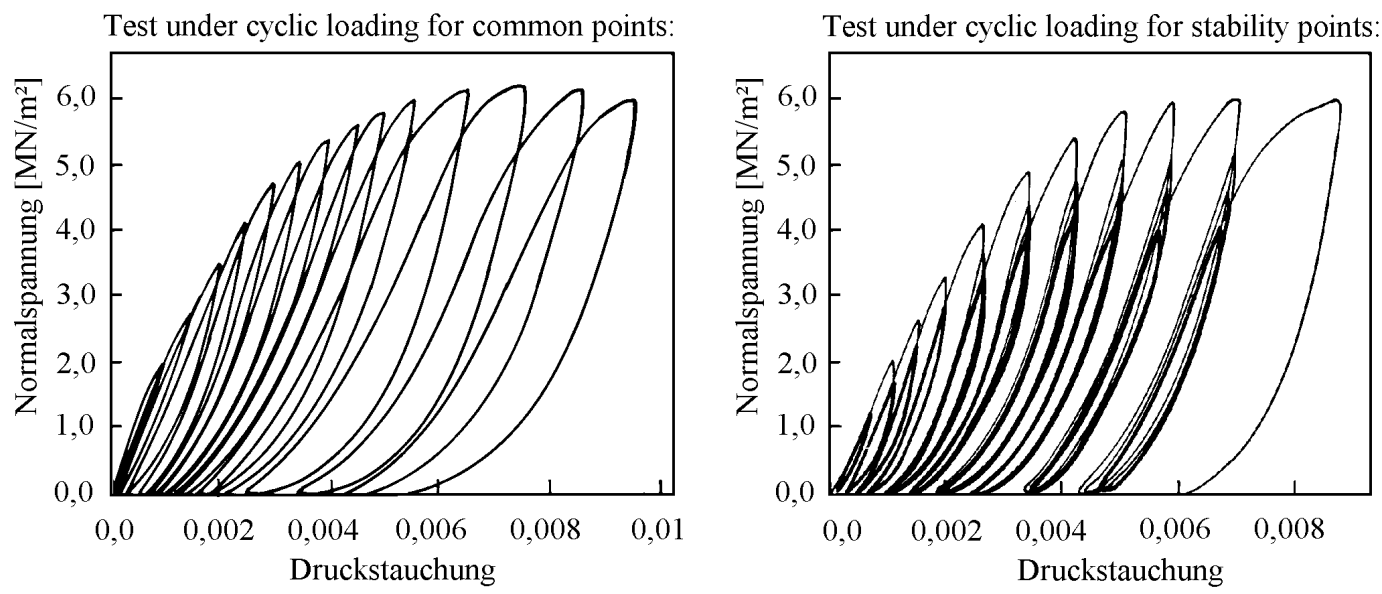

Comparison of envelope curve, common point curve, stability point curve

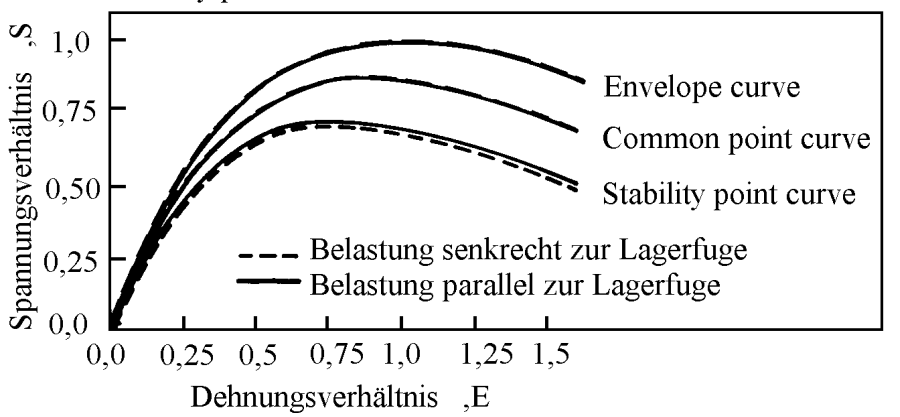

Abbildung 21: Spannungs-Dehnungslinien unter zyklischer Druckbelastung - aus Naraine / Sinha [93] 


\section{Bosiljkov, Zarnic und Bosiljkov}

Zur Untersuchung der Abhängigkeit des Schubtragverhaltens von Mauerwerk unter Erdbebenbelastung von den Mörteleigenschaften wurden von Bosiljkov / Zarnic / Bosiljkov [7] insgesamt 15 zyklische Schubversuche durchgeführt.

Für die Versuche an den Wänden mit den Abmessungen von 0,95 m $\cdot 1,4 \mathrm{~m} \cdot 0,12 \mathrm{~m}(l \cdot h \cdot d)$ wurden Vollziegel mit den Steinabmessungen $250 \mathrm{~mm} \cdot 65 \mathrm{~mm} \cdot 120 \mathrm{~mm}$ und voll vermörtelten Stoßfugen verwendet. Die Vertikallast betrug 1/6 der Vertikaltraglast, d.h. entsprechend der Mörtelfestigkeit zwischen 1,16 und 2,72 N/mm². Die Steindruckfestigkeit betrug im Mittel $19,52 \mathrm{~N} / \mathrm{mm}^{2}$ und die Spaltzugfestigkeit der Steine 1,89 N/mm². Es wurden die in Tabelle 11 aufgeführten fünf verschiedenen Mörtelarten verwendet.

\begin{tabular}{|c|c|c|c|c|c|}
\hline T. & Mörtel „Mix1““ & Mörtel „Mix2“ & Mörtel „Mix3“ & Mörtel „Mix4“ & Mörtel „Mix5“ \\
\hline Mischung & $\begin{array}{l}\text { Zement-Sand, } \\
\text { 1:4 in Vol.- } \\
\text { Anteilen }\end{array}$ & $\begin{array}{l}\text { Kalk-Zement- } \\
\text { Sand,1:1:6 in } \\
\text { Vol.-Anteilen }\end{array}$ & $\begin{array}{l}\text { Kalk-Sand, 1:3 } \\
\text { in Vol.-Anteilen }\end{array}$ & $\begin{array}{l}\text { Kalk-Zement- } \\
\text { Sand, } 1: 1: 6 \text { in } \\
\text { Vol.-Anteilen } \\
\end{array}$ & $\begin{array}{l}\text { Kalk-Zement- } \\
\text { Sand, } 1: 1: 6 \text { in } \\
\text { Vol.-Anteilen }\end{array}$ \\
\hline Vergütung & - & - & - & $\begin{array}{l}\text { Polypropylen- } \\
\text { fasern im } \\
\text { Mörtel }{ }^{1)}\end{array}$ & $\begin{array}{c}\text { Glasfaser- } \\
\text { gewebe in } \\
\text { Lagerfugen }\end{array}$ \\
\hline $\begin{array}{l}\text { Druckfestigkeit } \\
f_{m o}\left[\mathbf{N} / \mathbf{m m}^{2}\right]\end{array}$ & 13,85 & 9,47 & 1,13 & 7,69 & 9,57 \\
\hline $\begin{array}{l}\text { Biegezugfestigkeit } \\
f_{m o, B Z}\left[\mathrm{~N} / \mathbf{m m}^{2}\right]\end{array}$ & 3,72 & 2,76 & 0,37 & 2,31 & 2,85 \\
\hline $\begin{array}{l}\text { Haftzugfestigkeit } \\
f_{m o, H Z}\left[\mathbf{N} / \mathbf{m m}^{2}\right]\end{array}$ & 0,41 & 0,342 & 0,127 & 0,404 & 0,369 \\
\hline \multicolumn{6}{|c|}{$\begin{array}{l}\text { 1) Polypropylenfasern Typ Krenit, Faserlänge } 6 \mathrm{~mm} \text {, Querschnitt } 35 \cdot 250 \div 600 \mu \mathrm{m} \text {, Zugfestigkeit } \\
340 \div 500 \mathrm{~N} / \mathrm{mm}^{2} \text {, E-Modul } 8.500 \div 12.500 \mathrm{~N} / \mathrm{mm}^{2} \text {, Bruchdehnung } 8 \div 10 \% \\
\text { 2) Glasfasergewebe mit Öffnungen von } 5 \mathrm{~mm} \cdot 5 \mathrm{~mm} \text {, maximale Zugkraft } 1,5 \mathrm{kN} \text {, Bruchdehnung }<3,5 \%\end{array}$} \\
\hline
\end{tabular}

Tabelle 11: Eigenschaften der verwendeten Mörtelarten von Bosiljkov / Zarnic / Bosiljkov [7]

Aus der Versuchsdurchführung ergaben sich die in Abbildung 22 beispielhaft dargestellten Hysteresekurven.

Der qualitative Verlauf der fünf Hysteresekurven ist im Wesentlichen miteinander vergleichbar. Nach einem linear-elastischen Bereich erfolgt ein Abflachen der Kurven und Aufweiten der Hysteresen. Signifikante, bleibende Verformungen sind erst bei den höheren Laststufen in der Größenordnung von der Maximallast erkennbar.

Bei der Wand mit dem Mörtel „Mix 3“ weicht die Form der Kurve dagegen von der der anderen Versuche etwas stärker ab. Der Grund liegt in der deutlich geringeren Druckfestigkeit und Haftscherfestigkeit (s. Tabelle 11). Dadurch kam es während dem Versuch zur Zerstörung des Mörtels in den Lagerfugen.

Bei dem Wandversuch mit Glasfaserbewehrung im Mörtel („Mix 5“) ist das Verhalten im Vergleich zu den anderen Wänden deutlich duktiler. Nach dem Erreichen der Maximallast von $\left|H_{\max }\right|=92,2 \mathrm{kN}$ können noch weitere drei Zyklen durchlaufen werden. Im gegenüberliegenden, ersten Quadranten korrespondiert die Form der Hysterese mit der des Versuches mit dem Referenzmörtel Typ (,Mix 2“). 


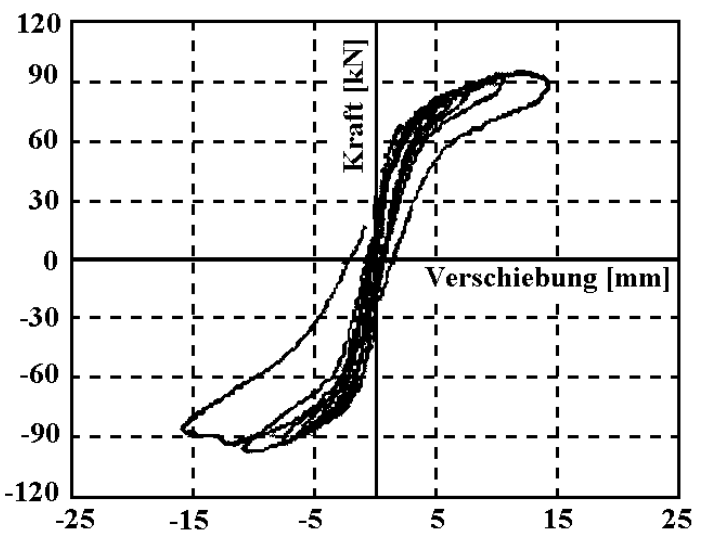

a) Mix 1

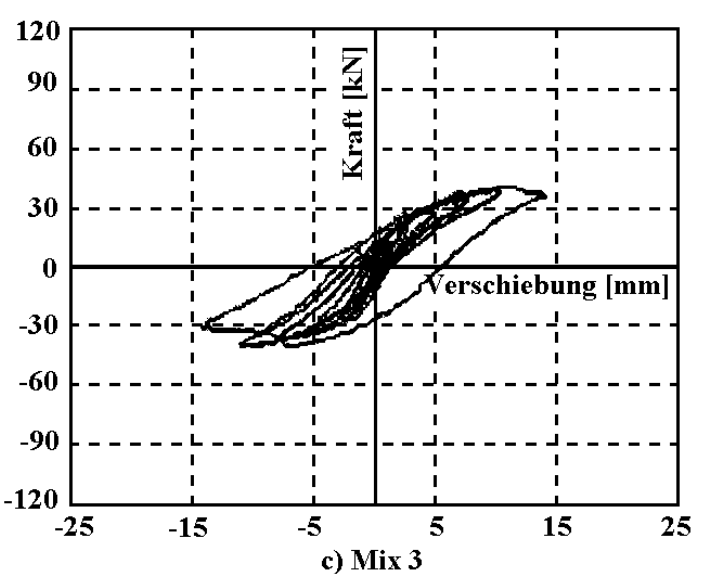

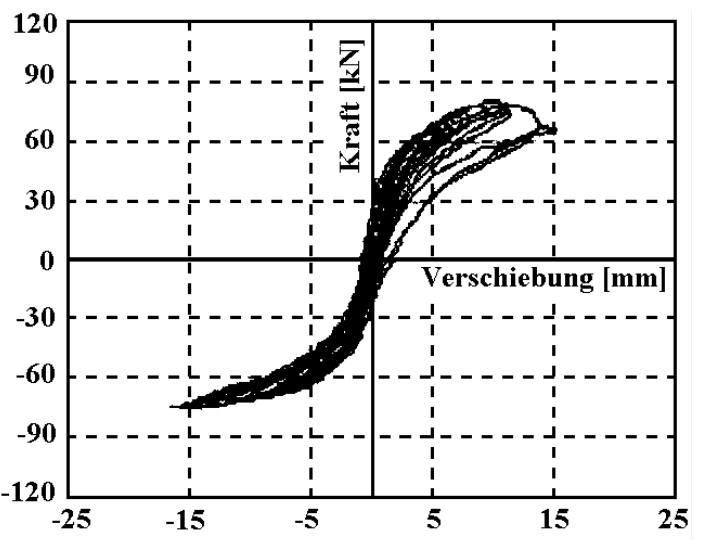

b) Mix 2

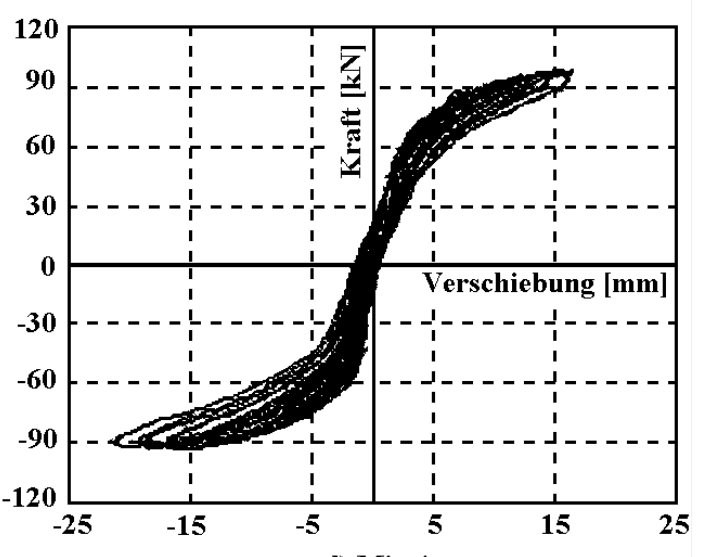

d) $\operatorname{Mix} 4$

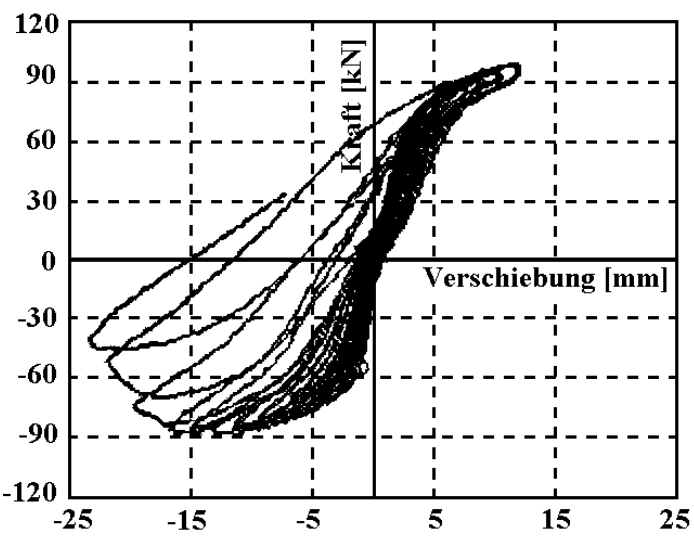

e) $\operatorname{Mix} 5$

Abbildung 22: Kraft-Verformungs-Diagramm der Versuche von Bosiljkov / Zarnic / Bosiljkov [7]

Die Rückführung der Hysteresekurven auf die in Abbildung 23 dargestellte idealisierte Form ergibt die in Tabelle 12 zusammengefassten Parameter. 


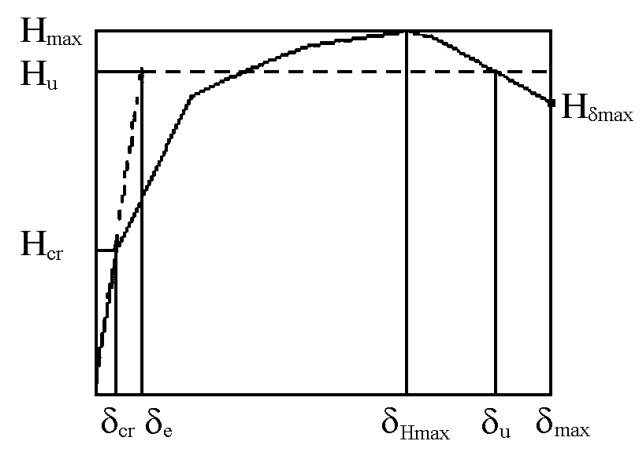

Abbildung 23: Idealisierte Form der Kraft-Verformungs-Beziehungen der Versuche von Bosiljkov / Zarnic /Bosiljkov [7]

\begin{tabular}{|l|c|c|c|c|c|c|c|c|c|c|c|}
\hline $\begin{array}{c}\text { Versuch } \\
\text { Nr. }\end{array}$ & $\begin{array}{c}\text { Teil- } \\
\text { versuch } \\
\text { Nr. }\end{array}$ & $\begin{array}{c}\text { Normal- } \\
\text { spannung } \\
{\left[\mathbf{N} / \mathbf{m m}^{2}\right]}\end{array}$ & $\begin{array}{c}\boldsymbol{H}_{\boldsymbol{m a x}} \\
{[\mathbf{k N ]}]}\end{array}$ & $\begin{array}{c}\boldsymbol{\delta}_{\boldsymbol{H} \boldsymbol{x}} \\
{[\mathbf{m m}]}\end{array}$ & $\begin{array}{c}\boldsymbol{\delta}_{\boldsymbol{m} \boldsymbol{x}} \\
{[\mathbf{m m}]}\end{array}$ & $\begin{array}{c}\boldsymbol{H}_{\boldsymbol{u}} \\
{[\mathbf{k N}]}\end{array}$ & $\begin{array}{c}\boldsymbol{H}_{\boldsymbol{f}} \\
{[\mathbf{k N}]}\end{array}$ & $\left.\begin{array}{c}\boldsymbol{k}_{\boldsymbol{e}} \\
{[\mathbf{k N} /}\end{array}\right]$ & $\begin{array}{c}\boldsymbol{\delta}_{\boldsymbol{e}} \\
{[\mathbf{m m}]}\end{array}$ & $\begin{array}{c}\boldsymbol{\mu}_{\boldsymbol{u} \boldsymbol{k}} \\
{[-]}\end{array}$ & $\begin{array}{c}\boldsymbol{H}_{\boldsymbol{s}} \\
{[\mathbf{k N}]}\end{array}$ \\
\hline Mix 1 & 2 & 2,72 & 96,6 & 8,3 & 11,7 & 90,0 & 59,5 & 39,2 & 2,7 & 4,5 & 65,6 \\
\hline Mix 2 & 3 & 2,00 & 71,6 & 10,4 & 12,0 & 63,8 & 33,5 & 39,8 & 1,6 & 7,5 & 67,5 \\
\hline Mix 3 & 3 & 1,16 & 44,6 & 9,5 & 12,5 & 39,8 & 23,3 & 18,4 & 2,6 & 5,9 & 27,9 \\
\hline Mix 4 & 3 & 2,42 & 87,8 & 13,9 & 15,5 & 80,0 & 47,2 & 33,6 & 2,4 & 6,7 & 81,1 \\
\hline Mix 5 & 2 & 2,54 & 92,2 & 13,4 & 20,5 & 81,7 & 51,5 & 33,4 & 2,5 & 8,5 & 67,6 \\
\hline
\end{tabular}

Tabelle 12: Versuchsergebnisse und Parameter der idealisierten Hysteresekurven von Bosiljkov / Zarnic / Bosiljkov [7]

\section{$\underline{\text { ETH Zürich }}$}

An der ETH Zürich wurden über einen längeren Zeitraum verschiedene Untersuchungen zum Trag- und Verformungsverhalten kombiniert beanspruchter Mauerwerksscheiben durchgeführt, von denen die wichtigsten nachfolgend kurz beschrieben werden.

Ganz und Thürlimann [33] testeten zu Beginn der 80er Jahre 12 Mauerwerkskörper aus Hochlochziegeln mit Zementmörtel. Ziel war die Ermittlung von Bruchbedingungen zweiachsig beanspruchten Mauerwerkes (s. Abschnitt 3.1). Variiert wurden an den 1,2 m·1,2 m großen Körpern die Lagerfugenneigung unter kombinierter Druck-Druck bzw. Druck-ZugBeanspruchung.

In Erweiterung dazu wurden [34] an sieben geschosshohen Wänden der Länge 3,6 m mit jeweils einem Flansch an beiden Enden statische und zyklische Schubversuche durchgeführt. Bei den zyklischen Beanspruchungen lag die Bruchlast etwa 15\% tiefer als bei progressiver Laststeigerung. Der Bruch wurde hier durch Steinzugversagen in Wandmitte ausgelöst, wohingegen das Versagen bei monotoner Laststeigerung am Wandende zu beobachten war. Der elastische Bereich, bei dem keinerlei Einfluss der Zyklenzahl auf das Last-Verformungsbild erkennbar war, lag bei etwa 50\% der Maximallast. Der Einfluss der Normalkraft auf die Bruchkraft [32, 140] konnte bestätigt werden. Die Duktilität der Wände war bei geringen Normaldruckspannungen deutlich höher als bei hoher Normalkraft. Versuche mit Lagerfugenbewehrung konnten die Traglast nicht bedeutend steigern, wohingegen die Duktilität dadurch deutlich verbessert wurde.

Lurati und Thürlimann führten an drei Wänden aus Zementstein und Zementmörtel Schubversuche unter Variation der Normalkraft analog zu [33] durch. Der Einfluss der Normalkraft auf das Verformungsvermögen und die Bruchquerkraft zeigte sich auch bei diesen Steinen deutlich. Die Versuchsnachrechnung mit Hilfe der Bruchbedingungen von Ganz [32, 140] ergab eine gute Übereinstimmung. 
Für die Bestimmung der Rechenwerte der Mauerwerksfestigkeiten in Abhängigkeit der SteinMörtel-Kombination wurden von Guggisberg und Thürlimann [40] als Erweiterung und analogem Versuchprogramm zu [33] insgesamt 63 Kleinversuchskörper geprüft. Anhand der Ergebnisse konnten die Eingansparameter der Bruchbedingungen [32, 140] bestimmt werden. Ergänzend dazu erfolgten von Lurati, Graf und Thürlimann [69] und Lurati und Thürlimann [70] Untersuchungen an Körpern und geschosshohen Wänden aus Zementsteinen.

Mojsilović und Marti [87] untersuchten Anfang der 90er Jahre an 20 Kleinprüfkörpern und 28 geschosshohen Wänden das Verhalten von Mauerwerk unter kombinierter Scheiben- und Plattenbeanspruchung. Als Materialien wurden Hochlochziegel, Kalksand- und Zementsteine verwendet. Einige Versuche wurden mit Bewehrung oder mit Vorspannung durchgeführt. Die Versuchskörper mit den Abmessungen 1,29 m · 1,30 m (Kleinprüfkörper) bzw. 1,03 m · 2,6 m und $1,03 \mathrm{~m} \cdot 5,0 \mathrm{~m}$ (geschosshohe Wände) wurden mit einer Neigung der Lagerfuge von $\alpha=0 \div 45^{\circ}$ hergestellt. Die Prüfung der Kleinprüfkörper erfolgte unter monoton gesteigerter zentrischer Belastung. Infolge der Neigung der Lagerfugen konnten die Mauerwerksfestigkeiten $f_{m x}, f_{m y}$ sowie die Fugenparameter Kohäsion $k$ und Reibungswinkel $\varphi$ bestimmt werden. Bei den geschosshohen Wänden wurde zuerst die unter dem Winkel $\alpha$ zu den Lagerfugen wirkende Scheibenkraft aufgebracht und anschließend über eine Fußverdrehung ein Moment in Plattenebene eingeleitet. Diese Verdrehung wurde bis zum Bruch gesteigert. Als Ergebnis zeigte sich, dass mit gesteigerter Normalkraft, der erste Riss bei einer größeren Fußverdrehung auftrat, die maximal mögliche Verdrehung jedoch abnahm. Es trat größtenteils sprödes Versagen auf - duktiler versagte lediglich Kalksandstein- und Zementsteinmauerwerk mit horizontalen Lagerfugen $\left(\alpha=0^{\circ}\right)$.

\section{Vratsanou / Langer}

Vratsanou und Langer [154, 156] führten an insgesamt 12 unbewehrten Porenbetonwänden mit den Abmessungen 2,0 $\mathrm{m} \cdot 0,24 \mathrm{~m} \cdot 2,5 \mathrm{~m}(1 \cdot \mathrm{b} \cdot \mathrm{h})$ statische Schubversuche durch. Als Materialien wurden Plansteine der Festigkeitsklasse 2 und 4 mit Dünnbettmörtel verwendet. Neben der Auflast wurde auch das Überbindemaß ( $\ddot{u} / \Delta y=1,0$ bzw. 0,4) variiert. Bei drei Wänden wurden die Stoßfugen vermörtelt, um diesen Einfluss auf das Schubtragverhalten zu untersuchen. Die Anordnung der Wand im Versuchsrahmen ergab eine mechanische Behinderung der Kopfverdrehung mit Hilfe von Spannstäben, wodurch im Bruchzustand eine zusätzliche Normalkraft $\Delta N_{U}$ resultierte. Diese betrug das Vielfache der im Anfangszustand aufgebrachten Soll-Normalkraft $N_{0}$. So lag bei Versuch 4.5 beispielsweise mit $\Delta N_{U}=173,7 \mathrm{kN}$ und $N_{0}=52,8 \mathrm{kN}$ der Verhältniswert bei 4,3.

Das Versagen trat bei allen Versuchen schlagartig ein - eine Steigerung der Horizontallast nach dem ersten Bruch war anschließend nicht mehr möglich. Das Versagensbild äußerte sich bei allen Wänden durch schräge Risse, die mehrheitlich wahllos durch Steine und Fugen verliefen. Reibungsversagen in den Fugen war aufgrund der hohen Haftscherfestigkeit und des hohen Reibbeiwertes nicht maßgebend. Die Bruchlasten der Wände mit vermörtelten Stoßfugen lagen $40 \% \div 100 \%$ über denen ohne Stoßfugenvermörtelung, was jedoch auch auf die in diesen Fällen deutlich höheren zusätzlich aktivierten Normalkräfte $\Delta N_{U}$ zurückzuführen war.

\section{$\underline{\text { Ötes }}$}

Ötes beschreibt in [55] die zurzeit laufenden statisch-zyklischen Versuche an unbewehrtem Mauerwerk mit verschiedenen Stein-Mörtel-Kombinationen. Im Rahmen dieser Versuchsserie an der Universität Dortmund werden des Weiteren die Randbedingungen der Wände, d.h. die 
Einspannung am Wandkopf, und die Normalkraft variiert. Allgemein stellt sich auch hier die Problematik der bei mechanischer Behinderung der Kopfverdrehung einhergehenden Aktivierung von Normalkräften unbekannten Betrages (s. Versuche von Langer/Vratsanou und Abschnitt 5.1.2). Abschließende Ergebnisse wurden noch nicht veröffentlicht.

\subsection{Untersuchungen zur Bruchenergie}

\section{Zugbeanspruchung}

Für die Bestimmung der Bruchenergie von Mauerwerk unter Zugbeanspruchung führte van der Pluijm [104] umfangreiche Untersuchungen an Mauerwerk senkrecht zu den Lagerfugen sowie an Einzelsteinen durch. Als Materialien wurden hier Vollziegel und Kalksandsteine mit Kalk-Zement-Mörtel verwendet.

Bei den Zugversuchen an Mauerwerkskörpern senkrecht zu den Lagerfugen trat das Versagen in der Kontaktfläche von Stein und Mörtel auf. Die Haftzugfestigkeit lag dabei zwischen 0,13 und $0,5 \mathrm{~N} / \mathrm{mm}^{2}$ und die Bruchenergie $G_{f}$ bezogen auf die Bruttoquerschnittsfläche zwischen 0,004 und $0,012 \mathrm{Nmm} / \mathrm{mm}^{2}$. Es zeigte sich jedoch nach den Versuchen, dass nicht die gesamte Lagerfläche der Steine von Mörtel bedeckt war. Wird die Bruchenergie auf die resultierende, benetzte Nettoflächen bezogen, ergibt sich ein Wertebereich von $0,01 \div 0,03 \mathrm{Nmm} / \mathrm{mm}^{2}$. Wird dieser Wert wiederum auf einen mittleren Benetzungsgrad in der Wand von $59 \%$ umgerechnet, so resultiert eine Bruchenergie $G_{f}$ zwischen 0,006 und $0,018 \mathrm{Nmm} / \mathrm{mm}^{2}$. Für die Haftzugfestigkeit ergibt sich bei analoger Vorgehensweise ein Wertebereich von $0,3 \div 0,87 \mathrm{~N} / \mathrm{mm}^{2}$. Die Normalspannungs-Verschiebungs-Beziehung einiger ausgewählter Versuche ist in Abbildung 24 dargestellt.

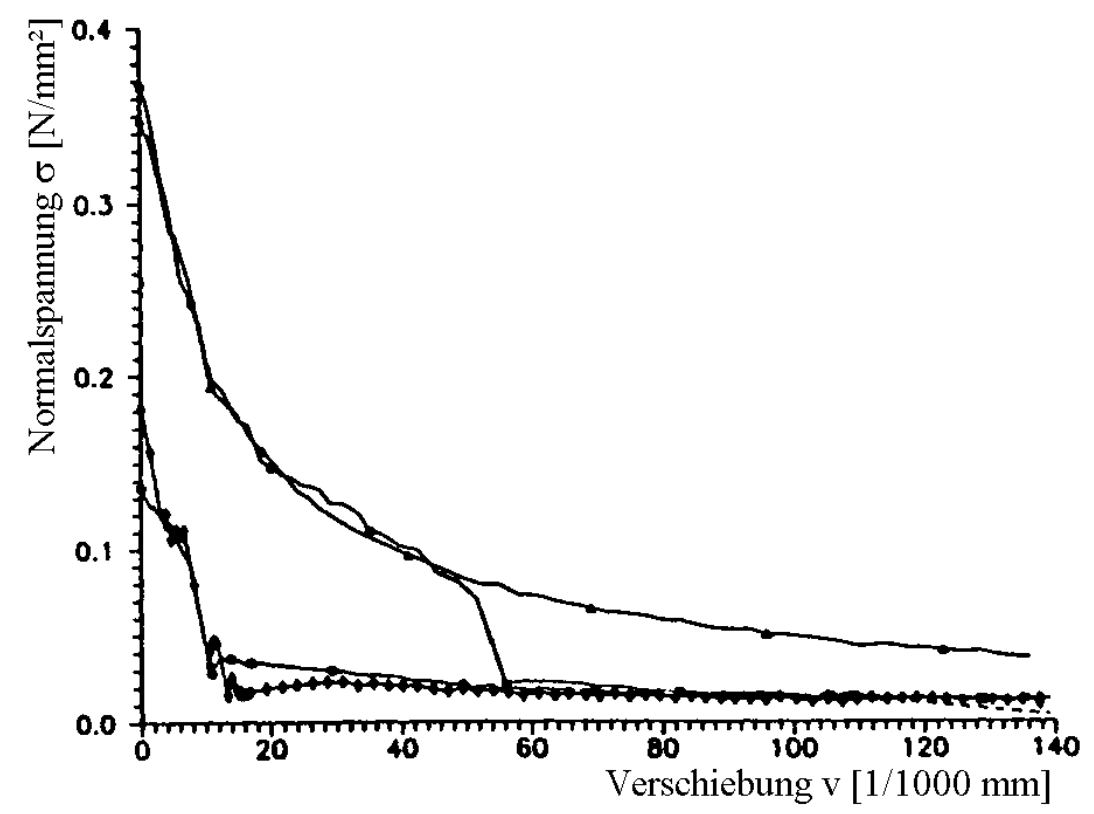

Abbildung 24: Normalspannungs-Verschiebungs-Diagramm bei zugbeanspruchtem Mauerwerk senkrecht zu den Lagerfugen - aus van der Pluijm [104]

Die Prüfung der Steine auf Zug senkrecht und parallel zu den Lagerfugen ergab bei den Untersuchungen von van der Pluijm [104] Zugfestigkeiten zwischen 1,5 und 3,5 N/mm². Die Bruchenergie $G_{f}$ betrug zwischen 0,061 und $0,128 \mathrm{Nmm} / \mathrm{mm}^{2}$. Der qualitative Verlauf der Normalspannungs-Verschiebungs-Beziehung ist dabei ähnlich zu Abbildung 24. 


\section{Schubbeanspruchung}

Untersuchungen zum Verbundverhalten zwischen Stein und Mörtel einschließlich des Lastbereiches nach Überschreitung der Maximallast wurden von Lourenco / Rots [68], van der Pluijm [104, 105, 106], Hansen / Nykänen / Gottfredsen [45] und Molnar [89] durchgeführt. Die prinzipielle Versuchseinrichtung für die Einleitung einer Schubspannung und konstanten Normalspannung über den Kontaktbereich ist in Abbildung 25 dargestellt.
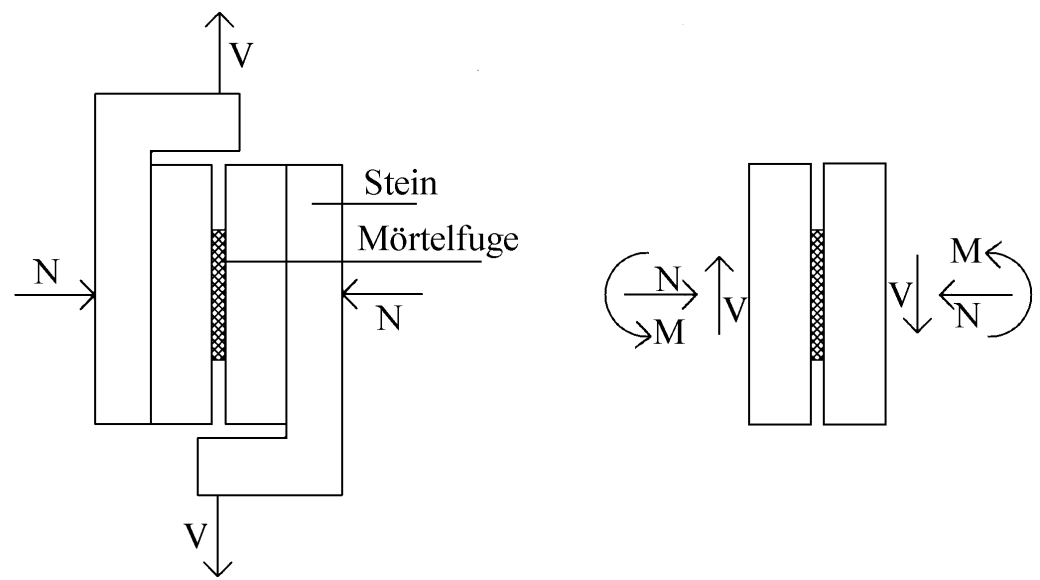

Abbildung 25: Schematische Darstellung der Zweistein-Schubversuche von Hansen / Nykänen / Gottfredsen [45] mit angreifenden Schnittgrößen

In Abbildung 26 ist der Verlauf der Schubspannung in Abhängigkeit der Schubverformung dargestellt. Die Normalspannung wurde während der Versuche konstant gehalten.

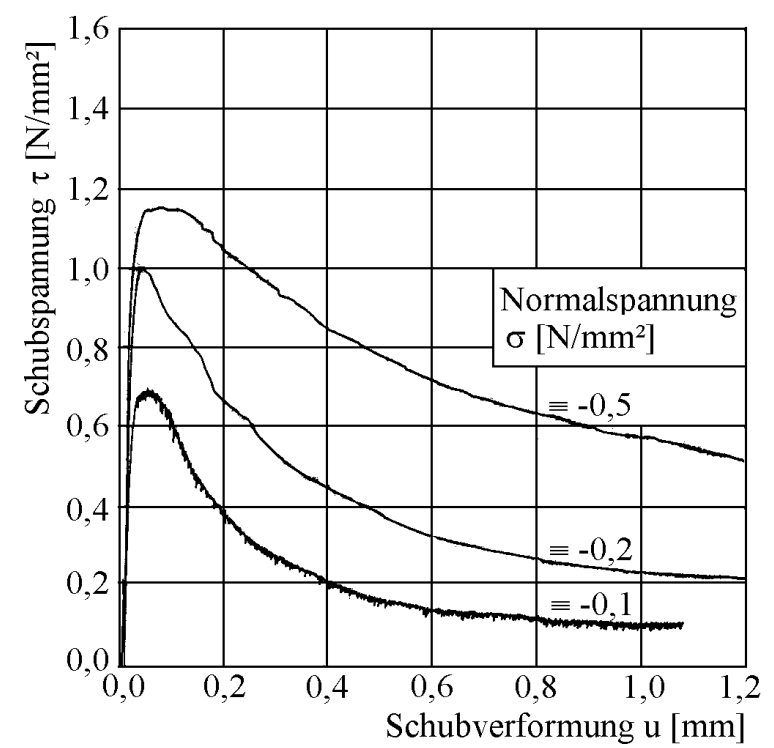

Abbildung 26: Schubspannungs-Verformungs-Diagramm der Zweistein-Schubversuche - Versuch DP60 (Hochlochziegel / Kalk-Zementmörtel) von Hansen / Nykänen / Gottfredsen [45]

In allen Untersuchungen zeigte sich nach Überschreiten der Maximallast ein exponentieller Abfall der Kraft-Verformungslinie bis auf einen konstanten Reibanteil. Die Höhe der Resttragfähigkeit war proportional zu der vorhandenen Normalspannung.

Van der Pluijm [105] und Lourenco / Rots / van der Pluijm [68] geben des Weiteren in Abhängigkeit der Normaldruckspannung den Dilatationswinkel $\psi$ an, der das Richtungsverhältnis von Schub- und Normalspannungsverformung über $\tan \psi=u / v$ beschreibt. Es ist eine deutliche Abhängigkeit von der Höhe der Auflast erkennbar. Bei kleinen Normaldruckspan- 
nungen in der Fuge liegt $\tan \psi$ mit großer Streuung zwischen 0,2 und 0,7, während mit zunehmender Normaldruckspannung der Wert auf $0,2 \div 0,3$ absinkt. Dieses ist durch die glättere Oberflächenstruktur der entstehenden Bruch- bzw. Gleitfläche zu erklären. Bei weiterer Steigerung der Schubverformung kann davon ausgegangen werden, dass eine reine Schubgleitung stattfindet und der Anteil der Verformung senkrecht zu den Lagerfugen zu vernachlässigen ist.

Die Bestimmung der Bruchenergie $G_{f}$ ergibt einen deutlichen Zusammenhang zu der Normaldruckspannung (s. Abbildung 27). Mit steigender Druckspannung nimmt die Bruchenergie infolge der größeren aktivierten Rissreibung zu.

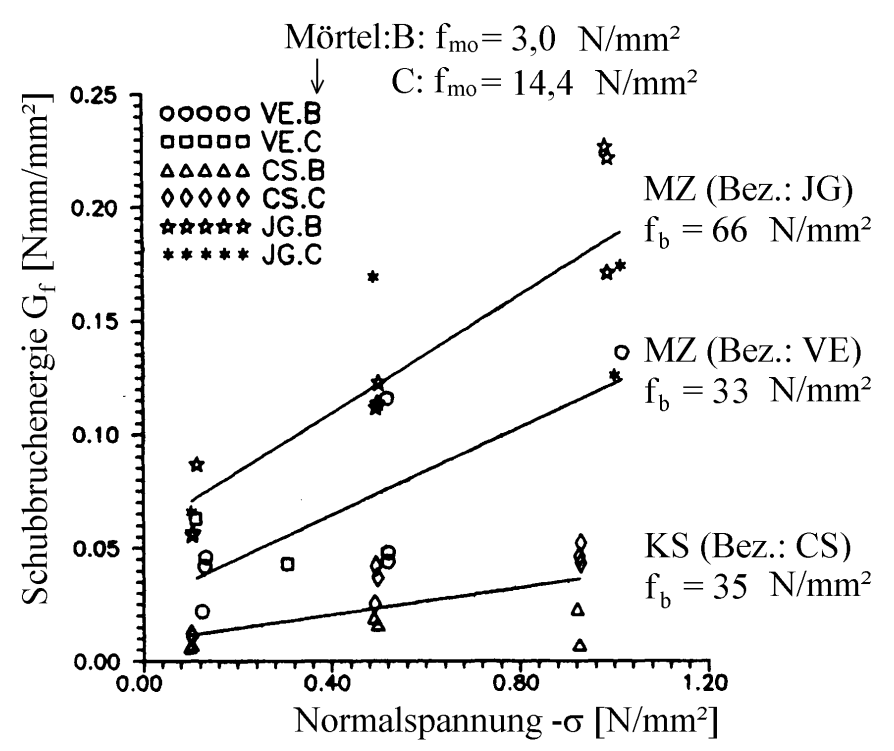

Abbildung 27: Bruchenergie für Schubversagen in der Lagerfuge in Abhängigkeit der Normaldruckspannung, ausgewählte Ergebnisse aus van der Pluijm [104]

\subsection{Schadensbilder - allgemeine Untersuchungen}

\section{$\underline{\text { Page }}$}

Page beschreibt in [101] das Verhalten unbewehrter Mauerwerkskonstruktionen beim Erdbeben von Newcastle / Australien, (150 km nördlich von Sydney) am 28.12.1989. Das betreffende Gebiet wurde in der australischen Norm von 1979 als Gebiet geringer Seismizität eingestuft, in welchem für Mauerwerksbauten keine besonderen konstruktiven Vorgaben für den Lastfall Erdbeben gefordert sind. Die Intensität bei dem aufgetretenen Beben betrug in der weiteren Umgebung von Newcastle nach der Modifizierten-Mercalli-Skala (MM) etwa 6 und im Bereich der Stadtmitte - bei ungünstigen Baugrundverhältnissen mit Erhöhungsfaktoren von $3 \div 6$ - bis maximal $8 \div 9$ (die Europäische Intensitätsskala EMS 1992 (s. Tabelle 1) ist in diesen Intensitätsstufen mit der Modifizierten-Mercalli-Skala vergleichbar). Bei der Mehrzahl der Schäden an tragendem Mauerwerk oder Ausfachungsmauerwerk von Stahlbetonrahmen waren allgemeine, minimale, konstruktive Vorgaben nicht eingehalten worden. So wurden bei Gebäuden mit Holzbalkendecken ohne Scheibenwirkung, sowohl bei Belastungsrichtung in Wandrichtung als auch bei Belastung senkrecht zu Wandebene, vermehrt Schäden beobachtet. Neue, gut ausgesteifte Gebäude hielten dem aufgetretenen Erdbeben jedoch im Wesentlichen gut stand. 


\section{Schwarz / Hampe}

Das Verhalten von Mauerwerksbauten unter seismischen Einwirkungen wurde von Hampe und Schwarz [44] behandelt. Die Untersuchungen erstrecken sich neben unbewehrtem Mauerwerk auch auf bewehrtes, historisches, Naturstein- und Ausfachungsmauerwerk sowie den Einfluss konstruktiver Maßnahmen. Nach einer Darstellung der qualitativen Erfassung der seismischen Einwirkungen geben die Autoren einen Überblick über die international üblichen Berechnungsverfahren. Anschließend werden Schädigungsmuster und Schädigungsgrade für Mauerwerk erläutert. Anhand einer Literaturauswertung von Versuchen an maßstäblichen Wandscheiben (bewehrt und unbewehrt) sowie an Modellmauerwerk (Einzelbauteile und Gesamtbauwerke) wird der Einfluss der Größe der Erdbebeneinwirkung auf den Schädigungsgrad angegeben.

Für die Ausführung von Neubauten werden Entwurfsgrundsätze, z. B. Regularitätskriterien, sowie konstruktive Maßnahmen zur Erhöhung der Erdbebentragfähigkeit bei bestehenden Mauerwerksgebäuden vorgeschlagen.

\section{Bernardini et. al. / Sandi}

Im Rahmen eines internationalen Symposiums [6] zum Thema Erdbebenschäden an Mauerwerksbauten wurden von mehreren Autoren aufgetretene Schäden dokumentiert und beschrieben. Es zeigte sich, dass Schäden im Wesentlichen bei Gebäuden ohne ausreichende horizontale Aussteifungselemente auftraten. Bei geschädigten älteren Bauwerken waren üblicherweise Holzbalkendecken vorhanden, die bei der horizontalen Lastverteilung eine allgemein deutlich geringere Tragfähigkeit aufweisen und auch erheblich weicher als Stahlbetondecken sind. Dieses wurde auch durch das Schadensbild des Herausklappens von Außenwandecken deutlich, bei denen keinerlei Rückverankerungen in Form von Ringbalken oder Ringankern wirksam waren. Bei größeren Erdbebenlasten traten zum Teil auch bei neueren Konstruktionen mit aussteifenden Stahlbetongeschossdecken Schäden auf. Diese waren insbesondere gekreuzte Diagonalrisse im Bereich von Fensterbrüstungen und Wandpfeilern.

Zusätzlich wurde in mehreren Beiträgen der Zusammenhang von Erdbebenkenngrößen und Bodenbewegungskenngrößen zu den aufgetretenen Schadensintensitäten untersucht. Die Beziehung zwischen Intensität und den Bodenbewegungsgrößen maximale Beschleunigung, Geschwindigkeit und Verschiebung, speziell im Hinblick auf den Einfluss auf Mauerwerksbauten, wurde in einem Beitrag von Sandi [112] diskutiert.

\section{Bachmann / Lang}

Von Bachmann und Lang [3, 65] wurde für die Bemessung von Mauerwerksbauten ein Kapazitätsverfahren vorgeschlagen, bei dem der Verschiebeduktilitätsbedarf des Gebäudes dem maximal zulässigen Wert gegenübergestellt wird. Die Kapazitätskurve eines Gesamtgebäudes setzt sich dabei aus denen der einzelnen Tragwände zusammen. Für die einzelnen Wände wird eine Vereinfachung über einen bi-linearen Ansatz mit reduzierter Anfangssteifigkeit durchgeführt. Die Stockwerksschiefstellung wird über eine empirische Funktion in Abhängigkeit der Normalspannung beschrieben. Nach der Berechnung als äquivalenten Einmassenschwinger kann der Verschiebeduktilitätsbedarf ermittelt werden. Bei der Modellierung der Gesamtstruktur wurde eine Rahmenwirkung durch Einbezug des Brüstungs- bzw. Sturzmauerwerkes und der Stahlbetongeschossdecken angesetzt.

Im Gegensatz zu den momentan vorhandenen Normansätzen konnten mit Hilfe dieses Verfahrens einzelne Bauwerke in der schweizer Erdbebenzone 1 nachgewiesen werden. 


\section{Materialeigenschaften}

Nachfolgend wird eine Übersicht über die im Rahmen der Versuche verwendeten Materialien und ihre Eigenschaften gegeben. Die durch eigene Versuche bestimmten Materialparameter werden durch Angaben aus der Literatur ergänzt.

Für die insgesamt 10 Versuchswände wurden zwei Arten von Hochlochziegel sowie zwei Arten von Kalksandsteinen verwendet. Als Mörtel kamen sowohl Normalmörtel als auch verschiedene Dünnbettmörtelarten zur Anwendung. Die Abmessungen der Wände betrugen 2,5 m (Wand E, F: 2,43 m) $\cdot 2,50 \mathrm{~m} \cdot 0,175 \mathrm{~m}$ - sie wurden im regelmäßigen Läuferverband mit einem Überbindemaß von einer halben Steinlänge erstellt.

Für die hoch bewehrten Fertigteilfundamente sowie Ortbetonkopfbalken wurde die Betonfestigkeitsklasse zu C 30/35 gewählt. Die Zielfestigkeit konnte mit Prüfhammerversuchen bestätigt werden.

Zur Bestimmung des Einflusses einer Stoßfugenvermörtelung wurden die von den Materialparametern her identischen Wände KS1 und KS2 mit bzw. ohne Stoßfugenvermörtelung hergestellt. Der Einfluss einer geringeren Haftscherfestigkeit auf das Trag- und Verformungsverhalten wurde an Wand KS3 mit einem speziell angefertigten Mörtel untersucht. Die Unterschiede der gemessenen Haftscherfestigkeitswerte im Vergleich zur Referenzwand KS4 betrugen dabei etwa $20 \%$ (s. Tabelle 19).

Bei Wand F wurden zur Vermeidung von lokalen Spannungsspitzen im Randbereich der untersten Lagerfuge handelsübliche Elastomerplatten auf einer Länge von jeweils $40 \mathrm{~cm}$ angeordnet. Dadurch sollte das in der ersten Versuchsstufe aufgetretene Versagensbild in Form von schrägen Rissen in den Randsteinen verhindert werden.

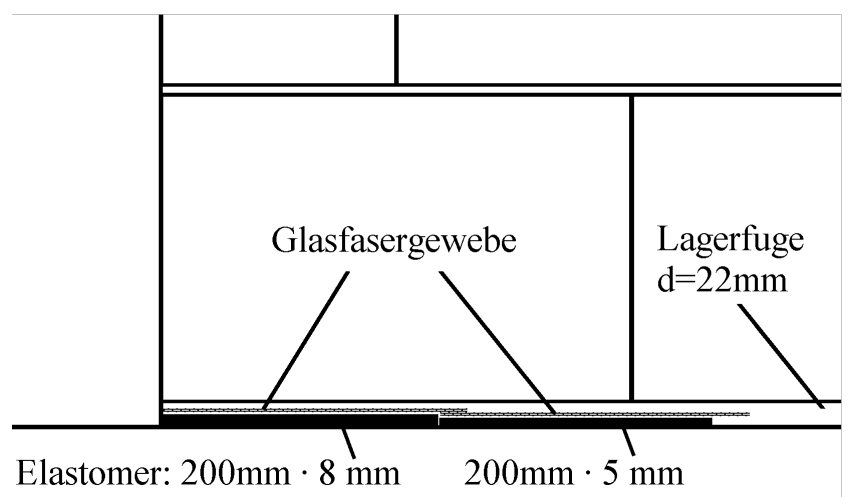

Abbildung 28: Detail der Anordnung der Elastomere in den Eckbereichen am Wandfuß von Wand F

Durch die Anordnung mehrlagigen Glasfasergewebes wurden die aus der Querverformung der Elastomere resultierenden Querzugspannungen aufgenommen. Die unbewehrten Elastomere besaßen entsprechend den Herstellerangaben einen Druck-Modul von $E_{D}=145 \mathrm{~N} / \mathrm{mm}^{2}$ (Dicke $8 \mathrm{~mm}$ ) bzw. $291 \mathrm{~N} / \mathrm{mm}^{2}$ (Dicke $5 \mathrm{~mm}$ ).

Ein Überblick über die geprüften Wände und die verwendeten Materialien ist nachfolgend in Tabelle 13 gegeben. 


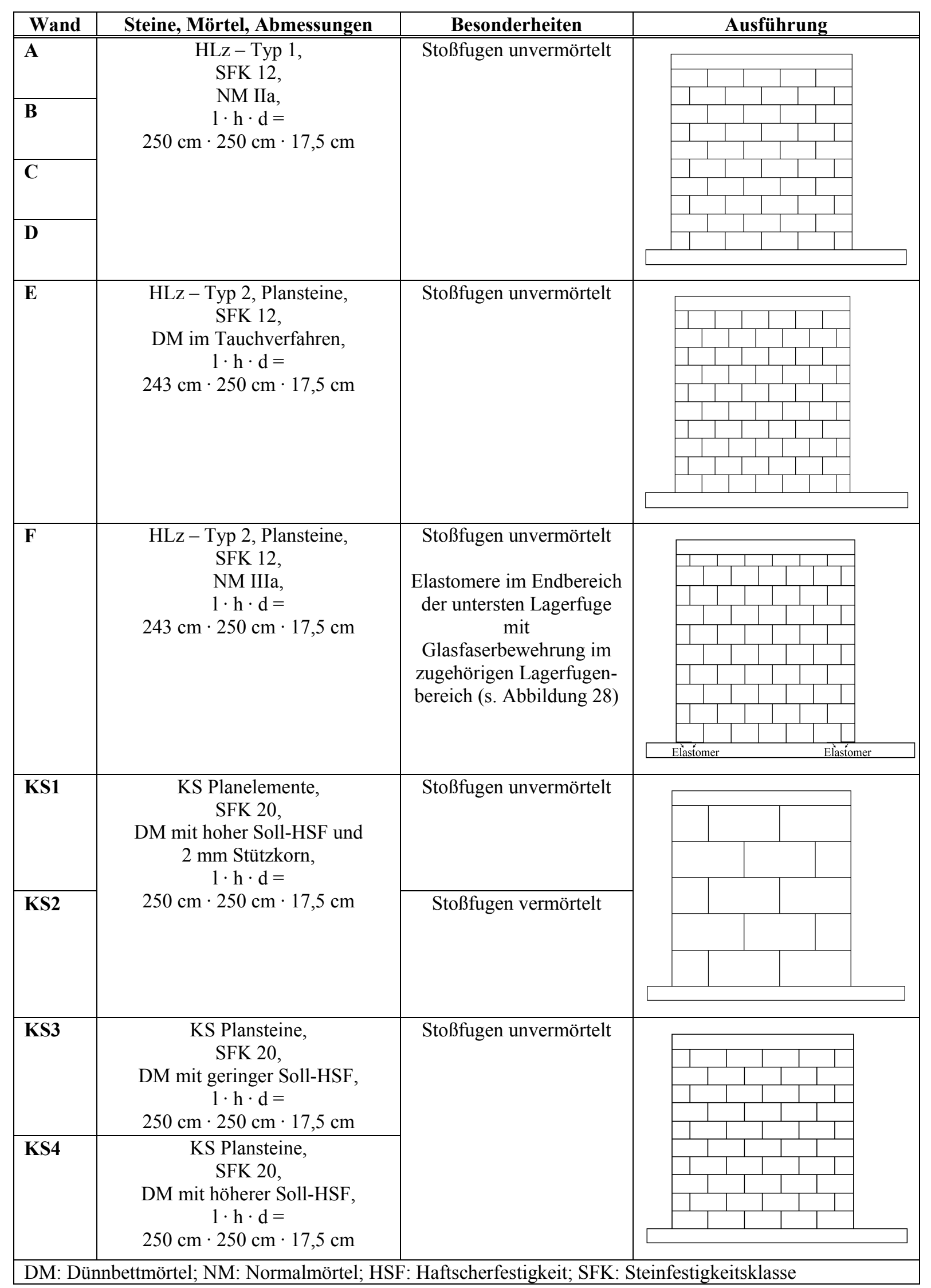

Tabelle 13: Übersicht über die Versuchswände und die verwendeten Materialien 


\subsection{Steine}

Insgesamt wurden vier verschiedene Steinarten verwendet, deren Materialeigenschaften in Nebenversuchen bestimmt wurden. Dabei wurde neben der reinen Festigkeitsbestimmung in einigen Versuchen auch die zugehörige Last-Verformungs-Beziehung unter Druckbeanspruchung gemessen. Nachfolgend wird in Tabelle 14 ein Überblick über die verwendeten Steine gegeben.

\begin{tabular}{|c|c|c|c|}
\hline Wand & $\begin{array}{l}\text { Klassifizie- } \\
\text { rung }\end{array}$ & Steinabmessungen und Lochbild & $\begin{array}{l}\text { Lochanteil bzw. } \\
\text { Lochanzahl }\end{array}$ \\
\hline $\mathbf{A}$ & \multirow{4}{*}{$\begin{array}{l}\text { HLz Typ } 1 \\
\text { SFK } 12, \\
\text { Rohdichte- } \\
\text { klasse } 0,9\end{array}$} & $\begin{array}{c}1 \cdot \mathrm{h} \cdot \mathrm{d}=490 \mathrm{~mm} \cdot 238 \mathrm{~mm} \cdot 175 \mathrm{~mm} \\
(\text { einschl. Verzahnung: } 1=508 \mathrm{~mm})\end{array}$ & \multirow[t]{4}{*}{$42 \%$} \\
\hline $\mathbf{B}$ & & & \\
\hline $\mathbf{C}$ & & & \\
\hline $\mathbf{D}$ & & $\begin{array}{l}\text { Die erforderlichen halben Steine wurden vor Ort ge- } \\
\text { schnitten. }\end{array}$ & \\
\hline $\mathbf{E}$ & \multirow[t]{2}{*}{$\begin{array}{l}\text { HLz Typ } 2 \\
\text { Plansteine } \\
\text { SFK } 12, \\
\text { Rohdichte- } \\
\text { klasse } 0,9\end{array}$} & $\begin{array}{l}\mathrm{l} \cdot \mathrm{h} \cdot \mathrm{d}=365 \mathrm{~mm} \cdot 248 \mathrm{~mm} \cdot 175 \mathrm{~mm} \\
(\text { einschl. Verzahnung: } \mathrm{l}=378 \mathrm{~mm})\end{array}$ & \multirow[t]{2}{*}{$43 \%$} \\
\hline $\mathbf{F}$ & & $\begin{array}{l}\text { Die erforderlichen halben Steine wurden vor Ort ge- } \\
\text { schnitten. }\end{array}$ & \\
\hline KS1 & \multirow[t]{2}{*}{$\begin{array}{l}\text { KS Planele- } \\
\text { mente } \\
\text { SFK 20, } \\
\text { Rohdichte- } \\
\text { klasse 2,0 }\end{array}$} & $\begin{array}{c}\mathrm{l} \cdot \mathrm{h} \cdot \mathrm{d}=998 \mathrm{~mm} \cdot 498 \mathrm{~mm} \cdot 175 \mathrm{~mm} \\
(\text { einschl. Verzahnung: } 1=1003 \mathrm{~mm})\end{array}$ & \multirow[t]{2}{*}{$\begin{array}{l}2 \text { Grifföffnungen an der } \\
\text { Steinoberseite: } \\
\phi 50 \mathrm{~mm} \text { mit einer } \\
\text { Tiefe von } 100 \mathrm{~mm}\end{array}$} \\
\hline KS2 & & $\begin{array}{l}\text { Die halben Planelemente wurden im Werk vorkonfektio- } \\
\text { niert (Bemerkung zu Festigkeit: s. Abschnitt 4.1.1). }\end{array}$ & \\
\hline KS3 & \multirow[t]{2}{*}{$\begin{array}{l}\text { KS Plansteine } \\
\text { SFK 20, } \\
\text { Rohdichte- } \\
\text { klasse } 2,0\end{array}$} & $\begin{array}{l}\mathrm{l} \cdot \mathrm{h} \cdot \mathrm{d}=498 \mathrm{~mm} \cdot 248 \mathrm{~mm} \cdot 175 \mathrm{~mm} \\
(\text { einschl. Verzahnung: } 1=502 \mathrm{~mm})\end{array}$ & \multirow{2}{*}{ 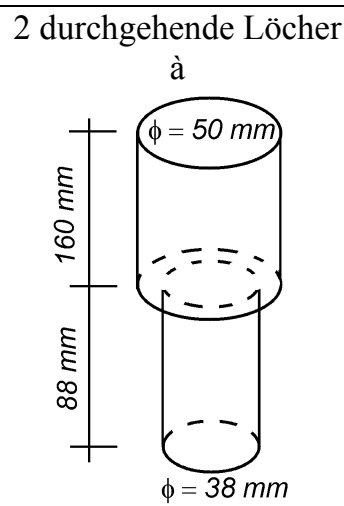 } \\
\hline KS4 & & $\begin{array}{l}\text { Die halben Plansteine mit einer Länge von } 248 \mathrm{~mm} \\
\text { (einschl. Verzahnung: } 1=253 \mathrm{~mm} \text { ) besaßen - abwei- } \\
\text { chend vom Lochbild der ganzen Steine }- \text { drei durchge- } \\
\text { hende Löcher (Abmessungen der einzelnen Löcher } \\
\text { analog zu den ganzen Steinen } \rightarrow \text { s. rechts) }\end{array}$ & \\
\hline
\end{tabular}

Tabelle 14: Übersicht über die verwendeten Steine

\subsubsection{Druckversuche}

Im Rahmen der Nebenversuche wurden einige Steine senkrecht und parallel zur Lagerfuge auf ihre Druckfestigkeit geprüft. Für Vergleichszwecke wurden zusätzlich an Bohrkernen von 
KS-Planelementen Druckversuche für die Bestimmung der Festigkeit und des E-Moduls durchgeführt. Es zeigte sich, dass die im Werk vorkonfektionierten halben Planelemente offensichtlich einer anderen Produktionscharge als der der ganzen Planelemente entstammten, da die normierten Festigkeitsunterschiede etwa 23\% betrugen.
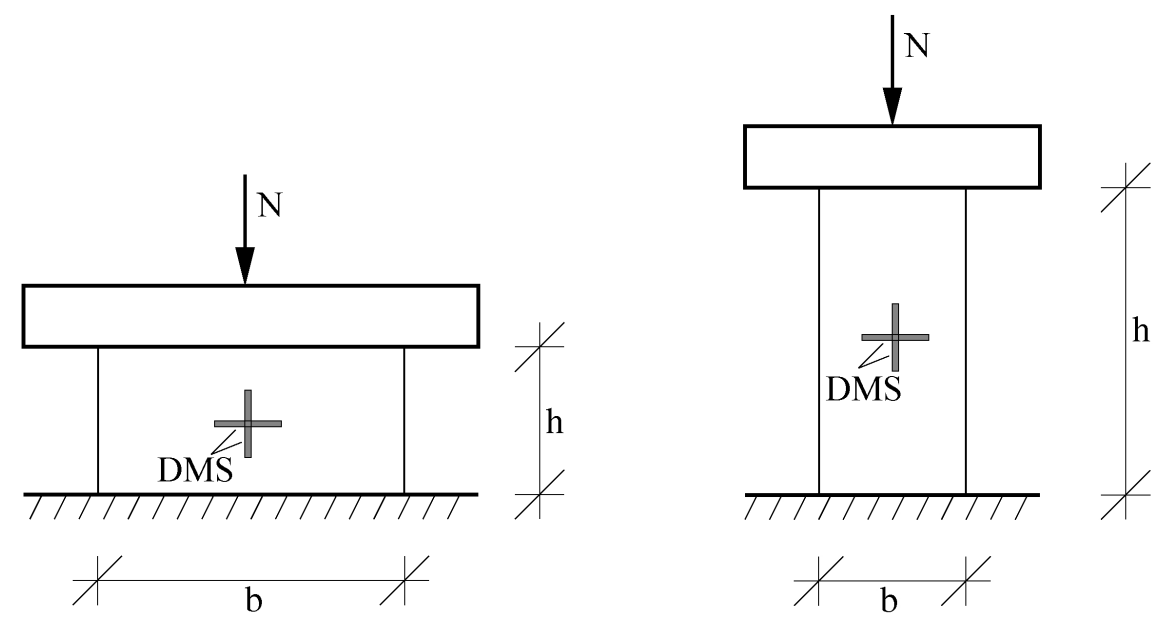

Abbildung 29: Schematische Darstellung der Steindruckversuche senkrecht und parallel zur Lagerfuge

In der Zusammenstellung in Tabelle 15 sind die Druckfestigkeiten der Probekörper $f_{b, P r}$ und die resultierenden Steindruckfestigkeiten $f_{b}$ (mit Formfaktor) angegeben. Der Formfaktor für die Prüfung der Steine wurde sowohl für die Prüfung senkrecht als auch parallel zu der Lagerfläche analog zu DIN 105-1 [14], DIN 105-2 [15] und DIN 106-1 [16] zu $f=1,2$ angenommen. Auf genauere Differenzierung, z. B. nach Kirtschig / Kasten [56], wurde aus Gründen der besseren Vergleichbarkeit verzichtet.

Für die Versuche an den Bohrkernen der KS-Planelemente wurden die Formfaktoren in Anlehnung an die Verhältnisse bei Beton mit niedriger Festigkeit festgelegt (Grübl / Weigler / Karl [37]). Hier entspricht bei einer Schlankheit von $h / d=2$ sowie dehn- und biegesteifen Druckplatten die ermittelte Festigkeit etwa der einachsigen Druckfestigkeit. Bei einer Schlankheit von $h / d=1,0$ reduziert sich dieses Verhältnis auf etwa 1/1,2=0,83.

Es zeigte sich, dass bei den verwendeten Hochlochziegeln die Druckfestigkeit in Steinlängsrichtung - bei relativ großer Streuung - im Mittel 13\% der Festigkeit senkrecht zur Lagerfuge beträgt. Bei den Versuchen erfolgte der Abgleich der Stoßfugenverzahnung mit Zementmörtel in Anlehnung an DIN 105-1 [14]. Infolge der Verzahnung an der Stirnseite der Steine betrug die Dicke der Ausgleichsschicht bei Typ 1 zwischen $5 \mathrm{~mm}$ und $20 \mathrm{~mm}$ und bei Typ 2 zwischen $5 \mathrm{~mm}$ und $13 \mathrm{~mm}$ (s. Tabelle 14). Aus den Dickenversprüngen und der keilförmigen Mörtelfüllung zwischen den Scherbenzähnen ergeben sich bei der Prüfung in den Steinen zusätzliche lokale Querzugkräfte, wodurch die Druckfestigkeit reduziert wird. Bei Vergleichsuntersuchungen von Meyer [85] an Hochlochziegeln mit abgetrennten Zähnen und demzufolge gleichmäßiger Schichtdicke des Ausgleichsmörtels lagen die Prüfwerte etwa 40\% höher als bei der oben beschriebenen Vorgehensweise.

Bei den verwendeten KS-Plansteinen (KS3, 4) betrug das Verhältnis der Druckfestigkeiten senkrecht und parallel zu den Lagerfugen im Mittel 63\%. Die Abweichung zu 100\%, was dem ideal-isotropen Fall entsprechen würde, ist primär auf den größeren Einfluss der Lochung senkrecht zu der Lagerfuge zurückzuführen (s. Tabelle 14). Wird dieser Einfluss durch Bezug der Bruchlast auf die Nettoquerschnittsflächen herausgerechnet, so beträgt der Verhältniswert $83 \%$. Für die verbleibende Abweichung sind neben den üblichen Streuungen noch die durch 
den Herstellvorgang bedingte Anisotropie sowie die Pauschalierung des Formfaktors $f$ verantwortlich.

\begin{tabular}{|c|c|c|c|c|c|}
\hline $\begin{array}{l}\text { Steine } \\
\text { (Wand) }\end{array}$ & $\begin{array}{c}\text { Richtung } \\
\text { ( } \perp \text { oder } \| \text { zur } \\
\text { Lagerfuge), } \\
\text { Versuchsanzahl } \\
\end{array}$ & $\begin{array}{c}\text { Abmessung } \\
b \cdot h \\
(d \equiv 175 \mathrm{~mm}) \\
{[\mathrm{mm}]}\end{array}$ & $\begin{array}{c}\text { Mittlere Festigkeit } \\
\text { des Probekörpers } \\
\text { (Wertebereich) } \\
f_{b, P r}\left[\mathbf{N} / \mathbf{m m}^{2}\right]\end{array}$ & $\begin{array}{c}\text { Form- } \\
\text { faktor } \\
f[-]\end{array}$ & $\begin{array}{c}\text { Mittlere rech- } \\
\text { nerische Stein- } \\
\text { druckfestigkeit } \\
f_{b}\left[\mathbf{N} / \mathbf{m m}^{2}\right]\end{array}$ \\
\hline \multirow{2}{*}{$\begin{array}{l}\text { HLz Typ } 1 \\
\text { SFK } 12 \\
\text { (Wand A } \div \text { D) }\end{array}$} & $\begin{array}{c} \\
\mathrm{n}=11\end{array}$ & $508 \cdot 238$ & $\begin{array}{c}16,5 \\
(13,5 \ldots 19,5)\end{array}$ & 1,2 & 19,8 \\
\hline & $\begin{array}{c}\| \\
\mathrm{n}=5\end{array}$ & $238 \cdot 510$ & $\begin{array}{c}2,27 \\
(1,76 \ldots 3,07)\end{array}$ & 1,2 & 2,72 \\
\hline \multirow{2}{*}{$\begin{array}{l}\text { HLz Typ 2 } \\
\text { Plansteine } \\
\text { SFK 12 } \\
\text { (Wand E, F) }\end{array}$} & $\begin{array}{c}\perp \\
\mathrm{n}=3\end{array}$ & $378 \cdot 248$ & $\begin{array}{c}13,1 \\
(12,2 \ldots 13,6)\end{array}$ & 1,2 & 15,7 \\
\hline & $\begin{array}{c}\| \\
n=3\end{array}$ & $248 \cdot 378$ & $\begin{array}{c}1,75 \\
(1,02 \ldots 2,39)\end{array}$ & 1,2 & 2,1 \\
\hline \multirow{3}{*}{$\begin{array}{l}\text { KS- } \\
\text { Planelemente } \\
\text { (halbe PE) } \\
\text { SFK } 20 \\
\text { (Wand KS1, 2) }\end{array}$} & $\begin{array}{l}\perp \\
\mathrm{n}=1\end{array}$ & $500 \cdot 498$ & $\begin{array}{c}27,0 \\
\text { (zyklischer Druck- } \\
\text { versuch) }\end{array}$ & 1,2 & 32,4 \\
\hline & $\begin{array}{c}\perp \\
\mathrm{n}=3\end{array}$ & $\begin{array}{c}\text { Bohrkern: } \\
\phi=99 \mathrm{~mm} \\
\mathrm{~h}=198 \mathrm{~mm}\end{array}$ & $\begin{array}{c}29,3 \\
(25,4 \ldots 31,5)\end{array}$ & 1,0 & 29,3 \\
\hline & $\begin{array}{c}\perp \\
\mathrm{n}=4\end{array}$ & $\begin{array}{l}\text { Bohrkern: } \\
\phi=99 \mathrm{~mm} \\
\mathrm{~h}=97 \mathrm{~mm}\end{array}$ & $\begin{array}{c}33,0 \\
(31,7 \ldots 34,7)\end{array}$ & 0,83 & 27,5 \\
\hline \multirow{2}{*}{$\begin{array}{l}\text { KS- } \\
\text { Planelemente } \\
\text { (ganze PE) } \\
\text { SFK 20 } \\
\text { (Wand KS1, 2) }\end{array}$} & $\begin{array}{c}\perp \\
\mathrm{n}=3\end{array}$ & $\begin{array}{c}\text { Bohrkern: } \\
\phi=99 \mathrm{~mm} \\
\mathrm{~h}=198 \mathrm{~mm}\end{array}$ & $\begin{array}{c}24,0 \\
(23,6 \ldots 24,5)\end{array}$ & 1,0 & 24,0 \\
\hline & $\begin{array}{c}\perp \\
\mathrm{n}=4\end{array}$ & $\begin{array}{c}\text { Bohrkern: } \\
\phi=99 \mathrm{~mm} \\
\mathrm{~h}=97 \mathrm{~mm}\end{array}$ & $\begin{array}{c}23,6 \\
(22,8 \ldots 24,5)\end{array}$ & 0,83 & 19,6 \\
\hline \multirow{2}{*}{$\begin{array}{l}\text { KS-Plansteine } \\
\text { (ganze Steine) } \\
\text { SFK 20 } \\
\text { (Wand KS3, 4) }\end{array}$} & $\begin{array}{c}\perp \\
\mathrm{n}=3\end{array}$ & $502 \cdot 248$ & $\begin{array}{c}32,5 \\
(31,5 \ldots 33,2)\end{array}$ & 1,2 & 38,9 \\
\hline & $\begin{array}{c}\| \\
n=3\end{array}$ & $248 \cdot 502$ & $\begin{array}{c}20,5 \\
(19,5 \ldots 21,1)\end{array}$ & 1,2 & 24,6 \\
\hline $\begin{array}{l}\text { KS-Plansteine } \\
\text { (halbe Steine) } \\
\text { SFK } 20 \\
\text { (Wand KS3, 4) }\end{array}$ & $\begin{array}{c}\perp \\
\mathrm{n}=3\end{array}$ & $253 \cdot 248$ & $\begin{array}{c}24,3 \\
(23,6 \ldots 24,8)\end{array}$ & 1,2 & 29,1 \\
\hline
\end{tabular}

Tabelle 15: Druckfestigkeiten der verwendeten Steine

\subsubsection{Biegezugversuche}

Für die Bestimmung der zentrischen Steinzugfestigkeit, deren versuchstechnischer Aufwand als sehr hoch anzusehen ist, existiert zurzeit keine Prüfnorm. Üblicherweise wird die zentrische Zugfestigkeit daher über die Biegezug- oder Spaltzugfestigkeit ermittelt. Im Nachfolgenden wurden daher für die Bestimmung der Steinzugfestigkeit parallel zu den Lagerfugen Biegezugversuche in Anlehnung an die Normen ÖNORM B 3200 [97] und DIN 1048-5 [19] durchgeführt. Neben der Prüfung der Biegezugfestigkeit in Scheibenebene wurden bei zwei ganzen KS-Planelementen auch Biegezugversuche in Plattenebene durchgeführt. 

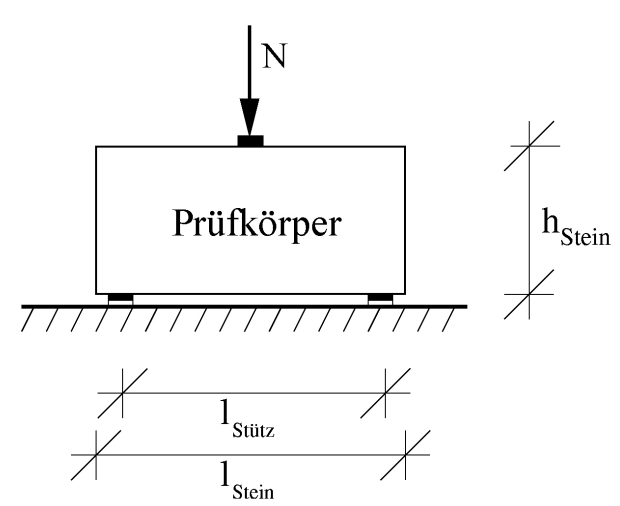
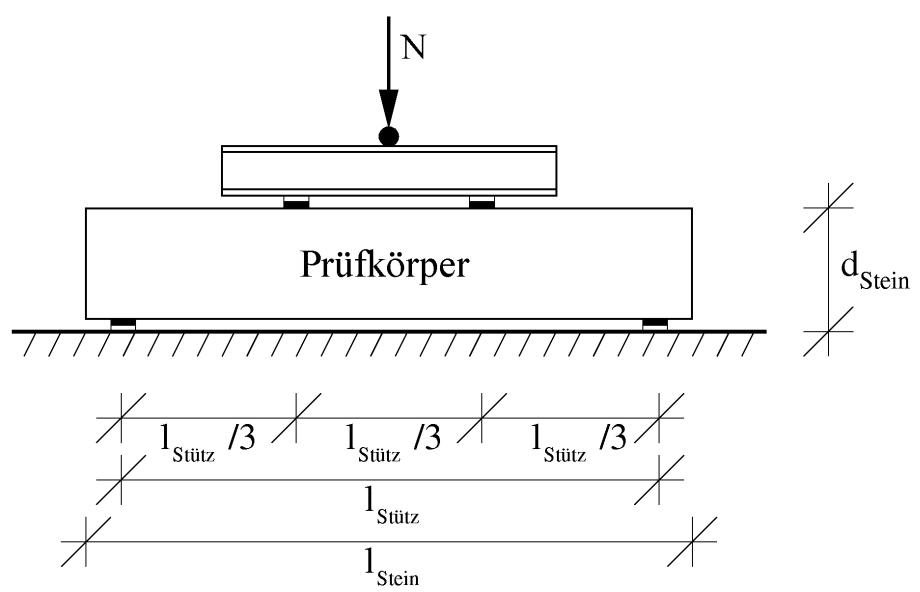

Abbildung 30: Schematische Darstellung der Biegezugversuche in Scheiben- und Plattenebene

\begin{tabular}{|c|c|c|c|c|}
\hline $\begin{array}{c}\text { Steine } \\
\text { (Wand) }\end{array}$ & $\begin{array}{l}\text { Richtung, } \\
\text { Versuchsan- } \\
\text { zahl }\end{array}$ & $\begin{array}{c}\text { Abmessung } \\
l_{\text {Stein }} \cdot \boldsymbol{h}_{\text {Stein }}\left(d_{\text {Stein }}\right) \\
l_{\text {Stütz }} \\
{[\mathrm{mm}]} \\
\end{array}$ & $\begin{array}{c}\text { Biegezugfestigkeit } \\
\text { Mittelwert } \\
\text { (Wertebereich) } \\
f_{b t, B Z}\left[\mathbf{N} / \mathbf{m m}^{2}\right] \\
\end{array}$ & $\begin{array}{c}\text { Verhältnis zur zugeh. } \\
\text { Druckfestigkeit }(\perp \text { bzw. } \\
\text { || zu den Lagerfugen })^{1)} \\
{[-]}\end{array}$ \\
\hline $\begin{array}{l}\text { HLz Typ } 1 \\
\text { (Wand A } \div \text { D) }\end{array}$ & $\begin{array}{l}\text { Scheibenebene } \\
\qquad n=6\end{array}$ & $\begin{array}{c}508 \cdot 238 \\
1_{\text {Stütz }}=445\end{array}$ & $\begin{array}{c}0,74 \\
(0,61 \ldots 0,92)\end{array}$ & $0,037 / 0,272$ \\
\hline $\begin{array}{l}\text { HLz Typ } 2 \\
\text { (Wand E, F) }\end{array}$ & $\begin{array}{l}\text { Scheibenebene } \\
n=6\end{array}$ & $\begin{array}{c}378 \cdot 248 \\
1_{\text {Stütz }}=330\end{array}$ & $\begin{array}{c}0,38 \\
(0,25 \ldots 0,63) \\
\end{array}$ & $0,024 / 0,181$ \\
\hline \multirow{2}{*}{$\begin{array}{l}\text { KS- } \\
\text { Planelemente } \\
\text { (Wand KS1, 2) }\end{array}$} & $\begin{array}{l}\text { Scheibenebene } \\
n=3\end{array}$ & $\begin{array}{l}1003 \cdot 498 \\
1_{\text {Stütz }}=900\end{array}$ & $\begin{array}{c}3,39 \\
(3,32 \ldots 3,44) \\
\end{array}$ & \multirow[t]{2}{*}{$0,161 / \quad-$} \\
\hline & $\begin{array}{c}\text { Plattenebene } \\
n=2\end{array}$ & $\begin{array}{l}1003 \cdot 175 \\
1_{\text {Stütz }}=900\end{array}$ & $\begin{array}{c}3,59 \\
(3,70 \ldots 3,47)\end{array}$ & \\
\hline $\begin{array}{l}\text { KS-Plansteine }{ }^{2)} \\
(\text { Wand KS3, 4) }\end{array}$ & $\begin{array}{c}\text { Scheibenebene } \\
n=3 \\
\end{array}$ & $\begin{array}{c}502 \cdot 248 \\
1_{\text {Stütz }}=445 \\
\end{array}$ & $\begin{array}{c}3,33 \\
(2,96 \ldots 3,60) \\
\end{array}$ & $0,086 / 0,135$ \\
\hline
\end{tabular}

Tabelle 16: Biegezugfestigkeiten der verwendeten Steine

Schubert [119, 120] und Schubert / Bohne [121] geben für den Zusammenhang zwischen zentrischer Zugfestigkeit in Steinlängsrichtung $f_{b t, \|}$ und Druckfestigkeit senkrecht zur Lagerfuge $f_{b}$ (einschließlich eines Formfaktors $f$ ) folgende Zusammenhänge an:

- KS:

- MZ:

$f_{b t, \|}=0,051 \cdot f_{b}$

- HLz:

$f_{b t, \|}=0,026 \cdot f_{b}$

- L-HLz:

$f_{b t, \|}=0,03 \cdot f_{b}$

- Porenbeton:

$f_{b t, \|}=0,01 \cdot f_{b}$

- Leichtbeton:

$f_{b t, \|}=0,182 \cdot f_{b}$

$f_{b t, \|}=0,092 \cdot f_{b}$

(Festigkeitsklasse 2)

(Festigkeitsklasse $4 \div 8$ )

Für Leichthochlochziegel (L-HLz) wird der Verhältniswert von Längszugfestigkeit und Normdruckfestigkeit (ohne Formfaktor $f$ ) zu 0,01 angegeben [120]. Die in den eigenen Versuchen ermittelten Verhältniswerte der Biegezugfestigkeiten entsprachen etwa dem $2 \div 4$-fachen. Die Unterschiede zwischen Biegezug- und zentrischer Zugfestigkeit sind im Allgemeinen auf den nichtlinearen Zusammenhang von Spannungen und Dehnungen über die Prüfkörperhöhe bei Biegebeanspruchung, sowie auf das unterschiedliche Umlagerungsvermögen (sog. Reißverschlusseffekt) zurückzuführen. 
Die Biegezugfestigkeit von Kalksandsteinprismen wird von Schubert [120] mit 16\% der Druckfestigkeit angegeben, was auch den Ergebnissen der eigenen Versuche entspricht (KSPlanelemente). Bei den Plansteinen ist aufgrund des Formfaktors $f=1,2$ bei der Druckfestigkeitsumrechnung der Verhältniswert von Biegezug- zu resultierender einaxialer Druckfestigkeit deutlich geringer. Eine Ursache der hohen gemessenen Biegezugfestigkeiten der KSPlanelemente ist teilweise in dem Herstellprozess zu suchen. Durch die Pressung der Ausgangsstoffe in der Stahlschalung ist der Randbereich, d.h. die Seiten-, Stirn- und Lagerflächen, stärker verdichtet und besitzt demzufolge auch eine höhere Druck- und Zugfestigkeit als der Kern. Bei der Biegezugprüfung - sowohl in Scheiben- als auch in Plattenebene - ist dieser Randbereich entscheidend für die Festigkeit, wohingegen bei zentrischen Zugprüfungen in Scheibenebene der Kern einen größeren Anteil besitzt.

Die Abweichungen der Verhältniswerte senkrecht und parallel zu den Lagerfugen bei den KSPlansteinen (KS3, 4) sind auf den Einfluss der Lochung bei der Druckfestigkeitsprüfung in Steinlängsrichtung zurückzuführen. Bestätigt durch das Bruchbild, haben im Gegensatz dazu die Löcher bei der Biegezugprüfung in Scheibenebene aufgrund der vorgegebenen Laststellung keinen nennenswerten Einfluss.

\section{Scherbenzugfestigkeit}

Eine weitere Möglichkeit der Bestimmung der Steinlängszugfestigkeit bei Hochlochziegeln besteht in der Abschätzung aus der Scherbenzugfestigkeit. Dabei wird ein Scherben mit den Abmessungen von $40 \mathrm{~mm} \cdot 40 \mathrm{~mm}$ und der betreffenden Stegdicke mit Hilfe einer Prüfzange zentrisch auf Zug geprüft (Metzemacher [82]). Über den Bezug auf den minimalen Querschnittsanteil des Steins kann eine Längszugfestigkeit bestimmt werden. Der Einfluss der Umleitung der Zugkräfte bei in Längsrichtung versetzten Stegen wird hierbei vereinfacht vernachlässigt.

Aus den Lochbildern der verwendeten Hochlochziegeln (s. Tabelle 14) und den Angaben von Meyer [85] zu den verwendeten Hochlochziegeln ergeben sich nachfolgende Festigkeitswerte.

\begin{tabular}{|l|c|c|c|}
\hline \multicolumn{1}{|c|}{$\begin{array}{c}\text { Steine } \\
\text { (Wand) }\end{array}$} & $\begin{array}{c}\text { Minimaler Quer- } \\
\text { schnittsflächenanteil } \\
{[-]}\end{array}$ & $\begin{array}{c}\text { Scherbenzugfestigkeit } \\
\text { nach Meyer }[\mathbf{8 5}] \\
\boldsymbol{f}_{\mathbf{S}, \boldsymbol{t}}\left[\mathbf{N} / \mathbf{m m}^{2}\right] \text { (Wertebereich) }\end{array}$ & $\begin{array}{c}\text { zugehörige rechnerische } \\
\text { Steinlängszugfestigkeit } \\
\boldsymbol{f}_{b, t, \|}\left[\mathbf{N} / \mathbf{m m}^{2}\right]\end{array}$ \\
\hline \hline $\begin{array}{l}\text { HLz Typ 1 } \\
\text { (Wand A } \div \text { D) }\end{array}$ & $60 / 175=0,34$ & $\begin{array}{c}2,5 \\
(1,5 \div 2,5)\end{array}$ & 0,86 \\
\hline $\begin{array}{l}\text { HLz Typ 2 } \\
\text { (Wand E, F) }\end{array}$ & $54 / 175=0,31$ & $\begin{array}{c}3,5 \\
(2,0 \div 5,0)\end{array}$ & 1,08 \\
\hline
\end{tabular}

Tabelle 17: Scherbenzugfestigkeit und zugehörige rechnerische Steinlängszugfestigkeit

Für die Steine des Typs 1 passen die Werte der Abschätzung über die Scherbenzugfestigkeit und die gemessenen Biegezugfestigkeiten (Tabelle 16) mit einer relativen Abweichung von $16 \%$ gut überein.

Im Gegensatz dazu fallen beim Vergleich der Werte für den Steintyp 2 die Unterschiede deutlich größer aus. Die aus der Scherbenzugfestigkeit ermittelte Steinlängszugfestigkeit beträgt mit $1,08 \mathrm{~N} / \mathrm{mm}^{2}$ das 2,8-fache der mittleren Biegezugfestigkeit von $0,38 \mathrm{~N} / \mathrm{mm}^{2}$. Als Ursache kommen im Wesentlichen die aus dem Schleifen der Plansteine herrührenden Risse, die vornehmlich in der Nähe der Lagerfläche zu finden sind, in Frage. Für die Festlegung einer zentrischen Steinlängszugfestigkeit in größerem Abstand vom oberen und unteren Stein- 
rand wird daher in Anlehnung an Tabelle 17 der Wert zu $f_{b t}=0,9 \mathrm{~N} / \mathrm{mm}^{2}$ gewählt (s. a. Abschnitt 4.3.2).

\subsubsection{Verformungskenngrößen}

Bei einem Teil der Steindruckversuche wurden mit Hilfe jeweils auf der Vorder- und Rückseite in Steinmitte aufgeklebter Dehnmessstreifen (DMS) die Dehnungen in und senkrecht zur Kraftrichtung gemessen (s. Abbildung 29). Die Versuchsdurchführung und Auswertung erfolgte in Anlehnung an DIN 1048-5 [19] und DIN 18555-4 [24]. Daraus ergaben sich die in Tabelle 18 zusammengefassten Verformungskennwerte, die für eine Oberspannung von 1/3 der zugehörigen Druckfestigkeit ermittelt wurden.

\begin{tabular}{|c|c|c|c|c|c|}
\hline $\begin{array}{l}\text { Steine } \\
\text { (Wand) }\end{array}$ & $\begin{array}{c}\text { Richtung } \\
(\perp \text { oder } \| \text { zur } \\
\text { Lagerfuge }), \\
\text { Versuchsanzahl }^{3)} \\
\end{array}$ & $\begin{array}{c}\begin{array}{c}\text { Abmessung } \\
b \cdot h^{1)}\end{array} \\
{[\mathrm{mm}]} \\
\end{array}$ & $\begin{array}{c}\text { Druck-E-Modul } \\
\text { (Wertebereich) } \\
E_{D} 1^{3}\left[\mathrm{~N} / \mathbf{m m}^{2}\right] \\
\end{array}$ & $\begin{array}{c}\begin{array}{c}\text { Querdehnungs- } \\
\text { modul }\end{array} \\
(\text { Wertebereich) } \\
E_{Q} 1^{3}\left[\mathrm{~N} / \mathbf{m m}^{2}\right] \\
\end{array}$ & $\begin{array}{c}\text { Quer- } \\
\text { dehnzahl } \\
v[-]\end{array}$ \\
\hline \multirow[t]{2}{*}{$\begin{array}{l}\text { HLz Typ } 1 \\
\text { (Wand A } \div \text { D) }\end{array}$} & $\begin{array}{c}\perp \\
\mathrm{n}=3\end{array}$ & $508 \cdot 238$ & $\begin{array}{c}9,05 \\
(6,00 \ldots 12,35)\end{array}$ & $\begin{array}{c}62,3 \\
(42,8 \ldots 90,1) \\
\end{array}$ & $\begin{array}{c}0,12 \ldots 0,15 \\
(0,24)^{2)}\end{array}$ \\
\hline & $\begin{array}{c}\| \\
\mathrm{n}=3\end{array}$ & $238 \cdot 508$ & $\begin{array}{c}2,24 \\
(1,73 \ldots 2,78) \\
\end{array}$ & $\begin{array}{c}22,8 \\
(14,5 \ldots 31,8) \\
\end{array}$ & $0,07 \ldots 0,13$ \\
\hline \multirow{2}{*}{$\begin{array}{l}\text { HLz Typ } 2 \\
\text { Plansteine } \\
\text { (Wand E, F) }\end{array}$} & $\begin{array}{c}\perp \\
\mathrm{n}=3\end{array}$ & $378 \cdot 248$ & $\begin{array}{c}6,00 \\
(4,71 \ldots 7,21)\end{array}$ & $\begin{array}{c}62,2 \\
(39,4 \ldots 90,6)\end{array}$ & $0,07 \ldots 0,14$ \\
\hline & $\begin{array}{c}\| \\
n=2\end{array}$ & $248 \cdot 378$ & $\begin{array}{c}1,91 \\
(1,23 \ldots 2,73)\end{array}$ & $\begin{array}{c}20,1 \\
(16,9 \ldots 23,8)\end{array}$ & $0,07 \ldots 0,14$ \\
\hline $\begin{array}{l}\text { KS-Planelemente } \\
\text { (halbe PE) } \\
\text { (Wand KS1, 2) }\end{array}$ & $\begin{array}{c}\perp \\
\mathrm{n}=3\end{array}$ & $\begin{array}{c}\text { Bohrkern: } \\
\phi=99 \\
\mathrm{~h}=198\end{array}$ & $\begin{array}{c}14,15 \\
(8,61 \ldots 23,69)\end{array}$ & n.d. ${ }^{4)}$ & n.d. ${ }^{4)}$ \\
\hline $\begin{array}{l}\text { KS-Planelemente } \\
\text { (ganze PE) } \\
\text { (Wand KS1, 2) }\end{array}$ & $\begin{array}{c}\perp \\
\mathrm{n}=3\end{array}$ & $\begin{array}{c}\text { Bohrkern: } \\
\phi=99 \\
\mathrm{~h}=198\end{array}$ & $\begin{array}{c}10,92 \\
(8,94 \ldots 13,46)\end{array}$ & n.d. ${ }^{4)}$ & n.d. ${ }^{4)}$ \\
\hline \multirow{2}{*}{$\begin{array}{l}\text { KS-Plansteine } \\
\text { (ganze Steine) } \\
\text { (Wand KS3, 4) }\end{array}$} & $\begin{array}{c}\perp \\
\mathrm{n}=3\end{array}$ & $502 \cdot 248$ & $\begin{array}{c}12,61 \\
(10,49 \ldots 14,33)\end{array}$ & $\begin{array}{c}170 \\
(127 \ldots 227) \\
\end{array}$ & $0,06 \ldots 0,10$ \\
\hline & $\begin{array}{c}\| \\
\mathrm{n}=3\end{array}$ & $248 \cdot 502$ & $\begin{array}{c}11,17 \\
(10,70 \ldots 12,20)\end{array}$ & $\begin{array}{c}80,4 \\
(48,8 \ldots 96,3) \\
\end{array}$ & $\begin{array}{c}0,12 \ldots 0,14 \\
(0,24)^{2)}\end{array}$ \\
\hline
\end{tabular}

Tabelle 18: Verformungskennwerte der verwendeten Steine

\subsection{Mörtel}

Für die Bestimmung weiterer Materialparameter wurden an den Mörteln der einzelnen Wände und Herstelltage verschiedene Standard-Materialversuche vorgenommen. Die Prüfung der Druck-, Biegezugfestigkeit und Rohdichte wurde nach DIN 18555-3 [23] und die der Haftscherfestigkeit nach DIN 18555-5 [25] durchgeführt. Ein Überblick der ermittelten Festigkeitswerte ist in Tabelle 19 gegeben. 


\begin{tabular}{|c|c|c|c|c|}
\hline $\begin{array}{c}\text { Wand } \\
\text { (Mörtel) }\end{array}$ & $\begin{array}{c}\text { Druckfestigkeit } \\
f_{m o}\left[\mathbf{N} / \mathbf{m m}^{2}\right] \\
\end{array}$ & $\begin{array}{l}\text { Haftscherfestigkeit } \\
k\left[\mathbf{N} / \mathbf{m m}^{2}\right] \\
\end{array}$ & $\begin{array}{c}\text { Biegezugfestigkeit } \\
f_{m o, B Z}\left[\mathrm{~N} / \mathrm{mm}^{2}\right] \\
\end{array}$ & $\begin{array}{l}\text { Rohdichte } \\
\rho_{f}\left[\mathbf{k g} / \mathbf{d m}^{3}\right]\end{array}$ \\
\hline \multirow[t]{2}{*}{$\begin{array}{l}\text { A } \\
\text { (NM IIa) }\end{array}$} & $\begin{array}{c}4,4,7 \\
(\mathrm{n}=6: 4,4 \ldots 4,9)\end{array}$ & n.d. & $\begin{array}{c}1,5 \\
(\mathrm{n}=3: 1,2 \ldots 1,8)\end{array}$ & $\begin{array}{c}1,71 \\
(\mathrm{n}=3: 1,70 \ldots 1,72)\end{array}$ \\
\hline & \multicolumn{4}{|c|}{ HT: 16.10 .2000$, PT: 13.11 .2000} \\
\hline \multirow[t]{2}{*}{$\begin{array}{l}\text { B \& C } \\
\text { (NM IIa) }\end{array}$} & $\begin{array}{c}5,9 \\
(\mathrm{n}=6: 5,7 \ldots 6,1) \\
\end{array}$ & n.d. & $\begin{array}{c}2,0 \\
(\mathrm{n}=3: 1,9 \ldots 2,1)\end{array}$ & $\begin{array}{c}1,76 \\
(\mathrm{n}=3: 1,75 \ldots 1,77) \\
\end{array}$ \\
\hline & \multicolumn{4}{|c|}{ HT: 18.10 .2000$, PT: 15.11 .2000} \\
\hline \multirow[t]{2}{*}{$\begin{array}{l}\text { C \& D } \\
\text { (NM IIa) }\end{array}$} & $\begin{array}{c}4,4 \\
(\mathrm{n}=6: 4,3 \ldots 4,6)\end{array}$ & n.d. & $\begin{array}{c}1,6 \\
(\mathrm{n}=3: 1,5 \ldots 1,8)\end{array}$ & $\begin{array}{c}1,71 \\
(\mathrm{n}=3: 1,70 \ldots 1,71)\end{array}$ \\
\hline & \multicolumn{4}{|c|}{ HT: 19.10 .2000$, PT: 16.11 .2000} \\
\hline \multirow{3}{*}{$\begin{array}{l}\text { E } \\
(\text { DM Typ E / } \\
\text { Rot) }\end{array}$} & $\begin{array}{c}12,6 \\
(\mathrm{n}=6: 12,2 \ldots 13,2)\end{array}$ & $\begin{array}{c}0,88 \\
(\mathrm{n}=12: 0,74 \ldots 0,98)\end{array}$ & $\begin{array}{c}4,8 \\
(\mathrm{n}=3: 4,6 \ldots 5,0) \\
\end{array}$ & $\begin{array}{c}1,22 \\
(n=3: 1,22 \ldots 1,23)\end{array}$ \\
\hline & $\begin{array}{l}\text { Unterste Lagerfuge: } \\
\qquad \begin{array}{l}4,7 \\
(\mathrm{n}=6: 4,4 \ldots 4,9)\end{array}\end{array}$ & $\begin{array}{l}\text { Unterste Lagerfuge: } \\
\text { n.d. }\end{array}$ & $\begin{array}{l}\text { Unterste Lagerfuge: } \\
\qquad \begin{array}{l}1,5 \\
(\mathrm{n}=3: 1,4 \ldots 1,6)\end{array}\end{array}$ & $\begin{array}{l}\text { Unterste Lagerfuge: } \\
\qquad 1,67 \\
(\mathrm{n}=3: 1,66 \ldots 1,67)\end{array}$ \\
\hline & \multicolumn{4}{|c|}{$\begin{array}{l}\text { In der untersten Lagerfuge wurde als Ausgleichsmörtel NM IIa benutzt (s.o.). } \\
\text { HT: 23.07.2001, PT: 20.08.2001 (für beide Mörtelarten) }\end{array}$} \\
\hline \multirow[t]{2}{*}{$\begin{array}{l}\text { F } \\
(\text { NM IIIa) }\end{array}$} & $\begin{array}{c}52,9 \\
(\mathrm{n}=6: 52,1 \ldots 54,1)\end{array}$ & n.d. & $\begin{array}{c}8,1 \\
(\mathrm{n}=3: 7,6 \ldots 8,8)\end{array}$ & $\begin{array}{c}1,99 \\
(\mathrm{n}=3: 1,99 \ldots 2,0)\end{array}$ \\
\hline & \multicolumn{4}{|c|}{$\begin{array}{l}\text { Die Planziegel wurden über die ganze Wandhöhe mit Normalmörtel NM IIIa vermauert. In der } \\
\text { untersten Lagerfuge wurden im Bereich der Elastomere mehrere Lagen Glasfasergewebe in den } \\
\text { Mörtel eingelegt, um die Querdehnung in der Fuge zu reduzieren (s. Abbildung 28). } \\
\text { HT: 25.07.2001, PT: 22.08.2001 }\end{array}$} \\
\hline \multirow{2}{*}{$\begin{array}{l}\text { KS1 \& KS2 } \\
(\text { DM Typ } \\
\text { KMS 100) }\end{array}$} & $\begin{array}{c}5,7 \\
(\mathrm{n}=6: 5,3 \ldots 5,9)\end{array}$ & $\begin{array}{c}1,72 \\
(\mathrm{n}=12: 1,24 \ldots 1,95)\end{array}$ & $\begin{array}{c}2,5 \\
(\mathrm{n}=3: 2,4 \ldots 2,6)\end{array}$ & $\begin{array}{c}1,09 \\
(\mathrm{n}=3: 1,08 \ldots 1,09)\end{array}$ \\
\hline & \multicolumn{4}{|c|}{$\begin{array}{l}\text { Dünnbettmörtel mit } 2 \text { mm Stützkorn für Planelemente } \\
\text { HT: } 12.07 .2001, \text { PT: 09.08.2001 }\end{array}$} \\
\hline \multirow[t]{2}{*}{$\begin{array}{l}\text { KS3 } \\
\text { (DM Spezial) }\end{array}$} & $\begin{array}{c}6,7 \\
(\mathrm{n}=6: 6,5 \ldots 7,0)\end{array}$ & $\begin{array}{r}0,65 \\
(\mathrm{n}=12: 0,57 \ldots 0,80)\end{array}$ & $\begin{array}{r}2,9 \\
(\mathrm{n}=3: 2,8 \ldots 3,0)\end{array}$ & $\begin{array}{c}1,17 \\
(\mathrm{n}=3: 1,17 \ldots 1,18)\end{array}$ \\
\hline & \multicolumn{4}{|c|}{$\begin{array}{l}\text { Spezielle Mischung des Dünnbettmörtels mit niedriger Soll-Haftscherfestigkeit } \\
\text { HT: } 18.12 .2001 \text {, PT: } 15.01 .2002\end{array}$} \\
\hline \multirow{2}{*}{$\begin{array}{l}\text { KS4 } \\
\text { (DM Typ } \\
\text { KS4) }\end{array}$} & $\begin{array}{c}10,1 \\
(n=6: 9,6 \ldots 10,7)\end{array}$ & $\begin{array}{r}0,82 \\
(\mathrm{n}=12: 0,56 \ldots 1,12)\end{array}$ & $\begin{array}{c}3,7 \\
(\mathrm{n}=3: 3,6 \ldots 3,8)\end{array}$ & $\begin{array}{c}1,32 \\
(\mathrm{n}=3: 1,32 \ldots 1,32)\end{array}$ \\
\hline & \multicolumn{4}{|c|}{ HT: 18.12 .2001$, PT: 15.01 .2002} \\
\hline
\end{tabular}

Tabelle 19: Festigkeiten der verwendeten Mörtel

\subsection{Mauerwerk}

Eigene Versuche an Mauerwerkskörpern zur Bestimmung der Eigenschaftswerte wurden nicht durchgeführt. Daher werden diese im Weiteren entweder aus den Eigenschaften der Einzelkomponenten Stein und Mörtel anhand empirischer Beziehungen bestimmt oder aus den Literaturangaben entnommen.

Die für die nachfolgenden Untersuchungen maßgebenden Eigenschaftswerte sind:

- Druck- und Zugfestigkeit senkrecht und parallel zu den Lagerfugen

- Elastizitätsmodul senkrecht und parallel zu den Lagerfugen

- Schubmodul und Querdehnzahl

- Spannungs-Dehnungs-Linie unter Druckbeanspruchung senkrecht zu den Lagerfugen

- Haftscherfestigkeit, Reibbeiwert und schräge Steinzugfestigkeit 
Eine umfassende Übersicht über international vorhandene empirische Ansätze zur Beschreibung der Mauerwerksdruckfestigkeit, des E-Moduls und der Spannungs-Dehnungs-Linie unter monotoner Druckbeanspruchung wird von Vratsanou [155] gegeben.

\subsubsection{Druck- und Zugfestigkeit}

Die Beschreibung der Mauerwerksdruckfestigkeit senkrecht zu den Lagerfugen $f_{m y}$ erfolgt üblicherweise nach Gleichung (14) in Abhängigkeit der Steindruckfestigkeit $f_{b}$ und der Mörteldruckfestigkeit $f_{m o}$ (Meyer / Schubert [83], Schubert [118, 120]):

$$
f_{m y}=a \cdot f_{b}^{b} \cdot f_{m o}^{c}
$$

Die Parameter $a, b$ und $c$ hängen dabei von der Stein-Mörtel-Kombination ab. Nachfolgend sind in Tabelle 20 die damit für die verwendeten Materialien ermittelten rechnerischen Mauerwerksdruckfestigkeiten angegeben.

\begin{tabular}{|c|c|c|c|c|c|c|}
\hline \multirow[t]{2}{*}{ Wand } & \multicolumn{2}{|c|}{ Mittlere Druckfestigkeiten } & \multicolumn{3}{|c|}{$\begin{array}{c}\text { Parameter } \\
\text { aus Gleichung (14) }\end{array}$} & \multirow{2}{*}{$\begin{array}{l}\text { Resultierende rechnerische } \\
\text { Mauerwerksdruckfestigkeit } \\
\text { cal } f_{m v}\left[\mathbf{N} / \mathbf{m m}^{2}\right]\end{array}$} \\
\hline & $f_{b}\left[\mathbf{N} / \mathbf{m m}^{2}\right]$ & $f_{m o}\left[\mathbf{N} / \mathbf{m m}^{2}\right]$ & $a[-]$ & $b[-]$ & $c[-]$ & \\
\hline $\mathbf{A}$ & \multirow[t]{4}{*}{19,8} & 4,7 & \multirow{4}{*}{$\begin{array}{l}0,25^{1)} \\
(0,55)\end{array}$} & \multirow{4}{*}{$\begin{array}{l}0,82^{1)} \\
(0,56)\end{array}$} & \multirow{4}{*}{$\begin{array}{l}0,41^{1)} \\
(0,46)\end{array}$} & $5,5^{1)}(6,0)$ \\
\hline B & & 5,9 & & & & $6,0^{1)}(6,6)$ \\
\hline $\mathbf{C}$ & & 5,9 bzw. 4,4 & & & & 6,0 bzw. $5,3^{1)}(6,6$ bzw. 5,8$)$ \\
\hline $\mathbf{D}$ & & 4,4 & & & & $5,3^{1)}(5,8)$ \\
\hline $\mathbf{E}$ & \multirow[t]{2}{*}{15,7} & 12,6 & 0,76 & 0,72 & 0 & 5,5 \\
\hline $\mathbf{F}$ & & $\begin{array}{c}52,9 \\
\left(25^{22}\right)\end{array}$ & 0,25 & 0,82 & 0,41 & $\left.\begin{array}{r}12,2 \\
(8,9)\end{array}\right\} \rightarrow 10,6$ \\
\hline KS 1 & \multirow[t]{2}{*}{$25,4^{3)}$} & \multirow[t]{2}{*}{5,7} & \multirow[t]{2}{*}{0,55} & \multirow[t]{2}{*}{0,98} & \multirow[t]{2}{*}{0} & \multirow[t]{2}{*}{13,1} \\
\hline KS 2 & & & & & & \\
\hline KS 3 & \multirow[t]{2}{*}{$34,1^{4)}$} & 6,7 & 0,55 & 0,98 & 0 & 17,5 \\
\hline KS 4 & & 10,1 & 0,55 & 0,98 & 0 & 17,5 \\
\hline \multicolumn{7}{|c|}{$\begin{array}{l}\text { 1) Werte für L-HLz, Werte in Klammern für HLz } \\
\text { 2) Mindestdruckfestigkeit bei Eignungsprüfung nach DIN 1053-1 (1996-11), Anhang } 3 \text { [17] } \\
\text { 3) Mittelwert aus allen } 15 \text { Versuchen } \\
\text { 4) Mittelwert von halben und ganzen Steinen }\end{array}$} \\
\hline
\end{tabular}

Tabelle 20: Resultierende Mauerwerksdruckfestigkeiten senkrecht zur Lagerfuge

Bei der Bestimmung der Druckfestigkeit von Wand F wurden zusätzlich die relativ hohen gemessenen Mörteldruckfestigkeiten durch den Mindestwert bei der Eignungsprüfung nach DIN 1053-1 (1996-11), Anhang 3 [17] ersetzt. Für die weiteren Berechnungen wird der Mittelwert aus den beiden Einzelergebnissen verwendet, da davon ausgegangen werden kann, dass die empirische Gleichung (14) den Bereich dieser hohen Mörtelfestigkeit nicht ausreichend abdecken kann, und die Tragfähigkeit dadurch überschätzt werden würde. 
Die Zugfestigkeit für die Beanspruchung senkrecht zu den Lagerfugen wird im Wesentlichen von der Haftzugfestigkeit zwischen Stein und Mörtel beschrieben (s. DIN 18555-6 [26]). Eine Ausnahme davon stellt Porenbeton mit Dünnbettmörtel dar, bei dem die Steinzugfestigkeit teilweise geringer als die Haftzugfestigkeit sein kann. In Tabelle 21 sind Anhaltswerte für die Haftzugfestigkeit nach Schubert [120] angegeben.

\begin{tabular}{|l|c|c|}
\hline \multicolumn{1}{|c|}{$\begin{array}{c}\text { Stein } \\
\text { (Wand) }\end{array}$} & Mörtel & $\begin{array}{c}\text { Mittelwert der } \\
\text { Haftzugfestigkeit } \\
\left.\boldsymbol{f}_{\boldsymbol{m o}, \boldsymbol{H Z}} \text { [N/mm } \mathbf{m}^{2}\right]\end{array}$ \\
\hline $\begin{array}{l}\text { HLz Typ 1 } \\
\text { (A, B, C, D) }\end{array}$ & NM IIa & 0,48 bzw. 0,44 ${ }^{2)}$ \\
\hline $\begin{array}{l}\text { KS PE } \\
\text { (KS1, KS2) }\end{array}$ & DM & $0,69\left(0,29^{3)}\right)$ \\
\hline $\begin{array}{l}\text { KS PS } \\
\text { (KS3, KS4) }\end{array}$ & DM & 0,61 \\
\hline $\begin{array}{l}\text { 1) } \\
\text { für den Steintyp von Wand E und F (HLz Typ 2) liegen } \\
\text { keine passenden Angaben aus der Literatur vor }\end{array}$ \\
2) $\begin{array}{l}\text { Unterschiede infolge verschiedener Prüfverfahren } \\
\text { 3) } \text { Prüfalter unter 14 Tagen }\end{array}$ \\
\hline
\end{tabular}

Tabelle 21: Anhaltswerte für die Haftzugfestigkeit verschiedener Stein-Mörtel-Kombinationen - aus Schubert [120]

Die Druck- und Zugfestigkeit parallel zu den Lagerfugen ist für die hier untersuchten Beanspruchungsfälle von untergeordneter Bedeutung.

Untersuchungen zur Mauerwerksfestigkeit bei Beanspruchung in Wandlängsrichtung wurde von Glitza [36] und Schubert / Hoffmann [122] durchgeführt. Die Druckfestigkeit kann dabei mit Hilfe der Gleichung (14) mit modifizierten Parametern durchgeführt werden. Dabei ist, im Unterschied zur Beschreibung der Festigkeit senkrecht zu den Lagerfugen, eine deutlich stärkere Differenzierung nach Steinart und -sorte notwendig.

Die Mauerwerkszugfestigkeit parallel zu den Lagerfugen kann durch die beiden Grenzbedingungen Steinzugversagen und Überschreitung des Haftscherverbundes zwischen Stein und Mörtel beschrieben werden. Durch Druckspannungen senkrecht zu den Lagerfugen wird letzterer deutlich erhöht. Unter Vernachlässigung der Lagerfugendicke ergibt sich ohne Normalspannungen senkrecht zur Lagerfuge mit der Steinlängszugfestigkeit $f_{b t, \|}$, der Haftscherfestigkeit $k$ und dem Verhältniswert von Überbindelänge zu Steinhöhe $\ddot{u} / \Delta y$ als Mauerwerkszugfestigkeit $f_{m t x}$ parallel zur Lagerfuge:

$$
f_{m t x}=\min \left\{\begin{array}{c}
0,5 \cdot f_{b t, \|} \\
k \cdot \frac{\ddot{u}}{\Delta y}
\end{array}\right\}
$$




\subsubsection{Kennwerte zur Schubfestigkeit}

\section{Haftscherfestigkeit}

Die Haftscherfestigkeit der verwendeten Dünnbettmörtel wurde am KS-Referenzstein nach DIN 18555-5 [25] geprüft (s. Tabelle 19). Da die Festigkeit bei anderen Steinarten erheblich abweichen kann, finden die Prüfwerte aus Tabelle 19 lediglich für die Wände KS $1 \div 4$ Verwendung. Bei den gemessenen Haftscherfestigkeiten ist zu beachten, dass die am KSReferenzstein ermittelten Werte etwa um den Faktor 1/1,2 unter denen an üblichen KSSteinen liegen (s. DIN 1053-1, Anhang 3 [17]).

Bei Dünnbettmörteln stellt die Lagerfugendicke einen entscheidenden Parameter für die Haftscherfestigkeit dar. Mit abnehmender Lagerfugendicke sinkt auch die Haftscherfestigkeit, was sich aus dem kleineren Verhältnis von Lagerfugendicke zu saugender Steinoberfläche begründet. Weicht die ausgeführte Fugendicke in der Wand von dem Sollwert aus der Prüfung stärker ab, können sich deutliche Haftscherfestigkeitsunterschiede ergeben.

Bei Hochlochziegeln und Dünnbettmörtel kann im Fall von vertikal zwischen zwei Steinreihen versetzten Stegen, insbesondere bei in Querrichtung verlaufenden Innenstegen, nicht automatisch von einem vollflächigen Kontakt in der Lagerfuge ausgegangen werden. Die Anhaltswerte zur Haftscherfestigkeit (Wand E) sind für die Nachrechnung entsprechend zu reduzieren.

\begin{tabular}{|c|c|c|c|c|}
\hline \multirow{2}{*}{$\begin{array}{l}\text { Wand } \\
\text { (Stein) }\end{array}$} & \multirow[t]{2}{*}{ Mörtel } & \multicolumn{3}{|c|}{ Haftscherfestigkeit $k\left[\mathrm{~N} / \mathrm{mm}^{2}\right]$} \\
\hline & & $\begin{array}{l}1,2 \cdot \text { Mittelwert } \\
\text { aus Tabelle } 19^{2)}\end{array}$ & $\begin{array}{c}\text { nach } \\
\text { Schubert }[120]\end{array}$ & $\begin{array}{c}\text { aus } \\
\text { DIN 1053-1 } 1{ }^{1), 2)}\end{array}$ \\
\hline A, B, C, D (HLz Typ 1) & NM IIa & n.d. & 0,5 & 0,288 \\
\hline E (HLz Typ 2) & DM & $1,2 \cdot 0,88=1,06$ & 0,63 & $0,352(0,704)^{3)}$ \\
\hline F (HLz Typ 2) & NM IIIa & n.d. & 1,0 & 0,416 \\
\hline KS1, 2 (PE) & DM (Typ: KMS 100) & $1,2 \cdot 1,72=2,06$ & 1,70 & $0,352(0,704)^{3)}$ \\
\hline KS3 (PS) & DM (Typ: Spezial) & $1,2 \cdot 0,65=0,78$ & $(1,70)$ & $0,352(0,704)^{3)}$ \\
\hline KS4 (PS) & DM (Typ: KS 4) & $1,2 \cdot 0,82=0,98$ & 1,70 & $0,352(0,704)^{3)}$ \\
\hline \multicolumn{5}{|c|}{$\begin{array}{l}\text { 1) Rechenwert }=1,6 \cdot \beta_{R H S}=1,6 \cdot 2 \cdot \sigma_{o H S}\left(\text { mit } \sigma_{o H S} \text { nach } D I N \text { 1053-1, Tab. 5), infolge des Ansatzes nicht abge- }\right. \\
\text { minderter Haftscherfestigkeitswerte bei } \ddot{u} / \Delta y=1 \text { und } \mu=0,6 \rightarrow \text { Faktor } 1 /(1+0,6 \cdot \Delta y / \ddot{u}) \\
\text { (s. a. E DIN 1053-5 (2002-04), Tab. } 4 \text { [18]) } \\
\text { geprüft am KS-Referenzstein } \\
\left.\text { aus dem Rechenwert für NM III (s. }{ }^{1)}\right) \text { und dem Verhältnis der Mindestwerte bei Eignungsprüfung nach } \\
\text { DIN 1053-1, A3 für NM III und DM [17] }\end{array}$} \\
\hline
\end{tabular}

Tabelle 22: Haftscherfestigkeit, mittlere Prüfergebnisse und Anhaltswerte aus der Literatur

Für die Werte der Wände aus Hochlochziegeln wird im Weiteren auf Literaturangaben zurückgegriffen [120]. Dabei ist zu beachten, dass es bei Hochlochziegeln mit Normalmörtel infolge der eindringenden Mörteldollen zu einer mechanischen Verzahnung von Mörtel und Steinrandbereich kommt. Die Größe dieses Verbundes kann, abhängig von der Lochgeometrie, der Scherben- und Mörtelfestigkeit sowie dem daraus resultierenden Bruchbild, stark variieren (Hofmann, Stöckl, Mainz [49, 136]).

\section{$\underline{\text { Reibbeiwert }}$}

Der Reibbeiwert $\mu$ beschreibt das Verhältnis von Schub- und Normalspannung nach dem Überschreiten der Haftscherfestigkeit. Für Vollziegel und Kalksandsteine mit Kalk-ZementMörtel gibt van der Pluijm [105] einen mittleren Reibbeiwert $\mu=0,75$ an. Mann [74] 
empfiehlt nach der Übersicht von Versuchsergebnissen verschiedener Autoren einen Reibbeiwert für Hohlblocksteine / NM IIa $\mu=0,56$, für KS-Vollsteine / NM IIa $\mu=0,51$ und für Vollziegel $\mu=0,75$. Für KS-Steine mit Normalmörtel wird bei den Versuchen von König / Mann / Ötes [60] ein Wertebereich zwischen 0,67 und 0,86 bestimmt.

\section{Steinzugfestigkeit}

Die schräge Steinzugfestigkeit wird für die Beschreibung des Schubbruchkriteriums Steinzugversagen benötigt. Bei den Lochsteinen spielt die Richtungsorientierung der untersuchten Zugbeanspruchung eine entscheidende Rolle, da sich die Zugfestigkeiten senkrecht und parallel zur Lagerfuge um etwa eine Zehnerpotenz unterscheiden können. Bei den eigenen Versuchen (s. Kapitel 5) wurden beim Versagensbild Steinzugversagen die Risse in den Hochlochziegeln unter einem mittleren Winkel von ca. $\alpha_{0}=15 \div 30^{\circ}$ zur Vertikalen beobachtet.

Die tatsächlich maßgebende mehraxiale Festigkeit unter kombinierter Druck-ZugBeanspruchung konnte aufgrund der dafür erforderlichen aufwendigen zweiaxialen Versuchseinrichtung hier nicht bestimmt werden. Es wurde daher ein vereinfachter trigonometrischer Ansatz nach Gleichung (16) gewählt, der durch den Vergleich der Ergebnisse für die Fälle $\alpha_{0}=0^{\circ}$ und $\alpha_{0}=90^{\circ}$ sowie den isotropen Fall überprüft werden kann.

$$
f_{b t}=f_{b t, \|} \cdot \sin ^{2} \alpha_{0}+f_{b t, \perp} \cdot \cos ^{2} \alpha_{0}
$$

Für die Steinzugfestigkeit parallel zu den Lagerfugen $\left(\alpha_{0}=0^{\circ}\right)$ wird für den Typ 1 vereinfacht die in Abschnitt 4.1.2 ermittelte Biegezugfestigkeit angesetzt. Für Steintyp 2, bei dem die Biegezugfestigkeit infolge von Rissen im Randbereich zu den Lagerflächen deutlich reduziert ist, wird 0,9 N/mm² als Steinzugfestigkeit in Anlehnung an den über die Scherbenzugfestigkeit ermittelten Wert festgelegt.

Für die Bestimmung der Zugfestigkeit der Hochlochziegel senkrecht zu den Lagerfugen $\left(\alpha_{0}=90^{\circ}\right)$ wurde von der Annahme ausgegangen, dass die Zugfestigkeiten senkrecht und parallel zur Lagerfuge sich analog zu den zugehörigen Druckfestigkeiten verhalten.

Daraus ergibt sich eine Zugfestigkeit senkrecht zu den Lagerfugen bei Typ 1 von 5,39 N/mm² und bei Typ 2 von $6,74 \mathrm{~N} / \mathrm{mm}^{2}$.

Für Kalksandsteine spielt die Neigung der Hauptzugbeanspruchung für den Wert der Steinzugfestigkeit nur eine untergeordnete Rolle, da vereinfacht isotrope Festigkeiten angenommen werden können. 


\begin{tabular}{|c|c|c|c|c|c|c|}
\hline \multirow[t]{3}{*}{$\begin{array}{l}\text { Stein } \\
\text { (Wand) }\end{array}$} & \multicolumn{2}{|c|}{$\begin{array}{c}\text { Steinzugfestigkeit } \\
f_{b t}\left[\mathbf{N} / \mathbf{m m}^{2}\right]\end{array}$} & \multirow{2}{*}{\multicolumn{2}{|c|}{$\begin{array}{c}\text { Schräge Steinzugfestigkeit } \\
\text { cal } f_{b t}\left[\mathrm{~N} / \mathbf{m m}^{2}\right] \\
\text { für } \alpha_{0}\left[{ }^{\circ}\right] \text { nach Gl. (16) }\end{array}$}} & \multirow{2}{*}{\multicolumn{2}{|c|}{$\begin{array}{l}\text { Angaben der resultierenden } \\
\text { Steinzugfestigkeit } f_{b t}\left[\mathrm{~N} / \mathbf{m m}^{2}\right]\end{array}$}} \\
\hline & $\perp$ zu den & || zu den & & & & \\
\hline & LaFu ${ }^{1)}$ & $\mathrm{LaFu}^{2)}$ & 15 & 30 & DIN 1053-1 ${ }^{3)}$ & Mann $[60]$ \\
\hline $\begin{array}{l}\text { HLz Typ } 1 \\
\text { (Wand A } \div \text { D) }\end{array}$ & 5,39 & 0,74 & 1,05 & 1,90 & $\begin{array}{c}0,033 \cdot 19,8= \\
0,65\end{array}$ & $\begin{array}{c}0,06 \cdot 19,8= \\
1,19\end{array}$ \\
\hline $\begin{array}{l}\text { HLz Typ } 2 \\
\text { (Wand E, F) }\end{array}$ & 6,74 & 0,90 & 1,29 & 2,36 & $\begin{array}{c}0,033 \cdot 15,7= \\
0,52\end{array}$ & $\begin{array}{c}0,06 \cdot 15,7= \\
0,94\end{array}$ \\
\hline $\begin{array}{l}\text { KS PE } \\
\text { (Wand KS1, 2) }\end{array}$ & \multicolumn{2}{|c|}{$3,5^{2)}$} & \multicolumn{2}{|c|}{$3,5^{2)}$} & $\begin{array}{c}0,04 \cdot 25,4= \\
1,02\end{array}$ & $\begin{array}{c}0,038 \cdot 25,4= \\
0,97\end{array}$ \\
\hline $\begin{array}{l}\text { KS PS } \\
(\text { Wand KS3, 4) }\end{array}$ & \multicolumn{2}{|c|}{$3,3^{2)}$} & \multicolumn{2}{|c|}{$3,3^{2)}$} & $\begin{array}{c}0,04 \cdot 34,1= \\
1,36\end{array}$ & $\begin{array}{c}0,038 \cdot 34,1= \\
1,30\end{array}$ \\
\hline
\end{tabular}

Tabelle 23: Resultierende schräge Steinzugfestigkeiten

Mann [60] gibt als Verhältnis von Steinzug- zu Steindruckfestigkeit bei Hochlochziegeln ohne in Steinlängsrichtung durchgehende Innenstege als Faktor 0,06 und für KS-Vollsteine 0,038 an.

\section{Bruchenergie}

Für die Bestimmung der Bruchenergie der beiden Versagensbilder Steinzugversagen und Haftscherfestigkeitsversagen wird auf die Angaben in der Literatur zurückgegriffen (s. Abschnitt 3.3).

Nach den Untersuchungen von van der Pluijm [104] beträgt bei einer Steinzugfestigkeit zwischen 1,5 und 3,5 N/mm² - was etwa auch die Größenordnung der Festigkeiten der eigenen Versuche darstellt - die Zugbruchenergie $G_{f}$ zwischen 0,061 und $0,128 \mathrm{Nmm} / \mathrm{mm}^{2}$.

Bei dem Versagensbild Haftscherfestigkeit ist ein deutlicher Zusammenhang zu der Normaldruckspannung erkennbar (s. Abbildung 27). Bei einer mittleren Normalspannung von $-0,6 \mathrm{~N} / \mathrm{mm}^{2}$ bei den eigenen Versuchen kann bei den Wänden $\mathrm{KS} 1 \div \mathrm{KS} 4$ die Bruchenergie $G_{f}$ mit $0,03 \mathrm{Nmm} / \mathrm{mm}^{2}$ und für die Wände $\mathrm{A} \div \mathrm{F} \mathrm{zu} 0,12 \mathrm{Nmm} / \mathrm{mm}^{2}$ angenommen werden.

\subsubsection{Verformungskenngrößen}

Für die Bestimmung der Verformungskennwerte des Mauerwerkes wird auf die Angaben aus der Literatur zurückgegriffen (Schubert [116, 117, 120]). Die maßgebenden Parameter Druck-E-Modul und die Querdehnzahl - sind nachfolgend in Tabelle 23 dargestellt. 


\begin{tabular}{|c|c|c|c|c|c|c|}
\hline \multirow[t]{2}{*}{ Wand } & \multirow[t]{2}{*}{ Mörtel } & \multicolumn{3}{|c|}{$\begin{array}{l}\text { Druck-E-Modul nach verschiedenen } \\
\text { Ansätzen - aus Schubert [120] }\end{array}$} & \multirow{2}{*}{$\begin{array}{l}\text { Querdehnzahl } \\
\text { (Wertebe- } \\
\text { reich) } \\
\text { v [-] }\end{array}$} & \multirow{2}{*}{$\begin{array}{c}\text { Dehnung bei Maxi- } \\
\text { malspannung } \\
\text { (Wertebereich) } \\
\varepsilon_{u, D}[-]\end{array}$} \\
\hline & & $\begin{array}{l}E_{y}=a \cdot f_{m y} \\
{\left[\mathbf{N} / \mathbf{m m}^{2}\right]}\end{array}$ & $\begin{array}{l}E_{y}=b \cdot f_{b} \\
{\left[\mathbf{N} / \mathbf{m m}^{2}\right]}\end{array}$ & $\begin{array}{l}\text { Festwert } \\
{\left[\mathbf{N} / \mathbf{m m}^{2}\right]}\end{array}$ & & \\
\hline $\mathbf{A}$ & NM IIa & 6.600 & - & 6.000 & \multirow{6}{*}{$\begin{array}{c}0,1 \\
(0,05 \ldots 0,23)\end{array}$} & \multirow{6}{*}{$\begin{array}{c}-0,0018 \\
(-0,001 \ldots-0,0026)\end{array}$} \\
\hline B & NM IIa & 7.200 & - & 6.000 & & \\
\hline $\mathrm{C}$ & NM IIa & 7.200 bzw. 6.360 & - & 6.000 & & \\
\hline D & NM IIa & 6.360 & - & 6.000 & & \\
\hline $\mathbf{E}$ & DM & 7.315 & 6.594 & 7.500 & & \\
\hline $\mathbf{F}$ & NM IIIa & 12.720 & - & 10.000 & & \\
\hline KS1, 2 & DM & 7.860 & - & 10.000 & \multirow{2}{*}{$\begin{array}{c}0,1 \\
(0,07 \ldots 0,12)\end{array}$} & \multirow{2}{*}{$\begin{array}{c}-0,0025 \\
(-0,0013 \ldots-0,0039)\end{array}$} \\
\hline KS3, 4 & DM & 10.500 & - & - & & \\
\hline
\end{tabular}

Tabelle 24: Verformungskenngrößen von Mauerwerk - aus Schubert [120]

Für den Schubmodul $G_{x y}$ kann als Anhaltswert nachfolgende von Mojsilović [88] vorgeschlagene Vereinfachung mit einer Querdehnzahl von $v=0,25$ verwendet werden.

$$
G_{x y}=\frac{\left(E_{x}+E_{y}\right)}{4(1+v)}
$$

Für die genaue Bestimmung des E-Moduls senkrecht und parallel zu den Lagerfugen sowie der Querdehnzahl sind in der Regel Versuche an größeren Mauerwerkskörpern notwendig (s. Ganz und Thürlimann [32, 33, 140], Lurati, Graf und Thürlimann [69, 70], Mojsilović und Marti $[87,88])$. 


\section{Experimentelle Untersuchungen}

\section{1. Übersicht}

\subsubsection{Allgemeines}

Soll das Trag- und Verformungsverhalten von Konstruktionen im Lastfall Erdbeben experimentell untersucht werden, stellen Rütteltischversuche die mit Abstand realitätsnächste Methodik dar. Jedoch stößt man schon bei geringen Prüfmassen und Bodenbeschleunigungswerten rasch an die technischen Grenzen der in Deutschland vorhandenen Anlagen. Einzelne leistungsstarke und entsprechend kostenintensive Rütteltische finden sich lediglich im südeuropäischen Ausland, in Japan und in Nordamerika. Will man mit kleineren Anlagen Versuche an Gebäuden durchführen, sind lediglich Maßstabsversuche möglich. Besonders für Mauerwerk treten dabei, neben den allgemein bekannten Maßstabsfaktoren aus den Dimensionen Masse, Länge, Zeit und den daraus abgeleiteten Größen, zusätzlich noch die Problempunkte der Herstellung der Versuchskörper mit zielgenauen Eigenschaftswerten, z. B. orthotrope Steinfestigkeiten und Haftscherfestigkeit, hinzu. Umfangreiche Untersuchungen auf diesem Gebiet wurden von Tomazevic $[141 \div 150]$ durchgeführt.

\subsubsection{Statisch-zyklische Versuche}

Eine Möglichkeit das Verhalten erdbebenbeanspruchter Scheiben näherungsweise zu untersuchen besteht in der Form von statisch-zyklischen Schubversuchen. Dabei wird unter konstanter Normalkraft eine Horizontalverschiebung mit wechselndem Vorzeichen und schrittweise erhöhter Amplitude auf den Wandkopf aufgebracht. In der Regel wird dabei lediglich die Horizontalverschiebung gesteuert - die Soll-Vertikallast bleibt vom Betrag her konstant. Ohne weitere Maßnahmen würde es bei entsprechend großer Biegebeanspruchung der Wand zu einem Klaffen und einer nicht zu vernachlässigenden Verdrehung des Wandkopfes führen.

Da jedoch das Verhalten und Versagen unter dominierender Schubbeanspruchung zu untersuchen ist, wird die Kopfverdrehung üblicherweise durch einen mechanischen Anschlag, z. B. über einen steifen Riegel mit vertikalen Spannstäben, verhindert (s. Abbildung 31).

Beim Überschreiten einer vorgegebenen maximalen Verdrehung stützt sich der Wandkopf mit dem darauf angeordneten Lastverteilungsbalken gegen den steifen Anschlag ab. Dabei wird eine zusätzliche Normalkraft undefinierter Größe aktiviert. Besonders in dem entscheidenden Bereich hoher Horizontallasten tritt dieser Effekt in Erscheinung und kann die Ergebnisse durch die Erhöhung der Wandnormalkraft wesentlich verfälschen. Zusätzlich ergeben sich bei größeren Verschiebungen am Wandkopf zusätzliche horizontale Rückstellkräfte aus Theorie II. Ordnung. Diese Versuchsanordnung erfasst somit die Randbedingungen von Schubwänden in Gebäuden nicht realistisch, was bei der Bewertung der entsprechenden Ergebnisse zu beachten ist.

Nachfolgend ist in Abbildung 31 der konventionelle Versuchsaufbau dargestellt, wie er auch von Vratsanou / Langer [154, 156] und Ötes [55] in Anlehnung an Rilem LUMC3 [110] für Schubversuche benutzt wurde (s. a. Abschnitt 3.2). 


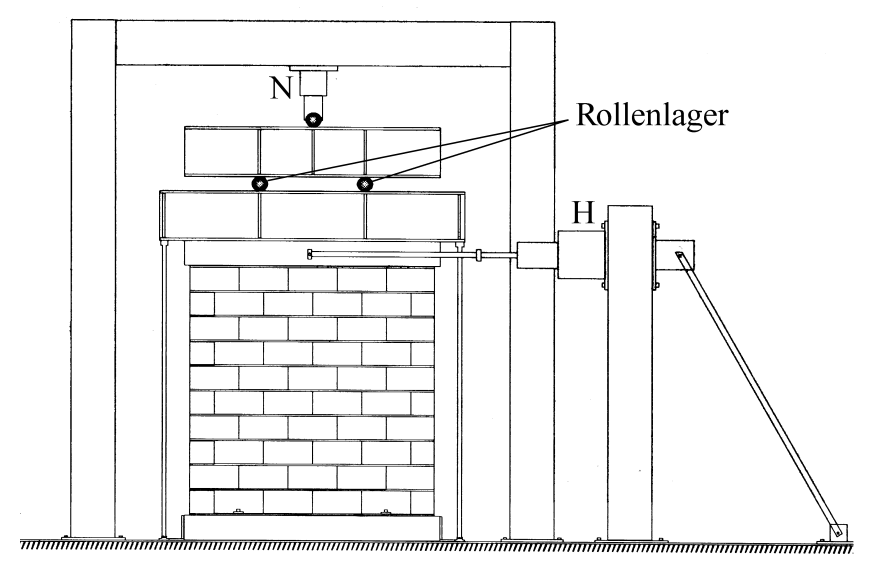

Abbildung 31: Herkömmlicher Versuchsstand für statisch-zyklische Versuche mit mechanischer Behinderung der Kopfverdrehung

Abhilfe davon kann die direkte Steuerung der Normalkraft mit der zugehörigen Ausmitte $(\rightarrow$ Kopfmoment) schaffen, z. B. über zwei symmetrisch zur Wandmitte angeordnete und unabhängig geregelte Zylinder. Dabei wird das Wandkopfmoment entsprechend der gemessenen Wandkopfverdrehung gegendrehend aufgebracht. So kann die Kopplung von Kopfmoment und gemessener Verdrehung, z. B. über eine Drehfeder mit definierter Federkennlinie erfolgen. Diese Vorgehensweise wurde im Rahmen der Vorversuche zu den pseudodynamischen Erdbebenversuchen der zweiten Stufe (s. Abschnitt 5.5.3) angewendet.

\subsubsection{Pseudodynamik}

\section{Allgemeines}

Bei der Methode der Pseudodynamik wird das dynamische Verhalten von Bauwerken oder einzelner Bauteile im Zeitbereich experimentell untersucht. Basis dabei ist die numerische, zeitschrittweise Lösung der Schwingungsdifferentialgleichung der zu untersuchenden Konstruktion. Die Versuchsdurchführung hat dabei nicht zwangsläufig am gesamten System zu erfolgen, sondern es genügt in der Regel das maßgebende Bauteil allein zu prüfen und die restliche Konstruktion als so genannte Sub-Struktur lediglich numerisch zu berücksichtigen. Als weiterer Vorteil der Methode ist zu nennen, dass sie mit verhältnismäßig einfachen Mitteln, wie sie in den meisten Prüflaboren vorhanden sind, durchzuführen ist. Die Gebäudemassen müssen z. B. nicht wie bei Rütteltischversuchen direkt bewegt werden, sondern deren Berücksichtigung erfolgt lediglich numerisch.

Als Nachteil ist die Tatsache zu werten, dass durch die verlangsamte Versuchsdurchführung zeitabhängige Materialeigenschaften, wie z. B. Relaxation oder Erhöhung des E-Moduls unter dynamischer Belastung nicht oder nur schwer zu erfassen sind (Shing / Mahin [129, 130]). Geschwindigkeitsabhängige Effekte können dagegen über einen Dämpfungsansatz abgedeckt werden. Dabei ist der Ansatz einer viskosen Dämpfung als Vereinfachung ausreichend.

Allgemein hat sich die Pseudodynamik als äußerst leistungsfähige Versuchsmethode für die Untersuchung von Bauwerken oder Bauteilen unter Erdbebeneinwirkung bewährt.

\section{Entwicklungsgeschichte}

Die Methode der Pseudodynamik wurde Ende der 70er Jahre in Japan als Alternative zu Rütteltischversuchen entwickelt (Takanashi et. al [137]). Die Grundlagen dieser Methode 
stellte Mahin [71] erstmals 1981 dar. Dabei wurden neben der theoretischen Basis und der prinzipiellen Vorgehensweise auch mögliche Fehlerquellen und deren Auswirkung diskutiert. Es erfolgte ein Vergleich der Güten der Integrationsmethoden Newmark- und ZentraleDifferenz-Methode für den Fall mit und ohne Dämpfung.

Seible et. al. [125, 126, 157] führten im Rahmen eines japanisch-amerikanischen Forschungsprojektes pseudodynamische Erdbebenversuche an dreigeschossigen Gebäuden aus bewehrtem Mauerwerk im Maßstab 1:1 durch. Durch die Anordnung als Mehrmassenschwinger mit Verschiebungsfreiheitsgraden auf Höhe der Stahlbetongeschossdecken konnten höhere Eigenformen der Struktur erfasst werden.

Mitte der 90er Jahre wurden am Europäischen Forschungslabor ELSA [39, 107] insgesamt sechs Brückenkonstruktionen mit der Methode der Pseudodynamik geprüft. Die drei Pfeiler der Brücke wurden im Maßstab 1:2,5 hergestellt und im Zuge der Versuchsdurchführung direkt geprüft. Die Erfassung des Überbaus dagegen erfolgte lediglich numerisch als linearelastische Sub-Struktur. Als Erdbebeneinwirkung wurde ein auf Eurocode 8 basierender und auf $0,35 \cdot \mathrm{g}$ skalierter Zeitverlauf mit der Gesamtdauer von $10 \mathrm{~s}$ künstlich generiert. Dabei wurden die Zeitverläufe der einzelnen Pfeiler jeweils modifiziert, um die Einflüsse der räumlichen Anordnung in Realität über zeitliche Kopplung und Änderung der Frequenzcharakteristik zu erfassen.

An dreigeschossigen Stahlbetonscheiben im Maßstab 1:3 wurden von Thiele [138] im Jahr 2000 an der ETH Zürich Erdbebenversuche mit der Methode der Pseudodynamik durchgeführt. Aus dem Vergleich mit den Ergebnissen gleicher, parallel auf dem Rütteltisch geprüfter Wandscheiben konnte eine gute Übereinstimmung und dementsprechend auch gute Eignung der Methode gezeigt werden. Daneben wurden numerische Aspekte der einzelnen Integrationsmethoden, insbesondere deren Eignung für nichtlineare Systeme mit großen Steifigkeitsänderungen diskutiert (s. Abschnitt 5.2.2).

\subsubsection{Zielsetzung und Vorgehensweise}

Ziel der hier beschriebenen pseudodynamischen Erdbebenversuche ist, das Verhalten unbewehrter Mauerwerkskonstruktionen unter seismischer Belastung realitätsnah zu untersuchen. Als Untersuchungsobjekt werden zwei repräsentative Gebäudemodelle des üblichen Hochbaus gewählt, die in Horizontalrichtung allein von unbewehrten Mauerwerksscheiben ausgesteift sind und deren Bemessung für Horizontallasten einen hohen Auslastungsgrad erwarten lässt.

Die Lasteinwirkung erfolgt im Grundriss in Richtung der schwachen Hauptachse - Torsionseinflüsse sowie Belastungen senkrecht zur Ebene der zu untersuchenden Wand werden vernachlässigt. Ebenfalls unberücksichtigt bleiben die sich durch eine Vertikalbeschleunigung ergebenden Änderungen der Wandnormalkraft (vgl. Abschnitt 2.5.3).

Als Prüfobjekt dient die für die Horizontaltragfähigkeit des Gebäudes entscheidende Schubwand im untersten Geschoss. Mit der Reduzierung des Gesamtgebäudes auf ein ebenes Ersatzsystem wird die zu untersuchende Wand als Tragelement erfasst. Die Versuchsdurchführung erfolgt an Wänden im Maßstab 1:1 unter kombinierter Normalkraft-, Biege- und Schubbeanspruchung.

Eine Übersicht über die Materialeigenschaften der geprüften Wände ist in Kapitel 4 gegeben. 


\subsection{Grundlagen der Pseudodynamik}

\subsubsection{Prinzip}

Die dynamische Gleichgewichtsbedingung eines fußpunkterregten Systems - nachfolgend auf einen viskos gedämpften Einmassenschwinger nach Abbildung 4 bezogen - bestimmt sich mit den relativen Verschiebungsgrößen $d_{r e l}=d-d_{g}$ in Abhängigkeit der Zeit $t \mathrm{zu}$ :

$$
m \cdot \ddot{d}_{r e l}(t)+c \cdot \dot{d}_{r e l}(t)+k \cdot d_{r e l}(t)=-m \cdot \ddot{d}_{g}(t)
$$

Die rechte Seite stellt den Anteil der absoluten Bodenbeschleunigung dar, die aus dem Zeitverlauf $a^{g}(t)$ resultiert. Im Nachfolgenden wird der Index rel der Einfachheit halber nicht mehr explizit angegeben. Die Dämpfungskraft resultiert hier aus einem geschwindigkeitsabhängigen, viskosen Dämpfer. Sie wird, ebenso wie die Massenträgheitskraft, im Experiment lediglich numerisch berücksichtigt, da die Versuchsgeschwindigkeit etwa um den Faktor 500 kleiner als in Echtzeit ist.

Soll die Untersuchung an einem Mehrmassenschwinger erfolgen, so lässt sich die Gleichung (18) in die allgemeine Matrizenform erweitern:

$$
\underline{m} \cdot \underline{a}+\underline{c} \cdot \underline{v}+\underline{r}(\underline{d})=-\underline{m} \cdot \underline{a}^{g}
$$

Dabei stellt $\underline{m}$ die Massen- und $\underline{c}$ die Dämpfungsmatrix dar. Der zu messende Rückstellkraftvektor unter der Auslenkung $\underline{d}$ wird mit $\underline{r}(\underline{d})$ bezeichnet.

\subsubsection{Numerik}

Im Rahmen der Pseudodynamik wird die Schwingungsdifferentialgleichung (19) der zu untersuchenden Konstruktion im Zeitbereich parallel zur Versuchsdurchführung numerisch gelöst. Die Versuchsdauer liegt infolge der Beschränkung von Rechengeschwindigkeit und Geschwindigkeit der Hydraulikeinrichtung für eine Erdbebendauer von 9,0 s zwischen 60 und 90 Minuten.

Für die numerische Lösung der Schwingungsdifferentialgleichung wird der Erdbebenzeitverlauf in $n_{\text {ges }}$ diskrete Zeitschritte der Länge $\Delta t$ unterteilt, in denen die Gleichgewichtsbeziehung (19) zu erfüllen ist. Die aus der Berechnung des vorangegangenen Zeitschrittes resultierenden Verschiebungsgrößen werden auf die Konstruktion aufgebracht und die sich einstellenden Reaktionen gemessen. Diese Werte gehen wiederum in die Berechnung der Zielwerte des nächsten Zeitschrittes ein.

Sind zum Zeitpunkt $t_{n}$ die Verschiebungs-, Geschwindigkeits- und Beschleunigungsgrößen $\underline{d}$, $\underline{v}, \underline{a}$ und $\underline{a}^{g}$ bekannt, so stellt sich die Problematik auf die Zielgrößen des nächsten Zeitschrittes $n+1 \mathrm{zu}$ schließen, bei denen die Gleichgewichtsbedingung (19) zu erfüllen ist. Im Zeitschritt $n+1$ ist von vorneherein lediglich der Wert der Bodenbeschleunigung $a^{g}(n+1)$ aus dem vorgegebenen Zeitverlauf bekannt.

Erfolgt der Schluss auf die Größen im Zeitpunkt $t_{n+1}$ lediglich aus denen des vorangegangenen Zeitschrittes $t_{n}$, so handelt es sich um ein explizites Verfahren. Gehen bei der Bestimmung der Zielgrößen im Zeitpunkt $t_{n+1}$ neben den Größen des Zeitschrittes $n$ zusätzlich auch die des 
Zeitschrittes $n+1$ selbst mit ein, so handelt es sich um ein implizites Verfahren, das iterativ zu lösen ist.

Ein universelles Verfahren zur numerischen Integration geht auf Newmark [96] zurück:

$$
\begin{aligned}
& \underline{d}_{n+1}=\underline{d}_{n}+\underline{v}_{n} \cdot \Delta t+\left[\left(\frac{1}{2}-\beta\right) \cdot \underline{a}_{n}+\beta \cdot \underline{a}_{n+1}\right] \cdot \Delta t^{2} \\
& \underline{v}_{n+1}=\frac{\gamma}{\beta \cdot \Delta t} \cdot\left(\underline{d}_{n+1}-\underline{d}_{n}\right)-\left(\frac{\gamma}{\beta}-1\right) \cdot \underline{v}_{n}-\Delta t \cdot\left(\frac{\gamma}{2 \cdot \beta}-1\right) \cdot \underline{a}_{n} \\
& \underline{a}_{n+1}=\frac{1}{\beta \cdot \Delta t^{2}} \cdot\left(\underline{d}_{n+1}-\underline{d}_{n}\right)-\frac{1}{\beta \cdot \Delta t} \cdot \underline{v}_{n}-\left(\frac{1}{2 \cdot \beta}-1\right) \cdot \underline{a}_{n}
\end{aligned}
$$

Durch die Wahl der Parameter $\gamma$ und $\beta$ kann die Gewichtung der einzelnen Schritte festgelegt werden. Für $\gamma \geq 1 / 2$ und $\beta \geq 1 / 4$ ist das Verfahren unbedingt stabil [103], d.h. die Lösung wächst unabhängig von der Zeitschrittlänge $\Delta t$ für beliebige Anfangsbedingungen nicht unbegrenzt. Für $\gamma \geq 1 / 2$ und $\beta \leq \not 2$ ist das Verfahren stabil - die Zeitschrittlänge $\Delta t$ muss dabei in Abhängigkeit der kleinsten Eigenperiode $T_{\min }$ nachfolgender Bedingung gehorchen:

$$
\Delta t<\frac{T_{\min }}{2 \pi \sqrt{0,5 \cdot \gamma-\beta}}
$$

\subsubsection{Explizites Zeitschrittverfahren}

Explizite Verfahren besitzen den Vorteil, dass aus den Zustandsgrößen des Zeitschrittes $n$ direkt auf die des Nachfolgenden $n+1$ geschlossen werden kann. Nachteilig wirkt sich die Ungenauigkeit aus. Soll das Verfahren unbedingt stabil sein, ist als Bedingung eine maximale Zeitschrittlänge einzuhalten.

Nach dem Vorschlag von Shing [132] wurden für das verwendete explizite Verfahren als Parameter des Newmark-Verfahrens (20) $\beta=0$ und $\gamma=1 / 2$ festgelegt. Für die Verschiebung und Geschwindigkeit ergeben sich somit

$$
\underline{d}_{n+1}=\underline{d}_{n}+\Delta t \cdot \underline{v}_{n}+\frac{\Delta t^{2}}{2} \cdot \underline{a}_{n}
$$

und

$$
\underline{v}_{n+1}=\underline{v}_{n}+\frac{\Delta t}{2} \cdot\left(\underline{a}_{n}+\underline{a}_{n+1}\right)
$$

Werden obige Beziehungen in Gleichung (20c) eingesetzt, so bestimmt sich die Beschleunigung zu: 


$$
\underline{a}_{n+1}=\frac{\underline{m} \cdot \underline{a}_{n+1}^{g}-\underline{r}_{n+1}\left(\underline{d}_{n+1}\right)-\underline{c} \cdot\left(\underline{v}_{n}+\frac{\Delta t}{2} \cdot \underline{a}_{n}\right)}{\underline{m}+\frac{\Delta t}{2} \cdot \underline{c}}
$$

In der Versuchsdurchführung wird die berechnete Soll-Verschiebung $\underline{d}_{n+1}$ (22a) auf den Prüfkörper aufgebracht, und die sich einstellende Rückstellkraft $\underline{r}_{n+1}$ gemessen. Daraus kann abschließend die Geschwindigkeit $\underline{v}_{n+1}$ und Beschleunigung $\underline{a}_{n+1}$ berechnet werden. Als kritische Zeitschrittlänge ergibt sich nach Gleichung (21):

$$
\Delta t<\frac{T_{\min }}{\pi}
$$

Das oben dargestellte explizite Zeitschrittverfahren wurde lediglich für die Untersuchungen an dem Einmassenschwingersystem der ersten Versuchsstufe in Abschnitt 5.4 verwendet. Für Untersuchungen an Mehrmassenschwingern - insbesondere mit lediglich numerisch berücksichtigten Sub-Strukturen - kann das Verfahren nach Shing [132] nicht empfohlen werden. Bei den höheren Eigenformen ergeben sich aus der kritischen Zeitschrittlänge sehr kleine in einem Zeitschritt zu fahrende Pressenwege, die den Bereich der Mess- und Regelgenauigkeit der Versuchseinrichtungen erreichen können und somit zu großen relativen Fehlern führen.

Für die Berechnungen der zweiten Versuchsstufe an dem Mehrmassensystem mit SubStruktur-Technik wurde daher das im nachfolgenden Abschnitt dargestellte implizite Zeitschrittverfahren gewählt.

\subsubsection{Implizites Zeitschrittverfahren}

Aufbauend auf das Newmark-Verfahren wurde von Hilber / Hughes / Taylor [46] ein als $\alpha$ Methode bezeichnetes implizites Verfahren bei uneingeschränkter Stabilität entwickelt. Mit dem Parameter $-1 / 3 \leq \alpha \leq 0$ können höhere Eigenformen numerisch gedämpft werden, um eine unerwünschte Anregung zu verhindern. Eine ausführliche Darstellung und Herleitung der Beziehungen ist in Shing / Nakashima / Bursi [132] und Thiele [138] zu finden.

Die Schwingungsdifferentialgleichung (19) wird bei der $\alpha$-Methode durch nachfolgenden Ansatz ersetzt:

$$
\begin{aligned}
& \underline{m}_{g e s} \cdot \underline{a}_{n+1}+(1+\alpha) \cdot \underline{c}_{g e s} \cdot \underline{v}_{n+1}-\alpha \cdot \underline{c}_{g e s} \cdot \underline{v}_{n}-\alpha \cdot \underline{k}_{g e s} \cdot \underline{d}_{n} \\
& +(1+\alpha) \cdot \underline{k}_{g e s} \cdot \underline{d}_{n+1}=-(1+\alpha) \cdot \underline{m}_{g e s} \cdot \underline{a}_{n+1}^{g}+\alpha \cdot \underline{m}_{g e s} \cdot \underline{a}_{n}^{g}
\end{aligned}
$$

Als Parameter $\alpha, \beta$ und $\gamma$ wurden folgende Werte gewählt:

$$
\alpha=-\frac{1}{3} \quad \beta=\frac{(1-\alpha)^{2}}{4}=\frac{4}{9} \quad \gamma=\frac{1}{2}-\alpha=\frac{5}{6}
$$


Die Ansätze für die Bestimmung der Verschiebung, Geschwindigkeit und Beschleunigung (20a, b c) werden unverändert übernommen. Eingesetzt in Gleichung (23) ergibt sich:

$$
\underline{\bar{m}} \cdot \underline{d}_{n+1}=\underline{p}_{n+1}-\Delta t^{2} \cdot \beta \cdot(1+\alpha) \cdot \underline{r}_{n+1}
$$

mit:

$$
\begin{aligned}
\underline{p}_{n+1}= & \underline{\bar{m}} \cdot\left(\underline{d}_{n}+\Delta t \cdot \underline{v}_{n}+\Delta t^{2} \cdot(0,5-\beta) \cdot \underline{a}_{n}\right) \\
& +\Delta t^{2} \cdot \beta \cdot\left(\alpha \cdot \underline{m}_{g e s} \cdot \underline{a}_{n}^{g}-(1+\alpha) \cdot \underline{m}_{g e s} \cdot \underline{a}_{n+1}^{g}-\underline{c}_{g e s} \cdot \underline{v}_{n}\right. \\
& \left.-(1+\alpha) \cdot(1-\gamma) \cdot \Delta t \cdot \underline{c}_{g e s} \cdot \underline{a}_{n}+\alpha \cdot \underline{r}_{n}\right) \\
\underline{m}= & \underline{m}_{g e s}+(1+\alpha) \cdot \gamma \cdot \Delta t \cdot \underline{c}_{g e s}
\end{aligned}
$$

In der Hilfsgröße $\underline{p}_{n+1}$ stehen die aus dem vorangegangenen Zeitschritt bekannten Werte, wohingegen in $\bar{m}$ die im neuen Zeitschritt berechneten oder gemessenen Werte erfasst werden. Daher kann die Lösung nur iterativ erfolgen. Im Iterationsschritt $(k)$ ergibt sich aus dem Schritt $(k-1)$ :

$$
\underline{\bar{m}} \cdot \underline{d}_{n+1}^{(k)}=\underline{p}_{n+1}-\Delta t^{2} \cdot \beta \cdot(1+\alpha) \cdot \underline{r}_{n+1}^{(k)}
$$

mit:

$$
\begin{aligned}
& \underline{d}_{n+1}^{(k)}=\underline{d}_{n+1}^{(k-1)}+\Delta \underline{d}_{n+1}^{(k)} \\
& \underline{r}_{n+1}^{(k)}=\underline{r}_{n+1}^{(k-1)}+\Delta \underline{r}_{n+1}^{(k)}
\end{aligned}
$$

Wird der Zuwachs des Rückstellkraftvektors über die tangentiale Steifigkeit $\underline{k}_{\text {ges }}$ multipliziert mit dem Zuwachs des Verschiebungsvektors angenähert, so ergibt sich:

$$
\begin{aligned}
\bar{m} \cdot\left(\underline{d}_{n+1}^{(k-1)}+\Delta \underline{d}_{n+1}^{(k)}\right)= & \underline{p}_{n+1}-\Delta t^{2} \cdot \beta \cdot(1+\alpha) \\
& \cdot\left(\underline{r}_{n+1}^{(k-1)}+\underline{k}_{g e s}^{(k)} \cdot \Delta \underline{d}_{n+1}^{(k)}\right)
\end{aligned}
$$

Für die Steifigkeit wird bei hochgradig nichtlinearen Systemen - wie sie im Rahmen der hier beschriebenen Versuche anzutreffen sind - statt der unbekannten, aktuellen, tangentialen Steifigkeit $\underline{k}_{\text {ges }}$, eine zu Versuchsbeginn festzulegende Prädiktorsteifigkeit $\underline{k}_{0}$ angesetzt.

$$
\Delta \underline{d}_{n+1}^{(k)}=-\left(\underline{m}^{*}\right)^{-1} \cdot\left(\bar{m} \cdot \underline{d}_{n+1}^{(k-1)}-\underline{p}_{n+1}+\Delta t^{2} \cdot \beta \cdot(1+\alpha) \cdot \underline{r}_{n+1}^{(k-1)}\right)
$$

mit:

$$
\underline{m}^{*}=\underline{\bar{m}}+\Delta t^{2} \cdot \beta \cdot(1+\alpha) \cdot \underline{k}_{0}
$$


Der Iterationszyklus wird so lange wiederholt, bis der Zuwachs des Verschiebungsvektors die Größenordnung der Regelgenauigkeit erreicht. Im Rahmen dieser Versuche lag der Mindestfahrweg des Horizontalzylinders bei etwa $2 / 100 \mathrm{~mm}$. Zu beachten ist jedoch, dass in diesem Mindestfahrweg auch die Differenz zwischen dem im vorhergehenden Iterationsschritt zu erreichenden Sollwert und dem tatsächlich gefahrenen Weg eingeschlossen ist.

Nach Abschluss des Zeitschrittes erfolgt noch eine als I-Modifikation bezeichnete Korrektur des Rückstellkraftvektors $\Delta \underline{\underline{r}}{ }^{\text {rest }}=\underline{k}_{0} \cdot \Delta \underline{d}^{\text {rest }}$, mit der die verbleibende Differenz $\Delta \underline{d}^{\text {rest }}$ zwischen Soll-Weg und tatsächlich vorhandenem Ist-Weg abgedeckt wird.

\subsection{Erdbebeneinwirkung}

Zur Beschreibung der Erdbebeneinwirkung wurden vier Bodenbeschleunigungszeitverläufe künstlich generiert (Meskouris [79, 80, 81], Abschnitt 2.2.5). Als Zielspektren wurden die Boden-Baugrund-Kombinationen A1, A3, B3 und C3 nach E DIN 4149 (2002-10) [22] mit einem Grundwert der Bodenbeschleunigung von 0,4 bzw. $0,6 \mathrm{~m} / \mathrm{s}^{2}$ gewählt (s. Tabelle 25). Die Zeitverläufe wurden im Rahmen der Versuche zusätzlich noch mit einem Lastfaktor $f$ belegt (s. Tabelle $27-30$ bzw. $34-36$ ).

\begin{tabular}{|c|c|c|c|c|}
\hline Zeitverlauf & $\begin{array}{c}\text { Boden- } \\
\text { Baugrund- } \\
\text { Kombination }\end{array}$ & $\begin{array}{c}\text { Boden- } \\
\text { parameter } \\
S[-]\end{array}$ & $\begin{array}{l}\text { Grundwert der Bo- } \\
\text { denbeschleunigung } \\
\qquad a^{g}\left[\mathrm{~m} / \mathrm{s}^{2}\right]\end{array}$ & $\begin{array}{l}\text { Starkbeben- } \\
\text { dauer }^{1)} \\
T_{S B D}[\mathrm{~s}]\end{array}$ \\
\hline ZV1 & B3 & 1,25 & 0,4 & 5,9 \\
\hline ZV2 & A3 & 1,50 & 0,6 & 5,6 \\
\hline$\overline{Z V 3}$ & A1 & 1,00 & 0,4 & 5,7 \\
\hline ZV4 & $\mathrm{C} 3$ & 0,75 & 0,4 & 6,1 \\
\hline
\end{tabular}

Tabelle 25: Parameter der künstlich generierten Zeitverläufe ZV1 $\div$ ZV4

Für die Intensitätsfunktion wurde der in Abbildung 32 dargestellte Verlauf gewählt. Die Gesamterdbebendauer von 9,0 s setzt sich dabei aus einem linearen Anstieg von 1,5 s, einem Plateau von $3 \mathrm{~s}$ und einem linearen Abfall der Dauer 4,5 s zusammen. Die Zeitschrittlänge wurde $\mathrm{zu} \Delta t=0,01 \mathrm{~s}$ festgelegt $\left(n_{\text {ges }}=900\right)$.

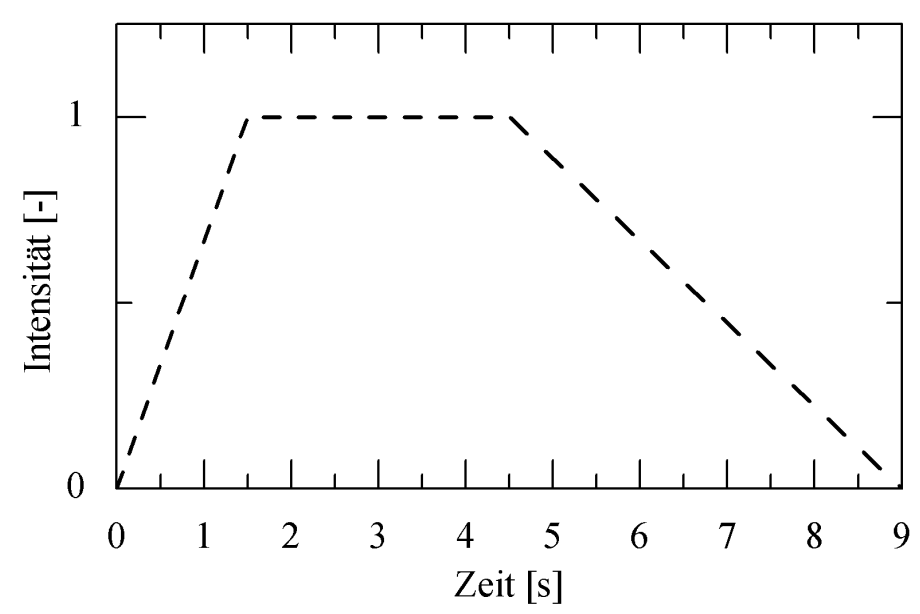

Abbildung 32: Intensitätsfunktion der generierten Zeitverläufe ZV1 $\div$ ZV 4 
Im Anhang A.1 sind die Zeitverläufe ZV1 $\div$ ZV4 mit den zugehörigen Antwortspektren, ZielAntwortspektren und Husid-Diagrammen dargestellt.

\subsection{Erste Simulationsstufe - Einmassenschwingersystem}

\subsubsection{Allgemeines}

Bei der ersten Simulationsstufe wurde das zu untersuchende Bauwerk auf die Form eines äquivalenten Einmassenschwingers reduziert. Der Versuch wurde an einer Kragscheibe durchgeführt, bei der die Kopfverdrehung planmäßig nicht behindert wurde (s. Abbildung $33)$.

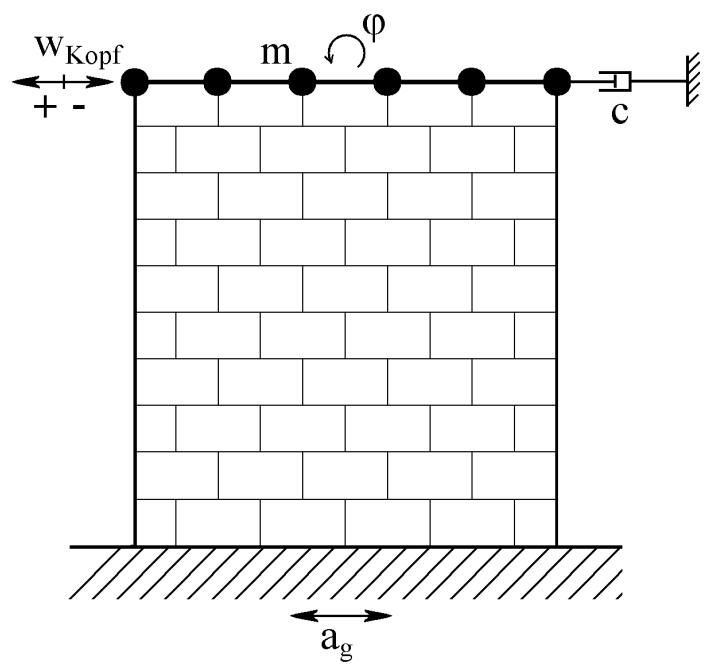

Abbildung 33: Kragscheiben-Ersatzsystem für die erste Versuchsstufe Wand $A \div C$

Die Gesamtmasse $m$ des Systems wurde im Zuge der Simulation rechnerisch am Wandkopf angeordnet. Geschwindigkeitsabhängige Dämpfungseffekte wurden mit Hilfe des viskosen Einzeldämpfers $c$ abgedeckt.

\section{Versuchsstand}

Die Wände wurden auf Fertigteilfundamenten mit aufgerauter Oberseite aufgemauert und nach dem Betonieren des Kopfbalkens in den Versuchsstand eingehoben. Die Verschiebungssicherung erfolgte durch Einspannen des Fundamentbalkens in das Prüffeld mit Hilfe von zwei Spannstäben (s. Abbildung 34).

Die während der Versuche konstant gehaltene Wandnormalkraft $N$ wurde mit Hilfe einer Handpumpe und sechs parallel geschalteten Einzelzylindern aufgebracht. Diese übertrugen die Druckkraft über Rollenlager und Lastverteilungsprofile auf den Kopfbalken. Über die gewählte Anordnung der Einzelpressen konnte eine gleichförmige Lastverteilung am Wandkopf sichergestellt werden.

Die Horizontalverschiebung wurde von dem Hydraulikzylinder mit angeschlossener Kalotte über eine Kopfplatte in den Wandkopfbalken eingeleitet. Druckkräfte wurden dabei direkt über Druckkontakt übertragen, wohingegen die Einleitung von Zugkräften mit Hilfe fünf angeschweißter Bewehrungsstäbe erfolgte. Diese wurden im ersten Drittel des Ortbetonbalkens abisoliert, um eine möglichst gleichmäßige Lasteinleitung über die Wandlänge zu gewährleisten. 
Für die Aufnahme der Horizontallasten musste der vorhandene und primär auf Vertikallasten ausgelegte Prüfrahmen mit Hilfe mehrerer Aussteifungsbleche verstärkt werden. Infolge der elastischen Verformungen und der Schlupfanteile in den Verbindungen von Stiel und Riegel war der Rahmen relativ biegeweich. Während der Vorversuche zeigte sich, dass unter Maximalbelastung die gesamte Rahmenverformung etwa das 2-fache der Wandkopfverschiebung betrug. Diese Tatsache musste bei den Festlegungen zu Steuerung und Regelung der Versuche berücksichtigt werden. Es erfolgte daher die Unterscheidung zwischen der Regelgröße Zylinderverschiebung und der Steuergröße Wandkopfverschiebung w Kopf. $_{\text {. }}$

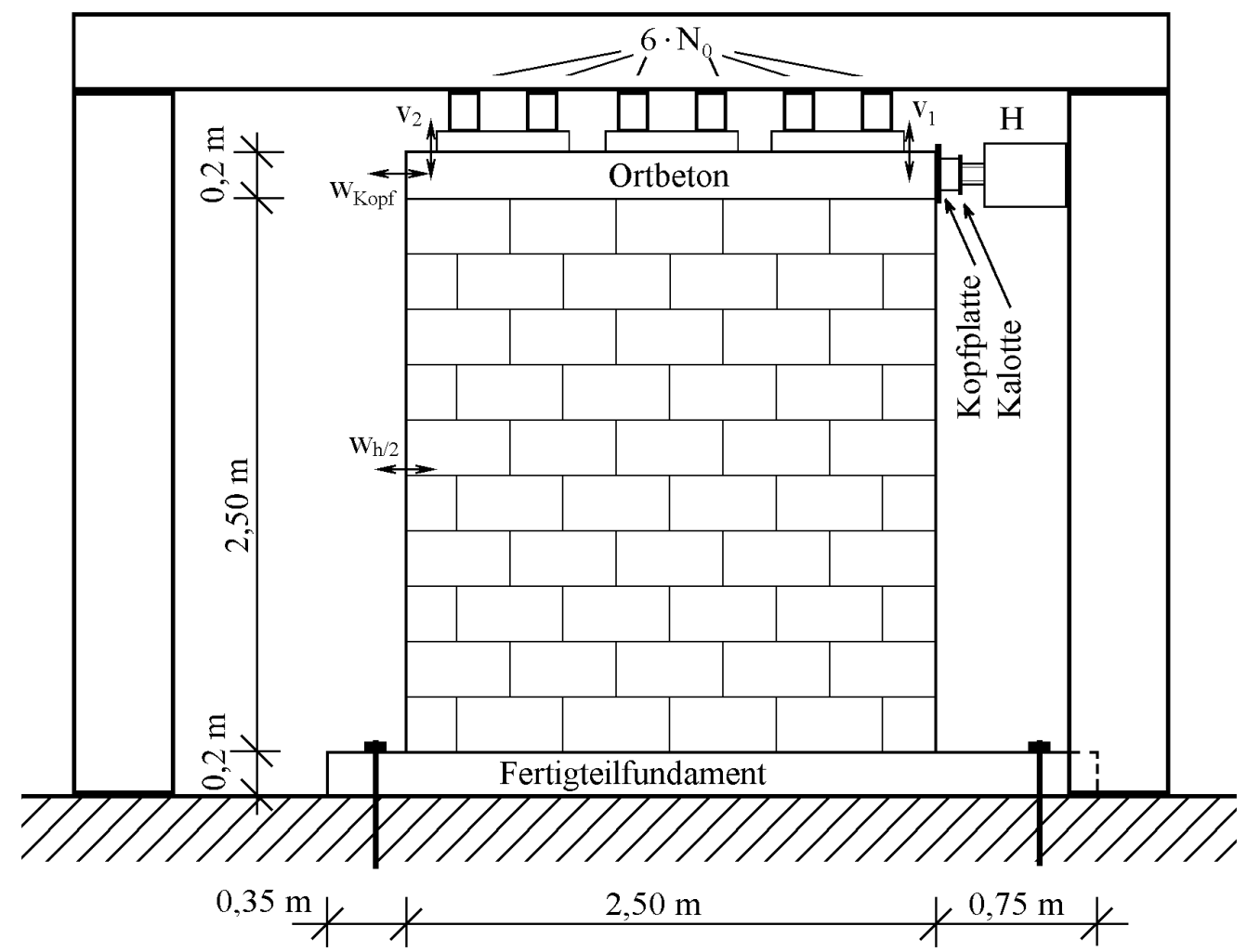

Abbildung 34: Versuchsstand für die erste Versuchsstufe - Wand $A \div C$

\section{Messprogramm}

Während der Versuche wurden nachfolgend angegebene Werte kontinuierlich mit einer Taktrate von $50 \mathrm{~Hz}$ erfasst:

- Horizontalverschiebung des Wandkopfes $w_{K o p f}$

- Horizontalkraft $H$

- Horizontalverschiebung auf halber Wandhöhe $w_{h / 2}$

- Vertikale Verschiebung am Wandkopf $v_{1}$ und $v_{2}$ durch 2 Messgeber im Abstand von 2,3 $\mathrm{m} \rightarrow$ Berechnung der Wandkopfverdrehung

- Pressenweg und Rahmenverschiebung (für Regelungs- und Steuerungsroutinen)

Der Druck in den sechs Vertikalpressen wurde mit Hilfe einer Handpumpe aufgebracht und über ein Manometer kontrolliert. Die Verschiebungen wurden im Bezug auf einen unabhängigen Messrahmen bestimmt. 


\section{Vorversuche - Ausschwingversuche}

Vor Beginn der Hauptversuche wurden für die Bestimmung der dynamischen Parameter der Wände mehrere Ausschwingversuche durchgeführt. Dazu wurde mit einem Metallgewicht auf den Wandkopf eine Impulslast in Scheibenebene aufgebracht und das Schwingungsverhalten mit einem Weggeber und einem Beschleunigungsaufnehmer gemessen. Die Versuche erfolgten ohne vertikale Auflast und ohne sonstige Festhaltungen an der eingespannten Kragscheibe. Als mitschwingende Massen agierten hierbei nur der Stahlbetonkopfbalken sowie das Wandeigengewicht $\left(\gamma=10 \mathrm{kN} / \mathrm{m}^{3}\right)$. Aus dem logarithmischen Dekrement $\Lambda$ der Abklingkurve wurde ein mittlerer Dämpfungsgrad $\zeta=3 \%$ bestimmt. Nachfolgend ist in Abbildung 35 beispielhaft ein Ausschnitt aus dem Verlauf der Wandkopfbeschleunigung in einem Ausschwingversuch an Wand B dargestellt.

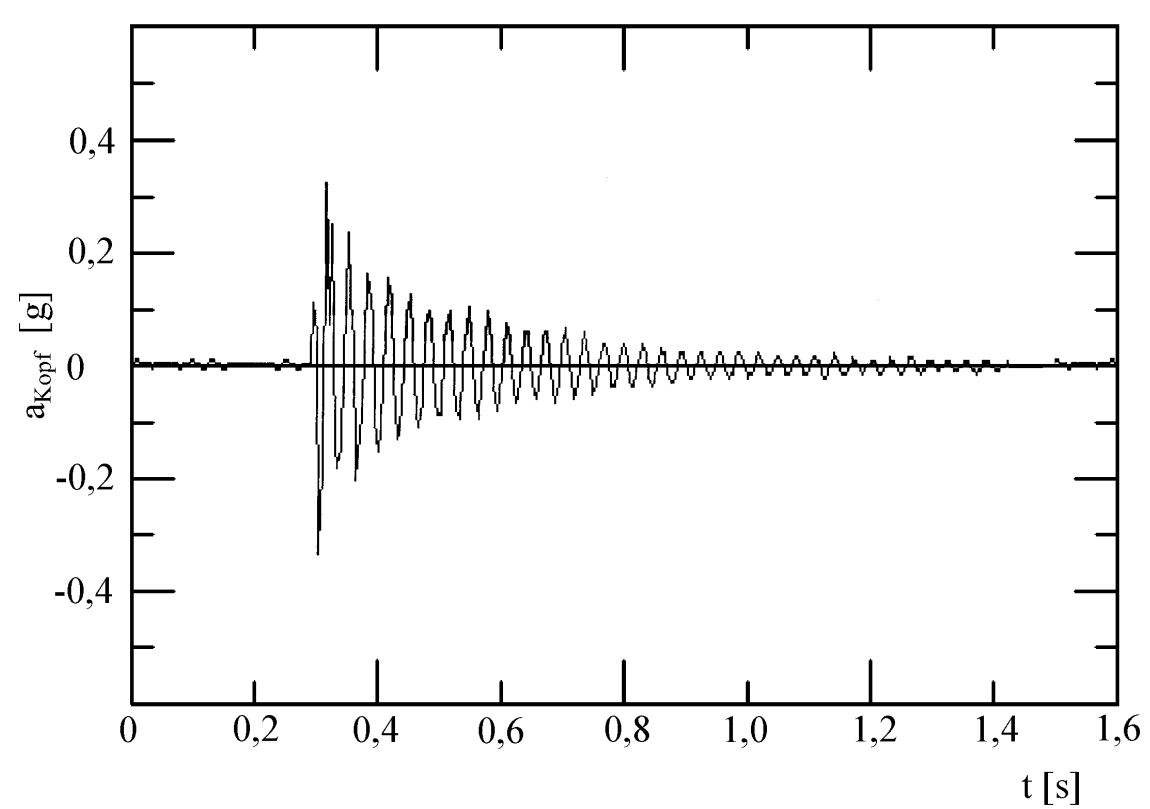

Abbildung 35: Zeitlicher Verlauf der Wandkopfbeschleunigung eines Ausschwingversuches an Wand B (Ausschnitt)

Mit Hilfe der Fast-Fourier-Transformation der Messwerte wurde die erste Eigenfrequenz bestimmt. In Abbildung 36 ist das Amplitudenspektrum eines Ausschwingversuches an Wand B dargestellt. 


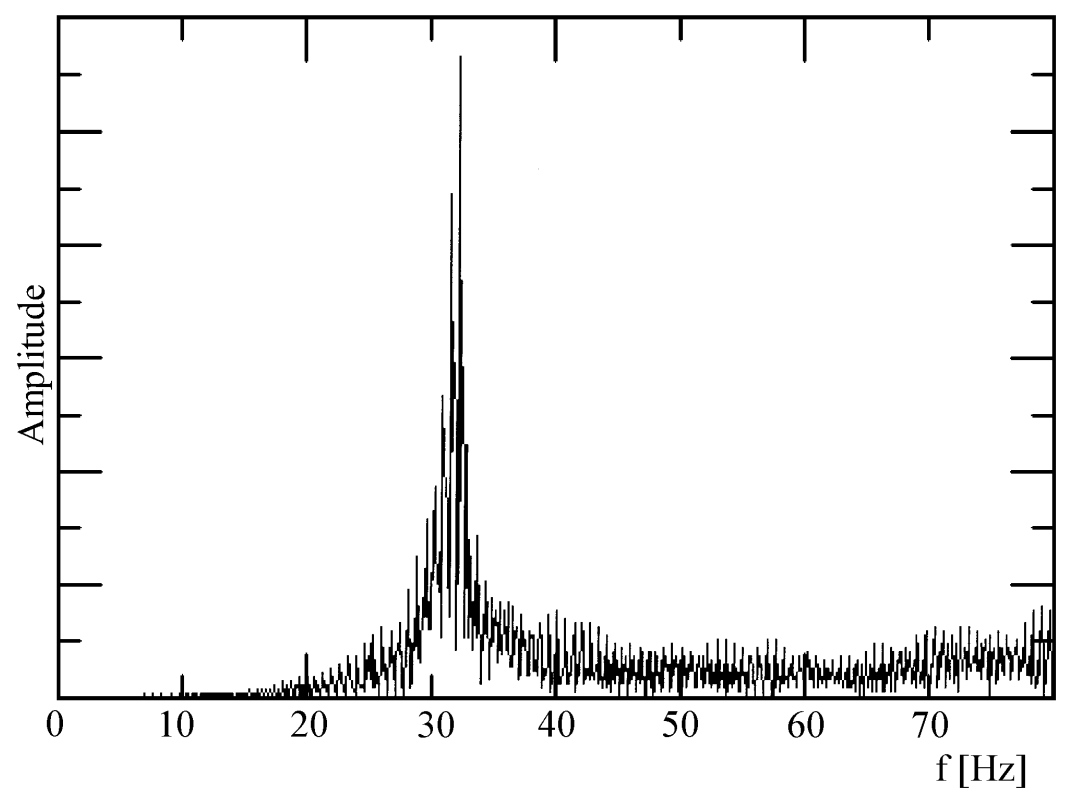

Abbildung 36: Ermittlung der Wandeigenfrequenz mit Hilfe der Fast-Fourier-Transformation der Wandkopfbeschleunigung eines Ausschwingversuches an Wand B

Mit Hilfe einer FE-Vergleichsrechnung an einer linear-elastischen Scheibe [135] wurde eine ideelle Wandsteifigkeit ermittelt. Die Rückrechnung auf den zugehörigen E-Modul erfolgte mit der bekannten Massenverteilung und der gemessenen ersten Eigenfrequenz. Die daraus bestimmten Wandsteifigkeiten im Bereich von $k=19,6 \div 36,2 \mathrm{kN} / \mathrm{mm}$ (s. Tabelle 26) lagen deutlich unter den erwarteten und nachfolgend im Zuge der statisch-zyklischen Vorversuche bestimmten Werten von $k=95 \div 120 \mathrm{kN} / \mathrm{mm}$ (s. Tabelle 27).

Als Ursachen dieser Abweichungen kommen im Wesentlichen zwei Punkte in Betracht. Zum einen tritt infolge fehlender Auflast ein Klaffen des Wandquerschnittes auf, was zu einer deutlichen Steifigkeitsreduktion führt. Zum anderen sind die maximalen Verschiebungsamplituden mit $\pm 0,11 \mathrm{~mm}$ relativ gering, was beim Vorhandensein von lokalen Schlupfanteilen im Kontaktbereich zwischen Stein und Mörtel zu deutlich geringeren resultierenden Sekantensteifigkeiten führt, als bei größeren Auslenkungen, bei denen derartige Schlupfanteile vernachlässigbar sind.

\begin{tabular}{|c|c|c|c|}
\hline \multirow[t]{2}{*}{ Wand } & \multirow{2}{*}{$\begin{array}{c}\text { Eigenfrequenz } \\
\text { aus Versuch } \\
f[\mathrm{~Hz}]\end{array}$} & \multicolumn{2}{|c|}{ Rückrechnung als linear-elastische Scheibe } \\
\hline & & $\begin{array}{l}\text { ideeller isotroper } \\
\text { E-Modul }\left[\mathbf{N} / \mathbf{m m}^{2}\right]\end{array}$ & $\begin{array}{c}\text { zugeh. Wandsteifigkeit } \\
k=H / w_{K o p f}[\mathrm{kN} / \mathrm{mm}]\end{array}$ \\
\hline $\mathbf{A}$ & 32,2 & 1320 & 27,4 \\
\hline B & 31,7 & 1265 & 26,3 \\
\hline $\mathbf{C}$ & 37,1 & 1740 & 36,2 \\
\hline D & 27,3 & 940 & 19,6 \\
\hline
\end{tabular}

Tabelle 26: Ergebnisse der Ausschwingversuche von Wand A $\div$ D und Steifigkeitsparameter aus einer linear-elastischen Nachrechnung als isotrope Kragscheibe mit Hilfe der FEM

\section{Vorversuche - statisch-zyklische Versuche}

Zur Bestimmung der Anfangssteifigkeit wurden an Wand $\mathrm{A} \div \mathrm{C}$ statisch-zyklische Vorversuche mit einer Normalkraft von $N=270 \mathrm{kN}$ durchgeführt. Die Grenzen der maximalen Horizontalkraft wurden zu $H_{\max }= \pm 30 \mathrm{kN}$ festgelegt. Entsprechend der Ermittlung als Tangenten- 
oder Sekantensteifigkeit ergab sich für die Wände $\mathrm{A} \div \mathrm{C}$ einheitlich als Anfangssteifigkeit ein Wertebereich von $k=95 \div 120 \mathrm{kN} / \mathrm{mm}$ (s. Tabelle 27).

\subsubsection{Gebäudemodell - Ersatzsystem}

Für die erste Simulationsstufe musste das im Aufriss als Mehrmassenschwinger wirkende System auf einen äquivalenten Einmassenschwinger reduziert werden. Als Randbedingung galt, dass die Eigenperiode des Einmassenschwinger-Ersatzsystems gleich der ersten Eigenperiode des ursprünglichen Gebäudemodells entspricht.

Als Untersuchungsobjekte wurden in der ersten Simulationsstufe ein 3-geschossiges Reihenmittelhaus (Wand A und C) und ein Wohngebäude (Wand B) mit 4 oberirdischen Stockwerken gewählt (s. Abbildung 37). Ein optionales steifes Kellergeschoss wurde bei beiden Systemen nicht berücksichtigt, da in diesem Fall der Erregungshorizont direkt auf Höhe der darüber liegenden Geschossdecke angesetzt werden kann (Korkut [62]).

Geschosswohngebäude

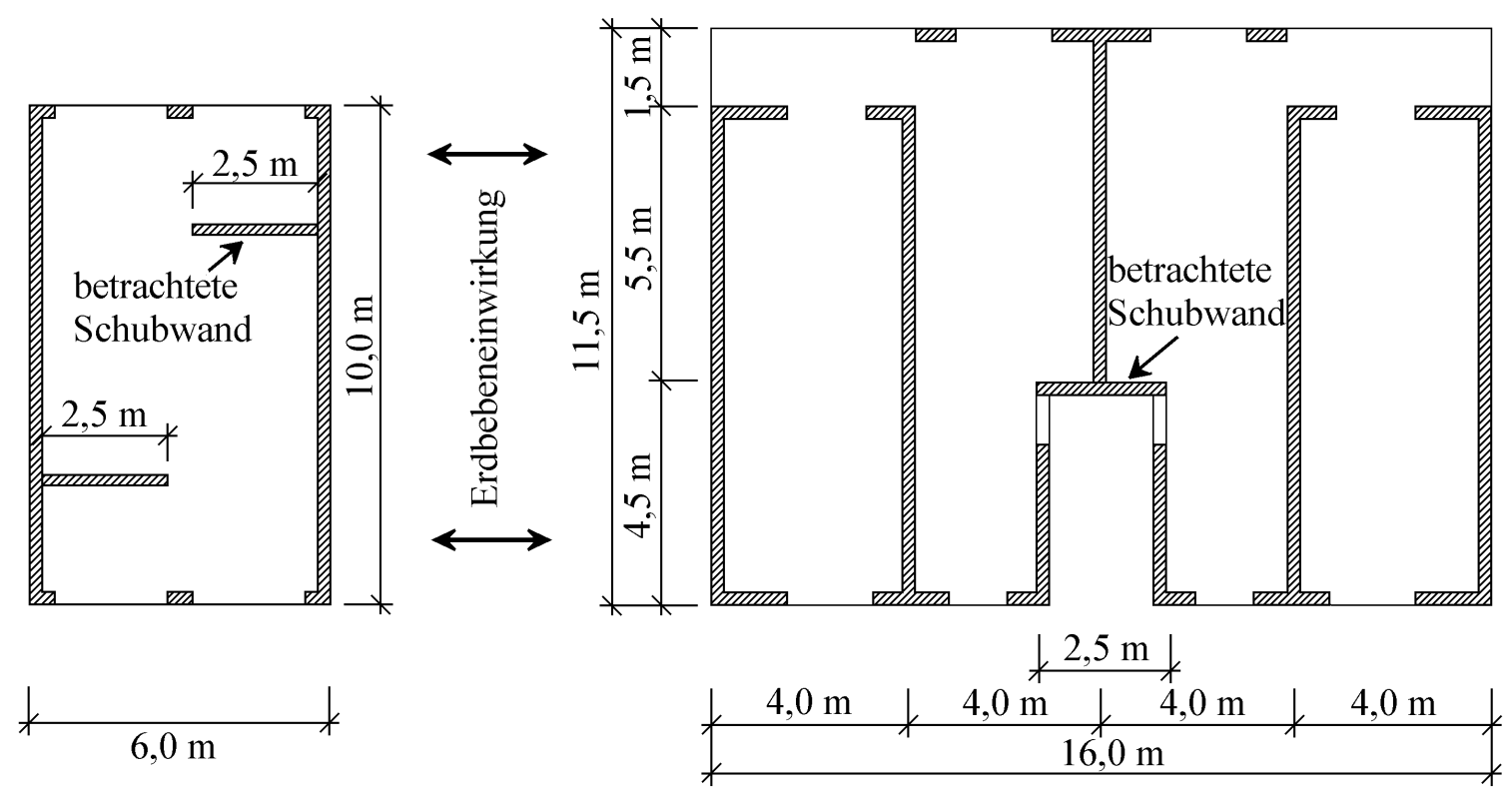

Abbildung 37: Grundriss der untersuchten Gebäudetypen Reihenmittelhaus und Geschosswohngebäude

Für die Bestimmung der Eigenschwingzeiten und der Horizontallastaufteilung auf die Schubwände wurden die beiden Systeme linear-elastisch als räumliche Faltwerke berechnet [135] die Berücksichtigung gerissener Querschnitte erfolgte nicht. Für die Wände $(d=17,5 \mathrm{~cm})$ wurde ein E-Modul von $6.000 \mathrm{~N} / \mathrm{mm}^{2}$ und für die Stahlbetongeschossdecken $(d=18 \mathrm{~cm})$ einer von $30.000 \mathrm{~N} / \mathrm{mm}^{2}$ angesetzt. Die Lastverteilung ergab für die untersuchte Schubwand im Reihenmittelhaus $(R M H)$ 45\% und für die im Geschosswohngebäude $(G W G)$ 24\% der Gesamthorizontallast. Für die Ermittlung der Eigenschwingzeiten wurde neben den ständigen Lasten ein Verkehrslastanteil von $q=0,5 \mathrm{kN} / \mathrm{m}^{2}$ als quasi-ständig vorhanden und mitschwingend berücksichtigt. Pro Geschoss betrug die mitschwingende Masse $m=64 \mathrm{t}$ (RMH) bzw. $195 \mathrm{t}$ (GWG). Die erste Eigenperiode ermittelte sich für das Reihenmittelhaus zu $T_{1, R M H}=0,24 \mathrm{~s}$ und für das Geschossgebäude $\mathrm{zu} T_{1, G W G}=0,4 \mathrm{~s}$.

Da Torsionseffekte und die Wirkung als zusammengesetzter Querschnitt vernachlässigt wurden, konnten die Versuche unter Ausnutzung der Symmetrie auf die betrachtete Schubwand 
reduziert werden (analog zu Abbildung 44 links). Als zentrische Wandnormalkraft wurde für beide Grundrisse die unter der quasi-ständigen Lastkombination vorhandene Vertikallast zu $N=270 \mathrm{kN}$ ermittelt.

Am äquivalenten Ersatzsystem (Einmassenschwinger mit der Anfangssteifigkeit nach Tabelle 27) bestimmten sich aus der Festlegung der Eigenperiode $\left(T_{1, R M H}=0,24 \mathrm{~s}\right.$ bzw. $T_{1, G W G}=0,4 \mathrm{~s}$ ) die Kopfmassen abweichend $\mathrm{zu}$ den tatsächlichen Gebäudemassen zu $150 \mathrm{t}$ (Wandversuche A1 \& A2), $180 \mathrm{t}$ (Wandversuche $\mathrm{A} 3 \div \mathrm{A} 8, \mathrm{C} 1 \div \mathrm{C} 3$ ) bzw. $385 \mathrm{t}$ (Wandversuch B).

\subsubsection{Ablauf}

Das Ablaufschema für die Versuche der ersten Simulationsstufe ist in Abbildung 38 dargestellt. Die sich daraus ergebenden Aufgaben können folgendermaßen zusammengefasst werden:

- Durchführung des Algorithmusses der Pseudodynamik

- Messen und Protokollieren der Daten (Messwerte inkl. abgeleiteter Größen)

- Steuern des Horizontalzylinders über den digitalen Servo-Controller

- Visualisierung maßgebender Zustandgrößen (Zeitschritt, Kraft-Verformungslinie, aktuelle Zustandgrößen)

Im Zuge der ersten Simulationsstufe wurden diese Aufgaben in der Skriptsprache des Systems CATMAN [13] programmiert und auf einem einzelnen Computer ausgeführt. Das Einlesen der Messwerte erfolgte über einen an der Parallel-Schnittstelle des Rechners angeschlossenen Messverstärker mit eingebauten Analog-Digital-Wandlern. Für die Steuerung des horizontalen Hydraulikzylinders über einen digitalen Servo-Controller wurde eine interne Signalausgangskarte genutzt.

\section{Visualisierung}

Für die Visualisierung der Ergebnisse wurden neben den protokollierten Rissaufnahmen zusätzlich die Versuche über die ganze Dauer mit einer Kamera aufgenommen. Durch die Anzeige der Erdbebenzeit konnten nach Abschluss der einzelnen Versuche die den 900 Zeitschritten zugehörigen Einzelbilder bestimmt werden und somit die Risse den einzelnen Zeitschritten zugeordnet werden. Für die Darstellung des Verhaltens der geprüften Wand in Echtzeit wurden die Einzelbilder anschließend zu einem Film zusammengesetzt. 


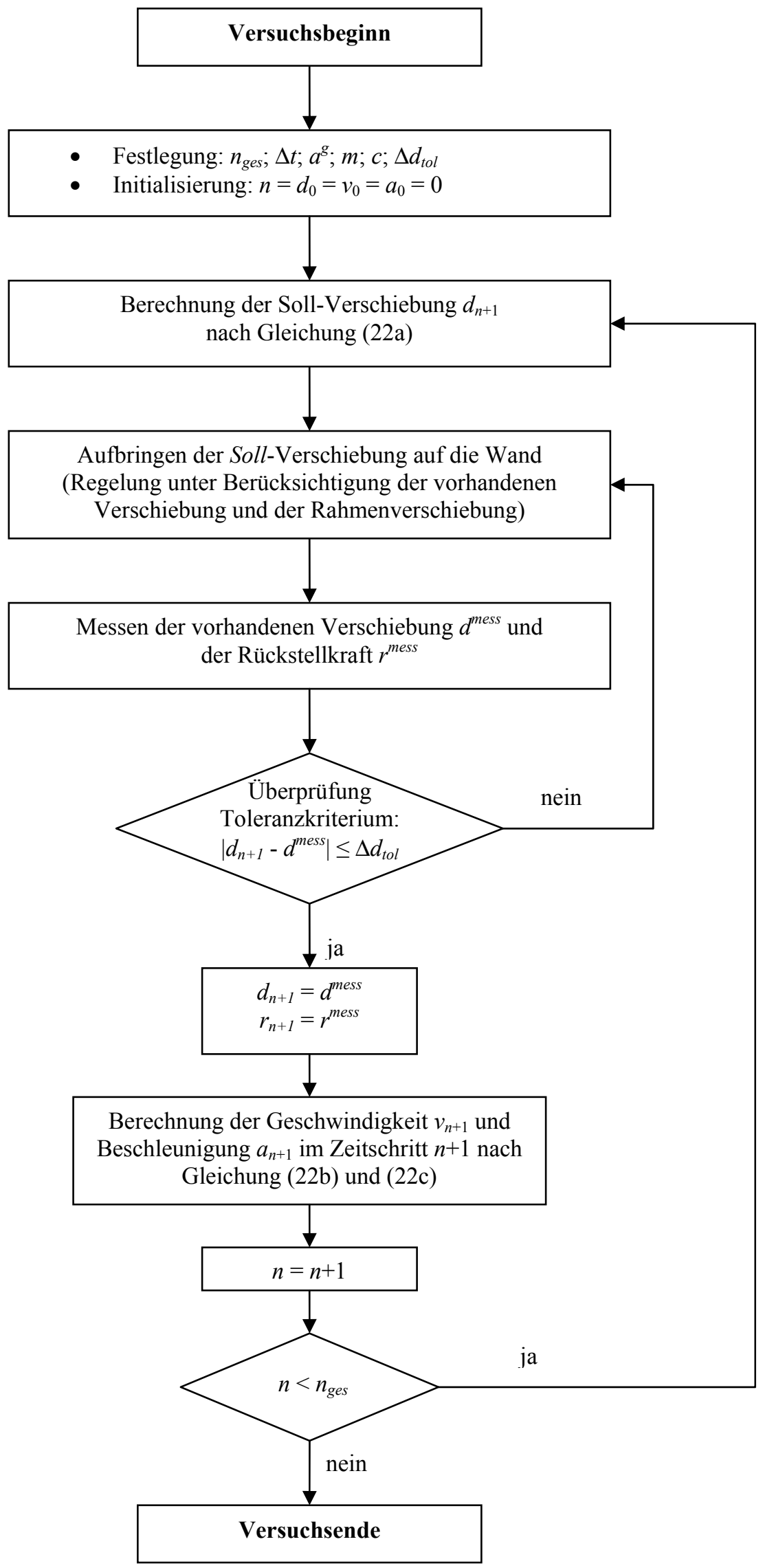

Abbildung 38: Ablaufschema für die erste Simulationsstufe 


\subsubsection{Ergebnisse}

Nachfolgend sind in Tabelle 27 die Parameter der Versuche und die Ergebnisse in Form maximaler Horizontalkräfte und Kopfverschiebungen angegeben. Die Kopfmasse $m$ und die Dämpferkonstante $c$ wurden so festgelegt, dass die erste Eigenperiode $T_{1}$ des resultierenden Einmassenschwingers mit der aus der Berechnung als Faltwerk (Abschnitt 5.4.2) übereinstimmt und der Dämpfungsgrad $\zeta=5 \%$ beträgt (s. Tabelle 29 ).

Die Kraft-Verformungs-Beziehungen sowie die abschließenden Rissbilder sind im Anhang A.2.1 und A.3.1 zusammengestellt.

\begin{tabular}{|c|c|c|c|c|c|}
\hline $\begin{array}{l}\text { Wand / } \\
\text { Versuch }\end{array}$ & $\begin{array}{l}\text { Kopfmasse } \\
\quad m[\mathrm{t}] \\
\text { Dämpfer } \\
c[\mathrm{kNs} / \mathrm{m}] \\
\end{array}$ & $\begin{array}{c}\text { BB-ZV }{ }^{1)} \\
\text { Lastfaktor } \\
f[-]\end{array}$ & $\begin{array}{l}\text { maximale } \\
\text { Kopfverschie- } \\
\text { bung } \\
\text { w }_{\text {Kopf }}[\mathrm{mm}] \\
\end{array}$ & $\begin{array}{c}\text { maximale } \\
\text { Horizontal- } \\
\text { kraft } \\
H[\mathrm{kN}] \\
\end{array}$ & $\begin{array}{c}\text { rechnerische } \\
\text { Anfangssteifig- } \\
\left.\text { keit }^{2}\right) \\
k[\mathrm{kN} / \mathrm{mm}] \\
\end{array}$ \\
\hline$\overline{\mathrm{A1}}$ & \multirow[t]{2}{*}{$150 ; 377$} & $\begin{array}{c}\text { ZV1-B3 }\left(\mathrm{a}_{\mathrm{g}}=0,4 \mathrm{~m} / \mathrm{s}^{2}\right) \\
\mathrm{f}=0,25\end{array}$ & 0,60 & 43,1 & \multirow[t]{2}{*}{95} \\
\hline $\mathbf{A 2}$ & & $\mathrm{f}=0,5$ & 1,13 & 69,7 & \\
\hline $\mathbf{A 3}$ & \multirow[t]{6}{*}{$180 ; 467$} & $\mathrm{f}=0,46$ & 1,14 & 73,2 & \multirow[t]{6}{*}{120} \\
\hline $\mathbf{A 4}$ & & $\mathrm{f}=0,8$ & 2,25 & 102,3 & \\
\hline A5 & & $\mathrm{f}=0,8$ & 2,25 & 103,6 & \\
\hline A6 & & $\mathrm{f}=1,07$ & 3,64 & 123,8 & \\
\hline A7 & & $\mathrm{f}=1,5$ & 6,33 & 147,8 & \\
\hline $\mathbf{A 8}$ & & $\mathrm{f}=2,16$ & 9,06 & 153,8 & \\
\hline $\mathrm{B}^{3)}$ & $385 ; 605$ & $\begin{array}{c}\text { ZV1-B3 }\left(\mathrm{a}_{\mathrm{g}}=0,4 \mathrm{~m} / \mathrm{s}^{2}\right) \\
\mathrm{f}=4\end{array}$ & $(20)^{3)}$ & $(150)^{3)}$ & 95 \\
\hline$C 1^{4)}$ & \multirow[t]{3}{*}{$180 ; 467$} & $\begin{array}{c}\mathrm{ZV} 2-\mathrm{A} 3\left(\mathrm{a}_{\mathrm{g}}=0,6 \mathrm{~m} / \mathrm{s}^{2}\right) \\
\mathrm{f}=0,27\end{array}$ & 1,58 & 55,6 & \multirow[t]{3}{*}{120} \\
\hline $\mathbf{C 2}$ & & $\mathrm{f}=0,54$ & 2,47 & 92,8 & \\
\hline $\mathbf{C 3}$ & & $\mathrm{f}=0,81$ & 4,59 & 116,6 & \\
\hline \multicolumn{6}{|c|}{$\begin{array}{l}\text { 1) Bodenbeschleunigungszeitverlauf, Boden-Baugrund-Kombination; Grundwert d } \\
\text { 2) Ermittlung im Zuge von statisch-zyklischen Vorversuchen (s. Abschnitt 5.4.1) } \\
\text { 3) Maximallast bei Zeitschritt } 220 \text { / Versuchsabbruch bei Zeitschritt } 707 \text { von } 900 \\
\text { 4) nicht ausreichende Verschiebesicherung der Fundamentplatte } \Rightarrow \text { aufgetretene G }\end{array}$} \\
\hline
\end{tabular}

Tabelle 27: Parameter und Ergebnisse der ersten Simulationsstufe

Die drei Wände $\mathrm{A} \div \mathrm{C}$ zeigten infolge gleichen Materials und Versuchsaufbaus ein ähnliches Last-Verformungs-Verhalten (Abbildung A-23 $\div$ A-33). Bei geringem Beanspruchungsniveau (Wandversuche $\mathrm{A} 1 \div \mathrm{A} 3, \mathrm{C} 1$ ) war die Kraft-Verformungs-Beziehung im Wesentlichen linear-elastisch bei relativ geringer Völligkeit. Mit zunehmender Belastung trat aufgrund der konstant gehaltenen und am Wandkopf mittig zentrierten Normalkraft $N$ ein Klaffen des Querschnittes am Wandfuß auf. Bei Vernachlässigung einer Haftzugfestigkeit und Abschätzung des Wandeigengewichtes mit $\Delta N=14 \mathrm{kN}$ bestimmt sich als Erstrisslast $H_{1}=45,5 \mathrm{kN}$. Nach deren Überschreitung erfolgte anschließend ein Abflachen und Aufweiten der Hysterese im oberen Lastniveau (s. Wandversuche A $4 \div \mathrm{A} 8, \mathrm{C} 2 \div \mathrm{C} 3$ ). Als rechnerische Grenzlast des Querschnittes ohne Zugfestigkeit senkrecht zu den Lagerfugen bestimmt sich $H_{2}=136,5 \mathrm{kN}$, bei der die Resultierende am Wandfuß auf dem Querschnittsrand zum liegen kommt.

Die tatsächlich in den Versuchen aufgetretenen höheren Lasten von maximal $H_{\max }=153,8 \mathrm{kN}$ (Wandversuch A8) sind auf die besonderen Randbedingungen im Versuchsstand zurückzuführen. Bei hoher horizontaler Druckbeanspruchung erfolgte infolge des Querschnittsklaffens ein Abheben der Wand auf Seite des Hydraulikzylinders. Da die Kalotte lediglich punktuelle Verdrehungen zwängungsfrei aufnehmen kann, wurde durch die vertikale Verschiebung nach 
oben eine zusätzliche Normaldruckkraft am Wandkopf aktiviert, die über ihre Lage am vorderen Wandende (in Abbildung 34 rechts) rückdrehend wirkte. Bei hohen Zugkräften im Horizontalzylinder erfolgte entsprechend am gegenüberliegenden Wandende (in Abbildung 34 links) eine Verschiebung nach oben. Wurde dabei der freie Pressenweg der Vertikalpressen überschritten, schlugen die Zylinder an den Querriegel und eine zusätzliche, exzentrische, rückdrehende Normaldruckkraft am Wandkopf wurde aktiviert. Durch diese Effekte bei hohen Horizontallasten - wobei der Betrag der zusätzlichen Normalkräfte nicht bestimmt werden konnte - wurden der Kräfte- und Spannungszustand in der Wand wesentlich verändert und es resultierten höhere aufnehmbare horizontale Beanspruchungen als die rechnerische Grenzlast $H_{2}$.

\section{Versagensbilder}

Die Versagensbilder der drei Wände (s. Anhang A.2.1, Abbildung A-9 $\div$ A-12) stellten sich entsprechend der Beanspruchungssituation ähnlich dar. Nach dem Klaffen des Querschnittes in der zweiten Lagerfuge von unten (Wand A: Riss 1 bzw. 2 und 3; Wand B: Riss 1; bei Wand C nicht dokumentiert) kam es bei höheren Horizontalkräften von ca. $115 \mathrm{kN}$ am stärker gedrückten Querschnittsrand zu Schub- und Druckspannungsspitzen, die bei Überschreitung der Steinfestigkeit zu schrägen Rissen führten. Diese waren in der Regel in den beiden äußeren Steinen anzutreffen. Die Gesamttragfähigkeit der Wand wurde durch dieses lokale Bruchbild infolge möglicher Lastumlagerung auf weiter innen liegende Nachbarsteine nicht signifikant reduziert.

Bei weiter gesteigerter Belastung erfolgte bei den Wänden A und B ein Fugenversagen in der zweiten Lagerfuge von unten (Wand A: Riss 12, Wand B: Risse 5 und 15). Dabei wurden durch die in die Löcher eingedrungenen Mörteldollen die Innenstege abgeschert. Infolge der reduzierten Schub- und Normalkrafttragfähigkeit erfolgte bei zunehmender Horizontalverschiebung ein Herausklappen der Außenstege mit anschließendem Totalversagen der Wand. Bei Wand C erfolgte vorzeitig ein Steinzugversagen (Risse $1 \div 9$ ) ausgehend von der unteren Wandecke. Bei Lastumkehr kam es hier anschließend zu einer Kombination von abgetrepptem Fugenversagen und Steinzugversagen in der untersten Reihe (Risse $27 \div 31$ und $\mathrm{R} 24 \div \mathrm{R} 30)$.

\section{Tragfähigkeit}

Bei allen drei Wänden traten bei einer Belastung von ca. $115 \mathrm{kN}$ zusammen mit einem Klaffen des Wandquerschnittes schräge Risse am stärker gedrückten Querschnittsrand infolge Spannungskonzentrationen auf. Abweichend zu den Wänden A und B, bei denen sich die Risse im Wesentlichen auf die beiden untersten Steinreihen beschränkten, erweiterten sich bei Wandversuch C3 diese Risse in Form eines Diagonalrisses über die ganze Wandhöhe.

Die Nachrechnung der maximalen Bruchlasten (Versuch A8: $154 \mathrm{kN}$, Versuch B: $150 \mathrm{kN}$ ) ist aufgrund der nicht bekannten zusätzlichen Normalkraft infolge großer Wandkopfverdrehungen nicht möglich (s. o.). Es wird daher nachfolgend die maximale Last der drei Wände beim Auftreten der ersten schrägen Steinrisse in der unteren Wandecke von ca. $115 \mathrm{kN}$ (Wand A8: 112,8 kN; Wand C: -116,6 kN) mit dem Schubmodell von Mann / Müller (s. Abschnitte 3.1, 5.5.7 und 6.2.1) abgeschätzt. Es wird davon ausgegangen, dass bei dem betrachteten Lastniveau keine zusätzliche, exzentrische, rückdrehende Normaldruckkraft am Wandkopf aktiviert wurde. Für die Nachrechnungen werden entsprechend den aufgetretenen Versagensbildern die Kriterien Druckversagen und Steinzugversagen betrachtet. 
Am Wandfuß ergibt sich bei einer Horizontallast von $H=115 \mathrm{kN}$ und einem Hebelarm von 2,6 m ein Biegemoment von $299 \mathrm{kNm}$. Mit der Gesamtnormalkraft $N_{F u \beta}=284 \mathrm{kN}$ (aus planmäßiger Last am Wandkopf $N=270 \mathrm{kN}$ und Wandeigengewicht $\Delta N=14 \mathrm{kN}$ ) verbleibt mit der Ausmitte $1,05 \mathrm{~m}$ bei dreiecksförmiger Normalspannungsverteilung eine überdrückte Länge von 0,59 m. Die maximale Schubspannung bestimmt sich mit einem Schubspannungsverteilungsfaktor von $1,25 \mathrm{zu} \tau_{\max }=1,39 \mathrm{~N} / \mathrm{mm}^{2}$ bei einer mittleren Normalspannung von $\sigma=2,74 \mathrm{~N} / \mathrm{mm}^{2}$.

Der Nachweis des Kriteriums Druckversagen nach Gleichung (48) erfolgt mit der Randeckspannung von 5,48 N/mm². Die auf Basis der empirischen Beziehung (14) abgeschätzte Mauerwerksdruckfestigkeit von $f_{m y}=5,3 \div 6,0 \mathrm{~N} / \mathrm{mm}^{2}$ (Tabelle 20) liegt aufgrund der Querdehnungsbehinderung der Steine der untersten Reihe durch die Betonplatte lokal deutlich höher (s. Abschnitt 5.5.7). Das Kriterium Druckversagen ist somit für das beobachtete Versagensbild offensichtlich nicht maßgebend.

Für das Kriterium Steinzugversagen bestimmt sich aus Gleichung (47) eine aufnehmbare Schubspannung von $\tau=1,29 \mathrm{~N} / \mathrm{mm}^{2}$, was mit einer relativen Abweichung von $7 \%$ gut mit dem vorhandenen Schubspannungsmaximum übereinstimmt. Es zeigt sich somit, dass mit dem Schubmodell von Mann / Müller und den gegebenen Materialparametern die Erstrissbzw. Bruchlast für das Kriterium Steinzugversagen gut beschrieben werden kann.

\section{Steifigkeit}

Mit zunehmender Beanspruchungshöhe in den Versuchen nahm auch die Steifigkeit der Wände ab. Die Auswertung dazu erfolgte ersatzweise an der 1. Systemeigenperiode, die mit Hilfe der Fast-Fourier-Transformation aus dem zeitlichen Verlauf der Wandkopfverschiebung ermittelt wurde. Hierbei wurde davon ausgegangen, dass die Einwirkung in Form des Bodenbeschleunigungszeitverlaufes im betreffenden Periodenbereich einem weißen Rauschen entspricht.

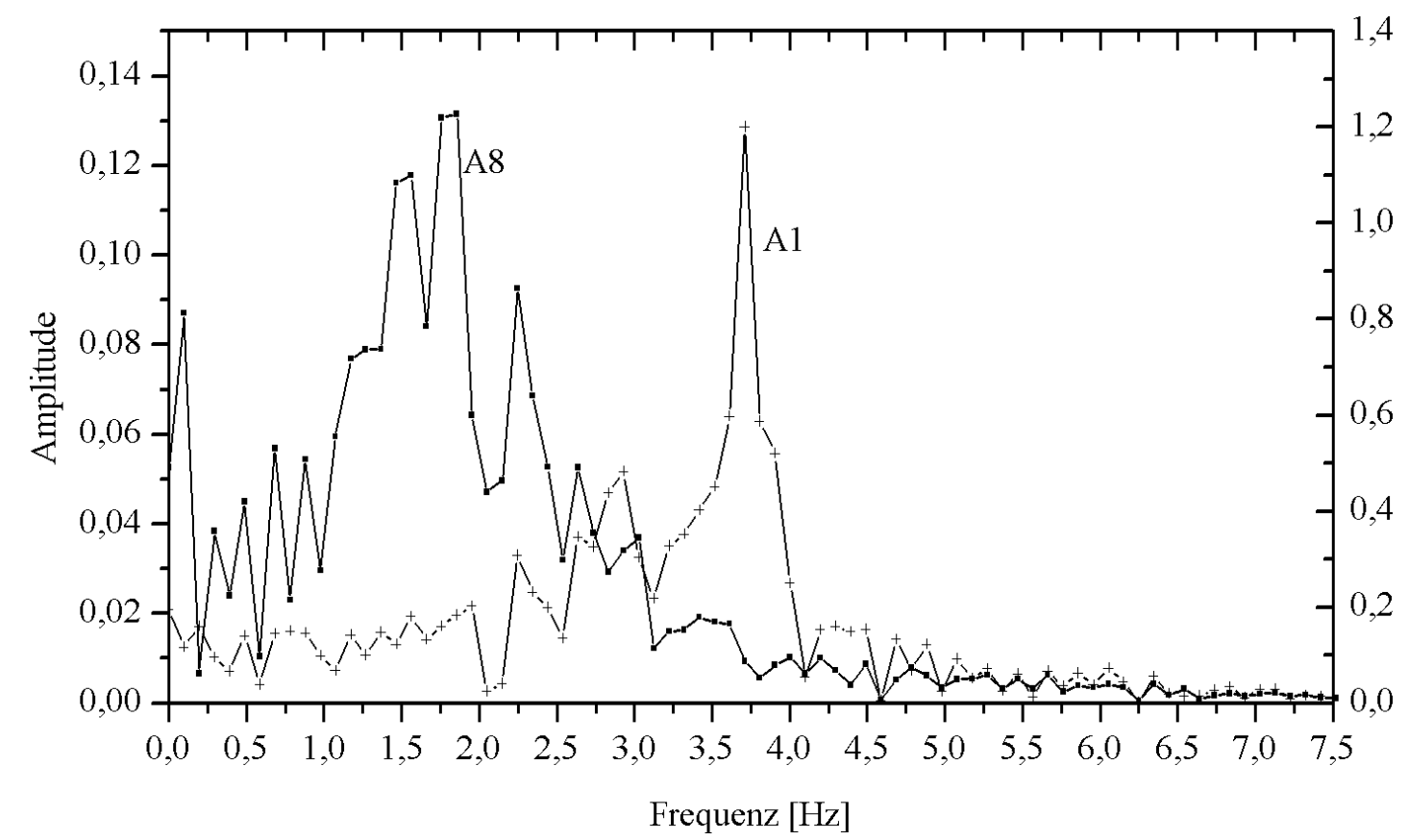

Abbildung 39: FFT des Wandkopfverschiebungsverlaufes der Versuche A1 und A8 
Die Bestimmung der Eigenfrequenz $f_{F F T}$ aus dem Amplitudenspektrum erfolgte durch Schwerpunktbildung der Werte in einem lokal eingegrenzten Frequenzfenster. Dieses wurde individuell auf Basis des Amplitudenverlaufes festgelegt (Tabelle 28).

In Abbildung 39 sind beispielhaft die Ergebnisse der Versuche A1 und A8 dargestellt. Es ist $\mathrm{zu}$ erkennen, dass die Eigenfrequenz in Versuch $\mathrm{A} 1(f=3,6 \mathrm{~Hz})$ mit einem Verhältniswert von 2 deutlich über der in Versuch A8 $(f=1,9 \mathrm{~Hz})$ liegt.

Die Übersicht der Ergebnisse aller Versuche der ersten Stufe ist in Tabelle 28 gegeben. Aus der Eigenperiode $T_{F F T}$ wurde mit der vorgegebenen Kopfmasse $m$ (Tabelle 27) eine rechnerische Wandsteifigkeit $k$ bestimmt. Im Vergleich mit den Anfangssteifigkeiten aus den Vorversuchen (Tabelle 27) mit $k=95 \div 120 \mathrm{kN} / \mathrm{mm}$ liegen die Werte der Versuche, insbesondere bei hohen Lasten, deutlich darunter.

\begin{tabular}{|c|c|c|c|c|}
\hline $\begin{array}{l}\text { Wand / } \\
\text { Versuch }\end{array}$ & $\begin{array}{c}\text { BB-ZV }^{1)} \\
\text { Lastfaktor } f[-]\end{array}$ & $T_{F F T}[\mathbf{s}]$ & $\begin{array}{c}\text { Frequenzbe- } \\
\text { reichsfenster } \\
\quad f[\mathrm{~Hz}]\end{array}$ & $\begin{array}{c}\text { rechnerische } \\
\text { Wandsteifigkeit }{ }^{2)} \\
k[\mathrm{kN} / \mathbf{m m}]\end{array}$ \\
\hline A1 & ZV1-B3 $\left(\mathrm{a}_{\mathrm{g}}=0,4 \mathrm{~m} / \mathrm{s}^{2}\right) ; \mathrm{f}=0,25$ & 0,276 & $3,12 \div 4,1$ & 77,7 \\
\hline A2 & $\mathrm{f}=0,5$ & 0,316 & $2,54 \div 3,9$ & 59,3 \\
\hline $\mathbf{A 3}$ & $\mathrm{f}=0,46$ & 0,339 & $2,14 \div 3,71$ & 61,8 \\
\hline A4 & $\mathrm{f}=0,8$ & 0,352 & $2,05 \div 3,8$ & 57,4 \\
\hline $\mathbf{A 5}$ & $\mathrm{f}=0,8$ & 0,354 & $2,05 \div 3,8$ & 56,7 \\
\hline A6 & $\mathrm{f}=1,07$ & 0,383 & $1,66 \div 3,8$ & 48,4 \\
\hline A7 & $\mathrm{f}=1,5$ & 0,485 & $1,07 \div 3,12$ & 30,2 \\
\hline A8 & $\mathrm{f}=2,16$ & 0,533 & $0,98 \div 3,12$ & 25,0 \\
\hline B $^{3)}$ & ZV1-B3 $\left(\mathrm{a}_{\mathrm{g}}=0,4 \mathrm{~m} / \mathrm{s}^{2}\right) ; \mathrm{f}=4$ & s. & - & - \\
\hline C1 & ZV2-A3 $\left(\mathrm{a}_{\mathrm{g}}=0,6 \mathrm{~m} / \mathrm{s}^{2}\right) ; \mathrm{f}=0,27$ & 0,342 & $2,15 \div 3,80$ & 60,8 \\
\hline $\mathrm{C2}$ & $f=0,54$ & 0,383 & $1,37 \div 3,71$ & 48,4 \\
\hline C3 & $\mathrm{f}=0,81$ & 0,46 & $1,07 \div 3,12$ & 33,6 \\
\hline \multicolumn{5}{|c|}{$\begin{array}{l}\text { 1) Bodenbeschleunigungszeitverlauf, Boden-Baugrund-Kombination; Grundwert der } \\
\text { Bodenbeschleunigung } \\
\text { 2) Ermittlung am Einmassenschwinger mit der Kopfmasse } m \text { aus Tabelle } 27 \text { für } T_{F F T}=T_{E M S} \\
\text { 3) Nur Extremwerte der Kopfverschiebung vorhanden }\end{array}$} \\
\hline
\end{tabular}

Tabelle 28: Eigenperioden und rechnerische Wandsteifigkeiten der Versuche der ersten Stufe

Bei der maximalen Beanspruchung (Wandversuche A8 und C3) fallen die Steifigkeitswerte weiter auf $1 / 2$ bis $1 / 3$ des ursprünglichen Wertes ab. Bezogen auf die rechnerischen Werte aus den Vorversuchen beträgt das Verhältnis 0,21 (Versuch A8, Tabelle 29) und bezogen auf die Ermittlung an einer linear-elastischen Kragscheibe 0,16 (Versuch A8, Tabelle 30).

Dieser Effekt ist im Wesentlichen auf das Klaffen der Fuge am Wandfuß zurückzuführen.

Für die Beurteilung der Ergebnisse wird zusätzlich in Abbildung 40 das Verhältnis der Eigenperioden der Versuche $T_{F F T}$ zu denen der Nachrechnung als linear-elastischer Einmassenschwinger (EMS) $T_{E M S}$ und Kragscheibe $T_{\text {Scheibe }}$ (s. Abschnitt 5.4.5) dargestellt. Als Bezugsgröße wird der Verhältniswert von maximal im Versuch erreichter Horizontallast zu maximaler Horizontallast aller Hauptversuche der betreffenden Wand gewählt. 


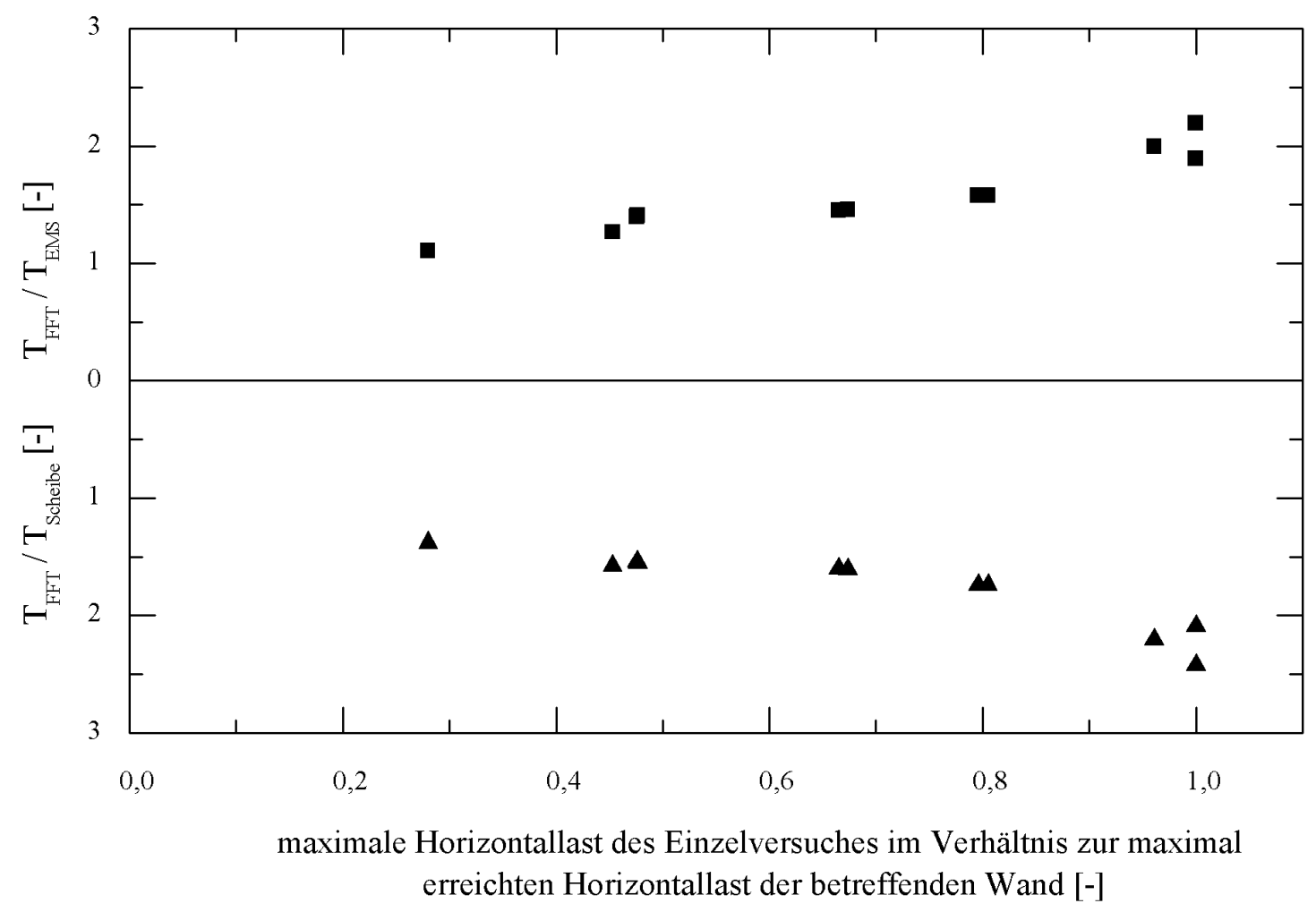

Abbildung 40: Verhältnis der Eigenperioden der Versuche zu denen der Nachrechnung als linearelastischer Einmassenschwinger $\left(T_{F F T} / T_{E M S}\right)$ und Kragscheibe $\left(T_{F F T} / T_{S c h e i b e}\right)$ in Abhängigkeit des Verhältnisses von maximal im Versuch erreichter Horizontallast zu maximaler Horizontallast aller Versuche der betreffenden Wand

Es ist deutlich zu erkennen, dass mit steigendem Belastungsniveau das Verhältnis der Eigenperioden der Versuche zu denen der linear-elastischen Nachrechnung zunimmt. Im Bereich der maximalen Horizontallasten beträgt der Wert bei Wandversuch A8 2,19 (EMS) bzw. 2,42 (Scheibe) und bei Wandversuch C3 1,89 (EMS) bzw. 2,09 (Scheibe).

Bei geringem Beanspruchungsniveau (Versuch A1) liegt der Verhältniswert für die Nachrechnung als linear-elastischer Einmassenschwinger bei 1,10, was die relativ gute Näherung der Wandsteifigkeit mit der Anfangssteifigkeit $k=95 \mathrm{kN} / \mathrm{mm}$ (Abschnitt 5.4.1 und Tabelle 27) bestätigt. Die Scheibenberechnung liegt mit einem Verhältniswert von 1,38 deutlich darüber und beschreibt die Wandsteifigkeit somit deutlich zu hoch.

\subsubsection{Vergleichsrechnungen}

\section{Vergleichsrechnung als linear-elastischer Einmassenschwinger (EMS)}

Zur Bewertung der Ergebnisse wurden die Versuche vergleichend als linear-elastischer Einmassenschwinger mit der in den statisch-zyklischen Vorversuchen ermittelten Anfangssteifigkeit aus Tabelle 27 im Zeitbereich berechnet. In Tabelle 29 sind die Ergebnisse in Form maximaler Horizontalkräfte und Kopfverschiebungen zusammengefasst.

Die Verhältnisse von rechnerischen und tatsächlich aufgetretenen maximalen Kraft- und Verschiebungswerten sind in Abbildung 41 in Abhängigkeit des Verhältniswertes von maximal im Versuch erreichter Horizontallast zu maximaler Horizontallast aller Hauptversuche der betreffenden Wand dargestellt. 


\begin{tabular}{|c|c|c|c|c|c|}
\hline $\begin{array}{l}\text { Wand / } \\
\text { Versuch }\end{array}$ & $\begin{array}{c}\text { rechnerische } \\
\text { Anfangssteifigkeit } \\
k[\mathrm{kN} / \mathrm{mm}]\end{array}$ & $\begin{array}{l}T[\mathbf{s}] \\
\zeta[\%]\end{array}$ & $\begin{array}{c}\text { BB-ZV }^{1)} \\
\text { Lastfaktor } f[-]\end{array}$ & $\begin{array}{c}\text { maximale } \\
\text { Kopfver- } \\
\text { schiebung }{ }^{2)} \\
w_{\text {Kopf, cal }}[\mathrm{mm}] \\
\end{array}$ & $\begin{array}{c}\text { maximale } \\
\text { Horizontal- } \\
\text { kraft }^{2)} \\
H_{c a l}[\mathrm{kN}] \\
\end{array}$ \\
\hline A1 & \multirow[t]{2}{*}{95} & \multirow[t]{2}{*}{$\begin{array}{c}0,25 \\
5,0\end{array}$} & $\begin{array}{c}\mathrm{ZV} 1-\mathrm{B} 3\left(\mathrm{a}_{\mathrm{g}}=0,4 \mathrm{~m} / \mathrm{s}^{2}\right) \\
\mathrm{f}=0,25\end{array}$ & $0,48(0,60)$ & $45,2(43,1)$ \\
\hline A2 & & & $\mathrm{f}=0,5$ & $0,95(1,13)$ & $90,4(69,7)$ \\
\hline A3 & \multirow[t]{6}{*}{120} & \multirow{6}{*}{$\begin{array}{c}0,243 \\
5,0\end{array}$} & $\mathrm{f}=0,46$ & $0,80(1,14)$ & $95,4(73,2)$ \\
\hline A4 & & & $f=0,8$ & $1,38(2,25)$ & $166,0(102,3)$ \\
\hline A5 & & & $\mathrm{f}=0,8$ & $1,38(2,25)$ & $166,0(103,6)$ \\
\hline A6 & & & $\mathrm{f}=1,07$ & $1,85(3,64)$ & $222,0(123,8)$ \\
\hline A7 & & & $\mathrm{f}=1,5$ & $2,59(6,33)$ & $311,2(147,8)$ \\
\hline A8 & & & $\mathrm{f}=2,16$ & $3,73(9,06)$ & $448,2(153,8)$ \\
\hline $\mathrm{B}^{3)}$ & 95 & $\begin{array}{l}0,4 \\
5,0 \\
\end{array}$ & $\begin{array}{c}\text { ZV1-B3 }\left(\mathrm{a}_{\mathrm{g}}=0,4 \mathrm{~m} / \mathrm{s}^{2}\right) \\
\mathrm{f}=4\end{array}$ & $11,88(20)^{3)}$ & $1129(150)^{3)}$ \\
\hline $\mathrm{C1}$ & 120 & $\begin{array}{c}0,243 \\
5,0\end{array}$ & $\begin{array}{c}\text { ZV2-A3 }\left(\mathrm{a}_{\mathrm{g}}=0,6 \mathrm{~m} / \mathrm{s}^{2}\right) \\
\mathrm{f}=0,27\end{array}$ & $0,81(1,58)$ & $104,4(55,6)$ \\
\hline $\mathrm{C2}$ & & & $\mathrm{f}=0,54$ & $1,74(2,47)$ & $208,7(92,8)$ \\
\hline $\mathbf{C 3}$ & & & $\mathrm{f}=0,81$ & $2,61(4,59)$ & $313,1(116,6)$ \\
\hline \multicolumn{6}{|c|}{$\begin{array}{l}\text { Kopfmasse } m \text { und Dämpferkonstante } c \text { : s. Tabelle } 27 \\
\text { 1) Bodenbeschleunigungszeitverlauf, Boden-Baugrund-Kombination; Grundwert der Bodenbeschleunigung } \\
\text { 2) Werte in Klammern: aus zugehörigem Versuch } \\
\text { 3) Versuche nicht vollständig durchgeführt } \Rightarrow \text { Berechnungsergebnisse bis zum betreffenden Zeitpunkt } \\
\text { Maximallast im Versuch bei Zeitschritt } 220 \text { / Versuchsabbruch bei Zeitschritt } 707 \text { von } 900\end{array}$} \\
\hline
\end{tabular}

Tabelle 29: Parameter und Ergebnisse der Vergleichsrechnung als linear-elastischer Einmassenschwinger

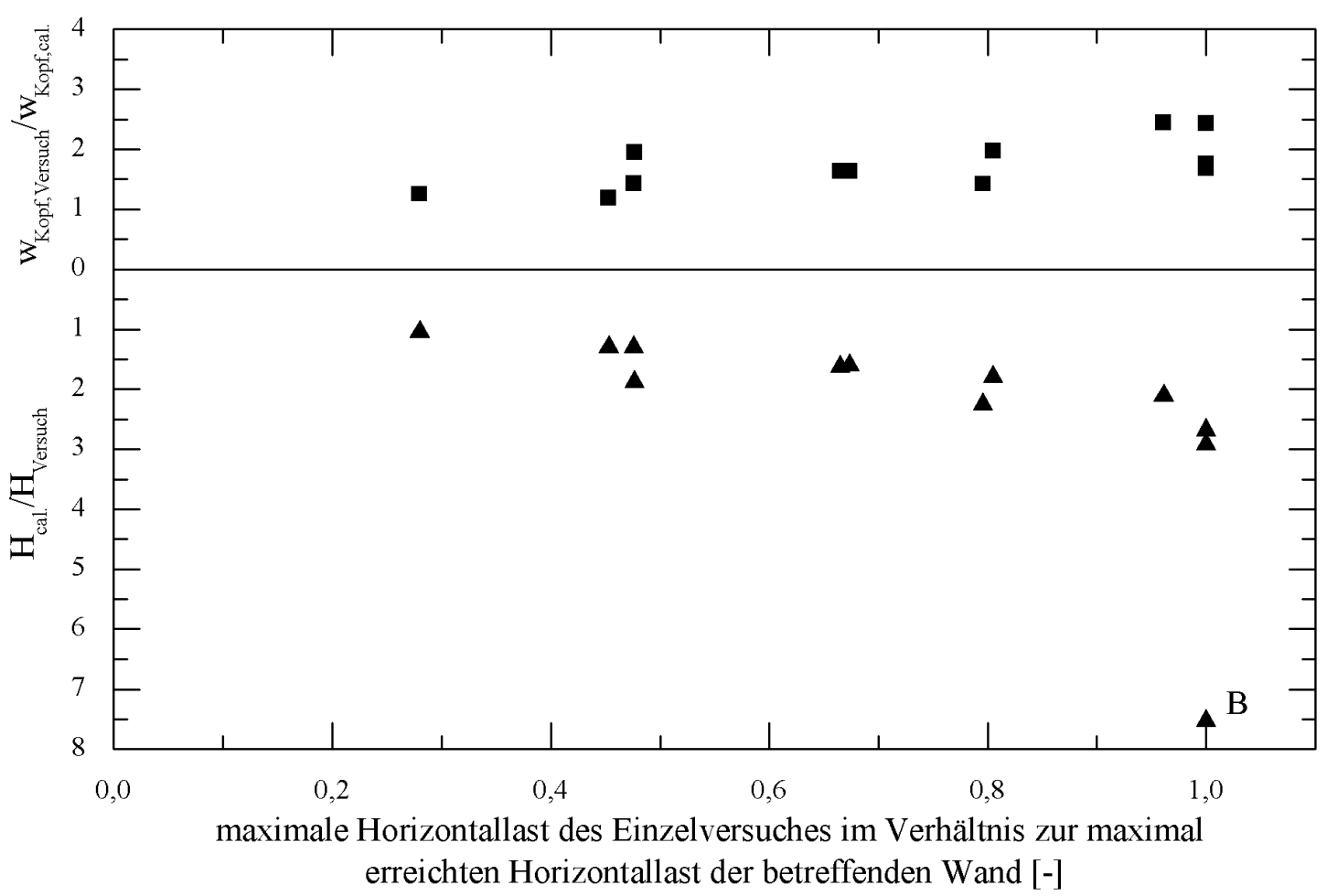

Abbildung 41: Verhältnis der bei linear-elastischer Berechnung als Einmassenschwinger ermittelten zu den im Versuch aufgetretenen Kopfverschiebungen und Horizontalkräfte in Abhängigkeit des Verhältnisses von maximal im Versuch erreichter Horizontallast zu maximaler Horizontallast aller Versuche der betreffenden Wand 
Es zeigt sich deutlich, dass mit steigender Beanspruchungshöhe das Verhältnis von tatsächlich aufgetretener zu rechnerischer Maximalverschiebung zunimmt. Die Werte liegen dabei zwischen 1,19 und 2,44 bei einem Mittelwert von 1,73. Die Wandsteifigkeit wird in der linearelastischen Berechnung mit den vorgegebenen Steifigkeiten - insbesondere bei höheren Belastungen - damit zu hoch beschrieben.

Bei der Betrachtung der Horizontalkraft zeigt sich ein ähnliches Bild. Mit zunehmender Beanspruchungshöhe steigt das Verhältnis von rechnerischer zu tatsächlich im Versuch aufgetretener Maximallast. Bleibt der Versuch an Wand B unberücksichtigt, so liegen die Werte zwischen 1,05 und 2,91 bei einem Mittelwert von 1,86. Daraus wird ersichtlich, dass der Einfluss der Lastreduktion durch das nichtlineare Verhalten der Wandscheiben im Versuch mit steigender Einwirkung zunimmt. Bei den Bruchversuchen A8 und C3 betrug der Reduktionsfaktor 2,91 bzw. 2,68.

\section{Vergleichsrechnung als linear-elastische Kragscheibe}

Zusätzlich wurden die Versuche vergleichend als isotropes, linear-elastisches Kragscheibensystem nachgerechnet (System s. Abbildung 33) [135]. Grund war die unterschiedliche Systemsteifigkeit bei Ansatz des E-Moduls $E=6.000 \mathrm{~N} / \mathrm{mm}^{2}$ entsprechend DIN 1053-1 [17] bzw. den Angaben in der Literatur (Schubert [120]). Dadurch wurde - wie der Vergleich der rechnerischen Eigenschwingzeiten aus Tabelle 30 mit den gemessenen Werten aus Tabelle 28 zeigt - die Wandsteifigkeit mit $k=156 \mathrm{kN} / \mathrm{mm}$ für horizontale Beanspruchung deutlich zu hoch abgeschätzt.

Die Auswertung der Berechnungsläufe erfolgte nach den maximal aufgetretenen Kopfverschiebungen sowie Horizontalkräften.

\begin{tabular}{|c|c|c|c|c|}
\hline $\begin{array}{l}\text { Wand / } \\
\text { Versuch }\end{array}$ & $\begin{array}{c}\text { Berechnung als linear- } \\
\text { elastische Scheibe } \\
T[\mathrm{~s}] ; \zeta[\%]\end{array}$ & $\begin{array}{c}\text { BB-ZV } \\
\text { Lastfaktor } f[-]\end{array}$ & $\begin{array}{c}\text { maximale Kopfver- } \\
\text { schiebung }{ }^{2} \\
w_{\text {Kopf, cal }}[\mathrm{mm}] \\
\end{array}$ & $\begin{array}{c}\text { maximale Hori- } \\
\text { zontalkraft }^{2)} \\
H_{c a l}[\mathrm{kN}] \\
\end{array}$ \\
\hline A1 & \multirow[t]{2}{*}{0,$2 ; 3,7$} & $\begin{array}{c}\text { ZV1-B3 }\left(a_{\mathrm{g}}=0,4 \mathrm{~m} / \mathrm{s}^{2}\right) \\
\mathrm{f}=0,25\end{array}$ & $0,31(0,60)$ & $44,6(43,1)$ \\
\hline A2 & & $\mathrm{f}=0,5$ & $0,622(1,13)$ & $89,2(69,7)$ \\
\hline A3 & \multirow[t]{6}{*}{0,$22 ; 4,2$} & $\mathrm{f}=0,46$ & $0,63(1,14)$ & $92,7(73,2)$ \\
\hline A4 & & $\mathrm{f}=0,8$ & $1,10(2,25)$ & $161,3(102,3)$ \\
\hline A5 & & $\mathrm{f}=0,8$ & $1,10(2,25)$ & $161,3(103,6)$ \\
\hline A6 & & $\mathrm{f}=1,07$ & $1,48(3,64)$ & $215,7(123,8)$ \\
\hline A7 & & $\mathrm{f}=1,5$ & $2,07(6,33)$ & $302,4(147,8)$ \\
\hline A8 & & $\mathrm{f}=2,16$ & $2,98(9,06)$ & $435,5(153,8)$ \\
\hline $\mathbf{B}^{3)}$ & 0,$324 ; 3,7$ & $\begin{array}{c}\text { ZV1-B3 }\left(\mathrm{a}_{\mathrm{g}}=0,4 \mathrm{~m} / \mathrm{s}^{2}\right) \\
\mathrm{f}=4\end{array}$ & $6,45(20)^{3)}$ & $1011(150)^{3)}$ \\
\hline C1 & 0,$22 ; 4,2$ & $\begin{array}{c}\text { ZV2-A3 }\left(\mathrm{a}_{\mathrm{g}}=0,6 \mathrm{~m} / \mathrm{s}^{2}\right) \\
\mathrm{f}=0,27\end{array}$ & $0,67(1,58)$ & $99,1(55,6)$ \\
\hline $\mathbf{C 2}$ & & $\mathrm{f}=0,54$ & $1,35(2,47)$ & $198,3(92,8)$ \\
\hline C3 & & $\mathrm{f}=0,81$ & $2,03(4,59)$ & $297,4(116,6)$ \\
\hline $\begin{array}{l}\text { Kopfmass } \\
\text { rechnerisc } \\
\text { 1) Bodent } \\
\text { 2) Werte } \\
\text { 3) Versuc } \\
\text { Maxim }\end{array}$ & $\begin{array}{l}m \text { und Dämpferkonstante } \\
\text { e Wandsteifigkeit } k=156 \\
\text { eschleunigungszeitverlauf, } \\
\text { Klammern: aus zugehörig } \\
\text { e nicht vollständig durchg } \\
\text { llast im Versuch bei Zeitsc }\end{array}$ & $\begin{array}{l}\text { s. Tabelle 27, Wandabn } \\
\text { N/mm } \\
\text { 3oden-Baugrund-Kombi } \\
\text { m Versuch } \\
\text { ührt } \Rightarrow \text { Berechnungserg } \\
\text { ritt } 220 \text { / Versuchsabbru }\end{array}$ & $\begin{array}{l}\text { sungen: } 2,5 \mathrm{~m} \cdot 2,5 \mathrm{~m} \text { · } \\
\text { ion; Grundwert der Bo } \\
\text { nisse bis zum betreffen } \\
\text { bei Zeitschritt } 707 \text { von }\end{array}$ & $\begin{array}{l}175 \mathrm{~m} \\
\text { nbeschleunigung } \\
\text { Zeitpunkt } \\
00\end{array}$ \\
\hline
\end{tabular}

Tabelle 30: Parameter und Ergebnisse der vergleichenden linear-elastischen Kragscheibenberechnung 
Werden die Verhältniswerte von rechnerischen und tatsächlich aufgetretenen Maximalwerten in Abhängigkeit des Verhältniswertes von maximal im Versuch erreichter Horizontallast zu maximaler Horizontallast aller Hauptversuche der betreffenden Wand dargestellt, ergibt sich folgendes Bild:

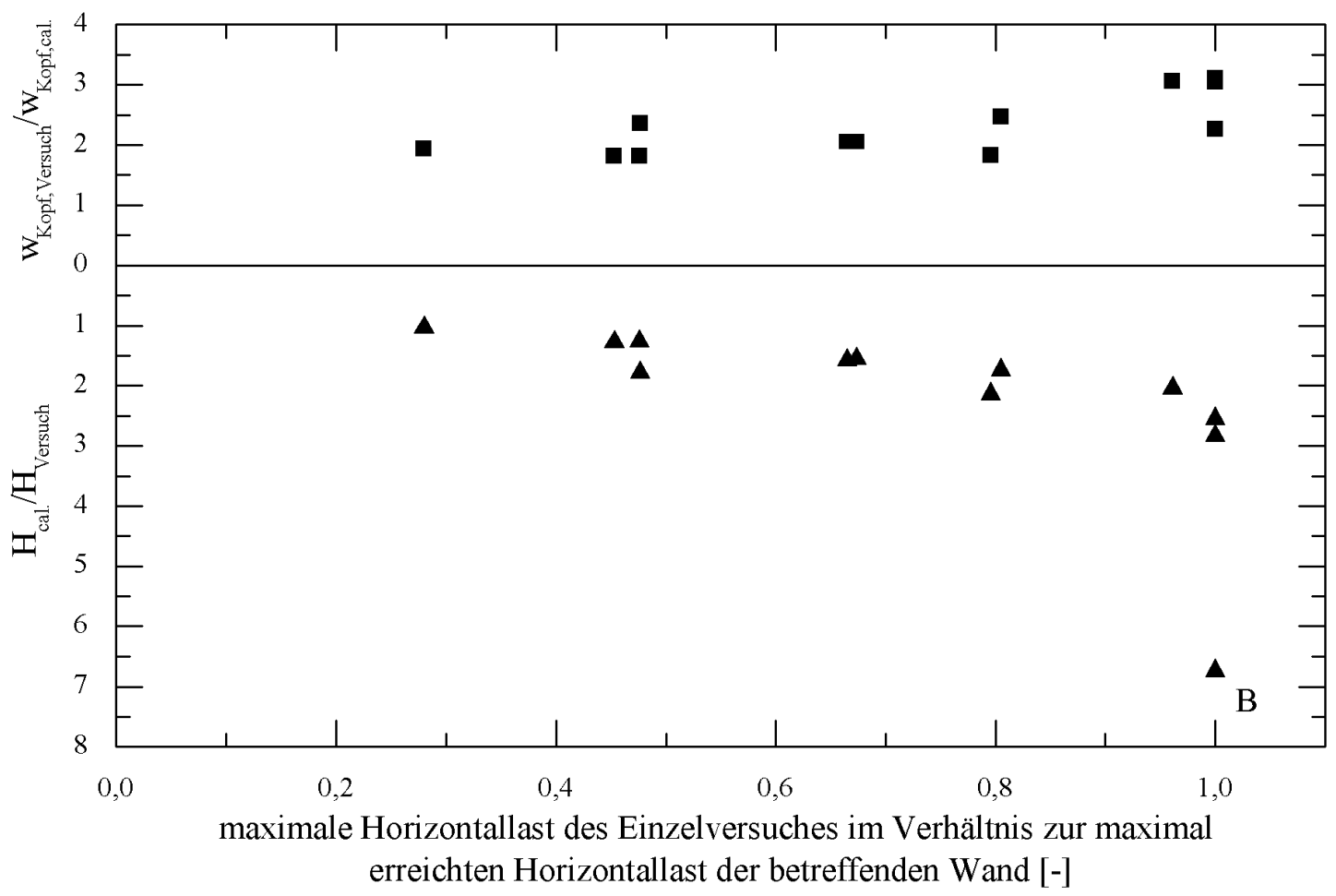

Abbildung 42:Verhältnis der bei linear-elastischer Scheibenberechnung ermittelten zu den im Versuch aufgetretenen Kopfverschiebungen und Horizontalkräfte in Abhängigkeit des Verhältnisses von maximal im Versuch erreichter Horizontallast zu maximaler Horizontallast aller Versuche der betreffenden Wand

Bei der Nachrechnung als linear-elastische Kragscheibe zeigt sich ein analoges Bild zur EMSBerechnung, jedoch weichen infolge der höheren Systemsteifigkeit die rechnerischen Verschiebungswerte (Tabelle 30) von denen des Einmassenschwingers unter gemessener Anfangssteifigkeit (Tabelle 29) stärker ab. Das Verhältnis von tatsächlich in den Versuchen aufgetretener zu rechnerischer Maximalverschiebung der Scheibenberechnung liegt zwischen 1,81 und 3,10 bei einem Mittelwert von 2,31.

Bei Betrachtung der Verhältniswerte von rechnerischer zu tatsächlich im Versuch aufgetretener Maximallast ergibt sich - bei Vernachlässigung des Versuches an Wand B - ein Wertebereich zwischen 1,04 und 2,83 bei einem Mittelwert von 1,80.

Es zeigt sich bei den Verhältniswerten der Horizontalkräfte eine gute Übereinstimmung mit den Werten der Einmassenschwingerberechnung, wohingegen die Verhältniswerte der Verschiebungen bei der Scheibenberechung etwa 38\% höher liegen. Dieses erklärt sich aus der Tatsache, dass die Eigenperioden beider Systeme - Einmassenschwinger und Scheibensystem - jeweils im Plateaubereich des Beschleunigungsantwortspektrums liegen. Bei gleichen Massen ergeben sich - unter Vernachlässigung der Ungleichförmigkeit des tatsächlichen Antwortspektrums - gleiche rechnerische Maximallasten. Die Reduzierung der Eigenperiode bei der Scheibenberechnung bewirkt jedoch in diesem Periodenbereich im Verschiebungsantwortspektrum eine Reduzierung der Ordinate, entsprechend der doppelt-logarithmischen Darstellung (s. Anhang A.1). 


\subsubsection{Zusammenfassung}

Im Zuge der Durchführung der ersten Versuchsstufe zeigte sich, dass das Gesamtverformungsbild der Kragwand von dem Biegeverformungsanteil dominiert wurde. Mit der konstanten und am Wandkopf mittig zentrierten Vertikalkraft $N$ resultierten ab einer Horizontalkraft $|H| \geq N \cdot h /(6 \cdot l)$ vertikale Zugspannungen am Wandfuß. Bei Überschreitung der i. d. R. geringen Haftzugfestigkeit zwischen Stein und Mörtel trat entsprechend ein Klaffen des Querschnittes auf und die Wandsteifigkeit reduzierte sich deutlich. Bei weiter zunehmender Horizontalkraft bzw. Kopfverschiebung nahm bei den Versuchen die überdrückte Querschnittsfläche am Wandfuß sukzessive weiter ab und die lokalen Druck- und Schubspannungen erhöhten sich. Neben dem lokalen Versagen der Steine in den unteren Eckbereichen, ohne wesentliche Reduzierung der Gesamttragfähigkeit der Wand, trat bei weiterer Laststeigerung ein Abscheren der Innenstege durch die eingreifenden Mörteldollen auf. Bei Wand C stellte sich das Steinzugversagen in Form eines Diagonalrisses über die ganze Wandhöhe ein. Das anschließend bei Lastumkehr aufgetretene abgetreppte Fugenversagen erklärte sich durch die Vorschädigung.

Allgemein kann festgestellt werden, dass das System einer Kragscheibe die Randbedingungen einer Schubwand in üblichen Hochbauten nicht ausreichend beschreibt. Infolge der in Wirklichkeit vorhandenen Behinderung der Kopfverdrehung durch Teileinspannung in die Stahlbetondecken wird am Wandkopf ein gegendrehendes Moment eingeleitet, wodurch ein von der Kragwand abweichender Spannungs- und Verformungszustand resultiert.

Infolgedessen wurde nach Abschluss der Versuche an den ersten drei Wänden $\mathrm{A} \div \mathrm{C}$ die Modifikation und Erweiterung des Versuchssystems - im Weiteren als Simulationsstufe 2 bezeichnet - festgelegt.

\subsection{Zweite Simulationsstufe-Mehrmassenschwingersystem}

\subsubsection{Allgemeines}

Ziel der Erweiterung des Versuchssystems war, den Kraft- und Verformungszustand der zu prüfenden Wand realitätsnäher zu erfassen. Entscheidende Bedeutung kam dabei dem Wandkopfmoment bzw. der Kopfverdrehung zu. Diese beiden Parameter mussten neben den bereits in der ersten Stufe berücksichtigten Größen Wandkopfverschiebung und Horizontalkraft noch in die Versuchseinrichtung einbezogen werden. Eine einfache mechanische Kopplung oder Festhaltung der Kopfverdrehung (z. B. über einen steifen Anschlag) wurde aufgrund der in Abschnitt 5.1.2 diskutierten Problempunkte nicht weiter in Betracht gezogen.

Die einfachste Möglichkeit die Kopfverdrehung gezielt zu behindern stellt prinzipiell der rechnerische Einbau einer Drehfeder am Wandkopf dar. Damit wären die beiden Größen Wandkopfverdrehung und Wandkopfmoment direkt gekoppelt und im Rahmen der Versuchssteuerung einfach zu behandeln (analog zu den durchgeführten Vorversuchen in Abschnitt 5.5.3). Bei dieser Vorgehensweise würde erneut ein äquivalenter Einmassenschwinger für die Beschreibung des Mehrmassenschwingers herangezogen werden (vgl. Abbildung 46 links).

Im Zuge der zweiten Simulationsstufe wurde daher die unabhängige Steuerung der beiden Vertikalzylinder - was einer Steuerung von Normalkraft und Kopfmoment entspricht - genutzt, um das Verhalten eines Mehrmassenschwingers zu beschreiben. 


\section{Versuchsstand}

Über die symmetrisch am Wandkopf angeordneten und unabhängig gesteuerten Vertikalzylinder $N_{1}$ und $N_{2}$ mit einem Achsabstand von 1,60 m wurden die Vertikalkraft und das Wandkopfmoment eingeleitet. Die Lastweiterleitung auf den Ortbeton-Kopfbalken erfolgte analog zur ersten Simulationsstufe über Rollenlager und Lastverteilungsprofile. Der Reaktionsrahmen wurde unverändert übernommen.

Für die momenten- und querkraftfreie Einleitung der Horizontalkraft in den Wandkopfbalken wurde darin mittig ein Gelenk in Form eines Passbolzens $\varnothing=60 \mathrm{~mm}$ angeordnet (Wand E, F \& KS1 $\div$ KS4). Die Übertragung der Horizontallast von dem Hydraulikzylinder mit angeschlossener Kalotte auf den Bolzen erfolgte über zwei seitliche Stahlprofile U 160 mit entsprechender Passbohrung. Da Wand D bereits vor Umplanung des Versuchsstandes hergestellt wurde, wurde die Horizontallast entsprechend Simulationsstufe 1 eingeleitet.

Die Versuche der zweiten Simulationsstufe wurden bezüglich der Vertikalzylinder kraftgesteuert und bezüglich des Horizontalzylinders weggesteuert durchgeführt. Analog zur ersten Stufe erfolgte bei letzterem eine Differenzierung von Regelgröße Zylinderverschiebungen und Steuergröße Wandkopfverschiebung w Kopf $_{\text {. }}$

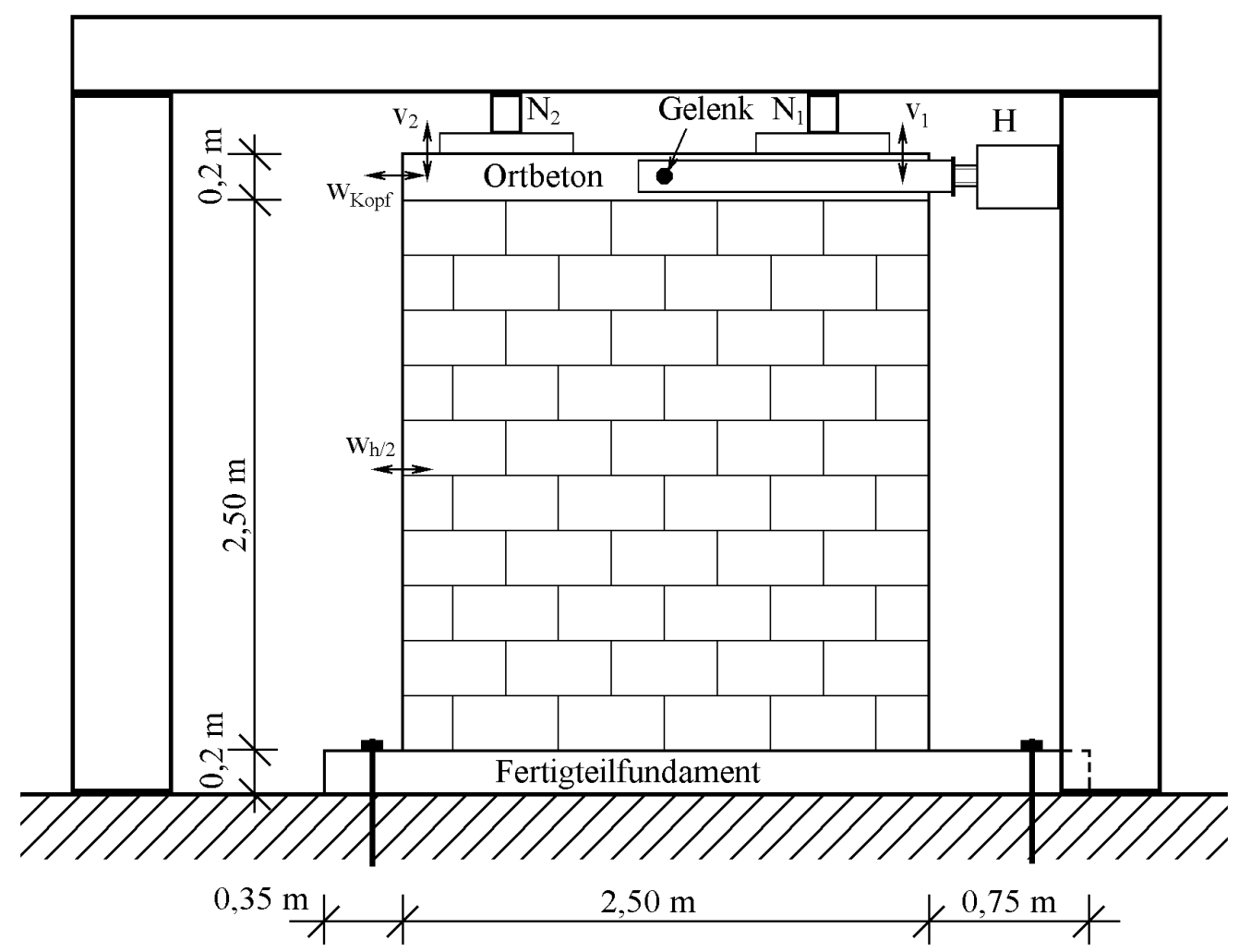

Abbildung 43: Versuchsstand für die zweite Versuchsstufe - Wand D $\div$ F und KS1 $\div$ KS4

\section{Messprogramm}

Während der Versuche wurden nachfolgend angegebene Werte kontinuierlich mit einer Taktrate von $50 \mathrm{~Hz}$ erfasst:

- Horizontalverschiebung des Wandkopfes $w_{K o p f}$

- Horizontalkraft $H$ 
- Horizontalverschiebung auf halber Wandhöhe $w_{h / 2}$

- Vertikale Verschiebungen am Wandkopf $v_{1}$ und $v_{2}$ durch 2 Messgeber im Abstand von $2,30 \mathrm{~m} \rightarrow$ Berechnung der Wandkopfverdrehung

- Pressenweg und Rahmenverschiebung (für Regelungs- und Steuerungsroutinen)

- Normalkraft der 2 Pressen $N_{1}$ und $N_{2} \rightarrow$ Berechnung der Gesamtnormalkraft $N$ und des Wandkopfmomentes $M_{\text {Kopf }}$

Die Verschiebungen wurden im Bezug auf einen unabhängigen Messrahmen bestimmt.

\subsubsection{Gebäudemodell - Ersatzsystem}

Als Untersuchungsobjekt wurde in der zweiten Simulationsstufe ein Reihenmittelhaus gewählt, das in der betrachteten Querrichtung im Wesentlichen durch zwei Querwände ausgesteift wird (s. Abbildung 44). Da Torsionseffekte vernachlässigt wurden, konnte der Versuch unter Ausnutzung der Symmetrie auf eine einzelne Schubwand reduziert werden.

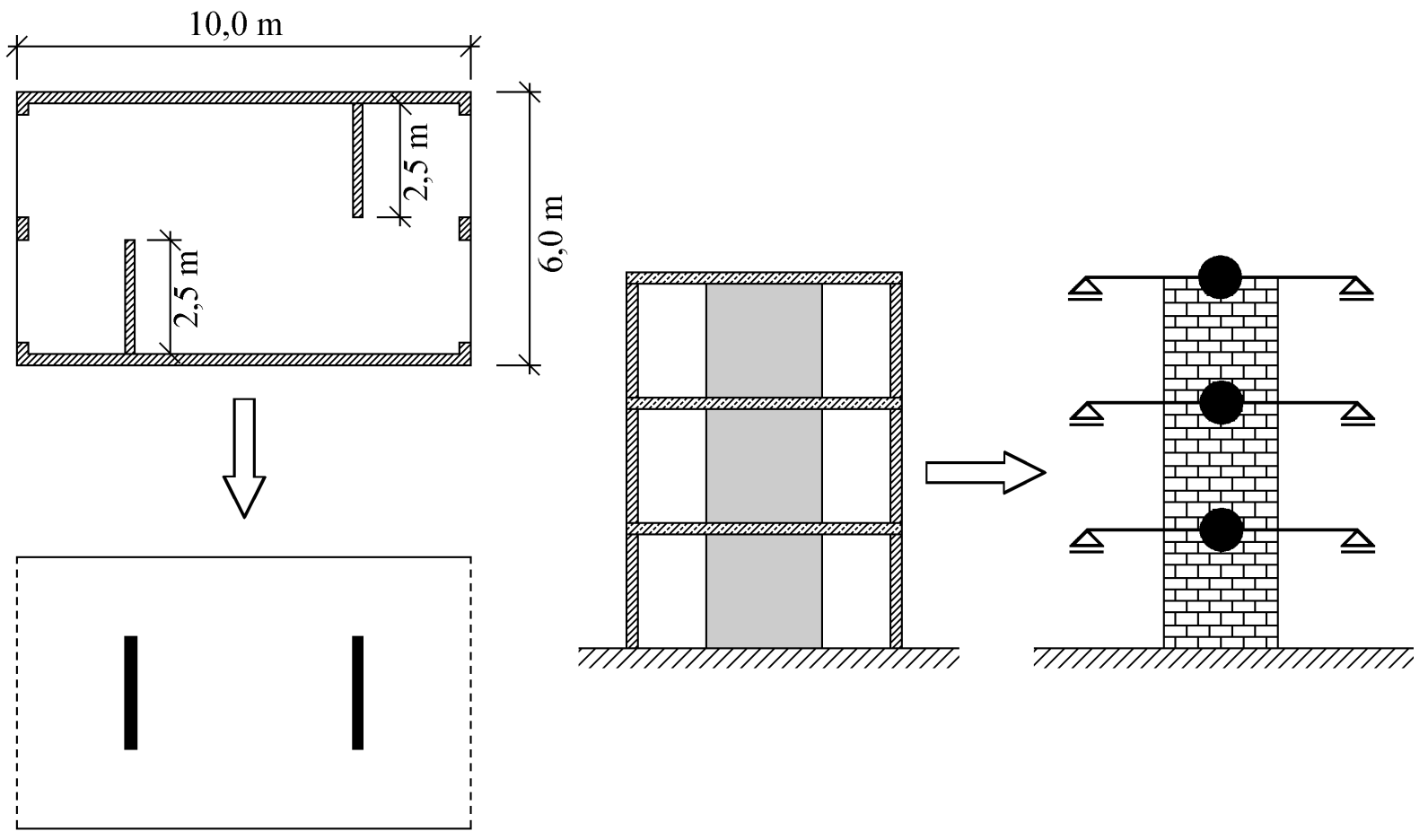

Abbildung 44: Untersuchtes Gebäudemodell und rechnerische Vereinfachung

Als zentrische Wandnormalkraft wurden die unter der quasi-ständigen Lastkombination vorhandenen Vertikallasten angesetzt (pro Geschoss: $90 \mathrm{kN}$ ). Das untersuchte Gebäude wurde im Aufriss auf einen 3-Massenschwinger mit elastischer Einspannung in die Geschossdecken auf Höhe der Einzelmassen reduziert. Die Abschätzung der Drehfederkonstanten $c_{D 1,2,3}$ erfolgte über eine Vergleichsberechnung an einer $18 \mathrm{~cm}$ starken Stahlbetonplatte mit einer pauschalen Abminderung infolge Steifigkeitsverlust bei bereichsweisem Übergang in den Zustand II.

Die Geschossmassen im Abstand von je $h_{1,2,3}=2,6 \mathrm{~m}$ betrugen je Gebäudehälfte $m_{1,2,3}=29 \mathrm{t}$. Der viskose Dämpfungsanteil wurde durch 3 Einzeldämpfer auf Höhe der Punktmassen beschrieben. Die Ermittlung der Dämpfungskonstanten $c_{D M P i}$ erfolgte über den ZielDämpfungsgrad der drei Eigenformen. 
Während die Tragwand im untersten Geschoss im Zuge der Simulation direkt geprüft wurde, wurden die oberen beiden Geschosse als Sub-Struktur lediglich numerisch durch jeweils eine Schub- und Biegefeder erfasst. Das betrachtete ebene Ersatzsystem sowie die kinematischen Beziehungen im ausgelenkten Zustand sind nachfolgend in Abbildung 45 dargestellt.
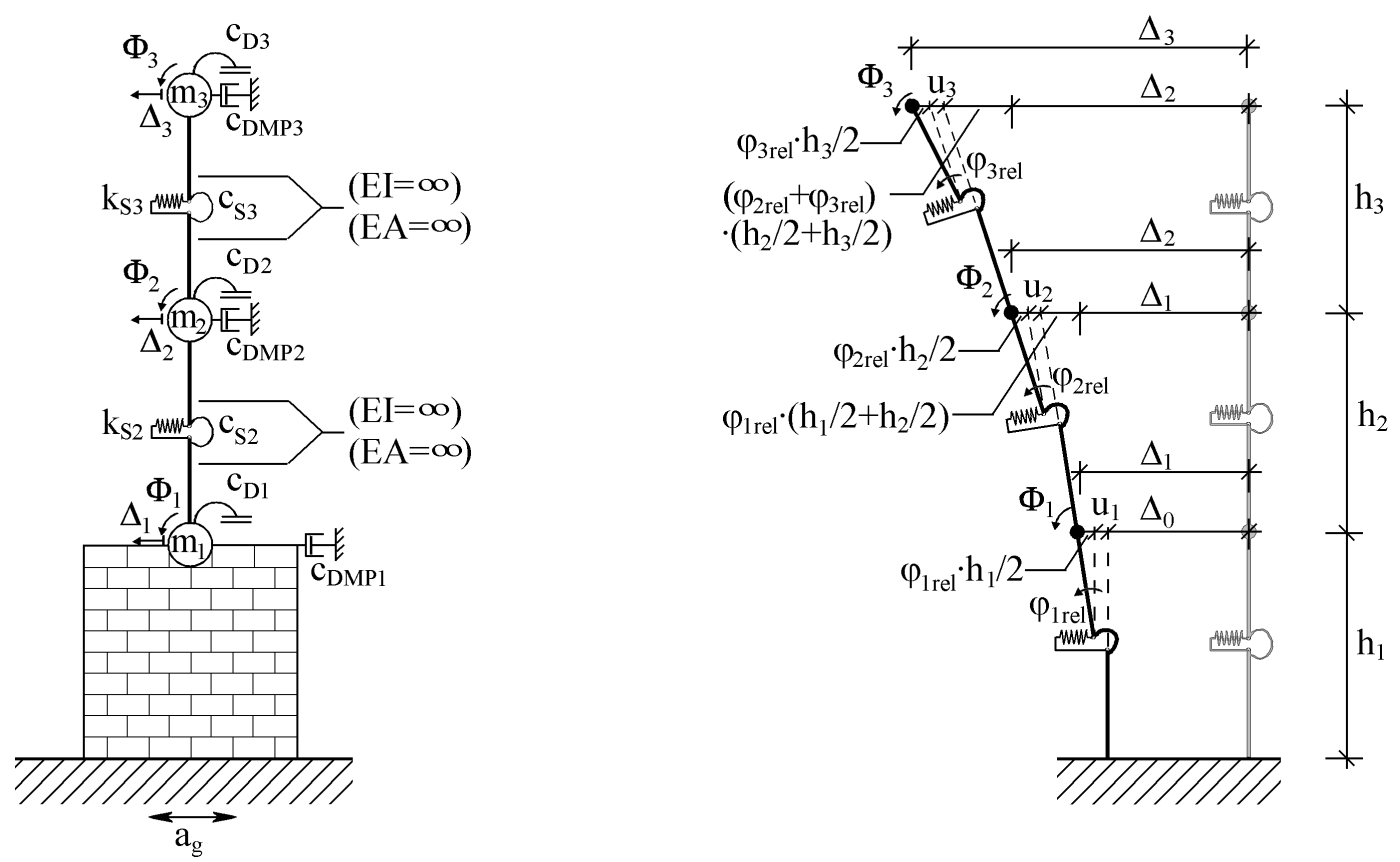

Abbildung 45: Ebenes Ersatzsystem und kinematische Beziehungen

Als Verformungsvektor $\underline{d}$ bestimmt sich

$$
\underline{d}=\left\{\begin{array}{llllll}
u_{1} & \varphi_{1 r e l} & u_{2} & \varphi_{1 r e l} & u_{3} & \varphi_{3 r e l}
\end{array}\right\}^{T}
$$

Die Umrechnung auf die kondensierten und den Fußpunkt bezogenen Knotenverschiebungen $\underline{\Delta}$ erfolgt unter Annahme kleiner Winkel mit der Beziehung

$$
\Delta=\left\{\begin{array}{l}
\Delta_{1}-\Delta_{0} \\
\Delta_{2}-\Delta_{0} \\
\Delta_{3}-\Delta_{0}
\end{array}\right\}=\left[\begin{array}{cccccc}
1 & \frac{h_{1}}{2} & 0 & 0 & 0 & 0 \\
1 & \frac{h_{1}}{2}+h_{2} & 1 & \frac{h_{2}}{2} & 0 & 0 \\
1 & \frac{h_{1}}{2}+h_{2}+h_{3} & 1 & \frac{h_{2}}{2}+h_{3} & 1 & \frac{h_{3}}{2}
\end{array}\right] \cdot \underline{d}
$$

Die Erdbebeneinwirkung in Form der horizontalen Massenbeschleunigung (s. a. Gleichungen (23) und (24a)) ermittelt sich zu: 


$$
\underline{m}_{g e s} \cdot \underline{a}_{n}^{g}=\left\{\begin{array}{c}
m_{1} \cdot a^{g}(n) \\
m_{2} \cdot a^{g}(n) \\
m_{3} \cdot a^{g}(n) \\
0 \\
0 \\
0
\end{array}\right\}
$$

Entsprechend bestimmen sich Massen- $\underline{m}_{g e s}$, Dämpfungs- $\underline{c}_{g e s}$ und Steifigkeitsmatrix $\underline{k}_{g e s} \mathrm{zu}$ :

$\underline{m}_{\text {ges }}=\left[\begin{array}{cccccc}m_{1} & m_{1} \cdot \frac{h_{1}}{2} & 0 & 0 & 0 & 0 \\ m_{2} & m_{2} \cdot\left(\frac{h_{1}}{2}+h_{2}\right) & m_{2} & m_{2} \cdot \frac{h_{2}}{2} & 0 & 0 \\ m_{3} & m_{3} \cdot\left(\frac{h_{1}}{2}+h_{2}+h_{3}\right) & m_{3} & m_{3} \cdot\left(\frac{h_{2}}{2}+h_{3}\right) & m_{3} & m_{3} \cdot \frac{h_{3}}{2} \\ 0 & 0 & 0 & 0 & 0 & 0 \\ 0 & 0 & 0 & 0 & 0 & 0 \\ 0 & 0 & 0 & 0 & 0 & 0\end{array}\right]$

$\underline{c}_{g e s}=\left[\begin{array}{cccccc}c_{D M P 1} & c_{D M P 1} \cdot \frac{h_{1}}{2} & 0 & 0 & 0 & 0 \\ c_{D M P 2} & c_{D M P 2} \cdot\left(\frac{h_{1}}{2}+h_{2}\right) & c_{D M P 2} & c_{D M P 2} \cdot \frac{h_{2}}{2} & 0 & 0 \\ c_{D M P 3} & c_{D M P 3} \cdot\left(\frac{h_{1}}{2}+h_{2}+h_{3}\right) & c_{D M P 3} & c_{D M P 3} \cdot\left(\frac{h_{2}}{2}+h_{3}\right) & c_{D M P 3} & c_{D M P 3} \cdot \frac{h_{3}}{2} \\ 0 & 0 & 0 & 0 & 0 & 0 \\ 0 & 0 & 0 & 0 & 0 & 0 \\ 0 & 0 & 0 & 0 & 0 & 0\end{array}\right]$

$$
\underline{k}_{g e s}=\left[\begin{array}{cccccc}
k_{S 1} & 0 & -k_{S 2} & 0 & 0 & 0 \\
0 & 0 & k_{S 2} & 0 & -k_{S 3} & 0 \\
0 & 0 & 0 & 0 & k_{S 3} & 0 \\
-k_{S 1} \cdot \frac{h_{1}}{2} & c_{D 1}+c_{S 1} & -k_{S 2} \cdot \frac{h_{2}}{2} & -c_{S 2} & 0 & 0 \\
0 & c_{D 2} & -k_{S 2} \cdot \frac{h_{2}}{2} & c_{D 2}+c_{S 2} & -k_{S 3} \cdot \frac{h_{3}}{2} & c_{S 3} \\
0 & c_{D 3} & 0 & c_{D 3} & -k_{S 3} \cdot \frac{h_{3}}{2} & c_{D 3}+c_{S 3}
\end{array}\right]
$$




\subsubsection{Sub-Struktur}

Für die numerische Erfassung der oberen Stockwerke der Konstruktion wurden im Vorfeld an den zu untersuchenden Wänden statisch-zyklische Vorversuche durchgeführt. Dabei erfolgte eine Anpassung der Normalkraft an die entsprechenden Randbedingungen, d.h. die Versuche für die Steifigkeitsbestimmung der oberen Geschosse wurden mit auf $1 / 3$ bzw. $2 / 3$ reduzierter Normalkraft durchgeführt. Zusätzlich wurden für die Bestimmung der Prädiktorsteifigkeit (s. Tabelle 34) Vorversuche mit voller Normalkraft $N=270 \mathrm{kN}$ durchgeführt.

Die Vorversuche wurden statisch-zyklisch und kraftgesteuert mit einer elastischen Kopfeinspannung durchgeführt. Durch den Ansatz einer rechnerischen Kopffeder $c_{\varphi}$ wurden die sich einstellende Wandkopfverdrehung $\varphi$ und das aufgebrachte Kopfmoment $M_{\text {Kopf }}$ direkt gekoppelt (Ausnahme: Wand D: s. u.). Die Größe der Beanspruchungen wurde so gewählt, dass keine relevante Vorschädigung der Wände resultierte.
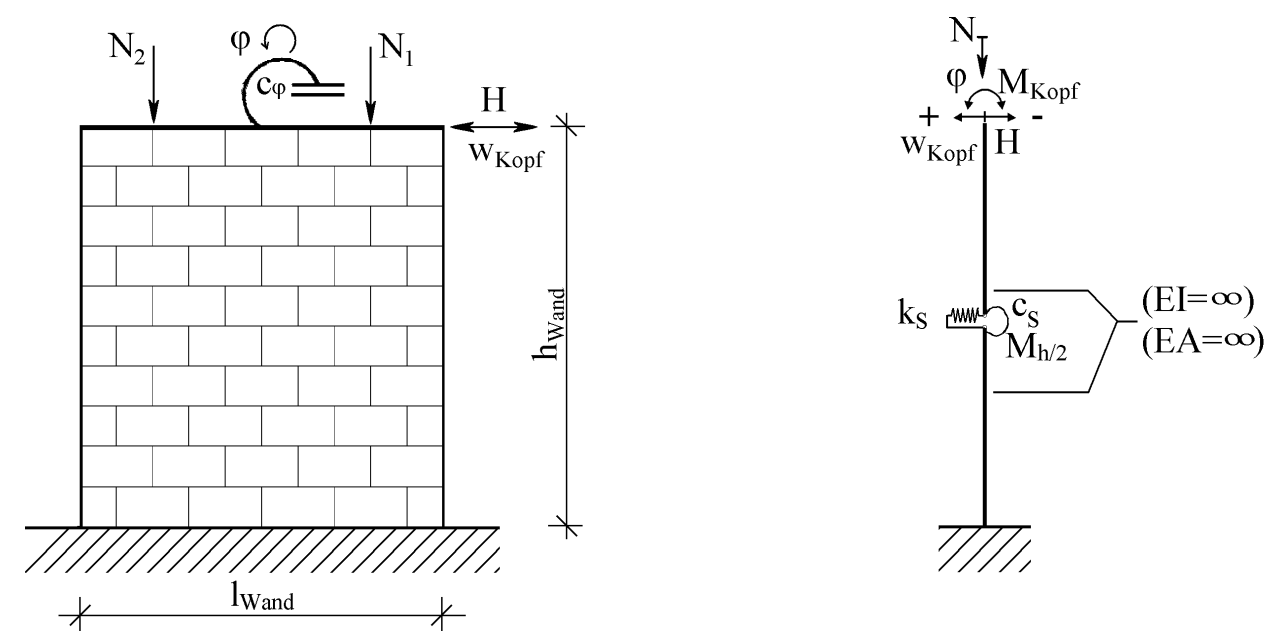

Abbildung 46: System der statisch-zyklischen Vorversuche und resultierende rechnerische Vereinfachung

Bei der Prüfung der insgesamt 7 Wände der zweiten Simulationsstufe waren aufgrund der fehlenden Erfahrungen die gewählten Lastgeschichten der Vorversuche teilweise unterschiedlich, wodurch sich auch abweichende Ansätze zur Erfassung der Wandsteifigkeiten der oberen Geschosse $k_{S i}$ und $c_{S i}$ ergaben.

Nachfolgend werden beispielhaft die Vorversuche an Wand D und KS2 dargestellt. Die Beschreibung der Federsteifigkeiten für alle Wände der Simulationsstufe 2 erfolgt anschließend in Tabelle 31.

\section{Vorversuche an Wand D}

An Wand D erfolgten die Untersuchungen für jede Auflaststufe von 90, 180 und $270 \mathrm{kN}$ getrennt. Dabei wurde das Kopfmoment nicht in Abhängigkeit der Wandkopfverdrehung festgelegt, sondern in 13 Stufen schrittweise variiert, in denen jeweils kraftgesteuert Lastzyklen gefahren wurden. Das mechanisch maximal mögliche Kopfmoment ergab sich aus der Normalkraft und dem Abstand der Vertikalpressen von der Wandmitte, z.B. bei mit einem Abstand von $0,8 \mathrm{~m}$ und $N=180 \mathrm{kN}$ (2. Geschoss) resultiert $\mid M_{\text {Kop } f \mid \max }=144 \mathrm{kNm}$. Als obere Grenze der Horizontalkraft in den Vorversuchen wurden $75 \mathrm{kN}$ bzw. eine resultierende maximale Schubspannung von $0,8 \mathrm{~N} / \mathrm{mm}^{2}$ und eine maximale Randdruckspannung von $4 \mathrm{~N} / \mathrm{mm}^{2}$ festgelegt. 
Nachfolgend ist in Abbildung 47 die Kraft-Verformungs-Beziehung von Wand D bei einer Normalkraft $N=180 \mathrm{kN}$ in Abhängigkeit des Wandkopfmomentes dargestellt.

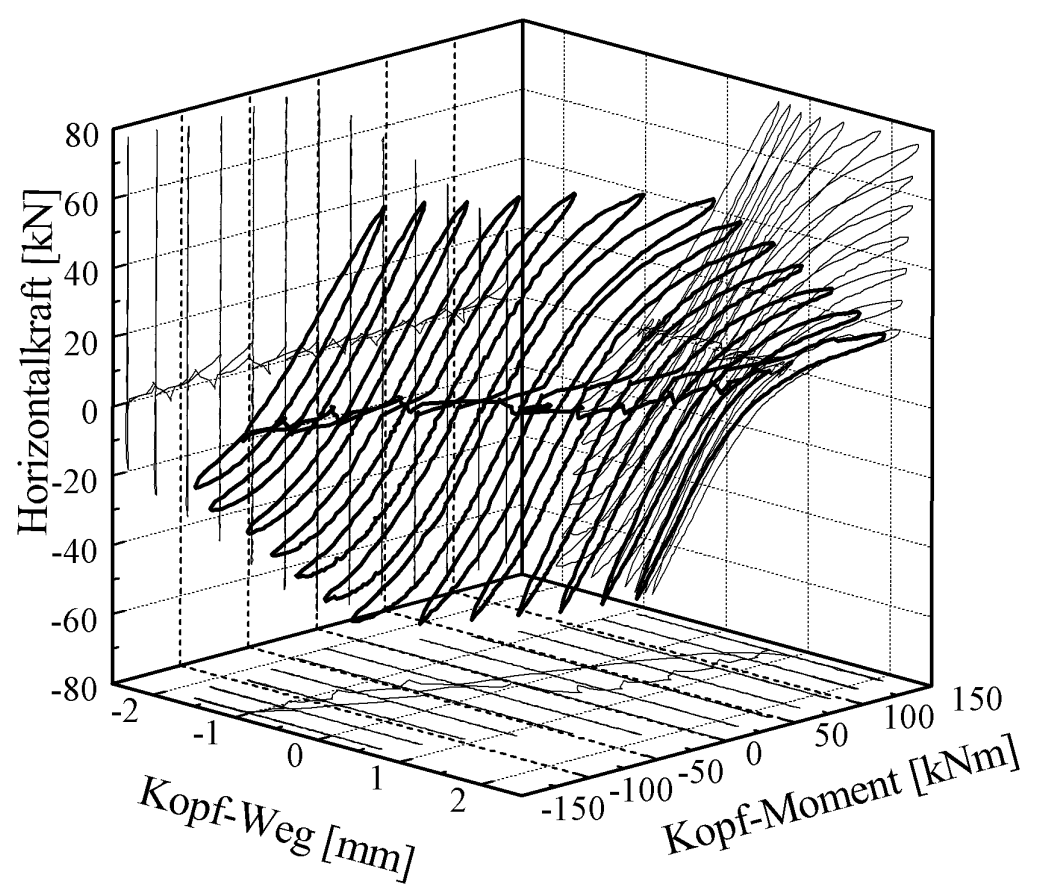

Abbildung 47: Kraft-Verformungs-Beziehung der Vorversuche an Wand D in Abhängigkeit des Wandkopfmomentes für $N=180 \mathrm{kN}$

\section{Vorversuche an Wand KS2}

Für die Vorversuche an den Wänden E, F und KS1 $\div$ KS4 wurde ein von der Vorgehensweise bei Wand D abweichender Weg gewählt. Die Vorversuche wurden statisch-zyklisch und kraftgesteuert mit einer elastischen Kopfeinspannung durchgeführt. Die Kopffeder $c_{\varphi}$ wurde dabei in jedem Durchgang schrittweise $(0,200,500,2.000 \mathrm{kNm} / \mathrm{mrad})$ variiert. In Abbildung 48 ist die Lastgeschichte des Vorversuches an Wand KS2 mit einer Drehfederkonstanten $c_{\varphi}=2.000 \mathrm{kNm} / \mathrm{mrad}$ dargestellt.

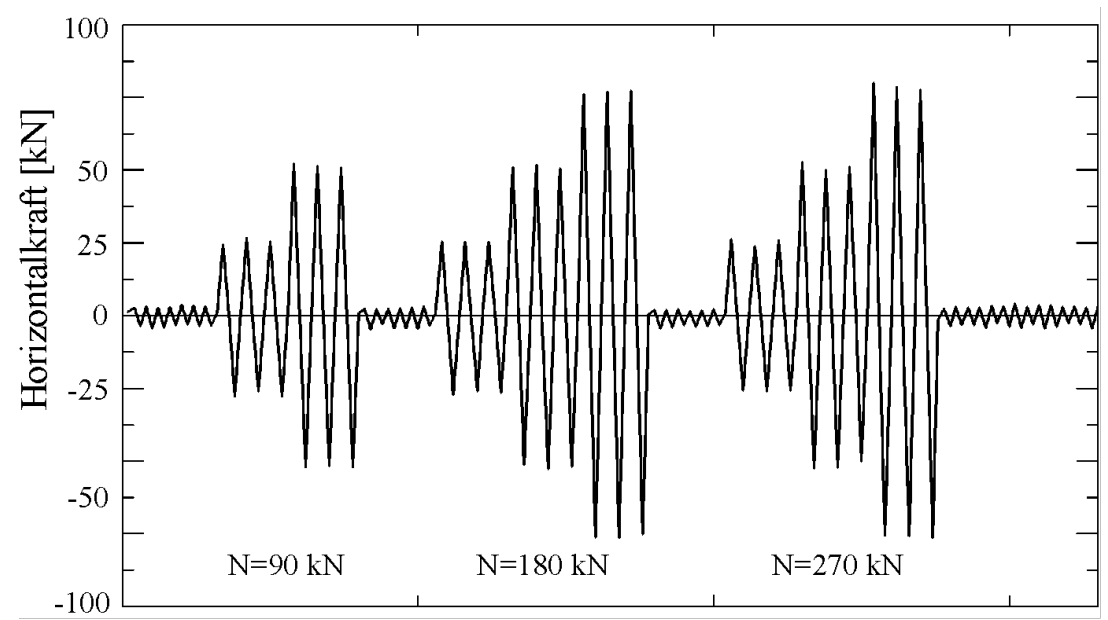

Abbildung 48: Lastgeschichte des Vorversuches an Wand KS2 mit $c_{\varphi}=\mathbf{2 . 0 0 0} \mathrm{kNm} / \mathrm{mrad}$ 
In Abbildung 49 ist die Horizontalkraft-Kopfverschiebungs-Beziehung von Wand KS2 bei einer Normalkraft $N=180 \mathrm{kN}$ in Abhängigkeit des Wandkopfmomentes für die Einspanngrade $c_{\varphi}=200,500$ und $2.000 \mathrm{kNm} / \mathrm{mrad}$ dargestellt.

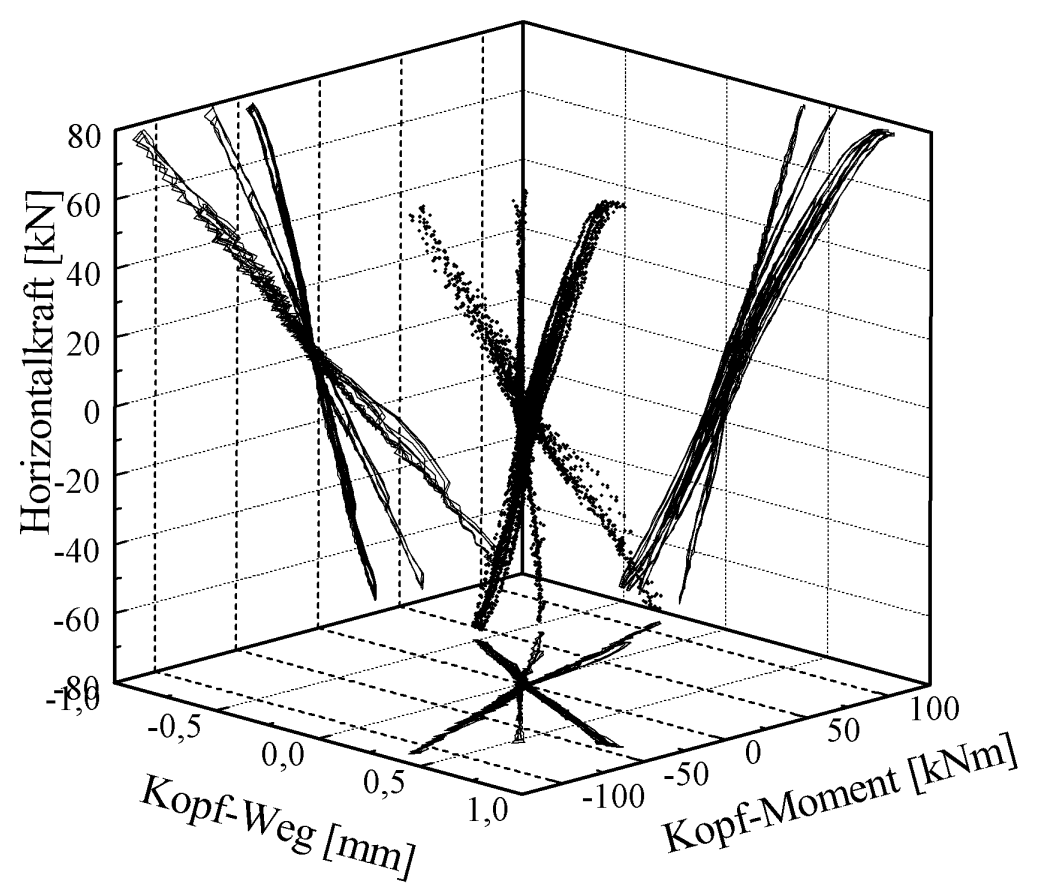

Abbildung 49: Kraft-Verformungs-Beziehung der Vorversuche an Wand KS2 in Abhängigkeit des Wandkopfmomentes für $N=180 \mathrm{kN}$

\section{Beschreibung der Federsteifigkeiten}

Die einzelnen Wände der Sub-Struktur wurden aufgrund des gewählten Ersatzsystems jeweils durch eine Schub- und eine Biegefeder auf halber Wandhöhe beschrieben (s. Abbildung 45). Die Schubverformung $u_{\text {Schub }}$ wurde dabei aus der gesamten Kopfverschiebung $w_{\text {Kopf }}$ abzüglich des aus der Kopfverdrehung $\varphi$ bzw. $\varphi_{\text {rel }}$ bestimmten Biegeverformungsanteils ermittelt.

Bereits zu Beginn der Untersuchungen zeigte sich das ausgeprägte nichtlineare Verhalten der Wände - sowohl der Schub- als auch der Biegeverformungsanteile. Eine exakte Beschreibung dieser Flächen im Raum war allgemein nicht möglich. Es stellte sich daher die Frage nach einer einfachen und zugleich die Ergebnisse gut beschreibenden Näherung für die Federsteifigkeiten.

Bei dem ersten Wandversuch der zweiten Simulationsstufe (Wand D) wurden die Steifigkeiten in Abhängigkeit der Momentenbeanspruchung auf halber Wandhöhe $\left(=M_{h / 2}\right)$ gesetzt. Hintergrund dieser Überlegung war, dass die Momentenbeanspruchung ein Maß für das Klaffen des Querschnittes in diesem Bereich darstellt, und somit indirekt auch für die Bestimmung des Steifigkeitsabfalls der ganzen Wand angesetzt werden kann. Anstelle der Berücksichtigung des Querschnittklaffens über den allgemeinen bilinearen Ansatz für Baustoffe ohne Zugfestigkeit in der Form $1 \geq f \geq 3 \cdot(0,5-|e / d|)$, wurde ein parabelförmiger Verlauf gewählt (s. Tabelle 31 und Abbildung 50, Typ 1).

Bei den nachfolgenden Vorversuchen an den Wänden E $\div$ KS4 zeigte sich, dass eine Abhängigkeit der Steifigkeiten von den zugehörigen Verformungsgrößen die Ergebnisse deutlich besser beschreibt. Die Schubsteifigkeit $k_{S}$ wurde dabei in Abhängigkeit des Schubverfor- 
mungsanteils $u_{S c h u b}$ und die Biegesteifigkeit $c_{S}$ in Abhängigkeit der Kopfverdrehung $\varphi$ bzw. $\varphi_{\text {rel }}$ gesetzt.

Die Beschreibung wurde in dem durch die Vorversuche abgedeckten Bereich an die Messwerte angepasst. Für die Bereiche außerhalb wurde ein Mindestwert als unterer Steifigkeitswert festgelegt. In Tabelle 31 mit Abbildung 50 sind die Steifigkeitsparameter der oberen beiden Geschosse (Sub-Struktur) angegeben. Die Anfangssteifigkeiten der untersten Geschosse sind in Tabelle $34 \mathrm{zu}$ finden.

\begin{tabular}{|c|c|c|c|c|}
\hline Wand & $\begin{array}{c}k_{S 2}{ }^{1)} \\
{[\mathrm{kN} / \mathbf{m m}]}\end{array}$ & $\begin{array}{c}\mathbf{k}_{\mathbf{S 3}}{ }^{1)} \\
{[\mathrm{kN} / \mathbf{m m}]}\end{array}$ & $\begin{array}{c}c_{S 2}{ }^{1)} \\
{[\mathrm{kNm} / \mathbf{m r a d}]}\end{array}$ & $\begin{array}{c}c_{S 3}{ }^{11} \\
{[\mathrm{kNm} / \mathrm{mrad}]}\end{array}$ \\
\hline D & $\begin{array}{c}\text { 70: }\left|M_{h / 2}\right|=0 \mathrm{kNm} \\
\text { 35: }\left|M_{h / 2}\right| \geq 145 \mathrm{kNm} \\
\text { Zwischenbereich: } \\
\text { parabolisch (s. Typ 1) }\end{array}$ & $\begin{array}{c}\text { 47: }\left|M_{h / 2}\right|=0 \mathrm{kNm} \\
\text { 23: }\left|M_{h / 2}\right| \geq 80 \mathrm{kNm} \\
\text { Zwischenbereich: } \\
\text { parabolisch (s. Typ 1) }\end{array}$ & $\equiv 200$ & $\equiv 125$ \\
\hline $\mathbf{E}$ & $\equiv 60$ & $\equiv 45$ & $\equiv 200$ & $\begin{array}{c}\text { 130: }\left|\varphi_{\text {rel }}\right|=0 \mathrm{mrad} \\
\text { 60: }\left|\varphi_{\text {rel }}\right| \geq 0,8 \mathrm{mrad} \\
\text { Zwischenbereich: } \\
\text { linear (s. Typ 2) }\end{array}$ \\
\hline $\mathbf{F}$ & $\equiv 42$ & $\begin{array}{l}\text { 35: }\left|u_{\text {Schub }}\right|=0 \mathrm{~mm} \\
\text { 25: }\left|u_{\text {Schub }}\right| \geq 2 \mathrm{~mm} \\
\text { Zwischenbereich: } \\
\text { linear (s. Typ 2) }\end{array}$ & $\equiv 80$ & $\begin{array}{c}\text { 55: }\left|\varphi_{\text {rel }}\right|=0 \mathrm{mrad} \\
\text { 28: }\left|\varphi_{\text {rel }}\right| \geq 1,5 \mathrm{mrad} \\
\text { Zwischenbereich: } \\
\text { linear (s. Typ 2) }\end{array}$ \\
\hline KS1 & $\equiv 220$ & $\equiv 120$ & $\equiv 320$ & $\begin{array}{c}\text { 220: }\left|\varphi_{\text {rel }}\right|=0 \mathrm{mrad} \\
\text { 120: }\left|\varphi_{\text {rel }}\right| \geq 0,4 \mathrm{mrad} \\
\text { Zwischenbereich: } \\
\text { linear (s. Typ 2) }\end{array}$ \\
\hline KS2 & $\begin{array}{c}\text { 330: }\left|u_{\text {Schub }}\right| \leq 0,1 \mathrm{~mm} \\
\text { 200: }\left|u_{\text {Schub }}\right| \geq 0,4 \mathrm{~mm} \\
\text { Zwischenbereich: } \\
\text { linear (s. Typ 3) }\end{array}$ & $\begin{array}{c}170:\left|u_{\text {Schub }}\right|=0 \mathrm{~mm} \\
\text { 100: }\left|u_{\text {Schub }}\right| \geq 0,5 \mathrm{~mm} \\
\text { Zwischenbereich: } \\
\text { linear (s. Typ 2) }\end{array}$ & $\begin{array}{c}\text { 400: }\left|\varphi_{\text {rel }}\right| \leq 0,1 \mathrm{mrad} \\
\text { 310: }\left|\varphi_{\text {rel }}\right| \geq 0,2 \mathrm{mrad} \\
\text { Zwischenbereich: } \\
\text { linear (s. Typ 3) }\end{array}$ & $\begin{array}{c}\text { 250: }\left|\varphi_{\text {rel }}\right|=0 \mathrm{mrad} \\
\text { 120: }\left|\varphi_{\text {rel }}\right| \geq 0,25 \mathrm{mrad} \\
\text { Zwischenbereich: } \\
\text { linear (s. Typ 2) }\end{array}$ \\
\hline $\begin{array}{l}\text { KS3; } \\
\text { KS4 }\end{array}$ & $\equiv 260$ & $\begin{array}{c}\text { 180: }\left|u_{\text {Schub }}\right|=0 \mathrm{~mm} \\
\text { 135: }\left|u_{\text {Schub }}\right| \geq 0,4 \mathrm{~mm} \\
\text { Zwischenbereich: } \\
\text { linear (s. Typ 2) }\end{array}$ & $\begin{array}{c}\text { 400: }\left|\varphi_{\text {rel }}\right|=0 \mathrm{mrad} \\
\text { 300: }\left|\varphi_{\text {rel }}\right| \geq 0,23 \mathrm{mrad} \\
\text { Zwischenbereich: } \\
\text { linear (s. Typ 2) }\end{array}$ & $\begin{array}{c}\text { 230: }\left|\varphi_{\text {rel }}\right|=0 \mathrm{mrad} \\
\text { 140: }\left|\varphi_{\text {rel }}\right| \geq 0,35 \mathrm{mrad} \\
\text { Zwischenbereich: } \\
\text { linear (s. Typ 2) }\end{array}$ \\
\hline
\end{tabular}

Tabelle 31: Steifigkeitsparameter der Sub-Struktur
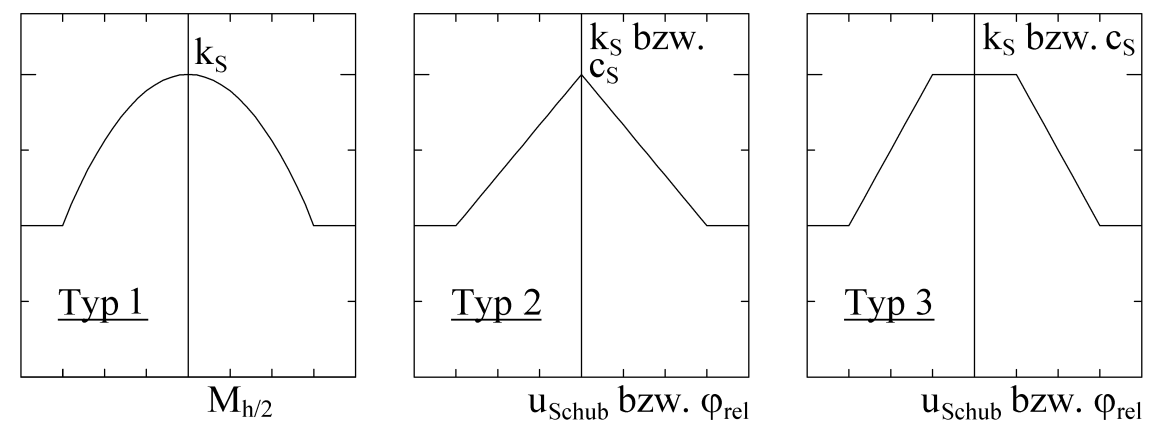

Abbildung 50: Qualitativer Verlauf der nichtlinearen Steifigkeitsbeziehungen Typ $1 \div$ Typ 3

Nachfolgend ist in Abbildung 51 beispielhaft die berechnete Kraft-Verformungs-Beziehung an Wand KS2 bei einer Normalkraft $N=180 \mathrm{kN}$ und den Einspanngraden $c_{\varphi}=200,500$ und $2.000 \mathrm{kNm} / \mathrm{mrad}$ dargestellt. 


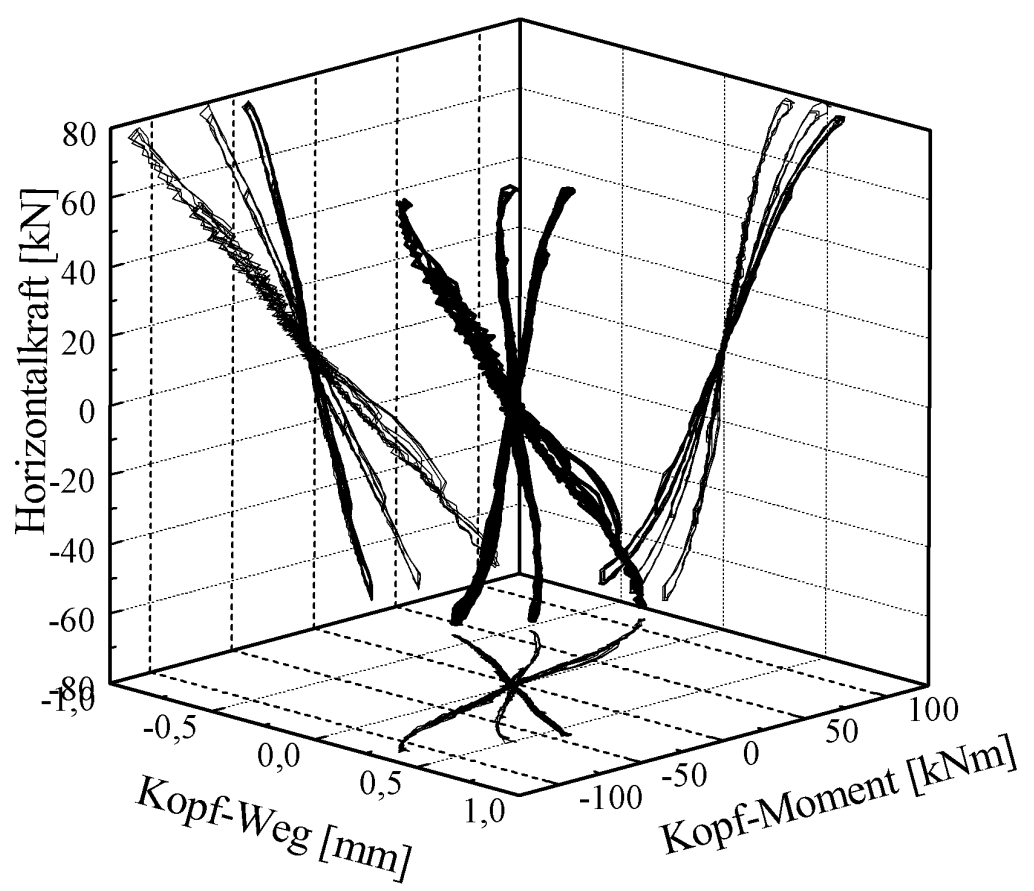

Abbildung 51: Rechnerische Kraft-Verformungs-Beziehung von Wand KS2 in Abhängigkeit des Wandkopfmomentes für $N=180 \mathrm{kN}$ - berechnete Werte in dem durch die Vorversuche abgedeckten $H$ - $M_{K o p f}$-Lastbereich

Der abgefahrene $H$ - $M_{K o p f}$-Bereich entspricht dem in Abbildung 49 Dargestellten (linke Projektionsebene). Der Vergleich von gemessenen und berechneten Graphen (Abbildung 49 und 51) zeigt eine gute Übereinstimmung im Rahmen der rechnerischen Vereinfachung. Wird der Kopfweg über dem $H-M_{K o p f}$-Lastbereich gerastert berechnet, ergibt sich folgendes Bild:

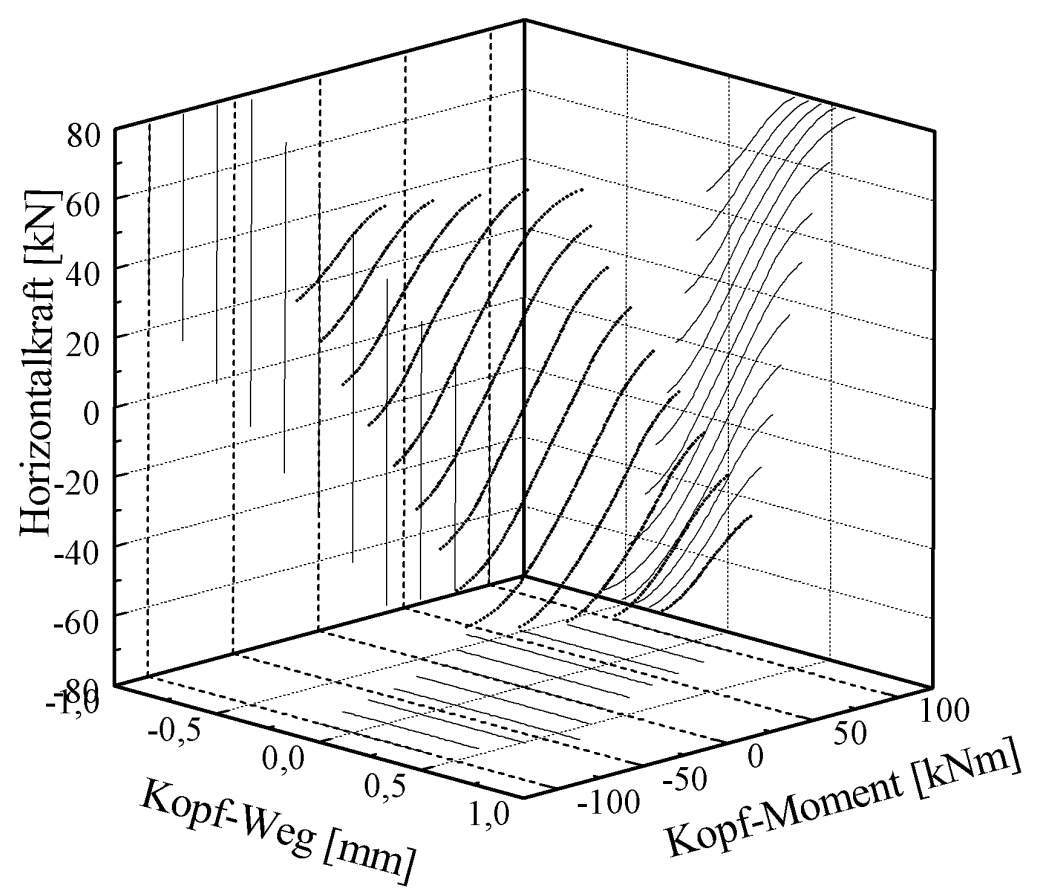

Abbildung 52: Rechnerische Kraft-Verformungs-Beziehung von Wand KS2 in Abhängigkeit des Wandkopfmomentes für $N=180 \mathrm{kN}$ 
Eine verbesserte Erfassung des Verhaltens der oberen Wände könnte grundsätzlich mit Hilfe einer Scheibenberechnung mit einem nichtlinearen Materialmodell erfolgen, die parallel zum Versuch mit der zugehörigen Lastgeschichte durchgeführt wird. Da zu Beginn der Versuche kein passendes Materialmodell zur Verfügung stand, bzw. erst im Rahmen dieser Arbeit entwickelt wurde (Kapitel 6), war dieser Weg hier nicht möglich. Des Weiteren ergeben sich daraus zusätzliche Problempunkte bezüglich der Kommunikation der einzelnen Programmteile, d.h. Mess- und Regelprogramm, Programm zur Lösung des Algorithmus der Pseudodynamik sowie der FE-Berechnung. Diese Möglichkeit kann im Zuge zukünftiger Arbeiten genutzt werden.

\section{Beurteilung der Steifigkeiten}

Für Vergleichszwecke werden die Schub- und Biegesteifigkeiten (Tabelle 31 und Tabelle 34) bei geringer Beanspruchungshöhe (= ungerissene Querschnitte) zu einer einzelnen Gesamtwandsteifigkeit

$$
k=\frac{1}{\frac{1}{k_{S i}}+\frac{h_{1}{ }^{2}}{4 \cdot c_{S i}}}
$$

bei Vernachlässigung einer Kopfeinspannung $\left(M_{\text {Kopf }}=0\right)$ zusammengefasst. Ergänzend dazu werden analog zur Vorgehensweise in Abschnitt 5.4.5 die Wände linear-elastisch und isotrop mit einem E-Modul in Anlehnung an Tabelle 24 mit Hilfe der FEM berechnet.

\begin{tabular}{|c|c|c|c|c|c|c|}
\hline \multirow[t]{2}{*}{ Wand } & \multicolumn{4}{|c|}{ Gesamtwandsteifigkeit $k[\mathrm{kN} / \mathrm{mm}]$} & \multirow[b]{2}{*}{$\begin{array}{c}E \\
{\left[\mathbf{N} / \mathbf{m m}^{2}\right]} \\
\end{array}$} & \multirow[b]{2}{*}{$\begin{array}{l}I_{\text {Wand }} \\
{[\mathrm{m}]} \\
\end{array}$} \\
\hline & $\begin{array}{c}1.0 G \\
(\mathrm{~N}=270 \mathrm{kN}) \\
\end{array}$ & $\begin{array}{c}2.0 G \\
(\mathrm{~N}=180 \mathrm{kN}) \\
\end{array}$ & $\begin{array}{c}\begin{array}{c}3.0 G \\
(\mathrm{~N}=90 \mathrm{kN})\end{array} \\
\end{array}$ & FEM & & \\
\hline D & 61,9 & 444,0 & 28,7 & 143,1 & 6000 & 2,5 \\
\hline $\mathbf{E}$ & 53,3 & 39,8 & 28,4 & 167,8 & 7500 & 2,43 \\
\hline $\mathbf{F}$ & 29,3 & 22,3 & 16,9 & 85,0 & 10000 & 1,63 \\
\hline KS1 & 144,3 & 101,8 & 62,4 & 238,7 & 10000 & 2,5 \\
\hline KS2 & 164,0 & 137,8 & 79,1 & 238,7 & 10000 & 2,5 \\
\hline KS3, KS4 & 160,3 & 123,9 & 77,5 & 238,7 & 10000 & 2,5 \\
\hline
\end{tabular}

Tabelle 32: Rechnerische Gesamtwandsteifigkeit $k$ für $M_{K o p f}=0$ und Ergebnis einer linear-elastischen Kragscheibenberechnung für eine Geschosshöhe $h=2,6 \mathrm{~m}$

Der Vergleich der Steifigkeit von Wand D im untersten Geschoss mit den Werten aus den Versuchen der ersten Stufe bei geringer Belastung von $60,8 \div 77,7 \mathrm{kN} / \mathrm{mm}^{2}$ (Tabelle 28) zeigt die gute Näherung der obigen Beschreibung (34).

Insgesamt ist im Vergleich mit der linear-elastischen Berechnung als Kragscheibe erkennbar, dass das FE-Modell im Zustand I mit ungerissenen Querschnitten die Wände deutlich zu steif erfasst. Im untersten Geschoss liegen die Verhältniswerte zwischen 1,5 und 3,3. Mit abnehmender Normalkraft nehmen die Unterschiede weiter $\mathrm{zu}$ - im obersten Geschoss beträgt das Verhältnis der Steifigkeiten zwischen 3,0 und 5,9.

Die Betrachtung ohne Kopfeinspannung stellt den Fall mit der geringsten rechnerischen Wandsteifigkeit dar. Als oberer Grenzwert ergibt sich bei starrer Kopfeinspannung die Wandsteifigkeit direkt zu der Schubsteifigkeit $k_{S, i}$ (Tabelle 34). Der Vergleich der Ergebnisse mit der zugehörigen FE-Berechnung bei verhinderter Kopfverdrehung ist in Tabelle 33 zusam- 
mengestellt. Das Steifigkeitsverhältnis im untersten Geschoss liegt zwischen 1,1 und 4,1 und nimmt bis zum oberen Geschoss auf 2,4 bis 7,0 zu.

\begin{tabular}{|c|c|c|c|c|c|c|}
\hline \multirow[t]{2}{*}{ Wand } & \multicolumn{4}{|c|}{ Gesamtwandsteifigkeit $k[\mathrm{kN} / \mathrm{mm}]$} & \multirow[b]{2}{*}{$\begin{array}{c}E \\
{\left[\mathbf{N} / \mathbf{m m}^{2}\right]}\end{array}$} & \multirow[b]{2}{*}{$\begin{array}{c}l_{\text {Wand }} \\
{[\mathrm{m}]} \\
\end{array}$} \\
\hline & $\begin{array}{c}1.0 G \\
(N=270 \mathrm{kN})\end{array}$ & $\begin{array}{c}2.0 G \\
(\mathrm{~N}=180 \mathrm{kN})\end{array}$ & $\begin{array}{c}3.0 G \\
(\mathrm{~N}=90 \mathrm{kN})\end{array}$ & FEM & & \\
\hline D & 100 & 70 & 47 & 263,8 & 6.000 & 2,5 \\
\hline $\mathbf{E}$ & 80 & 60 & 45 & 315,5 & 7.500 & 2,43 \\
\hline $\mathbf{F}$ & 50 & 42 & 35 & 207,0 & 10.000 & 1,63 \\
\hline KS1 & 300 & 220 & 120 & 440,5 & 10.000 & 2,5 \\
\hline KS2 & 400 & 330 & 170 & 440,5 & 10.000 & 2,5 \\
\hline KS3, KS4 & 350 & 260 & 180 & 440,5 & 10.000 & 2,5 \\
\hline
\end{tabular}

Tabelle 33: Rechnerische Gesamtwandsteifigkeit $k$ für $c_{\varphi, K o p f}=\infty$ und Ergebnis einer linear-elastischen Scheibenberechnung mit Kopfeinspannung für eine Geschosshöhe $h=2,6 \mathrm{~m}$

Bei den obigen vereinfachten Vergleichen zeigten sich größere Unterschiede zwischen den aus den Anfangsfedersteifigkeiten (= ungerissene Querschnitte) ermittelten Wandsteifigkeiten und denen einer linear-elastisches FE-Berechnung. Die tatsächliche Wandsteifigkeit für Schubbeanspruchung wird mit üblichen Annahmen (linear-elastische Berechnung, isotropes Material, zugeh. E-Modul) somit deutlich zu hoch abgeschätzt.

\subsubsection{Ablauf}

Aufgrund des im Vergleich zur ersten Simulationsstufe erheblich gestiegenen numerischen Aufwandes, wurden diese Aufgaben auf zwei Computer aufgeteilt. Die Kommunikation untereinander erfolgte mit Hilfe einer $C++$-Routine über ein serielles Nullmodemkabel. Die Programmierung des Algorithmusses der Pseudodynamik erfolgte mit dem Programmsystem MAPLE [76]. Durch die Vielzahl der darin implementierten Routinen für Matrizenoperationen konnte der Programmieraufwand in Grenzen gehalten werden. Für die Mess-, Steuer- und Regelungsroutinen kam das Programmsystem CATMAN [13] zur Anwendung. Das Einlesen der Messwerte sowie die Zylindersteuerung erfolgtem analog zur ersten Simulationsstufe.

Der Ablauf wird in dem in Abbildung 53 dargestellten Schema erläutert. Die grundlegende Aufteilung der Aufgaben auf die beiden Computer erfolgte folgendermaßen:

\section{Computer 1:}

- Messen und Protokollieren der Daten (Messwerte inkl. abgeleiteter Größen)

- Steuern der Zylinder über die drei digitalen Servo-Controller

- Visualisierung maßgebender Zustandgrößen (Zeitschritt, Kraft-Verformungs-Linie, aktuelle Zustandgrößen)

\section{Computer 2:}

- Durchführung des Algorithmusses der Pseudodynamik

- Leitung der Kommunikation

Die Visualisierung erfolgte analog zur ersten Simulationsstufe. 


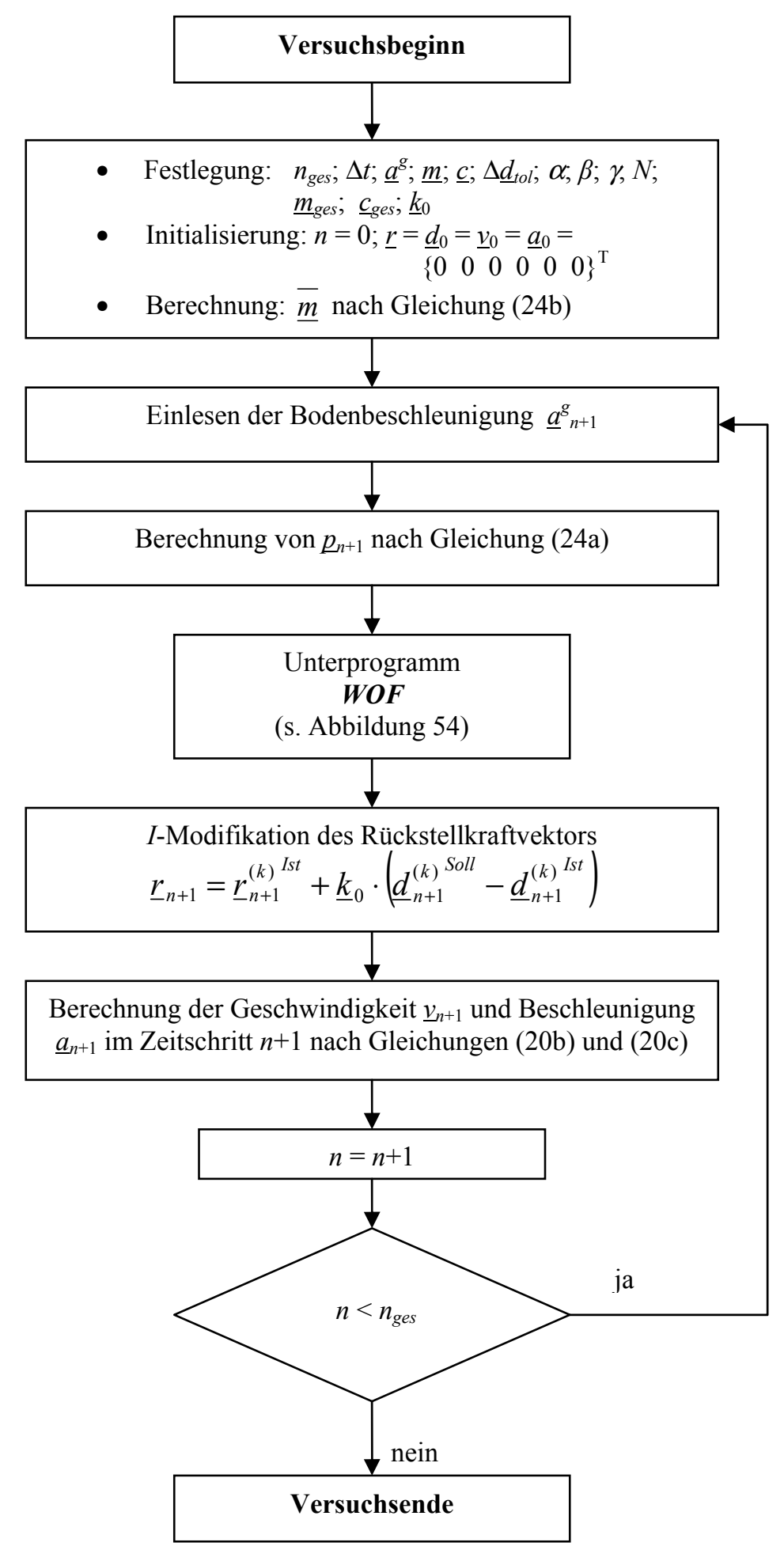

Abbildung 53: Ablaufschema für die Versuche der zweiten Simulationsstufe

Im Unterprogramm WOF ist das implizite Integrationsverfahren mit den Mess- und Regelungsroutinen zusammengefasst. 


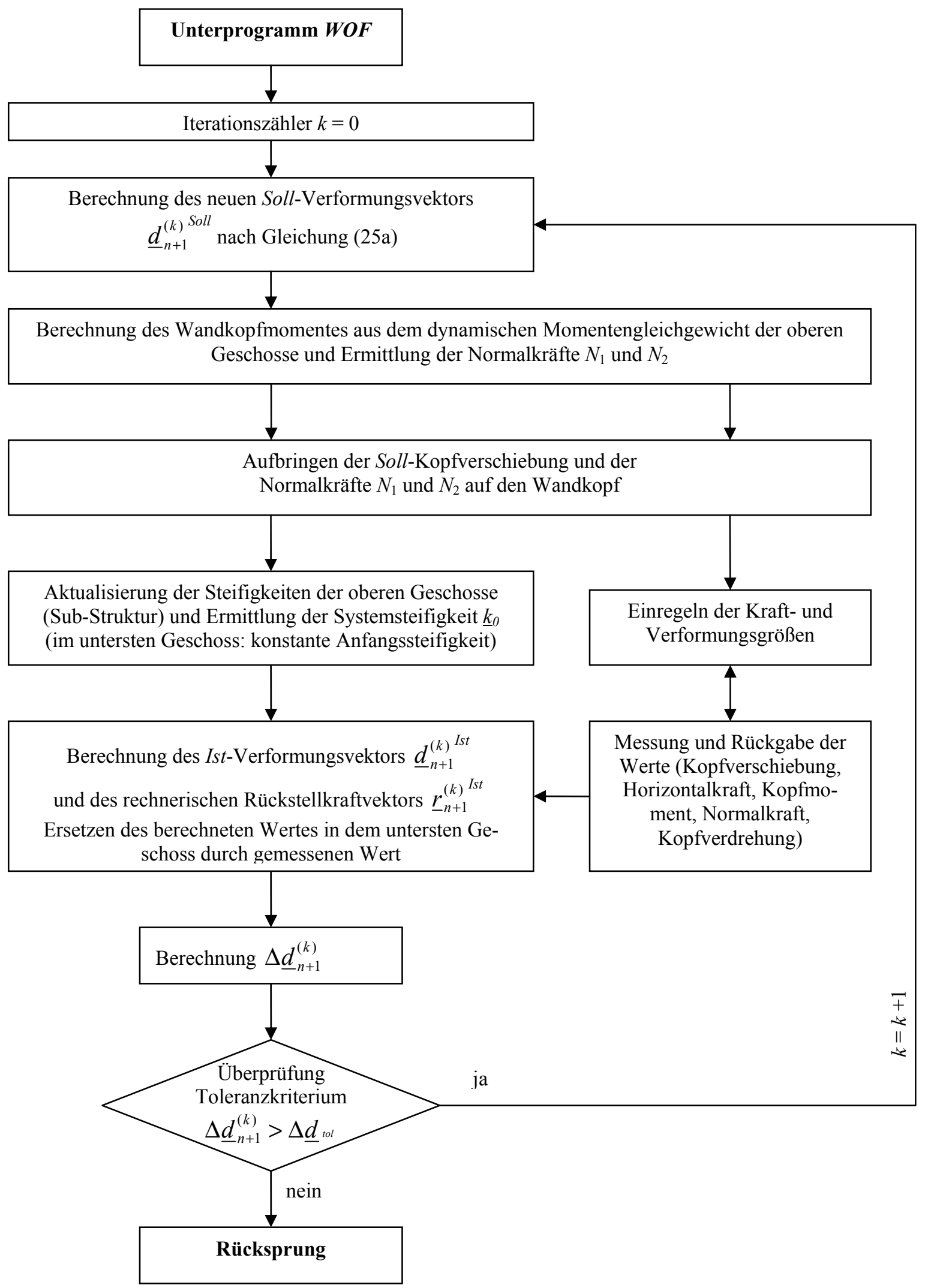

Abbildung 54: Ablaufschema des Unterprogramms WOF der zweiten Simulationsstufe 


\subsubsection{Ergebnisse}

Die Ausgangsparameter der Versuche und die Ergebnisse in Form maximaler Horizontalkräfte und Kopfverschiebungen sind nachfolgend in Tabelle 34 dargestellt. Die in Spalte 2 angegebenen Steifigkeiten $k_{S, i}$ und $c_{S, i}$ entsprechen dabei den Anfangssteifigkeiten aus Tabelle 31 .

\begin{tabular}{|c|c|c|c|c|c|}
\hline $\begin{array}{l}\text { Wand / } \\
\text { Versuch }\end{array}$ & $\begin{array}{c}\text { Anfangssteifigkeit }{ }^{1), 2)} \\
c_{S, i}[\mathrm{kNm} / \mathrm{mrad}] \\
k_{S, i}[\mathrm{kN} / \mathrm{mm}]\end{array}$ & $\begin{array}{c}\text { Dämpfer }{ }^{1)} \\
c_{D M P, i} \\
{[\mathbf{k N s} / \mathbf{m}]}\end{array}$ & $\begin{array}{c}\text { BB-ZV }^{3)} ; \\
\text { Lastfaktor } f \text { [-] }\end{array}$ & $\begin{array}{c}\text { maximale } \\
\text { Kopfver- } \\
\text { schiebung } \\
w_{\text {Kopf }}[\mathrm{mm}] \\
\end{array}$ & $\begin{array}{c}\text { maximale } \\
\text { Horizontal- } \\
\text { kraft } \\
H[\mathrm{kN}] \\
\end{array}$ \\
\hline D-1 & \multirow[t]{5}{*}{$\begin{array}{c}275 ; 200 ; 125 \\
100 ; 70 ; 47\end{array}$} & \multirow[t]{5}{*}{$\begin{array}{c}172,5 ; 2,8 ; \\
13,1\end{array}$} & $\begin{array}{c}\text { ZV1-B3 }\left(\mathrm{a}_{\mathrm{g}}=0,4 \mathrm{~m} / \mathrm{s}^{2}\right) \\
\mathrm{f}=0,15\end{array}$ & 0,33 & 22,8 \\
\hline D-2 & & & $\mathrm{f}=1$ & 2,11 & 109,9 \\
\hline D-3 & & & $\mathrm{f}=1,5$ & 3,46 & 140,0 \\
\hline D-4 & & & $\mathrm{f}=2$ & 4,11 & 154,9 \\
\hline D-5 & & & $\mathrm{f}=2,67$ & $4,52^{4)}$ & $145,5^{4)}$ \\
\hline E-1 & \multirow[t]{2}{*}{$\begin{array}{l}270 ; 200 ; 130 \\
80 ; 60 ; 45\end{array}$} & \multirow[t]{2}{*}{$35 ; 1 ; 4,3$} & $\begin{array}{c}\text { ZV4-C3 }\left(\mathrm{a}_{\mathrm{g}}=0,4 \mathrm{~m} / \mathrm{s}^{2}\right) \\
\mathrm{f}=1\end{array}$ & 1,5 & 61,8 \\
\hline E-2 & & & $\mathrm{f}=2,67$ & $4,27^{4)}$ & $112,1^{4)}$ \\
\hline F-1 & \multirow[t]{3}{*}{$\begin{array}{c}120 ; 80 ; 55 \\
50 ; 42 ; 35\end{array}$} & \multirow[t]{3}{*}{$240 ; 70 ; 6$} & $\begin{array}{c}\text { ZV3-A1 }\left(\mathrm{a}_{\mathrm{g}}=0,4 \mathrm{~m} / \mathrm{s}^{2}\right) \\
\mathrm{f}=2\end{array}$ & 1,85 & 55,7 \\
\hline F-2 & & & $\begin{array}{c}\text { ZV4-C3 }\left(\mathrm{a}_{\mathrm{g}}=0,4 \mathrm{~m} / \mathrm{s}^{2}\right) \\
\mathrm{f}=3\end{array}$ & 5,37 & 115,4 \\
\hline F-3 & & & $\begin{array}{c}\text { ZV3-A1 }\left(\mathrm{a}_{\mathrm{g}}=0,4 \mathrm{~m} / \mathrm{s}^{2}\right) \\
\mathrm{f}=7\end{array}$ & $8,0^{4)}$ & $110,6^{4)}$ \\
\hline KS1-1 & \multirow[t]{8}{*}{$\begin{array}{l}470 ; 320 ; 220 \\
300 ; 220 ; 120\end{array}$} & $700 ; 60 ; 40$ & $\begin{array}{c}\text { ZV3-A1 }\left(\mathrm{a}_{\mathrm{g}}=0,4 \mathrm{~m} / \mathrm{s}^{2}\right) \\
\mathrm{f}=2\end{array}$ & $0,87^{4)}$ & $76,6^{4)}$ \\
\hline KS1-2 & & \multirow[t]{2}{*}{$525 ; 200 ; 0$} & $\mathrm{f}=2$ & 1,18 & 108,2 \\
\hline KS1-3 & & & $\mathrm{f}=3$ & 1,68 & 144,7 \\
\hline KS1-4 & & \multirow[t]{5}{*}{$570 ; 85 ; 30$} & $\mathrm{f}=3$ & $1,76^{4)}$ & $120,7^{4)}$ \\
\hline KS1-5 & & & $\mathrm{f}=3$ & 1,74 & 120,6 \\
\hline KS1-6 & & & $\mathrm{f}=4$ & 2,40 & 143,4 \\
\hline KS1-7 & & & $\mathrm{f}=6$ & 3,36 & 165,7 \\
\hline KS1-8 & & & $\mathrm{f}=8$ & $3,21^{4)}$ & $149,9^{4)}$ \\
\hline KS2-1 & \multirow[t]{2}{*}{$\begin{array}{l}470 ; 400 ; 250 \\
400 ; 330 ; 170\end{array}$} & \multirow[t]{2}{*}{$700 ; 60 ; 40$} & $\begin{array}{c}\text { ZV3-A1 }\left(\mathrm{a}_{\mathrm{g}}=0,4 \mathrm{~m} / \mathrm{s}^{2}\right) \\
\mathrm{f}=1\end{array}$ & 0,37 & 47,3 \\
\hline KS2-2 & & & $\mathrm{f}=8$ & $3,62^{4)}$ & $209,4^{4)}$ \\
\hline KS3-1 & \multirow[t]{4}{*}{$\begin{array}{l}500 ; 400 ; 230 \\
350 ; 260 ; 180\end{array}$} & \multirow[t]{4}{*}{$675 ; 45 ; 50$} & $\begin{array}{c}\mathrm{ZV} 1-\mathrm{B} 3\left(\mathrm{a}_{\mathrm{g}}=0,4 \mathrm{~m} / \mathrm{s}^{2}\right) \\
\mathrm{f}=1,5\end{array}$ & 1,26 & 117,3 \\
\hline KS3-2 & & & $\mathrm{f}=2$ & 2,24 & 153,3 \\
\hline KS3-3 & & & $\mathrm{f}=2,5$ & 3,25 & 174,5 \\
\hline KS3-4 & & & $\mathrm{f}=3,5$ & $3,88^{4)}$ & $176,0^{4)}$ \\
\hline KS4-1 & \multirow[t]{4}{*}{$\begin{array}{l}500 ; 400 ; 230 \\
350 ; 260 ; 180\end{array}$} & \multirow[t]{4}{*}{$675 ; 45 ; 50$} & $\begin{array}{c}\text { ZV1-B3 }\left(\mathrm{a}_{\mathrm{g}}=0,4 \mathrm{~m} / \mathrm{s}^{2}\right) \\
\mathrm{f}=1,5\end{array}$ & 1,12 & 110,6 \\
\hline KS4-2 & & & $\mathrm{f}=2$ & 2,14 & 143,2 \\
\hline KS4-3 & & & $\mathrm{f}=2,5$ & 3,19 & 165,1 \\
\hline KS4-4 & & & $\mathrm{f}=3,5$ & $5,42^{4)}$ & $169,3^{4)}$ \\
\hline \multicolumn{6}{|c|}{$\begin{array}{l}\text { 1) Nummerierung / Reihenfolge: } 1,2,3: \text { unteres, mittleres, oberes Geschoss } \\
\text { Einspannung in Deckendrehfedern je Geschoss mit } c_{D, i}=300 \mathrm{kNm} / \mathrm{mrad} \text { (Ausnahme: KS1-2 \& 1-3: 600) } \\
\text { Geschosshöhe }=2,60 \mathrm{~m} ; 3 \text { Einzelmassen: } m_{1,2,3}=29 \mathrm{t} \\
\text { 2) Verlauf der Steifigkeiten } c_{S, i} \text { und } k_{S, i}: \mathrm{s} \text {. Tabelle } 31 \\
\text { 3) Bodenbeschleunigungszeitverlauf, Boden-Baugrund-Kombination; Grundwert der Bodenbeschleunigung } \\
\text { 4) Versuche nicht vollständig durchgeführt } \Rightarrow \text { Ergebnisse bis zum betreffenden Zeitpunkt }\end{array}$} \\
\hline
\end{tabular}

Tabelle 34: Parameter und Ergebnisse der zweiten Simulationsstufe 
Mit den Einzelmassen $m_{i}$ und den Dämpfern $c_{D M P, i}$ bestimmen sich am linear-elastischen Ersatzsystem nach Abbildung 57 die Eigenperioden $T_{i}$ und die Dämpfungsgrade $\zeta_{i}$ (Tabelle 36).

Die 28 Hauptversuche wurden mit verschiedenen Bodenbeschleunigungszeitverläufen und unterschiedlichen Lastfaktoren $f$ durchgeführt. Die Zeitverläufe mit den zugehörigen Antwortspektren sind im Anhang A.1 dargestellt.

Die im Anhang A.3.2 dargestellten Kraft-Verformungs-Linien waren in weiten Bereichen annähernd linear-elastisch mit geringer Völligkeit. Lediglich in der Nähe der Maximalbeanspruchung war bei einigen Versuchen ein leichtes Abflachen erkennbar, z.B. Wand KS1-7 und KS3-3.

Es zeigte sich, dass das aufgebrachte Kopfmoment einen entscheidenden Einfluss auf die Wandsteifigkeit und entsprechend bei vorgegebener Kopfverschiebung auf die sich einstellende Horizontalkraft hat. Während der Versuche wurde im Zuge des impliziten Zeitintegrationsverfahrens das Kopfmoment kontinuierlich über das Momentengleichgewicht in der SubStruktur berechnet und auf den Wandkopf aufgebracht. Über die Abhängigkeit der Wandsteifigkeit kam es somit in den Kraft-Verformungs-Linien zu teilweisen zackigen Verläufen, insbesondere im Bereich der Umkehrpunkte.

\subsubsection{Versagensbilder}

Im Gegensatz zu den Versuchen der ersten Simulationsstufe wurde in der zweiten Stufe die Kopfverdrehung durch den Ansatz der Deckeneinspannung $c_{D, i}$ im Zusammenwirken mit der Sub-Struktur behindert. Dieser Einfluss ist auch in den aufgetretenen Bruchbildern, d.h. im Ort und in der Art der Risse, deutlich zu erkennen (Rissbilder s. Anhang A.2.2).

\section{Wand D}

Bei den ersten drei Hauptversuchen an Wand D (Hochlochziegel und Normalmörtel IIa, unvermörtelte Stoßfugen) waren bis zu einem Lastfaktor $f=1,5$ keinerlei Risse oder sonstige Schädigungen erkennbar.

Im vierten Versuch D-4 (Lastfaktor $f=2$ ) traten im Zeitschritt 405 bei einer Horizontalkraft von $H=-128 \mathrm{kN}$ die ersten beiden Steinzugrisse 1 und 2 (bzw. R1 und R2) in Wandmitte und seitlich versetzt am Wandkopf auf (s. Abbildung A-13 und A-14). Bei der anschließenden Lastumkehr bildeten sich im Zeitschritt 428 bei $H=155 \mathrm{kN}$ seitlich am Wandfuß zusätzliche schräge Steinzugrisse aus (Riss 3 und 4 bzw. R3 und R4).

Im nachfolgenden Versuch D-5 mit erhöhtem Lastfaktor $(f=2,66)$ traten die Steinzugrisse 5, 6 und 7 (bzw. R5, R6 und R7) bei einer Horizontallast $H=129 \mathrm{kN}$ in Wandmitte auf (Zeitschritt 389). Nach Lastumkehr stellten sich im Zeitschritt 403 die nächsten Risse 8 und 9 (bzw. R8 und R9) am Wandfuß bei $H=-146 \mathrm{kN}$ ein. Das Gesamtversagen von Wand D erfolgte anschließend in Form eines durchgehenden Diagonalrisses, der größtenteils durch die Steine und bereichsweise auch abgetreppt durch Stoß- und Lagerfugen verlief (Bezeichnung „B“ in den Rissbildern).

\section{Wand E}

Bei Wand E (Plan-Hochlochziegel mit Dünnbettmörtel im Tauchverfahren, unvermörtelte Stoßfugen) waren nach Abschluss des ersten Versuches E-1 $(f=1,0)$ keinerlei Risse sichtbar. 
Im zweiten Versuch mit dem Lastfaktor $f=2,67$ traten die ersten Steinzugrisse 1 und 2 im Zeitschritt 253 bei einer Horizontallast von $H=94 \mathrm{kN}$ auf (s. Abbildung A-15). Im weiteren Verlauf bildeten sich bei alternierender Last $H=-107 \div 100 \mathrm{kN}$ die diagonal gekreuzten Risse $3 \div 42$ aus. Diese verliefen größtenteils abgetreppt durch die Stoß- und Lagerfugen. Im Zeitschritt 557 versagte schließlich die Wand bei einer Horizontallast von $H=-112 \mathrm{kN}$ in Form der Risse $43 \div 47$, die mehrheitlich schräg durch die Steine verliefen. Der Versuch musste zu diesem Zeitpunkt abgebrochen werden, da infolge der Wandschädigung und des starken Steifigkeitsabfalles kein Gleichgewicht im Lösungsalgorithmus gefunden werden konnte.

Da die Gesamttragfähigkeit der Wand in diesem Zustand noch teilweise gegeben war, wurde ein abschließender handgesteuerter Nachversuch mit zwei vollen Lastzyklen unter konstanter Normalkraft $N \equiv 270 \mathrm{kN}$ und Kopfmoment $M_{\text {Kopf }} \equiv 0 \mathrm{kNm}$ durchgeführt. Die maximale Horizontalkraft betrug $H=116 \mathrm{kN}$ und die maximale Kopfverschiebung $w_{\text {Kopf }}=13,5 \mathrm{~mm}$. Die zugehörigen Steinzugrisse - mehrheitlich in den äußeren Bereichen des Wandfußes - sind im Rissbild mit , $x$ “ bezeichnet.

\section{Wand F}

In den Eckbereichen der untersten Lagerfuge von Wand F (Plan-Hochlochziegel mit NM IIIa, unvermörtelte Stoßfugen) wurden zur Vermeidung von Spannungsspitzen Elastomere angeordnet (s. Abbildung 28). Dadurch verringerte sich die effektive Wandlänge am Fuß um etwa 1/3. Als Ergebnis reduzierten sich sowohl die rechnerischen Schubsteifigkeiten $k_{S, i}$ als auch die Biegesteifigkeiten $c_{S, i}$ (s. Tabelle 31 und Tabelle 34). Dieser Effekt war beim Biegeverformungsanteil infolge der sich teilweise ausgebildeten Rotation um den Fußpunkt mit einer Steifigkeitsabminderung von in Mittel 57\% deutlich stärker ausgeprägt als bei der Schubsteifigkeit mit einer Abminderung um in Mittel 30\%. Bei gleichen Geschossmassen und Deckendrehfedern lag die erste Eigenperiode im Vergleich zu Wand E um 18\% höher.

Im zweiten Versuch F-2 (Lastfaktor $f=3$ ) traten die ersten Risse $1 \div 19$ im Zeitschritt 564 bei einer Horizontallast $H=-105 \mathrm{kN}$ auf (s. Abbildung A-16). Diese verliefen diagonal vom Wandkopf bis zum Beginn der Elastomerlager in der gegenüberliegenden unteren Wandecke (Riss 19). Dabei handelte es sich größtenteils um schräge Steinzugrisse und nur vereinzelt um Risse durch Stoß- und Lagerfugen. Im weiteren Verlauf traten im übernächsten Lastzyklus bei $H=113 \mathrm{kN}$ im Zeitschritt 638 die Risse $20 \div 40$ auf. Diese bildeten sich spiegelbildlich zu den vorhergehenden Rissen und verliefen im Wesentlichen durch die Steine. Im Zeitschritt 739 schließlich traten die Steinzugrisse $41 \div 50$ bei einer Horizontallast von $H=105 \mathrm{kN}$ auf.

Im letzten Versuch F-3 (Lastfaktor $f=7$ ) erweiterte sich im Zeitschritt 260 der Riss 45 vertikal zu Riss $45+(H=107 \mathrm{kN})$. Im weiteren Verlauf traten die Risse $51 \div 57$ bei $H=109 \mathrm{kN}$ (Zeitschritt 543) sowie die Risse 58 und 59 bei $H=-89 \mathrm{kN}$ (Zeitschritt 563) auf. Der Versuchsabbruch erfolgte im Zeitschritt 563 bei $H=-102 \mathrm{kN}$ (Risse $60 \div 68$ ). Das abschließende Rissbild der Wand war durch Steindruckversagen in Form von senkrecht zur Wandebene aufgespalteten Steinen (Nummer $62 \div 65$ ) sowie durch schräge Steinzugrisse gekennzeichnet.

\section{Wand KS1}

Wand KS1 wurde mit KS-Planelementen und Dünnbettmörtel mit hoher Haftscherfestigkeit und $2 \mathrm{~mm}$ Stützkorn hergestellt. Die Stoßfugen waren im Gegensatz zur Referenzwand KS2 nicht vermörtelt. 
Im ersten Versuch KS1-1 (Lastfaktor $f=2$ ) wurde aufgrund eines fehlerhaften Vertikalmessgebers das maximal mögliche Kopfmoment im Zeitschritt 360 voll abtreibend aufgebracht, was im Zusammenwirken mit der Horizontalkraft zu einem Klaffen in der untersten Lagerfuge führte (Riss 1 in Abbildung A-17 bzw. R1 in Abbildung A-18).

Für die nächsten beiden Versuche KS1-2 (aufgetretene Gleitverschiebung zwischen Fundamentplatte und Hallenboden von 0,25 mm infolge zu geringer Vorspannung) und KS1-3 mit einem Lastfaktor $f=2$ bzw. $f=3$ wurde die Deckeneinspannung auf $c_{D, i}=600 \mathrm{kNm} / \mathrm{mrad}$ verdoppelt. Weitere Risse traten hier und in den folgenden Versuchen KS1-4 (musste aufgrund des Ausfalls eines Teils der Hydraulik im Zeitschritt 329 abgebrochen werden) und KS1-5 mit jeweils dem Lastfaktor $f=3$ nicht auf.

Im Versuch KS1-6 (Lastfaktor $f=4$ ) klaffte Riss 1 bzw. R1 in der untersten Lagerfuge im Zeitschritt 322 bei einer Horizontallast von $H=143 \mathrm{kN}$ sowie im Versuch KS1-7 (Lastfaktor $f=6$ ) im Zeitschritt 324 bei $H=149 \mathrm{kN}$.

Beim letzten Versuch KS1-8 mit dem Lastfaktor $f=8$ traten im Zeitschritt 123 bei einer Horizontalkraft $H=-150 \mathrm{kN}$ die Risse $2 \div 11(\mathrm{R} 2 \div \mathrm{R} 11)$ auf. Das Versagensbild mit einer Kopfverschiebung von $-3,2 \mathrm{~mm}$ war durch Gleitverschiebungen mit abgetrepptem Rissbild sowie einem einzelnen Steinriss in der unteren rechten Wandecke (Riss 11 bzw. R11) gekennzeichnet. Der Versuch musste im Zeitschritt 126 abgebrochen werden, da infolge der Wandschädigung und des starken Steifigkeitsabfalles kein Gleichgewicht im Lösungsalgorithmus gefunden werden konnte.

Anschließend wurde ein handgesteuerter Nachversuch unter Variation der Normalkraft und des Kopfmomentes durchgeführt. Zu Beginn wurde unter konstanter Normalkraft und einer elastischen Kopfeinspannung von $c_{\varphi, \text { Kopf }}=250 \mathrm{kNm} / \mathrm{mrad}$ die Horizontalkraft im Bereich von $-20 \div-120 \mathrm{kN}$ zyklisch variiert. Es zeigten sich infolge der aufgetretenen Gleitverschiebungen bleibende Verformungen. Anschließend wurde die Normalkraft auf $N=400 \mathrm{kN}$ erhöht und die Kopfverschiebung sukzessive bei einem konstanten Kopfmoment $M_{\text {Kopf }} \equiv 0 \mathrm{kNm}$ erhöht. Bei einer Horizontalkraft von $H=166 \mathrm{kN}$ klaffte der Wandfuß (Riss $1 \mathrm{bzw}$. R1) und bei $H=180 \mathrm{kN}$ traten zusätzlich die Risse $12 \div 15$ (bzw. R12 $\div \mathrm{R} 15$ ) auf. Dieses Versagensbild kann als Kombination aus Gleiten in der Lagerfuge (abgetreppt durch Stoßfuge) sowie einer Biegeverformung mit klaffendem Querschnitt am Wandfuß beschrieben werden. Anschließend wurde ein gegendrehendes Kopfmoment von $M_{\text {Kopf }}=-150 \mathrm{kNm}$ aufgebracht $(N=387 \mathrm{kN})$ und die Kopfverschiebung weiter erhöht. Bei einer Horizontalkraft von $H=208 \mathrm{kN}$ traten die weiteren Schubrisse $16 \div 21$ (bzw. $\mathrm{R} 16 \div \mathrm{R} 21$ ) auf, die teilweise durch

die Steine verliefen. Anschließend wurde nach einer Reduzierung der Normalkraft auf $N=200 \mathrm{kN}$ sowie einem Kopfmoment von $M_{\text {Kopf }}=0 \mathrm{kNm}$ die Verschiebung weiter gesteigert. Es stellten sich bei einem Horizontalkraftplateau von $H=100 \div 110 \mathrm{kN}$ die weiteren Schubrisse $22 \div 25$ (bzw. R22 $\div$ R25) in den Stoß- und Lagerfugen ein.

\section{Wand KS2}

Im Vergleich zu Wand KS1 wurden die Stoßfugen der Referenzwand KS2 voll vermörtelt. Im zweiten Versuch KS2-2 (Lastfaktor $f=8$ ) trat bei einer Horizontallast von $H=-141 \mathrm{kN}$ ein Klaffen in der untersten Lagerfuge auf (Riss 1 in Abbildung A-19 bzw. R1 in Abbildung A20). Bei Lastumkehr erfolgte im Zeitschritt $112(H=146 \mathrm{kN})$ ein Abheben der Wand an der gegenüberliegenden Wandecke (Riss 2 bzw. R2). Das Versagen der Wand trat bei der Maximallast von $H=209 \mathrm{kN}$ im Zeitschritt 124 auf. Das Versagensbild (Riss 3 bzw. R3) war 
durch eine Kombination von schrägen Steinzugrissen und abgetreppten Rissen durch Stoßund Lagerfugen gekennzeichnet.

Nach Abbruch des Hauptversuches wurde unter konstanter Normalkraft $N \equiv 270 \mathrm{kN}$ ein zusätzlicher Nachversuch durchgeführt. Zu Beginn wurde bei einem Kopfmoment $M_{\text {Kopf }} \equiv 0 \mathrm{kNm}$ die Kopfverschiebung bis zum Klaffen des Querschnitts am Wandfuß erhöht (Riss 4 und R4). Es stellte sich insgesamt ein Gleiten in der Lagerfuge ein (abgetreppter Riss 5 und R5) mit großen bleibenden Verformungen.

Nach dem Erreichen einer Kopfverschiebung von $20 \mathrm{~mm}$ wurde ein gegendrehendes Moment von $M_{\text {Kopf }}=-208 \mathrm{kNm}$ aufgebracht, wodurch sich die Kopfverschiebung reduzierte und bei konstantem Pressenweg infolge vergrößerter Rahmenverschiebung die Horizontalkraft erhöhte (s. a. Nachversuch an Wand KS4). Anschließend wurde die Kopfverschiebung weiter bis zum Maximalwert von 27 mm erhöht. Es stellten sich die Risse $6 \div 9$ (bzw. R6 $\div$ R9) ein, die infolge des gegendrehenden Kopfmomentes größtenteils durch die Steine verliefen.

Nach teilweiser Lastreduktion wurde das Kopfmoment auf $M_{\text {Kopf }} \equiv 0 \mathrm{kNm}$ festgelegt und die Kopfverschiebung weiter reduziert. Die sich einstellende Gleitverformung (Riss 3 und R3) ist in der Last-Verformungs-Beziehung durch ein Plateau gekennzeichnet. Nach Lastreduktion war eine bleibende Verformung in Form der Rissöffnung 3 bzw. R3 erkennbar.

\section{Wand KS3}

Bei Wand KS3 wurden KS-Plansteine in Dünnbettmörtel mit geringer Haftscherfestigkeit verlegt. Die Stoßfugen wurden nicht vermörtelt. Die ersten Risse (s. Abbildung A-21) traten im zweiten Versuch KS3-2 (Lastfaktor $f=2$ ) in Form eines Klaffens in der untersten Lagerfuge auf, nämlich Riss 1 (Zeitschritt 384, $H=124 \mathrm{kN}$ ), Riss 2 (Zeitschritt 399, $H=-131 \mathrm{kN}$ ) sowie Riss 3 (Zeitschritt 402, $H=-144 \mathrm{kN}$ ).

Im dritten Versuch KS3-3 (Lastfaktor $f=2,5$ ) öffnete sich im Zeitschritt 402 bei $H=-146 \mathrm{kN}$ der Riss 4 in der untersten Lagerfuge weiter. Nachfolgend bildete sich im Zeitschritt 406 bei $H=-164 \mathrm{kN}$ ein abgetreppter Riss durch Stoß- und Lagerfugen $(5 \div 14)$. Dadurch wurde die rechte obere Wandecke teilweise von dem restlichen Wandteil entkoppelt und es stellte sich eine bleibende Verformung von $0,5 \mathrm{~mm}$ ein. Bei Lastumkehr trat bei $H=146 \mathrm{kN}$ mit einem abgetreppten Riss $15 \div 23$ das analoge Bruchbild für die linke untere Wandecke auf.

Im vierten Versuch KS3-4 (Lastfaktor $f=3,5$ ) verlängerte sich im Zeitschritt 352 bei $H=-150 \mathrm{kN}$ der schräge Riss 5 weiter zu Riss 24 und es tauchten in der gegenüberliegenden unteren Ecke die Risse $25 \div 28$ auf. Im weiteren Verlauf traten im Zeitschritt 403 bei $H=-116 \mathrm{kN}$ die Risse $29 \div 34$ auf. Der Versuch musste bei einer Horizontalkraft von $170 \mathrm{kN}$ im Zeitschritt 405 mit den Rissen $35 \div 38$ abgebrochen werden, da kein Gleichgewicht gefunden werden konnte.

Im folgenden Nachversuch wurde ausgehend vom Endzustand des Hauptversuches $\left(H=9 \mathrm{kN}, w_{\text {Kopf }}=-1,2 \mathrm{~mm}\right)$ bei konstanter Normalkraft $N \equiv 270 \mathrm{kN}$ und einem Kopfmoment von $M_{\text {Kopf }} \equiv 0 \mathrm{kNm}$ die Kopfverschiebung sukzessive bis $w_{\text {Kopf }}=8,8 \mathrm{~mm}$ gesteigert. Es stellte sich eine Gleitverschiebung in den abgetreppten Rissen $39 \div 49$ bei einer maximalen Horizontalkraft von $H=125 \mathrm{kN}$ ein. Anschließend wurde ein gegendrehendes Kopfmoment $M_{\text {Kopf }}=-125 \mathrm{kNm}$ bei leicht verminderter Normalkraft $N=260 \mathrm{kN}$ aufgebracht, wodurch die Kopfverschiebung infolge der aufgetretenen Steinzugrisse $50 \div 56$ sprunghaft um $0,9 \mathrm{~mm}$ zunahm. Bei weiterer Erhöhung der Kopfverschiebung bis $w_{\text {Kopf }}=13,68 \mathrm{~mm}$ konnte die Horizontalkraft auf maximal $130 \mathrm{kN}$ gesteigert werden. Für die anschließenden beiden Lastzyklen 
wurde das Kopfmoment auf $M_{\text {Kopf }}=0 \mathrm{kNm}$ und die Normalkraft auf $N=300 \mathrm{kN}$ erhöht. Im zweiten Lastzyklus traten bei der Horizontalkraft $H=149 \mathrm{kN}$ die weiteren Risse $57 \div 60$ auf. Dadurch glitt der rechte obere Wandabschnitt auf dem schrägen abgetreppten Riss.

\section{Wand KS4}

Bei Wand KS4 wurden Plansteine in Dünnbettmörtel mit im Vergleich zu Wand KS3 höherer Soll-Haftscherfestigkeit verlegt. Die Stoßfugen wurden nicht vermörtelt. Die ersten Risse (s. Abbildung A-22) traten - ähnlich zu Wand KS3 - im zweiten Versuch KS4-2 in Form eines Klaffens in der untersten Lagerfuge auf, nämlich Riss 1 (Zeitschritt 385, $H=113 \mathrm{kN}$ ) und Riss 2 (Zeitschritt 403, $H=-142 \mathrm{kN}$ ).

Im dritten Versuch KS4-3 (Lastfaktor $f=2,5$ ) öffnete sich im Zeitschritt 402 ein weiterer horizontaler Riss in der untersten Lagerfuge (Riss 3) bei $H=-140 \mathrm{kN}$. Im weiteren Verlauf traten im Zeitschritt 406 die Risse $4 \div 18$ bei einer maximalen Horizontalkraft von $H=-165 \mathrm{kN}$ auf. Diese vom Kopfbalken ausgehenden Risse verliefen abgetreppt durch die Stoß- und Lagerfugen.

Im letzten Hauptversuch KS4-4 (Lastfaktor $f=3,5$ ) trat im Zeitschritt 348 bei einer Horizontallast von $H=-158 \mathrm{kN}$ der Steinzugriss 19 in Verlängerung der bereits vorher vorhandenen Risse $4 \div 18$ auf. Es stellte sich eine bleibende Verformung von ca. 0,4 mm ein. Im nächsten Zyklus im Zeitschritt 404 verlängerte sich bei einer Horizontalkraft von $H=$ 169 kN Riss 19 in Form von Riss 20, woraus sich der bleibende Verformungsanteil auf $2,1 \mathrm{~mm}$ erhöhte. Gleichzeitig öffnete sich auf der gegenüberliegenden Seite auf halber Wandhöhe ein Biegezugriss (Riss 22) senkrecht zu der Lagerfuge. Bei Lastumkehr öffneten sich bei der Rückverformung die horizontalen Risse 21 und 23 (Zeitschritt 423, $H=163 \mathrm{kN}$ ) sowie 24 (Zeitschritt 459, $H=138 \mathrm{kN}$ ). Der Versuchsabbruch erfolgte im Zeitschritt 496 bei einer Horizontalkraft $H=133 \mathrm{kN}$ und der zugehörigen Kopfverschiebung $w_{\text {Kopf }}=0,22 \mathrm{~mm}$. Hier konnte aufgrund der Schädigung und des unstetigen Steifigkeitsverlaufes der Wand kein rechnerisches Gleichgewicht mit der geforderten Genauigkeit mehr gefunden werden.

Der Nachversuch erfolgte mit einer Normalkraft $N \equiv 360 \mathrm{kN}$ und abschnittsweise erhöhtem rückdrehendem Kopfmoment. Zu Beginn wurde mit $M_{\text {Kopf }}=0 \mathrm{kNm}$ die Kopfverschiebung bis $\mathrm{zu} w_{\text {Kopf }}=5,3 \mathrm{~mm}$ erhöht. Bei einer Horizontalkraft $H=151 \mathrm{kN}$ kam es am Wandfuß zum Querschnittsklaffen in Form der Risse $25 \div 29$. Anschließend wurde schrittweise ein gegendrehendes Kopfmoment aufgebracht. Da als Steuergröße der Pressenweg des Horizontalzylinders agierte, kam es infolge der Reduktion der Biegeverformung durch das rückdrehende Moment bei konstantem Pressenweg zu einer höheren Prüfrahmenverformung. Dadurch wurde die Horizontalkraft $H$ bei reduzierter Wandkopfverformung $w_{\text {Kopf }}$ erhöht. Bei einem Kopfmoment $M_{\text {Kopf }}=-127 \mathrm{kNm}$ traten die Risse $30 \div 31$ am rechten Wandkopf in Form schräger Steinzugrisse auf. Bei weiter gesteigerter Kopfverschiebung und einem Kopfmoment von $M_{\text {Kopf }}=-183 \mathrm{kNm}$ traten die abschließenden Risse $32 \div 65$ auf. Diese verliefen fast ausschließlich abgetreppt in den Stoß- und Lagerfugen und nur vereinzelt in den Eckbereichen auch schräg durch die Steine (Risse 32, 33, 54, 47, 49, 65 in Abbildung A-22).

\subsubsection{Steifigkeit}

Die Wand- und damit auch die Systemsteifigkeit hängen entscheidend von der Momentenbeanspruchung am Wandkopf ab. Bei Betrachtung allein der HorizontalkraftKopfverformungs-Beziehungen (s. Anhang A.3.2) fehlt dagegen die Information über das zugehörige Kopfmoment. Da dieses während der Versuchsdauer in einem weiten Bereich 
variierte, ist eine einfache Beschreibung der Wandsteifigkeit allein aus der KraftVerformungs-Beziehung nicht ohne weiteres möglich.

Ersatzweise wurde daher für die Auswertung der zweiten Simulationsstufe bezüglich der Steifigkeit auf die erste Systemeigenperiode zurückgegriffen. Deren Ermittlung erfolgte vereinfacht mit der Fast-Fourier-Transformation der im Versuch aufgetretenen Geschossverschiebungen. Hierbei wurde davon ausgegangen, dass die Einwirkung in Form des Bodenbeschleunigungszeitverlaufes im betreffenden Periodenbereich einem weißen Rauschen entspricht.

Nachfolgend ist in Abbildung 55 das skalierte Amplitudenspektrum der Fast-FourierTransformation der drei Geschossverschiebungen des Versuches KS1-7 dargestellt.

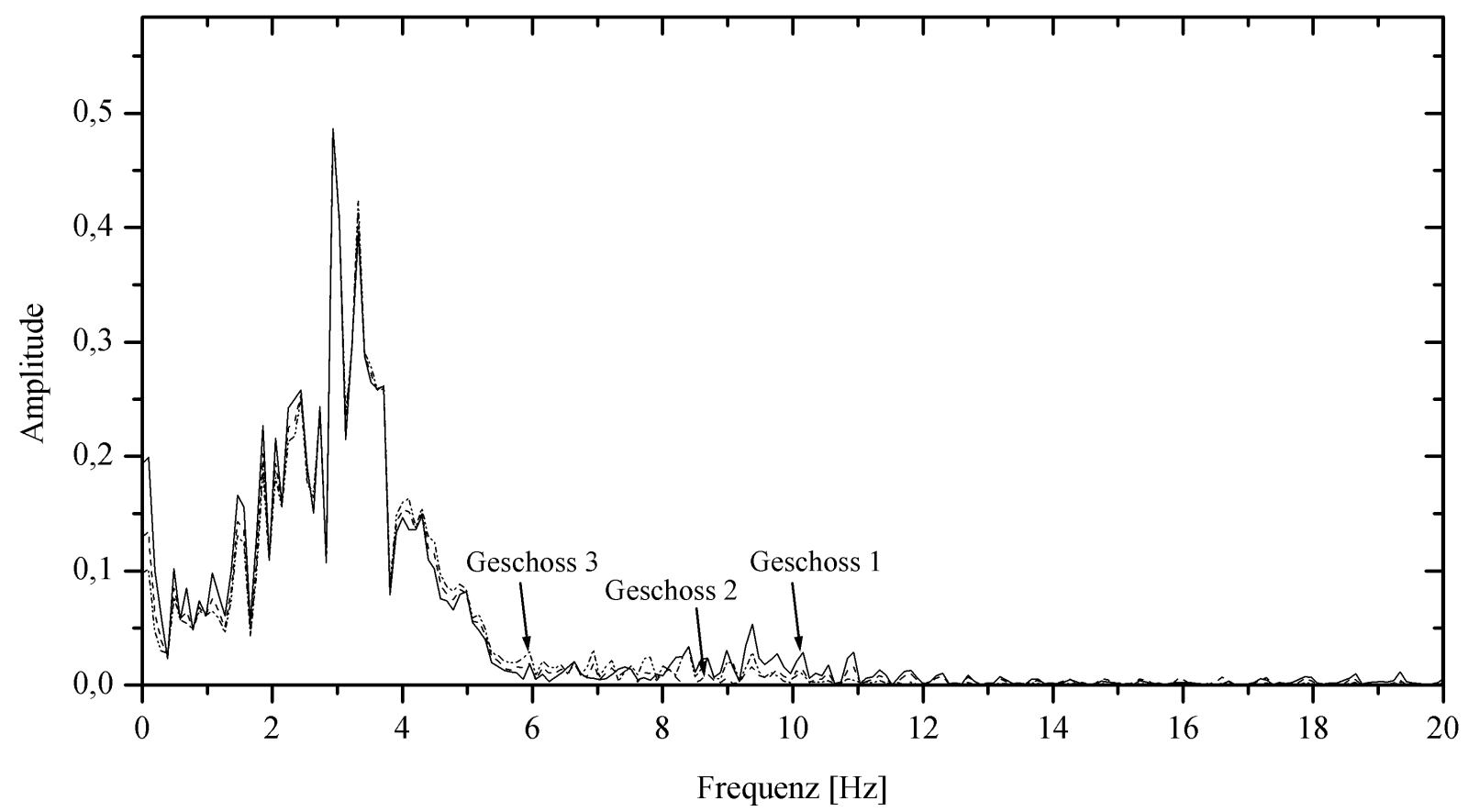

Abbildung 55: FFT der Geschossverschiebungsverläufe von Versuch KS1-7

Die drei Spektren sind besonders im Bereich um die erste Eigenfrequenz nahezu deckungsgleich. Neben der allgemein stark ausgeprägten ersten Eigenfrequenz im Bereich von ca. $3,2 \mathrm{~Hz}$ ist die zweite Eigenfrequenz von ca. 9,4 Hz lediglich aus der Untersuchung der untersten Geschossverschiebung (Geschoss 1) erkennbar. Die Ursache ist primär in dem größeren Amplitudenverhältnis von zweiter und erster Eigenform auf Höhe des Wandkopfes im untersten Geschoss im Vergleich zu der im zweiten und dritten Geschoss zu suchen.

Für die weiteren Untersuchungen wurden die ersten Eigenfrequenzen $f_{1, F F T}$ aus den Verschiebungsverläufen des untersten Geschosses ermittelt. Deren Bestimmung aus dem Amplitudenspektrum erfolgte durch Schwerpunktbildung der Werte in einem lokal eingegrenzten Frequenzfenster (Tabelle 35). Dieses wurde individuell auf Basis des Amplitudenverlaufes festgelegt.

Nachfolgend werden in Abbildung 56 die nach der oben dargestellten Methode rechnerisch ermittelten ersten Eigenperioden $T_{1, F F T}$ denen aus einer vergleichenden Stabberechnung (Tabelle 36) mit den Anfangssteifigkeiten aus den Vorversuchen (Tabelle 31) gegenübergestellt. Es zeigt sich deutlich, dass - bis auf zwei Ausnahmen - die in den Versuchen aufgetretenen Eigenperioden über denen der Stabberechnung liegen. Für die Beurteilung der Ergebnisse 
wird in Abbildung 56 das Verhältnis $T_{1, F F T} / T_{1, \text { Stabsystem }}$ in Abhängigkeit des Verhältniswertes von maximal im Versuch erreichter Horizontallast zu maximaler Horizontallast aller Hauptversuche der betreffenden Wand dargestellt.

\begin{tabular}{|c|c|c|c|c|}
\hline $\begin{array}{l}\text { Wand / } \\
\text { Versuch }\end{array}$ & BB-ZV ${ }^{1)}$; Lastfaktor $f[-]$ & $\begin{array}{c}T_{1, F F T} \\
{[\mathbf{s}]}\end{array}$ & $\begin{array}{l}\text { Frequenzbereichsfenster } \\
\qquad[\mathrm{Hz}]\end{array}$ & $\begin{array}{c}T_{1, \text { Stabsystem }} \\
{[\mathbf{s}]}\end{array}$ \\
\hline D-1 & ZV1-B3 $\left(\mathrm{a}_{\mathrm{g}}=0,4 \mathrm{~m} / \mathrm{s}^{2}\right) ; f=0,15$ & 0,360 & $2,15 \div 3,42$ & $0,0,367$ \\
\hline D-2 & $\mathrm{f}=1$ & 0,376 & $2,05 \div 3,12$ & 0,367 \\
\hline D-3 & $\mathrm{f}=1,5$ & 0,389 & $2,05 \div 3,12$ & 0,367 \\
\hline D-4 & $\mathrm{f}=2$ & 0,394 & $2,05 \div 3,12$ & 0,367 \\
\hline D-5 ${ }^{2)}$ & $\mathrm{f}=2,67$ & 0,437 & $1,66 \div 2,83$ & 0,367 \\
\hline E-1 & ZV4-C3 $\left(\mathrm{a}_{\mathrm{g}}=0,4 \mathrm{~m} / \mathrm{s}^{2}\right) ; \mathrm{f}=1$ & 0,427 & $1,76 \div 2,93$ & 0,384 \\
\hline E-2 ${ }^{2)}$ & $\mathrm{f}=2,67$ & 0,547 & $0,68 \div 3,12$ & 0,384 \\
\hline F-1 & ZV3-A1 $\left(\mathrm{a}_{\mathrm{g}}=0,4 \mathrm{~m} / \mathrm{s}^{2}\right) ; \mathrm{f}=2$ & 0,458 & $1,66 \div 2,83$ & 0,448 \\
\hline F-2 & ZV4-C3 $\left(\mathrm{a}_{\mathrm{g}}=0,4 \mathrm{~m} / \mathrm{s}^{2}\right) ; \mathrm{f}=3$ & 0,500 & $1,56 \div 2,54$ & 0,448 \\
\hline${\mathrm{F}-3^{2)}}^{2}$ & ZV3-A1 $\left(\mathrm{a}_{\mathrm{g}}=0,4 \mathrm{~m} / \mathrm{s}^{2}\right) ; \mathrm{f}=7$ & 0,520 & $1,17 \div 2,73$ & 0,448 \\
\hline KS1-1 ${ }^{2)}$ & ZV3-A1 $\left(\mathrm{a}_{\mathrm{g}}=0,4 \mathrm{~m} / \mathrm{s}^{2}\right) ; \mathrm{f}=2$ & 0,253 & $3,12 \div 4,78$ & 0,277 \\
\hline KS1-2 $^{3)}$ & $\mathrm{f}=2$ & 0,246 & $2,83 \div 5,66$ & 0,238 \\
\hline $\mathrm{KS}_{1-3^{3)}}$ & $\mathrm{f}=3$ & 0,244 & $3,12 \div 5,66$ & 0,238 \\
\hline KS1-4 ${ }^{2)}$ & $\mathrm{f}=3$ & 0,299 & $2,54 \div 4,20$ & 0,277 \\
\hline KS1-5 & $\mathrm{f}=3$ & 0,292 & $2,15 \div 4,78$ & 0,277 \\
\hline KS1-6 & $\mathrm{f}=4$ & 0,299 & $2,15 \div 4,59$ & 0,277 \\
\hline KS1-7 & $\mathrm{f}=6$ & 0,317 & $1,95 \div 4,59$ & 0,277 \\
\hline $\mathrm{KS}_{1-8}{ }^{2)}$ & $\mathrm{f}=8$ & 0,332 & $0,78 \div 5,47$ & 0,277 \\
\hline KS2-1 & ZV3-A1 $\left(\mathrm{a}_{\mathrm{g}}=0,4 \mathrm{~m} / \mathrm{s}^{2}\right) ; \mathrm{f}=1$ & 0,262 & $2,83 \div 4,79$ & 0,263 \\
\hline KS2-2 ${ }^{2)}$ & $\mathrm{f}=8 \mathrm{f}$ & 0,332 & $1,56 \div 4,69$ & 0,263 \\
\hline KS3-1 & ZV1-B3 $\left(\mathrm{a}_{\mathrm{g}}=0,4 \mathrm{~m} / \mathrm{s}^{2}\right) ; \mathrm{f}=1,5$ & 0,318 & $2,15 \div 4,10$ & 0,266 \\
\hline KS3-2 & $\mathrm{f}=2$ & 0,325 & $2,15 \div 4,10$ & 0,266 \\
\hline KS3-3 & $f=2,5$ & 0,334 & $2,15 \div 3,90$ & 0,266 \\
\hline KS3-4 ${ }^{2)}$ & $\mathrm{f}=3,5$ & 0,355 & $1,56 \div 4,10$ & 0,266 \\
\hline KS4-1 & ZV1-B3 $\left(\mathrm{a}_{\mathrm{g}}=0,4 \mathrm{~m} / \mathrm{s}^{2}\right) ; \mathrm{f}=1,5$ & 0,323 & $1,66 \div 4,59$ & 0,266 \\
\hline KS4-2 & $\mathrm{f}=2$ & 0,334 & $1,66 \div 4,39$ & 0,266 \\
\hline KS4-3 & $\mathrm{f}=2,5$ & 0,344 & $1,76 \div 4,10$ & 0,266 \\
\hline KS4-4 ${ }^{2)}$ & $f=3,5$ & 0,411 & $1,17 \div 3,71$ & 0,266 \\
\hline \multicolumn{5}{|c|}{$\begin{array}{l}\text { 1) Bodenbeschleunigungszeitverlauf, Boden-Baugrund-Kombination; Grundwert der } \\
\text { Bodenbeschleunigung } \\
\text { 2) Versuche nicht vollständig durchgeführt } \Rightarrow \text { FFT an reduzierter Messwertanzahl }\end{array}$} \\
\hline
\end{tabular}

Tabelle 35: Erste Systemeigenperioden der Versuche der zweiten Simulationsstufe - ermittelt aus der FFT des Wandkopfverschiebungsverlaufes des untersten Geschosses

Es zeigt sich deutlich, dass mit steigendem Belastungsniveau der Verhältniswert $T_{1, F F T} / T_{1, \text { Stabsystem }}$ zunimmt. Im Bereich der maximalen Horizontallasten der geprüften sieben Wände der zweiten Simulationsstufe beträgt der Wertebereich $1,07 \div 1,55$ bei einem Mittelwert von 1,27. Bei geringen Beanspruchungen liegen die Verhältniswerte bei etwa 1, was die gute Näherung der Wandsteifigkeiten mit den Anfangssteifigkeiten aus Tabelle 31 und Tabelle 34 bestätigt. Eine Ausnahme bildet hier Wandversuch KS1-1, der nach 3,6 Sekunden Erdbebenzeit abgebrochen werden musste. Hier bestimmte sich die Eigenperiode aus der FastFourier-Transformation der 360 Messwerte zu 91\% der rechnerischen Steifigkeit des Stabsystems. Bei weiterer Laststeigerung erreichte das Verhältnis $T_{1, F F T} / T_{1, \text { Stabsystem }}$ im Versuch KS1-7 den Wert 1,14 und im Wandversuch KS1-8 den Wert 1,2.

Als Ergebnis kann festgehalten werden, dass mit zunehmender Beanspruchungshöhe die Systemsteifigkeit und damit auch die Eigenfrequenz deutlich abnehmen. Bei den untersuchten 
Systemen erhöhte sich in den Versuchen mit der maximal aufgetretenen Horizontalkraft die erste Eigenperiode um in Mittel 27\% bezogen auf den ursprünglichen Zustand.

Unabhängig davon ergibt die Beschreibung der Wände mit ungerissenen Querschnitten und isotropem, linear-elastischem Verhalten deutlich geringere Eigenperioden als $T_{1, \text { Stabsystem }}$.

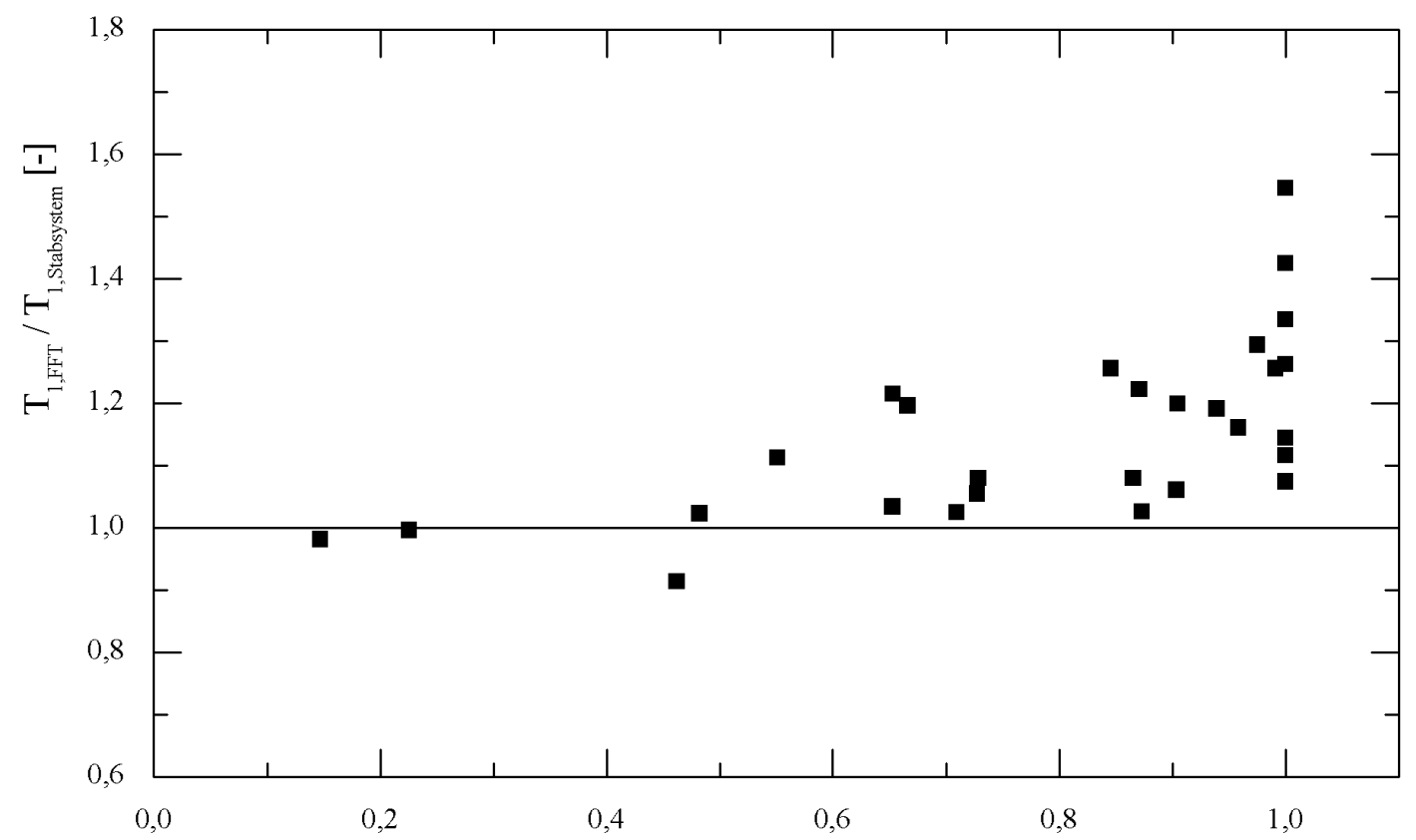

maximale Horizontallast des Einzelversuches im Verhältnis zur maximal erreichten Horizontallast der betreffenden Wand [-]

Abbildung 56: Verhältnis von erster Systemeigenperiode der Versuche zu der einer elastischen Stabberechnung in Abhängigkeit des Verhältnisses von maximal im Versuch erreichter Horizontallast zu maximaler Horizontallast aller Versuche der betreffenden Wand

\subsubsection{Vergleichsrechnung}

Zur Bewertung der Ergebnisse wurden die Versuche vergleichend als linear-elastische Mehrmassenschwinger (Abbildung 57) mit den in den statisch-zyklischen Vorversuchen ermittelten Anfangssteifigkeiten aus Tabelle 31 und Tabelle 34 berechnet.

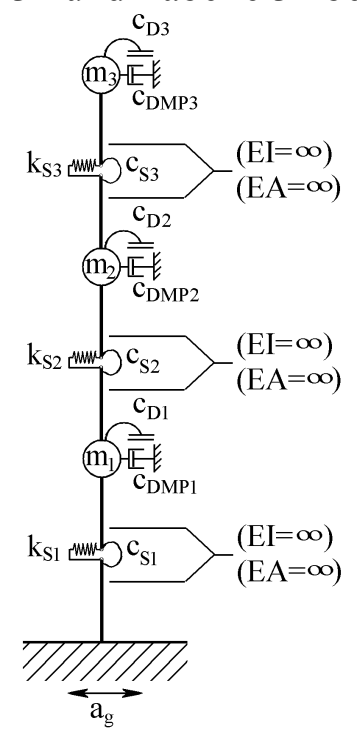

Abbildung 57: Mehrmassen-Stabsystem für die linear-elastische Nachrechnung der Versuche 
Nachfolgend sind in Tabelle 36 die Ergebnisse der Berechnungen in Form maximaler Horizontalkräfte und Kopfverschiebungen zusammengefasst.

\begin{tabular}{|c|c|c|c|c|c|}
\hline $\begin{array}{l}\text { Wand / } \\
\text { Versuch }\end{array}$ & $\begin{array}{c}\text { Parameter }{ }^{1), 2)} \\
c_{S, i}[\mathrm{kNm} / \mathrm{mrad}] \\
k_{S, i}[\mathrm{kN} / \mathbf{m m}] \\
c_{D M P, i}[\mathrm{kNs} / \mathbf{m}]\end{array}$ & $\begin{array}{l}T_{1,2,3}[\mathbf{s}] \\
\zeta_{1,2,3}[\%]\end{array}$ & $\begin{array}{c}\text { BB-ZV }^{3)} \\
\text { Lastfaktor } f[-]\end{array}$ & $\begin{array}{c}\text { maximale } \\
\text { Kopfver- } \\
\text { schiebung } \\
w_{\text {Kopf }}[\mathrm{mm}]\end{array}$ & $\begin{array}{c}\text { maximale } \\
\text { Horizontal- } \\
\text { kraft }^{4)} \\
H[\mathrm{kN}]\end{array}$ \\
\hline D-1 & \multirow{5}{*}{$\begin{array}{c}275 ; 200 ; 125 \\
100 ; 70 ; 47 \\
172,5 ; 2,8 ; 13,1\end{array}$} & \multirow[t]{5}{*}{$\begin{array}{c}0,37 ; 0,12 ; 0,07 \\
1,7 ; 2 ; 2,3\end{array}$} & $\begin{array}{c}\text { ZV1-B3 }\left(\mathrm{a}_{\mathrm{g}}=0,4 \mathrm{~m} / \mathrm{s}^{2}\right) \\
\mathrm{f}=0,15\end{array}$ & $0,36(0,33)$ & $23,0(22,8)$ \\
\hline D-2 & & & $\mathrm{f}=1$ & $2,39(2,11)$ & $153,1(109,9)$ \\
\hline D-3 & & & $f=1,5$ & $3,59(3,46)$ & $229,7(140,0)$ \\
\hline D-4 & & & $\mathrm{f}=2$ & $4,78(4,11)$ & $306,2(154,9)$ \\
\hline D-5 & & & $\mathrm{f}=2,67$ & $3,25(4,52)^{5)}$ & $215,2(145,5)^{5)}$ \\
\hline E-1 & \multirow{2}{*}{$\begin{array}{c}270 ; 200 ; 130 \\
80 ; 60 ; 45 \\
35 ; 1 ; 4,3\end{array}$} & \multirow[t]{2}{*}{$\begin{array}{c}0,38 ; 0,13 ; 0,08 \\
0,51 ; 0,5 ; 0,47\end{array}$} & $\begin{array}{c}\text { ZV4-C3 }\left(\mathrm{a}_{\mathrm{g}}=0,4 \mathrm{~m} / \mathrm{s}^{2}\right) \\
\mathrm{f}=1\end{array}$ & $1,16(1,5)$ & $65,1(61,8)$ \\
\hline E-2 & & & $\mathrm{f}=2,67$ & $2,75(4,27)^{5)}$ & $155,4(112,1)^{5)}$ \\
\hline F-1 & \multirow{3}{*}{$\begin{array}{c}120 ; 80 ; 55 \\
50 ; 42 ; 35 \\
240 ; 70 ; 6\end{array}$} & \multirow[t]{3}{*}{$\begin{array}{c}0,45 ; 0,15 ; 0,10 \\
5 ; 5 ; 4,1\end{array}$} & $\begin{array}{c}\text { ZV3-A1 }\left(\mathrm{a}_{\mathrm{g}}=0,4 \mathrm{~m} / \mathrm{s}^{2}\right) \\
\mathrm{f}=2\end{array}$ & $1,89(1,85)$ & $68,4(55,7)$ \\
\hline F-2 & & & $\begin{array}{c}\text { ZV4-C3 }\left(\mathrm{a}_{\mathrm{g}}=0,4 \mathrm{~m} / \mathrm{s}^{2}\right) \\
\mathrm{f}=3\end{array}$ & $4,64(5,37)$ & $171,0(115,4)$ \\
\hline F-3 & & & $\begin{array}{c}\text { ZV3-A1 }\left(\mathrm{a}_{\mathrm{g}}=0,4 \mathrm{~m} / \mathrm{s}^{2}\right) \\
\mathrm{f}=7\end{array}$ & $6,63(8,0)^{5)}$ & $239,4(110,6)^{5)}$ \\
\hline KS1-1 & \multirow{8}{*}{$\begin{array}{l}470 ; 320 ; 220 \\
300 ; 220 ; 120 \\
\text { 700; } 60 ; 40(1) \\
\text { bzw. } \\
525 ; 200 ; 0(2 ; 3) \\
\text { bzw. } \\
570 ; 85 ; 30(4-8)\end{array}$} & $\begin{array}{c}0,28 ; 0,08 ; 0,04 \\
5,1 ; 5,5 ; 5,9\end{array}$ & $\begin{array}{c}\text { ZV3-A1 }\left(\mathrm{a}_{\mathrm{g}}=0,4 \mathrm{~m} / \mathrm{s}^{2}\right) \\
\mathrm{f}=2\end{array}$ & $0,78(0,87)^{5)}$ & $100,7(76,6)^{5)}$ \\
\hline KS1-2 & & \multirow{2}{*}{$\begin{array}{c}0,24 ; 0,08 ; 0,04 \\
4,9 ; 5,1 ; 4,9\end{array}$} & $\mathrm{f}=2$ & $1,07(1,18)$ & $168,2(108,2)$ \\
\hline KS1-3 & & & $\mathrm{f}=3$ & $1,60(1,68)$ & $252,4(144,7)$ \\
\hline KS1-4 & & \multirow{5}{*}{$\begin{array}{c}0,28 ; 0,08 ; 0,04 \\
4,7 ; 4,8 ; 4,9\end{array}$} & $\mathrm{f}=3$ & $1,22(1,76)^{5)}$ & $156,3(120,7)^{5)}$ \\
\hline KS1-5 & & & $f=3$ & $1,42(1,74)$ & $182,8(120,6)$ \\
\hline KS1-6 & & & $\mathrm{f}=4$ & & $243,6(143,4)$ \\
\hline KS1-7 & & & $\mathrm{f}=6$ & $2,85(3,36)$ & $365,6(165,7)$ \\
\hline KS1-8 & & & $\mathrm{f}=8$ & $\left.1,53(3,21)^{5}\right)$ & $194,4(149,9)^{5)}$ \\
\hline KS2-1 & \multirow{2}{*}{$\begin{array}{c}470 ; 400 ; 250 \\
400 ; 330 ; 170 \\
700 ; 60 ; 40\end{array}$} & \multirow[t]{2}{*}{$\begin{array}{c}0,26 ; 0,08 ; 0,04 \\
4,8 ; 5 ; 5\end{array}$} & $\begin{array}{c}\text { ZV3-A1 }\left(\mathrm{a}_{\mathrm{g}}=0,4 \mathrm{~m} / \mathrm{s}^{2}\right) \\
\mathrm{f}=1\end{array}$ & $0,37(0,37)$ & $50,7(47,3)$ \\
\hline KS2-2 & & & $\mathrm{f}=8$ & $1,49(3,62)^{5)}$ & $215,2(209,4)^{5)}$ \\
\hline KS3-1 & \multirow{4}{*}{$\begin{array}{c}500 ; 400 ; 230 \\
350 ; 260 ; 180 \\
675 ; 45 ; 50\end{array}$} & \multirow[t]{4}{*}{$\begin{array}{c}0,27 ; 0,08 ; 0,04 \\
5 ; 5 ; 5\end{array}$} & $\begin{array}{c}\mathrm{ZV1-B} 3\left(\mathrm{a}_{\mathrm{g}}=0,4 \mathrm{~m} / \mathrm{s}^{2}\right) \\
\mathrm{f}=1,5\end{array}$ & $0,96(1,26)$ & $136,5(117,3)$ \\
\hline KS3-2 & & & $\mathrm{f}=2$ & $1,27(2,24)$ & $182,0(153,3)$ \\
\hline KS3-3 & & & $\mathrm{f}=2,5$ & $1,59(3,25)$ & $227,5(174,5)$ \\
\hline KS3-4 & & & $\mathrm{f}=3,5$ & $1,54(3,88)^{5)}$ & $\left.220,5(176,0)^{5}\right)$ \\
\hline KS4-1 & \multirow{4}{*}{$\begin{array}{c}500 ; 400 ; 230 \\
350 ; 260 ; 180 \\
675 ; 45 ; 50\end{array}$} & \multirow[t]{4}{*}{$\begin{array}{c}0,27 ; 0,08 ; 0,04 \\
5 ; 5 ; 5\end{array}$} & $\begin{array}{c}\text { ZV1-B3 }\left(\mathrm{a}_{\mathrm{g}}=0,4 \mathrm{~m} / \mathrm{s}^{2}\right) \\
\mathrm{f}=1,5\end{array}$ & $0,96(1,12)$ & $136,5(110,6)$ \\
\hline KS4-2 & & & $\mathrm{f}=2$ & $1,27(2,14)$ & $182,0(143,2)$ \\
\hline KS4-3 & & & $f=2,5$ & $1,59(3,19)$ & $227,5(165,1)$ \\
\hline KS4-4 & & & $\mathrm{f}=3,5$ & $1,63(5,42)^{5)}$ & $\left.246,1(169,3)^{5}\right)$ \\
\hline \multicolumn{6}{|c|}{$\begin{array}{l}\text { 1) Nummerierung / Reihenfolge: } 1,2,3 \text { : unteres, mittleres, oberes Geschoss } \\
\text { Verlauf der Steifigkeiten } c_{S, i} \text { und } k_{S, i}: \mathrm{s} \text {. Tabelle } 31 \\
\text { 2) Einspannung in Deckendrehfedern je Geschoss mit } c_{D, i}=300 \mathrm{kNm} / \mathrm{mrad} \text { (Ausnahme: KS1-2 \& 1-3: 600) } \\
\text { Geschosshöhe }=2,60 \mathrm{~m} ; 3 \text { Einzelmassen: } m_{1,2,3}=29 \mathrm{t} \\
\text { 3) Bodenbeschleunigungszeitverlauf, Boden-Baugrund-Kombination; Grundwert der Bodenbeschleunigung } \\
\text { 4) Werte in Klammern: aus zugehörigem Versuch } \\
\text { 5) Versuche nicht vollständig durchgeführt } \Rightarrow \text { Berechnungsergebnisse bis zum betreffenden Zeitpunkt (analog } \\
\text { Tabelle 34) }\end{array}$} \\
\hline
\end{tabular}

Tabelle 36: Parameter der Vergleichsrechnung am linear-elastischen Stabsystem

Der Vergleich von im Versuch aufgetretenen maximalen Kraft- und Verschiebungsgrößen mit Größen aus der linear-elastischen Stabberechnung ist nachfolgend in Abbildung 58 darge- 
stellt. Als Bezugsgröße wird der Verhältniswert von maximal im Versuch erreichter Horizontallast zu maximaler Horizontallast aller Hauptversuche der betreffenden Wand angesetzt.

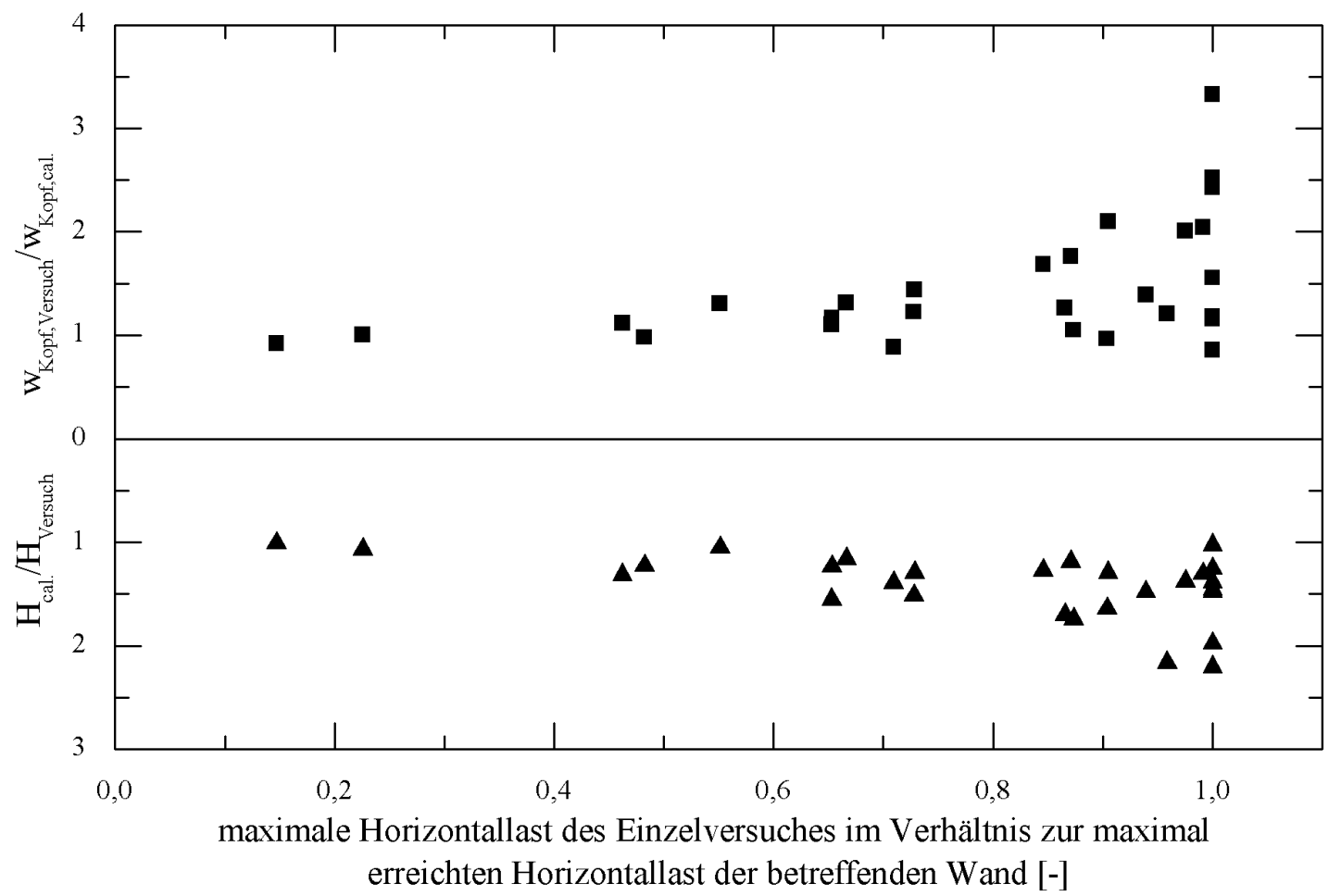

Abbildung 58: Verhältnis der bei linear-elastischer Stabberechnung ermittelten zu den im Versuch aufgetretenen Kopfverschiebungen und Horizontalkräften in Abhängigkeit des Verhältnisses von maximal im Versuch erreichter Horizontallast zu maximaler Horizontallast aller Versuche der betreffenden Wand

Es zeigt sich deutlich, dass mit steigender Beanspruchungshöhe die Reduktion der rechnerischen Horizontalkraft und die Erhöhung der rechnerischen Kopfverschiebung zunehmen. Der mittlere Verhältniswert von $H_{\text {cal }} / H_{\text {Versuch }}$ ist mit 1,42 (Wertebereich $1,0 \div 2,21$ ) etwas kleiner als der von im Versuch aufgetretener maximaler Kopfverschiebung und rechnerischer Kopfverschiebung $w_{\text {Kopf,Versuch }} / w_{\text {cal }}$ von 1,46 (Wertebereich $0,86 \div 3,33$ ). Des Weiteren ist die Zunahme des Verhältniswertes $H_{\text {cal }} / H_{\text {Versuch }}$ in Abhängigkeit der Beanspruchungshöhe deutlich geringer ausgeprägt als die von $w_{\text {Kopf,Versuch }} / w_{\text {cal }}$.

Im Bereich der maximalen Horizontallasten liegt das Verhältnis von $H_{\text {cal }} / H_{\text {Versuch }}$ zwischen 1,03 (KS2-2) und 2,21 (KS1-7) (Mittelwert 1,54) und das Verhältnis von $w_{\text {Kopf,Versuch }} / w_{\text {cal }}$ zwischen 0,86 (D-4) und 3,33 (KS4-4) (Mittelwert 1,86).

\subsubsection{Tragfähigkeit}

Nachfolgend werden die Einwirkungszustände, bei denen die charakteristischen Versagensbilder der einzelnen Wände auftraten, mit Hilfe des Schubmodells von Mann / Müller (s. Abschnitte 3.1 und 6.2.1) nachgerechnet und einer Bewertung unterzogen. Bei diesem Rechenmodell wird von annähernd konstanter Normaldruckspannung über die Steinlänge und vernachlässigbarem Einfluss von Randzonen ausgegangen. Beiden Bedingungen entspricht ein kleines Verhältnis von Stein- zu Wandabmessung. Dieses kann für die Wände $\mathrm{D} \div \mathrm{F}, \mathrm{KS} 3$ und KS4 als erfüllt angesehen werden, wohingegen bei den Wänden KS1 und KS2 lokale Einflüsse dominieren und ergänzende Betrachtungen erforderlich werden. Am Wandkopf und 
-fuß wird der Spannungszustand durch die Randbalken, d.h. Fertigteilfundament und Ortbeton-Kopfbalken, zusätzlich beeinflusst, was bei der Beurteilung der Ergebnisse zu berücksichtigen ist.

Die Spannungszustände in den Wänden werden vereinfacht mit Hilfe der technischen Biegelehre unter Ausschaltung von Zugspannungen senkrecht zu den Lagerfugen bestimmt. Die Nachrechnung der Versagenskriterien erfolgt grundsätzlich am Ort des Schubspannungsmaximums mit einem angenommenen Schubspannungsverteilungsfaktor von 1,25.

Die Wände KS1 und KS2 werden ergänzend mit einem Mikromodell, in dem die Steine und die Lagerfugen diskret abgebildet werden, berechnet.

\begin{tabular}{|c|c|c|c|c|}
\hline $\begin{array}{l}\text { Wand / } \\
\text { Versuch }\end{array}$ & $\begin{array}{c}H \\
{[\mathrm{kN}]}\end{array}$ & $\begin{array}{c}M_{\text {Kopf }} \\
{[\mathbf{k N m}]}\end{array}$ & $\begin{array}{c}\text { Zeitschritt } \\
n[-]\end{array}$ & Versagensbild \\
\hline D-4 & 155 & 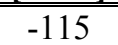 & 428 & Steinzugrisse am Wandfuß (Riss 3 und 4) \\
\hline E-2 & 104 & -55 & 538 & Diagonalriss in den Stoß- und Lagerfugen, (Risse $1 \div 42$ ) \\
\hline F-2 & -105 & 117 & 564 & Diagonaler Steinzugriss, (Risse $1 \div 19$ ) \\
\hline KS1-7 & -166 & 128 & 222 & Maximallast \\
\hline KS1-8 & -150 & 98 & 123 & Diagonalriss in den Stoß- und Lagerfugen, (Risse $2 \div 11$ ) \\
\hline KS2-2 & -209 & 211 & 126 & Diagonalriss in den Stoß- und Lagerfugen, (Riss 3 bzw. R3) \\
\hline KS3-3 & -164 & 111 & 406 & Diagonalriss in den Stoß- und Lagerfugen, (Risse $5 \div 14$ ) \\
\hline KS4-3 & -165 & 118 & 406 & Diagonalriss in den Stoß- und Lagerfugen, (Risse $4 \div 18$ ) \\
\hline
\end{tabular}

Tabelle 37: Versuchslasten für die Nachrechnung der Tragfähigkeit und der Bruchbilder

\section{Wandversuch D-4}

Die Nachrechnung des aufgetretenen Versagensbildes Steinzug im Versuch D-4 bei $H=155 \mathrm{kN}$ und $M_{\text {Kopf }}=-115 \mathrm{kNm}$ ergibt am Wandfuß mit der Normalkraft $N=284 \mathrm{kN}$ (= planmäßige Last: $270 \mathrm{kN}+$ Wandeigengewicht: $14 \mathrm{kN}$ ) eine Lastausmitte von 1,0 m. Der maximalen Schubspannung von $\tau_{\max }=1,56 \mathrm{~N} / \mathrm{mm}^{2}$ steht bei einer schrägen Steinzugfestigkeit $f_{b t}=1,9 \mathrm{~N} / \mathrm{mm}^{2}$ (Tabelle 23) eine aufnehmbare Schubspannung von 1,23 N/mm² gegenüber. Für die Übereinstimmung von aufnehmbarer und vorhandener Schubspannung ist eine rechnerische Steinzugfestigkeit von 2,59 N/mm² erforderlich, was einer Rissneigung von $39^{\circ}$ nach Gleichung (16) entspricht. Alternativ ist der Nachweis mit einem Schubspannungsverteilungsfaktor 1,0 und einer schrägen Steinzugfestigkeit $f_{b t}=1,9 \mathrm{~N} / \mathrm{mm}^{2}$ erfüllt.

In Anbetracht der Streuung der Materialparameter kann für die Nachrechnung des aufgetretenen Versagensbildes Steinzug mit dem Modell Mann / Müller eine gute Übereinstimmung festgestellt werden.

\section{Wandversuch E-2}

Die Maximallast und das Versagen von Wand E-2 trat in Form gekreuzter Diagonalrisse bei einer Horizontallast von ca. $H=94 \div 107 \mathrm{kN}$ auf. Die Risse verliefen größtenteils abgetreppt durch die Stoß- und Lagerfugen. Die Nachrechnung des Zeitschrittes 538 mit $H=104 \mathrm{kN}$ und $M_{\text {Kopf }}=-55 \mathrm{kNm}$ erfolgt für die beiden Versagensarten Gleiten in der Lagerfuge und Steinzugversagen.

Am Wandfuß liegen die vorhandenen Schubspannungen für das Kriterium Steinzugversagen um den Faktor 1,5 (schräge Steinzugfestigkeit $f_{b t}=1,3 \mathrm{~N} / \mathrm{mm}^{2}$ entsprechend Nachrechnung 
von Wandversuch F-2) unter den maximal Aufnehmbaren, wodurch dieser Versagensfall nicht maßgebend wird.

Für den Nachweis des Gleitens in der Lagerfuge wird ein Reibbeiwert von $\mu=0,7$ und eine Haftscherfestigkeit $k=0,5 \mathrm{~N} / \mathrm{mm}^{2}$ angesetzt (Reduktion der Literaturangabe von $0,63 \mathrm{~N} / \mathrm{mm}^{2}$ infolge nicht vollflächigen Kontaktes, s. Abschnitt 4.3.2). Am Wandfuß ergibt sich mit der mittleren Druckspannung von $\sigma=1,19 \mathrm{~N} / \mathrm{mm}^{2}$ auf der überdrückten Länge von 1,36 $\mathrm{m}$ die maximale Schubspannung zu $\tau_{\max }=0,55 \mathrm{~N} / \mathrm{mm}^{2}$. Mit den abgeminderten Reib- und Haftscherfestigkeitswerten (Abschnitt 6.2.1) beträgt die maximal aufnehmbare Schubspannung im Kriterium Gleiten in der Lagerfuge $\tau=0,69 \mathrm{~N} / \mathrm{mm}^{2}$, was $25 \%$ über dem vorhandenen Wert liegt. Die Tragfähigkeit wird somit mit dem angenommenen Reibbeiwert und der Haftscherfestigkeit leicht überschätzt.

\section{Wandversuch F-2}

Beim Wandversuch F-2 trat ein durch die Steine verlaufender, schräger Diagonalriss bei einer Horizontalkraft von $H=-105 \mathrm{kN}$ und einem Kopfmoment $M_{\text {Kopf }}=117 \mathrm{kNm}$ auf. Die Neigung der Risse $(1 \div 19$ in Abbildung A-16) war aufgrund der ungünstigeren Steingeometrie $(\Delta x / \Delta y=0,375 / 0,25)$ steiler als bei den Versuchen an Wand $\mathrm{D}(\Delta x / \Delta y=0,50 / 0,25)$. Für die schräge Steinzugfestigkeit kann daher, insbesondere für den Nachweis am Wandfuß, der Wert für eine geringere Neigung bei $\alpha_{0} \approx 15^{\circ}$ mit $f_{b t}=1,3 \mathrm{~N} / \mathrm{mm}^{2}$ angenommen werden (Tabelle 23). Für die Nachrechnung wird die Wandlänge um die Länge der Elastomerabschnitte auf 1,63 reduziert.

Unter den gegebenen Lasten bestimmt sich am Wandfuß die vorhandene maximale Schubspannung auf der überdrückten Abschnittslänge von $0,8 \mathrm{~m}$ zu $\tau_{\max }=0,94 \mathrm{~N} / \mathrm{mm}^{2}$. Dieser Wert stimmt mit der im Kriterium Steinzugversagen aufnehmbaren Spannungen von 0,91 N/mm² gut überein.

\section{Mikromodell für Nachrechnung der Wände KS1 und KS2}

Bei den Wänden KS1 und KS2 aus großformatigen Planelementen gelten aufgrund der geringen Steinanzahl die Voraussetzungen des Schubmodells von Mann / Müller nicht mehr. Die Traglast und die Bruchbilder können somit mit diesem Modell nur ungenügend beschrieben werden. Daher werden diese Wände als vereinfachtes Mikromodell mit Hilfe der FEM [135] nichtlinear berechnet.

Die Beschreibung der Steine erfolgt hierbei linear-elastisch und isotrop mit einem E-Modul von $11.000 \mathrm{~N} / \mathrm{mm}^{2}$. In den Lagerfugen werden die Steine durch nichtlineare Schub- und Normalfedern gekoppelt. Den Normalfedern wird infolge vernachlässigbarer Haftzugfestigkeit keine Zugfestigkeit zugewiesen. Bei vorhandenen Normaldruckspannungen können die Querfedern eine maximale Schubspannung von $\tau_{F l i e \beta}=\sigma \cdot \mu+k$ aufnehmen. Aus numerischen Gründen erhalten beide Federrichtungen eine im Vergleich zur Steinsteifigkeit sehr geringe Bettungssteifigkeit, wodurch Spannungsspitzen in den Lagerfugen vermieden werden. Die Lasteinleitung am Wandkopf in Form einer Normalkraft, eines Biegemomentes und einer Horizontalkraft erfolgt über einen starren Kopfbalken. Der Kontakt vom Kopfbalken zu der obersten Steinreihe sowie von der untersten Steinreihe zum Fundamentbalken erfolgt analog zu den Lagerfugen. 


\section{Wand KS1}

Das Versagensbild Gleiten in der Lagerfuge mit abgetreppten Rissen durch Stoß- und Lagerfugen (Riss $3 \div 10$ ) sowie einem einzelnen Steinzugriss am Wandfuß (Riss 11) trat im Wandversuch KS1-8 bei einer Horizontalkraft $H=-150 \mathrm{kN}$ und einem Kopfmoment $M_{\text {Kopf }}=98 \mathrm{kNm}$ auf. Die tatsächliche Reihenfolge des Eintretens der Risse konnte im Nachhinein nicht bestimmt werden - in den Filmaufnahmen (s. Abschnitt 5.5.4) tauchten sie gleichzeitig auf.

Bei der Nachrechnung werden unter den gegebenen Lasten in den Lagerfugen im Bereich der späteren Risse 4, 6 und 8 (Abbildung A-17) die maximal aufnehmbare Schubspannung erreicht und es treten am Ort des oberen Endes des späteren Risses 11 Steinzugspannungen in der Größenordnung von $3 \mathrm{~N} / \mathrm{mm}^{2}$ auf (Abbildung 59).
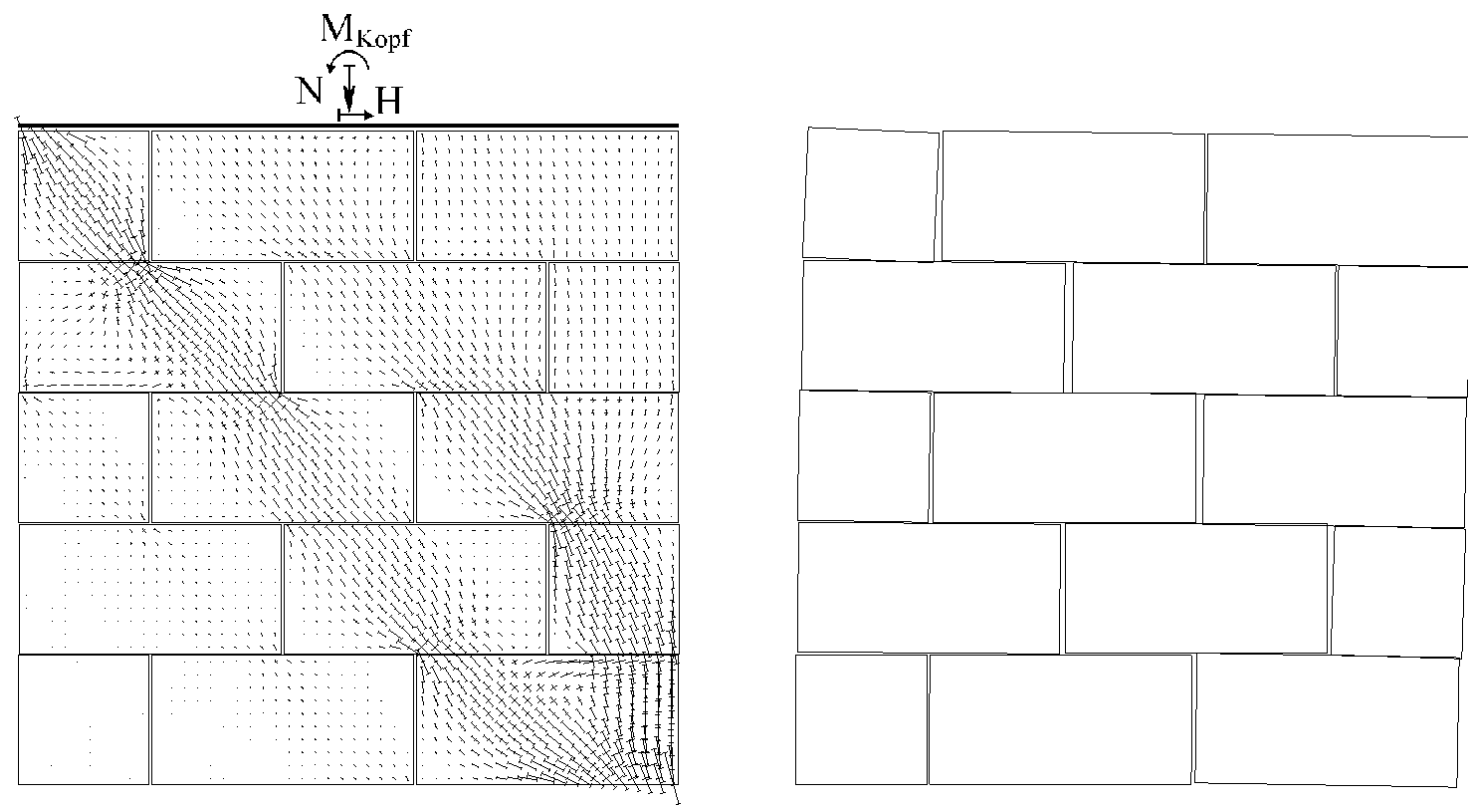

Abbildung 59: Trajektorienverlauf und verschobenes System bei der Nachrechnung von Versuch KS1-8

Beim vorhergehenden Wandversuch KS1-7 wurde die Maximallast von $H=-166 \mathrm{kN}$ im Zeitschritt 222 mit einem Kopfmoment $M_{\text {Kopf }}=128 \mathrm{kNm}$ erreicht. Es trat hier kein Versagen in Form eines Gleitens in der Lagerfuge oder Steinzugversagens auf.

Der qualitative Verlauf der Normal- und Schubspannungen in den Lagerfugen ist vergleichbar zu Versuch KS1-8. Die maximalen rechnerischen Steinzugspannungen im Bereich des späteren Risses 11 liegen bei der Nachrechung von Versuch KS1-7 in der Größenordnung von $3,5 \div 4,0 \mathrm{~N} / \mathrm{mm}^{2}$.

Die Tatsache, dass trotz geringerer rechnerischer Hauptzugspannungen das Versagen in Versuch KS1-8 eintrat, kann durch die Vorschädigung, z.B. über den lokalen Abbau der Haftscherfestigkeit im Vorfeld begründet werden. Dieses konnte im obigen vereinfachten Modell nicht berücksichtigt werden.

\section{Wandversuch KS2-2}

Wand KS2 mit vermörtelten Stoßfugen versagte im zweiten Hauptversuch in Form des diagonalen Risses 3 bzw. R3 (s. Abbildung A-19 und A-20), der größtenteils durch die Steine 
verlief. Die zugehörigen Lasten im Zeitschritt 126 betrugen $H=-209 \mathrm{kN}$ und $M_{\text {Kopf }}=211 \mathrm{kNm}$.

Infolge der Vermörtelung der Stoßfugen von Wand KS2, über die Schub- und Normalspannungen teilweise übertragen werden können, wird bei Verwendung des obigen Mikromodells die tatsächliche Tragfähigkeit unterschätzt. Für die Eingrenzung der rechnerischen Traglast wird als untere Grenze der Nachweis mit unvermörtelten Stoßfugen (analog zu Wand KS1, Mikromodell s. o.) und als obere Grenze der Nachweis des Gleitens in der untersten Lagerfuge für eine homogene Wand durchgeführt.

Als Mindestwert beim Nachweis des Gleitens bestimmt sich mit dem nicht abgeminderten Reibbeiwert $\mu=0,7$ unter Vernachlässigung des Kohäsionsanteils eine aufnehmbare Horizontalkraft von $\mu \cdot N_{F u \beta}=206 \mathrm{kN}$. Der Ansatz der Kohäsion erfolgt auf der am Wandfuß überdrückten Länge von $0,36 \mathrm{~m}$, woraus sich bei durchplastifizierter Fuge eine maximal aufnehmbare Horizontalkraft von $335 \mathrm{kN}$ ergibt.

Die Nachrechnung mit dem Mikromodell führt unter den gegebenen Maximallasten zu einer Gleitverschiebung, abgetreppt durch Stoß- und Lagerfugen. Das Verschiebungsbild ist vergleichbar zu Wandversuch KS1-8 (entsprechend zu den Rissen 4, 6 und 8 in Abbildung A17), jedoch tritt ein stärker ausgeprägtes Klaffen in den Lagerfugen zwischen den einzelnen Steinen auf. Entsprechend erhöhen sich die zugehörigen Steinzugspannungen deutlich. Der Maximalwert tritt in der untersten Steinreihe im rechten Stein mit ca. 6,0 N/mm ${ }^{2}$ auf (s. Abbildung 60). Im Vergleich zu Wandversuch KS1-7 liegt der Maximalwert näher am äußeren Querschnittsrand, da die überdrückte Länge der Lagerfuge unter dem halben Planelement kleiner ist.

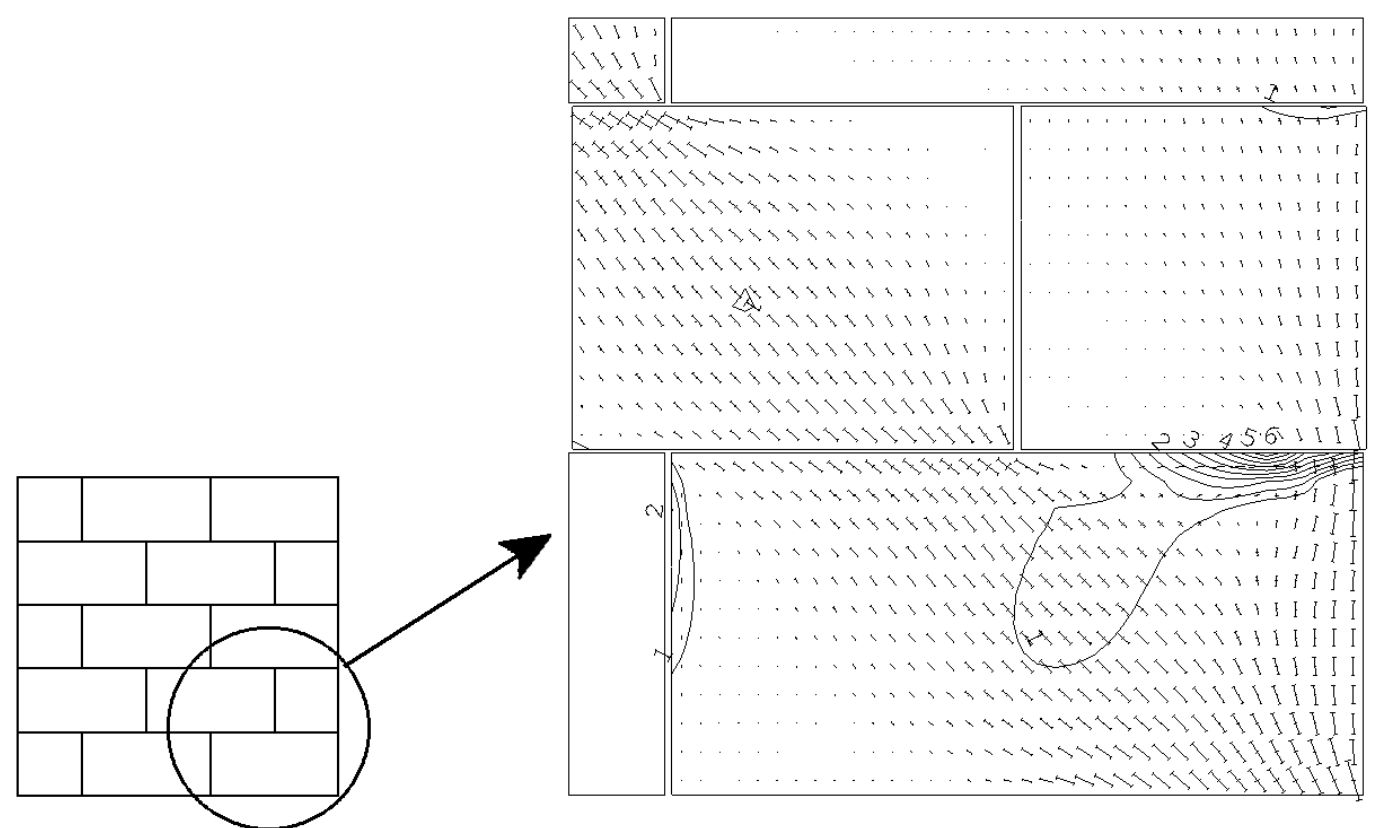

Abbildung 60: Trajektorienverlauf mit Hauptzugspannungen $\left[\mathrm{N} / \mathrm{mm}^{2}\right]$ der Nachrechnung von Versuch KS2-2 (Systemausschnitt)

Wird angenommen, dass durch die vermörtelten Stoßfugen ein Teil der Spannungen übertragen werden kann, lässt sich die zwischen dem unteren und oberen rechnerischen Grenzwert aufgetretene Horizontalkraft erklären. Entsprechend kann auch der Steinzugriss 3, ausgehend von der rechten unteren Wandecke, begründet werden. Aufgrund der teilweise vorhandenen 
Zugfestigkeit in den Stoßfugen verlaufen die Risse nicht über eine ganze Steinhöhe in den Stoßfugen vertikal, sondern zweigen vorher in die Steine ab (s. Abbildung A-19 und A-20).

\section{Wandversuch KS3-3}

Das Versagensbild im Wandversuch KS3-3 $\left(H=-164 \mathrm{kN}, M_{\text {Kopf }}=111 \mathrm{kNm}\right)$ war durch einen abgetreppten Riss in den Stoß- und Lagerfugen in der oberen Wandhälfte gekennzeichnet (Abbildung A-21). Die zugehörigen Schubspannungen am Wandkopf und auf halber Wandhöhe bestimmen sich zu jeweils $0,47 \mathrm{~N} / \mathrm{mm}^{2}$ und am Wandfuß zu 2,20 N/mm². Mit der gemessenen Haftscherfestigkeit von $k=0,78 \mathrm{~N} / \mathrm{mm}^{2}$ und einem abgeschätzten Reibbeiwert von $\mu=0,7$ betragen nach dem Schubmodell von Mann / Müller im Kriterium Gleiten in der Lagerfuge die aufnehmbaren Schubspannungen am Wandkopf und auf halber Wandhöhe $0,72 \mathrm{~N} / \mathrm{mm}^{2}$ sowie am Wandfuß $1,76 \mathrm{~N} / \mathrm{mm}^{2}$.

Die vorhandenen Schubspannungen liegen somit am Wandkopf und auf halber Wandhöhe um $35 \%$ unter den aufnehmbaren. Am Wandfuß dagegen werden die aufnehmbaren Schubspannungen um 25\% überschritten, ohne dass das Versagensbild Gleiten in der Lagerfuge hier auftrat.

Das Kriterium Steinzugversagen ist auf halber Wandhöhe und am Wandkopf infolge des Abstandes mit einem Faktor von 3,3 nicht maßgebend (Steinzugfestigkeit $f_{b t}=3,3 \mathrm{~N} / \mathrm{mm}^{2}$, Tabelle 23). Am Wandfuß dagegen wird die maximal aufnehmbare Schubspannung von $2,0 \mathrm{~N} / \mathrm{mm}^{2}$ um $10 \%$ ebenfalls überschritten, ohne dass dieses Versagensbild im Versuch unter den gegebenen Lasten tatsächlich auftrat.

Der sich ergebende Widerspruch lässt sich nur durch den Einfluss lokaler Effekte bei der Lasteinleitung am Wandkopf erklären. Im Gegensatz zu den Wänden aus Hochlochziegeln, die infolge des in die Steinlöcher eingedrungenen Betons eine sehr gute Verzahnung von oberster Steinreihe und Wandkopfbalken besaßen und damit eine gleichförmige Lastweiterleitung ermöglichten, konnten bei den KS-Wänden die Horizontallasten im Wesentlichen nur durch Reibung und Haftscherfestigkeit übertragen werden. Zusätzlich ergab sich durch die pro Stein vorhandenen zwei Grifflöcher $(\phi=50 \mathrm{~mm}$, Tabelle 14) eine lokale Dübelwirkung, die zu Spannungskonzentrationen - insbesondere im Umkreis der Lasteinleitung durch den Gelenkbolzen - führte.

Die Rückrechnung des Reibungsversagens in Riss 6 (s. Abbildung A-21) erfolgt mit der zugehörigen Normalspannung von $\sigma=0,74 \mathrm{~N} / \mathrm{mm}^{2}$ und einer um den Faktor 1,62 erhöhten Schubspannung $\tau_{\max }=0,76 \mathrm{~N} / \mathrm{mm}^{2}$. Dadurch wird die Schubspannungskonzentration in der obersten Steinreihe in Wandmitte erfasst (entspricht einem von 1,25 auf 2,0 erhöhten Schubspannungsverteilungsfaktor). Infolge des dadurch eingeleiteten Gleitens in den Rissen $6 \div 8$ und des damit verbundenen Abbaus der Haftscherfestigkeit wird die Gleitverschiebung in den abgetreppten Rissen $9 \div 14$ und anschließend der Steinzugriss 5 initiiert.

\section{Wandversuch KS4-3}

Das Versagen von Wand KS4-3 trat bei annähernd gleicher Einwirkungshöhe $(H=-165 \mathrm{kN}$, $M_{\text {Kopf }}=118 \mathrm{kNm}$ ) und in vergleichbarer Form wie bei Wandversuch KS3-3 ein. Trotz der um 26\% höheren Haftscherfestigkeit (Tabelle 23) waren keine signifikanten Unterschiede im Versagensbild erkennbar. 
Die zugehörigen Schubspannungen am Wandkopf und auf halber Wandhöhe liegen bei $0,48 \mathrm{~N} / \mathrm{mm}^{2}$ und am Wandfuß bei $2,04 \mathrm{~N} / \mathrm{mm}^{2}$. Mit der gemessenen Haftscherfestigkeit von $k=0,98 \mathrm{~N} / \mathrm{mm}^{2}$ und einem abgeschätzten Reibbeiwert von $\mu=0,7$, bestimmt sich im Kriterium Gleiten in der Lagerfuge als aufnehmbare Schubspannung am Wandkopf und auf halber Wandhöhe $0,84 \mathrm{~N} / \mathrm{mm}^{2}$ sowie am Wandfuß 1,78 N/mm².

Die vorhandenen Schubspannungen liegen am Wandkopf und auf halber Wandhöhe um 43\% unter den Aufnehmbaren. Am Wandfuß dagegen werden die aufnehmbaren Schubspannungen um 13\% überschritten, ohne dass das Versagensbild Gleiten in der Lagerfuge hier eintrat.

Das Kriterium Steinzugversagen ist auf halber Wandhöhe und am Wandkopf infolge des Abstandes mit einem Faktor von 3,3 nicht maßgebend (Steinzugfestigkeit $f_{b t}=3,3 \mathrm{~N} / \mathrm{mm}^{2}$, Tabelle 23). Am Wandfuß dagegen wird die maximal aufnehmbare Schubspannung von $1,97 \mathrm{~N} / \mathrm{mm}^{2}$ um $4 \%$ überschritten, ohne dass dieses Versagensbild im Versuch unter den gegebenen Lasten tatsächlich auftrat.

Der sich ergebende Widerspruch lässt sich nur durch den Einfluss lokaler Effekte bei der Lasteinleitung am Wandkopf entsprechend Wandversuch KS3-3 erklären.

Die Rückrechnung des Reibungsversagens in Riss 6 (s. Abbildung A-22) erfolgt mit der zugehörigen Normalspannung von $\sigma=0,75 \mathrm{~N} / \mathrm{mm}^{2}$ und der um den Faktor 1,85 erhöhten Schubspannung $\tau_{\max }=0,89 \mathrm{~N} / \mathrm{mm}^{2}$. Dadurch wird die Schubspannungskonzentration in der obersten Steinreihe in Wandmitte erfasst (entspricht einem von 1,25 auf 2,3 erhöhten Schubspannungsverteilungsfaktor). Infolge des dadurch eingeleiteten Gleitens in den Rissen $6 \div 8$ und des damit verbundenen Abbaus der Haftscherfestigkeit werden die Gleitverschiebung in den abgetreppten Rissen $9 \div 18$ und anschließend die Steinzugrisse 4 und 5 initiiert.

\subsubsection{Zusammenfassung}

Der Verformungs- und Spannungszustand der geprüften Wände wurde durch die Berücksichtigung der Sub-Struktur mit der Einspannwirkung in den Geschossdecken des 3Massenschwingers den Gegebenheiten in üblichen Hochbauten weitestgehend angenähert.

Durch die Erweiterung des Versuchsstandes in Form gesteuerter Wandkopfmomente und -normalkräfte konnten annähernd beliebige Kraft- und Verformungszustände auf die Wände aufgebracht, und die Nachteile konventioneller Prüfstände vermieden werden. Mit Hilfe statisch-zyklischer Vorversuche wurde die Charakteristik der in den Hauptversuchen lediglich numerisch berücksichtigten Sub-Struktur bestimmt.

Der Einfluss einer Stoßfugenvermörtelung (KS2) auf die Traglast war mit einer Erhöhung um $26 \%$ begrenzt. Das Versagensbild war hier durch einen diagonal durchlaufenden Riss gekennzeichnet. Die Wandsteifigkeit lag 14\% $\div 33 \%$ über der bei unvermörtelten Stoßfugen (KS1).

Durch die Anordnung der Elastomere im Randbereich der untersten Lagerfuge von Wand F kam es zu einer deutlichen Reduzierung der Steifigkeit. Die Bereiche direkt über den Elastomeren waren aufgrund der geringen Schub- und Drucksteifigkeit der Platten an der Lastabtragung nicht beteiligt. Entsprechend gingen die Steinzugrisse vom Ende der Elastomere aus.

Bei reduzierter Haftscherfestigkeit (KS3) trat als primäres Versagensbild ein abgetrepptes Gleiten in den Stoß- und Lagerfugen auf. Aufgrund des geringen Unterschiedes der gemessenen Haftscherfestigkeiten zu der der Referenzwand KS4, konnte der Einfluss einer Änderung der Haftscherfestigkeit auf das Bruchbild nicht detailliert untersucht werden. 
Die Nachrechnung der aufgetretenen Versagensbilder Klaffen in der Lagerfuge, Gleiten in der Lagerfuge und Steinzugversagen bei den Wänden D - F, KS3 und KS4 mit dem Schubmodell von Mann / Müller führte in Anbetracht der vorhandenen Streuungen der Materialparameter zu einer guten Übereinstimmung. Für die zutreffende Abschätzung der Schubtragfähigkeit sind daher die entscheidenden Kennwerte schräge Steinzugfestigkeit, Haftscherfestigkeit und Reibbeiwert den gewählten Stein-Mörtel-Kombinationen angepasst und realitätsnah zu bestimmen. Hier ist noch Forschungsbedarf erkennbar.

Bei großformatigen Elementen (Wand KS1 und KS2) setzten sich die Wände nur aus einer geringen Steinanzahl zusammen, wodurch die Voraussetzungen des Schubmodells von Mann / Müller - insbesondere bei großen Exzentrizitäten - verletzt wurden. Bei der Nachrechnung der Versagensbilder und Traglasten mit einem Mikromodell, bei dem die Steine und die Mörtelfuge diskret abgebildet wurden, konnten die Versuchsergebnisse bestätigt werden.

Als Ergebnis der zweiten Versuchsstufe zeigte sich, dass der Steifigkeitsabfall in Form einer Erhöhung der ersten Eigenperiode unter Maximalbeanspruchung von im Mittel 27\% deutlich geringer als in der ersten Stufe von im Mittel 104\% war. Als Ursache sind die geänderten Randbedingungen zu nennen, die den Biegeverformungsanteil der Wand deutlich reduzierten, was dem Verhalten unter realistischen Bedingungen entspricht. Entsprechend waren die aufgetretenen Versagensbilder in Form von Steinzugrissen oder Gleiten abgetreppt in den Stoßund Lagerfugen gleichmäßiger über die gesamte Wandhöhe verteilt.

Unabhängig vom Steifigkeitsabfall in Abhängigkeit der Belastungshöhe lagen die tatsächlichen Anfangssteifigkeiten der Wände bei ungerissenen Querschnitten deutlich unter denen einer linear-elastischen Vergleichsberechnung bei isotropen Eigenschaften. Die Unterschiede waren bei den Wänden der oberen Geschosse mit geringer Normalkraft besonders stark ausgeprägt. Bei der üblichen Ermittlungsweise werden die Systemsteifigkeiten und damit auch die Eigenfrequenzen der Konstruktionen somit zu hoch abgeschätzt. Im Antwortspektrum führt eine Erhöhung der Eigenfrequenz im Bereich des abfallenden Astes zu direkt proportionaler Lasterhöhung.

Für die Beurteilung der Ergebnisse wurden die Versuche vergleichend als linear-elastische Mehrmassenschwinger mit den in den statisch-zyklischen Vorversuchen ermittelten Anfangssteifigkeiten berechnet. Es zeigte sich, dass die in den Versuchen aufgetretenen Horizontalkräfte deutlich unter denen der linear-elastischen Berechnung lagen. Dieser Effekt nahm mit steigender Einwirkungshöhe zu - bei maximaler bezogener Horizontalkraft lag der Verhältniswert zwischen 1,03 und 2,21 bei einem Mittelwert von 1,54. Der unterste Wert von 1,03 trat bei der Wand KS2 mit vermörtelten Stoßfugen auf, wohingegen der oberste Wert von 2,21 zu der Referenzwand KS1 mit unvermörtelten Stoßfugen gehörte.

Zusätzlich ist dabei jedoch der oben dargestellte Einfluss der Steifigkeitsermittlung auf die Systemeigenperiode und damit indirekt auf die Einwirkungen zu berücksichtigen. 


\section{Materialmodell}

\subsection{Einleitung}

\subsubsection{Anisotropie}

Das Trag- und Verformungsverhalten von Mauerwerk ist ausgeprägt anisotrop. Dieses resultiert zum einen aus der Anisotropie der Mauersteine selbst - z. B. bei gelochten Steinen aus dem Lochbild oder bei Vollsteinen infolge des Herstellprozesses - und zum anderen aus der Anordnung der Stoß- und Lagerfugen im Mauerwerksverband. Insgesamt ergeben sich orthotrope Materialeigenschaften. Für den ebenen Spannungszustand stellt sich im linearelastischen Fall mit $x$ und $y$ als den Hauptachsen der Orthotropie die Beziehung $\underline{\sigma}=\underline{C} \cdot \underline{\varepsilon}$, mit

$$
\begin{aligned}
& \underline{\sigma}=\left\{\begin{array}{lll}
\sigma_{x} & \sigma_{y} & \tau_{x y}
\end{array}\right\}^{T} \\
& \underline{\varepsilon}=\left\{\begin{array}{lll}
\varepsilon_{x} & \varepsilon_{y} & \gamma_{x y}
\end{array}\right\}^{T} \\
& \underline{C}=\frac{1}{1-v_{y x} \cdot v_{x y}}\left[\begin{array}{ccc}
E_{x} & v_{y x} \cdot E_{x} & 0 \\
v_{x y} \cdot E_{y} & E_{y} & 0 \\
0 & 0 & \left(1-v_{y x} \cdot v_{x y}\right) \cdot G_{x y}
\end{array}\right]
\end{aligned}
$$

Mit der Vereinfachung bei transversaler Orthotropie $E_{y} / E_{x}=v_{\mathrm{yx}} / \mathrm{v}_{\mathrm{xy}}=n$ verbleiben für die symmetrische Matrix $\underline{C}$ die vier unabhängigen Parameter $E_{x}, E_{y}, v_{\mathrm{xy}}$ und $G_{x y}$.

Bei orthotropen Materialeigenschaften kann allein mit Angabe der Hauptspannungen der Spannungszustand in einer Scheibe nicht ausreichend beschrieben werden. Entweder ist als weiterer Parameter der Winkel der Hauptspannungen zu den orthotropen Hauptrichtungen der Orthotropie bekannt oder es werden die in das Hauptachsensystem der Orthotropie transformierten drei Spannungskomponenten $\sigma_{x}, \sigma_{y}$ und $\tau_{x y}$ angegeben. Da die Materialparameter von Mauerwerk bereits auf die Hauptrichtungen der Orthotropie - d.h. parallel bzw. senkrecht zur Lagerfuge - bezogen sind, wird nachfolgend letztere Möglichkeit gewählt.

\subsubsection{Makro- und Mikromodellierung}

Für numerische Untersuchungen zum Trag- und Verformungsverhalten bieten sich Mikrooder Makromodelle an. Bei ersteren werden die Steine und die Mörtelfugen mit Kontinuumselementen sowie die Kontaktfläche von Stein und Mörtel mit speziellen Kontaktelementen diskretisiert. Diese Vorgehensweise erfordert die genaue Kenntnis der geometrischen Parameter und der einzelnen Materialkennwerte. Auf diesem Weg können lokale Effekte, wie z. B. Fugenklaffen oder die Spannungs- und Dehnungsverteilung in den einzelnen Komponenten genau untersucht werden. Aufgrund des sehr hohen Diskretisierungs- und Auswertungsaufwandes eignet sich dieses Verfahren lediglich zur Nachrechnung einzelner Versuche, wie z.B. 
der Wandversuche KS1-7, KS1-8 und KS2-2 in Abschnitt 5.5.7. Größere Untersuchungen, beispielsweise im Zuge der Anwendung in der Praxis, scheiden daher in der Regel aus.

Mit Makromodellen werden die Eigenschaften der einzelnen Materialien sowie deren Zusammenwirken verschmiert abgebildet. Dadurch entfallen die wesentlichen Nachteile von Mikromodellen. Nachteilig wirkt sich hier jedoch die Tatsache aus, dass lokale Effekte, wie z. B. ein Klaffen der Lagerfuge, ebenfalls nur verschmiert abgebildet werden können.

Nachfolgend wird ein einfaches und robustes Materialmodell auf Makroebene nach der Plastizitätstheorie entwickelt, mit welchem das Trag- und Verformungsverhalten unter Erdbebenbeanspruchung realitätsnah beschrieben werden kann. Es eignet sich aufgrund der geringen Anzahl zu bestimmender Eingangswerte auch prinzipiell für die Anwendung in der Praxis.

\subsubsection{Grundlagen der Plastizitätstheorie}

\section{Allgemeines}

Beim Nachweis des Lastfalls Erdbeben im Grenzzustand der Tragfähigkeit liegen die Spannungen und Verformungen in Bereichen, in denen das Materialverhalten nicht mehr linearelastisch beschrieben werden kann. Bei Versuchen mit zyklischer Normalkraft- und Schubbeanspruchung treten neben elastischen Anteilen auch bleibende, plastische Dehnungsanteile auf. Die Grundlagen der Plastizitätstheorie mit ihrer Implementierung in die Methode der Finiten Elemente ist in Chen [9], Hofstetter / Mang [50], Owen / Hinton [98], Shah / Swartz / Ouyang [128] dargestellt.

Nach der Plastizitätstheorie setzen sich die Dehnungen bzw. die Dehninkremente aus einem reversiblen, elastischen und einem bleibenden, plastischen Anteil zusammen.

$$
d \varepsilon=d \varepsilon_{e l}+d \varepsilon_{p l}
$$

Mit Hilfe einer Fließbedingung $F(\sigma, \kappa)$ in Abhängigkeit des Spannungszustandes $\sigma$ und des Verfestigungsparameters $\kappa$ erfolgt die Unterscheidung zwischen den beiden Bereichen, d.h. ist $F<0$ liegt der elastische Fall vor. Der Bereich $F>0$ ist hier nicht zulässig.

In einem Spannungszustand an der Fließgrenze $F=0$ unterscheiden sich bei einem verfestigenden Material mit der Ableitung nach der Lastzeit folgende drei Fälle:

$$
\begin{aligned}
& F=0, \quad\left(\frac{\partial F}{\partial \sigma}\right)^{T} \dot{\sigma}>0, \quad \dot{\lambda}>0 \Rightarrow \text { Belastung } \\
& F=0, \quad\left(\frac{\partial F}{\partial \sigma}\right)^{T} \dot{\sigma}=0, \quad \dot{\lambda}=0 \Rightarrow \text { neutrale Spannungsumlagerung } \\
& F=0, \quad\left(\frac{\partial F}{\partial \sigma}\right)^{T} \dot{\sigma}<0, \quad \dot{\lambda}=0 \Rightarrow \text { Entlastung }
\end{aligned}
$$

Unter Berücksichtigung einer Verfestigung folgt aus der Bedingung, dass $F=0$ auch am Inkrementende gelten muss 


$$
d F=\frac{\partial F}{\partial \sigma} d \sigma+\frac{\partial F}{\partial \kappa} d \kappa=0
$$

\section{Fließgesetz}

Mit dem Fließgesetz werden die Richtungen und die Beträge der plastischen Verzerrungsinkremente festgelegt. Mit $G(\sigma, \kappa)$ als Funktion des plastischen Potentials und dem plastischen Multiplikatorinkrement $d \lambda$ als skalarem Faktor bestimmt sich das plastische Verzerrungsinkrement zu

$$
d \varepsilon_{p l}=\frac{\partial G}{\partial \sigma} d \lambda
$$

Bei einem assoziierten Fließgesetz wird die Funktion des plastischen Potentials $G(\sigma, \kappa)$ der der Fließfunktion $F(\sigma, \kappa)$ gleichgesetzt.

Entsprechend der Normalenregel steht der Vektor des plastischen Verzerrungsinkrementes senkrecht auf der Fließfläche. Als Gesamtverzerrungsinkrement ergibt sich einschließlich des elastischen Anteils

$$
d \varepsilon=C^{-1} d \sigma+\frac{\partial F}{\partial \sigma} d \lambda
$$

\section{Verfestigung}

Ändern sich die Fließgrenzen infolge der Lastgeschichte, so werden die Fließflächen bzw. die Potentialfunktionen modifiziert - es erfolgt eine Verfestigung bzw. Entfestigung. Bei einer gleichmäßigen Aufweitung bzw. Kontraktion der Fließfläche handelt es sich um eine isotrope Verfestigung, während eine Verschiebung als kinematische Verfestigung bezeichnet wird. Die Fließbedingung bestimmt sich unter Einschluss der Verfestigungsbeziehung zu

$$
F(\sigma ; \kappa)=f(\sigma)-k(\kappa)=0
$$

Die Berücksichtigung einer Verfestigung kann üblicherweise nach der Arbeits- oder der Dehnungsverfestigung erfolgen. Bei ersterer wird der Verfestigungsparameter als Funktion der gesamten plastischen Arbeit

$$
\kappa=W_{p l}=\int \sigma d \varepsilon_{p l}
$$

und beim Ansatz einer Dehnungsverfestigung zur effektiven, plastischen Dehnung $\varepsilon_{p l}$ angenommen. 


\subsection{Bruch- und Fließbedingungen}

Auf Basis der Plastizitätstheorie wird nachfolgend ein auf die untersuchte Beanspruchungssituation angepasstes Materialmodell entwickelt. Da Mauerwerk wegen der Vielzahl möglicher Versagenskriterien nicht durch ein einfaches Fließkriterium beschrieben werden kann, werden die Fließflächen aus den Bruchbedingungen abgeleitet. Als Versagenskriterien unter kombinierter Schub- und Vertikalbeanspruchung werden die Bruchbedingungen des Schubmodells von Mann / Müller angesetzt.

Eine Beschränkung der plastischen Dehnungen im Fall des Reibungsversagens in den Lagerfugen erfolgt nicht, da sich in Versuchen bei diesem Kriterium ein nahezu ideal-plastisches Verhalten ohne erkennbaren Tragfähigkeitsverlust zeigt. Für das Kriterium Druckversagen wird eine aus einaxialen Versuchen hergeleitete Arbeitsverfestigungsbeziehung angesetzt. Die Erfassung des Nachbruchverhaltens erfolgt dabei über die Entfestigung nach dem Erreichen der Maximalbeanspruchung sowie durch die Beschränkung der maximalen Dehnung. Die im Fall des Steinzugversagens auftretenden Risse werden durch eine lineare Entfestigungsbeziehung verschmiert abgebildet.

Für die Beanspruchungsrichtung parallel zu den Lagerfugen erfolgt die Annahme eines linearelastisches Verhaltens. Da die Spannungen und Dehnungen in $x$-Richtung bei den hier betrachteten Beanspruchungssituationen gering sind und deutlich unter experimentell ermittelten Festigkeitswerten liegen (Backes [4], Schubert / Hoffmann [122]), werden dahingehend keine Versagenskriterien oder Fließregeln angesetzt.

\subsubsection{Schubtragmodell}

Für die Beschreibung der Tragfähigkeit unter kombinierter Beanspruchung wird auf das Schubmodell von Mann / Müller [73, 74] zurückgegriffen. Hierbei wird davon ausgegangen, dass in den Stoßfugen keinerlei Schub- oder Normalspannungen übertragen werden können. Dieses begründet sich im Fehlen höherer Normalspannungen parallel zu den Lagerfugen zusammen mit dem Ablösen des Mörtels von den Stirnflächen der Steine, beispielsweise infolge Schwindverkürzung. Zum anderen werden heutzutage aus Wärmeschutz- und Rationalisierungsgründen vermehrt Stoßfugen planmäßig unvermörtelt hergestellt.

Die Untersuchungen im Rahmen dieser Arbeit erfolgen auf Basis eines regelmäßigen Läuferverbandes mit einem Überbindemaß von einer halben Steinlänge sowie einem Verhältnis von Steinhöhe zu -länge von annähernd 1:2. Soll der Einfluss anderer Überbindemaße oder Steingeometrien berücksichtigt werden, so ist dieses problemlos durch Modifikation der vorhandenen oder Einbau zusätzlicher Kriterien möglich (s. Kapitel 4).

Die vom Betrag her relativ kleinen globalen Druck- und Zugspannungen in Wandlängsrichtung werden bei den Bruchkriterien vernachlässigt. Dieses ist für den untersuchten Fall von schubbeanspruchten Wandscheiben zu vertreten, bei denen im wesentlichen Normalspannungen senkrecht zu den Lagerfugen aus den Vertikallasten sowie Schubspannungen aus den Horizontallasten in bemessungsrelevanten Größenordnungen auftreten.

Für die Untersuchung der Spannungsverteilung an einem Einzelstein wird ein Element aus der Wandscheibe herausgeschnitten und die angreifenden mittleren Schub- und Normalspannungen angetragen. Da in den Stoßfugen keinerlei Spannungen übertragen werden können, muss das Moment infolge angreifender Schubspannungen durch eine blockförmige Verteilung von $\Delta \sigma_{y}$-Normalspannungen in der Lagerfuge - mit unterschiedlichem Vorzeichen in der linken und rechten Steinhälfte - ausgeglichen werden. Zusammen mit den mittleren Normalspan- 
nungen $\sigma_{\mathrm{y}}$ infolge Vertikalauflast und den Schubspannungen $\tau$ ergibt sich folgender vereinfachter Einwirkungszustand am Einzelstein:

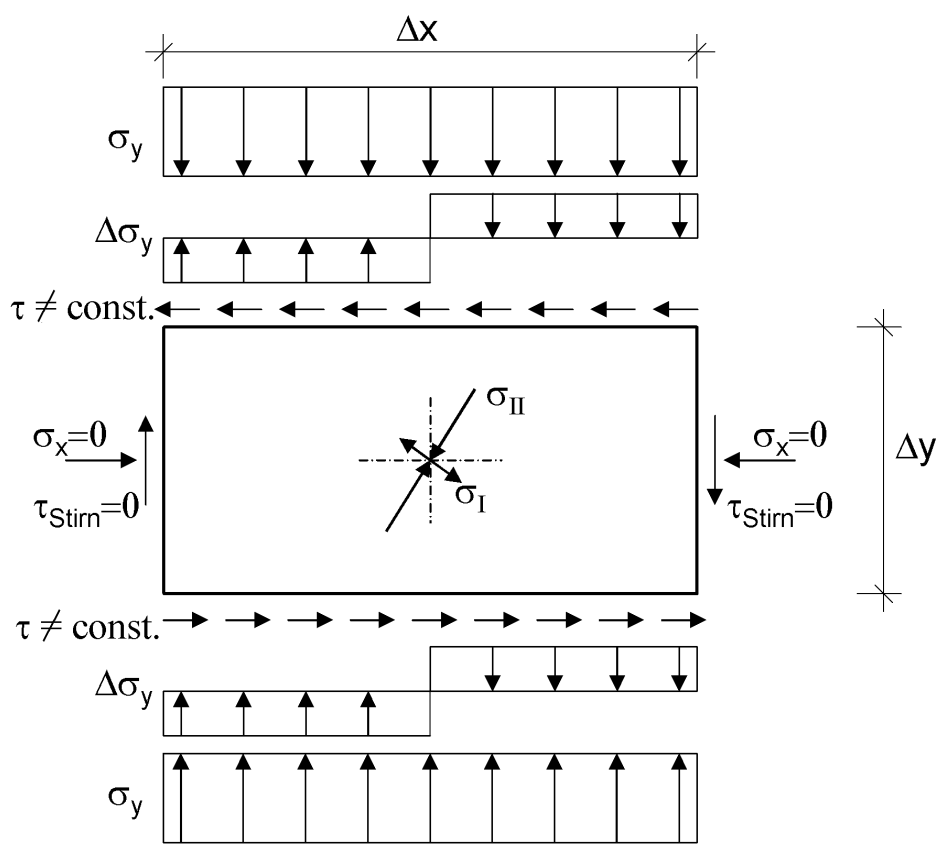

Abbildung 61: Spannungszustand am Einzelstein unter kombinierter Schub- und Druckbeanspruchung nach Mann [73]

Als Versagensformen können dabei folgende Fälle auftreten:

- Reibungsversagen / Gleiten in der Lagerfuge

- Überschreitung der schrägen Steinzugfestigkeit

- Druckversagen

- Lagerfugenzugversagen / Klaffen in der Lagerfuge

Die Tragfähigkeiten unter kombinierter Schub- und Normalkraftbeanspruchung der einzelnen Versagenskriterien werden im Folgenden beschrieben:

\section{Kriterium 1 - Gleiten in der Lagerfuge}

Die Normalspannungen in der Lagerfuge haben in den beiden Steinhälften aufgrund des zusätzlichen $\Delta \sigma_{y}$-Anteils unterschiedliche Beträge, d.h. $\sigma_{l, 2}=\sigma_{y} \pm|\tau| \cdot 2 \cdot \Delta y / \Delta x$. Für das Reibungsversagen in der Lagerfuge wird die Steinhälfte mit der geringeren Normaldruckspannung $\sigma_{l}$ maßgebend. Die Beschreibung erfolgt mit Hilfe der Coulomb'schenReibungsgeraden unter Berücksichtigung der Haftscherfestigkeit $k$ und des Reibbeiwertes $\mu$. Es ergibt sich eine maximal aufnehmbare Schubspannung von $|\tau|=k-\mu \cdot \sigma_{l}$. Durch die Einführung abgeminderter Haftscherfestigkeits- und Reibbeiwerte

$$
\bar{k}=k \cdot \frac{1}{1+\mu \cdot \frac{2 \cdot \Delta y}{\Delta x}}
$$


und

$$
\bar{\mu}=\mu \cdot \frac{1}{1+\mu \cdot \frac{2 \cdot \Delta y}{\Delta x}}
$$

wodurch die unterschiedliche Normalspannungsverteilung in den beiden Steinhälften berücksichtig wird, ergibt sich mit der mittleren Normalspannung $\sigma_{y}$ als maximal aufnehmbare Schubspannung im Kriterium 1:

$$
\left|\tau_{\text {Krit_ } 1}\right|=\bar{k}-\bar{\mu} \cdot \sigma_{y}
$$

\section{Kriterium 2 - Steinzugversagen}

$\mathrm{Da}$ in den Stoßfugen keinerlei Schubspannungen übertragen werden können, muss in jeder Steinlage die doppelte bezogene Schubspannung aufgenommen werden. Zusammen mit der mittleren Normalspannung $\sigma_{y}$ ergibt sich bei einem regelmäßigen Läuferverband mit einem Überbindemaß von einer halben Steinlänge in Steinmitte eine schiefe Hauptzugspannung $\sigma_{I}$, die beim Überschreiten der schrägen Steinzugfestigkeit $f_{b t}$ zum Steinzugversagen führt. Die maximalen Hauptzugspannungen ergeben sich in Steinmitte [73, 74] zu

$$
\sigma_{I}=-\frac{\sigma_{y}}{2}+\sqrt{\left(\frac{\sigma_{y}}{2}\right)^{2}+(2,3 \cdot \tau)^{2}}=f_{b t}
$$

Damit beträgt die maximale aufnehmbare Schubspannung im Kriterium 2:

$$
\left|\tau_{\text {Krit_2 }}\right|=\frac{f_{b t}}{2,3} \cdot \sqrt{1-\frac{\sigma_{y}}{f_{b t}}}
$$

$\mathrm{Zu}$ beachten ist, dass die schräge Steinzugfestigkeit $f_{b t}$ entsprechend der Steinart und Lochform richtungsabhängig ist und durch den vorhandenen Querdruck $\sigma_{I I}$ beeinflusst wird (s. Abbildung 61). 


\section{$\underline{\text { Kriterium } 3 \text { - Druckversagen }}$}

In der Steinhälfte mit der vom Betrag her höheren Normaldruckspannung $\sigma_{2}$ kommt es bei Überschreitung der Mauerwerksdruckfestigkeit $f_{m y}$ zum Druckversagen. Zurückgerechnet auf eine aufnehmbare Schubspannung bestimmt sich:

$$
\left|\tau_{\text {Krit_3 } 3}\right|=\left(f_{m y}+\sigma_{y}\right) \cdot \frac{\Delta x}{2 \cdot \Delta y}
$$

\section{Kriterium 4 - Klaffen in der Lagerfuge}

Bei geringer Drucknormalkraft in der Wand können in einer Steinhälfte Zugspannungen auftreten, welche die Haftzugfestigkeit zwischen Stein und Mörtel $f_{m o, H Z}$ überschreiten. Es kommt zum Klaffen in der Lagerfuge. Aufgelöst nach der Schubspannung ergibt sich:

$$
\left|\tau_{\text {Krit_ } 4}\right|=\left(f_{m o, H Z}-\sigma_{y}\right) \cdot \frac{\Delta x}{2 \cdot \Delta y}
$$

Dieses Versagenskriterium ist in der Regel durch den Ansatz des Kriteriums 1 (Gleiten in der Lagerfuge) sowie durch die Ausschaltung von globalen Zugspannungen senkrecht zu den Lagerfugen abgedeckt und wird im Weiteren vernachlässigt.

\subsubsection{Fließregeln}

Für die Beschreibung der Richtungen der plastischen Dehninkremente werden in dem entwickelten Materialmodell entsprechend dem Versagenstyp assoziierte und nicht assoziierte Fließregeln angesetzt. Die Unterscheidung erfolgt nach dem aktiven Versagenskriterium.

Beim Versagenstyp Gleiten in der Lagerfuge ist in Versuchen im Wesentlichen eine reine Schubgleitung zu beobachten. Verformungen senkrecht zu den Lagerfugen, was einem Dillatationswinkel $\tan \psi>0$ entsprechen würde (van der Pluijm [105], Abschnitt 3.3) und die bei einer assoziierten Fließbedingung zu berücksichtigen wären, sind in der Regel gering und können vernachlässigt werden. Daher wird für dieses Kriterium als plastisches Dehninkrement ausschließlich eine Schubverformung mit $\frac{\partial G_{K r i t \_} 1}{\partial \sigma}=\left\{\begin{array}{lll}0 & 0 & 1\end{array}\right\}^{T}$ festgelegt.

Im Fall der Kriterien Steinzugversagen und Druckversagen werden assoziierte Fließregeln angesetzt mit der Richtung der plastischen Dehninkremente senkrecht zu der aktiven Fließfläche.

Für die Beanspruchungsrichtung parallel zu den Lagerfugen wird ein linear-elastisches Verhalten angesetzt. Da die Spannungen und Dehnungen in $x$-Richtung bei den hier betrachteten Beanspruchungssituationen gering sind und deutlich unter experimentell ermittelten Festigkeitswerten liegen (Schubert / Hoffmann [122]), werden dahingehend keine Versagenskriterien oder Fließregeln angesetzt. 


\subsubsection{Verfestigung}

Die Verfestigungs- bzw. Entfestigungsbeziehungen in den beiden Beanspruchungsrichtungen Normalkraftbeanspruchung senkrecht zu den Lagerfugen und Schubbeanspruchung werden entkoppelt und unabhängig behandelt. Ver- und Entfestigung werden symmetrisch zur $\sigma_{\mathrm{y}}$ Achse angenommen (s. Abbildung 70).

\subsubsection{Normalbeanspruchung}

Unter Druckbeanspruchung in $y$-Richtung senkrecht zu den Lagerfugen verhält sich Mauerwerk in weiten Bereichen nahezu linear elastisch. Abhängig von der Stein-MörtelKombination erfolgt je nach Mauerwerksart ab ca. $60 \div 90 \%$ der Maximalspannung ein leichtes Abflachen der Spannungs-Dehnungs-Linie. Nach dem Erreichen der Maximalspannung findet sich ein in der Regel nur gering ausgeprägter Nachbruchbereich. Das Druckversagen kann allgemein als spröd bezeichnet werden. Die Arbeitslinie kann einfach durch verformungsgesteuerte Versuche ermittelt oder aus der Literatur entnommen werden, z. B. Meyer / Schubert [84], Schubert [120], Vratsanou [155].
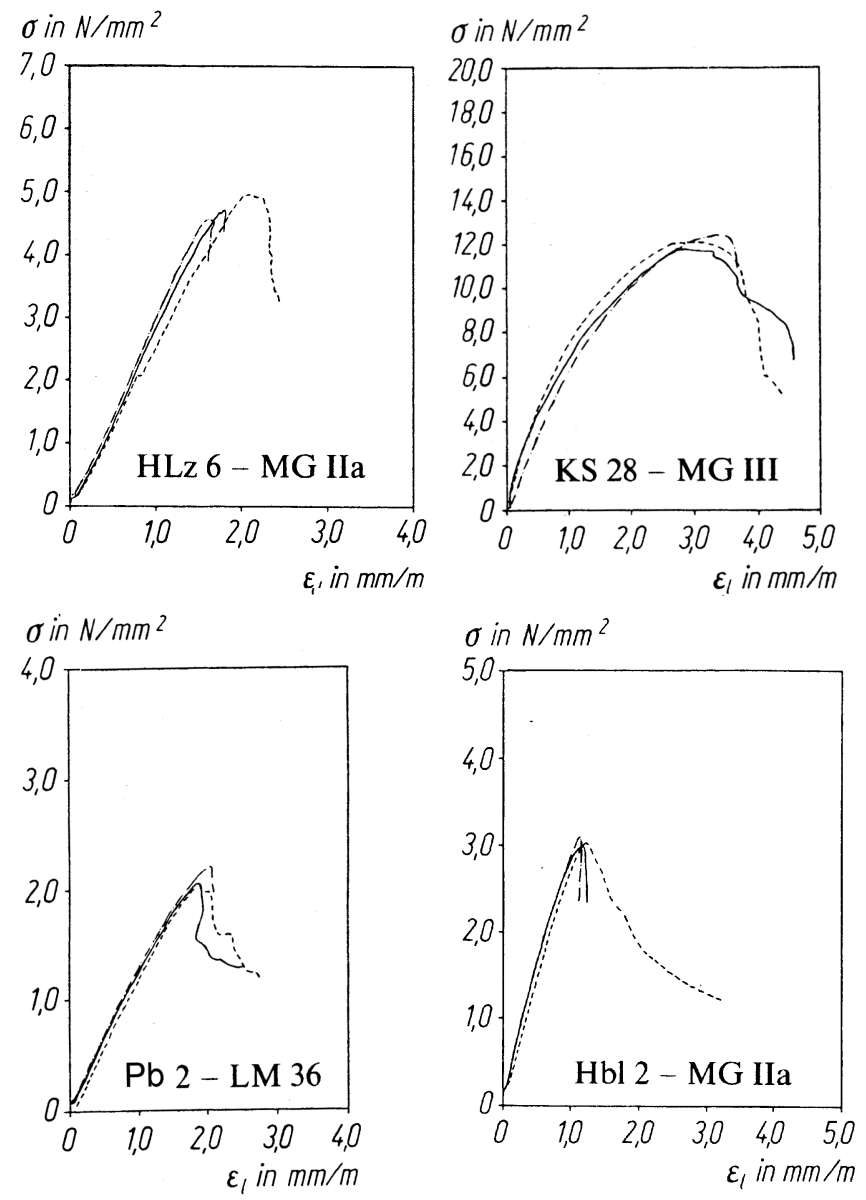

Abbildung 62: Spannungs-Dehnungs-Linien verschiedener Stein-Mörtel-Kombinationen unter Druckbeanspruchung senkrecht zu den Lagerfugen - aus Meyer / Schubert [84]

Die oben dargestellten Spannungs-Dehnungs-Linien stellen die Ergebnisse bei monotoner Belastungssteigerung dar. Erfolgt vor dem Erreichen der maximalen Dehnung eine Teilentlastung, so sind bleibende Dehnungen zu beobachten (Abrams / Noland / Atkinson [1], Naraine / 
Sinha $[93,94,95])$. Liegt die Ausgangslaststufe über der Elastizitätsgrenze, d.h. bereits im Bereich des abflachenden Astes, und erfolgt anschließend eine Teilentlastung, so liegt die Entlastungslinie etwa parallel zur Anfangssteigung der Erstbelastungslinie (s. Abbildung 21 und 63).

Nachfolgend ist die Spannungs-Dehnungs-Linie eines halben KS-Planelementes unter zyklischer Druckbelastung dargestellt. Da die Planelemente mit Dünnbettmörtel verlegt werden, besitzt der Mörtel nur einen untergeordneten Einfluss auf die Spannungs-Dehnungs-Linie des Mauerwerkes. Es kann in diesem Fall daher, insbesondere unter qualitativen Gesichtspunkten, vereinfacht von den Eigenschaften des Steins auf die des Gesamtmauerwerkes geschlossen werden. In dem in Abbildung 63 dargestellten Diagramm sind im Rahmen einer Nullpunktskorrektur die ersten beiden Zyklen durch eine Gerade ersetzt worden.

Die zyklischen Belastungskurven zeigen im Bereich kleiner Spannungen - hier etwa bis $4 \mathrm{~N} / \mathrm{mm}^{2}$, was etwa 1/7 der Maximalspannung entspricht - ein leichtes Abflachen der Spannungs-Dehnungs-Linie. Die Einhüllende der Zyklenkurven entspricht dabei im Wesentlichen der monotonen Belastungskurve. Sie ist vergleichbar mit der monotonen Belastungskurve von KS 28 - MG III in Abbildung 62.

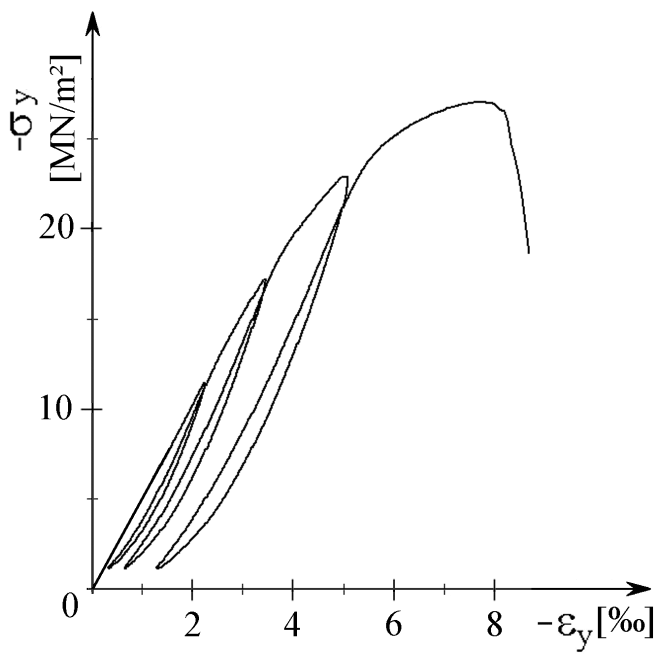

Abbildung 63: Spannungs-Dehnungs-Linie eines halben KS-Planelementes $(500 \mathrm{~mm} \cdot 500 \mathrm{~mm} \cdot 175 \mathrm{~mm})$ aus Wand KS3 unter zyklischer Druckbelastung senkrecht zu den Lagerfugen

Für die numerische Erfassung der Spannungs-Dehnungs-Linien sind die Versuchergebnisse weiter $\mathrm{zu}$ vereinfachen. Als ausreichend hat sich die Kombination aus einem linearelastischen Ast und einem anschließenden parabelförmigen Bereich erwiesen. Als Parameter für die Beschreibung sind dabei erforderlich:

- Elastizitätsmodul des Mauerwerkes senkrecht zu den Lagerfugen $E_{y}$ bzw. $E_{0}$

- Mauerwerksfestigkeit senkrecht zu den Lagerfugen $f_{m y}$

- Grenzdehnung elastischer Bereich $\varepsilon_{e l \text {-grenz }}$

- Dehnung bei maximaler Spannung $\varepsilon_{f_{-} \max }$

- Maximaldehnung $\varepsilon_{\max }$

Für die Beanspruchungsrichtung senkrecht zu den Lagerfugen in $y$-Richtung werden keinerlei Zugfestigkeiten angesetzt. Durch diese Vereinfachung werden vorhandene Haftzugfestigkeiten vernachlässigt, da sie zum einen eine unsichere und stark streuende Materialgröße sind, und zum anderen für das Trag- und Verformungsverhalten nur eine untergeordnete Bedeutung 
haben. Die zugehörigen voll reversiblen Zugdehnungen werden nicht begrenzt. Tritt im Rahmen der Berechnung in einem Prädiktorschritt eine Zugspannung senkrecht zu den Lagerfugen auf, so wird diese zusammen mit der zugehörigen Schubspannung zu Null gesetzt, was der Beobachtung eines spröden Zugverhaltens im Versuch entspricht.

Nachfolgend ist die resultierende qualitative Spannungs-Dehnungs-Linie bei Druckbeanspruchung senkrecht zu den Lagerfugen dargestellt:

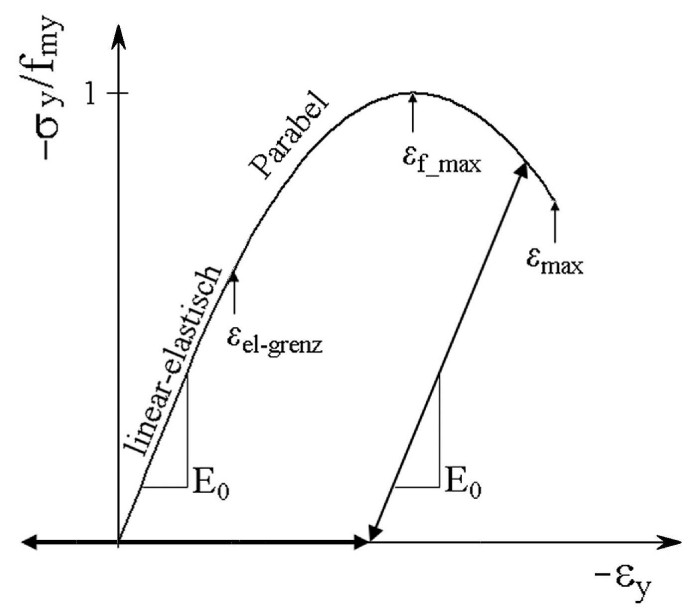

Abbildung 64: Qualitative Spannungs-Dehnungs-Beziehung für die Beanspruchung senkrecht zu den Lagerfugen

Die Funktion bei monotoner Laststeigerung ist in zwei Abschnitte aufgeteilt:

- Gerade: bei $\varepsilon_{p l}=0$

- Parabel bei $\varepsilon_{p l}<0$

(bzw. bei monotoner Erstbelastung: $\varepsilon_{y} \geq \varepsilon_{\text {el-grenz }}$ )

(bzw. bei monotoner Erstbelastung: $\varepsilon_{\mathrm{y}}<\varepsilon_{e l \text {-grenz }}$ )

Bei zyklischer Beanspruchung erfolgt die Entlastung auf einer gegenüber dem Ursprung um $\varepsilon_{p l}$ verschobenen Parallelen mit der Steigung $E_{0}$.

Die Beschreibung der einhüllenden Parabel in Abhängigkeit von $\varepsilon, \varepsilon_{f_{-} m a x}, \varepsilon_{e l \text {-grenz }}$ und $f_{m y}$ erfolgt mit

$$
\sigma_{y}(\varepsilon)=\frac{f_{m y}}{\varepsilon_{f_{-} \max }^{2}-\varepsilon_{\text {el-grenz }}^{2}} \cdot\left(\varepsilon^{2}-2 \cdot \varepsilon \cdot \varepsilon_{f_{-} \max }+\varepsilon_{\text {el-grenz }}^{2}\right)
$$

Für die Verfestigungsbeziehung wird als Bezugsgröße die Arbeit der plastischen Dehnung in $y$-Richtung mit den zugehörigen Spannungen $\sigma_{y}$ gesetzt. Als plastische Arbeit resultiert

$$
d W_{p l}^{y}=\sigma_{y} d \varepsilon_{p l}^{y}
$$


Die Arbeit einer plastischen Schubverformung bleibt bei diesem Verfestigungsansatz für Druckbeanspruchung senkrecht zu den Lagerfugen unberücksichtigt. Die qualitative Darstellung der Verfestigungsbeziehung ist in Abbildung 65 dargestellt.

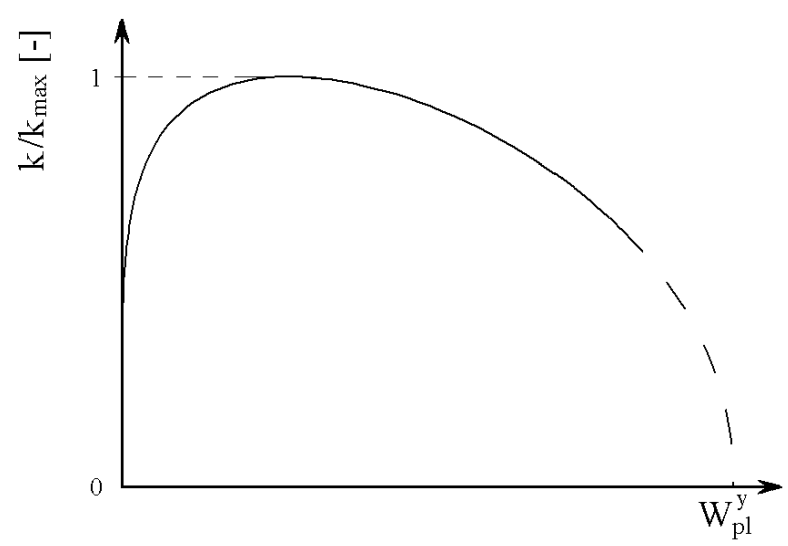

Abbildung 65: Qualitative Darstellung der Verfestigung $\boldsymbol{k}$ in Abhängigkeit der plastischen Arbeit $\boldsymbol{W}_{p} \boldsymbol{l}^{\boldsymbol{y}}$

Aus numerischen Gründen erfolgte nach dem Überschreiten der sich aus der Maximaldehnung $\mathcal{E}_{\max }$ ergebenden maximalen plastischen Arbeit der Ansatz einer minimalen Resttragfähigkeit.

\subsubsection{Schubbeanspruchung}

Beim Schubversagen wird nach den beiden Fällen und Versagensarten Steinzugversagen sowie Gleiten in der Lagerfuge unterschieden:

\section{Steinzugversagen}

Beim Schubversagen im Stein tritt an der Stelle mit der höchsten Hauptzugspannung (im regelmäßigen Läuferverband i. d. R. in Steinmitte) ein Riss infolge Überschreitung der schrägen Steinzugfestigkeit auf. Die Beschreibung dieses lokalen, quasi-spröden Verhaltens erfolgt mit Hilfe der bruchmechanischen Kenngröße der Bruchenergie $G_{f}$. Diese beschreibt den irreversiblen Arbeitsanteil, der für die vollständige Trennung eines Zugrisses - auch als Versagensart Mode I bezeichnet (s. Abbildung 66) - erforderlich ist. Global ist zwar ein Schubversagen der Wand zu beobachten, die lokale Betrachtung ergibt jedoch die Versagensart Zug.

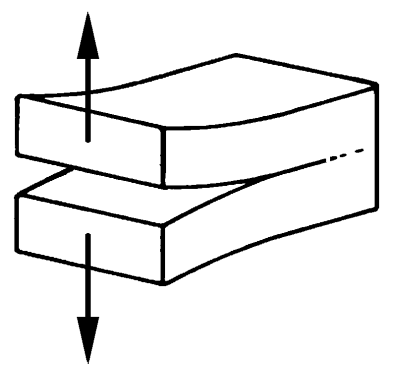

Mode I Zugversagen

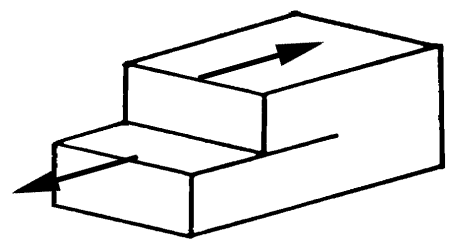

Mode II Schubversagen 
Wird für die Betrachtung eine lineare Spannungs-Rissöffnungs-Beziehung angesetzt (Hillerborg / Modeer / Petersson [47], Hofstetter / Mang [50], Shah / Swartz / Ouyang [128]) ergibt sich folgender Zusammenhang von Rissspannung und Rissöffnung $w$ :

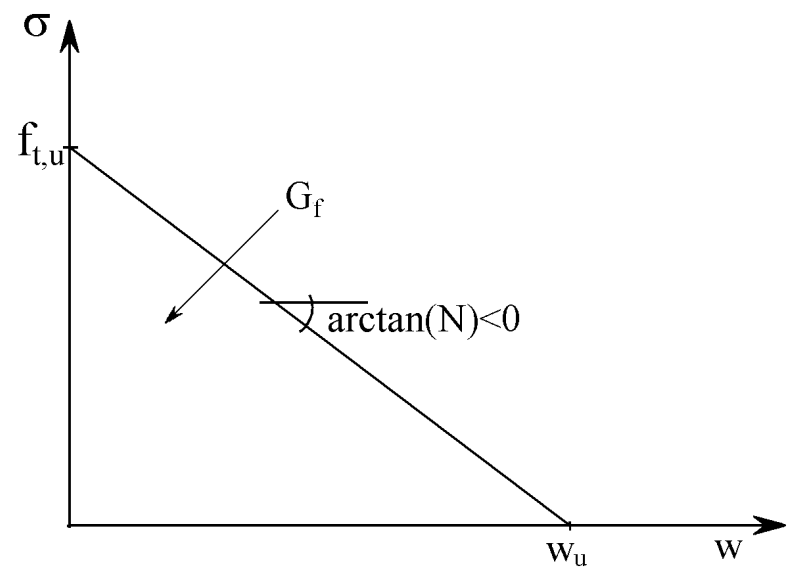

Abbildung 67: Lineare Spannungs-Rissöffnungsbeziehung

In Gleichungsform dargestellt:

$$
\begin{aligned}
& G_{f}=\int_{w=0}^{w=w_{u}} \sigma \cdot d w=\frac{1}{2} \cdot f_{t, u} \cdot w_{u} \\
& N=\frac{-f_{t, u}^{2}}{2 \cdot G_{f}}
\end{aligned}
$$

Für den Einbau in ein Finite-Elemente-Modell ist die Rissöffnungsbreite in eine Rissdehnung zu verschmieren. Als Bedingung gilt, dass unabhängig von der Maschenweite des Elementnetzes bei einem auftretenden Riss immer die gleiche Bruchenergie freigesetzt wird. Die Abhängigkeit von der Elementfläche und dem Ansatz wird durch eine äquivalente Länge beschrieben. Für den linearen Elementansatz resultiert bei annähernd quadratischen Elementen nach Rots [111] mit der Elementfläche $A_{e}$ die äquivalente Länge $L_{e q} \mathrm{zu}$

$$
L_{e q}=\alpha \cdot \sqrt{A_{e}}=\sqrt{2} \cdot \sqrt{A_{e}}
$$

Im Kriterium Steinzugversagen tritt die Rissöffnung bzw. die zugehörigen Dehnungen $\varepsilon_{I}$ senkrecht zu dem unter dem Winkel $\alpha_{0}$ zur Vertikalen liegenden Riss auf. Wird dieser irreversible Dehnungsanteil in das $x$ - $y$-Koordinatensystem transformiert, ergibt sich als zugehörige Schubverformung

$$
\gamma_{x y}=\varepsilon_{I} \cdot 2 \cdot \sin \alpha_{0} \cdot \cos \alpha_{0}
$$

Für das Kriterium Steinzugversagen kann somit die schräge Steinzugfestigkeit $f_{b t}$ in Abhängigkeit der Schubverformung dargestellt werden. 


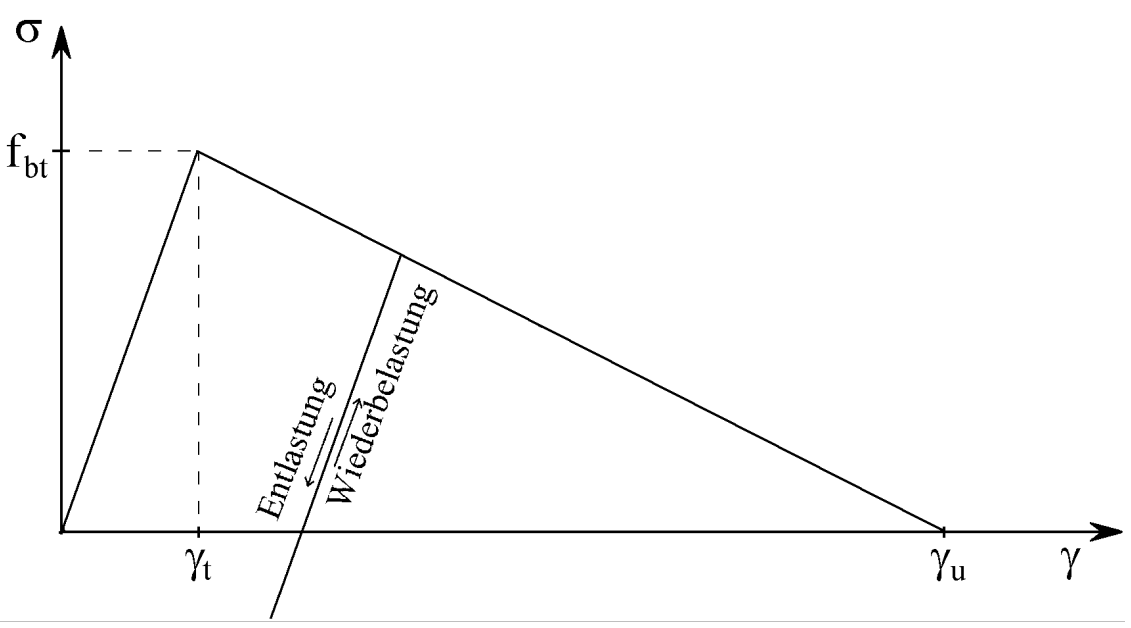

Abbildung 68: Lineare Entfestigung beim Steinzugversagen - Kriterium 2

Als zugehörige Grenzen resultieren:

$$
\begin{aligned}
& \gamma_{t}=\frac{f_{b t}}{2,3 \cdot G_{x y}} \cdot \sqrt{1-\frac{\sigma_{y}}{f_{b t}}} \\
& \gamma_{u}=\frac{4 \cdot G_{f}}{f_{b t} \cdot L_{e q}} \cdot \sin \alpha_{0} \cdot \cos \alpha_{0}
\end{aligned}
$$

\section{Gleiten in der Lagerfuge}

Nach dem Überschreiten der Schubfestigkeit des Kriteriums 1 tritt ein Gleiten in der Lagerfuge auf. Bei verformungsgesteuerten Schubversuchen an Zweisteinkörpern unter konstanter Normalspannung ist zu beobachten, dass die Schubspannung nach dem Überschreiten des Maximalwertes sukzessive auf einen Plateauwert abfällt, der dem Reibanteil entspricht (s. Kapitel 4). Der auf die Haftscherfestigkeit entfallende Schubtraganteil wird entsprechend abgebaut. Untersuchungen zur Bruchenergie von Mauerwerk unter diesem Versagensbild auch als Mode II bezeichnet (s. Abbildung 66) - sind in Kapitel 4 beschrieben. Die lineare Vereinfachung erfolgt mit Hilfe der Bedingung der gleichen Bruchenergie. 


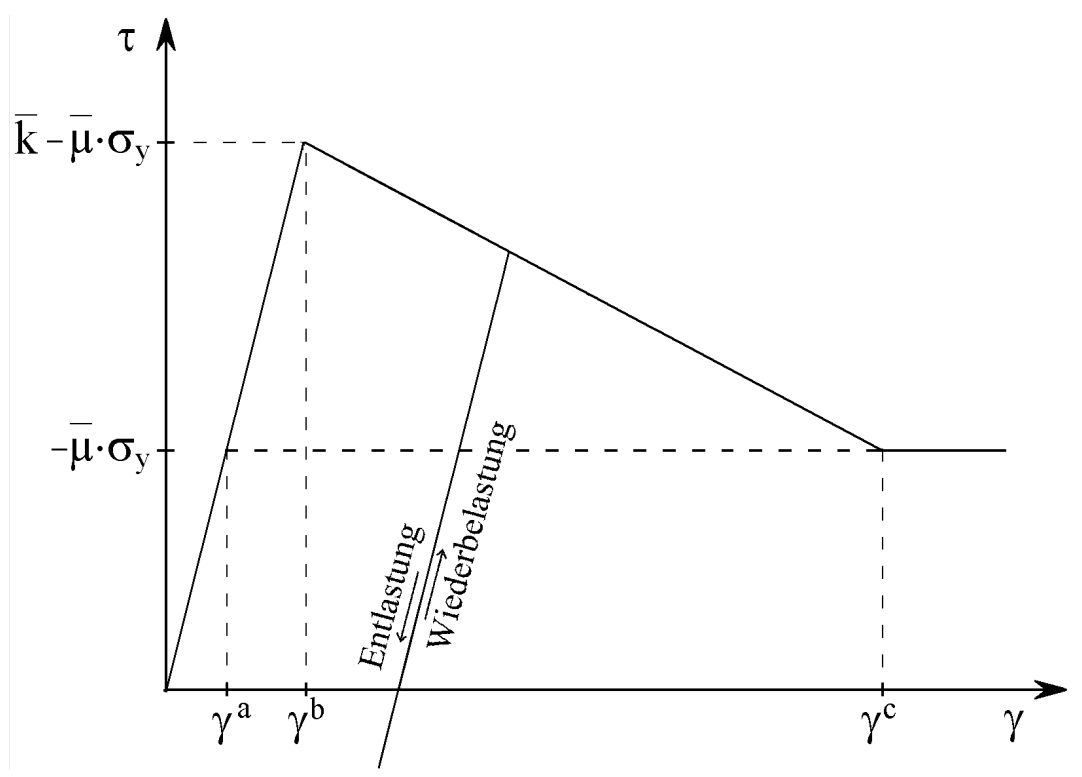

Abbildung 69: Entfestigung beim Reibungsversagen in der Lagerfuge - Kriterium 1

Als zugehörige Grenzen resultieren:

$$
\begin{aligned}
& \gamma^{a}=-\frac{\bar{\mu} \cdot \sigma_{y}}{G_{x y}} \\
& \gamma^{b}=\frac{\bar{k}-\bar{\mu} \cdot \sigma_{y}}{G_{x y}} \\
& \gamma^{c}=\gamma^{a}+\frac{2 \cdot G_{f}}{\bar{k} \cdot L_{e q}}=-\frac{\bar{\mu} \cdot \sigma_{y}}{G_{x y}}+\frac{2 \cdot G_{f}}{\bar{k} \cdot L_{e q}}
\end{aligned}
$$

Die Verfestigung wird symmetrisch zur $\sigma_{y}$-Achse angesetzt. Insgesamt ergeben sich folgende zusammengesetzte Fließflächen im $\sigma_{y}$ - $\tau$-Spannungsraum: 


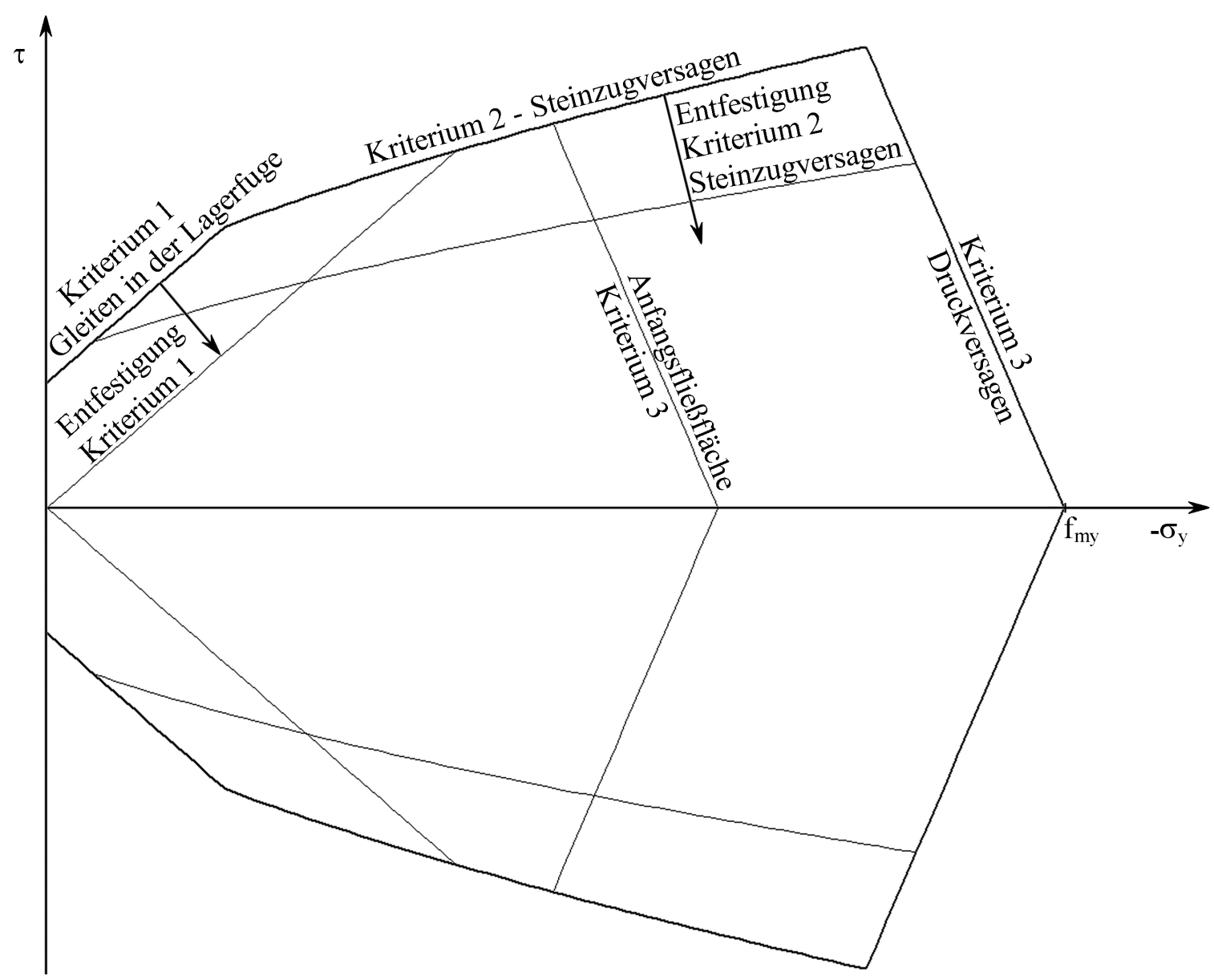

Abbildung 70: Fließ- und Versagensflächen im $\sigma_{y}-\tau$-Spannungsraum

\subsection{Implementierung des Stoffgesetzes}

\subsubsection{Allgemeines}

Im Zuge der Implementierung des Stoffgesetzes in das FE-Programm MARC [75] mit Hilfe einer Fortran-Benutzerroutine werden die in einem Zeitschritt angreifenden Systemlasten entsprechend der Systemsteifigkeit in einem iterativen Prozess in Knotenverschiebungen bzw. Dehnungen in den Integrationspunkten umgerechnet. Zusammen mit den Spannungen und Dehnungen des vorhergehenden Lastschrittes werden diese Zustandsgrößen der Benutzerroutine für jeden Integrationspunkt übergeben. Als Rückgabewert an das Hauptprogramm werden die resultierenden Spannungen und die lokale Steifigkeit $C$ erwartet.

Auf Systemebene werden am Ende des Lastschrittes $i$ die Residualkräfte ermittelt und für den Fall, dass diese die festgelegten Toleranzschranken nicht überschreiten, wird der nächste Zeitschritt $i+1$ angegangen. Sind die Toleranzkriterien nicht erfüllt, so wird ein neuer Iterationsschritt mit aktualisierten Zustandgrößen aus der Benutzerroutine durchgeführt.

Für die Berechnungen werden isoparametrische Elemente mit vier Knoten und vier Integrationspunkten verwendet. Die Verteilung der Ungleichgewichtskräfte auf Systemebene erfolgt mit Hilfe des Newton-Raphson-Verfahrens. Als Zeitintegrationsverfahren wird das NewmarkVerfahren mit $\beta=0,25$ und $\gamma=0,5$ gewählt. 


\subsubsection{Ablauf}

Der Zusammenhang von inkrementellen Dehnungsänderungen und zugehöriger Spannungsänderung wird mit folgender Differentialgleichung mit Ableitung nach der Lastzeit beschrieben:

$$
\dot{\sigma}=C^{e p}(\sigma ; \kappa) \cdot \dot{\varepsilon}
$$

Da die Beziehung $C^{e p}$ hochgradig nichtlinear ist (u. a. ist sie vom Erreichen der Fließkriterien abhängig) erfolgt die Lösung der Differentialgleichung mit Hilfe numerischer Verfahren. Neben expliziten Verfahren, z. B. dem Euler-Vorwärtsverfahren haben sich vermehrt implizite Verfahren durchgesetzt, da hier bei Verletzung eines Fließkriteriums die zugehörigen Randbedingungen, z. B. die Normalenregel, der zufolge das Verschiebungsinkrement senkrecht auf der Fließ- bzw. Potentialfläche stehen muss, eingehalten werden. Der Nachteil der impliziten Verfahren liegt in dem höheren numerischen Aufwand. Im Rahmen dieser Arbeit wird im Weiteren das implizite Euler-Rückwärtsverfahren angewendet.
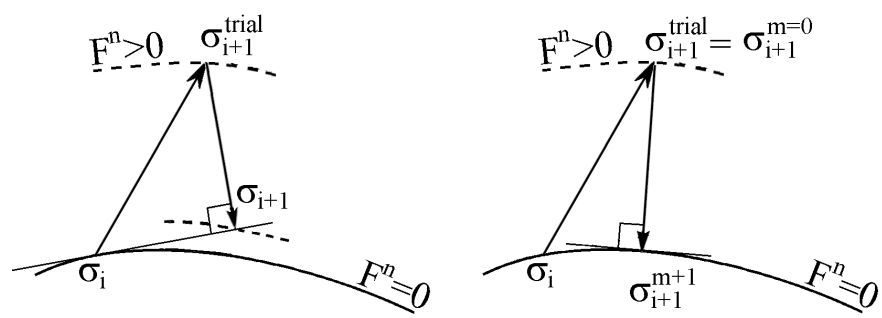

\section{Abbildung 71: Euler-Vorwärts- und -Rückwärtsverfahren (Darstellung ohne Verfestigungseinfluss)}

Die Ermittlung der Rückzugsspannungen auf der Fließfläche in Abbildung 71 rechts für den Lastschritt $i+1$ erfolgt dabei mit Hilfe der Linearisierung des Stoffgesetzes, der Fließfunktion und der Ver- und Entfestigungsbeziehung iterativ (s. Schneider [114], Pravida [108]).

$$
\begin{aligned}
& \sigma_{i+1}^{m+1}=C \cdot\left[\mathcal{E}-\frac{\partial F}{\partial \sigma_{i+1}^{m+1}} \lambda_{i+1}^{m+1}\right] \\
& F\left(\sigma_{i+1}^{m+1} ; \kappa_{i+1}^{m+1}\right)=F\left(\sigma_{i+1}^{m} ; \kappa_{i+1}^{m}\right)+\left.\frac{\partial F}{\partial \sigma_{i+1}^{m}}\right|^{T} \cdot \Delta \sigma_{i+1}^{m+1}+\left.\frac{\partial F}{\partial \kappa_{i+1}^{m}}\right|^{T} \cdot \Delta \kappa_{i+1}^{m+1}=0 \\
& \boldsymbol{\kappa}_{i+1}^{m+1}=\left[\psi_{i+1}^{m}+\frac{\partial \psi}{\partial \sigma_{i+1}^{m}} \Delta \sigma_{i+1}^{m+1}+\frac{\partial \psi}{\partial \kappa_{i+1}^{m}} \Delta \kappa_{i+1}^{m+1}\right] \cdot \lambda_{i+1}^{m+1}
\end{aligned}
$$




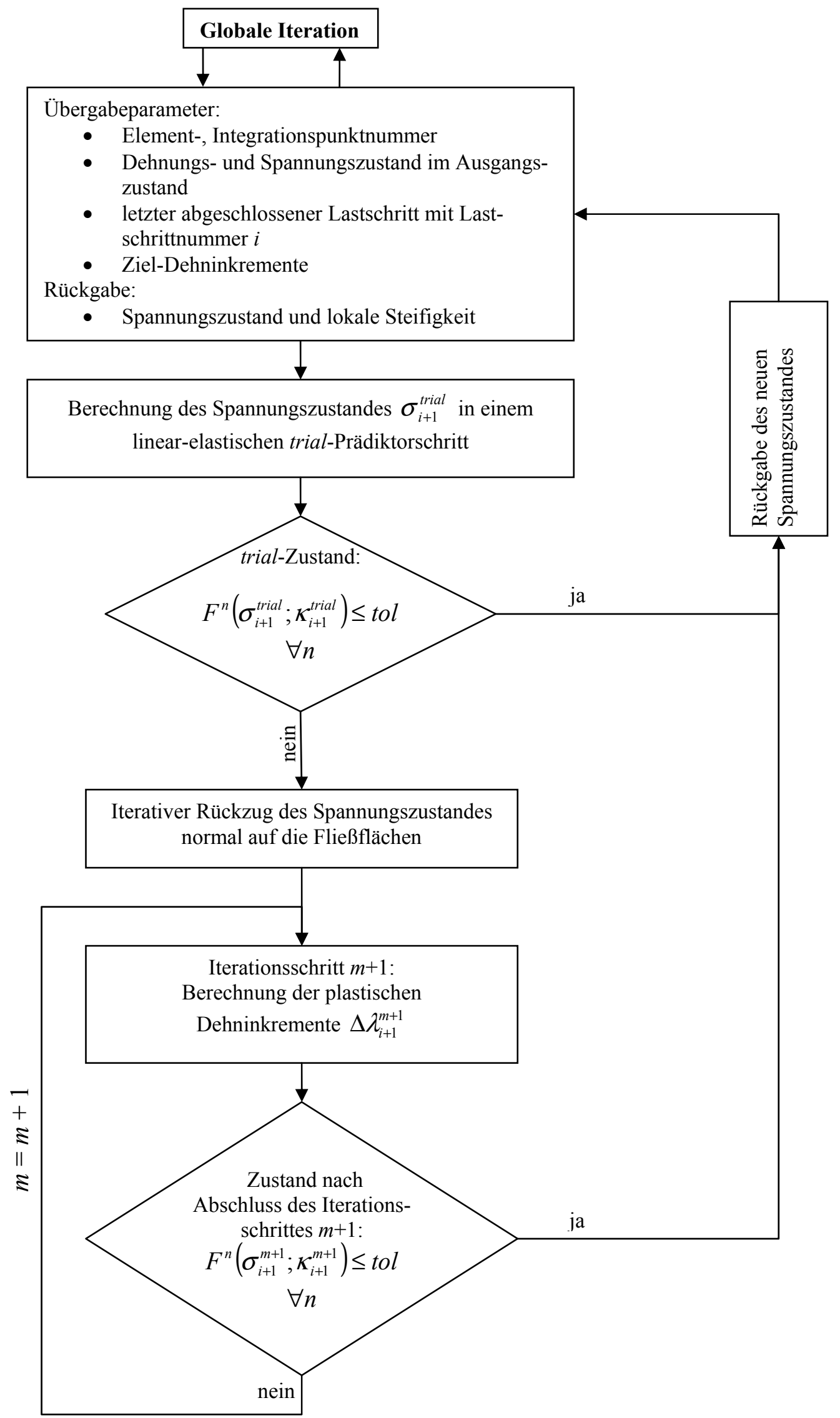

Abbildung 72: Ablaufschema für die Implementierung des Stoffgesetzes als Sub-Routine in das Programmsystem MARC [75] 


\subsection{Verifizierung des Modells}

Die Verifizierung des entwickelten Materialmodells erfolgt über die Nachrechnung eigener Simulationen. Als repräsentativ werden die Versuche an Wand A (Kragwand, Hochlochziegel mit NM), Wand E (Hochlochziegel mit DM) und an Wand KS4 (KS-PS mit DM) angesehen. Die beiden Wände E und KS4 weisen daneben einen unterschiedlichen Dämpfungsgrad infolge der viskosen Einzeldämpfer auf Höhe der Geschossdecken auf.

Die Nachrechnung der Wandversuche KS1 und KS2 aus großformatigen Planelementen ist aufgrund der hier nicht gegebenen Voraussetzungen des Schubmodells Mann / Müller nicht sinnvoll.

Für die Untersuchungen wird das Mehrmassensystem der zweiten Stufe (Wände E und KS4) auf einen äquivalenten Einmassenschwinger reduziert. An der betrachteten Kragscheibe wird am Wandkopf vereinfacht eine elastische Einspannung angesetzt, deren Federkonstante $c_{\varphi, K o p f}=200 \mathrm{kNm} / \mathrm{mrad}$ (Wand A: $c_{\varphi, K o p f}=0$ ) sich aus den während der Versuche eingestellten maximalen Kopfmomenten und den zugehörigen Kopfverdrehungen bestimmt. Zur Vermeidung von Vertikalschwingungen wird die Kopfmasse über einen zusätzlichen vertikalen Einzeldämpfer an die Gründung gekoppelt (in Abbildung 73 nicht dargestellt).

Als Normalkraft am Wandkopf wird die in den Versuchen aufgebrachte Last von $N=270 \mathrm{kN}$ angesetzt. Die Kopfmasse $m$ bestimmt sich aus der Bedingung, dass die Eigenperiode des neuen Ersatzsystems der ersten Eigenperiode des ursprünglichen Systems entspricht. Die Festlegung der Dämpferkonstanten $c$ erfolgt analog über den Ziel-Dämpfungsgrad $\zeta$.

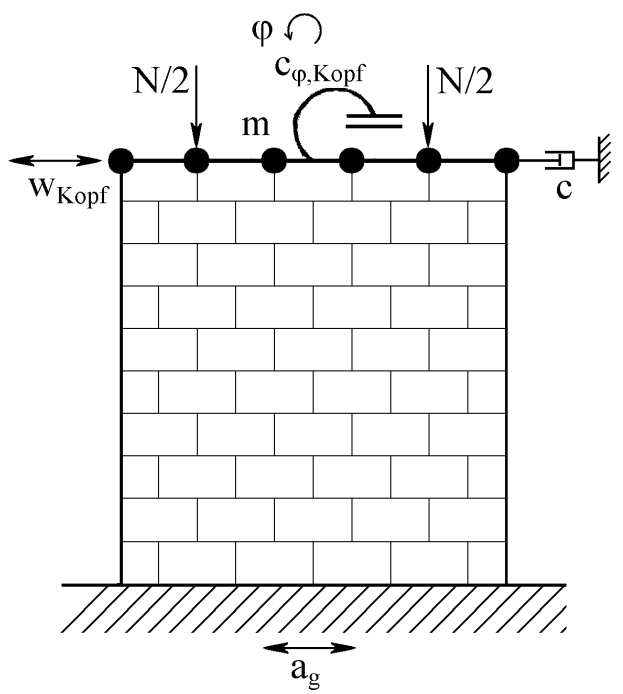

Abbildung 73: Ersatzsystem für die rechnerischen Untersuchungen

Für die FE-Berechnungen wird die untersuchte Wandscheibe mit den Abmessungen $2,5 \mathrm{~m} \cdot 2,5 \mathrm{~m} \cdot 0,175 \mathrm{~m}$ in $10 \cdot 10$ quadratische Elemente unterteilt. Die Knotenfesthaltungen am Wandkopf und am Wandfuß erfolgen über Quer- und Normalfedern. Deren Steifigkeit wird so gewählt, dass eine möglichst gleichförmige Lasteinleitung gewährleistet wird, ohne die Systemeigenperiode bei sehr weichen Federn zu stark zu verfälschen.

Die Eingangswerte des Modells in Form der Materialparameter werden weitestgehend aus Kapitel 4 unverändert übernommen. Eine eventuelle Vorschädigung der Wände infolge vorhergehender Versuche mit geringerer Laststufe wird vernachlässigt. 


\subsubsection{Wand A}

Zur Beschreibung der Eigenperiode $T=0,24$ s (Tabelle 29) wird mit der Anfangswandsteifigkeit von $k=89,2 \mathrm{kN} / \mathrm{mm}$ die Kopfmasse $\mathrm{zu} m=130,1 \mathrm{t}$ und die Dämpferkonstante $\mathrm{zu}$ $c=341 \mathrm{Ns} / \mathrm{mm}(\zeta=5 \%)$ bestimmt. Die rechnerische Wandsteifigkeit liegt somit in der Größenordnung des in den Versuchen bei geringer Laststufe aus der FFT des Wandkopfverschiebungsverlaufes ermittelten Wertes von 77,7 kN/mm (Wandversuch A1, Tabelle 28). Jedoch ist sie deutlich größer als in den Versuchen bei höherem Lastniveau (Wandversuche A8 und A4) mit $25,0 \div 57,4 \mathrm{kN} / \mathrm{mm}$. Im Vergleich dazu liegt die aus den statisch-zyklischen Vorversuchen ermittelte Anfangswandsteifigkeit von Wand D zwischen $61,9 \mathrm{kN} / \mathrm{mm} \quad\left(M_{\text {Kopf }}=0\right.$, Tabelle 32$)$ und $100 \mathrm{kN} / \mathrm{mm}\left(c_{\varphi, K o p f}=0\right.$, Tabelle 33$)$.

Aus der Ordinate des elastischen Antwortspektrums $S_{e, Z V I, \zeta=5 \%}\left(T_{I}=0,24 \mathrm{~s}\right)=1,16 \mathrm{~m} / \mathrm{s}^{2}$ ergibt sich mit obiger Kopfmasse eine rechnerische Horizontalkraft von $H_{\max }=151,2 \mathrm{kN}$. Bezogen auf die Maximallast bei linear-elastischer Berechnung als Einmassenschwinger von 45,2 kN / 0,25 = $170 \mathrm{kN}$ (Tabelle 29) bestimmt sich der Skalierungsfaktor der Wandversuche A4 und A5 für den Zeitverlauf ZV1-B3 zu $f=0,8 \cdot 170 / 151,2=0,9$. Zu gleichem Ergebnis kommt man bei der Ermittlung des Skalierungsfaktors über das Verhältnis der Kopfmassen im Versuch und in der Nachrechnung.

Die Wandversuche A7 und A8 können aufgrund der in den Versuchen aufgetretenen hohen Horizontalkräfte von $147,8 \mathrm{kN}$ bzw. $153,8 \mathrm{kN}$, die über der rechnerischen Grenzlast von $H_{2}=136,5 \mathrm{kN}$ liegen, nicht abgebildet werden. Bei vernachlässigbarer Haftzugfestigkeit liegt bei einer Horizontalkraft $\mathrm{H}_{2}$ die Resultierende am Wandfuß am Querschnittsrand (s. Abschnitt 5.4.4). In den Versuchen mit hohen Laststufen wurde infolge der großen Wandkopfverdrehung eine zusätzliche exzentrische Normalkraft am Wandkopf durch Anschlag an den Querriegel aktiviert, was in dieser Berechnung nicht erfasst werden kann.

\begin{tabular}{|c|c|c|}
\hline Größe & Wert & Einheit \\
\hline Mauerwerksdruckfestigkeit senkrecht zur Lagerfuge $f_{m v}$ & 5,5 & $\mathrm{~N} / \mathrm{mm}^{2}$ \\
\hline Grenzdehnung elastischer Bereich $\varepsilon_{\text {el-grenz }}$ & $-0,0011$ & - \\
\hline Dehnung bei maximaler Spannung $\varepsilon_{f \max }$ & $-0,0018$ & - \\
\hline E-Modul senkrecht zur Lagerfuge $\boldsymbol{E}_{v}$ & 3800 & $\mathrm{~N} / \mathrm{mm}^{2}$ \\
\hline Orthotropiefaktor $n$ & 2 & - \\
\hline Schubmodul $G_{x v}$ & 800 & $\mathrm{~N} / \mathrm{mm}^{2}$ \\
\hline Querdehnzahl $v_{y x}$ & 0,15 & - \\
\hline Reibbeiwert $\mu$ & 0,7 & - \\
\hline Haftscherfestigkeit $k$ & 2 & $\mathrm{~N} / \mathrm{mm}^{2}$ \\
\hline Resultierende schräge Steinzugfestigkeit $f_{b t}$ & 1,9 & $\mathrm{~N} / \mathrm{mm}^{2}$ \\
\hline Bruchenergie Haftscherversagen (Kriterium 1) $G_{f, 1}$ & 0,12 & $\mathrm{Nmm} / \mathrm{mm}^{2}$ \\
\hline Bruchenergie Steinzug (Kriterium 2) $\boldsymbol{G}_{f, 2}$ & 0,06 & $\mathrm{Nmm} / \mathrm{mm}^{2}$ \\
\hline Steinlänge $\Delta x$ & 500 & $\mathrm{~mm}$ \\
\hline Steinhöhe $\Delta y$ & 250 & $\mathrm{~mm}$ \\
\hline Kopfeinspannung $c_{\varphi, K o p f}$ & 0 & $\mathrm{kNm} / \mathrm{mrad}$ \\
\hline Normalkraft $N$ & 270 & $\mathrm{kN}$ \\
\hline Kopfmasse $m$ & 130,1 & $\mathrm{t}$ \\
\hline Dämpferkonstante $c$ & 341,0 & $\mathrm{Ns} / \mathrm{mm}$ \\
\hline Bodenbeschleunigungszeitverlauf & ZV1-B3 & - \\
\hline
\end{tabular}

Tabelle 38: Parameter der Versuchsnachrechnung Wand A4 bzw. A5

Bei der Nachrechnung von Versuch A6 (Lastfaktor $f=1,07 \cdot 170 / 151,2=1,2$ ) trat aufgrund eines geringeren Steifigkeitsabfalles in den Berechnungen eine Horizontalkraft in der Größenordnung von $120 \mathrm{kN}$ auf. Das System wurde infolge der geringen überdrückten Quer- 
schnittslänge am Wandfuß instabil, wodurch kein direkter Vergleich mit den Versuchsergebnissen möglich ist.

Daher wird sich im Folgenden auf die Nachrechnung des Wandversuches A5 beschränkt. Die Haftscherfestigkeit wird aufgrund des in den Versuchen aufgetretenen Verzahnungseffektes von Mörteldollen und Steinlöchern zu $k=2,0 \mathrm{~N} / \mathrm{mm}^{2}$ festgelegt.

Der E-Modul wird mit $3800 \mathrm{~N} / \mathrm{mm}^{2}$ deutlich geringer als in den Literaturangaben (Tabelle 24) gewählt - entsprechendes gilt auch für den Schubmodul. Trotzdem wird die Wandsteifigkeit für die untersuchte Beanspruchungssituation zu hoch beschrieben.

Aufgrund der nicht vorhandenen Einspannwirkung am Wandkopf kommt es teilweise zum Klaffen des Querschnittes am Wandfuß und es tritt ein vertikaler Verschiebungsanteil auf. Die Massenträgheitskräfte führen zu einer Änderung der Wandnormalkraft. Abweichend davon wurde in den Versuchen die Wandnormalkraft konstant gehalten. Nachfolgend ist in Abbildung 74 der zeitliche Verlauf der Horizontalkraft und der Wandnormalkraft bei der Nachrechnung dargestellt. Das Abklingen der Vertikalschwingungen wurde durch den vertikalen Einzeldämpfer in Wandmitte bewirkt.

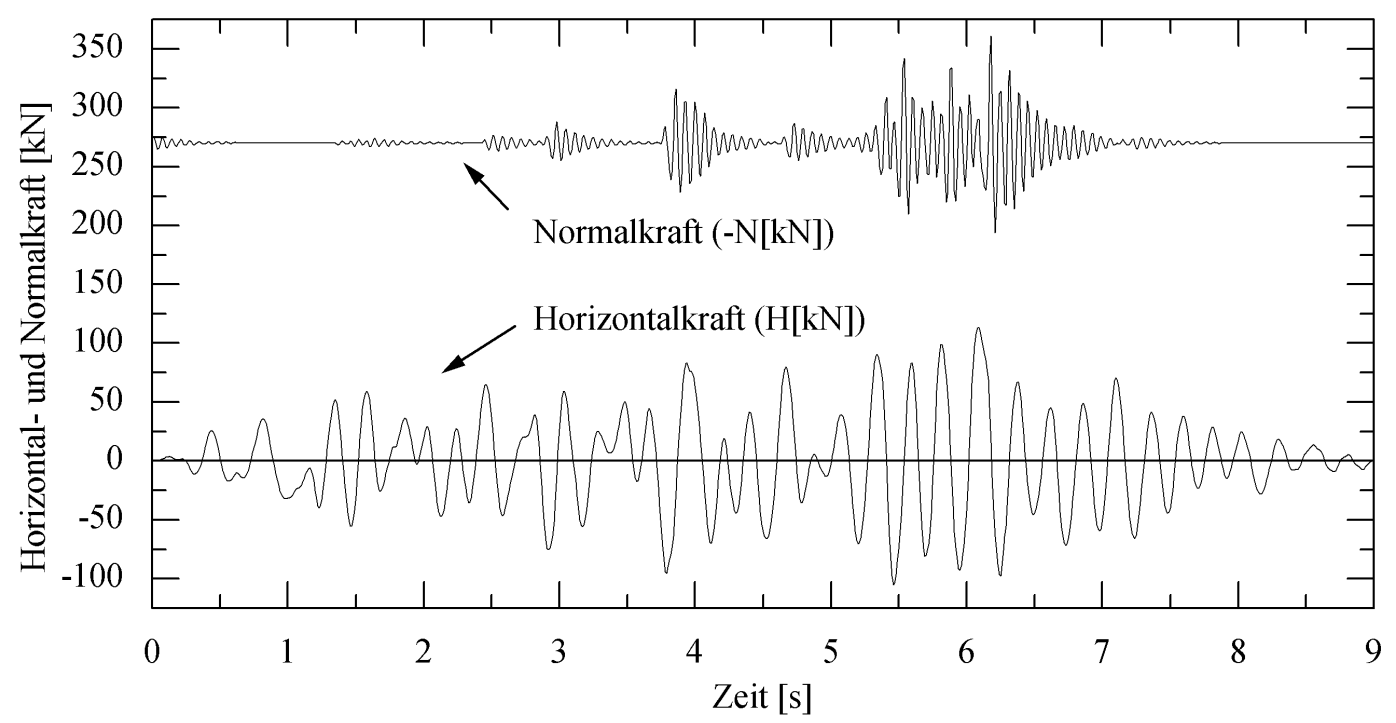

Abbildung 74: Zeitlicher Verlauf der Horizontal- und Normalkraft bei der Nachrechnung von Wandversuch A4 bzw. A5

Die Kraft-Verformungslinien von Versuch und Nachrechnung in Abbildung 75 zeigen trotz der höheren Steifigkeit im Modell insgesamt eine relativ gute Übereinstimmung.

Im Bereich der Umkehrpunkte läuft die Hysterese in den Versuchen relativ spitz zu, wohingegen in der Nachrechnung diese Bereiche stärker ausgerundet ausfallen. Ein Grund hierfür kann die Änderung der Normalkraft bei Umkehr der Verschiebungsrichtung sein. Bei dem Rechenlauf traten zum Zeitpunkt 5,46 s bei $H=105 \mathrm{kN}$ schräge Steinzugrisse in dem stärker gedrückten Bereich am Wandfuß auf, was gut mit der Abschätzung der Erstrisslast in Abschnitt 5.4.4 übereinstimmt. 

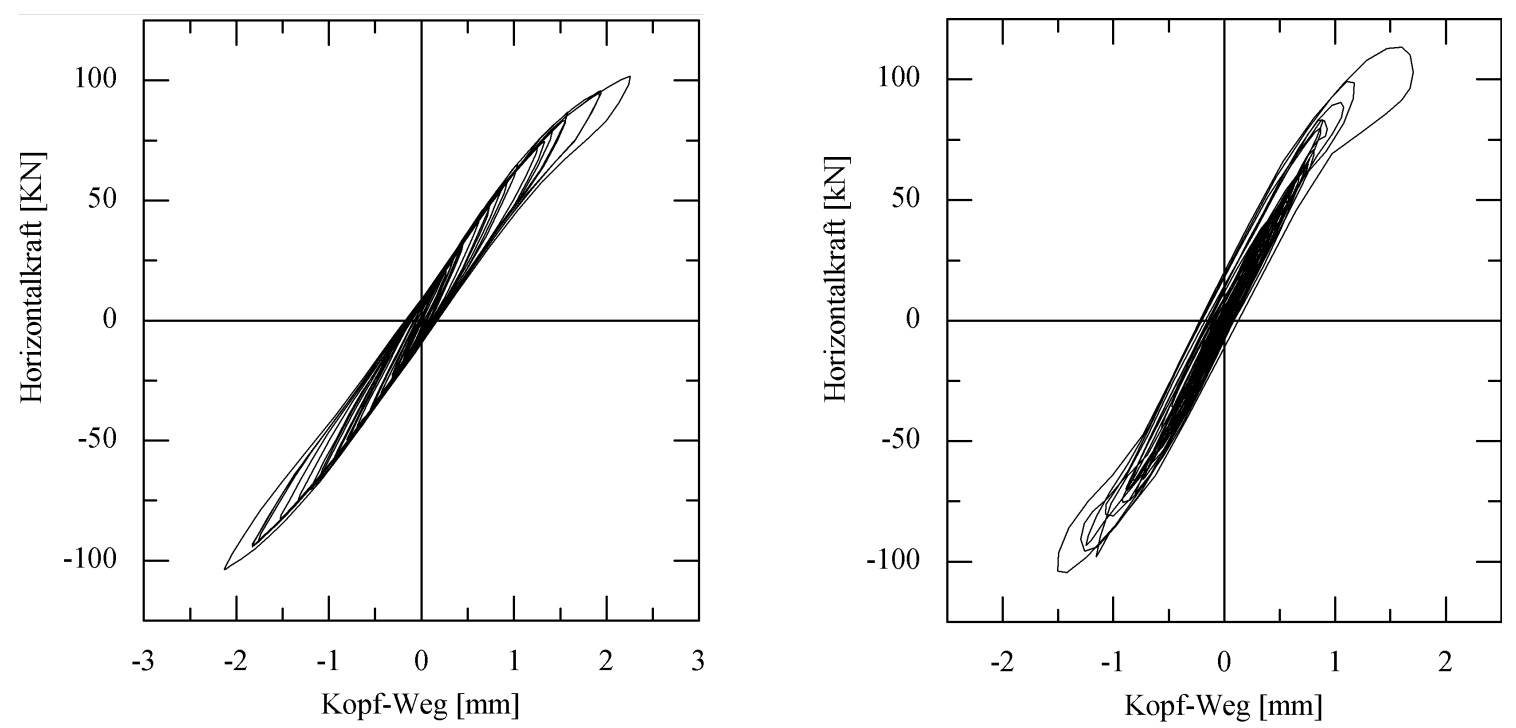

Abbildung 75: Kraft-Verformungs-Beziehung des Versuches A5 (links) und der Nachrechnung (rechts)

\subsubsection{Wand E}

Zur Beschreibung der ersten Eigenperiode $T=0,38 \mathrm{~s}$ (Tabelle 36) wird mit der Anfangswandsteifigkeit von $k=72,4 \mathrm{kN} / \mathrm{mm}$ die Kopfmasse $\mathrm{zu} m=266 \mathrm{t}$ und die Dämpferkonstante zu $c=44 \mathrm{Ns} / \mathrm{mm}(\zeta=0,5 \%)$ bestimmt (vgl. rechnerische Wandsteifigkeit aus statisch-zyklischen Vorversuchen: 53,3 kN/mm, Tabelle $32 \mathrm{bzw}$. $80 \mathrm{kN} / \mathrm{mm}$, Tabelle 33 ).

Aus der Ordinate des elastischen Antwortspektrums $S_{e, Z V 4, \zeta=0,5 \%}\left(T_{I}=0,38 \mathrm{~s}\right)=1,01 \mathrm{~m} / \mathrm{s}^{2}$ ergibt sich eine rechnerische Horizontalkraft von $H_{\max }=268,6 \mathrm{kN}$. Bezogen auf die Maximallast bei linear-elastischer Stabberechnung als Mehrmassenschwinger (Tabelle 36) von $65,1 \mathrm{kN}$ bestimmt sich der Skalierungsfaktor des Wandversuches E1 für den Zeitverlauf ZV4-C3 zu $f=65,1 / 268,6=0,242$ und des Wandversuches E2 zu $f=2,67 \cdot 65,1 / 268,6=0,647$.

\begin{tabular}{|c|c|c|}
\hline Größe & Wert & Einheit \\
\hline Mauerwerksdruckfestigkeit senkrecht zur Lagerfuge $f_{m v}$ & 25,5 & $\mathrm{~N} / \mathrm{mm}^{2}$ \\
\hline Grenzdehnung elastischer Bereich $\varepsilon_{\text {el-grenz }}$ & $-0,0011$ & - \\
\hline Dehnung bei maximaler Spannung $\varepsilon_{f \max }$ & $-0,0018$ & - \\
\hline E-Modul senkrecht zur Lagerfuge $E_{v}$ & 3800 & $\mathrm{~N} / \mathrm{mm}^{2}$ \\
\hline Orthotropiefaktor $n$ & 2 & - \\
\hline Schubmodul $G_{x y}$ & 800 & $\mathrm{~N} / \mathrm{mm}^{2}$ \\
\hline Querdehnzahl $v_{v x}$ & 0,15 & - \\
\hline Reibbeiwert $\mu$ & 0,7 & - \\
\hline Haftscherfestigkeit $k$ & 0,5 & $\mathrm{~N} / \mathrm{mm}^{2}$ \\
\hline Resultierende schräge Steinzugfestigkeit $f_{b t}$ & 1,29 & $\mathrm{~N} / \mathrm{mm}^{2}$ \\
\hline Bruchenergie Haftscherversagen (Kriterium 1) $G_{f t}$ & 0,12 & $\mathrm{Nmm} / \mathrm{mm}^{2}$ \\
\hline Bruchenergie Steinzug (Kriterium 2) $\boldsymbol{G}_{f, 2}$ & 0,06 & $\mathrm{Nmm} / \mathrm{mm}^{2}$ \\
\hline Steinlänge $\Delta x$ & 375 & $\mathrm{~mm}$ \\
\hline Steinhöhe $\Delta y$ & 250 & $\mathrm{~mm}$ \\
\hline Kopfeinspannung $c_{\varphi, K o p f}$ & 200 & $\mathrm{kNm} / \mathrm{mrad}$ \\
\hline Normalkraft $N$ & 270 & $\mathrm{kN}$ \\
\hline Kopfmasse $m$ & 266 & $\mathrm{t}$ \\
\hline Dämpferkonstante $c$ & 44 & $\mathrm{Ns} / \mathrm{mm}$ \\
\hline Bodenbeschleunigungszeitverlauf & ZV4-C3 & - \\
\hline
\end{tabular}

Tabelle 39: Parameter der Versuchsnachrechnung Wand E 
Obwohl der E-Modul senkrecht zu den Lagerfugen mit $3800 \mathrm{~N} / \mathrm{mm}^{2}$ nur etwa die Hälfte des Wertes der Literaturangaben aus Tabelle 24 beträgt, liegt die rechnerische Wandsteifigkeit erkennbar über dem Versuchswert.
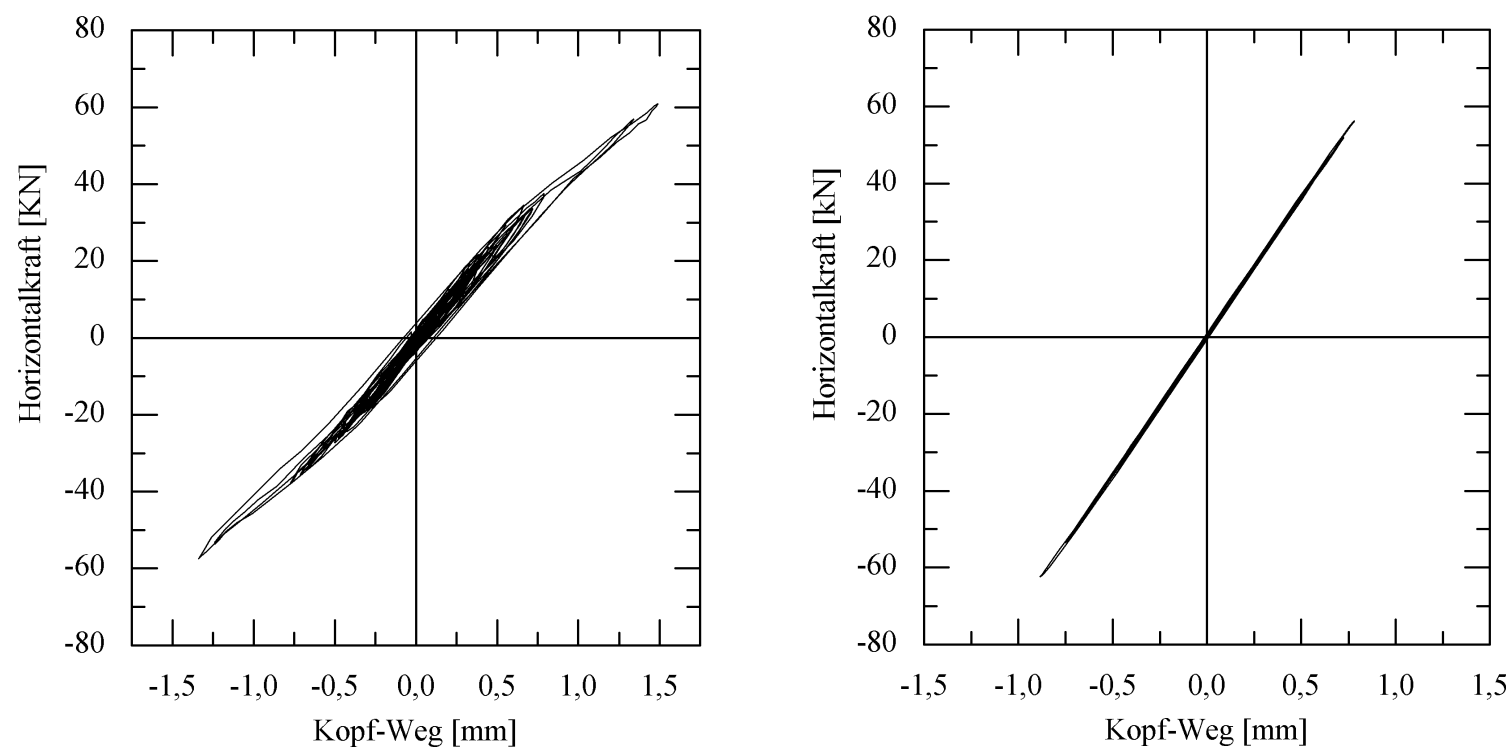

Abbildung 76: Kraft-Verformungs-Beziehung des Versuches E1 (links) und der Nachrechnung (rechts)
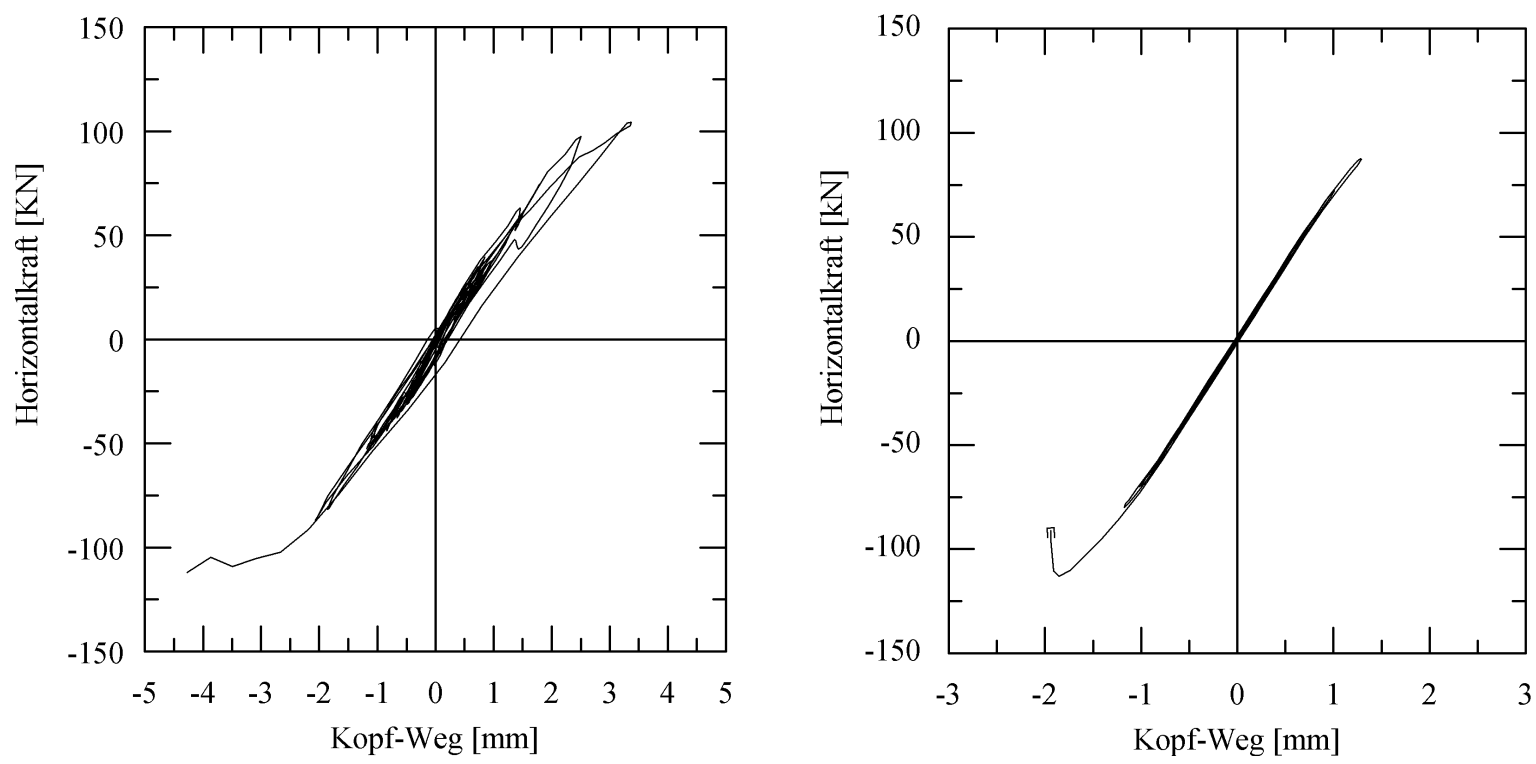

Abbildung 77: Kraft-Verformungs-Beziehung des Versuches E2 (links) und der Nachrechnung (rechts)

Die qualitative Übereinstimmung der rechnerischen Kraft-Verformungs-Linien mit den Versuchsergebnissen in Abbildung 76 und 77 ist gut erkennbar. Die Maximallasten und die Bruchlast im Versuch E2 konnten gut erfasst werden.

Bei Versuch E1 blieb die Wand in der Nachrechnung durchgängig im linear-elastischen Zustand - die geringe Völligkeit der Hysterese ist auf den geringen Dämpfungsgrad von $\zeta=0,5 \%$ zurückzuführen. 
Bei der Nachrechnung des Wandversuches E2 wurde das Kriterium Steinzugversagen im unteren Wandbereich aktiv, was anschließend zum Tragfähigkeitsverlust führte.

\subsubsection{Wand KS4}

Zur Beschreibung der ersten Eigenperiode $T_{1}=0,27 \mathrm{~s}$ (Tabelle 36) wird mit der Anfangswandsteifigkeit von $k=139,4 \mathrm{kN} / \mathrm{mm}$ die Kopfmasse $\mathrm{zu} m=257,4 \mathrm{t}$ und die Dämpferkonstante $\mathrm{zu} c=599 \mathrm{Ns} / \mathrm{mm}(\zeta=5 \%)$ festgelegt. Aus der Ordinate des elastischen Antwortspektrums $S_{e, Z V 1, \zeta=5 \%}\left(T_{1}=0,27 \mathrm{~s}\right)=1,23 \mathrm{~m} / \mathrm{s}^{2}$ ergibt sich eine rechnerische Horizontalkraft von $H_{\max }=316,6 \mathrm{kN}$. Bezogen auf die Maximallast bei linear-elastischer Stabberechnung als Mehrmassenschwinger (Tabelle 36) von 136,5 kN/1,5=91 kN bestimmt sich der Skalierungsfaktor des Wandversuches KS4-1 für den Zeitverlauf ZV1-B3 zu $f=1,5 \cdot 91 / 316,6=0,431$ und des Wandversuches KS4-3 zu 0,719.

\begin{tabular}{|c|c|c|}
\hline Größe & Wert & Einheit \\
\hline Mauerwerksdruckfestigkeit senkrecht zur Lagerfuge $\boldsymbol{f}_{\boldsymbol{m}}$ & 17,5 & $\mathrm{~N} / \mathrm{mm}^{2}$ \\
\hline Grenzdehnung elastischer Bereich $\varepsilon_{\text {el-grenz }}$ & $-0,00075$ & - \\
\hline Dehnung bei maximaler Spannung $\varepsilon_{f \max }$ & $-0,0025$ & - \\
\hline E-Modul senkrecht zur Lagerfuge $E_{y}$ & 10770 & $\mathrm{~N} / \mathrm{mm}^{2}$ \\
\hline Orthotropiefaktor $n$ & 2 & - \\
\hline Schubmodul $G_{x y}$ & 2500 & $\mathrm{~N} / \mathrm{mm}^{2}$ \\
\hline Querdehnzahl $v_{y x}$ & 0,2 & - \\
\hline Reibbeiwert $\mu$ & 0,7 & - \\
\hline Haftscherfestigkeit $k$ & 0,98 & $\mathrm{~N} / \mathrm{mm}^{2}$ \\
\hline Resultierende schräge Steinzugfestigkeit $f_{b t}$ & 3,3 & $\mathrm{~N} / \mathrm{mm}^{2}$ \\
\hline Bruchenergie Haftscherversagen (Kriterium 1) $G_{f, 1}$ & 0,03 & $\mathrm{Nmm} / \mathrm{mm}^{2}$ \\
\hline Bruchenergie Steinzug (Kriterium 2) $G_{f, 2}$ & 0,12 & $\mathrm{Nmm} / \mathrm{mm}^{2}$ \\
\hline Steinlänge $\Delta x$ & 500 & $\mathrm{~mm}$ \\
\hline Steinhöhe $\Delta y$ & 250 & $\mathrm{~mm}$ \\
\hline Kopfeinspannung $c_{\varphi, \text { Kopf }}$ & 200 & $\mathrm{kNm} / \mathrm{mrad}$ \\
\hline Normalkraft $N$ & 270 & $\mathrm{kN}$ \\
\hline Kopfmasse $m$ & 257,4 & $\mathrm{t}$ \\
\hline Dämpferkonstante $c$ & 599 & $\mathrm{Ns} / \mathrm{mm}$ \\
\hline Bodenbeschleunigungszeitverlauf & ZV1-B3 & - \\
\hline
\end{tabular}

Tabelle 40: Parameter der Versuchsnachrechnung Wand KS4

Wie aus dem Vergleich der Kraft-Verformungs-Linien des Versuches KS4-1 und der Nachrechnung in Abbildung 78 und 79 deutlich erkennbar ist, wird bei dieser geringen Laststufe die Maximallast mit dem Rechenmodell und den gegebenen Materialparametern als Eingangsgrößen gut erfasst. Die Wandsteifigkeit liegt bei der Nachrechnung etwa $30 \%$ höher als in den Versuchen (Abbildung 78), obwohl sie mit 139,4 $\mathrm{kN} / \mathrm{mm}$ unter dem rechnerischen Wert aus den Vorversuchen von 160,3 kN/mm (bei $M_{\text {Kopf }}=0$, Tabelle 32) bzw. $350 \mathrm{kN} / \mathrm{mm}$ (bei $c_{\varphi, \text { Kopf }}=0$, Tabelle 33) liegt.

Die Betrachtung der Kraft-Verformungs-Beziehung von Versuch KS4-3 zeigt eine bleibende Verformung infolge des aufgetretenen Gleitens in der Lagerfuge im abgetreppten Riss (Risse $4 \div 18$ in Abbildung A-22 im Bereich der oberen Wandhälfte, s. a. Abschnitte 5.5.5.1 und 5.5.7).

Bei der Nachrechnung trat ebenfalls ein Gleiten in der Lagerfuge ein. Infolge der am Wandfuß durch das Klaffen lokal erhöhten Schubspannungen wurde die maximal aufnehmbare Schubspannung überschritten. Durch den Abbau der Haftscherfestigkeit traten anschließend 
plastische Schubgleitungen auf. Im weiteren Verlauf pflanzten sich die plastischen Bereiche über die ganze Wandlänge fort und es trat eine bleibende Verformung auf.
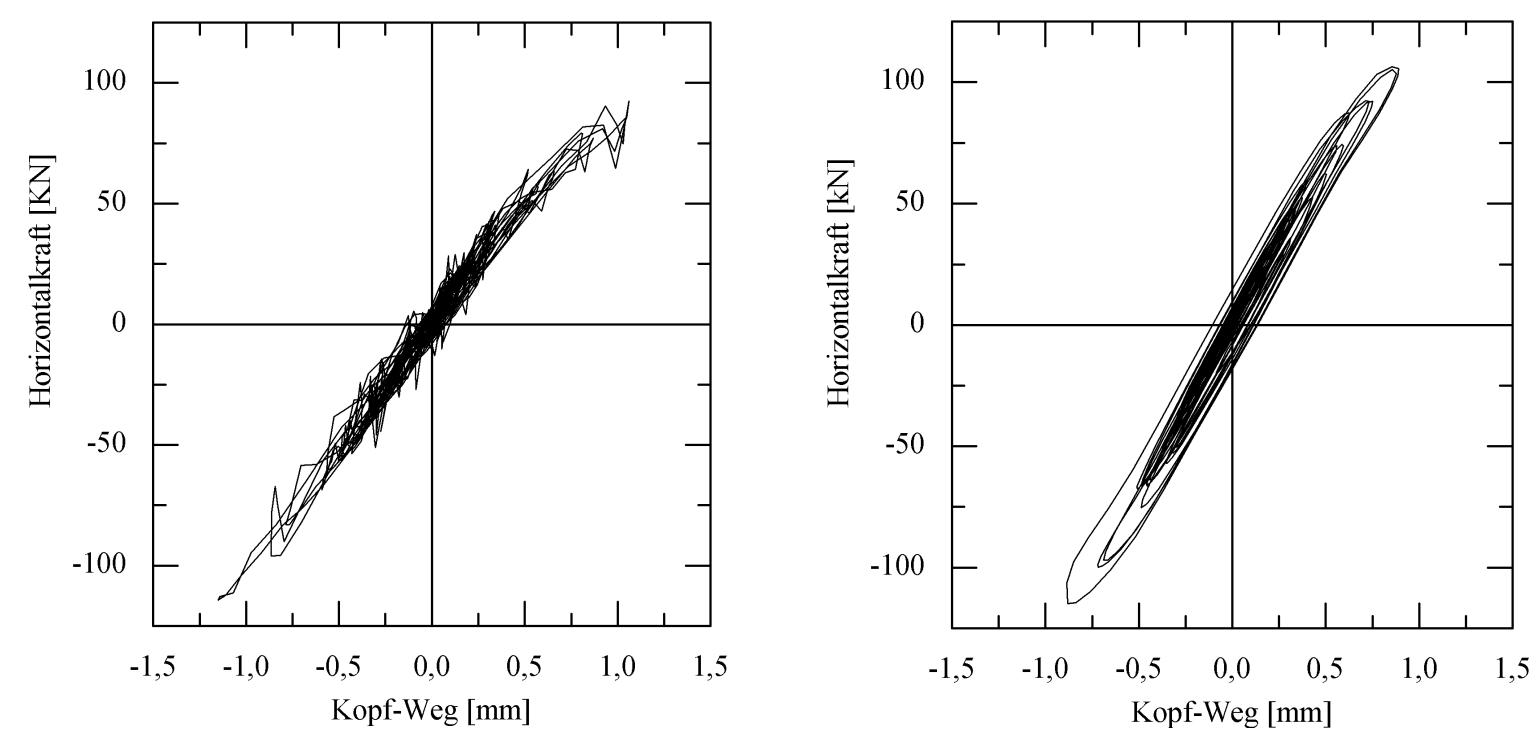

Abbildung 78: Kraft-Verformungs-Beziehung des Versuches KS4-1 (links) und der Nachrechnung (rechts)
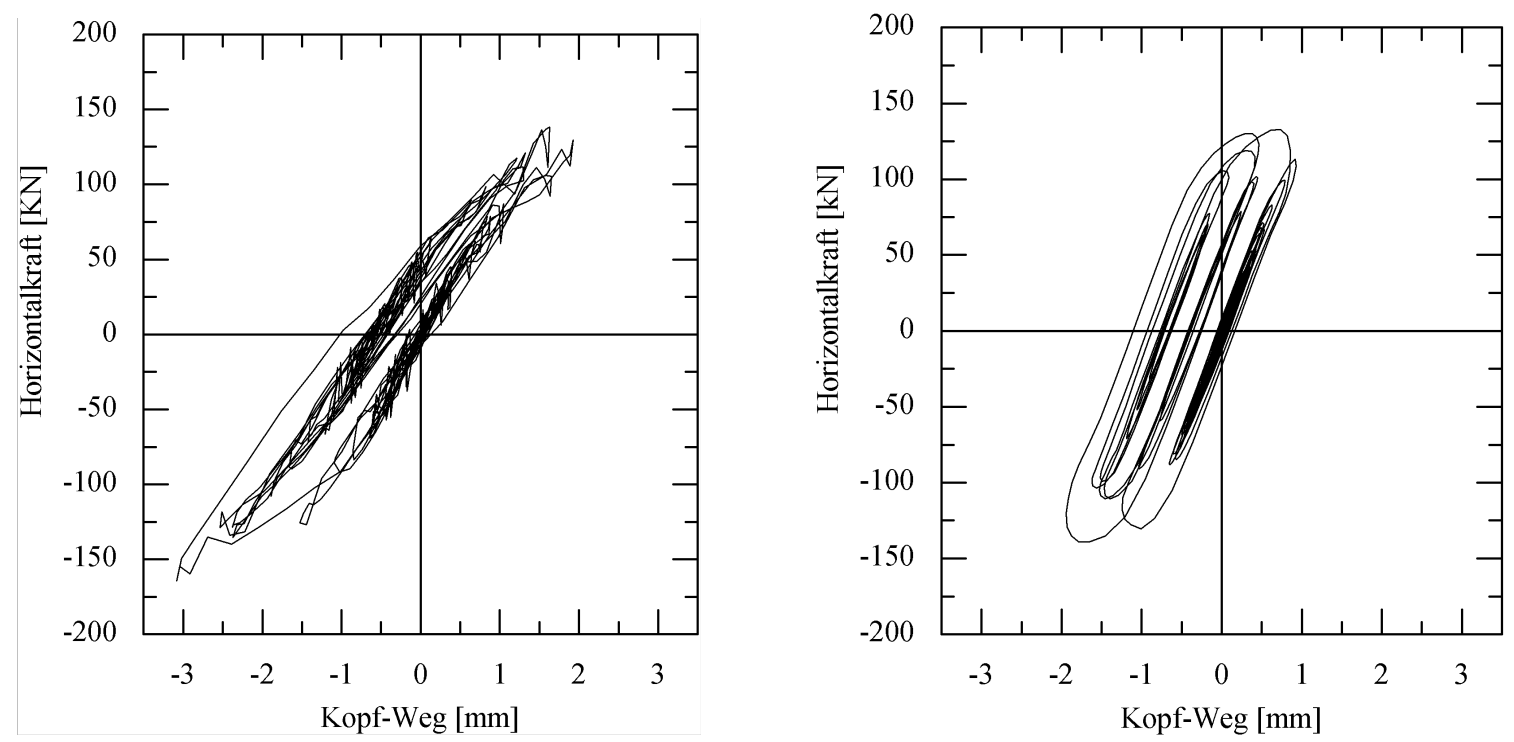

Abbildung 79: Kraft-Verformungs-Beziehung des Versuches KS4-3 (links) und der Nachrechnung (rechts)

\subsection{Parameterstudie}

Mit dem entwickelten Materialmodell wird anschließend eine Parameterstudie durchgeführt. Die Untersuchungen konzentrieren sich auf einige in Deutschland übliche Stein-MörtelKombinationen (Tabelle 41). Das Überbindemaß wird durchgängig zur halben Steinlänge festgelegt. 
Die Kopfmasse $m$ wird in allen Rechenläufen so gewählt, dass die erste Eigenperiode des ungeschädigten Systems $T_{0}=0,3 \mathrm{~s}$ beträgt.

\begin{tabular}{|c|c|c|c|c|c|}
\hline Bezeichnung & Steinart & Mörtelart & SFK & $\begin{array}{l}\text { Wanddicke } \\
d_{\text {Wand }}[\mathrm{cm}]\end{array}$ & $\begin{array}{c}\text { Grundwert der zul. } \\
\text { Normalspannung } \\
\sigma_{0}\left[\mathrm{~N} / \mathrm{mm}^{2}\right]^{1)} \\
\end{array}$ \\
\hline KS-PS & KS-Plansteine & $\mathrm{DM}$ & 20 & 17,5 & 3,2 \\
\hline HLz-DM & Leichthochlochziegel & DM & 12 & 17,5 & $1,8^{2)}$ \\
\hline PB-DM & Porenbeton & DM & 4 & 30 & 1,1 \\
\hline $\begin{array}{l}\text { 1) nach DIN } 10 \\
\text { 2) Richtwert au } \\
\text { Materialparam }\end{array}$ & $\begin{array}{l}3-1 \\
\text { Allgemeinen Bauaufsic } \\
\text { er: s. Tabelle } 42\end{array}$ & lichen Zula & ngen & & \\
\hline
\end{tabular}

Tabelle 41: Untersuchte Materialkombinationen

\begin{tabular}{|c|c|c|c|c|}
\hline Größe & KS-PS & HLz-DM & PB-DM & Einheit \\
\hline Mauerwerksdruckfestigkeit senkrecht zur Lagerfuge $\boldsymbol{f}_{\boldsymbol{m}}$ & 15,5 & 5,5 & 2,9 & $\mathrm{~N} / \mathrm{mm}^{2}$ \\
\hline Grenzdehnung elastischer Bereich $\varepsilon_{\text {el-grenz }}$ & $-0,00075$ & $-0,0007$ & $-0,0015$ & - \\
\hline Dehnung bei maximaler Spannung $\varepsilon_{f \max }$ & $-0,0025$ & $-0,0012$ & $-0,002$ & - \\
\hline E-Modul senkrecht zur Lagerfuge $E_{y}$ & 10770 & 6111 & 1650 & $\mathrm{~N} / \mathrm{mm}^{2}$ \\
\hline Orthotropiefaktor $n$ & 2 & 2 & 1,8 & - \\
\hline Schubmodul $G_{x y}$ & 2500 & 1100 & 320 & $\mathrm{~N} / \mathrm{mm}^{2}$ \\
\hline Querdehnzahl $v_{y x}$ & 0,2 & 0,1 & 0,2 & - \\
\hline Reibbeiwert $\mu$ & 0,65 & 0,75 & 0,65 & - \\
\hline Haftscherfestigkeit $k$ & 1,4 & 1,0 & 1,2 & $\mathrm{~N} / \mathrm{mm}^{2}$ \\
\hline Resultierende schräge Steinzugfestigkeit $f_{b t}$ & 3,3 & 1,0 & 0,4 & $\mathrm{~N} / \mathrm{mm}^{2}$ \\
\hline Bruchenergie Haftscherversagen (Kriterium 1) $G_{f, 1}$ & 0,1 & 0,06 & 0,010 & $\mathrm{Nmm} / \mathrm{mm}^{2}$ \\
\hline Bruchenergie Steinzug (Kriterium 2) $G_{f, 2}$ & 0,03 & 0,12 & 0,003 & $\mathrm{Nmm} / \mathrm{mm}^{2}$ \\
\hline Steinlänge $\Delta x$ & 500 & 500 & 500 & $\mathrm{~mm}$ \\
\hline Steinhöhe $\Delta y$ & 250 & 250 & 250 & $\mathrm{~mm}$ \\
\hline
\end{tabular}

Tabelle 42: Materialparameter der untersuchten Stein-Mörtel-Kombinationen

Nachfolgend erfolgt die Auswertung der Berechnungsläufe für die angegebenen Kombinationen nach dem (Verschiebe)-Duktilitätsfaktor $\mu$ bzw. dem daraus abgeleiteten Verhaltensfaktor $q_{\mu}$. Die Kraft-Verformungs-Linien werden dazu in die elasto-plastische bilineare Form nach Abbildung 6 überführt. Die Ergebnisse sind in Tabelle 43 zusammengefasst.

Im Versagensbild Gleiten in der Lagerfuge erfolgt - im Gegensatz zu dem Fall des Steinzugversagens - keine wesentliche Beeinträchtigung der lokalen Drucktragfähigkeit. Dieses ,gutmütige" Versagensbild ergibt daher teilweise sehr hohe Verschiebeduktilitätswerte, z.B. bei Steinen mit hoher Zugfestigkeit, sowie geringem Reibbeiwert und Haftscherfestigkeitswert. Jedoch resultieren durch das Gleiten allgemein auch Problempunkte in Nachbarbauteilen. Besonders das Zusammenwirken mit stark druckbeanspruchten und im Verband hergestellten Querwänden führt bei lokalen Schubverformungen der untersuchten Wand zu einer Reduzierung der Tragfähigkeit der Querwand. Daraus ergeben sich erforderliche Beschränkungen der zulässigen Geschossverschiebungen.

Der Einfluss der Größe der Deckeneinspannung $c_{\varphi, K o p f}$ auf die Traglast und die Verschiebeduktilität ist gering. Mit zunehmender Höhe der Horizontalbeanspruchung einer Wand nimmt erwartungsgemäß auch der Duktilitätsfaktor zu (Tabelle 43). Gleiches gilt auch bei kleinerem Verhältnis von Wandlänge zu Wandhöhe, wodurch der Biegeverformungsanteil an der Gesamtverformung zunimmt, und das Verhalten des Wandpfeilers deutlich duktiler wird. 


\begin{tabular}{|c|c|c|c|c|c|c|c|c|}
\hline S-M-K & $\begin{array}{c}\sigma \\
{\left[\mathbf{N} / \mathbf{m m}^{2}\right]}\end{array}$ & $\begin{array}{c}c_{\varphi, \text { Kopf }} \\
{[\mathrm{kNm} / \mathrm{mrad}]}\end{array}$ & $\begin{array}{c}\text { Zeitverlauf; } \\
\text { Faktor } f \\
\end{array}$ & $\begin{array}{l}m \\
{[t]}\end{array}$ & $\begin{array}{l}\boldsymbol{H}_{\max }[\mathrm{kN}] ; \\
\boldsymbol{w}_{\max }[\mathrm{mm}] \\
\end{array}$ & $\begin{array}{c}\text { Ver- } \\
\text { sagen }{ }^{1)} \\
\end{array}$ & $\mu[-]^{2)}$ & $q_{\mu}{ }^{3)}$ \\
\hline \multirow{15}{*}{$\begin{array}{l}\text { KS-PS } \\
1=2,5 \mathrm{~m}\end{array}$} & " 0,61 & 200 & בZV1;0,53 & $\begin{array}{ll}191 \\
\end{array}$ & $\begin{array}{l}111 ; 1,44 \\
\end{array}$ & $\overline{\mathrm{LaFu}}$ & 1,3 & 1,26 \\
\hline & 1,2 & 200 & ZV1;0,79 & 191 & $131 ; 1,48$ & & 1,0 & 1 \\
\hline & 1,2 & 200 & ZV1;1,06 & 191 & $181 ; 2,0$ & & 1,0 & 1 \\
\hline & 1,2 & 200 & ZV1;1,29 & 191 & $225 ; 2,72$ & $\mathrm{LaFu}$ & 1,2 & 1,18 \\
\hline & 1,2 & 200 & ZV1;1,59 & 191 & $247 ; 3,1$ & $\mathrm{LaFu}$ & $\begin{array}{l}1,45 \\
(1,9)\end{array}$ & $\begin{array}{c}1,38 \\
(1,67)\end{array}$ \\
\hline & 1,2 & 500 & ZV1;1,29 & 191 & $223 ; 3,03$ & $\mathrm{LaFu}$ & 1,25 & 1,22 \\
\hline & 1,2 & 500 & ZV1;1,59 & 191 & $246 ; 3,90$ & $\mathrm{LaFu}$ & 1,70 & 1,55 \\
\hline & 1,2 & 500 & ZV1;1,8 & 191 & $215 ; 3,64$ & $\mathrm{LaFu}$ & $\begin{array}{l}1,85 \\
(2,7)\end{array}$ & $\begin{array}{l}1,64 \\
(2,1) \\
\end{array}$ \\
\hline & 1,2 & 500 & ZV2;1,5 & 191 & $280 ; 3,28$ & $\mathrm{LaFu}$ & $\begin{array}{l}1,95 \\
(3,6) \\
\end{array}$ & $\begin{array}{c}1,7 \\
(2,49)\end{array}$ \\
\hline & 1,2 & 500 & ZV3;2,25 & 191 & $206 ; 2,84$ & $\mathrm{LaFu}$ & 1,35 & 1,3 \\
\hline & 1,2 & 500 & ZV4;3,0 & 191 & $279 ; 4,62$ & $\mathrm{LaFu}$ & 2,2 & 1,84 \\
\hline & 2,4 & 500 & ZV1; 1,8 & 191 & $378 ; 4,43$ & & 1,0 & 1 \\
\hline & 2,4 & 500 & ZV1; 2,47 & 191 & $432 ; 6,03$ & $\mathrm{LaFu}$ & 1,4 & 1,34 \\
\hline & 2,4 & 500 & ZV1; 3,6 & 191 & $429 ; 6,17$ & $\mathrm{LaFu}$ & $1,9(4)$ & $\begin{array}{c}1,67 \\
(2,65)\end{array}$ \\
\hline & 2,4 & 500 & ZV1; 4,2 & 191 & $433 ; 8,88$ & $\mathrm{LaFu}$ & $2,9(5)$ & $2,19(3)$ \\
\hline \multirow{4}{*}{$\begin{array}{l}\text { HLz-DM } \\
\mathrm{l}=2,5 \mathrm{~m}\end{array}$} & 0,9 & 500 & ZV1; 0,5 & 175 & $111 ; 1,48$ & & 1,05 & 1,05 \\
\hline & 0,9 & 500 & ZV1; 0,55 & 175 & $120 ; 1,63$ & StZ-sp & 1,05 & 1,05 \\
\hline & 0,9 & 500 & ZV1; 0,65 & 175 & $133 ; 1,94$ & StZ-sp & $1,05(*)$ & $1,05(*)$ \\
\hline & 0,9 & 500 & ZV1; 1,0 & 175 & $128 ; 2,1$ & StZ-sp & $1,1(*)$ & $1,1(*)$ \\
\hline \multirow{4}{*}{$\begin{array}{l}\text { HLz-DM } \\
\mathrm{l}=1,0 \mathrm{~m}\end{array}$} & 1,2 & 500 & ZV1; 0,18 & 61 & 14,$3 ; 0,53$ & & 1,05 & 1,05 \\
\hline & 1,2 & 500 & ZV1; 0,6 & 61 & 43,$6 ; 1,78$ & & 1,2 & 1,18 \\
\hline & 1,2 & 500 & ZV1; 0,63 & 61 & 45,$1 ; 1,87$ & & 1,2 & 1,18 \\
\hline & 1,2 & 500 & ZV1; 0,66 & 61 & 46,$7 ; 1,96$ & StZ-sp & $1,25(*)$ & $1,22(*)$ \\
\hline \multirow{4}{*}{$\begin{array}{l}\text { PB-DM, } \\
1=2,5 \mathrm{~m}\end{array}$} & 0,6 & 500 & ZV1; 0,8 & 110 & 112,$0 ; 2,34$ & & 1,05 & 1,05 \\
\hline & 0,6 & 500 & ZV1; 0,85 & 110 & $119 ; 2,49$ & & 1,05 & 1,05 \\
\hline & 0,6 & 500 & ZV1; 1,0 & 110 & $127 ; 2,57$ & StZ-sp & $1,10(*)$ & $1,1(*)$ \\
\hline & 0,6 & 500 & ZV1; 1,2 & 110 & $149 ; 4,37$ & StZ-sp & $1,15(*)$ & $1,14(*)$ \\
\hline
\end{tabular}

Tabelle 43: Berechnungsergebnisse der Parameterstudie

Bei dem Versagensbild Steinzug erfolgt das Versagen spröde mit entsprechend sukzessiver bis zum Kollaps abnehmender Schubtragfähigkeit. Die Ergebnisse der entsprechenden Rechengänge können bei der Bestimmung des Verhaltensfaktors somit nicht berücksichtigt werden.

Allgemein zeigt sich, dass das Auftreten des Versagensbildes Gleiten in der Lagerfuge zu hohen Duktilitätswerten $\mu$ führt, die einen Verhaltensfaktor deutlich über 1,5 zulassen würden. Im Fall des Steinzugversagens liegt der Verhaltensfaktor mit ca. 1,2 jedoch deutlich unter 1,5 .

Ergänzend werden die Ergebnisse der rechnerischen Untersuchungen in Form maximaler Horizontalkräfte $\left(H_{\max , F E M}\right)$ denen des Antwortspektrenverfahrens $\left(H_{\max , A S P}\right)$ gegenübergestellt (Tabelle 44). Die Maximallast nach letzterem bestimmt sich als Produkt aus der Ordinate des elastischen horizontalen Antwortspektrums des betreffenden Zeitverlaufes $S_{e}\left(T_{0}=0,3 \mathrm{~s}\right)$, des Lastfaktors $f$ und der Kopfmasse $m$. 


\begin{tabular}{|c|c|c|c|c|c|c|}
\hline S-M-K & $\begin{array}{c}\text { Zeitverlauf; } \\
\text { Faktor } f\end{array}$ & $\begin{array}{l}S_{e}\left(T_{0}\right) \\
{\left[\mathrm{m} / \mathbf{s}^{2}\right]}\end{array}$ & $\begin{array}{l}m \\
{[t]}\end{array}$ & $\begin{array}{c}H_{\max , \text { FEM }} \\
{[\mathrm{kN}]}\end{array}$ & $\begin{array}{c}H_{\max , A S P} \\
{[\mathrm{kN}]}\end{array}$ & $\begin{array}{c}q_{A S P} \\
{[-]}\end{array}$ \\
\hline \multirow{15}{*}{$\begin{array}{l}\text { KS-PS } \\
1=2,5 \mathrm{~m}\end{array}$} & ZV1; 0,53 & 0,685 & 191 & 111 & 130,9 & 1,18 \\
\hline & ZV1; 0,79 & 1,02 & 191 & 131 & 195,1 & 1,49 \\
\hline & ZV1; 1,06 & 1,37 & 191 & 181 & 261,8 & 1,45 \\
\hline & ZV1; 1,29 & 1,67 & 191 & 225 & 318,6 & 1,42 \\
\hline & ZV1; 1,59 & 2,06 & 191 & 247 & 392,7 & 1,59 \\
\hline & ZV1; 1,29 & 1,67 & 191 & 223 & 318,6 & 1,43 \\
\hline & ZV1; 1,59 & 2,06 & 191 & 246 & 392,7 & 1,60 \\
\hline & ZV1; 1,8 & 2,33 & 191 & 215 & 444,5 & 2,07 \\
\hline & ZV2; 1,5 & 3,38 & 191 & 280 & 646,1 & 2,31 \\
\hline & ZV3; 2,25 & 1,71 & 191 & 206 & 325,8 & 1,58 \\
\hline & ZV4; 3,0 & 2,20 & 191 & 279 & 419,4 & 1,50 \\
\hline & ZV1; 1,8 & 2,33 & 191 & 378 & 444,5 & 1,18 \\
\hline & ZV1; 2,47 & 3,19 & 191 & 432 & 610,0 & 1,41 \\
\hline & ZV1; 3,6 & 4,65 & 191 & 429 & 889,1 & 2,07 \\
\hline & ZV1; 4,2 & 5,43 & 191 & 433 & 1037,2 & 2,39 \\
\hline \multirow{4}{*}{$\begin{array}{l}\text { HLz-DM } \\
1=2,5 \mathrm{~m}\end{array}$} & ZV1; 0,5 & 0,65 & 175 & 111 & 113,1 & 1,02 \\
\hline & ZV1; 0,55 & 0,71 & 175 & 120 & 124,4 & 1,04 \\
\hline & ZV1; 0,65 & 0,84 & 175 & 133 & $147,1(*)$ & $*$ \\
\hline & ZV1; 1,0 & 1,29 & 175 & 128 & $226,3(*)$ & $*$ \\
\hline \multirow{4}{*}{$\begin{array}{l}\text { HLz-DM } \\
\mathrm{l}=1,0 \mathrm{~m}\end{array}$} & ZV1; 0,18 & 0,23 & 61 & 14,3 & 14,2 & 1,00 \\
\hline & ZV1; 0,6 & 0,78 & 61 & 43,6 & 47,3 & 1,09 \\
\hline & ZV1; 0,63 & 0,81 & 61 & 45,1 & 49,7 & 1,10 \\
\hline & ZV1; 0,66 & 0,85 & 61 & 46,7 & $52,1(*)$ & $*$ \\
\hline \multirow{4}{*}{$\begin{array}{l}\text { PB-DM } \\
l=2,5 \mathrm{~m}\end{array}$} & ZV1; 0,8 & 1,03 & 110 & 112,0 & 113,8 & 1,02 \\
\hline & ZV1; 0,85 & 1,10 & 110 & 119 & 120,9 & 1,02 \\
\hline & ZV1; 1,0 & 1,29 & 110 & 127 & $142,2(*)$ & $*$ \\
\hline & ZV1; 1,2 & 1,55 & 110 & 149 & $170,7(*)$ & $*$ \\
\hline
\end{tabular}

Tabelle 44: Vergleich der Ergebnisse der rechnerischen Untersuchung mit denen des Antwortspektrenverfahrens

In der dynamischen Berechnung wird der Steifigkeitsabfall des Systems über das Materialmodell mit erfasst. Durch die Verschiebung der Eigenperiode im Antwortspektrum in Richtung des abfallenden Astes resultieren geringere Lasten. Dieser Effekt lässt sich über den Vergleich der aufgetretenen maximalen Horizontallasten mit denen nach dem Antwortspektrenverfahren bestimmen.

Werden die nach den beiden Verfahren ermittelten Verhaltensfaktoren $q_{\mu}$ und $q_{A S P}$ gegenübergestellt, so ergibt sich folgendes Bild: 


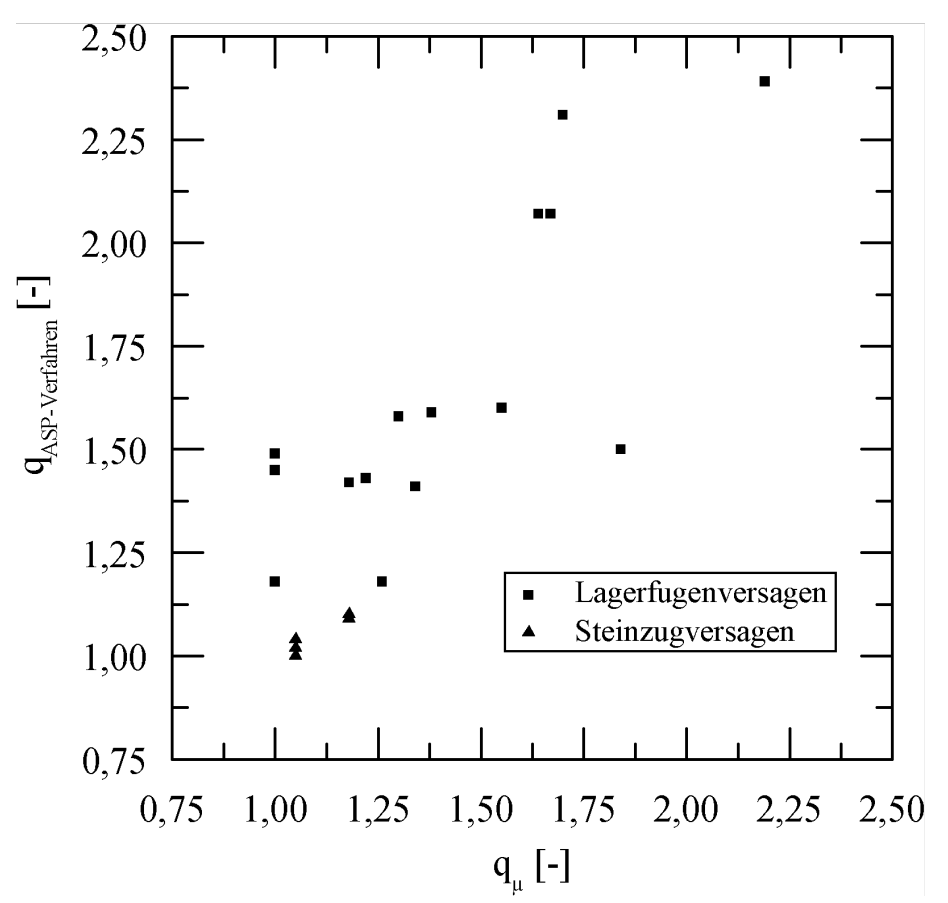

Abbildung 80: Gegenüberstellung der aus den Verschiebeduktilitäten und den Vergleichsrechnungen nach dem Antwortspektrenverfahren ermittelten Verhaltensfaktoren

Deutlich wird hierbei, dass die aus der Vergleichrechnung nach dem Antwortspektrenverfahren ermittelten Verhaltensfaktoren $q_{A S P}$ im Mittel um $10 \%$ größer als jene aus der Verschiebeduktilität bestimmten $q_{\mu}$ sind. Dieses ist insbesondere für das Versagensbild Lagerfugenversagen mit einer maximalen Differenz von 50\% stärker ausgeprägt, wohingegen im Fall des Steinzugversagens beide Verfahren annähernd gleiche Werte liefern.

\subsection{Bewertung}

Aufbauend auf das Schubmodell von Mann / Müller wurde ein Materialmodell nach der Plastizitätstheorie entwickelt und in ein FE-Programm [75] als Benutzerroutine implementiert. Das Materialmodell wurde anhand eigener Versuche überprüft und es konnte insgesamt eine gute Übereinstimmung festgestellt werden. Es zeigte sich, dass auch bei der Wahl eines Elastizitäts- und Schubmoduls an der unteren Grenze des in der Literatur angegebenen Wertebereiches, die Wandsteifigkeit teilweise deutlich zu hoch beschrieben wurde. Für die betrachtete Beanspruchungsart scheinen in diesem Bereich weitere Untersuchungen unter Berücksichtigung der Orthotropie erforderlich.

Im Rahmen einer Parameterstudie an einem Ersatzsystem wurden dynamische Berechnungen durchgeführt und die Ergebnisse ausgewertet. Die aus der Verschiebeduktilität bestimmten Verhaltensfaktoren lagen insgesamt zwischen $q_{\mu}=1,1$ und 2,2. Einzelne höhere rechnerische Werte ergaben sich bei Reibungsversagen in der Lagerfuge, z.B. bei Steinen mit hoher Zugfestigkeit und Mörteln mit geringen Reib- und Haftscherfestigkeitswerten. Das Zulassen hoher plastischer Schubverformungen würde jedoch die Tragfähigkeit von Nachbarbauteilen gefährden, z.B. im Verband hergestellter Querwände. Für den Fall des Steinzugversagens dagegen konnte der Verhaltensfaktor mit etwa $q_{\mu}=1,1 \div 1,2$ eingegrenzt werden.

Bei der Ermittlung des Verhaltensfaktors über den Vergleich mit den Ergebnissen nach dem Antwortspektrenverfahren ergaben sich, insbesondere für den Fall des Lagerfugenversagens, 
etwa 25\% höhere Werte als bei der Ermittlung aus der Verschiebeduktilität. Die Ursache lag in dem Steifigkeitsabfall der Wand mit einhergehender Lastreduktion infolge Verschiebung der Eigenperiode im Antwortspektrum in Richtung des abfallenden Astes, was lediglich in der nichtlinearen, dynamischen Berechnung erfasst werden konnte.

Die Höhe der rechnerischen Abminderung war von einer Vielzahl von Faktoren abhängig, u.a. vom Versagensbild, der Boden-Baugrund-Kombination $(\rightarrow$ Form des Antwortspektrums), den Wandabmessungen ( $\rightarrow$ Schub- / Biegeverformungsanteil) und den Randbedingungen im Bauwerk ( $\rightarrow$ Normalspannungsniveau, Einspanngrad in den Geschossdecken).

Nicht eingeschlossen in den oben dargestellten Ergebnissen ist der Einfluss der prinzipiell zu hohen Beschreibung der Wandsteifigkeiten. 


\section{Zusammenfassung und Ausblick}

Durch die Überarbeitung von europäischer und nationaler Erdbebennorm resultieren für übliche Hochbauten in deutschen Erdbebengebieten deutlich höhere Einwirkungen. Viele bewährte Mauerwerkskonstruktionen lassen sich infolge des geringen Verhaltensfaktors von $q=1,5$ rechnerisch nicht mehr nachweisen.

Ziel der Arbeit war, das Trag- und Verformungsverhalten unbewehrter Mauerwerkskonstruktionen für den Lastfall Erdbeben realitätsnah zu untersuchen und zu beschreiben. Im Rahmen einer Literatursichtung wurde deutlich, dass systematische experimentelle Untersuchungen an

zurzeit in Deutschland üblichen Stein-Mörtel-Kombinationen nicht vorhanden sind. Die Mehrzahl der Erdbebenversuche wurde in der Vergangenheit an Mauerwerkswänden aus kleinformatigen Vollsteinen mit Normalmörtel durchgeführt. Die direkte Übertragbarkeit der Ergebnisse auf modernes Mauerwerk, wie z. B. Lochsteine oder großformatige Elemente mit Dünnbettmörtel, erscheint hierbei fraglich.

Im Zuge der eigenen experimentellen Untersuchungen an zehn geschosshohen Wänden wurde die Versuchsmethode der Pseudodynamik angewendet. Bei dieser wird das dynamische Verhalten von Bauwerken oder einzelner Bauteile im Zeitbereich experimentell untersucht. Basis ist die numerische, zeitschrittweise Lösung der Schwingungsdifferentialgleichung der zu untersuchenden Konstruktion. Als repräsentative Bauwerke wurden ein 3-geschossiges Reihenmittelhaus sowie ein Wohngebäude mit 4 oberirdischen Stockwerken gewählt. Unter Ausnutzung von Symmetrieeffekten konnten die Versuche jeweils auf die maßgebende Schubwand im untersten Geschoss reduziert werden. Bei den angesetzten, künstlich generierten Bodenbeschleunigungszeitverläufen wurden die Besonderheiten deutscher Erdbebengebiete, in erster Linie Erdbebendauer und Form des Antwortspektrums, berücksichtigt.

In der ersten Simulationsstufe wurden die zu untersuchenden Bauwerke auf einen äquivalenten Einmassenschwinger in Form einer Kragscheibe reduziert, wohingegen in der zweiten Simulationsstufe die Untersuchungen an einem Mehrmassenschwinger durchgeführt wurden. Die oberen Geschosse wurden hierbei als Sub-Struktur lediglich numerisch berücksichtigt. Deren nichtlineare Charakteristik wurde in statisch-zyklischen Vorversuchen bestimmt. Die Erweiterung des Versuchsstandes ermöglichte die Steuerung von Wandkopfmoment und -normalkraft. Dadurch konnten annähernd beliebige Kraft- und Verformungszustände aufgebracht und die Problematik konventioneller Prüfstände umgangen werden.

Einen entscheidenden Eingangsparameter bei der Bestimmung der resultierenden Erdbebenbelastung einer Konstruktion stellen die Systemeigenperioden dar. Deren Ermittlung erfolgt entsprechend den neuen Erdbebennormen bei Mauerwerksbauten mit gegebenenfalls gerissenen Querschnitten. Jedoch zeigten die eigenen Versuche, dass bereits bei ungerissenen Querschnitten die Beschreibung mit isotropen Eigenschaften und E-Moduln aus der Beanspruchungsrichtung senkrecht zu den Lagerfugen, deutlich zu hohe Steifigkeitswerte liefert. Dieser Effekt war bei geringer Wandnormalkraft besonders stark ausgeprägt. Zusätzlich trat in den Versuchen mit zunehmender Horizontalbeanspruchung eine weitere Abnahme der Wandsteifigkeiten auf. Bei maximaler bezogener Horizontalkraft erhöhte sich bei den Kragscheibensystemen die erste Eigenperiode um den Faktor 2,0 und bei den Mehrmassensystemen der zweiten Versuchsstufe um den Faktor 1,3.

Bei der Untersuchung des Einflusses des nichtlinearen Verhaltens auf die Lastreduktion wurde ebenfalls eine Abhängigkeit des Systems erkennbar. Bei den Kragscheibensystemen der ersten Stufe lag der Verhältniswert zwischen rechnerischer Einwirkung mit gemessenen 
Anfangswandsteifigkeiten und tatsächlich in den Versuchen aufgetretener Horizontalkraft im Mittel bei 2,8. Bei den Versuchen am Mehrmassenschwinger war dieser Effekt geringer ausgeprägt. Hier trat die Lastreduktion in einem Bereich von 1,0 $\div 2,2$ mit einem Mittelwert von 1,5 auf. Tendenziell war bei dominierendem Lagerfugenversagen die Lastreduktion stärker ausgeprägt als bei vorwiegendem Steinzugversagen.

Für weitergehende Untersuchungen wurde aufbauend auf das Schubmodell von Mann / Müller ein Materialmodell nach der Plastizitätstheorie entwickelt und anhand der eigenen Versuchsergebnisse überprüft. Die Übereinstimmung von Versuch und Nachrechung war im Wesentlichen gut - jedoch wurden die Wandsteifigkeiten mit den gegebenen Eingangsparametern aus der Literatur allgemein zu hoch beschrieben. Die Bruchlasten und die Versagensbilder konnten mit dem Modell gut abgebildet werden. Anschließend wurde eine Parameterstudie durchgeführt und die Lastreduktion in Form des Verhaltensfaktors $q$ über die Verschiebeduktilität der Einzelwand ermittelt. Dabei zeigte sich eine entscheidende Abhängigkeit vom aufgetretenen Versagensbild. Beim Gleiten in der Lagerfuge ergaben sich aufgrund des ,gutmütigen“ Verhaltens teilweise sehr hohe rechnerische Verschiebeduktilitätswerte - aufgrund der negativen Beeinflussung der Tragfähigkeit von Nachbarbauteilen erwies sich deren Beschränkung jedoch als notwendig. Beim Versagensbild Steinzug ergab sich in den Berechnungen ein relativ sprödes Verhalten, was zu geringeren Duktilitätswerten führte.

Zusammenfassend kann festgestellt werden, dass den Ergebnissen der eigenen Untersuchungen zufolge für unbewehrte Mauerwerksbauten höhere Verhaltensfaktoren, als momentan in den Normen vorgeschlagen, gerechtfertigt erscheinen. Weiterer Untersuchungsbedarf - neben Bauteilversuchen unter Variation von Materialien, geometrischen Parametern und Randbedingungen im Gesamtbauwerk - wird noch in folgenden Bereichen gesehen:

- Schubbemessungsansatz in den Mauerwerksnormen und Beschreibung der Eingangsparameter (schräge Steinzugfestigkeit, Haftscherfestigkeit, Reibbeiwert)

- realistischer Steifigkeitsansatz unter Berücksichtigung der Orthotropie

In der Arbeit unberücksichtigt blieben Sicherheitsüberlegungen, die für den Lastfall Erdbeben von großer Bedeutung sind. Hier wird ebenfalls Forschungsbedarf gesehen, insbesondere in Zusammenhang mit dem Schubbemessungsansatz und der Streuung der Eingangsgrößen. 


\section{Literaturverzeichnis}

[1] Abrams, D.P.; Noland, J.L.; Atkinson, R.H.: Response of clay-unit masonry to repeated compressive forces. In: McNeilly, T.; Scrivener, J. C. (Hrsg.): Proceedings of the $7^{\text {th }}$ international brick masonry conference. Melbourne: 1985.

[2] Bachmann, H.: Erdbebensicherung von Hochbauten. Basel: Birkhäuser, 1995.

[3] Bachmann, H.; Lang, K.: Zur Erdbebensicherung von Mauerwerksbauten. (Bericht Nr. 274 des Institutes für Baustatik und Konstruktion, ETH Zürich). Zürich: vdfHochschulverlag AG, 2002.

[4] Backes, H.-P.: Zugfestigkeit von Mauerwerk und Verformungsverhalten unter Zugbeanspruchung. Berlin: Ernst \& Sohn, 1985. (Mauerwerk-Kalender 1985)

[5] Bernardini, A.; Castellato, A.; Modena, C.; Vitaliani, R.: Post-elastic Behaviour of Plain masonry Shear Walls. In: McNeilly, T.; Scrivener, J. C. (Hrsg.): Proceedings of the $7^{\text {th }}$ International Brick Masonry Conference. Melbourne: 1985.

[6] Bernardini, A. (Hrsg.): Seismic Damage to Masonry Buildings. Proceedings of the International Workshop on Measures of Seismic Damage to Masonry Buildings Monselice / Padova / Italien, 25.-26. Juli 1998. Rotterdam: A- A. Balkema, 1999.

[7] Bosiljkov, V.; Zarnic, R.; Bosiljkov V. B.: Shear Tests of the turn Panels made from different Types of Mortar - an experimental Study. In: Adell, J. M. (Hrsg.): Proceedings of the $12^{\text {th }}$ International Brick/Block Masonry Conference. Madrid: Universidad polytecnica, 2000.

[8] Brüstle, W.; Geyer, M.; Stange, S.; Schneider, G.: Geologische Untergrundklassen und Normspektren für DIN 4149-neu. In: Savidis, S. (Hrsg.): Tagungsband der Dreiländertagung D-A-CH der DGEB 1999 in Berlin, Entwicklungsstand in Forschung und Praxis auf dem Gebiet des Erdbebeningenieurwesens, der Boden- und Baudynamik, Berlin: DGEB, 1999.

[9] Chen, W. F.: Plasticity in Reinforced Concrete. New York: McGraw-Hill Book Company, 1982.

[10] Clough, R.W.; Gülkan, P.; Mayes, R.L.: Earthquake behaviour of one-story Masonry Houses. In: Hart, G. (Hrsg.): Proceedings of the $2^{\text {nd }}$ speciality conference on dynamic response of structure: Experimentation, observation, prediction and control. Atlanta, 1981.

[11] Dialer, C.: Bruch- und Verformungsverhalten von schubbeanspruchten Mauerwerksscheiben, zweiachsige Versuche an verkleinertem Modellmauerwerk. München, Diss. 1990 (Berichte aus dem konstruktiven Ingenieurbau der TU München Nr. 1/90)

[12] Dhanasekar, M.; Pager, A.W.; Kleeman, P.W.: The Behaviour of Brick Masonry under biaxial Stress with particular Reference to infilled Frames. In: McNeilly, T.; Scrivener, J. C. (Hrsg.): Proceedings of the $7^{\text {th }}$ international Brick Masonry Conference. Melbourne: 1985

[13] CATMAN. Version 3.1. Hottinger Baldwin Messtechnik GmbH, Darmstadt: 2001.

[14] DIN 105-1 (1989-08): Mauerziegel; Vollziegel und Hochlochziegel. August 1989. 
[15] DIN 105-2 (1989-08): Mauerziegel; Leichthochlochziegel. August 1989.

[16] DIN 106-1 (1980-09): Kalksandsteine; Vollsteine, Lochsteine, Blocksteine, Hohlblocksteine. September 1980.

[17] DIN 1053-1 (1996-11): Mauerwerk: Teil 1: Berechnung und Ausführung. November 1996.

[18] E DIN 1053-5 (2002-04): Mauerwerk - Teil 5: Mauerwerk aus großformatigen Planbauelementen. Entwurf, April 2002.

[19] DIN 1048-5 (1991-06): Prüfverfahren für Beton, Festbeton, gesondert hergestellte Probekörper, Juni 1991.

[20] DIN 4149-1 (1981-04): Bauten in deutschen Erdbebengebieten: Lastannahmen, Bemessung und Ausführung üblicher Hochbauten. April 1981

[21] DIN 4149-1/A-1 (1992-12): Bauten in deutschen Erdbebengebieten: Lastannahmen, Bemessung und Ausführung üblicher Hochbauten: Änderung 1, Karte der Erdbebenzonen. Dezember 1992

[22] E DIN 4149 (2002-10): Bauten in deutschen Erdbebengebieten: Auslegung von Hochbauten gegen Erdbeben. Entwurf, Oktober 2002.

[23] DIN 18555-3 (1982-09): Prüfung von Mörteln mit mineralischen Bindemitteln, Festmörtel, Bestimmung der Biegezugfestigkeit, Druckfestigkeit und Rohdichte, September 1982 .

[24] DIN 18555-4 (1986-03): Prüfung von Mörteln mit mineralischen Bindemitteln, Festmörtel, Bestimmung der Längs- und Querdehnung sowie Verformungskenngrößen von Mauermörtel im statischen Druckversuch, März 1986.

[25] DIN 18555-5 (1986-03): Prüfung von Mörteln mit mineralischen Bindemitteln, Festmörtel, Bestimmung der Haftscherfestigkeit von Mauermörteln, März 1986.

[26] DIN 18555-6 (1987-11): Prüfung von Mörteln mit mineralischen Bindemitteln, Festmörtel, Bestimmung der Haftzugfestigkeit, November 1987.

[27] DIN V ENV 1998-1-1 (1997-06): Eurocode 8 - Auslegung von Bauwerken gegen Erdbeben - Teil 1-1: Grundlagen; Erdbebeneinwirkungen und allgemeine Anforderungen an Bauwerke; Deutsche Fassung ENV 1998-1-1:1994.

[28] DIN V ENV 1998-1-2 (1997-06): Eurocode 8 - Auslegung von Bauwerken gegen Erdbeben - Teil 1-2: Grundlagen; Allgemeine Regeln für Hochbauten; Deutsche Fassung ENV 1998-1-2:1994.

[29] DIN V ENV 1998-1-3 (1997-06): Eurocode 8 - Auslegung von Bauwerken gegen Erdbeben - Teil 1-3: Grundlagen; Baustoffspezifische Regeln für Hochbauten; Deutsche Fassung ENV 1998-1-3:1995.

[30] Eurocode 8: Design of structures for earthquake resistance - Part 1: General rules, seismic Actions and Rules for Buildings. Draft No 6 - Stage 49. Entwurf, Januar 2003.

[31] Fehling, E.; Bunje, K.; Schmidt, T. J. H.: Zur Frage des realitätsnahen Nachweises von Mauerwerksbauten unter Erdbebenbeanspruchung in mitteleuropäischen Erdbebengebieten. In: Savidis, S. (Hrsg.): Tagungsband der Dreiländertagung D-A-CH der 
DGEB 1999 in Berlin, Entwicklungsstand in Forschung und Praxis auf dem Gebiet des Erdbebeningenieurwesens, der Boden- und Baudynamik, Berlin: DGEB, 1999.

[32] Ganz, H.-R.: Mauerwerksscheiben unter Normalkraft und Schub. (Bericht Nr. 148 des Institutes für Baustatik und Konstruktion, ETH Zürich) Basel: Birkhäuser, 1985.

[33] Ganz, H.-R.; Thürlimann, B.: Versuche über die Festigkeit von zweiachsig beanspruchtem Mauerwerk. (Bericht Nr. 7502-3 des Institutes für Baustatik und Konstruktion, ETH Zürich) Basel: Birkhäuser, 1982.

[34] Ganz, H.-R.; Thürlimann, B.: Versuche an Mauerwerksscheiben unter Normalkraft und Querkraft. (Bericht Nr. 7502-4 des Institutes für Baustatik und Konstruktion, ETH Zürich) Basel: Birkhäuser, 1984.

[35] Ganz, H.-R.; Guggisberg, R.; Schwartz, J.; Thürlimann, B.: Contribution to the design of masonry walls. (Bericht Nr. 168 des Institutes für Baustatik und Konstruktion, ETH Zürich) Basel: Birkhäuser, 1989.

[36] Glitza, H.: Druckbeanspruchung parallel zur Lagerfuge. Berlin: Ernst \& Sohn, 1988. (Mauerwerk-Kalender 1988)

[37] Grübl, P.; Weigler, H.; Karl, S.: Beton, Arten, Herstellung und Eigenschaften. Berlin: Ernst \& Sohn, 2001.

[38] Grünthal, G.; Mayer-Rosa, D.; Lenhardt, W. A.: Abschätzung der Erdbebengefährdung für die D-A-CH-Staaten - Deutschland, Österreich, Schweiz. Berlin: Ernst \& Sohn. 1998 (Die Bautechnik, Heft 10, 1998)

[39] Guedes, J.; Pegon, P.; Pinto, A.V.: PSD testing of Bridges - A numerical Validation study. In: Savidis, S.A: (Hrsg.): Proceedings of the $2^{\text {nd }}$ International Conference on Earthquake Resistant Construction and Design -Vol. 2, TU Berlin 1994. Rotterdam: Balkema, 1994.

[40] Guggisberg, R.; Thürlimann, B.: Versuche zur Festlegung der Rechenwerte von Mauerwerksfestigkeiten. (Bericht Nr. 7502-5 des Institutes für Baustatik und Konstruktion, ETH Zürich) Basel: Birkhäuser, 1987.

[41] Gülkan, P.; Mayes, R.L.; et. al.: An experimental on the seismic Behaviour of the single-story Masonry house. In: Proceedings of the north American Masonry Conference. Colorado: 1978.

[42] Hamid, A. A.; Drysdale, R.G.: Proposed Failure Criteria for Concrete Block Masonry under biaxial Stresses. ASCE - Journal of the Structural Division, 107, 1981.

[43] Hamid, A. A.; Drysdale, R.G.: Proposed Failure Criteria for Brick Masonry under combined Stresses. In: Proceedings of the second North American Masonry Conference, Maryland 1982. Maryland: The Masonry Society, 1982.

[44] Hampe; Schwarz, J.: Verhalten von Mauerwerksbauten unter seismischen Einwirkungen. Berlin: Ernst \& Sohn, 1991. (Mauerwerk-Kalender 1991)

[45] Hansen, K.F.; Nykänen, E.; Gottfredsen, F.R.: Shear Behaviour of Bed Joints at different levels of Precompression. In: Masonry International, Vol. 12, No. 2, 1998.

[46] Hilber, H. M.; Hughes, T. J. R.; Taylor, R. L.: Improved numerical Dissipation for time Integration Algorithms in Structural Dynamics, In: Earthquake Engineering and Structural Dynamics, 1977. 
[47] Hillerborg, A.; Modeer, M.; Petersson, P.E.: Analysis of Crack Formation and Crack Growth in Concrete by means of Fracture Mechanic and Finite Elements. In: Cement and Concrete Research 6, 1976.

[48] Hinzen, K.-G.: Erdbeben bei Alsdorf am 22.7.2002. In: D-A-CH-Mitteilungsblatt, Band 21, Nr. 2, August 2002.

[49] Hofmann, P.; Stöckl, S.: Versuche zum Haftscherverhalten der Lagerfugen von Mauerwerk. Forschungsbericht, Lehrstuhl für Massivbau, TU München. 1985.

[50] Hofstetter, G.; Mang, H. A.: Computational Mechanics of Reinforced Concrete Structure. Wien: Vieweg, 1995.

[51] Hosser, D.: Realistische seismische Lastannahmen für Bauwerke: Ergebnisse einer interdisziplinären Forschungsarbeit. Berlin: Springer, 1987 (Bauingenieur 62)

[52] Innenministerium Baden- Württemberg (Hrsg.): Erdbebensicher Bauen : Planungshilfe für Bauherren, Architekten und Ingenieure,1986.

[53] Kaiser, D.: Bodenbewegungen in der Nähe mittelgroßer Erdbeben. In: Savidis, S. (Hrsg.): Tagungsband der Dreiländertagung D-A-CH der DGEB 1999 in Berlin, Entwicklungsstand in Forschung und Praxis auf dem Gebiet des Erdbebeningenieurwesens, der Boden- und Baudynamik, Berlin: DGEB, 1999.

[54] Kanai, K.: Semi-empirical Formula for the Seismic Characteristics of the Ground. In: Bulletin of the Earthquake Research Institute, University of Tokyo, Vol. 35, 1957.

[55] Kasten, D.; Vratsanou,, V.; Meyer, U.; Ötes, A.: Mauerwerksbau in deutschen Erdbebengebieten, Berlin: Ernst \& Sohn, 2002. (Mauerwerk-Kalender 2002)

[56] Kirtschig, K.; Kasten, D.: Formfaktoren für die Prüfung von Mauersteinen. Berlin: Ernst \& Sohn, 1981. (Mauerwerk-Kalender 1981)

[57] Klein, G.: Bodendynamik und Erdbeben, In: Smoltczyk, U. (Hrsg.): GrundbauTaschenbuch, Teil 1: Geotechnische Grundlagen, Berlin: Ernst \& Sohn, 2001.

[58] Klein, H.-H.: Kenngrößen zur Beschreibung der Erdbebeneinwirkung, Mitteilung aus dem Institut für Massivbau der TH Darmstadt. Berlin: Ernst \& Sohn, 1985.

[59] Klein, H.-H.: Realistische seismische Lastannahmen für Bauwerke: Alternative Lastbeschreibung mittels Fourierspektren. Berlin: Springer, 1988 (Bauingenieur 66)

[60] König, G.; Mann, W.; Ötes, A.: Untersuchungen zum Verhalten von Mauerwerksbauten unter Erdbebeneinwirkung. Frankfurt/M.: IRB-Verlag, 1988.

[61] König, G.; Mann, W.; Ötes, A.: Experimental Investigations on the Behaviour of unreinforced Masonry Walls under seismically induced Loads and Lessons derived. In: Proceedings of the $9^{\text {th }}$ World Conference on Earthquake Engineering. Tokyo: 1988.

[62] Korkut, K.: Das seismische Verhalten von Gebäuden mit steif ausgebildeten Kellergeschossen. Berlin: Springer, 1991. (Bauingenieur 66)

[63] KTA 2201.1: Auslegung von Kernkraftwerken gegen seismische Einwirkungen; Teil 1: Grundsätze, Juni 1975.

[64] KTA 2201.3: Auslegung von Kernkraftwerken gegen seismische Einwirkungen; Teil 3: Auslegung der baulichen Anlagen, November 1980. 
[65] Lang, K.: Seismic vulnerability of existing buildings. (Bericht Nr. 273 des Institutes für Baustatik und Konstruktion, ETH Zürich) Zürich: vdf Hochschulverlag AG, 2002.

[66] Lourenco, P.B.: Computational Strategies for Masonry Structures. Diss., Delft University Press, 1996.

[67] Lourenco, P.B. ; Rots, J.G. : Understanding the Behaviour of Shear Walls: A numerical Approach. In: Shrive, N.G.; Huizer, A. (Hrsg.): Proceedings of the $10^{\text {th }}$ international Brick/Block Masonry Conference, Calgary 1994. Calgary: Masonry Council of Canada, 1994.

[68] Lourenco, P.B.; Rots, J.G.; van der Pluijm, R.: Understanding the Tensile Behaviour of Masonry parallel to the Bed Joints: A Numerical Approach. In: Masonry International, Vol. 12, No. 3, 1999.

[69] Lurati, F.; Graf, H.; Thürlimann, B: Versuche zur Festlegung der Festigkeitswerte von Zementsteinmauerwerk. (Bericht Nr. 8401-2 des Institutes für Baustatik und Konstruktion ETH Zürich) Basel: Birkhäuser, 1990.

[70] Lurati, F.; Thürlimann, B: Versuche an Mauerwerkswänden aus Zementstein. (Bericht Nr. 8401-3 des Institutes für Baustatik und Konstruktion ETH Zürich) Basel: Birkhäuser, 1990.

[71] Mahin, S. A.; Williams, M. E.: Computer controlled seismic Performance Testing. In: Hart, G. (Hrsg.): Proceedings of the $2^{\text {nd }}$ speciality Conference on dynamic Response of Structure: Experimentation, Observation, Prediction and Control. Atlanta, 1981.

[72] Mann, W.; König, G. et al.: Versuche zum Verhalten von Mauerwerk unter seismischer Beanspruchung. Berlin: Ernst \& Sohn, 1989. (Mauerwerk-Kalender 1989)

[73] Mann, W.; Müller, H.: Schubtragfähigkeit von Mauerwerk. Berlin: Ernst \& Sohn, 1978. (Mauerwerk-Kalender 1978)

[74] Mann, W.; Müller, H.: Schubtragfähigkeit von gemauerten Wänden und Voraussetzungen für das Entfallen des Windnachweises. Berlin: Ernst \& Sohn, 1985. (Mauerwerk-Kalender 1985)

[75] MARC. Version 2001. MSC.Software Corporation. Santa Ana: 2001.

[76] MAPLE. Version 6.02a. Waterloo Maple Inc.. Waterloo: 2001.

[77] Mayes, R.L.; Clough R.W.: A literature Survey - Compressive, Tensile, Bond and Shear Strength of Masonry. Report EERC 75-15 - Earthquake Engineering Research Center, University of California, Berkeley, 1975.

[78] Mayes, R.L.; Clough R.W.: State-of-the-art in seismic Shear Strength of Masonry - an Evaluation and Review. Report EERC 75-21 - Earthquake Engineering Research Center, University of California, Berkeley, 1975.

[79] Meskouris, K.: Beitrag zur Erdbebenuntersuchung von Tragwerken des Konstruktiven Ingenieurbaus. (Mitteilung Nr. 82-12). Bochum: Ruhr-Universität 1982.

[80] Meskouris, K.; Krätzig, W. B.; Elenas, A.; Heiny, L.; Meyer, I. F.: Mikrocomputerunterstützte Erdbebenuntersuchung von Tragwerken. (SFB 151 - Bericht Nr. 8). Bochum: Ruhr-Universität 1988.

[81] Meskouris, K.: Baudynamik. Berlin: Ernst \& Sohn, 1999. 
[82] Metzemacher, H.: Versuchseinrichtung zur Prüfung der Zugfestigkeit von Steinprismen. In: Neunast, A. (Hrsg.): Proceedings of the $9^{\text {th }}$ international Brick/Block Masonry Conference, Berlin 1991. Berlin: DGfM, 1991.

[83] Meyer, U.; Schubert, P.: Druck- und Schubfestigkeit von Mauerwerk aus Hochlochziegeln. Berlin: Ernst \& Sohn, 1999. (das Mauerwerk, Heft 1)

[84] Meyer,U.; Schubert, P.: Spannungs-Dehnungs-Linien von Mauerwerk. Berlin: Ernst \& Sohn, 1992. (Mauerwerk-Kalender 1992)

[85] Meyer,U.: Steinlängsdruckfestigkeit und Scherbenzugfestigkeit bei Hochlochziegeln. Mündliche Mitteilung. Januar 2003

[86] Modena, C.: Seismic Behaviour of Masonry Structures: Experimentally based Modelling. Masonry International, Vol. 6, 1992.

[87] Mojsilović, N.; Marti, P.: Versuche an kombiniert beanspruchten Mauerwerkswänden. (Bericht Nr. 203 des Institutes für Baustatik und Konstruktion, ETH Zürich) Basel: Birkhäuser, 1994.

[88] Mojsilović, N.: Zum Tragverhalten von kombiniert beanspruchtem Mauerwerk. (Bericht Nr. 216 des Institutes für Baustatik und Konstruktion, ETH Zürich) Basel: Birkhäuser, 1995.

[89] Molnár, M.: Shear and horizontal Tension Behaviour of Masonry Model Development and Verification. In: Masonry International, Vol. 15, No. 2, 2002.

[90] Motta, F.; D’Amore, E.: Numerical Modelling of the Structural Behaviour of Masonry Buildings. In: McNeilly, T.; Scrivener, J. C. (Hrsg.): Proceedings of the $7^{\text {th }}$ international Brick Masonry Conference. Melbourne: 1985.

[91] Müller, F. P.; Keintzel, E.: Erdbebensicherung von Hochbauten. Berlin: Ernst \& Sohn, 1984.

[92] NABau (Hrsg.): Richtlinie zur Anwendung von DIN V ENV 1998 (Vorschlag für ein Nationales Anwendungsdokument (V-NAD), Dokumentennummer: NABau 00.06.00 Nr. 1-98). Januar 1998.

[93] Naraine, K.; Sinha, S.: Test of Brick Masonry Models under cyclic Compressive Loading. In: De Courcy, J. W.: (Hrsg.): Proceedings of the $8^{\text {th }}$ international Brick and Block Masonry Conference, Dublin 1988. London: Elsevier, 1988.

[94] Naraine, K.; Sinha, S.: Geometrical Model for Brick Masonry under Cyclic Compressive Loading. In: West, H.W.H. (Hrsg.): Proceedings of the second International Masonry Conference - Part 4, London, 1989. London: British Masonry Society, 1990.

[95] Naraine, K.; Sinha, S.: Energy Dissipation in Brick Masonry under Cyclic compressive Loading. In: West, H.W.H. (Hrsg.): Proceedings of the second International Masonry Conference - Part 4, London, 1989. London: British Masonry Society, 1990.

[96] Newmark, N. M.: A method of Computation for structural Dynamics. ASCE - Journal of Engineering Mechanics Division, 1959.

[97] ÖNORM B 3200 (1987-07): Mauer- und Hochlochziegel, Anforderungen und Prüfungen, Normkennzeichnung, Juli 1987.

[98] Owen, D. R. J.; Hinton, E.: Finite Elements in Plasticity: Theory and Practice. Swansea: Pieridge Press Ltd., 1980. 
[99] Page, A. W.: An experimental Investigation of the biaxial Strength of Brick Masonry, Proceedings of the $6^{\text {th }}$ international Brick Masonry Conference, Rom 1982.

[100] Page, A. W.: The Strength of Brick Masonry under biaxial Tension-Compression. International Journal of Masonry Construction, Vol. 3, 1983.

[101] Page, A. W.: The Behaviour of unreinforced Masonry in the Newcastle Earthquake. In: Neunast, A. (Hrsg.): Proceedings of the $9^{\text {th }}$ international Brick/Block Masonry Conference, Berlin 1991. Berlin: DGfM, 1990.

[102] Paulay, T.; Bachmann, H.; Moser, K.: Erdbebensicherung von Stahlbetonhochbauten. Basel: Birkhäuser, 1990.

[103] Petersen, Ch.: Dynamik der Baukonstruktionen. Braunschweig: Vieweg, 2000

[104] van der Pluijm, R.: Material Properties of Masonry and its Components under Tension and Shear. In: Proceedings of the $6^{\text {th }}$ Canadian Masonry Symposium. Saskatoon, Saskatchevan: University of Saskatchevan, 1992

[105] van der Pluijm, R.: Shear Behaviour of Bed Joints. In: Hamind, A. A. (Hrsg.): Proceedings of the $6^{\text {th }}$ North American Masonry Conference. Philadelphia: Nat. Science Found., 1993.

[106] van der Pluijm, R.: Shear behaviour of Bed Joints. In: Adell, J. M. (Hrsg.): Proceedings of the $12^{\text {th }}$ international Brick/Block Masonry Conference. Madrid: Universidad polytecnica, 2000.

[107] Pinto, A.V.; Verzeletti, G.; Pegon, P.; Magonette, G.; Negro, P.; Guedes, J.: Pseudodynamic Testing of large-scale R/C Bridges. (ELSA-Report EUR 16378 EN). Luxembourg: ECSC, 1996.

[108] Pravida, J.: Zur nichtlinearen adaptiven Finite-Elemente-Analyse von Stahlbetonscheiben. München, Diss. 1999 (Berichte aus dem konstruktiven Ingenieurbau der TU München Nr. 6/99)

[109] Rackwitz, R.: Einwirkungen auf Bauwerke. In: Mehlhorn, G. (Hrsg.): Der Ingenieurbau - Tragwerkszuverlässigkeit und Einwirkungen. Berlin: Ernst \& Sohn, 1997.

[110] RILEM: Technical Recommendations for the Testing and Use of Construction Material. London: Spon, 1994.

[111] Rots, J.G.: Computational Modeling of Concrete in Fracture. Diss. TU Delft, 1988.

[112] Sandi, H.: Some methodological Aspects related to Vulnerability Analysis. In: Bernardini, A. (Hrsg.): Seismic Damage to Masonry Buildings. (Proceedings of the international Workshop on Measures of seismic Damage to Masonry Buildings - Monselice / Padova / Italien, 25. - 26. Juli 1998). Rotterdam: A- A. Balkema, 1999.

[113] Schlegel, R.; Rautenstrauch, K.; Zrost, H.: Dreidimensionale Berechnung ein- und mehrschaliger Mauerwerkkonstruktionen unter Einbeziehung des anisotropen Rißund Entfestigungsverhaltens. Berlin: Ernst \& Sohn, 2003. (das Mauerwerk, Heft 1)

[114] Schneider, R.: Modellierung des Tragverhaltens flächenhafter Stahlbetontragwerke unter Dauerlast. Diss. TU München, eingereicht in 2002.

[115] Schneider, K. H.; Wiegand, E.; Jucht, K.: Innerer Spannungszustand bei Mauerwerk mit nicht vermörtelten Stoßfugen. Kurzbericht über ein vom Bundesministerium für 
Raumordnung, Bauwesen und Städtebau gefördertes Forschungsvorhaben. Frankfurt/Main 1976.

[116] Schubert, P.: Zur Prüfung von Mauerwerk auf Druckfestigkeit und E-Modul nach DIN 18554. Berlin: Ernst \& Sohn, 1985. (Mauerwerk-Kalender 1985)

[117] Schubert, P.: E-Moduln von Mauerwerk in Abhängigkeit von der Druckfestigkeit des Mauerwerks, der Mauersteine und des Mauermörtels. Berlin: Ernst \& Sohn, 1985. (Mauerwerk-Kalender 1985)

[118] Schubert, P.: Beurteilung der Druckfestigkeit von ausgeführtem Mauerwerk aus künstlichen Steinen und Natursteinen. Berlin: Ernst \& Sohn, 1995. (Mauerwerk-Kalender 1995)

[119] Schubert, P.: Zur Schubfestigkeit von Mauerwerk. Berlin: Ernst \& Sohn, 1998. (Mauerwerk-Kalender 1998)

[120] Schubert, P.: Eigenschaftswerte von Mauerwerk, Mauersteinen und Mauermörtel. Berlin: Ernst \& Sohn, 2002. (Mauerwerk-Kalender 2002)

[121] Schubert, P.; Bohne, D.: Schubfestigkeit von Mauerwerk aus Leichtbetonsteinen. Berlin: Ernst \& Sohn, 2002. (das Mauerwerk, Heft 3)

[122] Schubert, P.; Hoffmann, G.: Druckfestigkeit von Mauerwerk parallel zu den Lagerfugen. Berlin: Ernst \& Sohn, 1994. (Mauerwerk-Kalender 1994)

[123] Schwarz, J.; Grünthal, G.: Zukunftsorientierte Konzepte zur Beschreibung seismischer Einwirkungen für das Erdbebeningenieurwesen. Berlin: Ernst \& Sohn, 1998. (Die Bautechnik, Heft 10)

[124] Schwarz. J. (Hrsg.): Ingenieurseismologie und Erdbebeningenieurwesen. Weimar: Bauhaus-Universität Weimar, 2001.

[125] Seible, F.; Igarashi, A.; Nagy G.: Full-scale Testing of Structural Masonry Systems under simulated seismic Loads. In: Neunast, A. (Hrsg.): Proceedings of the $9^{\text {th }}$ international Brick/Block Masonry Conference, Berlin 1991. Berlin: DGfM, 1991.

[126] Seible, F.; Hegemier, G.; Priestley, N.; Kingsley, G.; Igarashi, A.; Kürkchübasche, A.: Preliminary Results from the TCCMAR 5-Story full-scale reinforced Masonry Research Building Test. In: Hamind, A. A. (Hrsg.): Proceedings of the $6^{\text {th }}$ North American Masonry Conference. Philadelphia: Nat. Science Found., 1993.

[127] Seim, W.: Numerische Modellierung des anisotropen Versagens zweiachsig beanspruchter Mauerwerksscheiben. Diss. 1995 (Aus Forschung und Lehre, Institut für Tragkonstruktionen - Universität Karlsruhe (TH), Heft 27)

[128] Shah, S. P.; Swartz, S. E.; Ouyang, C.: Fracture Mechanics of Concrete. New York, John Wiley \& Sons, 1995.

[129] Shing, P.B.; Mahin, S. A.: Rate-of-loading Effects on pseudodynamic Tests. ASCE Journal of Structural Engineering, 1988.

[130] Shing, P.B.; Mahin, S. A.: Experimental error Effects in pseudodynamic Testing. ASCE - Journal of Structural Engineering, 1990.

[131] Shing, P.B.; Lofti, H. R.: Experimental and Finite Element Analyses of single-Story reinforced Masonry Shear Walls. In: Middleton, J.; Pande, G. N. (Hrsg.): Computer methods in Structural Masonry - Proceedings of the international Symposium on 
Computer Methods in Structural Masonry, Swansea, Books \& Journal International Ltd., Swansea, 1991.

[132] Shing, P.B.; Nakashima, M.; Bursi, O.S.: Application of pseudodynamic Test Method to structural Research. Earthquake Spektra, 1996.

[133] Simon, E.: Schubtragverhalten von Mauerwerk aus großformatigen Steinen. Diss. TU Darmstadt, 2002.

[134] Simon, E.: Schubtragfähigkeit von Mauerwerk aus großformatigen Steinen. Berlin: Ernst \& Sohn, 2003. (das Mauerwerk, Heft 1)

[135] SOFISTiK-ASE. Version 10.68-99. Oberschleißheim, SOFiSTiK AG, 2001.

[136] Stöckl, S. ; Hofmann, P. ; Mainz, J.: Methoden für Haftscherversuche. Berlin: Ernst \& Sohn, 1990 (Mauerwerk-Kalender 15)

[137] Takanashi, K.; Udagawa, K.; Seki, M.; Okada, T.; Tanaka, H.: Nonlinear Earthquake Response Analysis of structures by a Computer-Actuator on-line System. Bulletin of Earthquake resistant Structure Research Center 8, Institute of industrial Science, University of Tokio, 1975.

[138] Thiele, K.: Pseudodynamische Versuche an Tragwerken mit grossen Steifigkeitsänderungen und mehreren Freiheitsgraden. (Bericht Nr. 253-2000 des Institutes für Baustatik und Konstruktion der ETH Zürich), Basel: Birkhäuser, 2000.

[139] Thürlimann, B.; Ganz, H.: Versuche über die Festigkeit von zweiachsig beanspruchtem Mauerwerk. (Bericht Nr. 7502-3 des Institutes für Baustatik und Konstruktion ETH Zürich) Basel: Birkhäuser, 1982.

[140] Thürlimann, B.; Ganz, H.: Bruchbedingungen für zweiachsig beanspruchtes Mauerwerk. (Bericht Nr. 143 des Institutes für Baustatik und Konstruktion ETH Zürich) Basel: Birkhäuser, 1984.

[141] Tomazevic, M.: Experimental and analytical Study of dynamic Behaviour of a fourstoreyed Masonry Building Model. In: Proceedings of the $8^{\text {th }}$ European Conference on Earthquake Engineering. Lissabon: 1986

[142] Tomazevic, M.: Dynamic modelling of Masonry Buildings: Storey Mechanism Model as a simple Alternative. In: Earthquake Engineering and structural Dynamics, Vol. 15, 1987. London: John Wiley \& Sons, 1987.

[143] Tomazevic, M.: Experimental Basis for Earthquake resistant Design of Masonry Walls and Buildings. In: Flesch, R.G.; Ziegler, F. (Hrsg.): Proceedings of the $14^{\text {th }}$ European regional Seminar on Earthquake Engineering, Ossiach, 1988. Wien: OGE, 1989.

[144] Tomazevic, M.; Lutman, M.: Seismic Resistance of Reinforced Masonry Walls. In: Proceedings of the $9^{\text {th }}$ World Conference on Earthquake Engineering. Tokyo: 1988

[145] Tomazevic, M.; Lutman, M.: Simulation of the Seismic Behaviour of reinforced Masonry Walls by Laboratory Tests. In: Shrive, N.G.; Huizer, A. (Hrsg.): Proceedings of the $10^{\text {th }}$ international Brick/Block Masonry Conference. Calgary: Masonry Council of Canada, 1994.

[146] Tomazevic, M.; Turnsek, V.: Lateral Load Distribution as a Basis for the Seismic Resistance Analysis of Masonry Buildings. In: Proceedings of the international Re- 
search Conference on Earthquake Engineering. Skopje: National Science Foundation, 1980.

[147] Tomazevic, M.; Velechovsky, T.: Some aspects of testing small-scale Masonry Models on simple Earthquake Simulators. In: Earthquake Engineering and structural Dynamics, Vol. 21, 1992. London: John Wiley \& Sons, 1992.

[148] Tomazevic, M.; Weiss, P.: Seismic behaviour of Masonry Buildings: Reinforced versus unreinforced Masonry. In: Neunast, A. (Hrsg.): Proceedings of the $9^{\text {th }}$ international Brick/Block Masonry Conference, Berlin 1991. Berlin: DGfM, 1991.

[149] Tomazevic, M.; Weiss, P.: Seismic behaviour of plain and reinforced-Masonry Buildings. In: Journal of Structural Engineering 120, Heft 2, 1994

[150] Tomazevic, M.; Zarnic, R.: The Effect of horizontal Reinforcement on the Strength and Ductility of Masonry Walls in Shear Failure. In: McNeilly, T.; Scrivener, J. C. (Hrsg.): Proceedings of the $7^{\text {th }}$ international Brick Masonry Conference. Melbourne: 1985

[151] Tomazevic, M.; Zarnic, R.: The Behaviour of horizontally reinforced Masonry Walls subjected to cyclic lateral in-plane Load Reversals. In: Proceedings of the $8^{\text {th }}$ European Conference on Earthquake Engineering. Lissabon: 1986.

[152] Vanmarcke, E. H.; Cornell, C. A.; Gasparini, D. A.; Hou, S. N.: SIMQKE: A Program for artificial Motion Generation - User's Manual and Documentation, Department of Civil Engineering, Massachusetts Institute of Technology, Cambridge, 1976.

[153] Vanmarcke, E. H.; Fenton, E. H.; Heredia-Zavoni, E.: SIMQKE-II: Conditioned Earthquake Ground Motion Simulator - User's manual, Version 2.1, Department of Civil Engineering and Operations Research, Princeton University, 1999.

[154] Vratsanou, V., Langer, P.: Schubtragfähigkeit von Porenbeton - Plansteinmauerwerk. Berlin: Ernst \& Sohn, 1998. (das Mauerwerk, Heft 3)

[155] Vratsanou, V.: Das nichtlineare Verhalten unbewehrter Mauerwerksscheiben unter Erdbebenbeanspruchung - Hilfsmittel zur Bestimmung der q-Faktoren. Diss. 1992. (Schriftenreihe des Instituts für Massivbau und Baustofftechnologie - Universität Karlsruhe (TH), Heft 16 - 1992)

[156] Vratsanou, V.; Langer, P.: Untersuchung des Schubtragverhaltens von Wänden aus Porenbeton - Plansteinmauerwerk. Berlin: Ernst \& Sohn, 2001. (das Mauerwerk, Heft 6)

[157] Yamazaki, Y.; Seible, F.; Mizuno, H.; Kaminosono, T.; Teshigawara, M.: The Japanese 5-Story full Scale reinforced Concrete Masonry Test - forced Vibration and cyclic Test Results. The Masonry Society Journal, Vol. 7, No. 1, 1988.

[158] Zarnic, R.; Tomazevic, M.: Study of the Behaviour of Masonry infilled Reinforced Concrete Frames subjected to seismic Loading. In: Proceedings of the $7^{\text {th }}$ international Brick Masonry Conference. Melbourne, Australia: 1985.

[159] Zhuge, Y.; Thambiratnam, D., Corderoy J.: Experimental Testing of Masonry Walls under in-plane cyclic Loads. (In: Shrive, N.G.; Huizer, A. (Hrsg.): Proceedings of the $10^{\text {th }}$ international Brick/Block Masonry Conference, Calgary 1994), Calgary: Masonry Council of Canada, 1994. 
[160] Zilch, K.: Bemessung von Bauwerken gegen Erdbebenbelastung - Ein Bericht zum Stand der Forschung und Praxis. Berlin: 1974. (Die Bautechnik, Heft 5)

[161] Zilch, K.; Pietzonka, R.: Zur dynamischen Berechnung ebener regelmäßiger Rahmen im Lastfall Erdbeben. Berlin: 1980. (Die Bautechnik, Heft 3) 


\section{Bezeichnungen}

Nachfolgend werden die wichtigsten Bezeichnungen zusammengestellt. Matrizen und Vektoren werden in der Arbeit durch Unterstreichung gekennzeichnet.

\section{Lateinische Buchstaben}

a Beschleunigung, Parameter zur Beschreibung der Mauerwerksfestigkeit

$a_{0} \quad$ Maximalwert der Bodenbeschleunigung

$a^{g} \quad$ Bodenbeschleunigung

$a_{g} \quad$ Bemessungswerte der Bodenbeschleunigung für die Referenzwiederkehrperiode

$b \quad$ Breite, Parameter zur Beschreibung der Mauerwerksfestigkeit

$c$ Dämpfung, Biegefedersteifigkeit, Parameter zur Beschreibung der Mauerwerksfestigkeit

C Steifigkeit

$d \quad$ Auslenkung, Schichtmächtigkeit, Dicke

$D \quad$ Dämpfungsmatrix

E Elastizitätsmodul, Bebenenergie

$f \quad$ Formfaktor, Frequenz, allgemeiner Faktor,

$f_{b} \quad$ normierte Steindruckfestigkeit

$f_{b, P r} \quad$ Druckfestigkeit des Steinprüfkörpers

$f_{b t} \quad$ (schräge) Steinzugfestigkeit

$f_{b t, B Z} \quad$ Steinbiegezugfestigkeit

$f_{\text {mo }} \quad$ Mörteldruckfestigkeit

$f_{m o, B Z} \quad$ Mörtelbiegezugfestigkeit

$f_{m o, H Z} \quad$ Haftzugfestigkeit

$f_{m x} \quad$ Mauerwerksdruckfestigkeit parallel zu den Lagerfugen

$f_{m y} \quad$ Mauerwerksdruckfestigkeit senkrecht zu den Lagerfugen

$f_{m t x} \quad$ Mauerwerkszugfestigkeit parallel zu den Lagerfugen

$f_{m y, R e s t}$ Mauerwerksrestfestigkeit senkrecht zu den Lagerfugen unter der maximalen Bruchstauchung $\varepsilon_{\text {Bruch }}$

$f_{S, t} \quad$ Scherbenzugfestigkeit

$F \quad$ Fließbedingung bzw. Fließfunktion

$G \quad$ plastische Potentialfunktion, Schubmodul

$G_{f} \quad$ Bruchenergie

$h \quad$ Herdtiefe, Höhe

$H \quad$ Horizontalkraft, Ordinate im Husid-Diagramm

$i \quad$ Zähler

I Intensität

$k \quad$ Haftscherfestigkeit, Federsteifigkeit, Iterationsschritt, Verfestigungsfunktion 
$\bar{k} \quad$ abgeminderte Haftscherfestigkeit

$l \quad$ Länge

$m \quad$ Masse, Zähler

$M \quad$ Magnitude

$n \quad$ Orthotropiefaktor, Anzahl

$N \quad$ Normalkraft

$q \quad$ Verhaltensfaktor, Verkehrslast

$r \quad$ Rückstellkraft

$s, s_{0} \quad$ Hypozentraldistanz, Parameter

$S \quad$ Bodenparameter

$S_{e} \quad$ Ordinate des elastischen Antwortspektrums

$S_{d} \quad$ Ordinate des Bemessungsantwortspektrums

$t \quad$ Zeit

$T \quad$ Eigenschwingzeit

$T_{S} \quad$ stationärer Teil des Beschleunigungszeitverlaufes

$T_{S B D} \quad$ Starkbebendauer

$\ddot{u} \quad$ Überbindemaß

v Geschwindigkeit, Vertikalverschiebung

w Verschiebung

$W_{p l} \quad$ plastische Arbeit

$\Delta x \quad$ Steinlänge

$\Delta y \quad$ Steinhöhe

\section{Griechische Buchstaben}

$\alpha \quad$ Parameter der numerischen Integration

$\alpha_{0} \quad$ Neigung der Steinzugrisse zur Vertikalen

$\beta \quad$ Parameter der numerischen Integration

$\beta_{0} \quad$ Verstärkungsbeiwert der Spektralbeschleunigung

$\gamma \quad$ Schubverformung, Bedeutungsbeiwert, Parameter der numerischen Integration, Wichte

$\delta \quad$ Geschossgleitung

$\varepsilon \quad$ Dehnung

$\zeta \quad$ Dämpfungsgrad (Lehr'sches Dämpfungsmaß)

$\eta \quad$ Dämpfungs-Korrekturbeiwert

$\psi \quad$ Dillatationswinkel

$\kappa \quad$ Verfestigungsparameter

$\lambda \quad$ plastischer Multiplikator

$\Lambda \quad$ logarithmisches Dekrement 
$\mu \quad$ Reibbeiwert, Duktilität

$\bar{\mu} \quad$ abgeminderter Reibbeiwert

$v \quad$ Querdehnzahl

$\rho_{f} \quad$ Rohdichte

$\sigma \quad$ Normalspannung

$\tau \quad$ Schubspannung

$\omega \quad$ Eigenkreisfrequenz

$\varphi \quad$ Reibungswinkel, Verdrehung, Eigenvektor 


\section{Anhang}

\section{A.1 Zeitverläufe und Antwortspektren}

Nachfolgend sind die Bodenbeschleunigungszeitverläufe ZV1 $\div$ ZV4 mit den zugehörigen elastischen Antwortspektren für eine Dämpfung von 5\% angegeben. Zusätzlich sind das ZielAntwortspektrum nach E DIN 4149 (2002-10) [22] sowie das Husid-Diagramm angegeben.

\section{Zeitverlauf ZV1 - Boden-Baugrund-Kombination B3}

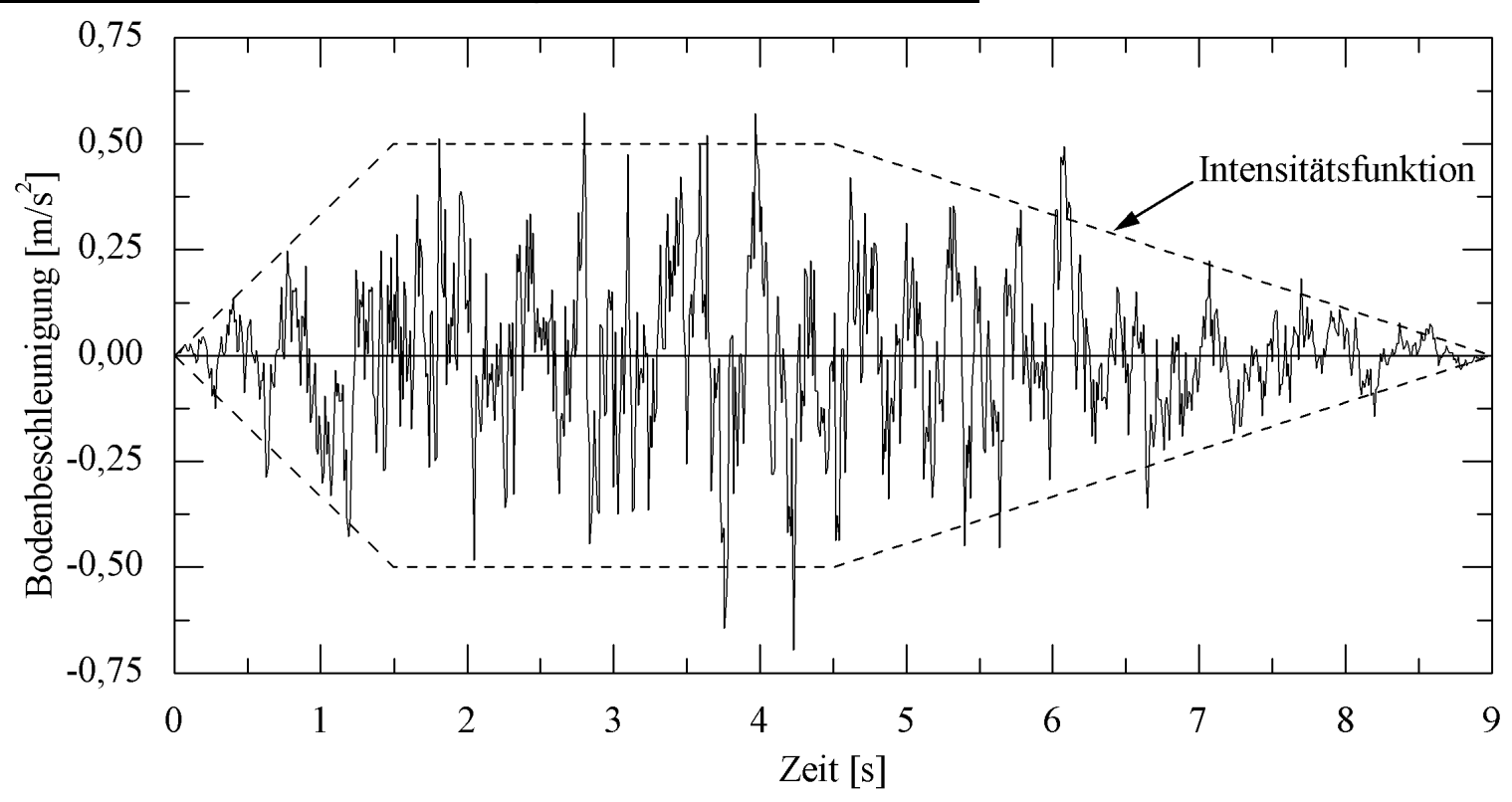

Abbildung A-1: Bodenbeschleunigungszeitverlauf ZV1 für die Boden-Baugrund-Kombination B3 $(S=1,25)$ mit einem Grundwert der Bodenbeschleunigung $a_{g}=0,4 \mathrm{~m} / \mathrm{s}^{2}$
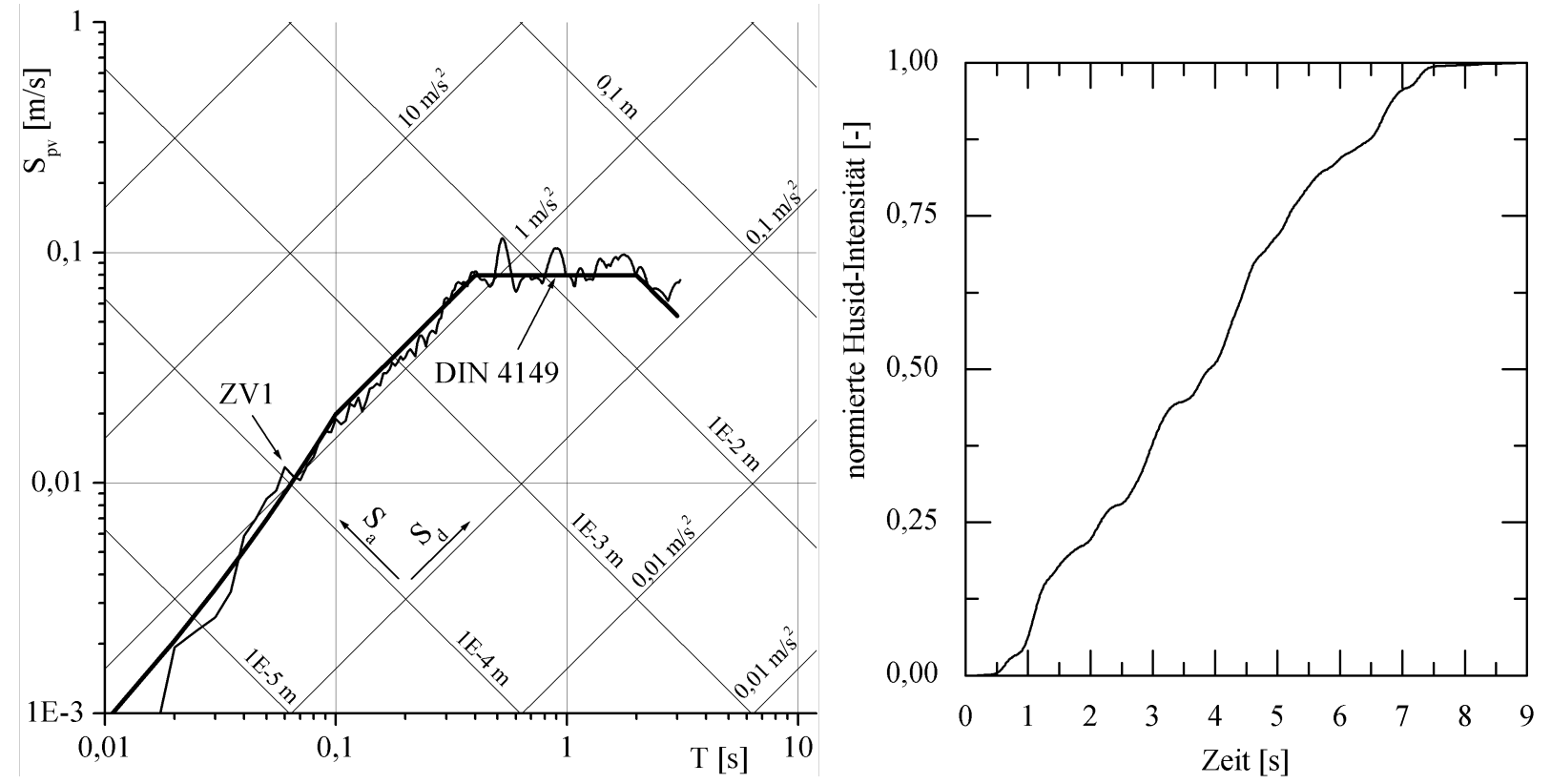

Abbildung A-2: Antwortspektrum mit Ziel-Spektrum und Husid-Diagramm des Zeitverlaufes ZV1 


\section{Zeitverlauf ZV2 - Boden-Baugrund-Kombination A3}

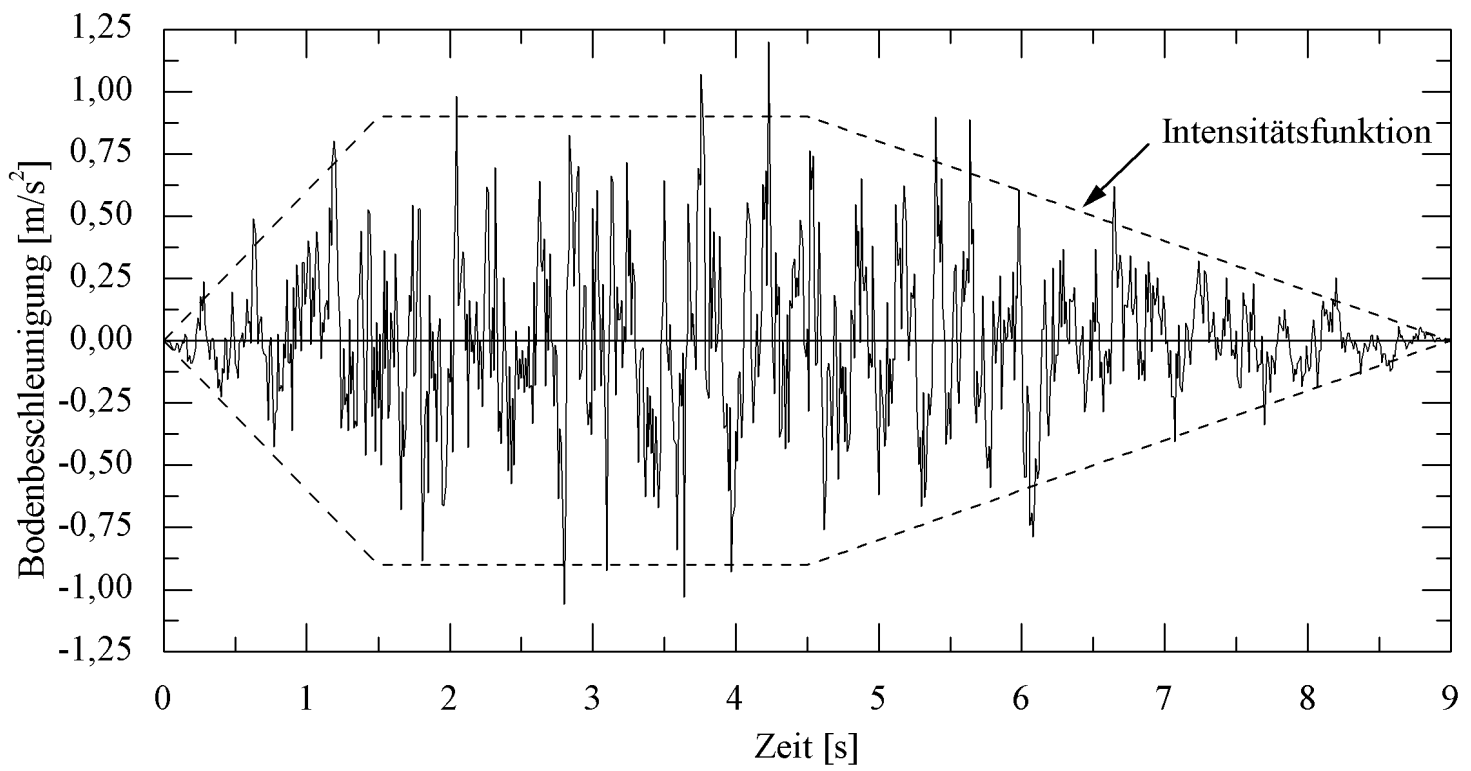

Abbildung A-3: Bodenbeschleunigungszeitverlauf ZV2 für die Boden-Baugrund-Kombination A3 (S=1,5) mit einem Grundwert der Bodenbeschleunigung $a_{g}=0,6 \mathrm{~m} / \mathrm{s}^{2}$
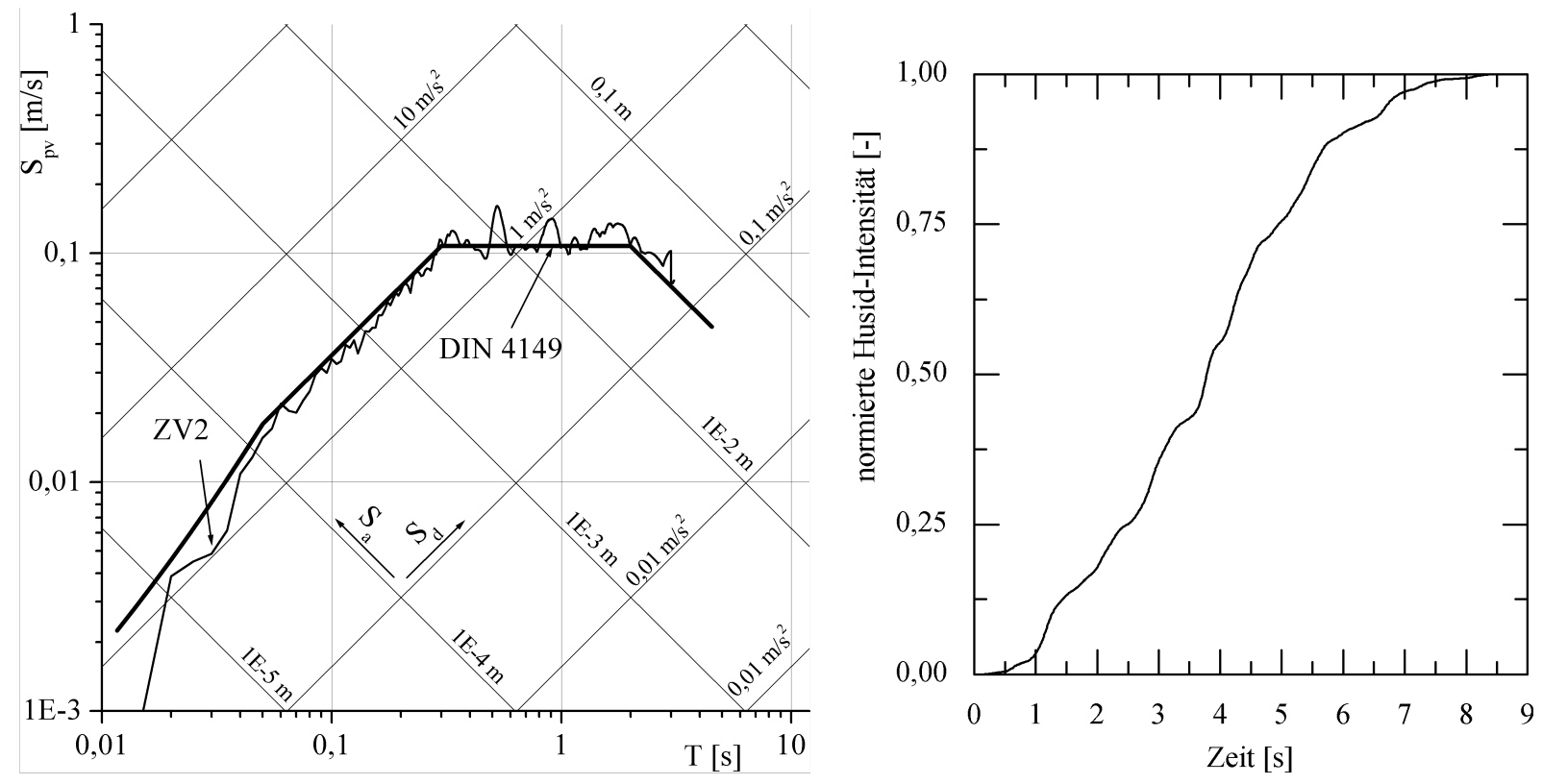

Abbildung A-4: Antwortspektrum mit Ziel-Spektrum und Husid-Diagramm des Zeitverlaufes ZV2 


\section{$\underline{\text { Zeitverlauf ZV3 - Boden-Baugrund-Kombination A1 }}$}

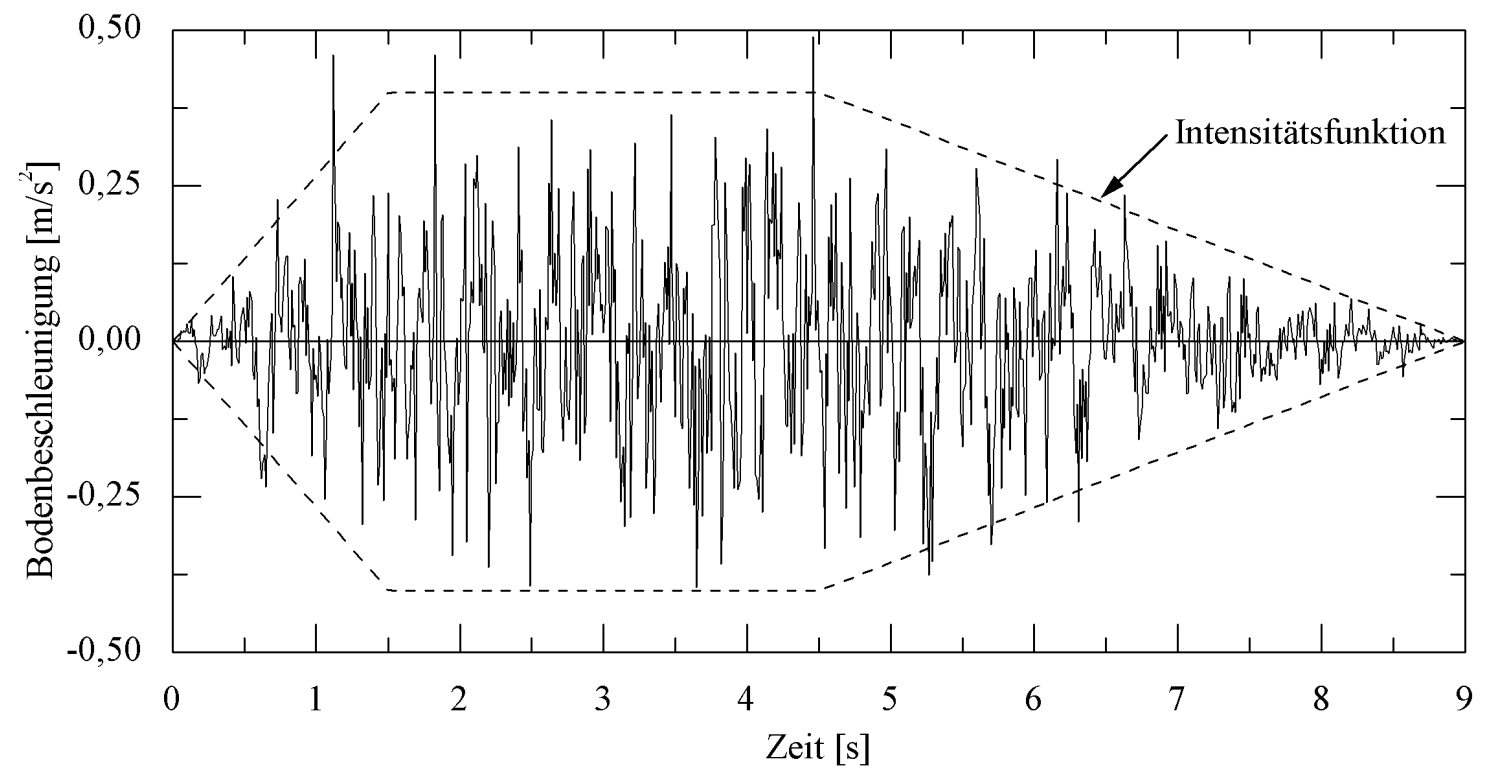

Abbildung A-5: Bodenbeschleunigungszeitverlauf ZV3 für die Boden-Baugrund-Kombination A1 (S=1,0) mit einem Grundwert der Bodenbeschleunigung $a_{g}=0,4 \mathrm{~m} / \mathrm{s}^{2}$
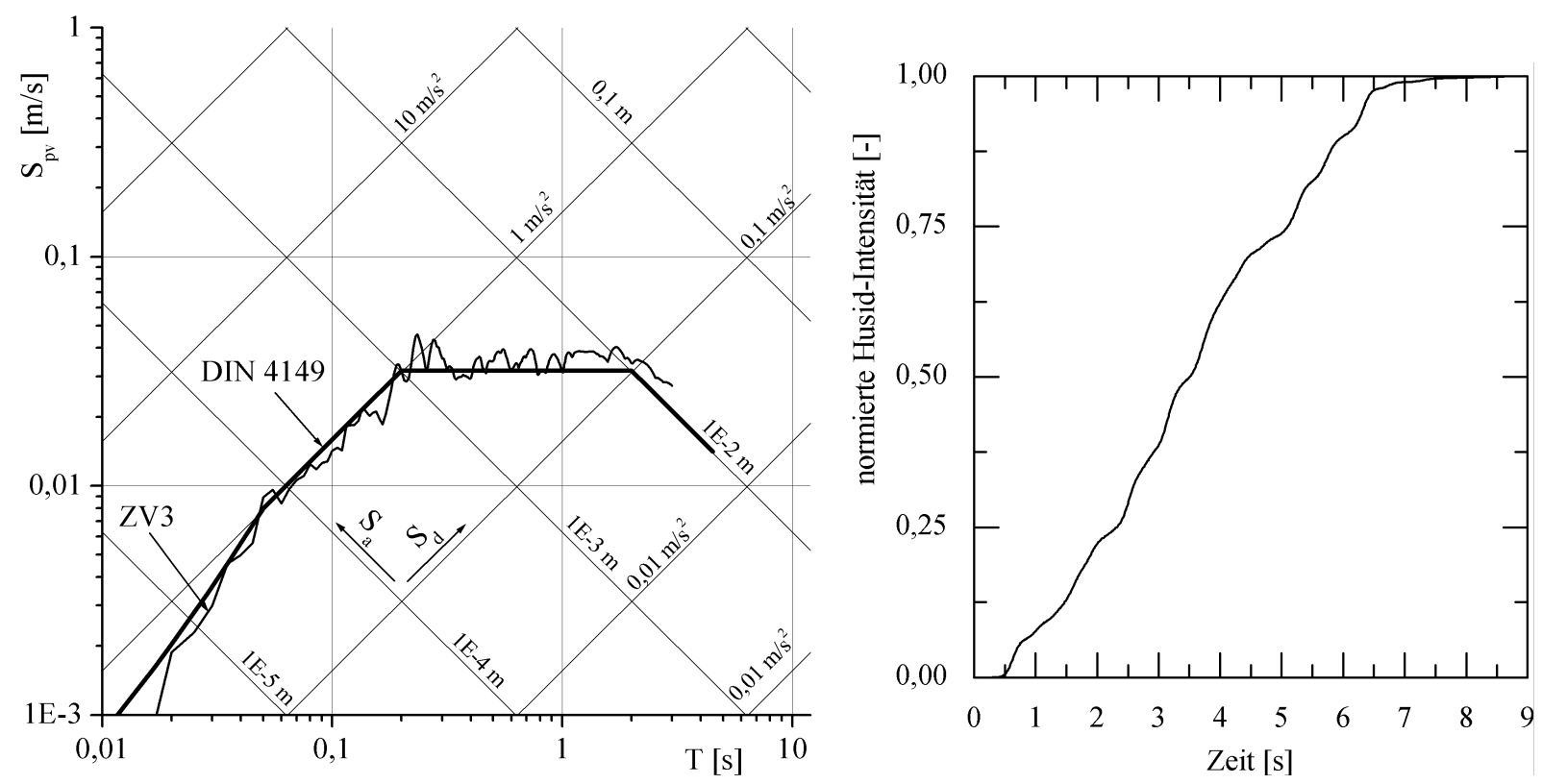

Abbildung A-6: Antwortspektrum mit Ziel-Spektrum und Husid-Diagramm des Zeitverlaufes ZV3 


\section{Zeitverlauf ZV4 - Boden-Baugrund-Kombination C3}

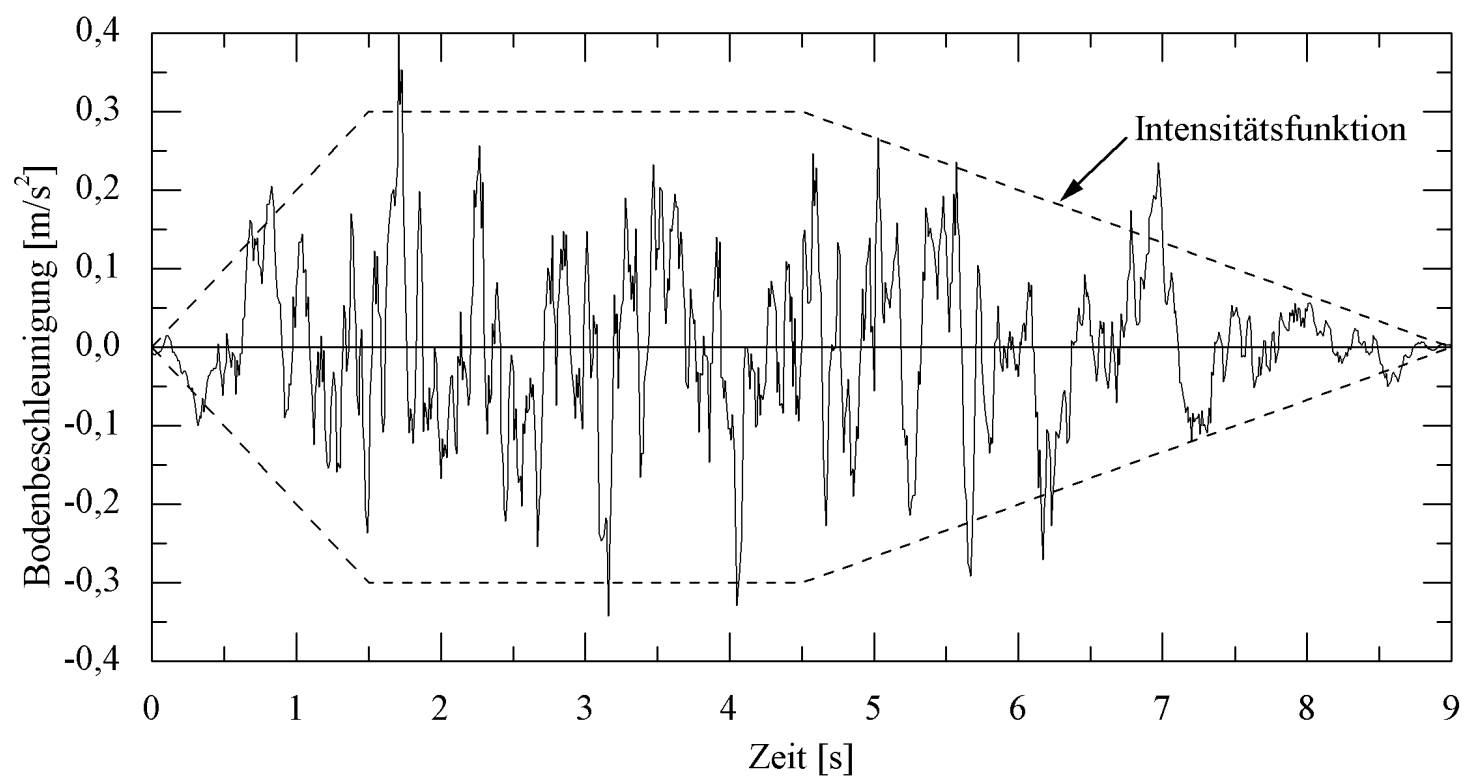

Abbildung A-7: Bodenbeschleunigungszeitverlauf ZV4 für die Boden-Baugrund-Kombination C3 $(\mathrm{S}=0,75)$ mit einem Grundwert der Bodenbeschleunigung $a_{g}=0,4 \mathrm{~m} / \mathrm{s}^{2}$
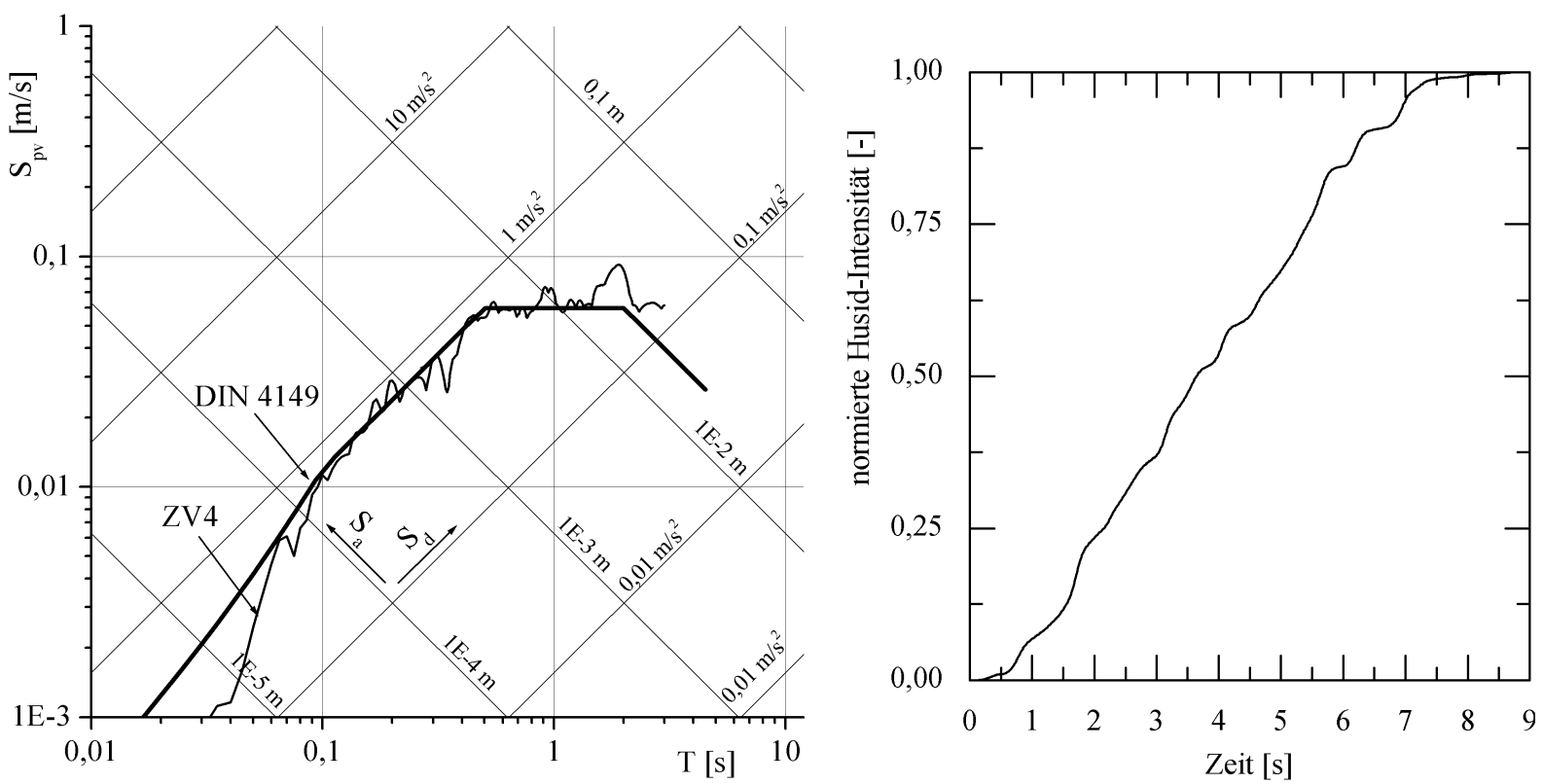

Abbildung A-8: Antwortspektrum mit Ziel-Spektrum und Husid-Diagramm des Zeitverlaufes ZV4 


\section{A.2 Rissbilder}

\section{A.2.1 Erste Simulationsstufe}

Nachfolgend werden die Rissbilder aus der ersten Simulationsstufe der Wände $A \div C$ nach Abschluss der Versuche dargestellt.

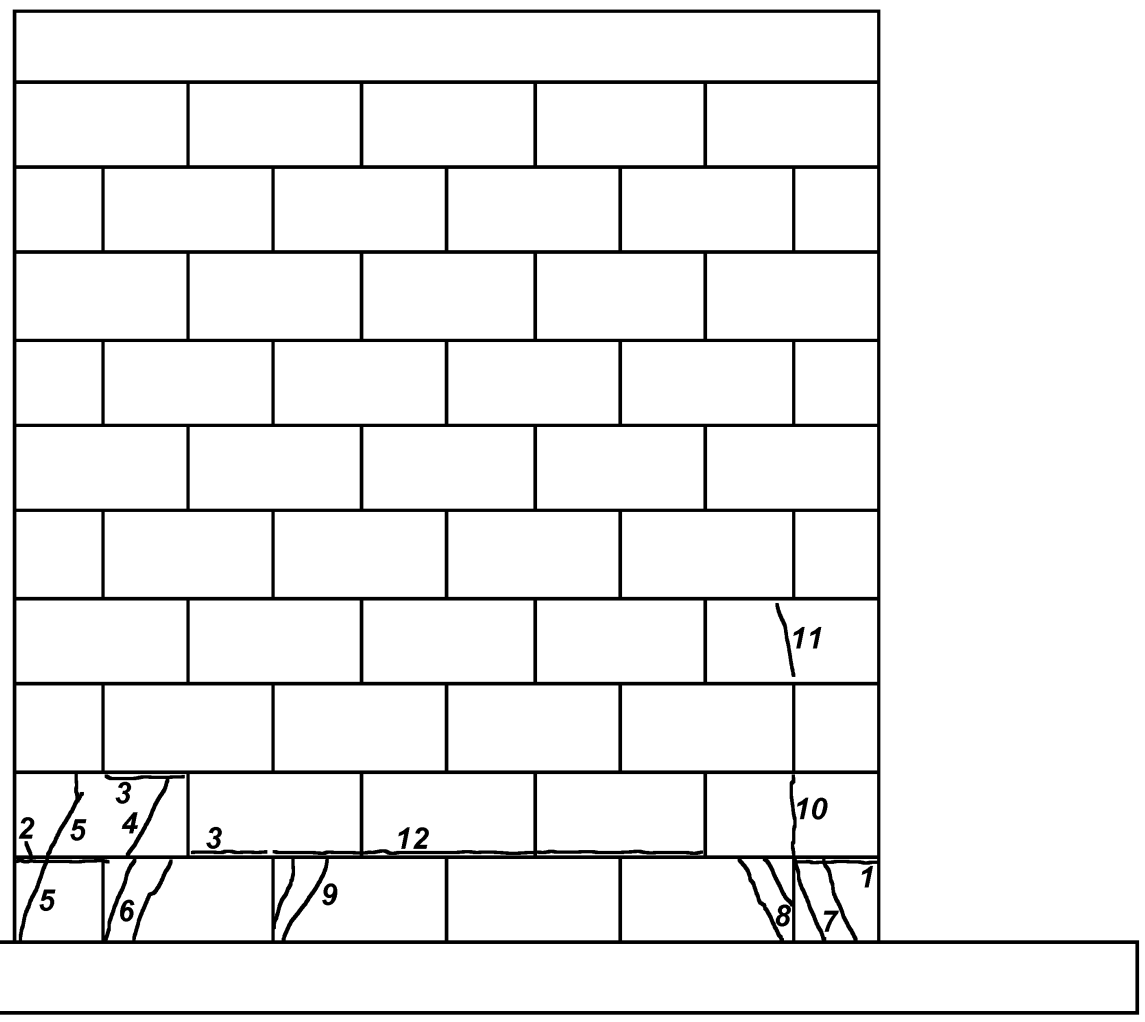

Abbildung A-9: Rissbild Wand A - Vorderseite (Rückseite analog) 


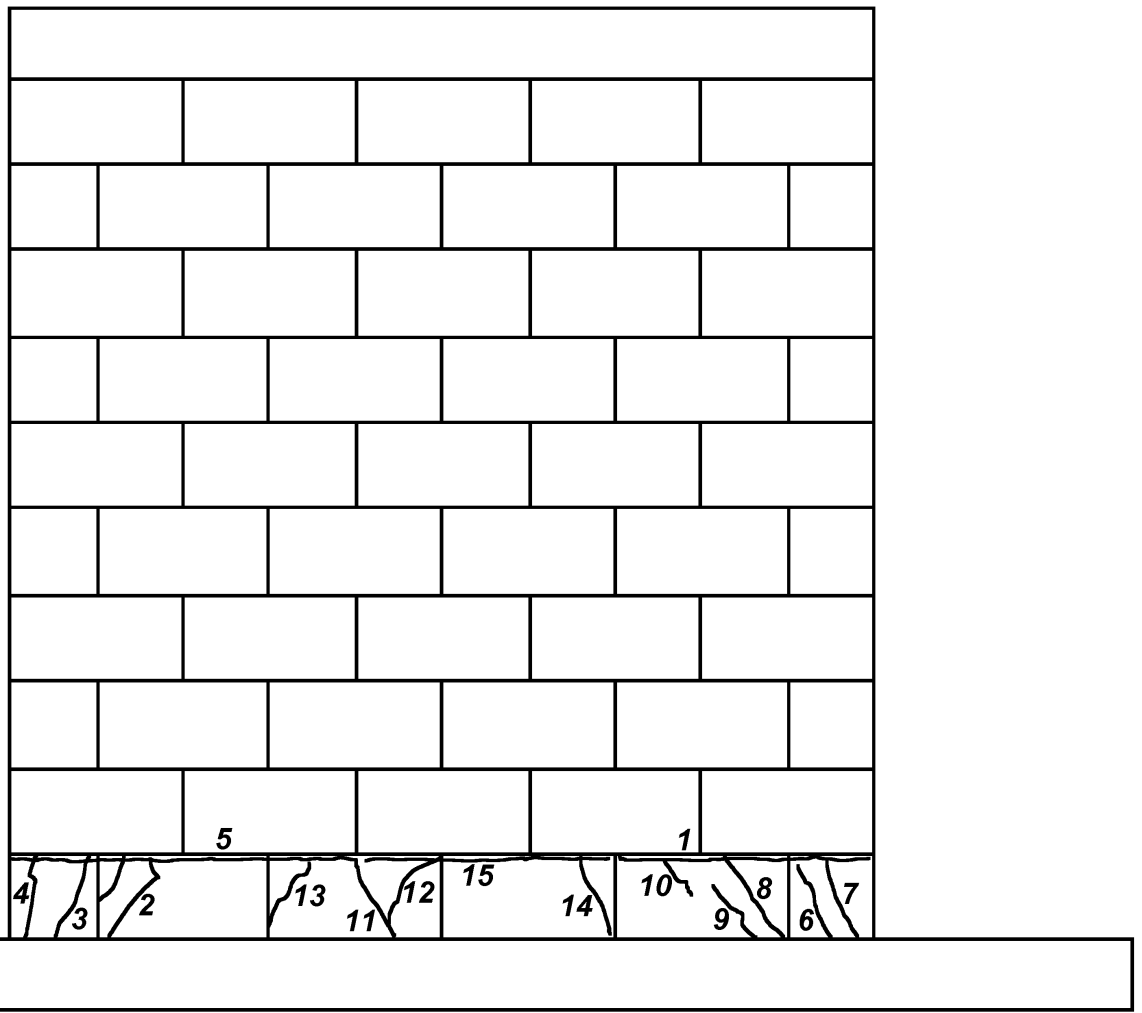

Abbildung A-10: Rissbild Wand B - Vorderseite (Rückseite analog)

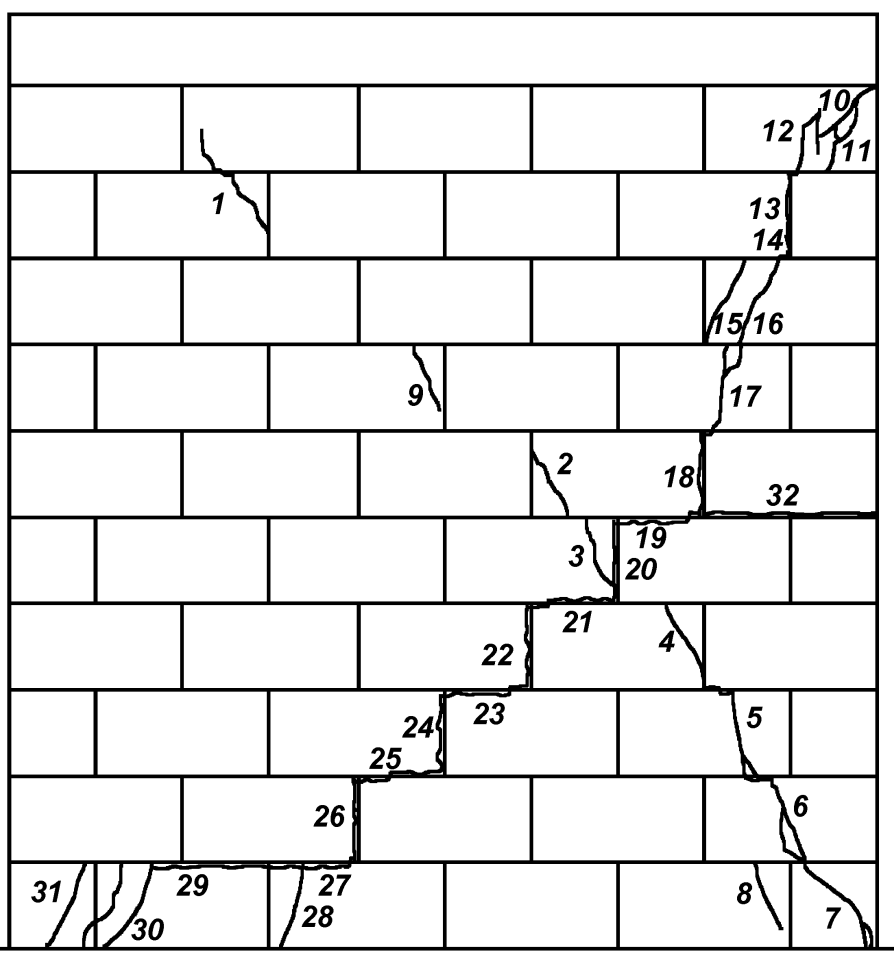

Abbildung A-11: Rissbild Wand C - Vorderseite 


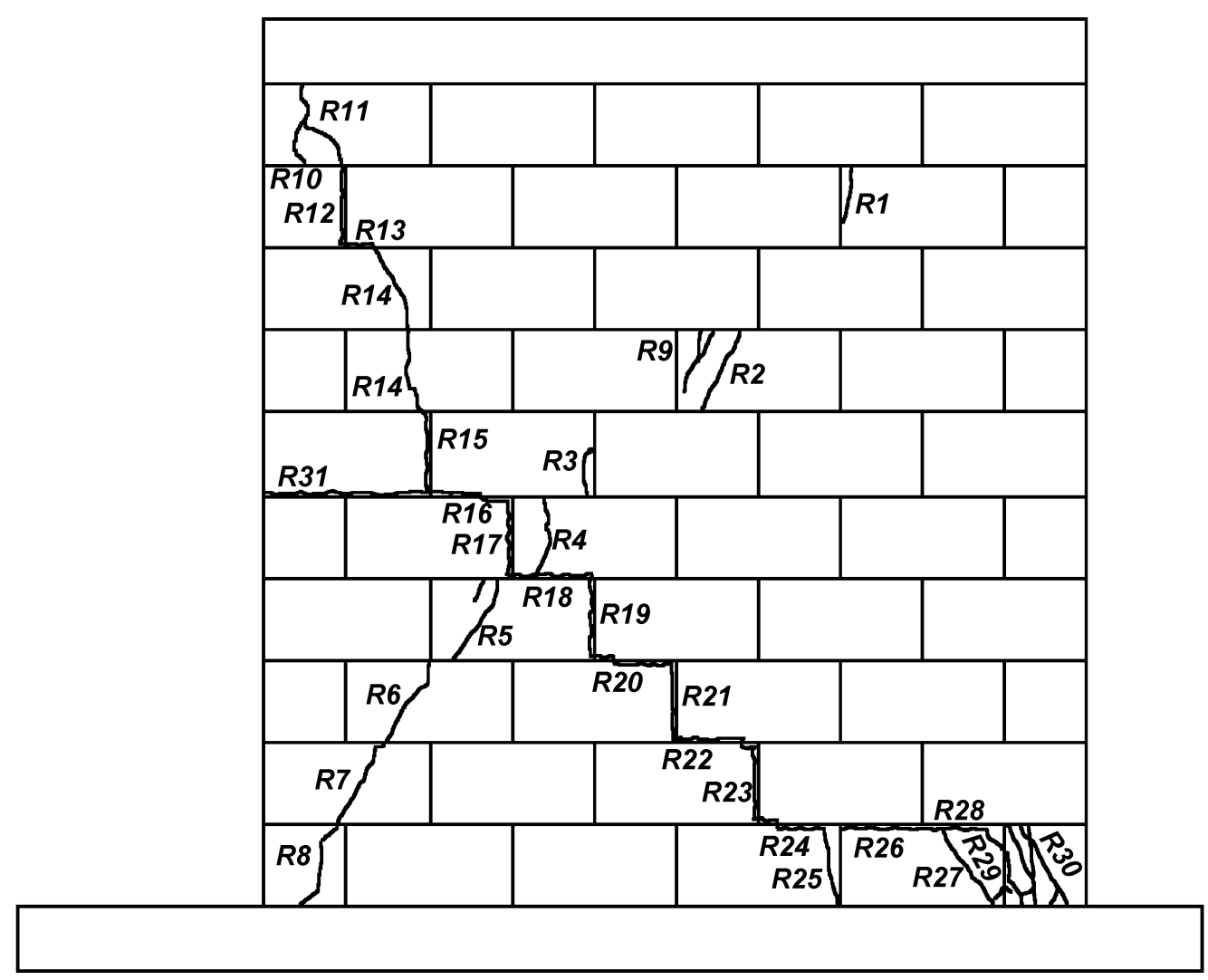

Abbildung A-12: Rissbild Wand C - Rückseite

\section{A.2.2 Zweite Simulationsstufe}

Nachfolgend werden die Rissbilder aus der zweiten Simulationsstufe der Wände D $\div$ F und KS1 $\div$ KS4 nach Abschluss der Versuche dargestellt. 


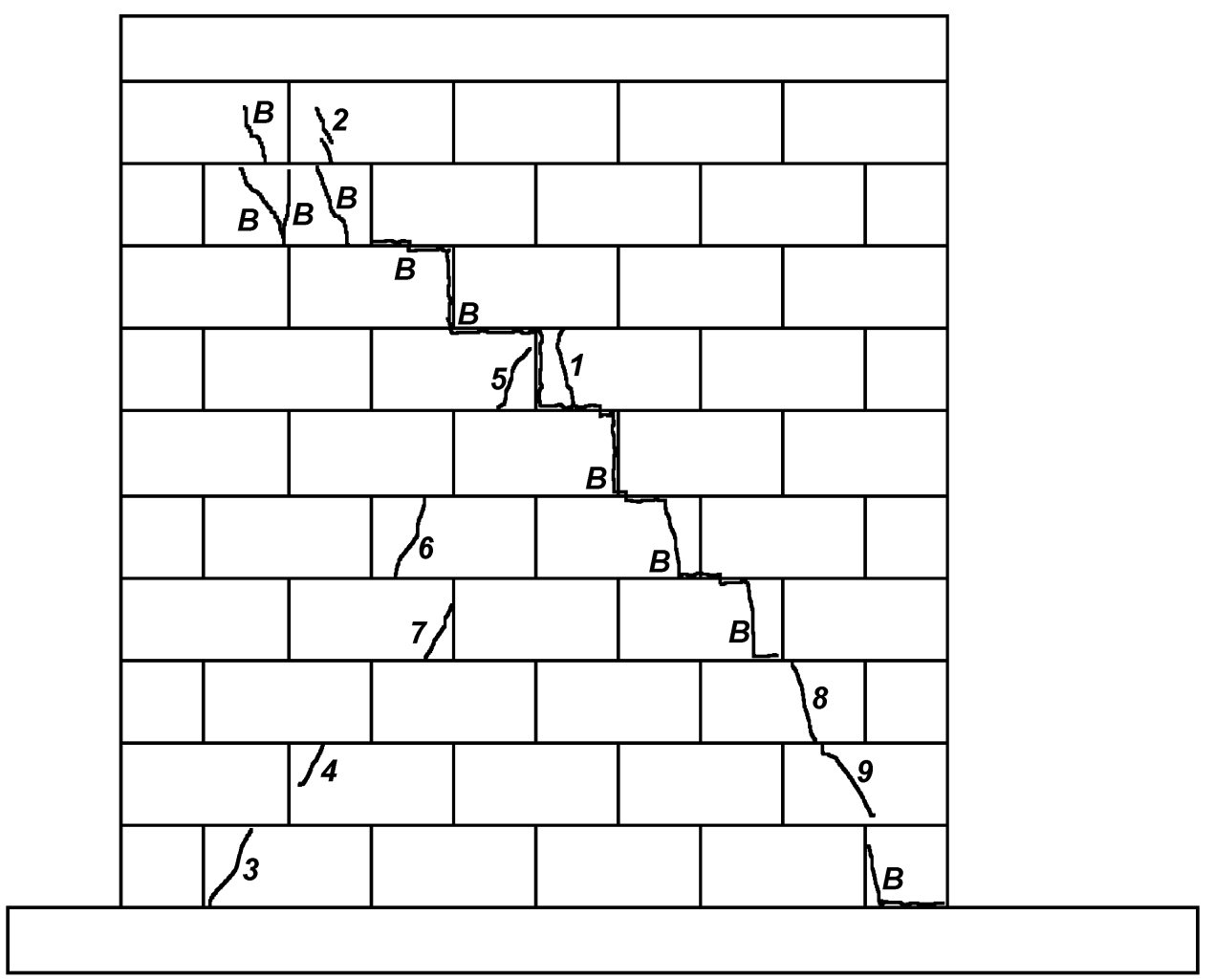

Abbildung A-13: Rissbild Wand D - Vorderseite (B: Risse beim Versagen in Versuch D5)

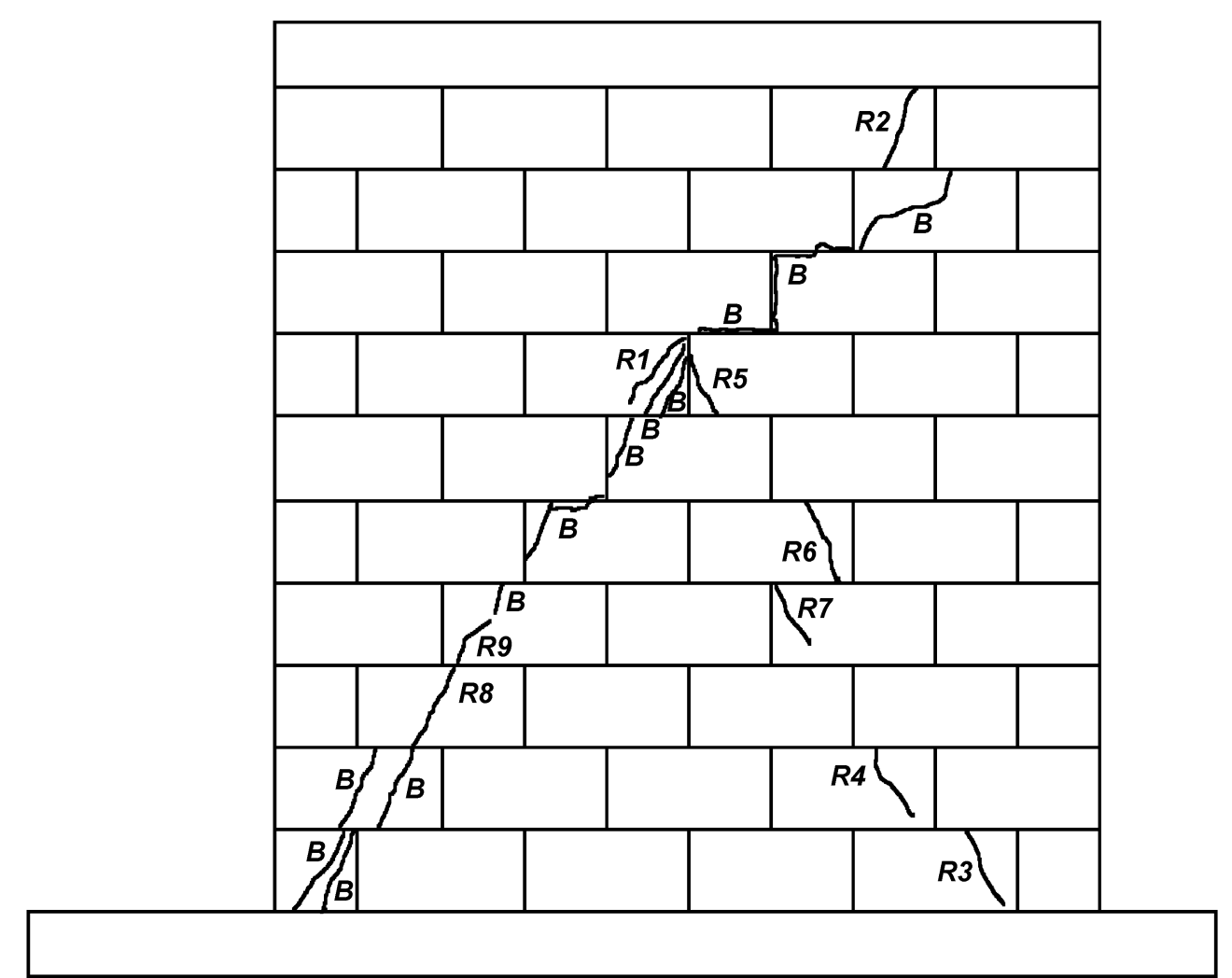

Abbildung A-14: Rissbild Wand D - Rückseite (B: Risse beim Versagen in Versuch D5) 


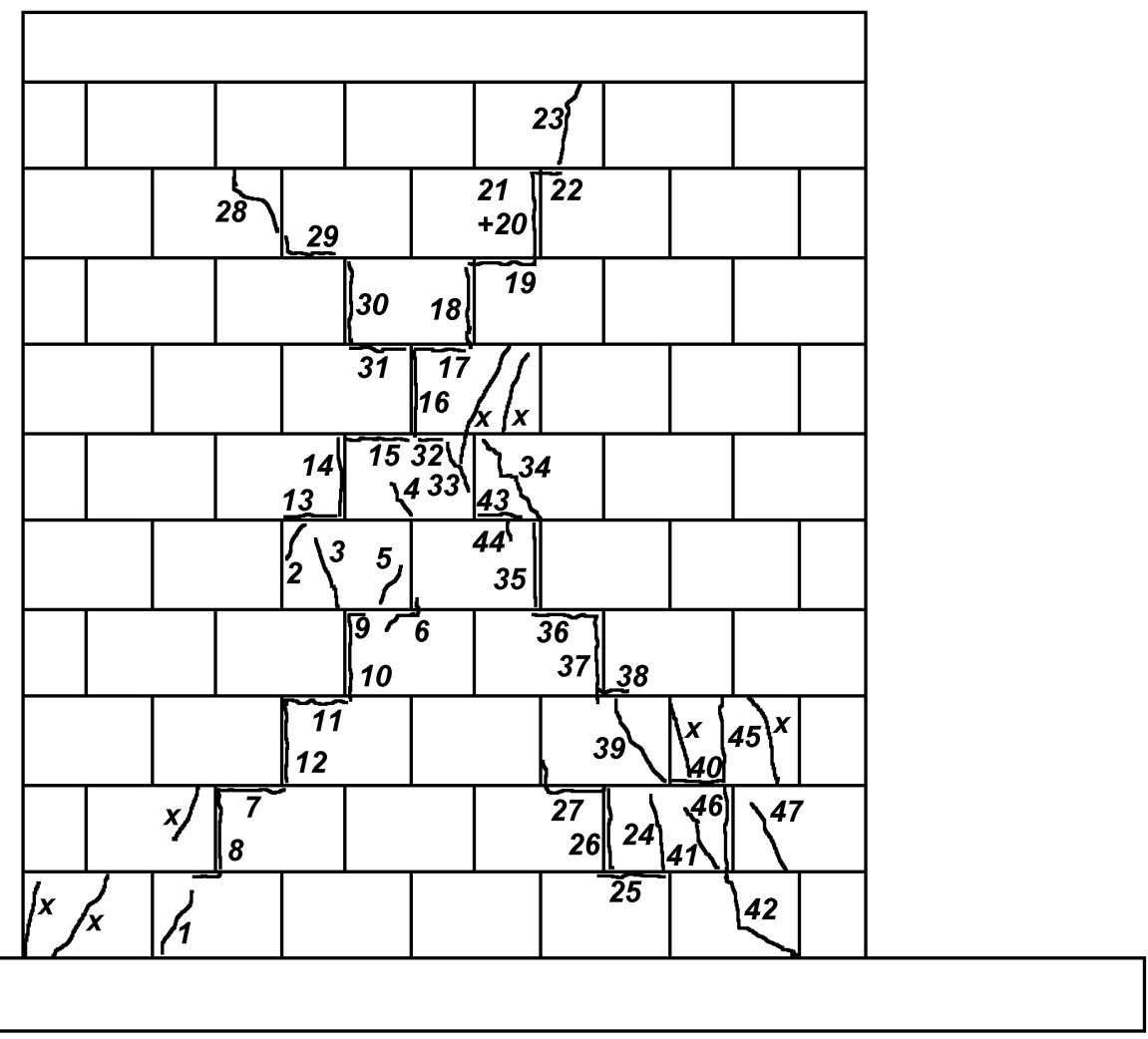

Abbildung A-15: Rissbild Wand E - Vorderseite (Rückseite analog) (X= Risse im Nachversuch)

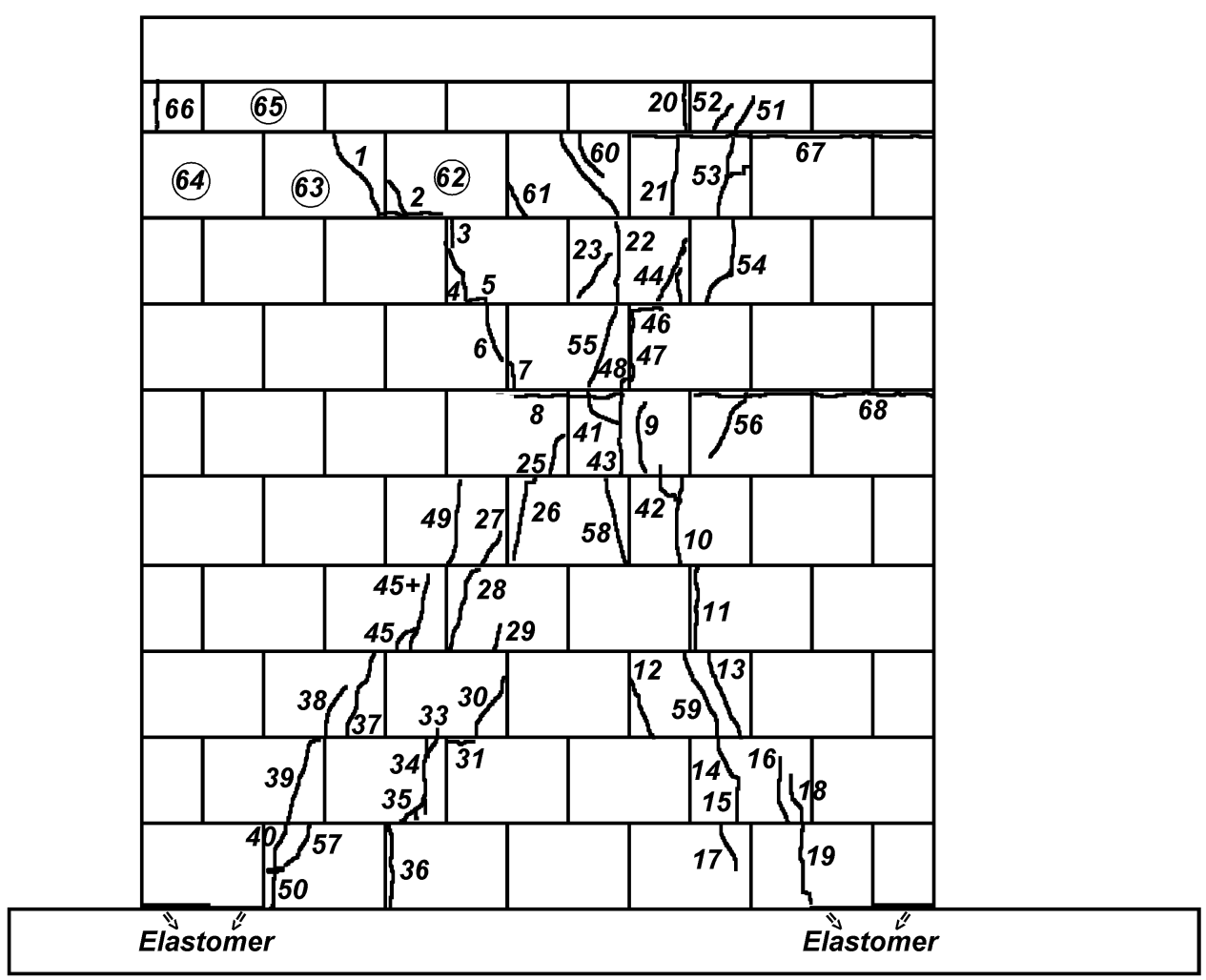

Abbildung A-16: Rissbild Wand F - Vorderseite (Rückseite analog) ( $X$ = totales Steinversagen) 


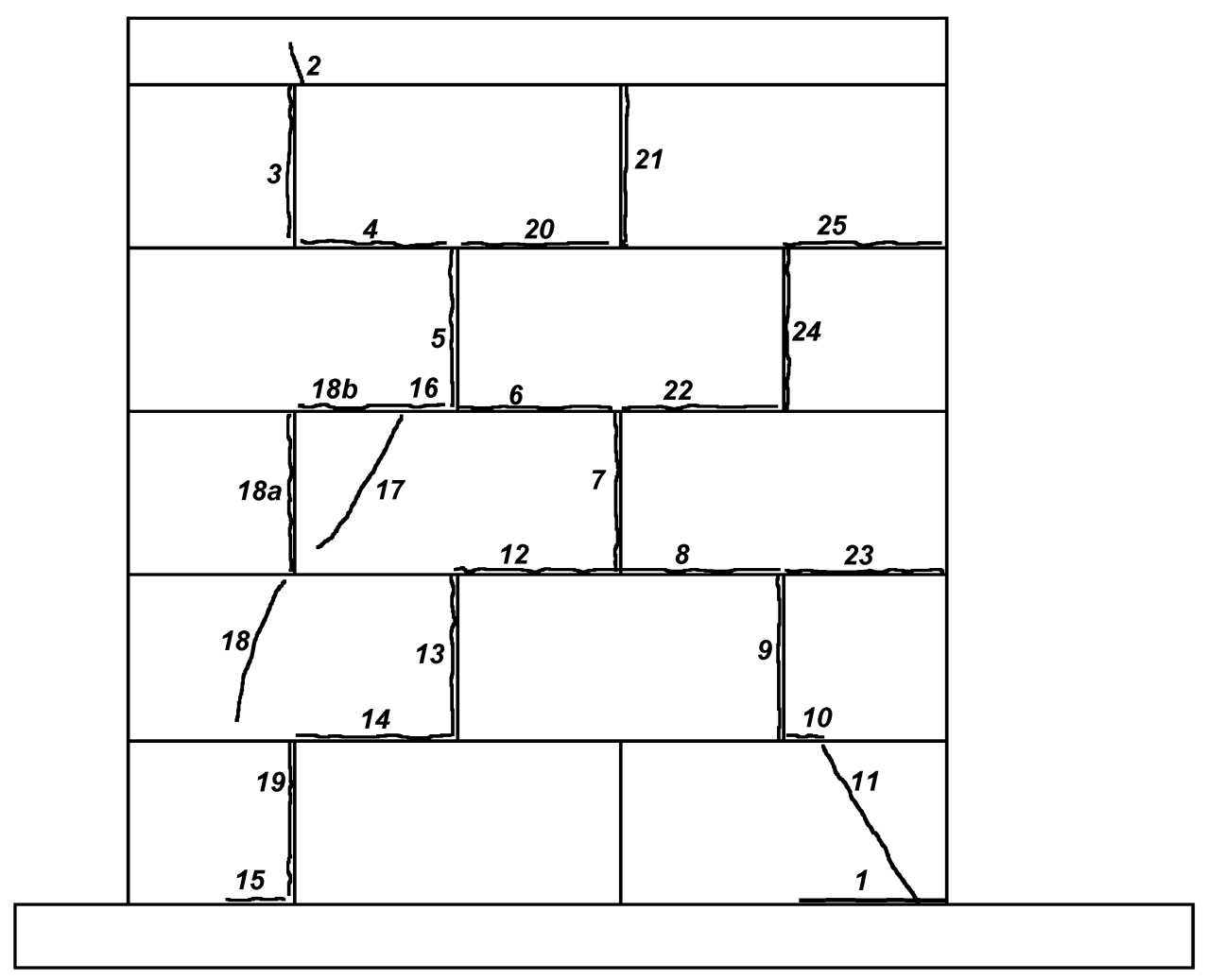

Abbildung A-17: Rissbild Wand KS 1 - Vorderseite

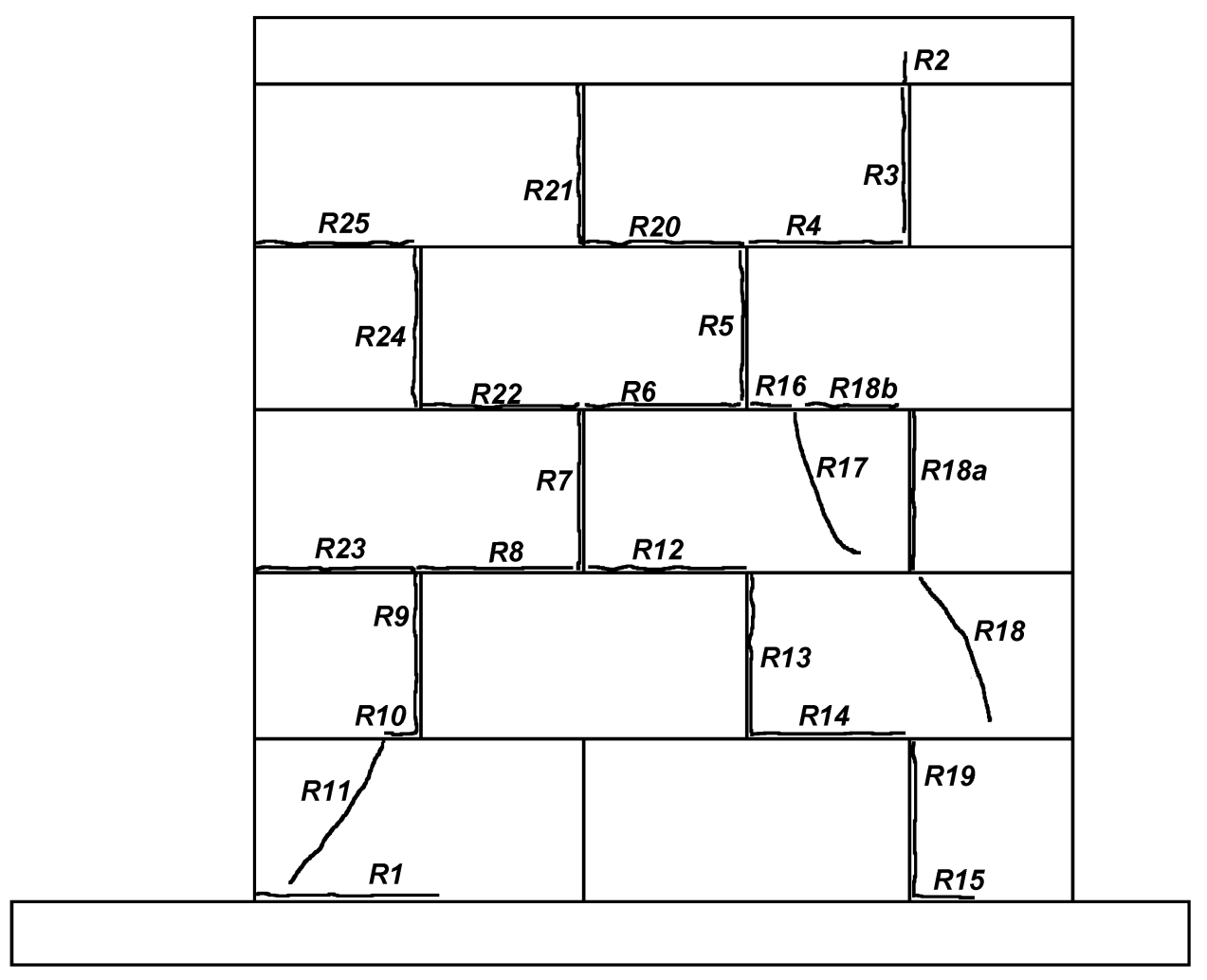

Abbildung A-18: Rissbild Wand KS 1 - Rückseite 


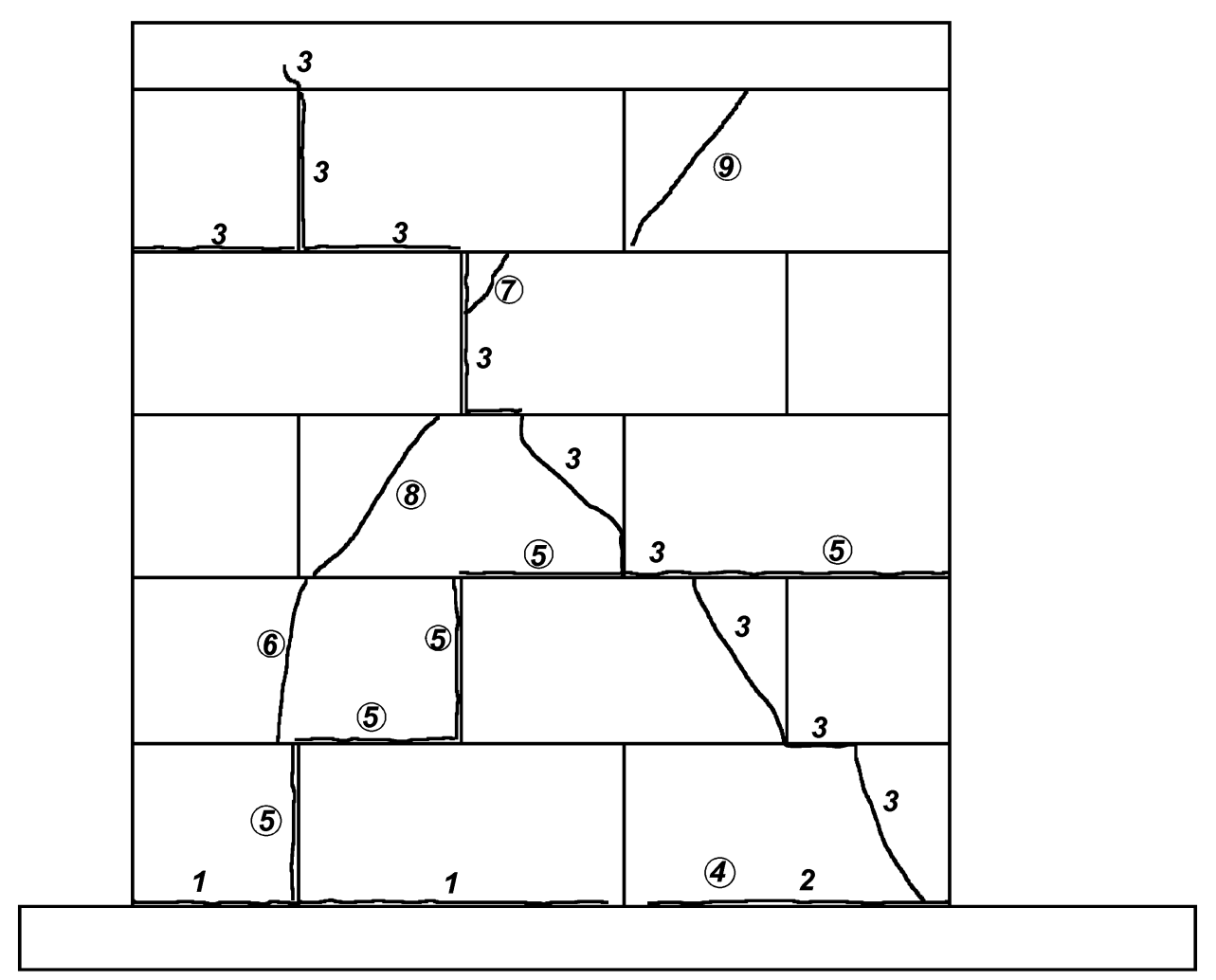

Abbildung A-19: Rissbild Wand KS 2 - Vorderseite (X = Risse im Nachversuch)

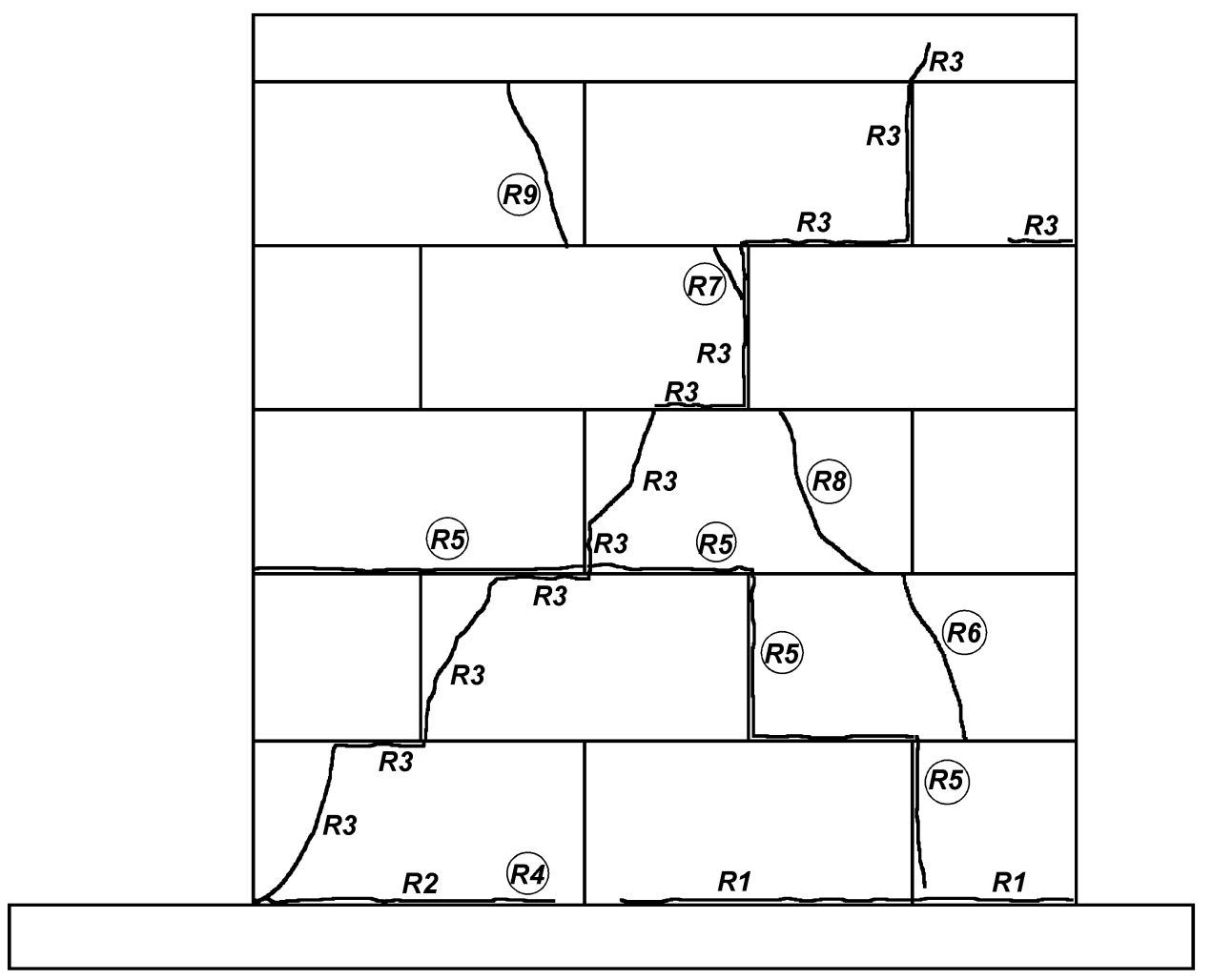

Abbildung A-20: Rissbild Wand KS 2 - Rückseite (X = Risse im Nachversuch) 


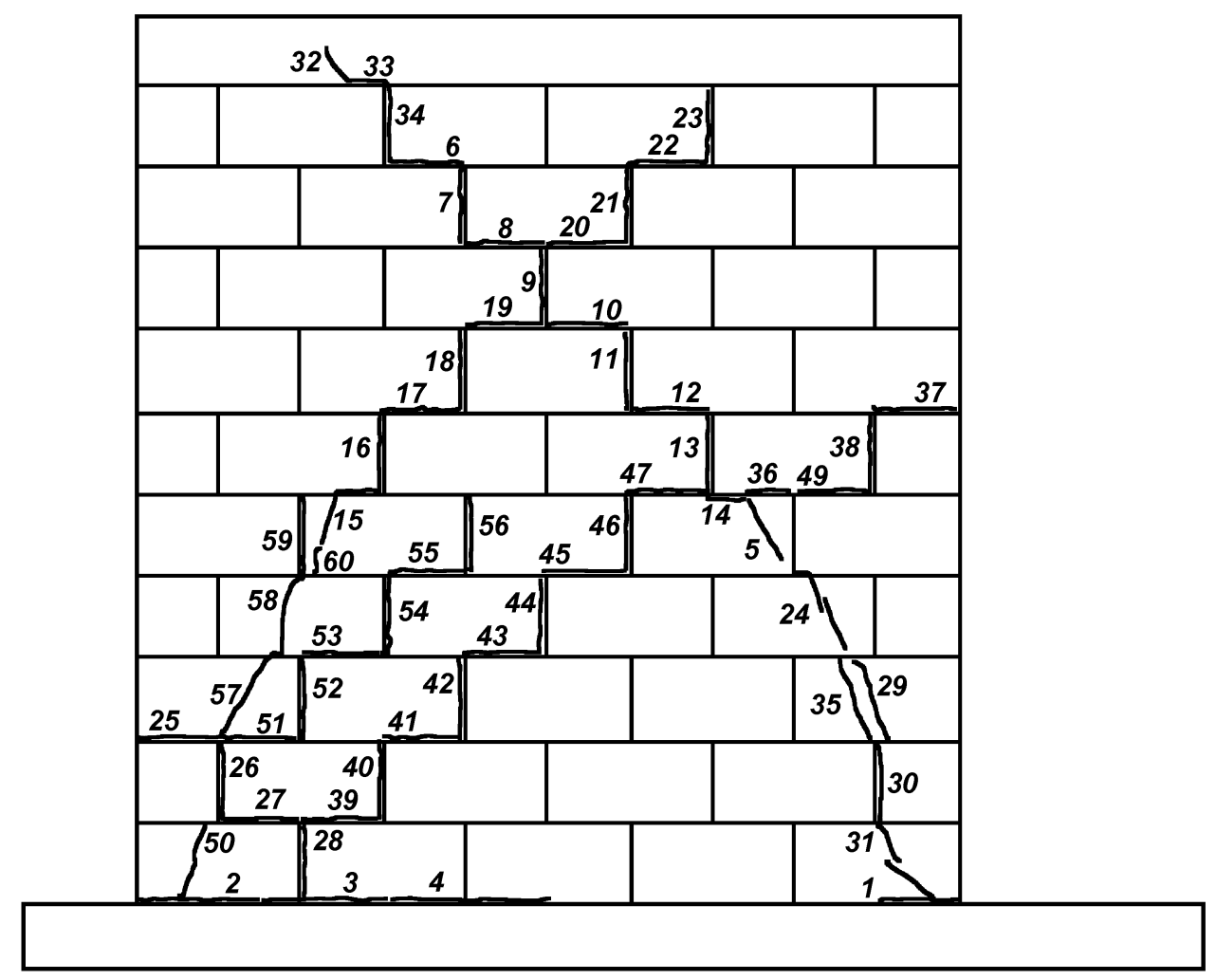

Abbildung A-21: Rissbild Wand KS 3 - Vorderseite (Rückseite analog)

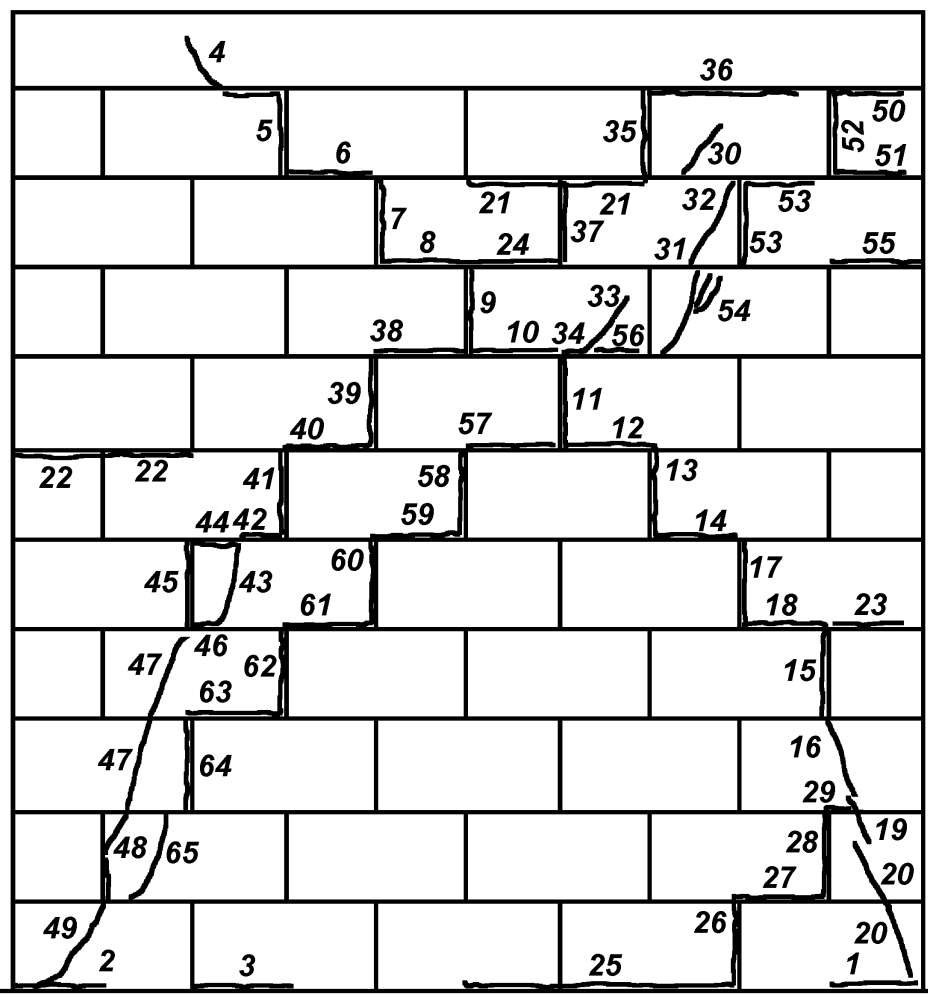

Abbildung A-22: Rissbild Wand KS 4 - Vorderseite (Rückseite analog) 


\section{A.3 Kraft-Verformungs-Beziehungen}

\section{A.3.1 Erste Simulationsstufe}

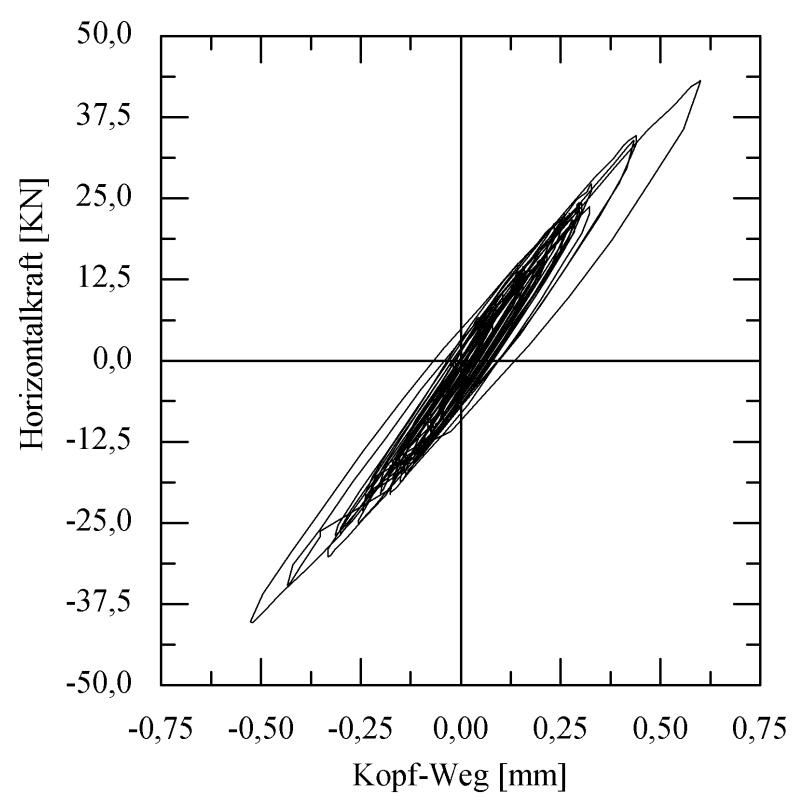

Abbildung A-23:Kraft-Verformungs-Beziehung Wand A, erster Hauptversuch (Versuch A1)

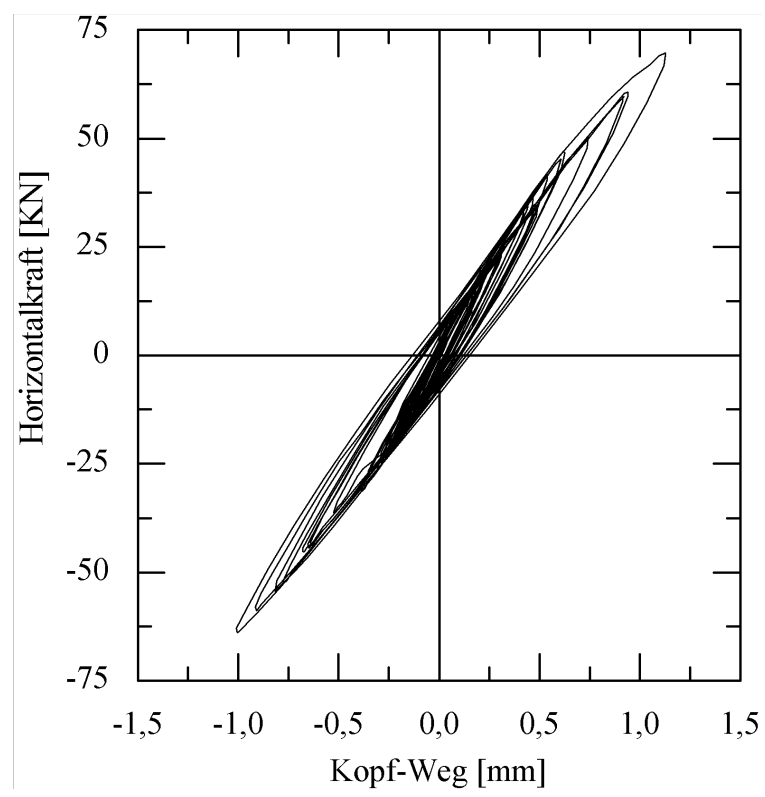

Abbildung A-24: Kraft-Verformungs-Beziehung Wand A, zweiter Hauptversuch (Versuch A2)

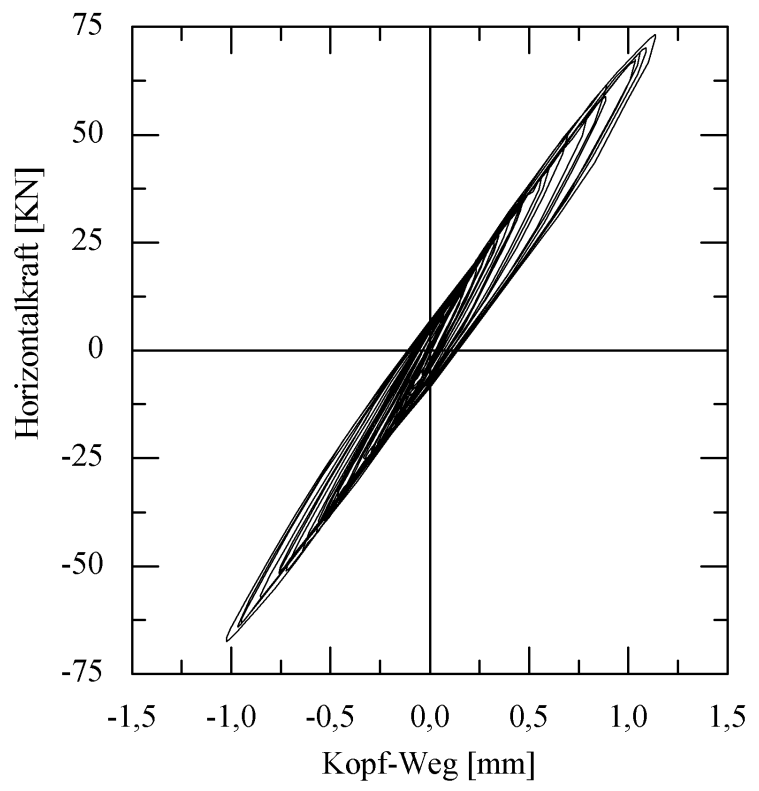

Abbildung A-25:Kraft-Verformungs-Beziehung Wand A, dritter Hauptversuch (Versuch A3)

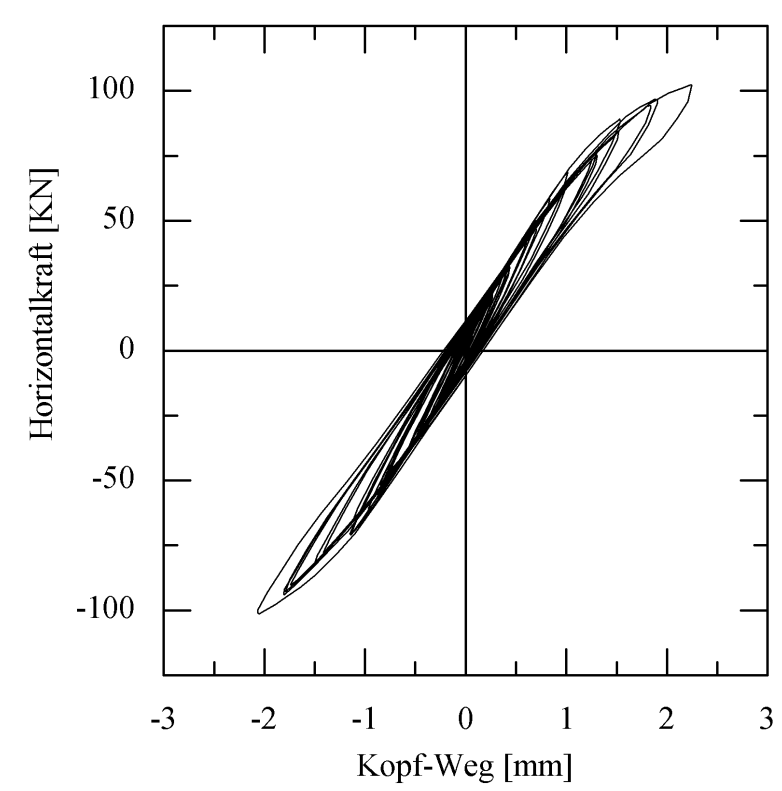

Abbildung A-26: Kraft-Verformungs-Beziehung Wand A, vierter Hauptversuch (Versuch A4) 


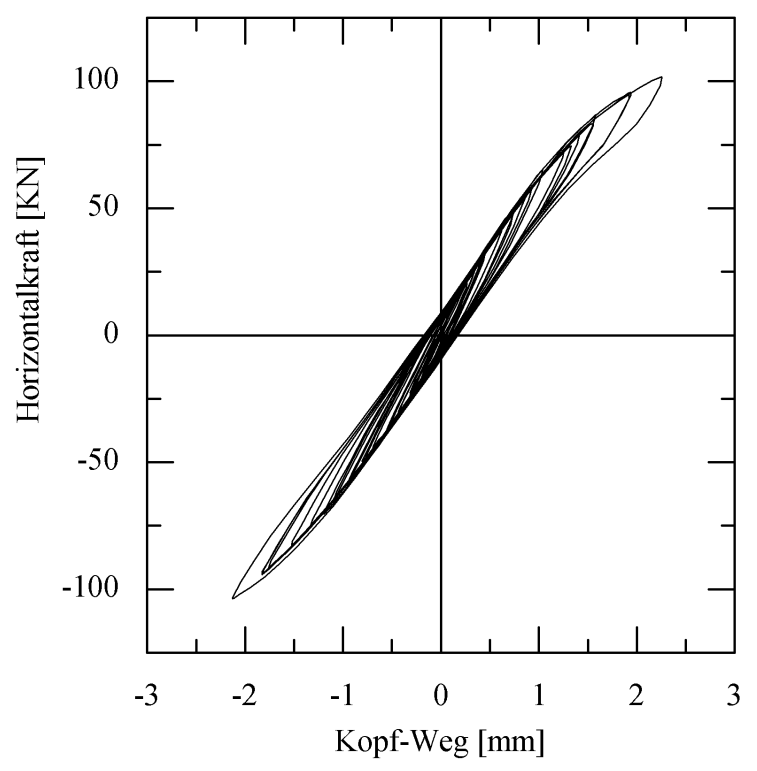

Abbildung A-27:Kraft-Verformungs-Beziehung Wand A, fünfter Hauptversuch (Versuch A5)

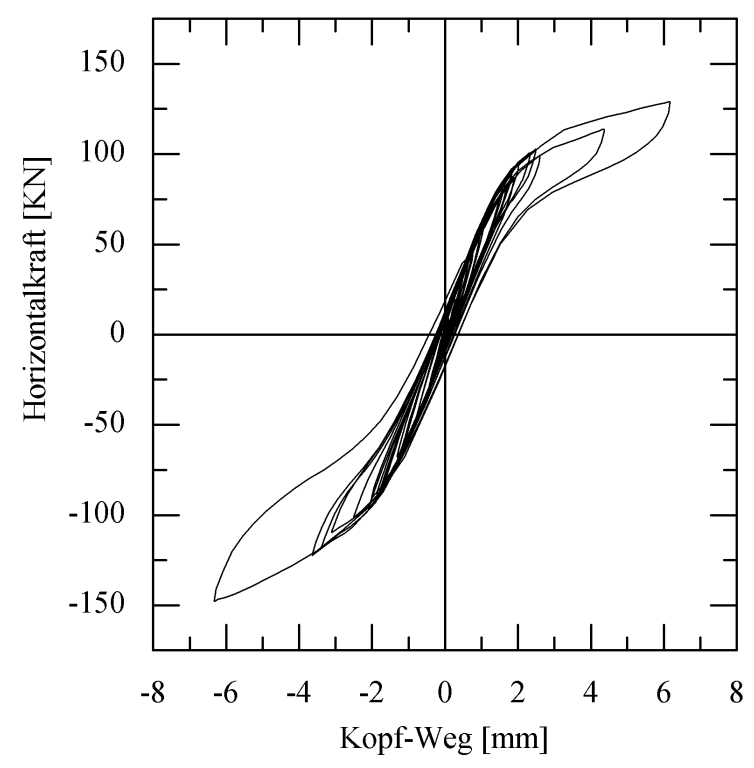

Abbildung A-29:Kraft-Verformungs-Beziehung Wand A, siebter Hauptversuch (Versuch A7)

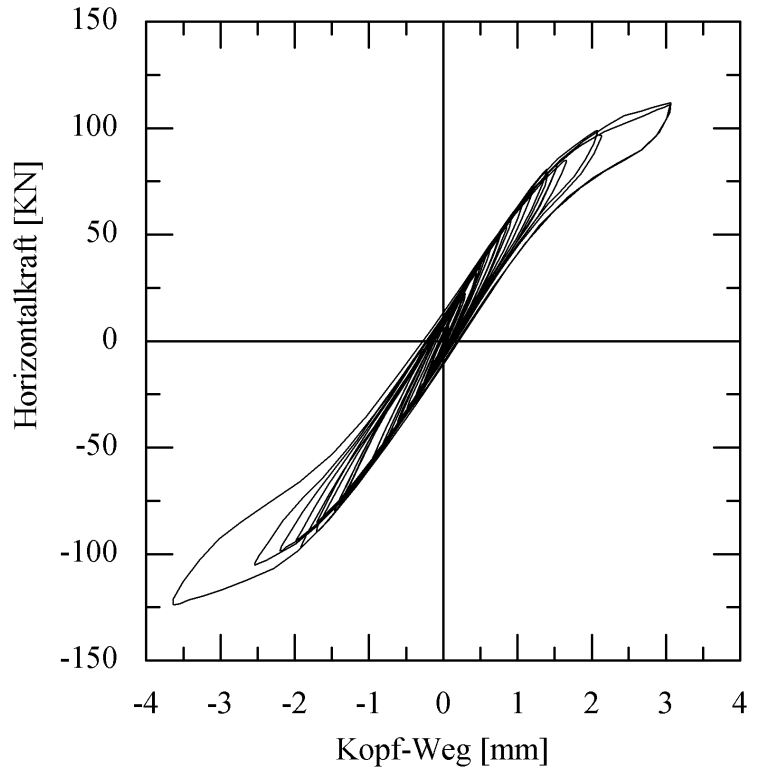

Abbildung A-28: Kraft-Verformungs-Beziehung Wand A, sechster Hauptversuch (Versuch A6)

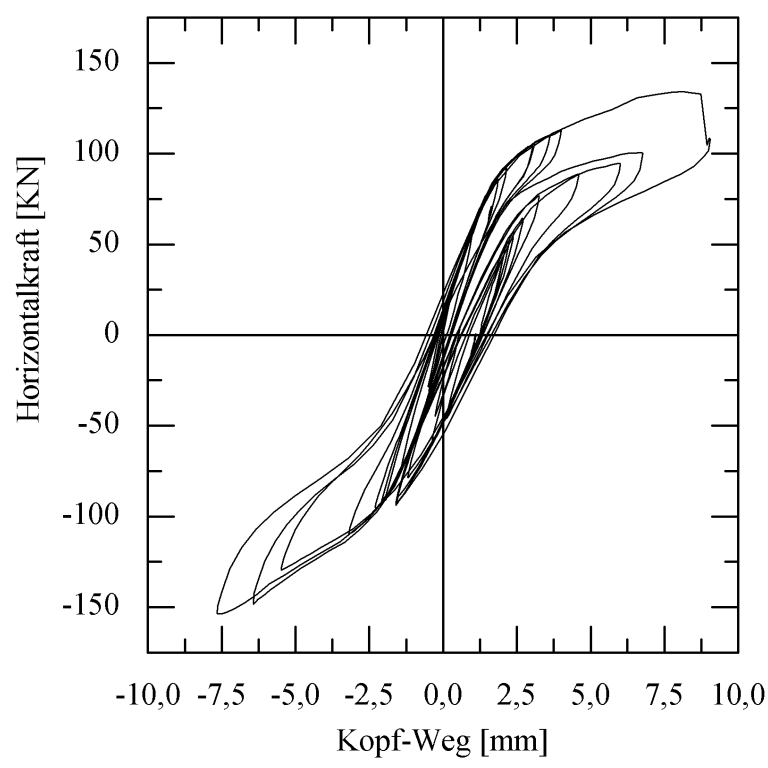

Abbildung A-30: Kraft-Verformungs-Beziehung Wand A, achter Hauptversuch (Versuch A8)

Aufgrund eines Computerausfalls konnten beim Hauptversuch von Wand B nur die Extremwerte von Horizontalkraft $(150 \mathrm{kN})$ und Kopfweg $(20 \mathrm{~mm})$ erfasst werden. 


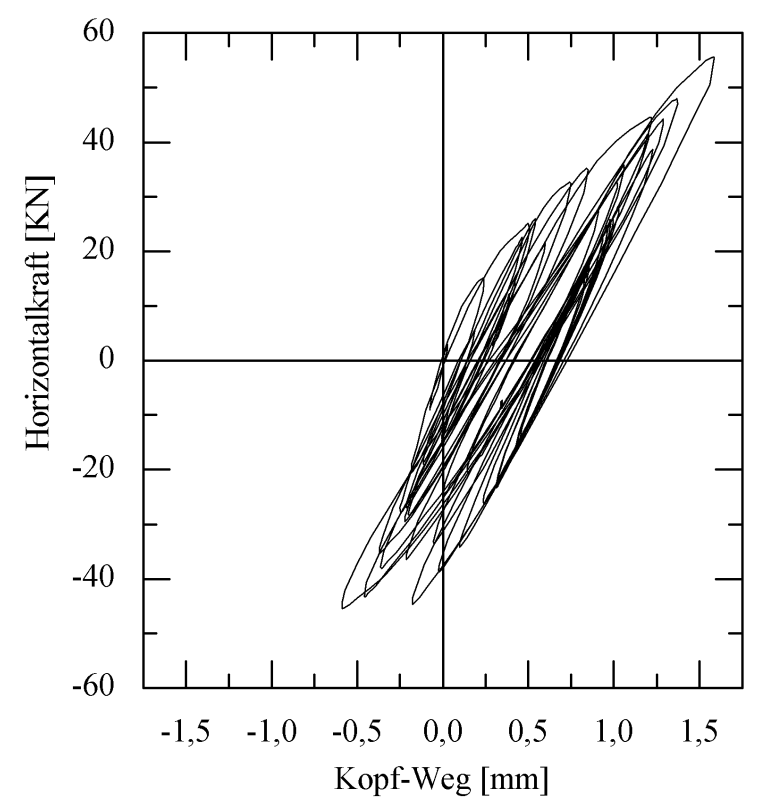

Abbildung A-31:Kraft-Verformungs-Beziehung Wand C, erster Hauptversuch (Versuch C1)

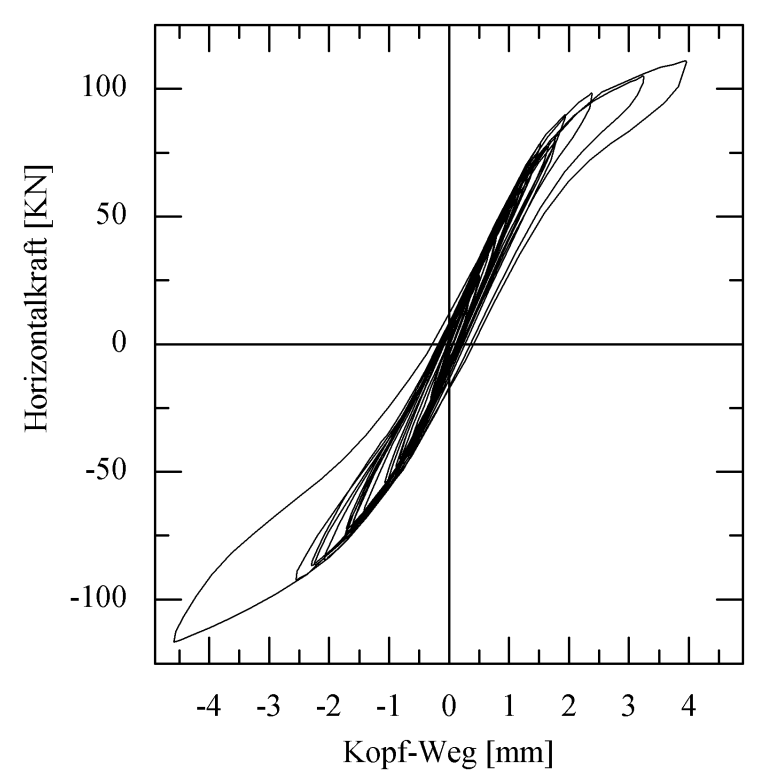

Abbildung A-33:Kraft-Verformungs-Beziehung Wand C, dritter Hauptversuch (Versuch C3)

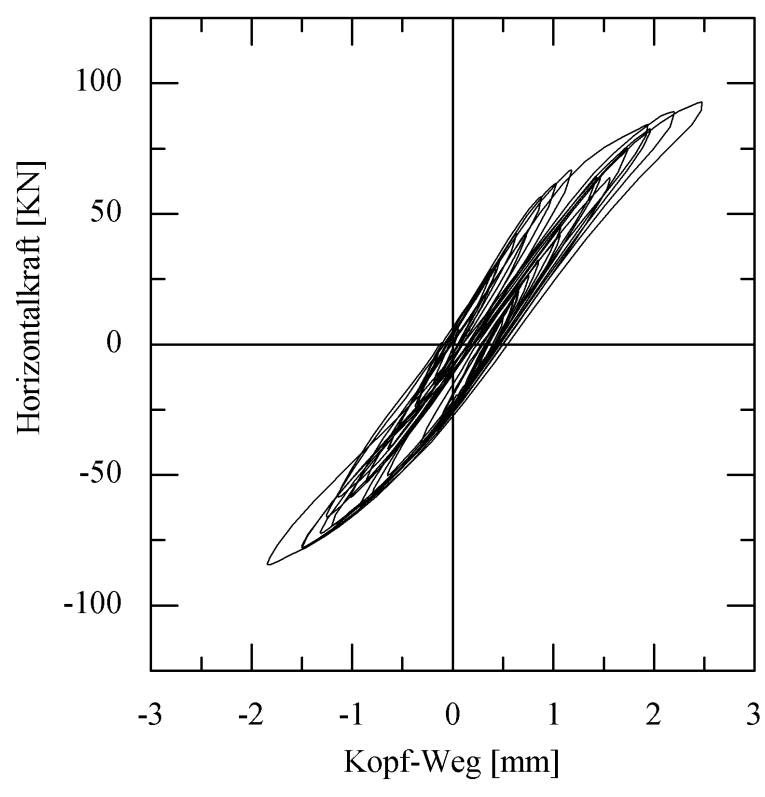

Abbildung A-32: Kraft-Verformungs-Beziehung Wand C, zweiter Hauptversuch (Versuch C2) 
A.3.2 Zweite Simulationsstufe

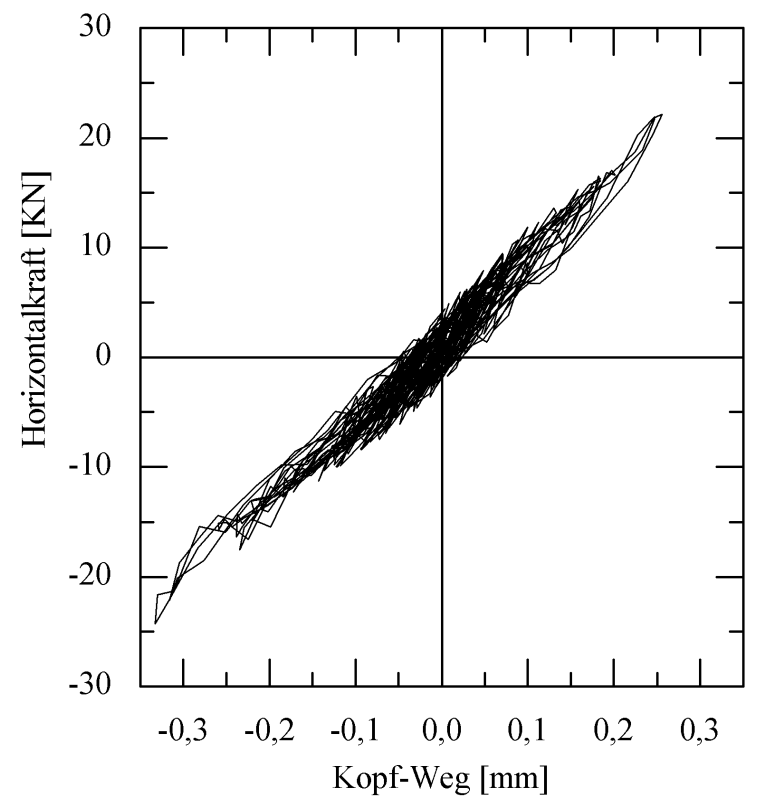

Abbildung A-34:Kraft-Verformungs-Beziehung Wand D, erster Hauptversuch (Versuch D1)

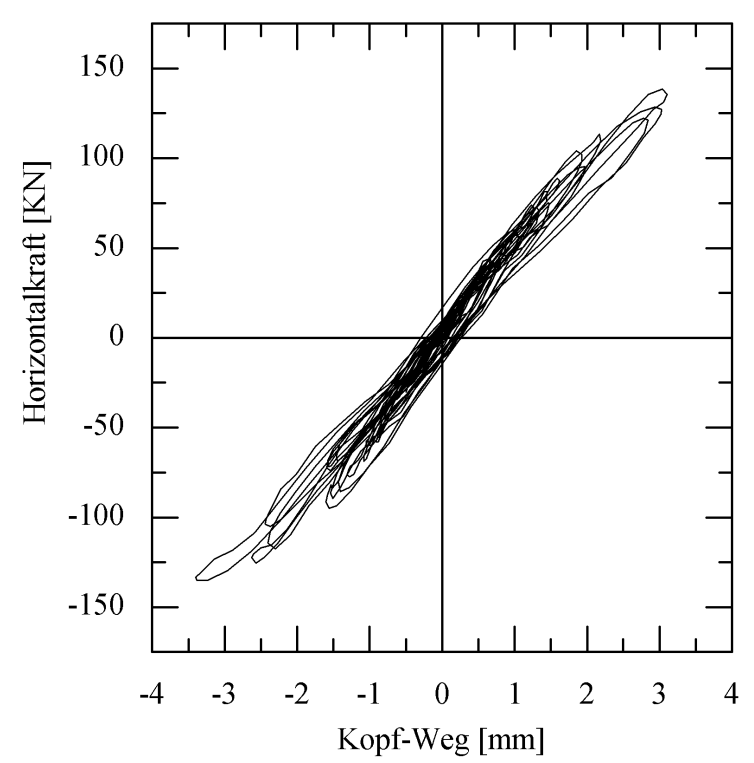

Abbildung A-36:Kraft-Verformungs-Beziehung Wand D, dritter Hauptversuch (Versuch D3)

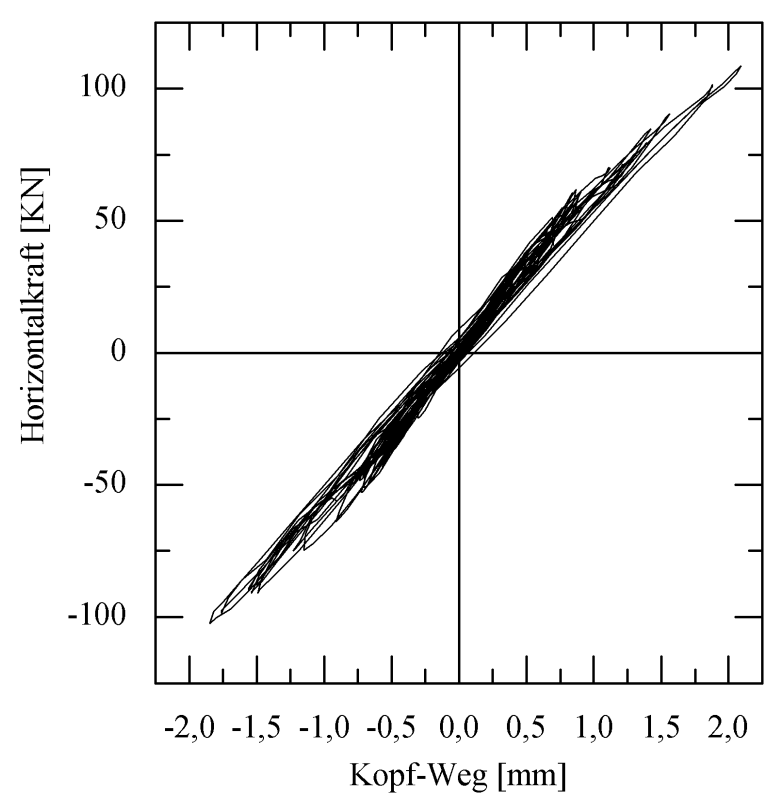

Abbildung A-35: Kraft-Verformungs-Beziehung Wand D, zweiter Hauptversuch (Versuch D2)

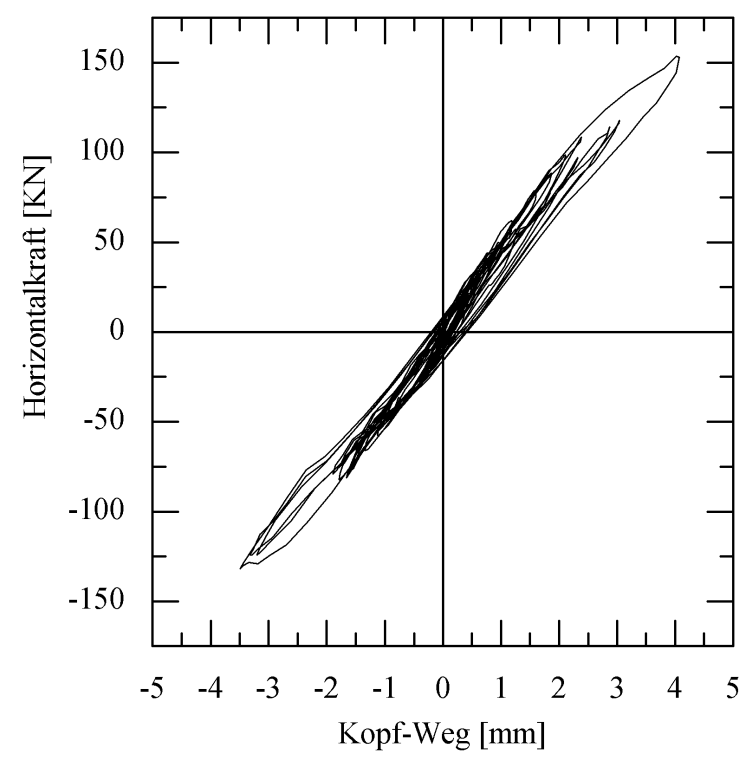

Abbildung A-37: Kraft-Verformungs-Beziehung Wand D, vierter Hauptversuch (Versuch D4) 


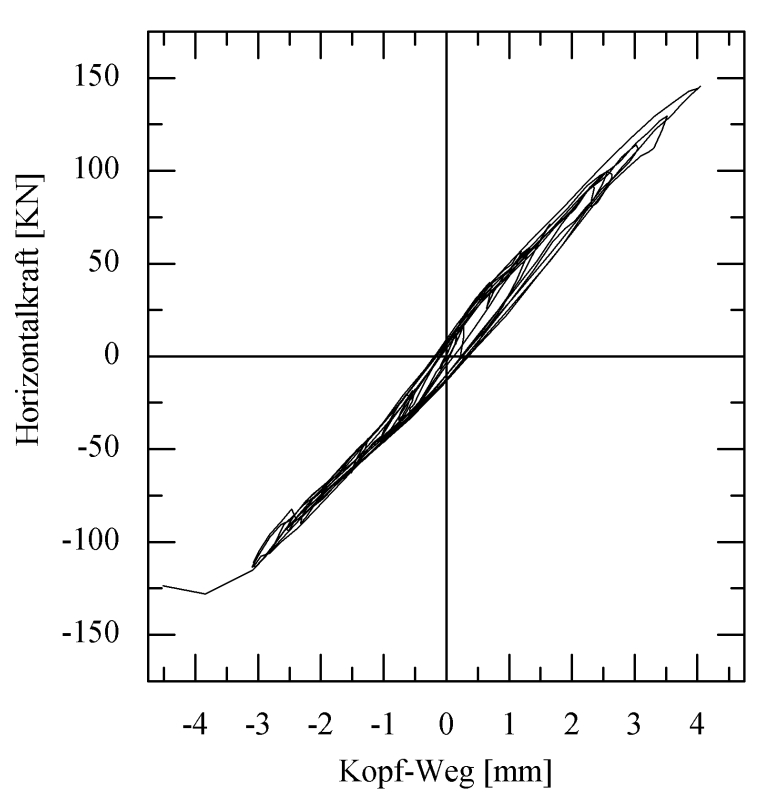

Abbildung A-38:Kraft-Verformungs-Beziehung Wand D, fünfter Hauptversuch (Versuch D5)

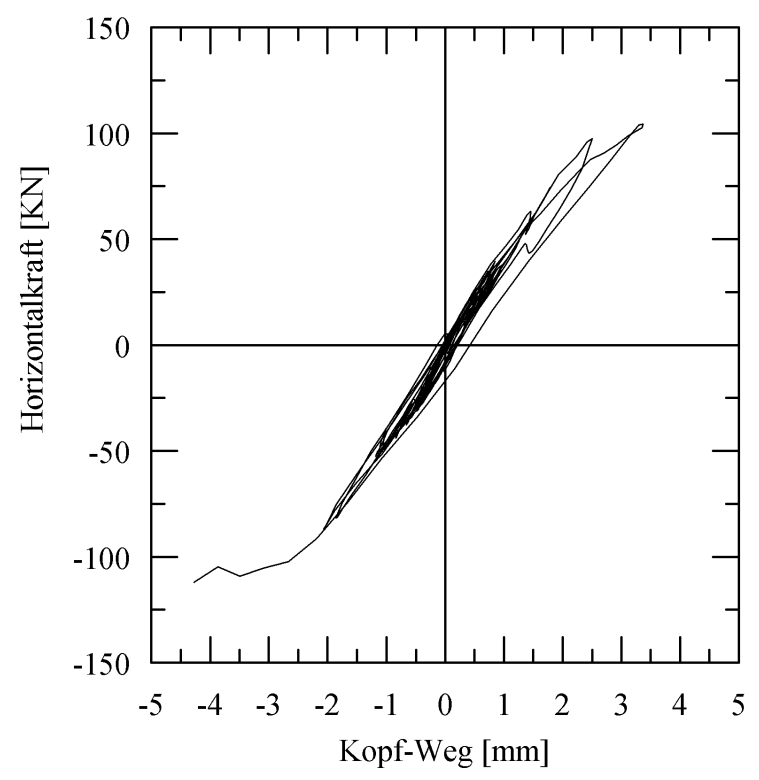

Abbildung A-40:Kraft-Verformungs-Beziehung Wand E, zweiter Hauptversuch (Versuch E2)

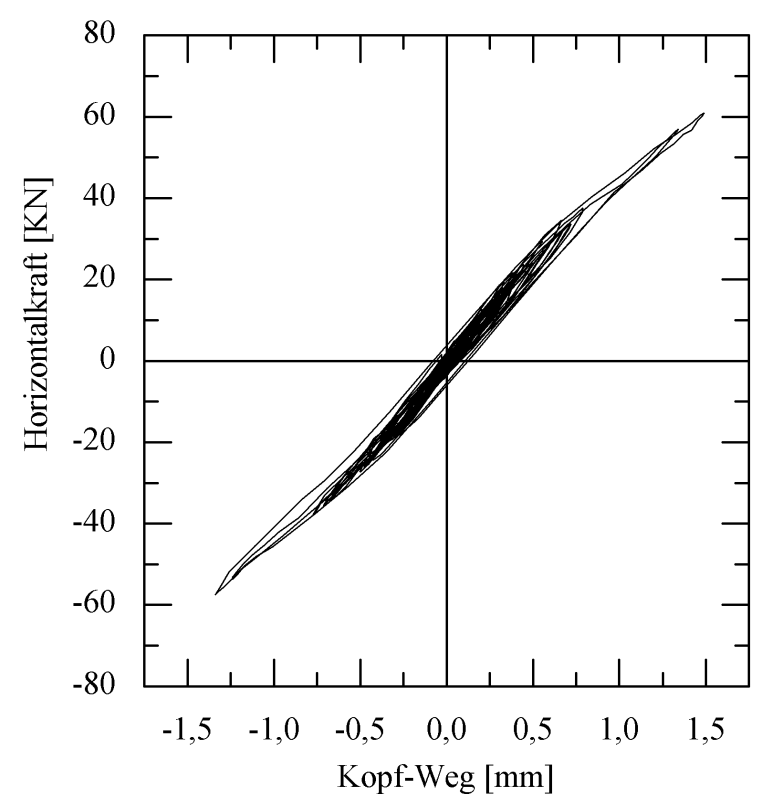

Abbildung A-39: Kraft-Verformungs-Beziehung Wand E, erster Hauptversuch (Versuch E1)

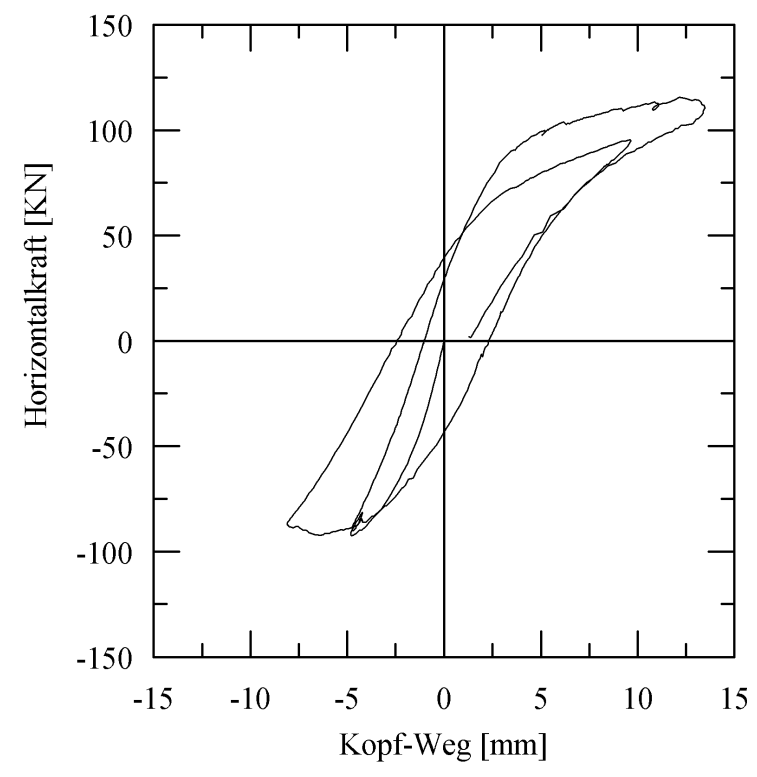

Abbildung A-41: Kraft-Verformungs-Beziehung Wand E, Nachversuch mit $N \equiv \mathbf{2 7 0} \mathrm{kN}$ und $M_{K o p f} \equiv \mathbf{0} \mathrm{kNm}$ 


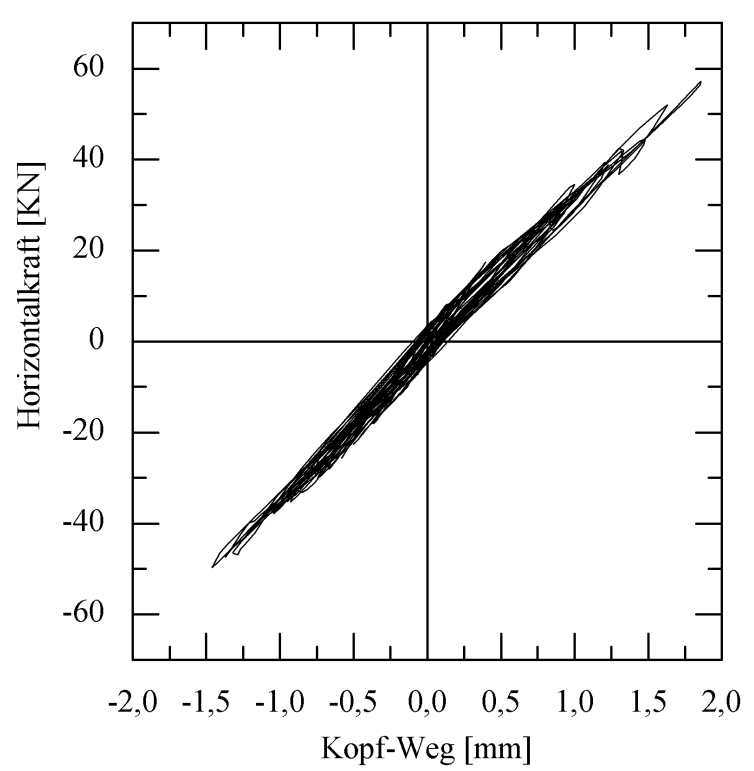

Abbildung A-42:Kraft-Verformungs-Beziehung Wand F, erster Hauptversuch (Versuch F1)

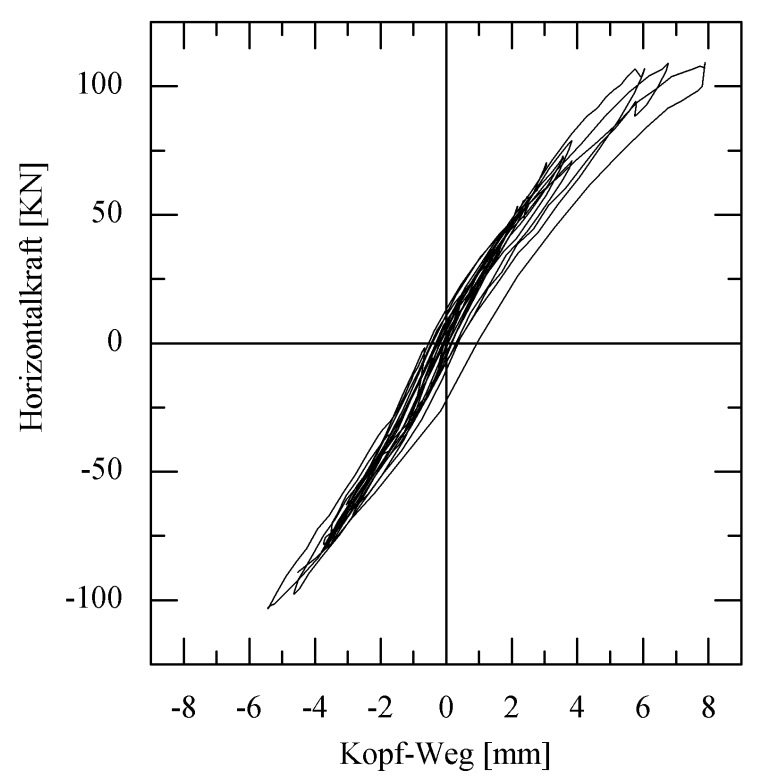

Abbildung A-44:Kraft-Verformungs-Beziehung Wand F, dritter Hauptversuch (Versuch F3)

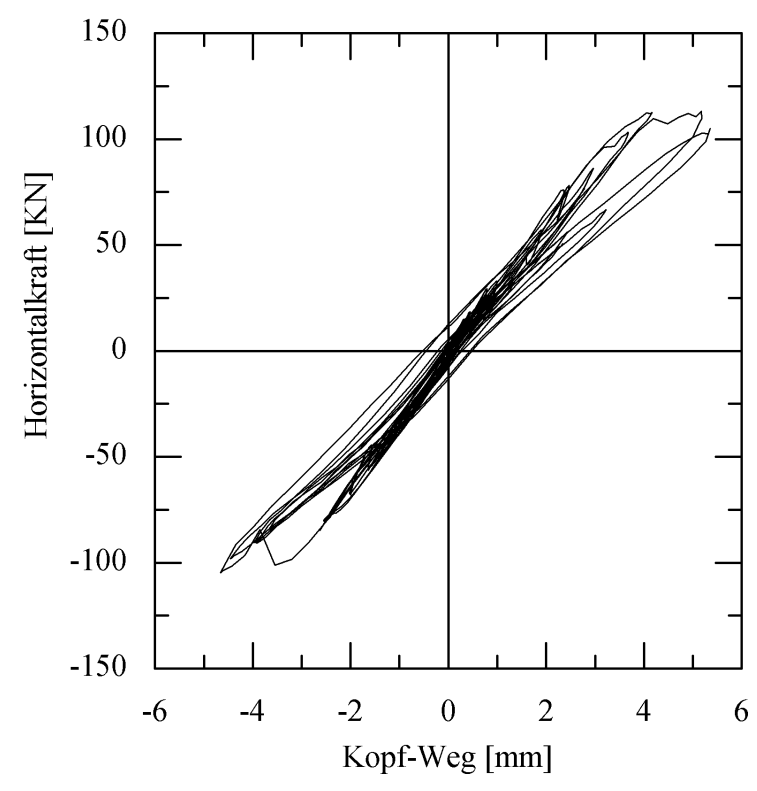

Abbildung A-43: Kraft-Verformungs-Beziehung Wand F, zweiter Hauptversuch (Versuch F2)

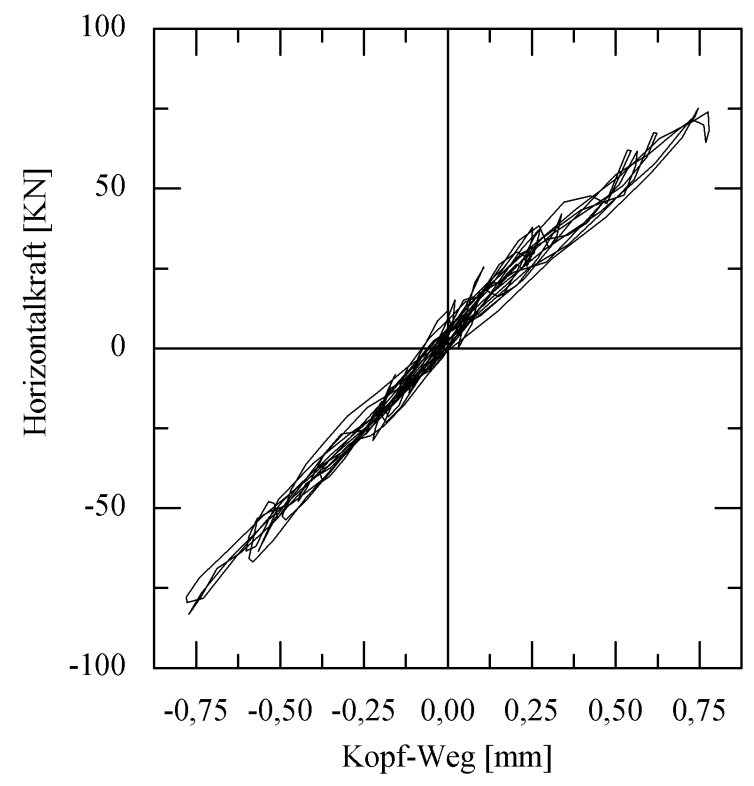

Abbildung A-45: Kraft-Verformungs-Beziehung Wand KS1, erster Hauptversuch (Versuch KS1-1) 


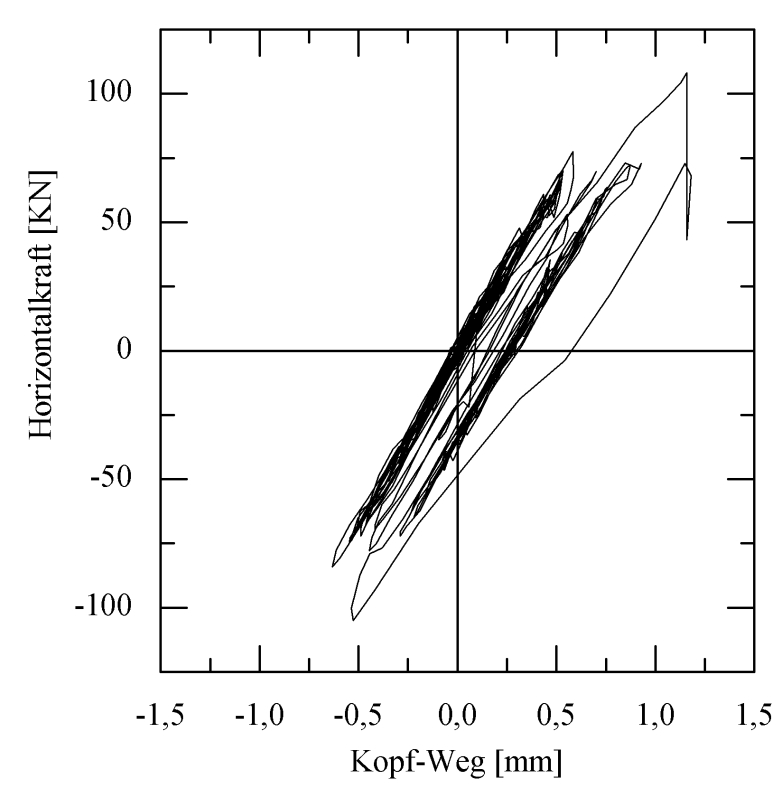

Abbildung A-46:Kraft-Verformungs-Beziehung Wand KS1, zweiter Hauptversuch (Versuch KS1-2)

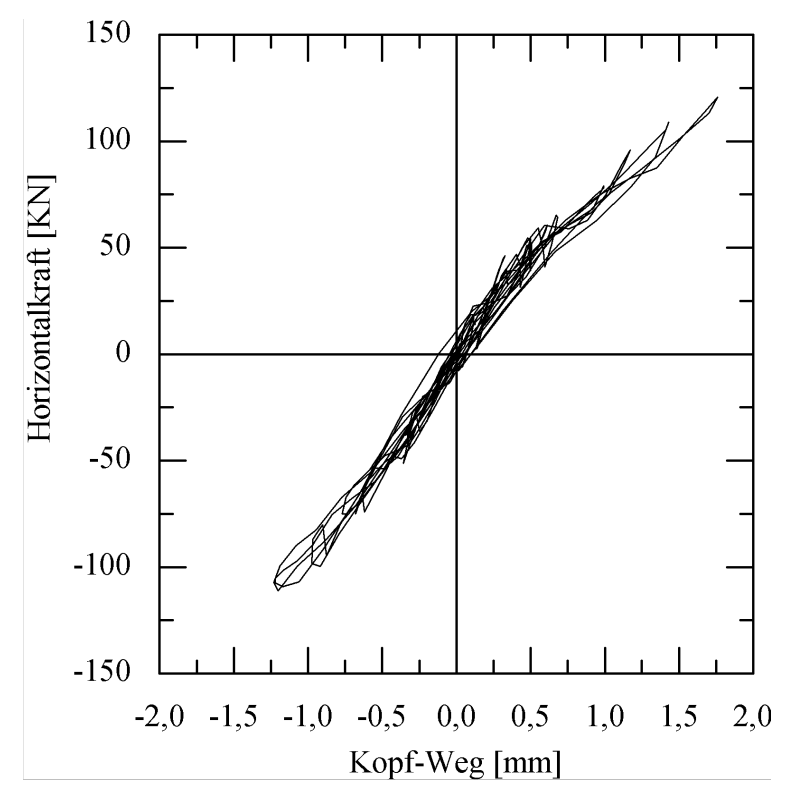

Abbildung A-48:Kraft-Verformungs-Beziehung Wand KS1, vierter Hauptversuch (Versuch KS1-4)

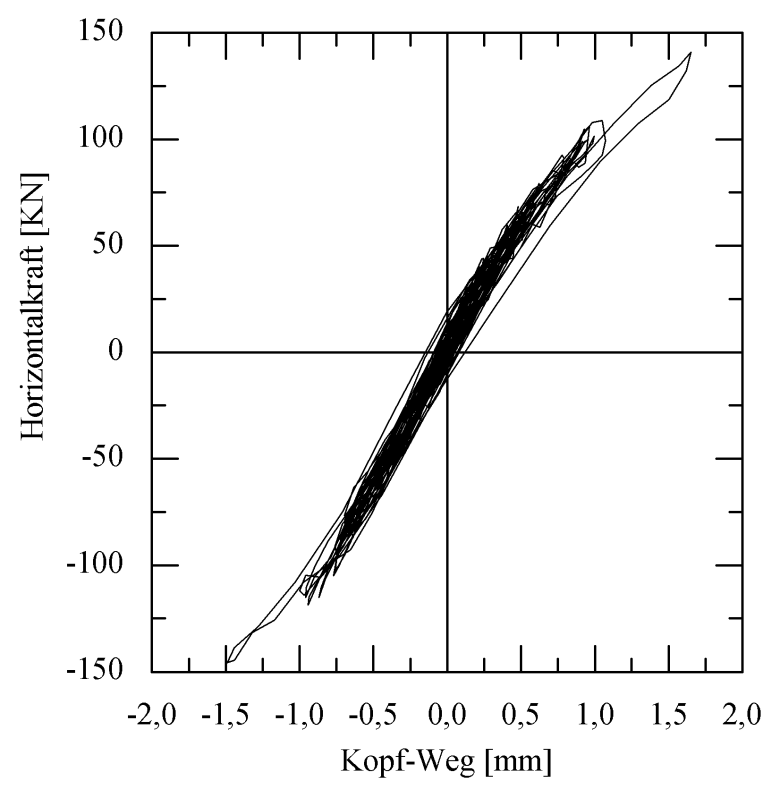

Abbildung A-47: Kraft-Verformungs-Beziehung Wand KS1, dritter Hauptversuch (Versuch KS1-3)

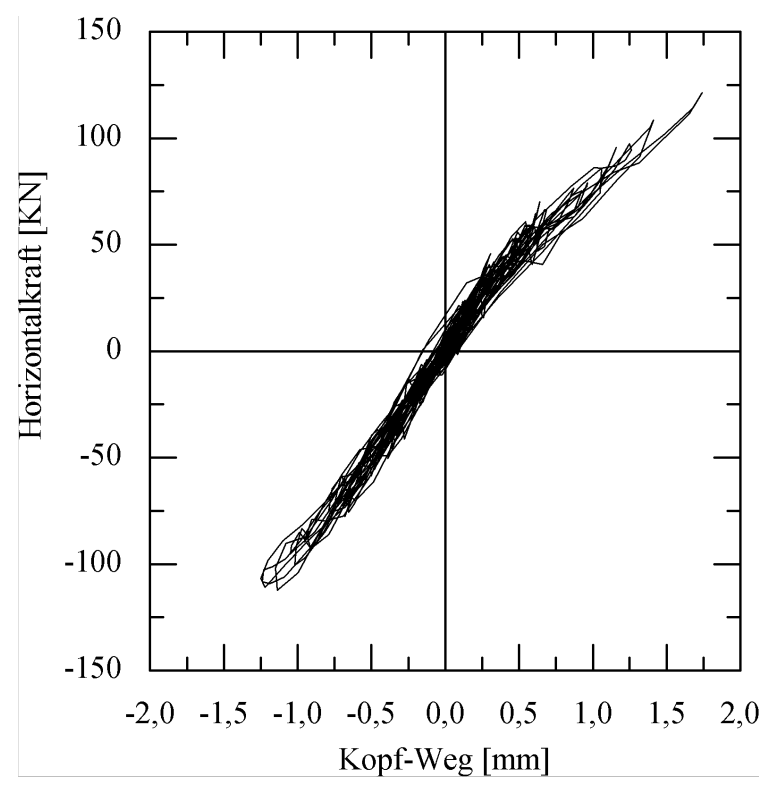

Abbildung A-49: Kraft-Verformungs-Beziehung Wand KS1, fünfter Hauptversuch (Versuch KS1-5) 


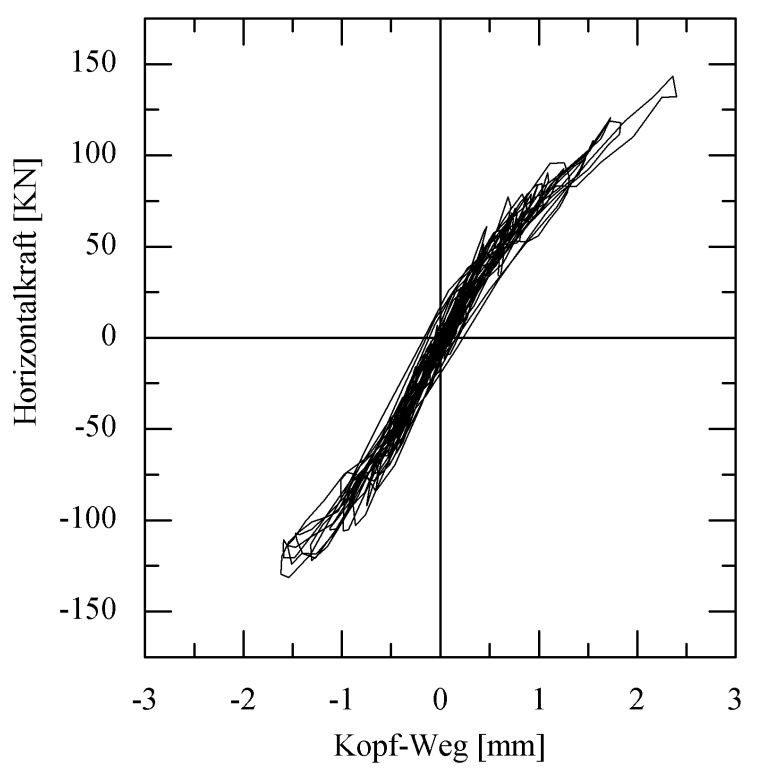

Abbildung A-50:Kraft-Verformungs-Beziehung Wand KS1, sechster Hauptversuch (Versuch KS1-6)

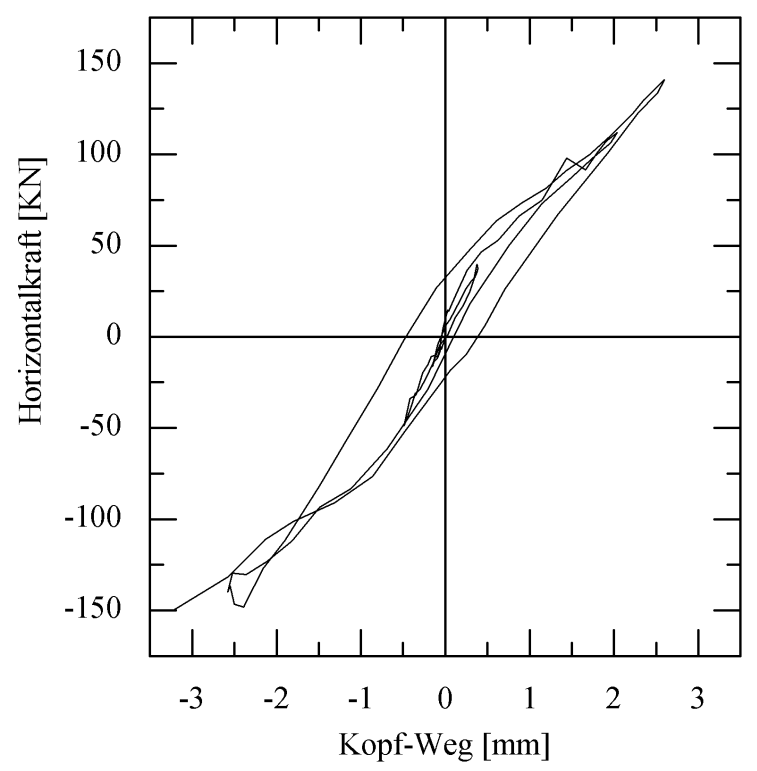

Abbildung A-52:Kraft-Verformungs-Beziehung Wand KS1, achter Hauptversuch (Versuch KS1-8)

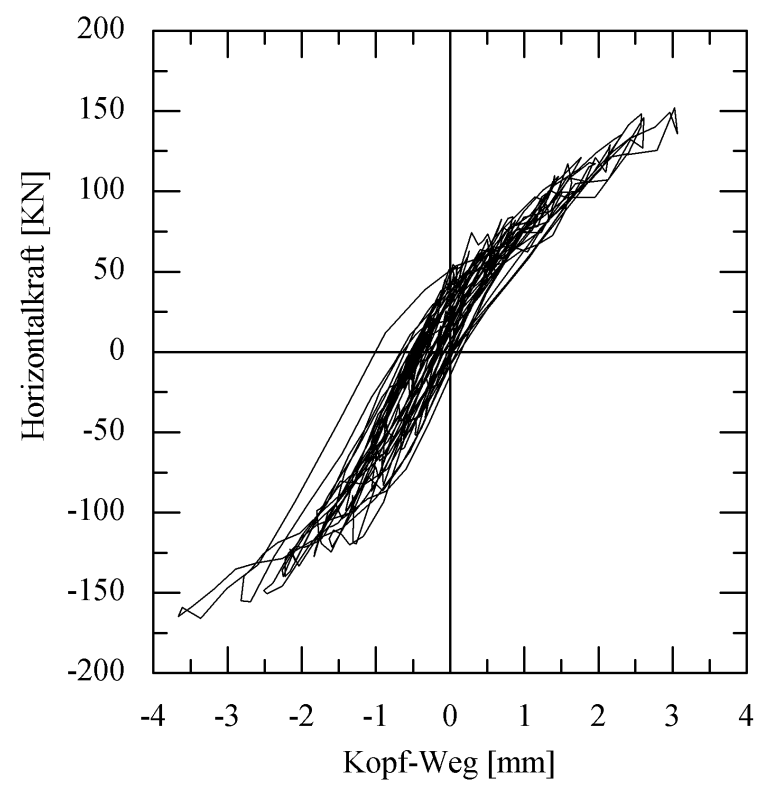

Abbildung A-51: Kraft-Verformungs-Beziehung Wand KS1, siebter Hauptversuch (Versuch KS1-7)

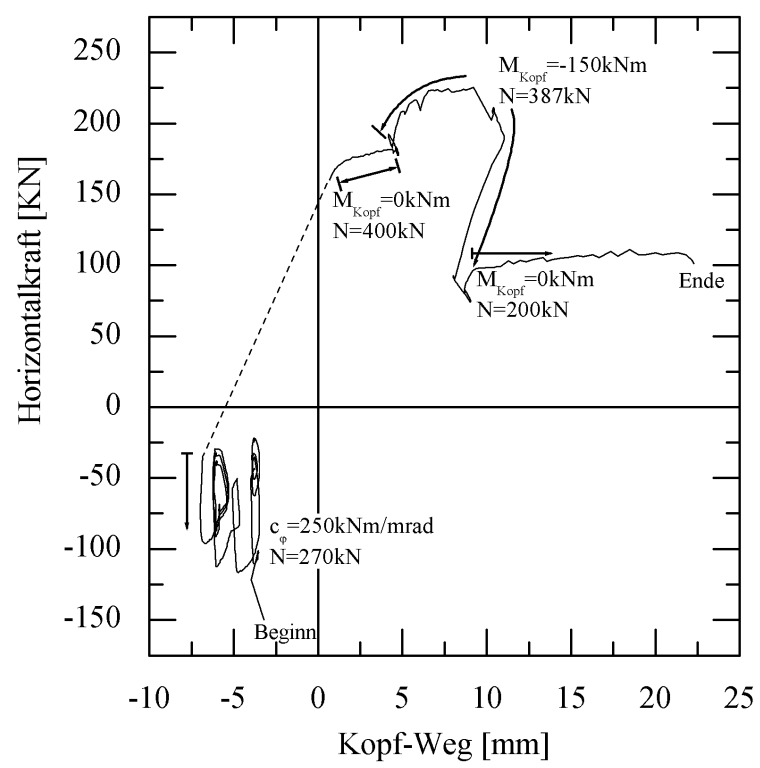

Abbildung A-53: Kraft-Verformungs-Beziehung Wand KS1, Nachversuch 


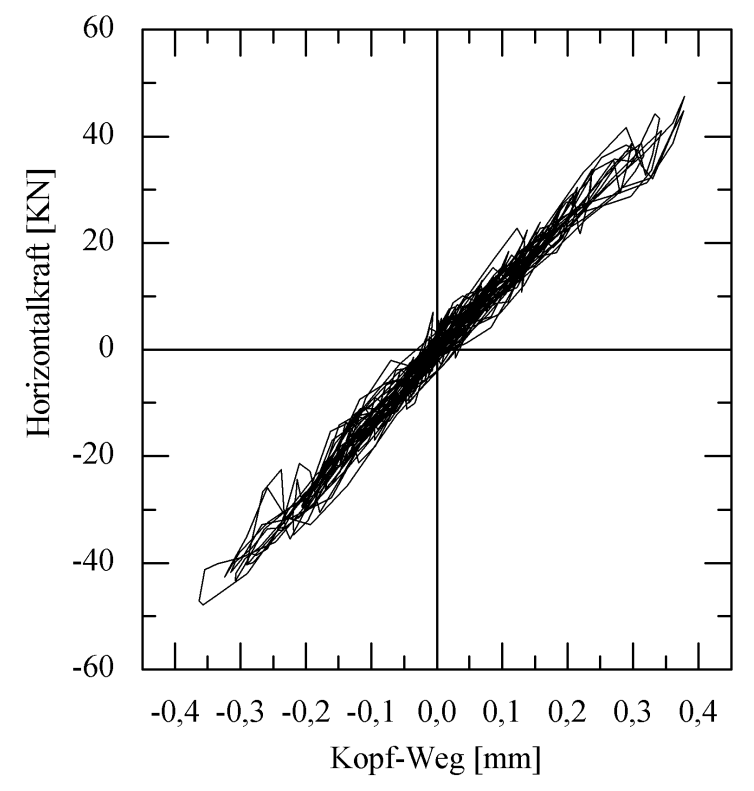

Abbildung A-54:Kraft-Verformungs-Beziehung Wand KS2, erster Hauptversuch (Versuch KS2-1)

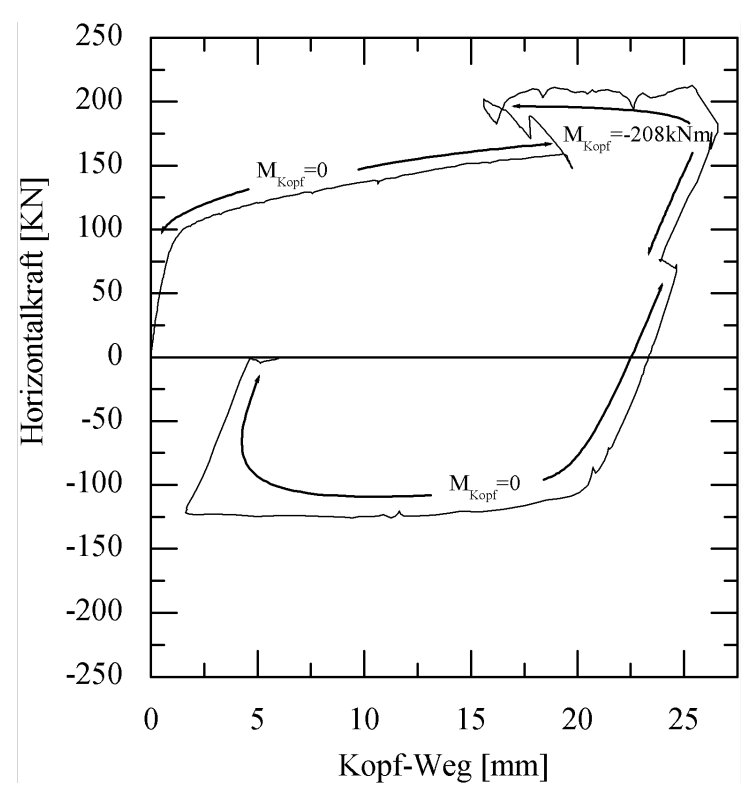

Abbildung A-56:Kraft-Verformungs-Beziehung Wand KS2, Nachversuch $N=270$ kN

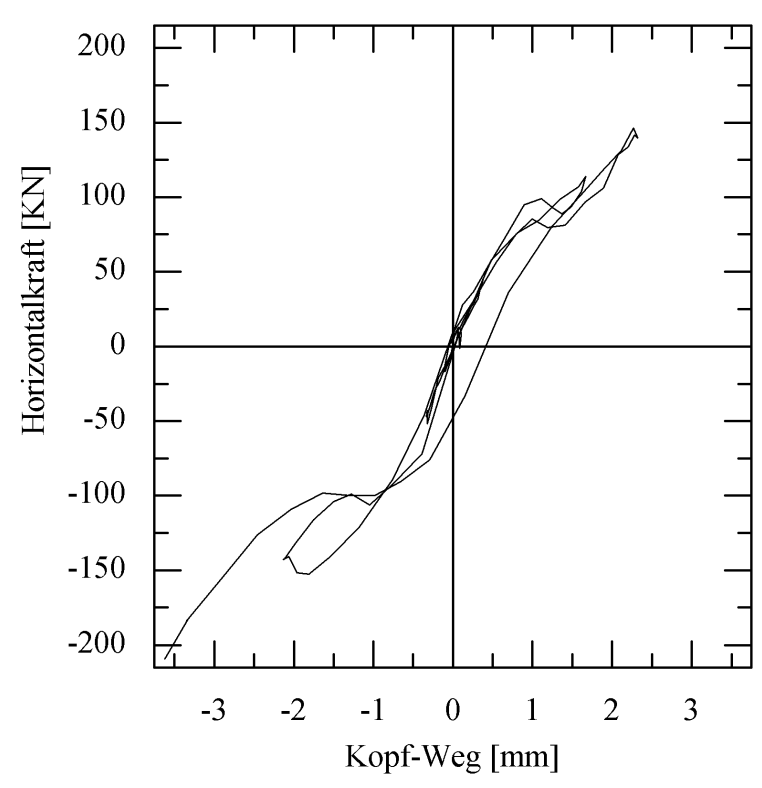

Abbildung A-55: Kraft-Verformungs-Beziehung Wand KS2, zweiter Hauptversuch (Versuch KS2-2)

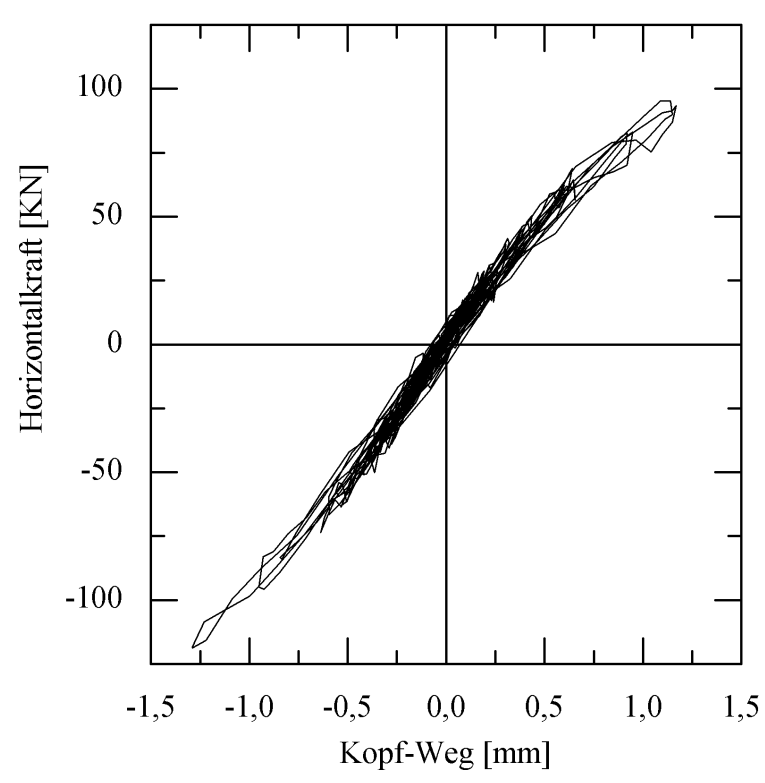

Abbildung A-57: Kraft-Verformungs-Beziehung Wand KS3, erster Hauptversuch (Versuch KS3-1) 


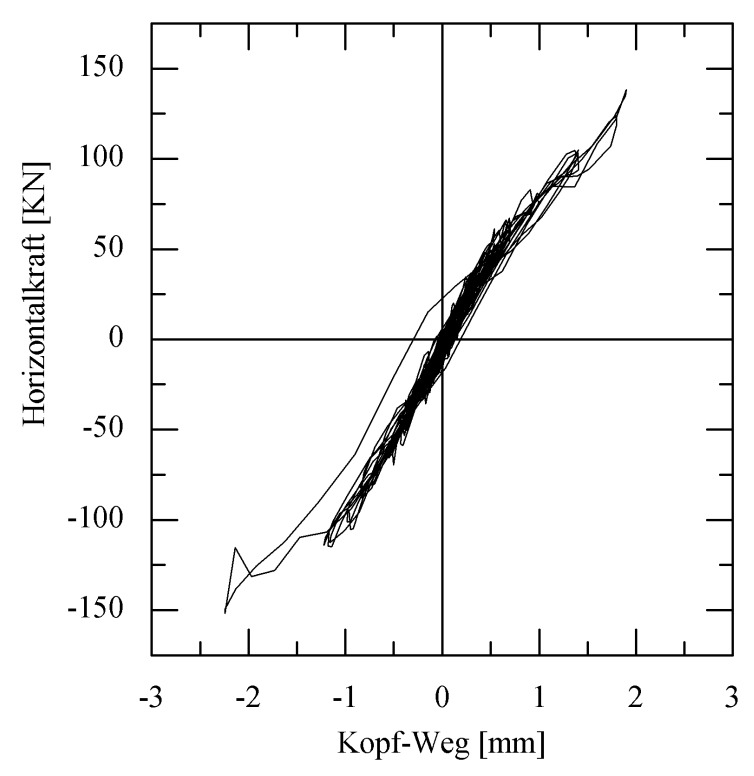

Abbildung A-58:Kraft-Verformungs-Beziehung Wand KS3, zweiter Hauptversuch (Versuch KS3-2)

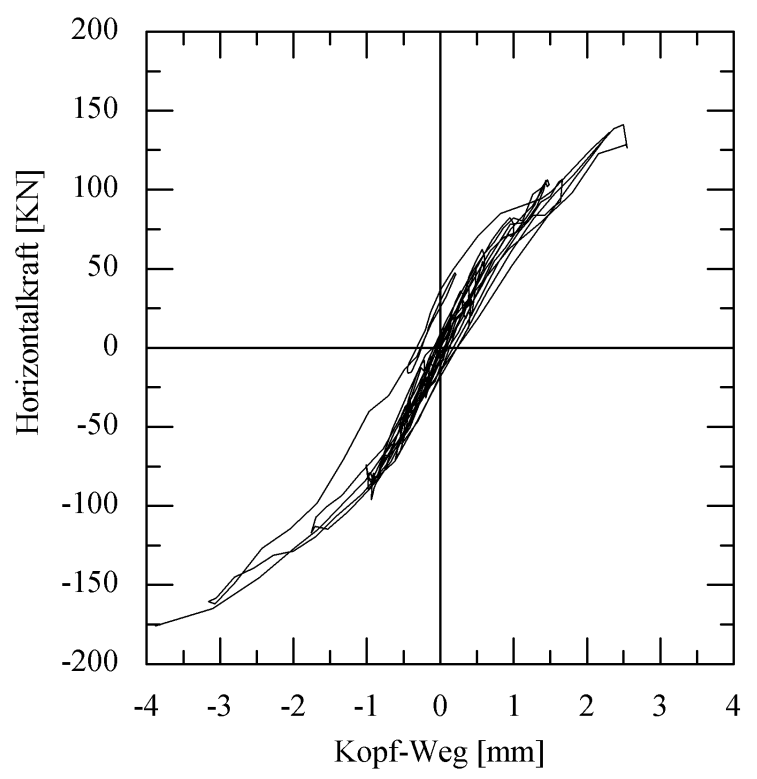

Abbildung A-60:Kraft-Verformungs-Beziehung Wand KS3, vierter Hauptversuch (Versuch KS3-4)

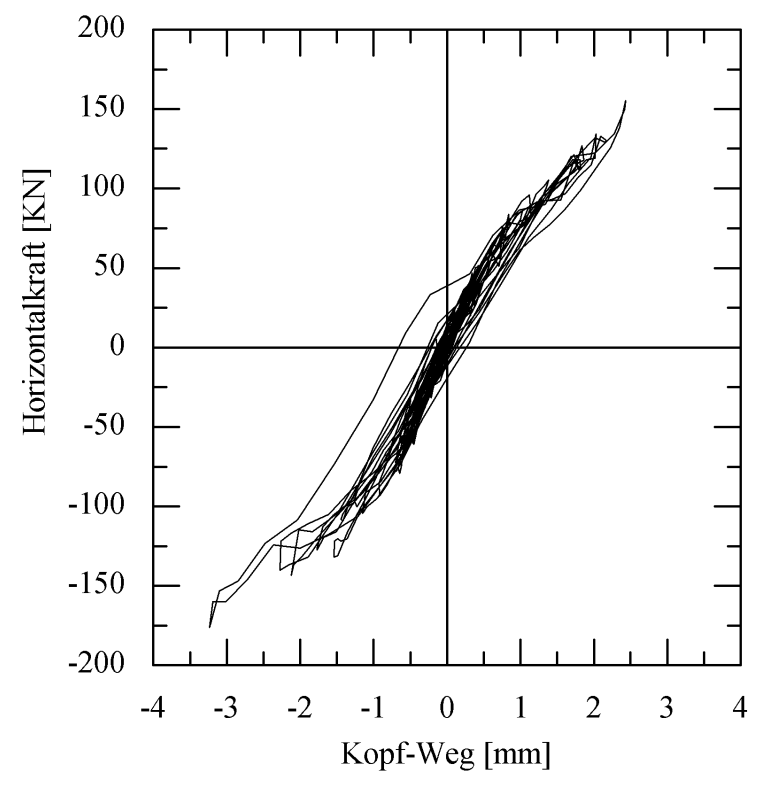

Abbildung A-59: Kraft-Verformungs-Beziehung Wand KS3, dritter Hauptversuch (Versuch KS3-3)

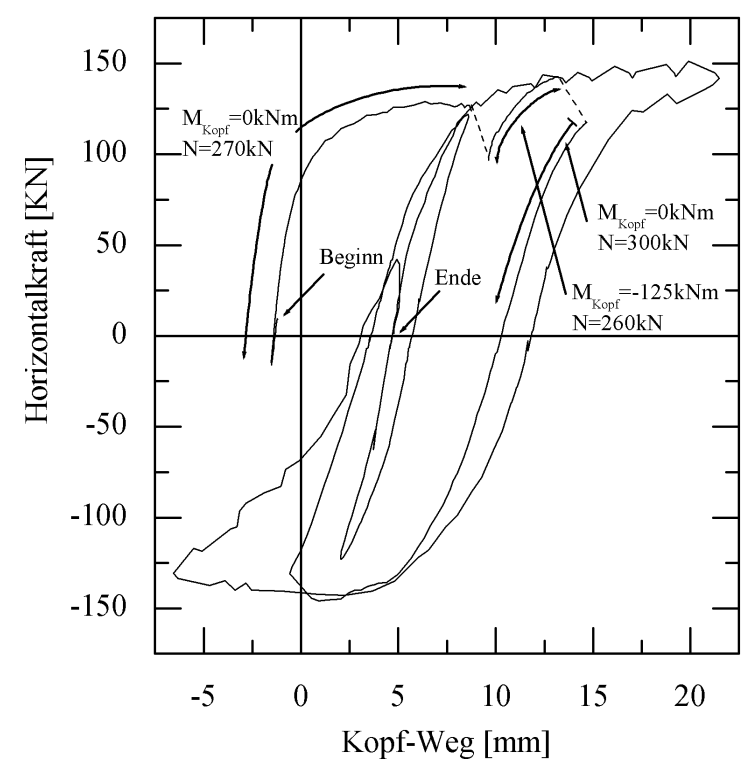

Abbildung A-61: Kraft-Verformungs-Beziehung Wand KS3, Nachversuch 


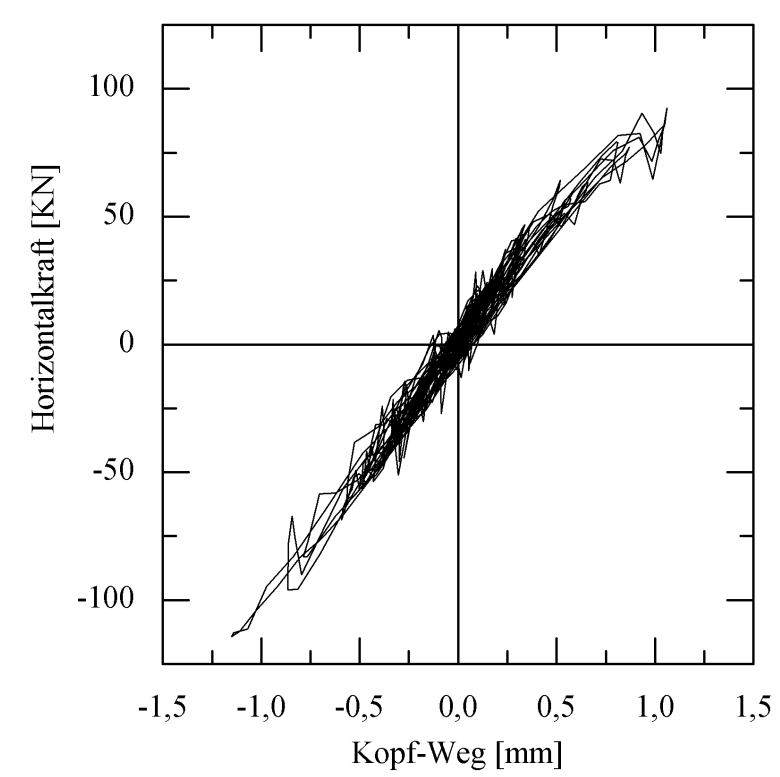

Abbildung A-62:Kraft-Verformungs-Beziehung Wand KS4, erster Hauptversuch (Versuch KS4-1)

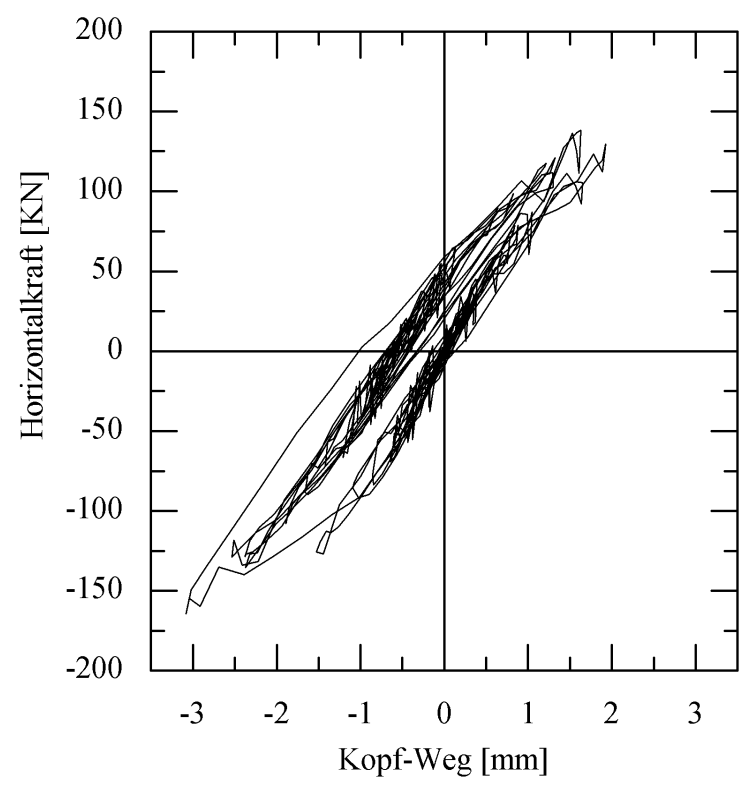

Abbildung A-64:Kraft-Verformungs-Beziehung Wand KS4, dritter Hauptversuch (Versuch KS4-3)

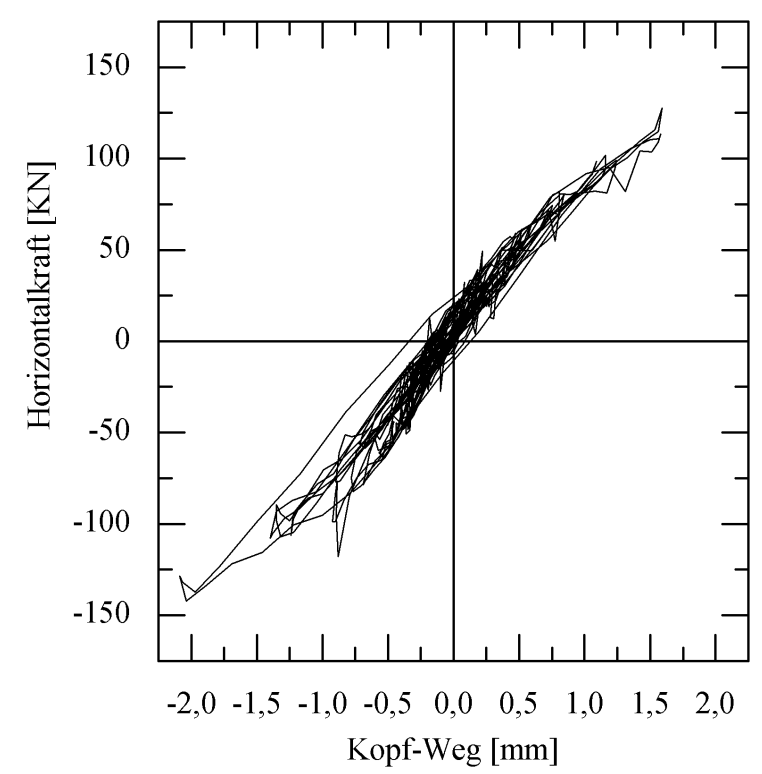

Abbildung A-63: Kraft-Verformungs-Beziehung Wand KS4, zweiter Hauptversuch (Versuch KS4-2)

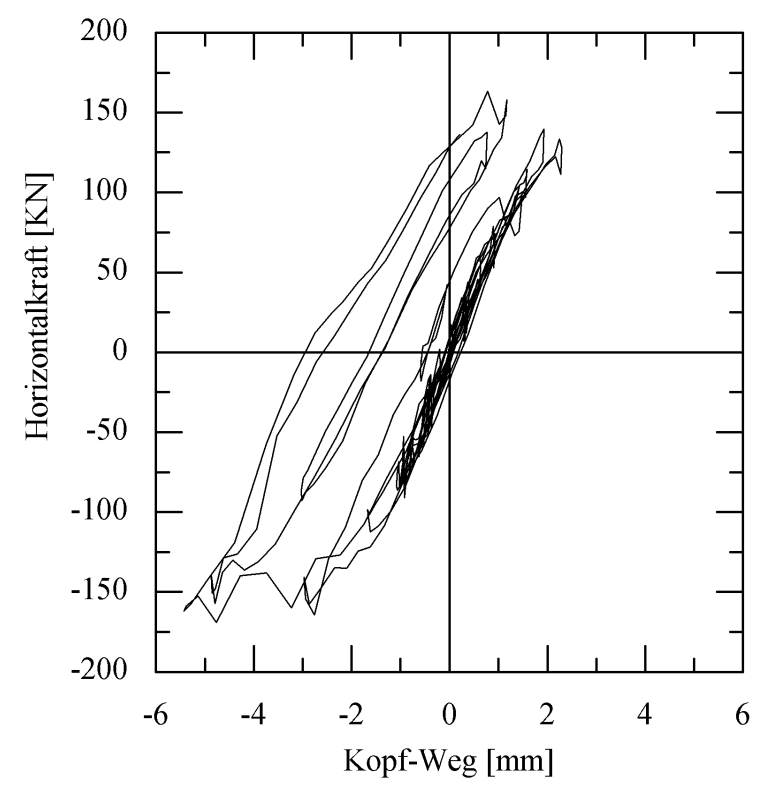

Abbildung A-65: Kraft-Verformungs-Beziehung Wand KS4, vierter Hauptversuch (Versuch KS4-4) 


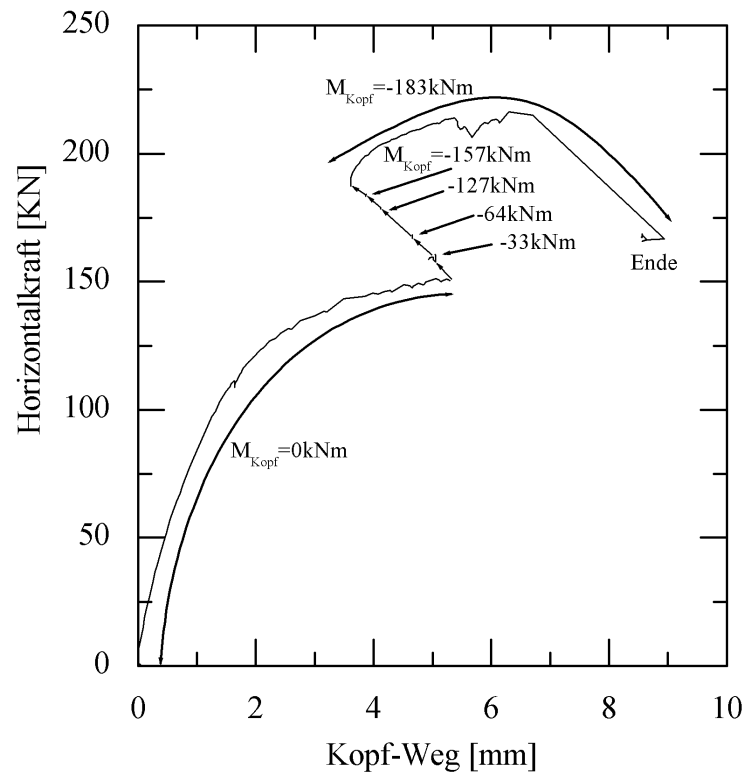

Abbildung A-66:Kraft-Verformungs-Beziehung Wand KS4, Nachversuch mit $N \equiv 360 \mathbf{k N}$ 
